WHOI-87--42

\title{
Midwater Fish Data Report for Warm-Core Gulf Stream Rings Cruises 1981-1982
}

\author{
by \\ James E. Craddock \\ Richard H. Backus \\ and \\ Mary Ann Daher
}

Woods Hole Oceanographic Institution

Woods Hole, Massachusetts 02543

October 1987

\section{Technical Report}

Funding was provided by the National Science Foundation under Grant Numbers OCE 80-17270 and OCE 86-20402.

Reproduction in whole or in part is permitted for any purpose of the United States Government. This report should be cited as:

Woods Hole Oceanog. Inst. Tech. Rept., WHOI-87-42.

Approved for publication; distribution unlimited.

Approved for Distribution:

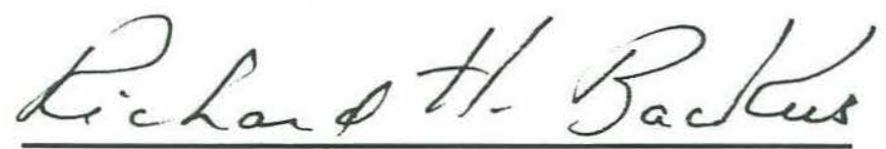

Richard H. Backus, Chairman

Department of Biology 

Introduction ........................... Acknowl edgements ........................ iv Literature cited ................. v Station data, Atlantis II cruise $110 \ldots \ldots$. . . . . . . 1 Station data, 0ceanus cruise $118 \ldots \ldots 2$ Station data, 0ceanus cruise $121 \ldots \ldots . \ldots 3$ Station data, 0ceanus cruise $125 \ldots \ldots 4$ Station data, Knorr cruise $98 \ldots \ldots \ldots$ Collection data, Atlantis II cruise $110 \ldots \ldots 7$ Collection data, 0ceanus cruise $118 \ldots . . . . . . . .88$

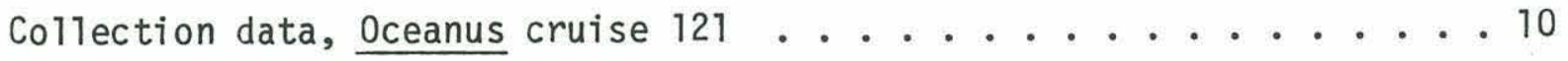
Collection data, 0ceanus cruise $125 \ldots \ldots 12$ Collection data, Knorr cruise $98 \ldots \ldots 15$ List of species ........................ 17 Depths $(m)$ at which nets were closed and opened ....... 23 Temperature $\left({ }^{\circ} \mathrm{C}\right)$ at depths where nets were closed and opened . . . 24 Salinity $(\%)$ at depths where nets were closed and opened . . . 2 25 Abundance of fishes (specimens $\left./ 10,000 \mathrm{~m}^{3}\right) \ldots 26$ Volume of fishes $\left(\mathrm{ml} / 10,000 \mathrm{~m}^{3}\right) \ldots \ldots 27$ Abundance and volume of certain families (alphabetically) .....28 Abundance and volume of certain species (alphabetically) ...... 40 



\section{INTRODUCTION}

This data report is for midwater fishes collected during the multidisciplinary Warm-Core Rings Program in 1981 and 1982.

Stations were made in and near three warm-core rings on five cruises within a period of 14 months. On Atlantis II cruise 110 (September-0ctober 1981) six stations were made in and around ring 81-D (age two months). Stations were made in the vicinity of ring 82-B on three cruises in 1982--twelve stations during Oceanus 118 (Apri1) when the ring was two months 01d, 15 stations during Oceanus 121 (June) at age four months, and 19 stations during Oceanus 125 (August) at age 5.5 months. Finally, twelve stations were made in and near meander/ring 82-H (age 0) during Knorr 98 in

September/0ctober 1982 (Tables 1-10).

The collections were made with a new midwater trawl - the MOCNESS-20 (MOC-20) (Wiebe et al., 1985), a scaled-up version of the MOCNESS-1 (an apparatus for collecting zooplankton; Wiebe et al., 1976) and successor to the MOCNESS-10 (1ike the MOC-20, a midwater traw1). (The number forming the distinctive part of the name of these nets is equal to the area of the projected mouth in square meters when the apparatus is in a common fishing attitude.) The $\mathrm{MOC}-20$ consists of a set of $3-\mathrm{mm}$ mesh rectangular nets that can be opened and closed by command from the surface via a signal-conducting towing warp. Apparatus attached to the net frame measures and transmits depth, temperature, conductivity, flow, and net-frame angle to the towing ship's laboratory. Flow (net speed), vertical velocity, and net-frame angle allow computation of the water volume filtered. On the WCR cruises a set of five or six nets was used. One net (not used for quantitative analyses) was fished down to $1000 \mathrm{~m}$, then closed and a second net opened. The second and successive nets were closed and opened sequentially at intervals as the apparatus was brought back to the surface. A surface-to-surface cycle with the gear is referred to as a station, the contents of a single net as a collection. In addition to being described by latitude and longitude, stations are located in the same radial coordinate system used to composite the warm-core rings physical data, that is, by distance and bearing from the moving ring center.

A typical 1000-m station (up nets) lasted about two and a half hours and filtered about $150,000 \mathrm{~m}^{3}$. Individual nets filtered between 30,000 and $40,000 \mathrm{~m}^{3}$. The MOC-20 was used at 64 stations during the 1981-1984 program; at 57 of these stations, complete 1000-m profiles were successfully made (two profiles were to $1250 \mathrm{~m})$ and a total of 312 collections secured. There were 21 stations in the cores of rings, 15 in the outer, high velocity regions (HVR) of the rings, 17 in the Slope Water, and two each in the Gulf Stream and Northern Sargasso Sea.

When sampled, ring 81-D was about three months old and was elliptically shaped (about 190 by $144 \mathrm{~km}$ ); it was interacting and exchanging waters with the Gulf Stream (Joyce et al., 1983; Joyce, 1984; Joyce et al., 1984). 
The following history of warm-core ring 82-B is taken from Evans et al. (1985), Joyce and Kennelly (1985), Schmitt and 01 son (1985), Boyd et al. (1986), and Conte et al. (1986). Ring 82-B was formed in mid-February 1982 and was centered at $38.5^{\circ} \mathrm{N}, 70^{\circ} \mathrm{W}$. Between then and mid June, while moving to the southwest about $450 \mathrm{~km}$, it changed $1 \mathrm{ittle}$ physically. Its diameter shrank from 160 to $130 \mathrm{~km}$; its core cooled from $17.7^{\circ} \mathrm{C}$ to $15.7^{\circ}$ but extended about as deep as before. By August, after interactions with shelf water, the Slope Water, and the Gulf Stream, its diameter had been reduced to only $45 \mathrm{~km}$. Waters in both the upper $100 \mathrm{~m}$ and deeper than $500 \mathrm{~m}$ had been completely exchanged. Finally, 82-B was swept away by the Gulf Stream in September at age seven months.

When first sampled by us, $82-\mathrm{H}$ was actually a large (190 km diameter) Gulf Stream meander; by October 9 the ring was separated from the stream, was about $220 \mathrm{~km}$ in diameter, and was moving to the WSW a about $10 \mathrm{~km} /$ day (Stalcup et a1., 1986).

A total of 142,630 specimens have been identified, most of them to species, some (Bathylagus, Bregmaceros) to genus only, a few (searsiids, paralepidids) to family only. At least 234 species are represented (Table 11).

Table 12 gives depths where nets were closed and opened (depth intervals fished by each net) and is thus a key for the remaining tables. Tables 13 and 14 give temperature and salinity limits, respectively, for the collections.

The remaining tables concern the abundance and biomass of fishes, and are in two parts. The upper section gives values for each collection (each depth interval) and corresponds with Table 12. Nighttime collections are underlined; daytime ones, not. The lower section gives integrated values for each station to a depth of $1000 \mathrm{~m}$ (1 specimen/10,000 $\mathrm{m}^{3}=1$ specimen $/ 10 \mathrm{~m}^{2}$ of sea surface for the $1000 \mathrm{~m}$ water column).

Volumes, measured on a lot-by-lot basis, are wet weight displacements of specimens in $70 \%$ ethyl alcohol (specimens had initially been preserved in $10 \%$ formaldehyde and transferred to $35 \%$ and then $70 \%$ alcohol).

\section{ACKNOWLEDGEMENTS}

We sincerely thank Valerie Barber, E. Bertelsen, Don Bourne, Steve Boyd, Bob Gibbs, Karsten Hartel, Charles Karnella, A1 Morton, John Pirie, Peter Wiebe, and Joe Wroblewski for all manner of help. The work was supported by NSF Grants OCE 80-17270 and 20402 


\section{LITERATURE CITED}

Boyd, S. H., P. H. Wiebe, R. H. Backus, J. E. Craddock, and M. A. Daher. 1986. Biomass of the micronekton in Gulf Stream ring 82-B and environs: changes with time. Deep-sea Res., 33:1885-1905.

Conte, M. H., J. K. B. Bishop, and R. H. Backus. 1986. 12-kHz nonmigratory deep scattering layers of Sargasso Sea origin in warm-core rings. Deep-sea Res., 33:1869-1884.

Evans, R. H., K. S. Baker, 0. B. Brown, and R. C. Smith. 1985. Chronology of warm-core ring 82-B. J. Geophys. Res., 90:8803-8811.

Joyce, T. M. 1984. Velocity and hydrographic structure of a Gulf Stream warm-core ring. J. Physical Oceanogr., 14:936-947.

Joyce, T., R. Backus, K. Baker, P. Blackwelder, 0. Brown, T. Cowles, R. Evans, G. Fryxe11, D. Mountain, D. 01 son, R. Schlitz, R. Schmitt, P. Smith, R. Smith, and P. Wiebe. 1984. Rapid evolution of a Gulf Stream warm-core ring. Nature, 308 (5962), 837-840.

Joyce, T. M., and M. A. Kennelly. 1985. Upper ocean velocity structure of Gulf Stream warm-core ring 82-B. J. Geophys. Res., 90:8839-8844.

Joyce, T. M., R. W. Schmitt, and M. C. Stalcup. 1983. Influence of the Gulf Stream on the short term evolution of a warm-core ring. Aust. J. Mar. Fresh Water Res., 34:515-524.

Schmitt, R. W., and D. B. 01son. 1985. Wintertime convection in warm core rings: thermocline ventilation and the formation of mesoscale eddies. J. Geophys. Res., 90:8823-8837.

Stalcup, M. C., T. M. Joyce, R. L. Barbour, and J. A. Dunworth. 1986. Hydrographic data from R/V Endeavor cruise \#90. Woods Hole Oceanogr. Inst. Tech. Rep. WHOI-86-15.

Wiebe, P. H., K. H. Burt, S. H. Boyd and A. W. Morton. 1976. A multiple opening/closing net and environmental sensing system for sampling zooplankton. J. Mar. Res., 34(3): 313-326.

Wiebe, P. H., A. W. Morton, A. M. Bradley, R. H. Backus, J. E. Craddock, V. A. Barber, T. J. Cowles, and G. R. Flierl. 1985. New developments in the MOCNESS, an apparatus for sampling zooplankton and micronekton. Mar. Biol., 87:313-323. 
Table 1. Summary of MOCNESS-20 stations, Atlantis II Cruise 110.

\begin{tabular}{|c|c|c|c|c|c|c|}
\hline MOCNESS & N & on $w$ & $\begin{array}{c}\text { Date } \\
\text { Sept-Oct } 1981\end{array}$ & Time $(+4)$ & $\begin{array}{l}\text { Depth } \\
\text { to } 10^{\circ} \mathrm{C}\end{array}$ & Area \\
\hline $20-1$ & $\begin{array}{l}40^{\circ} 14 \\
40^{\circ} 07\end{array}$ & $\begin{array}{l}63^{\circ} 28^{\prime} \\
63^{\circ} 34^{\prime}\end{array}$ & $23-24$ & (2144-) 0009-0242 & 615 & Ring $81-D$, Core \\
\hline $20-2$ & $\begin{array}{l}41^{\circ} 05^{\circ} \\
41^{\circ} 09^{\prime}\end{array}$ & $\begin{array}{l}63^{\circ} 33^{\prime} \\
63^{\circ} 34^{\prime}\end{array}$ & 28 & $(0006-) 0118-0320$ & 330 & Ring $81-D$, Edge \\
\hline $20-3$ & $\begin{array}{l}40^{\circ} 57^{\prime} \\
41^{\circ} 01^{\prime}\end{array}$ & $\begin{array}{l}63^{\circ} 35^{\prime} \\
63^{\circ} 31^{\prime}\end{array}$ & 28 & $(1408-) 1530-1736$ & 343 & Ring $81-D$, Edge \\
\hline $20-4$ & $\begin{array}{l}40^{\circ} 08^{\prime} \\
40^{\circ} 14^{\prime}\end{array}$ & $\begin{array}{l}66^{\circ} 00^{\prime} \\
65^{\circ} 57^{\prime}\end{array}$ & 3 & (0735-)0835-1118 & 240 & Slope Water \\
\hline $20-5$ & $\begin{array}{l}40^{\circ} 15^{\prime} \\
40^{\circ} 23^{\prime}\end{array}$ & $\begin{array}{l}66^{\circ} 07^{\prime} \\
66^{\circ} 08^{\prime}\end{array}$ & $3-4$ & $(2218-) 0015-0243$ & 240 & Slope Water \\
\hline $20-6$ & $\begin{array}{l}39^{\circ} 54^{\prime} \\
39^{\circ} 49^{\prime}\end{array}$ & $\begin{array}{l}67^{\circ} 42^{\prime} \\
67^{\circ} 36^{\prime}\end{array}$ & $5-6$ & $(2323-) 0038-0302$ & 240 & Slope Water \\
\hline
\end{tabular}


2

Table 2. Summary of MOCNESS-20 stations, Oceanus Cruise 118.

\begin{tabular}{|c|c|c|c|c|c|c|}
\hline MOCNESS & Posi & on & $\begin{array}{c}\text { Date } \\
\text { Apri1-May } 1982\end{array}$ & Time $(+5)$ & $\begin{array}{l}\text { Depth } \\
\text { to } 10^{\circ} \mathrm{C}\end{array}$ & Area \\
\hline $20-7$ & $\begin{array}{l}39^{\circ} 13.4^{\prime} \\
39^{\circ} 16.2^{\prime}\end{array}$ & $\begin{array}{l}69^{\circ} 35.2^{\prime} \\
69^{\circ} 34.5^{\prime}\end{array}$ & 19 & $(1354-) 1501-1607$ & 252 & Slope Water \\
\hline $20-8$ & $\begin{array}{l}39^{\circ} 17.3^{\prime} \\
39^{\circ} 21.3^{\prime}\end{array}$ & $\begin{array}{l}69^{\circ} 32.9^{\prime} \\
69^{\circ} 30.0^{\prime}\end{array}$ & 20 & $(0112-) 0250-0421$ & 224 & Slope Water \\
\hline $20-9$ & $\begin{array}{l}39^{\circ} 09.2^{\prime} \\
39^{\circ} 11.3^{\prime}\end{array}$ & $\begin{array}{l}69^{\circ} 41.7^{\prime} \\
69^{\circ} 38.9^{\prime}\end{array}$ & 21 & (1209-)1345-1510 & 234 & Slope Water \\
\hline $20-10$ & $\begin{array}{l}38^{\circ} 57.9^{\prime} \\
39^{\circ} 00.7^{\prime}\end{array}$ & $\begin{array}{l}71^{\circ} 15.8^{\prime} \\
71^{\circ} 15.7^{\prime}\end{array}$ & 22 & (0034-)0229-0357 & 568 & Ring $82-B$, Core \\
\hline $20-11$ & $\begin{array}{l}38^{\circ} 54.6^{\prime} \\
38^{\circ} 50.5^{\prime}\end{array}$ & $\begin{array}{l}71^{\circ} 36.8^{\prime} \\
71^{\circ} 35.1^{\prime}\end{array}$ & 23 & (1252-)1420-1559 & 561 & Ring 82-B, Core \\
\hline $20-12$ & $\begin{array}{l}38^{\circ} 39.4^{\prime} \\
38^{\circ} 38.4^{\prime}\end{array}$ & $\begin{array}{l}72^{\circ} 05.0^{\prime} \\
72^{\circ} 01.8^{\prime}\end{array}$ & 24 & (1231-) (1407-1623 & 442 & Ring 82-B, Edge \\
\hline \multirow[t]{2}{*}{$20-13$} & $\begin{array}{l}38^{\circ} 36.0^{\prime} \\
38^{\circ} 32.7^{\prime}\end{array}$ & $\begin{array}{l}72^{\circ} 03.8^{\prime} \\
72^{\circ} 05.4^{\prime}\end{array}$ & $24-25$ & $(2255-) 0001-0137$ & 452 & Ring $82-B$, Edge \\
\hline & & \multicolumn{5}{|c|}{ Time $(+4)$} \\
\hline $20-14$ & $\begin{array}{l}38^{\circ} 31.2^{\prime} \\
38^{\circ} 30.9^{\prime}\end{array}$ & $\begin{array}{l}72^{\circ} 23.6^{\prime} \\
72^{\circ} 19.5^{\prime}\end{array}$ & 26 & (0149-)0318-0504 & 370 & Ring 82-B, Edge \\
\hline $20-15$ & $\begin{array}{l}38^{\circ} 34.6^{\prime} \\
38^{\circ} 35.3^{\prime}\end{array}$ & $\begin{array}{l}72^{\circ} 31.7^{\prime} \\
72^{\circ} 27.5^{\prime}\end{array}$ & 26 & $(1358-) 1507-1650$ & 342 & Ring 82-B, Edge \\
\hline $20-16$ & $\begin{array}{l}38^{\circ} 49.4^{\prime} \\
38^{\circ} 50.0^{\prime}\end{array}$ & $\begin{array}{l}71^{\circ} 40.5^{\prime} \\
71^{\circ} 49.8^{\prime}\end{array}$ & 30 & $(0733-) 1014-1120$ & 548 & Ring 82-B, Core \\
\hline $20-17$ & $\begin{array}{l}38^{\circ} 48.3^{\prime} \\
38^{\circ} 51.0^{\prime}\end{array}$ & $\begin{array}{l}71^{\circ} 44.5^{\prime} \\
71^{\circ} 53.0^{\prime}\end{array}$ & 1 - 1 & $(2210-) 2341-0122$ & 553 & Ring $82-\mathrm{B}$, Core \\
\hline $20-18$ & $\begin{array}{l}39^{\circ} 27.7^{\prime} \\
39^{\circ} 28.0^{\prime}\end{array}$ & $\begin{array}{l}71^{\circ} 08.4^{\prime} \\
71^{\circ} 01.7^{\prime}\end{array}$ & $1-2$ & $(2155-) 2308-0056$ & 255 & Slope Water \\
\hline
\end{tabular}


Table 3. Summary of MOCNESS-20 stations, Oceanus Cruise 121.

\begin{tabular}{|c|c|c|c|c|c|c|}
\hline MOCNESS & Posit & on & $\begin{array}{l}\text { Date } \\
\text { June } 1982\end{array}$ & Time $(+4)$ & $\begin{array}{l}\text { Depth } \\
\text { to } 10^{\circ} \mathrm{C}\end{array}$ & Area \\
\hline $20-19$ & $\begin{array}{l}39^{\circ} 13.5^{\prime} \\
39^{\circ} 07.5^{\prime}\end{array}$ & $\begin{array}{l}71^{\circ} 17.6^{\prime} \\
71^{\circ} 17.4^{\prime}\end{array}$ & $15-16$ & $(2315-) 0032-0243$ & 285 & Slope Water \\
\hline $20-20$ & $\begin{array}{l}38^{\circ} 58.9^{\prime} \\
38^{\circ} 55.7^{\prime}\end{array}$ & $\begin{array}{l}71^{\circ} 29.5^{\prime} \\
71^{\circ} 34.2^{\prime}\end{array}$ & 16 & $(1131-) 1305-1454$ & 246 & Slope Water \\
\hline $20-21$ & $\begin{array}{l}37^{\circ} 05.7^{\prime} \\
36^{\circ} 59.7^{\prime}\end{array}$ & $\begin{array}{l}73^{\circ} 37.0^{\prime} \\
73^{\circ} 36.1^{\prime}\end{array}$ & 18 & $(0015-) 0141-0343$ & 549 & Ring 82-B, Core \\
\hline $20-23$ & $\begin{array}{l}36^{\circ} 58.6^{\prime} \\
37^{\circ} 01.7^{\prime}\end{array}$ & $\begin{array}{l}73^{\circ} 37.3^{\prime} \\
73^{\circ} 34.3^{\prime}\end{array}$ & 20 & $(0126-) 0249-0410$ & 543 & Ring 82-B, Core \\
\hline $20-24$ & $\begin{array}{l}36^{\circ} 54.6^{\prime} \\
36^{\circ} 57.1^{\prime}\end{array}$ & $\begin{array}{l}73^{\circ} 36.2^{\prime} \\
73^{\circ} 35.0^{\prime}\end{array}$ & 20 & $(1335-) 1439-1614$ & 530 & Ring $82-B$, Core \\
\hline $20-25$ & $\begin{array}{l}37^{\circ} 02.8^{\prime} \\
37^{\circ} 02.5^{\prime}\end{array}$ & $\begin{array}{l}73^{\circ} 16.2^{\prime} \\
73^{\circ} 10.5^{\prime}\end{array}$ & 21 & $(0005-) 0126-0255$ & 396 & Ring 82-B, Edge \\
\hline $20-26$ & $\begin{array}{l}37^{\circ} 00.3^{\prime} \\
37^{\circ} 03.4^{\prime}\end{array}$ & $\begin{array}{l}73^{\circ} 07.1^{\prime} \\
73^{\circ} 04.7^{\prime}\end{array}$ & 21 & $(1357-) 1510-1657$ & 353 & Ring 82-B, Edge \\
\hline $20-27$ & $\begin{array}{l}37^{\circ} 07.5^{\prime} \\
37^{\circ} 12.8^{\prime}\end{array}$ & $\begin{array}{l}73^{\circ} 08.7^{\prime} \\
73^{\circ} 11.8^{\prime}\end{array}$ & 23 & (1505-)1629-1834 & 215 & $\begin{array}{l}\text { Ring } 82-B \text {, } \\
\text { Entrainment Tongue }\end{array}$ \\
\hline $20-28$ & $\begin{array}{l}36^{\circ} 59.8^{\prime} \\
36^{\circ} 56.3^{\prime}\end{array}$ & $\begin{array}{l}73^{\circ} 48.9^{\prime} \\
73^{\circ} 56.1^{\prime}\end{array}$ & 24 & (0538-)0713-0926 & 514 & Ring $82-B$, Core \\
\hline $20-29$ & $\begin{array}{l}37^{\circ} 05.4^{\prime} \\
37^{\circ} 07.6^{\prime}\end{array}$ & $\begin{array}{l}73^{\circ} 46.9^{\prime} \\
73^{\circ} 51.8^{\prime}\end{array}$ & $25-26$ & $(2339-) 0128-0327$ & 460 & Ring 82-B, Edge \\
\hline $20-30$ & $\begin{array}{l}37^{\circ} 05.5^{\prime} \\
37^{\circ} 11.6^{\prime}\end{array}$ & $\begin{array}{l}73^{\circ} 45.7^{\prime} \\
73^{\circ} 46.7^{\prime}\end{array}$ & 26 & (1329-)1452-1711 & 441 & Ring 82-B, Edge \\
\hline $20-31$ & $\begin{array}{l}37^{\circ} 02.6^{\prime} \\
37^{\circ} 06.8^{\prime}\end{array}$ & $\begin{array}{l}73^{\circ} 31.3^{\prime} \\
73^{\circ} 30.2^{\prime}\end{array}$ & 27 & (1156-)1320-1523 & 406 & Ring 82-B, Edge \\
\hline $20-32$ & $\begin{array}{l}37^{\circ} 01.7^{\prime} \\
37^{\circ} 05.0^{\prime}\end{array}$ & $\begin{array}{l}73^{\circ} 32.3^{\prime} \\
73^{\circ} 35.4^{\prime}\end{array}$ & $27-28$ & $(2204-) 2340-0124$ & 430 & Ring 82-B, Edge \\
\hline $20-33$ & $\begin{array}{l}37^{\circ} 41.0^{\prime} \\
37^{\circ} 46.9^{\prime}\end{array}$ & $\begin{array}{l}72^{\circ} 54.8^{\prime} \\
72^{\circ} 50.7^{\prime}\end{array}$ & $28-29$ & $(2332-) 0053-0313$ & 224 & Slope Water \\
\hline $20-34$ & $\begin{array}{l}39^{\circ} 22.3^{\prime} \\
39^{\circ} 26.7^{\prime}\end{array}$ & $\begin{array}{l}71^{\circ} 36.0^{\prime} \\
71^{\circ} 34.4^{\prime}\end{array}$ & 30 & $(2050-) 2232-2358$ & 282 & Slope Water \\
\hline
\end{tabular}


Table 4. Summary of MOCNESS-20 stations, Oceanus Cruise 125.

\begin{tabular}{|c|c|c|c|c|c|c|}
\hline MOCNESS & Posit & on $w$ & $\begin{array}{l}\text { Date } \\
\text { August } 1982\end{array}$ & Time $(+4)$ & $\begin{array}{l}\text { Depth } \\
\text { to } 10^{\circ} \mathrm{C}\end{array}$ & Area \\
\hline $20-35$ & $\begin{array}{l}38^{\circ} 53.3^{\prime} \\
38^{\circ} 56.5^{\prime}\end{array}$ & $\begin{array}{l}71^{\circ} 47.6^{\prime} \\
71^{\circ} 42.1^{\prime}\end{array}$ & 7 & $(1316-) 1539-1720$ & 255 & $\begin{array}{l}\text { Slope Water? } \\
\text { (or old WCR?) }\end{array}$ \\
\hline $20-36$ & $\begin{array}{l}38^{\circ} 56.3^{\prime} \\
38^{\circ} 54.5^{\prime}\end{array}$ & $\begin{array}{l}71^{\circ} 39.8^{\prime} \\
71^{\circ} 32.0^{\prime}\end{array}$ & $7-8$ & $(2334-0248)$ & 247 & Slope water \\
\hline $20-37$ & $\begin{array}{l}36^{\circ} 37.8^{\prime} \\
36^{\circ} 34.6^{\prime}\end{array}$ & $\begin{array}{l}73^{\circ} 44.7^{\prime} \\
73^{\circ} 45.4^{\prime}\end{array}$ & 10 & $(1355-) 1529-1714$ & 398 & Ring 82-B, Core \\
\hline $20-38$ & $\begin{array}{l}36^{\circ} 40.9^{\prime} \\
36^{\circ} 38.3^{\prime}\end{array}$ & $\begin{array}{l}73^{\circ} 43.3^{\prime} \\
73^{\circ} 46.9^{\prime}\end{array}$ & 11 & $(0125-) 0231-0414$ & 399 & Ring 82-B, Core \\
\hline $20-39$ & $\begin{array}{l}36^{\circ} 52.9^{\prime} \\
36^{\circ} 51.0^{\prime}\end{array}$ & $\begin{array}{l}73^{\circ} 37.4^{\prime} \\
73^{\circ} 43.8^{\prime}\end{array}$ & 12 & $(0042-) 0155-0408$ & 398 & Ring 82-B, Core \\
\hline $20-41$ & $\begin{array}{l}37^{\circ} 01.0^{\prime} \\
37^{\circ} 07.3^{\prime}\end{array}$ & $\begin{array}{l}73^{\circ} 48.2^{\prime} \\
73^{\circ} 47.7^{\prime}\end{array}$ & 13 & $(0113-)$ 0243-0446 & 352 & Ring 82-B, Edge \\
\hline $20-42$ & $\begin{array}{l}36^{\circ} 54.3^{\prime} \\
36^{\circ} 58.0^{\prime}\end{array}$ & $\begin{array}{l}73^{\circ} 54.7^{\prime} \\
73^{\circ} 50.9^{\prime}\end{array}$ & 14 & $(0105-) 0228-0441$ & 295 & Ring 82-B, Edge \\
\hline $20-43$ & $\begin{array}{l}37^{\circ} 07.7^{\prime} \\
37^{\circ} 00.3^{\prime}\end{array}$ & $\begin{array}{l}73^{\circ} 39.9^{\prime} \\
73^{\circ} 39.9^{\prime}\end{array}$ & 14 & (1332-) 1516-1654 & 336 & Ring 82-B, Edge \\
\hline $20-44$ & $\begin{array}{l}37^{\circ} 07.0^{\prime} \\
37^{\circ} 04.3^{\prime}\end{array}$ & $\begin{array}{l}73^{\circ} 38.2^{\prime} \\
73^{\circ} 48.2^{\prime}\end{array}$ & 15 & $(0008-) 0156-0402$ & 327 & Ring 82-B, Edge \\
\hline $20-45$ & $\begin{array}{l}36^{\circ} 51.6^{\prime} \\
36^{\circ} 50.5^{\prime}\end{array}$ & $\begin{array}{l}73^{\circ} 53.7^{\prime} \\
73^{\circ} 40.4^{\prime}\end{array}$ & 15 & $(1304-) 1411-1758$ & 271 & Ring $82-B$, Edge \\
\hline $20-46$ & $\begin{array}{l}36^{\circ} 50.0^{\prime} \\
36^{\circ} 50.8^{\prime}\end{array}$ & $\begin{array}{l}73^{\circ} 40.5^{\prime} \\
73^{\circ} 49.8^{\prime}\end{array}$ & $15-16$ & $(2239-) 2312-0204$ & 256 & Ring 82-B, Edge \\
\hline $20-47$ & $\begin{array}{l}37^{\circ} 12.3^{\prime} \\
37^{\circ} 15.5^{\prime}\end{array}$ & $\begin{array}{l}74^{\circ} 18.1^{\prime} \\
74^{\circ} 25.5^{\prime}\end{array}$ & $16-17$ & $(2253-) 0012-0242$ & 233 & Slope Water \\
\hline $20-48$ & $\begin{array}{l}37^{\circ} 15.6^{\prime} \\
37^{\circ} 11.9^{\prime}\end{array}$ & $\begin{array}{l}74^{\circ} 24.9^{\prime} \\
74^{\circ} 17.3^{\prime}\end{array}$ & 17 & (1424-) (1550-1747 & 208 & Slope Water \\
\hline $20-49$ & $\begin{array}{l}36^{\circ} 02.2^{\prime} \\
36^{\circ} 05.6^{\prime}\end{array}$ & $\begin{array}{l}71^{\circ} 23.5^{\prime} \\
71^{\circ} 31.4^{\prime}\end{array}$ & 19 & $(0102-) 0225-0431$ & 972 & Sargasso Sea \\
\hline $20-50$ & $\begin{array}{l}36^{\circ} 04.4^{\prime} \\
36^{\circ} 00.6^{\prime}\end{array}$ & $\begin{array}{l}71^{\circ} 29.7^{\prime} \\
71^{\circ} 32.8^{\prime}\end{array}$ & (con & $\begin{array}{l}(1229-) 1343-1519 \\
\text { tinued) }\end{array}$ & 954 & Sargasso Sea \\
\hline
\end{tabular}


5

Table 4 (continued)

$\begin{array}{lllllll} & 37^{\circ} 00.9^{\prime} & 71^{\circ} 17.5^{\prime} & & & & \\ 20-51 & 37^{\circ} 03.7^{\prime} & 71^{\circ} 16.8^{\prime} & 20 & (1403-) 1526-1711 & 624 & \text { Gulf Stream } \\ & 37^{\circ} 05.3^{\prime} & 70^{\circ} 58.8^{\prime} & & & & \\ 20-52 & 37^{\circ} 04.6^{\prime} & 70^{\circ} 57.3^{\prime} & 21 & (0135-) 0240-0425 & 632 & \text { Gu1f Stream } \\ & 38^{\circ} 22.0^{\prime} & 70^{\circ} 52.7^{\prime} & & & & \\ 20-53 & 38^{\circ} 28.5^{\prime} & 70^{\circ} 55.5^{\prime} & 21-22 & (2346-) 0104-0308 & 202 & \text { Slope Water } \\ & 39^{\circ} 11.4^{\prime} & 70^{\circ} 59.1^{\prime} & & & & \\ 20-54 & 39^{\circ} 17.5^{\prime} & 70^{\circ} 58.8^{\prime} & 22 & (1235-) 1400-1556 & 230 & \text { S1ope Water }\end{array}$


Table 5. Summary of MOCNESS-20 stations, Knorr Cruise 98.

\begin{tabular}{|c|c|c|c|c|c|c|}
\hline MOCNESS & Posi & on & $\begin{array}{c}\text { Date } \\
\text { Sept-Oct } 1982\end{array}$ & Time $(+4)$ & $\begin{array}{l}\text { Depth } \\
\text { to } 10^{\circ} \mathrm{C}\end{array}$ & Area \\
\hline $20-55$ & $\begin{array}{l}39^{\circ} 16.7^{\prime} \\
39^{\circ} 17.2^{\prime}\end{array}$ & $\begin{array}{l}65^{\circ} 43.9 \\
65^{\circ} 43.4^{\prime}\end{array}$ & 28 & (0111-)0212-0355 & 568 & Ring $82-H$, Edge \\
\hline $20-56$ & $\begin{array}{l}39^{\circ} 28.0^{\prime} \\
39^{\circ} 22.2^{\prime}\end{array}$ & $\begin{array}{l}64^{\circ} 00.6^{\prime} \\
63^{\circ} 55.3^{\prime}\end{array}$ & 30 & $(0012-) 0131-0333$ & 834 & Ring $82-\mathrm{H}$, Core \\
\hline $20-57$ & $\begin{array}{l}39^{\circ} 30.1^{\prime} \\
39^{\circ} 21.9^{\prime}\end{array}$ & $\begin{array}{l}64^{\circ} 14.3^{\prime} \\
64^{\circ} 23.4^{\prime}\end{array}$ & $30-1$ & (2331-) 0040-0329 & 832 & Ring $82-\mathrm{H}$, Core \\
\hline $20-58$ & $\begin{array}{l}39^{\circ} 28.7^{\prime} \\
39^{\circ} 31.9^{\prime}\end{array}$ & $\begin{array}{l}64^{\circ} 36.3^{\prime} \\
64^{\circ} 43.8^{\prime}\end{array}$ & 2 & $(1241-) 1356-1627$ & 836 & Ring $82-\mathrm{H}$, Core \\
\hline $20-59$ & $\begin{array}{l}40^{\circ} 45.0^{\prime} \\
40^{\circ} 38.3^{\prime}\end{array}$ & $\begin{array}{l}65^{\circ} 03.0^{\prime} \\
64^{\circ} 57.7^{\prime}\end{array}$ & 5 & (0056-)0202-0431 & 307 & Slope Water \\
\hline $20-60$ & $\begin{array}{l}40^{\circ} 42.0^{\prime} \\
40^{\circ} 36.4^{\prime}\end{array}$ & $\begin{array}{l}65^{\circ} 00.3^{\prime} \\
64^{\circ} 53.2^{\prime}\end{array}$ & 5 & $(1344-) 1445-1658$ & 301 & Slope Water \\
\hline $20-61$ & $\begin{array}{l}39^{\circ} 46.7^{\prime} \\
39^{\circ} 40.6^{\prime}\end{array}$ & $\begin{array}{l}65^{\circ} 14.0^{\prime} \\
65^{\circ} 16.2^{\prime}\end{array}$ & 7 & (0019-)0119-0354 & 744 & Ring 82-H, Core \\
\hline $20-62$ & $\begin{array}{l}39^{\circ} 26.5^{\prime} \\
39^{\circ} 29.6^{\prime}\end{array}$ & $\begin{array}{l}65^{\circ} 21.7^{\prime} \\
65^{\circ} 25.6^{\prime}\end{array}$ & 8 & $(1121-) 1223-1533$ & 820 & Ring 82-H, Core \\
\hline $20-63$ & $\begin{array}{l}39^{\circ} 27.4^{\prime} \\
39^{\circ} 33.1^{\prime}\end{array}$ & $\begin{array}{l}65^{\circ} 35.2^{\prime} \\
65^{\circ} 36.1^{\prime}\end{array}$ & $8-9$ & (2313-) $0020-0325$ & 837 & Ring 82-H, Core \\
\hline $20-64$ & $\begin{array}{l}39^{\circ} 09.7^{\prime} \\
39^{\circ} 06.0^{\prime}\end{array}$ & $\begin{array}{l}65^{\circ} 57.9^{\prime} \\
66^{\circ} 04.0^{\prime}\end{array}$ & 13 & (1337-)1445-1647 & 816 & Ring $82-\mathrm{H}$, Core \\
\hline $20-65$ & $\begin{array}{l}39^{\circ} 04.9^{\prime} \\
39^{\circ} 02.9^{\prime}\end{array}$ & $\begin{array}{l}68^{\circ} 00.3^{\prime} \\
68^{\circ} 08.6^{\prime}\end{array}$ & 15 & $(0022-) 0155-0428$ & 214 & Slope Water \\
\hline $20-66$ & $\begin{array}{l}38^{\circ} 58.2^{\prime} \\
38^{\circ} 58.9^{\prime}\end{array}$ & $\begin{array}{l}68^{\circ} 18.6^{\prime} \\
68^{\circ} 26.2^{\prime}\end{array}$ & 15 & (1307-)1415-1628 & 242 & Slope Water \\
\hline
\end{tabular}


Table 6. Summary of MOCNESS-20 collections, Atlantis II Cruise 110.

\begin{tabular}{|c|c|c|c|c|}
\hline $\begin{array}{c}\text { MOCNESS } \\
20\end{array}$ & $\begin{array}{l}\text { Time } \\
(+4)\end{array}$ & $\begin{array}{c}\text { Depth } \\
\text { (m) }\end{array}$ & $\begin{array}{c}\text { Speed } \\
\text { (knots) }\end{array}$ & $\begin{array}{l}\text { Volume } \\
\left(10,000 \mathrm{~m}^{3}\right)\end{array}$ \\
\hline $\begin{array}{l}1,0 \\
1,1 \\
1,2 \\
1,3 \\
1,4\end{array}$ & $\begin{array}{l}2144-0009 \\
0009-0022 \\
0022-0057 \\
0057-0151 \\
0151-0242\end{array}$ & $\begin{array}{c}0-1260 \\
1000-750 \\
750-500 \\
500-250 \\
250-0\end{array}$ & $\begin{array}{l}1.2 \\
2.0 \\
1.8 \\
1.9 \\
2.1\end{array}$ & $\begin{array}{l}- \\
1.2 \\
3.2 \\
5.0 \\
5.0\end{array}$ \\
\hline $\begin{array}{l}2,0 \\
2,1 \\
2,2 \\
2,3 \\
2,4\end{array}$ & $\begin{array}{l}0006-0118 \\
0118-0134 \\
0134-0150 \\
0150-0223 \\
0223-0320\end{array}$ & $\begin{array}{c}0-1000 \\
1000-750 \\
750-500 \\
500-250 \\
250-0\end{array}$ & $\begin{array}{l}1.1 \\
1.9 \\
2.1 \\
2.4 \\
1.8\end{array}$ & $\begin{array}{l}- \\
1.6 \\
1.5 \\
3.7 \\
4.8\end{array}$ \\
\hline $\begin{array}{l}3,0 \\
3,1 \\
3,2 \\
3,3 \\
3,4\end{array}$ & $\begin{array}{l}1408-1530 \\
1530-1556 \\
1556-1625 \\
1625-1701 \\
1701-1736\end{array}$ & $\begin{array}{c}0-1030 \\
1000-750 \\
750-500 \\
500-250 \\
250-0\end{array}$ & $\begin{array}{l}1.1 \\
1.8 \\
2.0 \\
2.4 \\
1.8\end{array}$ & $\begin{array}{l}- \\
2.6 \\
2.8 \\
3.7 \\
2.8\end{array}$ \\
\hline $\begin{array}{l}4,0 \\
4,1 \\
4,2 \\
4,3 \\
4,4\end{array}$ & $\begin{array}{l}0735-0835 \\
0835-0910 \\
0910-0944 \\
0944-1035 \\
1035-1118\end{array}$ & $\begin{array}{c}0-1000 \\
1000-750 \\
750-500 \\
500-250 \\
250-0\end{array}$ & $\begin{array}{l}1.6 \\
2.1 \\
2.2 \\
2.1 \\
2.3\end{array}$ & $\begin{array}{l}- \\
3.4 \\
3.2 \\
4.6 \\
4.3\end{array}$ \\
\hline $\begin{array}{l}5,0 \\
5,1 \\
5,2 \\
5,3 \\
5,4\end{array}$ & $\begin{array}{l}2218-0015 \\
0015-0057 \\
0057-0134 \\
0134-0206 \\
0206-0243\end{array}$ & $\begin{array}{c}0-1000 \\
1000-750 \\
750-500 \\
500-250 \\
250-0\end{array}$ & $\begin{array}{l}1.4 \\
2.1 \\
2.1 \\
2.0 \\
2.1\end{array}$ & $\begin{array}{l}3.9 \\
3.2 \\
2.7 \\
3.0\end{array}$ \\
\hline $\begin{array}{l}6,0 \\
6,1\end{array}$ & $\begin{array}{l}2323-0038 \\
0038-0302\end{array}$ & $\begin{array}{l}0-1010 \\
1000-0\end{array}$ & $\begin{array}{l}1.3 \\
2.0\end{array}$ & 12.6 \\
\hline
\end{tabular}


Table 7. Summary of MOCNESS-20 collections, Oceanus Cruise 118.

\begin{tabular}{|c|c|c|c|c|c|c|}
\hline $\begin{array}{c}\text { MOCNESS } \\
20\end{array}$ & $\begin{array}{l}\text { Time } \\
(+5)\end{array}$ & $\begin{array}{l}\text { Depth } \\
(\mathrm{m})\end{array}$ & $\begin{array}{c}\text { Speed } \\
\text { (knots) }\end{array}$ & $\begin{array}{l}\text { Volume } \\
\left(10,000 \mathrm{~m}^{3}\right)\end{array}$ & $\begin{array}{l}\text { Distance } \\
\text { to Ring } \\
\text { Center } \\
(\mathrm{km})\end{array}$ & $\begin{array}{c}\text { Angle } \\
\text { from Ring } \\
\text { Center }\end{array}$ \\
\hline 7,0 & $1354-1607$ & $0-1022-0$ & 1.6 & - & 149 & 079 \\
\hline $\begin{array}{l}8,0 \\
8,1 \\
8,2 \\
8,3\end{array}$ & $\begin{array}{l}0112-0250 \\
0250-0308 \\
0308-0327 \\
0327-0344\end{array}$ & $\begin{array}{c}0-1026 \\
1000-750 \\
750-500 \\
500-250\end{array}$ & $\begin{array}{l}1.2 \\
2.4 \\
2.3 \\
2.1\end{array}$ & $\begin{array}{c}- \\
2.2 \\
2.2 \\
1.7\end{array}$ & 161 & 075 \\
\hline $\begin{array}{l}9,0 \\
9,1 \\
9,2 \\
9,3 \\
9,4\end{array}$ & $\begin{array}{l}1209-1345 \\
1345-1407 \\
1407-1427 \\
1427-1450 \\
1450-1510\end{array}$ & $\begin{array}{c}0-1029 \\
1000-750 \\
750-500 \\
500-250 \\
250-0\end{array}$ & $\begin{array}{l}1.1 \\
1.6 \\
1.7 \\
1.7 \\
1.6\end{array}$ & $\begin{array}{l}- \\
1.5 \\
1.4 \\
1.7 \\
1.5\end{array}$ & 152 & 079 \\
\hline $\begin{array}{l}10,0 \\
10,1 \\
10,2 \\
10,3 \\
10,4\end{array}$ & $\begin{array}{l}0034-0229 \\
0229-0252 \\
0252-0307 \\
0307-0327 \\
0327-0355\end{array}$ & $\begin{array}{c}0-1019 \\
1000-750 \\
750-500 \\
500-250 \\
250-0\end{array}$ & $\begin{array}{l}1.1 \\
1.5 \\
1.9 \\
2.1 \\
2.0\end{array}$ & $\begin{array}{l}- \\
1.7 \\
1.1 \\
1.7 \\
2.5\end{array}$ & 13 & 060 \\
\hline $\begin{array}{l}11,0 \\
11,1 \\
11,2 \\
11,3 \\
11,4\end{array}$ & $\begin{array}{l}1252-1420 \\
1420-1452 \\
1452-1509 \\
1509-1526 \\
1526-1559\end{array}$ & $\begin{array}{c}0-1039 \\
1000-750 \\
750-500 \\
500-250 \\
250-0\end{array}$ & $\begin{array}{l}1.6 \\
1.7 \\
2.0 \\
2.3 \\
2.0\end{array}$ & $\begin{array}{l}- \\
2.8 \\
1.6 \\
1.8 \\
3.4\end{array}$ & 11 & 275 \\
\hline $\begin{array}{l}12,0 \\
12,1 \\
12,2 \\
12,3 \\
12,4\end{array}$ & $\begin{array}{l}1231-1407 \\
1407-1444 \\
1444-1514 \\
1514-1544 \\
1544-1623\end{array}$ & $\begin{array}{c}0-1029 \\
1000-750 \\
750-500 \\
500-250 \\
250-0\end{array}$ & $\begin{array}{l}1.4 \\
1.5 \\
1.6 \\
1.9 \\
1.9\end{array}$ & $\begin{array}{l}- \\
2.9 \\
2.4 \\
2.6 \\
3.5\end{array}$ & 53 & 240 \\
\hline $\begin{array}{l}13,0 \\
13,1 \\
13,2 \\
13,3\end{array}$ & $\begin{array}{l}2255-0001 \\
0001-0053 \\
0053-0114 \\
0114-0137\end{array}$ & $\begin{array}{c}0-1010 \\
1000-500 \\
500-250 \\
250-0\end{array}$ & $\begin{array}{l}1.3 \\
1.9 \\
2.3 \\
2.2\end{array}$ & $\begin{array}{l}- \\
4.1 \\
1.8 \\
2.0\end{array}$ & 61 & 229 \\
\hline & Time $(+4)$ & & & & & \\
\hline $\begin{array}{l}14,0 \\
14,1 \\
14,2 \\
14,3 \\
14,4\end{array}$ & $\begin{array}{l}0149-0318 \\
0318-0336 \\
0336-0355 \\
0355-0426 \\
0426-0504\end{array}$ & $\begin{array}{c}0-1032 \\
1000-750 \\
750-500 \\
500-250 \\
250-0\end{array}$ & $\begin{array}{l}1.3 \\
1.6 \\
1.9 \\
2.2 \\
2.2\end{array}$ & $\begin{array}{l}- \\
1.4 \\
1.7 \\
3.0 \\
3.5\end{array}$ & 76 & 235 \\
\hline
\end{tabular}


Table 7 (continued)

$\begin{array}{lcccccc}15,0 & 1358-1507 & 0-1006 & 1.3 & - & 86 & 246 \\ 15,1 & 1507-1536 & 1000-750 & 1.8 & 2.1 & & \\ 15,2 & 1536-1600 & 750-500 & 1.9 & 1.7 & & \\ 15,3 & 1600-1622 & 500-245 & 2.1 & 1.7 & & \\ 15,4 & 1622-1650 & 245-0 & 1.9 & 1.8 & 80 & 160 \\ 16,0 & 0733-1014 & 0-1006 & 1.7 & - & 10 & \\ 16,1 & 1014-1033 & 800-600 & 1.9 & 1.5 & & 238 \\ 16,2 & 1033-1052 & 600-400 & 1.6 & 1.4 & & \\ 16,3 & 1052-1120 & 400-0 & 1.6 & 2.0 & 14 & 221 \\ 17,0 & 2210-2341 & 0-1005 & 1.4 & - & 15 & \\ 17,1 & 2341-0044 & 1017-500 & 2.3 & 6.7 & & \\ 17,2 & 0044-0102 & 500-250 & 2.3 & 1.9 & & \\ 17,3 & 0102-0122 & 250-0 & 2.0 & 1.8 & 22 & 034 \\ 18,0 & 2155-2308 & 0-1007 & 1.4 & - & 74 & \\ 18,1 & 2308-0056 & 1000-0 & 1.9 & 10.3 & 81 & 039\end{array}$


Table 8. Summary of MOCNESS-20 collections, Oceanus Cruise 121.

\begin{tabular}{|c|c|c|c|c|c|c|}
\hline $\begin{array}{l}\text { MOCNESS } \\
20\end{array}$ & $\begin{array}{l}\text { Time } \\
(+4)\end{array}$ & $\begin{array}{l}\text { Depth } \\
(\mathrm{m})\end{array}$ & $\begin{array}{c}\text { Speed } \\
\text { (knots) }\end{array}$ & $\begin{array}{c}\text { Volume } \\
\left(10,000 \mathrm{~m}^{3}\right)\end{array}$ & $\begin{array}{l}\text { Distance } \\
\text { to Ring } \\
\text { Center } \\
(\mathrm{km})\end{array}$ & $\begin{array}{c}\text { Angle } \\
\text { from Ring } \\
\text { Center }\end{array}$ \\
\hline $\begin{array}{l}19,0 \\
19,1 \\
19,2 \\
19,3 \\
19,4 \\
19,5\end{array}$ & $\begin{array}{l}2315-0032 \\
0032-0049 \\
0049-0106 \\
0106-0149 \\
0149-0213 \\
0213-0243\end{array}$ & $\begin{array}{c}0-1027 \\
1000-751 \\
751-503 \\
503-253 \\
253-103 \\
103-0\end{array}$ & $\begin{array}{l}1.1 \\
2.0 \\
2.2 \\
2.3 \\
2.6 \\
2.3\end{array}$ & $\begin{array}{l}- \\
1.9 \\
1.9 \\
5.6 \\
2.9 \\
4.1\end{array}$ & 300 & 042 \\
\hline $\begin{array}{l}20.0 \\
20,1 \\
20,2 \\
20,3 \\
20,4 \\
20,5\end{array}$ & $\begin{array}{l}1131-1305 \\
1305-1330 \\
1330-1352 \\
1352-1414 \\
1414-1432 \\
1432-1454\end{array}$ & $\begin{array}{r}0-1291 \\
1250-1001 \\
1001-751 \\
751-500 \\
500-251 \\
251-0\end{array}$ & $\begin{array}{l}1.1 \\
1.7 \\
1.7 \\
1.7 \\
1.8 \\
1.4\end{array}$ & $\begin{array}{l}- \\
2.5 \\
2.2 \\
2.3 \\
1.9 \\
1.9\end{array}$ & 265 & 041 \\
\hline $\begin{array}{l}21,0 \\
21,1 \\
21,2 \\
21,3 \\
21,4 \\
21,5\end{array}$ & $\begin{array}{l}0015-0141 \\
0141-0158 \\
0158-0220 \\
0220-0250 \\
0250-0334 \\
0334-0343\end{array}$ & $\begin{array}{c}0-1029 \\
1000-800 \\
800-601 \\
601-401 \\
401-54 \\
54-0\end{array}$ & $\begin{array}{l}1.2 \\
1.8 \\
2.0 \\
1.9 \\
2.0 \\
1.7\end{array}$ & $\begin{array}{l}- \\
1.8 \\
2.4 \\
3.3 \\
5.0 \\
1.0\end{array}$ & 8 & 167 \\
\hline $\begin{array}{l}23,0 \\
23,7 \\
23,2 \\
23,3 \\
23,4 \\
23,5\end{array}$ & $\begin{array}{l}0126-0249 \\
0249-0304 \\
0304-0322 \\
0322-0339 \\
0339-0354 \\
0354-0410\end{array}$ & $\begin{array}{c}0-1006 \\
1000-801 \\
801-600 \\
600-400 \\
400-151 \\
151-0\end{array}$ & $\begin{array}{l}1.1 \\
2.1 \\
2.3 \\
2.4 \\
2.5 \\
2.5\end{array}$ & $\begin{array}{l}- \\
1.7 \\
2.2 \\
2.0 \\
1.5 \\
1.7\end{array}$ & 15 & 112 \\
\hline $\begin{array}{l}24,0 \\
24,1 \\
24,2 \\
24,3 \\
24,4 \\
24,5\end{array}$ & $\begin{array}{l}1335-1439 \\
1439-1455 \\
1455-1509 \\
1509-1542 \\
1542-1544 \\
1544-1614\end{array}$ & $\begin{array}{c}0-1024 \\
1000-800 \\
800-600 \\
600-400 \\
400-382 \\
382-0\end{array}$ & $\begin{array}{l}1.1 \\
1.9 \\
1.9 \\
1.8 \\
1.8 \\
2.0\end{array}$ & $\begin{array}{l}- \\
1.8 \\
1.6 \\
3.7 \\
0.2 \\
3.4\end{array}$ & 17 & 126 \\
\hline $\begin{array}{l}25,4 \\
25,5\end{array}$ & $\begin{array}{l}0222-0246 \\
0246-0255\end{array}$ & $\begin{array}{c}301-51 \\
51-0\end{array}$ & $\begin{array}{l}2.5 \\
2.5\end{array}$ & $\begin{array}{l}2.9 \\
1.2\end{array}$ & $\overline{54}$ & $\overline{0} 3$ \\
\hline $\begin{array}{l}26,0 \\
26,1 \\
26,2 \\
26,3 \\
26,4 \\
26,5\end{array}$ & $\begin{array}{l}1357-1510 \\
1510-1527 \\
1527-1552 \\
1552-1610 \\
1610-1626 \\
1626-1657\end{array}$ & $\begin{array}{c}0-1016 \\
1000-750 \\
750-500 \\
500-374 \\
374-251 \\
251-0\end{array}$ & $\begin{array}{l}1.3 \\
2.3 \\
2.1 \\
2.0 \\
2.3 \\
2.3\end{array}$ & $\begin{array}{l}- \\
2.1 \\
2.9 \\
2.1 \\
1.9 \\
3.1\end{array}$ & 61 & 088 \\
\hline
\end{tabular}


Table 8 (continued)

\begin{tabular}{|c|c|c|c|c|c|c|}
\hline $\begin{array}{l}27,0 \\
27,1 \\
27,2 \\
27,3 \\
27,4 \\
27,5\end{array}$ & $\begin{array}{l}1505-1629 \\
1629-1714 \\
1714-1733 \\
1733-1751 \\
1751-1819 \\
1819-1834\end{array}$ & $\begin{array}{c}0-1010 \\
1001-750 \\
750-499 \\
499-301 \\
301-150 \\
150-0\end{array}$ & $\begin{array}{l}1.2 \\
2.3 \\
2.3 \\
2.3 \\
2.2 \\
2.3\end{array}$ & $\begin{array}{l}- \\
5.7 \\
2.4 \\
2.2 \\
3.4 \\
1.8\end{array}$ & 73 & 063 \\
\hline $\begin{array}{l}28,0 \\
28,1 \\
28,2 \\
28,3 \\
28,4 \\
28,5\end{array}$ & $\begin{array}{l}0538-0713 \\
0713-0742 \\
0742-0803 \\
0803-0827 \\
0827-0848 \\
0848-0926\end{array}$ & $\begin{array}{r}0-1262 \\
1250-1001 \\
1001-749 \\
749-501 \\
501-317 \\
317-0\end{array}$ & $\begin{array}{l}1.1 \\
2.4 \\
2.3 \\
2.1 \\
2.3 \\
2.3\end{array}$ & $\begin{array}{l}- \\
3.9 \\
2.6 \\
2.8 \\
2.6 \\
4.7\end{array}$ & 15 & 356 \\
\hline $\begin{array}{l}29.0 \\
29,1\end{array}$ & $\begin{array}{l}2339-0128 \\
0128-0327\end{array}$ & $\begin{array}{l}0-1027 \\
1000-0\end{array}$ & $\begin{array}{l}1.2 \\
2.1\end{array}$ & 14.5 & $\begin{array}{l}26 \\
30\end{array}$ & $\begin{array}{l}022 \\
003\end{array}$ \\
\hline $\begin{array}{l}30,0 \\
30,1 \\
30,2 \\
30,3 \\
30,4 \\
30,5\end{array}$ & $\begin{array}{l}1329-1452 \\
1452-1524 \\
1524-1554 \\
1554-1618 \\
1618-1638 \\
1638-1711\end{array}$ & $\begin{array}{c}0-1016 \\
1000-755 \\
800-600 \\
600-401 \\
401-301 \\
301-0\end{array}$ & $\begin{array}{l}1.3 \\
2.4 \\
2.2 \\
2.6 \\
2.5 \\
2.6\end{array}$ & $\begin{array}{l}- \\
4.3 \\
3.7 \\
3.2 \\
2.5 \\
3.9\end{array}$ & 30 & 012 \\
\hline $\begin{array}{l}31,0 \\
31,1 \\
31,2 \\
31,3 \\
31,4 \\
31,5\end{array}$ & $\begin{array}{l}1156-1320 \\
1320-1343 \\
1343-1410 \\
1410-1434 \\
1434-1449 \\
1449-1523\end{array}$ & $\begin{array}{c}0-1010 \\
1001-800 \\
800-600 \\
600-395 \\
395-300 \\
300-0\end{array}$ & $\begin{array}{l}1.1 \\
2.2 \\
2.2 \\
2.1 \\
2.4 \\
2.4\end{array}$ & $\begin{array}{l}2.9 \\
3.5 \\
2.8 \\
2.0 \\
4.5\end{array}$ & 45 & 044 \\
\hline $\begin{array}{l}32,0 \\
32,1 \\
32,3 \\
32,4 \\
32,5\end{array}$ & $\begin{array}{l}2204-2340 \\
2340-0005 \\
0005-0022 \\
0022-0041 \\
0041-0113\end{array}$ & $\begin{array}{c}0-1012 \\
1000-727 \\
727-499 \\
499-402 \\
402-50\end{array}$ & $\begin{array}{l}1.2 \\
2.2 \\
2.3 \\
2.4 \\
2.5\end{array}$ & $\begin{array}{l}- \\
2.8 \\
2.0 \\
2.5 \\
3.8\end{array}$ & 37 & 038 \\
\hline $\begin{array}{l}33,0 \\
33,1 \\
33,2 \\
33,3 \\
33,4 \\
33,5\end{array}$ & $\begin{array}{l}2332-0053 \\
0053-0156 \\
0156-0212 \\
0212-0230 \\
0230-0248 \\
0248-0313\end{array}$ & $\begin{array}{c}0-1013 \\
1000-501 \\
501-401 \\
401-200 \\
200-60 \\
60-0\end{array}$ & $\begin{array}{l}1.4 \\
2.2 \\
1.8 \\
2.3 \\
2.3 \\
2.0\end{array}$ & $\begin{array}{l}- \\
8.1 \\
1.8 \\
2.3 \\
2.4 \\
3.0\end{array}$ & 139 & 039 \\
\hline $\begin{array}{l}34,2 \\
34,3 \\
34,4 \\
34,5\end{array}$ & $\begin{array}{l}2110-2232 \\
2232-2314 \\
2314-2338 \\
2338-2358\end{array}$ & $\begin{array}{c}0-1015 \\
1000-400 \\
400-80 \\
80-0\end{array}$ & $\begin{array}{l}1.3 \\
2.4 \\
2.2 \\
1.6\end{array}$ & $\begin{array}{l}- \\
4.4 \\
2.7 \\
2.2\end{array}$ & 356 & 035 \\
\hline
\end{tabular}


Table 9. Summary of MOCNESS-20 collections, Oceanus Cruise 125.

\begin{tabular}{|c|c|c|c|c|c|c|}
\hline $\begin{array}{c}\text { MOCNESS } \\
20\end{array}$ & $\begin{array}{l}\text { Time } \\
(+4)\end{array}$ & $\begin{array}{l}\text { Depth } \\
\text { (m) }\end{array}$ & $\begin{array}{c}\text { Speed } \\
\text { (knots) }\end{array}$ & $\begin{array}{c}\text { Volume } \\
\left(10,000 \mathrm{~m}^{3}\right)\end{array}$ & $\begin{array}{l}\text { Distance } \\
\text { to Ring } \\
\text { Center } \\
(\mathrm{km})\end{array}$ & $\begin{array}{c}\text { Angle } \\
\text { from Ring } \\
\text { Center }\end{array}$ \\
\hline $\begin{array}{l}35,0 \\
35,1 \\
35,2 \\
35,3 \\
35,4 \\
35,5\end{array}$ & $\begin{array}{l}1316-1539 \\
1539-1551 \\
1551-1608 \\
1608-1633 \\
1633-1711 \\
1711-1720\end{array}$ & $\begin{array}{l}0-1042 \\
992-800 \\
800-601 \\
601-402 \\
402-201 \\
201-0\end{array}$ & $\begin{array}{l}1.0 \\
2.1 \\
1.9 \\
2.2 \\
2.4 \\
2.6\end{array}$ & $\begin{array}{l}- \\
1.4 \\
1.8 \\
3.0 \\
4.7 \\
1.0\end{array}$ & 318 & 035 \\
\hline 36,0 & $2334-0248$ & $0-1017-0$ & 2.0 & - & 318 & 037 \\
\hline $\begin{array}{l}37,0 \\
37,1\end{array}$ & $\begin{array}{l}1355-1529 \\
1529-1714\end{array}$ & $\begin{array}{l}0-1082 \\
998-0\end{array}$ & $\begin{array}{l}1.4 \\
2.1\end{array}$ & 13.0 & $\begin{array}{l}23 \\
29\end{array}$ & $\begin{array}{l}217 \\
211\end{array}$ \\
\hline $\begin{array}{l}38,0 \\
38,1\end{array}$ & $\begin{array}{l}0125-0231 \\
0231-0414\end{array}$ & $\begin{array}{l}0-1002 \\
1000-0\end{array}$ & $\begin{array}{l}1.3 \\
2.1\end{array}$ & 13.2 & $\begin{array}{l}21 \\
30\end{array}$ & $\begin{array}{l}220 \\
222\end{array}$ \\
\hline $\begin{array}{l}39,1 \\
39,2\end{array}$ & $\begin{array}{l}0042-0155 \\
0155-0408\end{array}$ & $\begin{array}{c}0-1004 \\
1009-0\end{array}$ & $\begin{array}{l}1.1 \\
2.1\end{array}$ & 17.0 & $\begin{array}{l}10 \\
22\end{array}$ & $\begin{array}{l}264 \\
255\end{array}$ \\
\hline $\begin{array}{l}41,0 \\
41,1 \\
41,2 \\
41,3\end{array}$ & $\begin{array}{l}0113-0243 \\
0243-0320 \\
0320-0350 \\
0350-0446\end{array}$ & $\begin{array}{c}0-1004 \\
1004-750 \\
750-500 \\
500-0\end{array}$ & $\begin{array}{l}1.4 \\
2.0 \\
1.9 \\
2.2\end{array}$ & $\begin{array}{l}- \\
4.7 \\
3.5 \\
7.7\end{array}$ & 27 & 281 \\
\hline $\begin{array}{l}42,0 \\
42,1 \\
42,2 \\
42,3 \\
42,4\end{array}$ & $\begin{array}{l}0105-0228 \\
0228-0244 \\
0244-0313 \\
0313-0339 \\
0339-0441\end{array}$ & $\begin{array}{c}0-1070 \\
1000-800 \\
800-601 \\
601-383 \\
383-0\end{array}$ & $\begin{array}{l}1.3 \\
2.3 \\
2.5 \\
2.5 \\
2.4\end{array}$ & $\begin{array}{l}- \\
2.1 \\
4.1 \\
3.4 \\
8.0\end{array}$ & 28 & 246 \\
\hline $\begin{array}{l}43,0 \\
43,1 \\
43,2 \\
43,3 \\
43,4 \\
43,5\end{array}$ & $\begin{array}{l}1332-1516 \\
1516-1531 \\
1531-1548 \\
1548-1607 \\
1617-1641 \\
1641-1654\end{array}$ & $\begin{array}{l}\quad 0-1037 \\
994-797 \\
797-597 \\
597-399 \\
399-115 \\
115-0\end{array}$ & $\begin{array}{l}1.5 \\
2.4 \\
2.3 \\
2.2 \\
2.6 \\
2.3\end{array}$ & $\begin{array}{l}- \\
2.1 \\
2.3 \\
2.4 \\
4.8 \\
1.8\end{array}$ & 8 & 180 \\
\hline $\begin{array}{l}44,0 \\
44,1 \\
44,2 \\
44,3\end{array}$ & $\begin{array}{l}0008-0156 \\
0156-0217 \\
0217-0239 \\
0239-0402\end{array}$ & $\begin{array}{c}0-1028 \\
1001-797 \\
797-598 \\
598-0\end{array}$ & $\begin{array}{l}1.5 \\
2.0 \\
2.2 \\
2.4\end{array}$ & $\begin{array}{r}- \\
2.6 \\
3.0 \\
10.8\end{array}$ & 15 & 042 \\
\hline
\end{tabular}


Table 9 (continued)

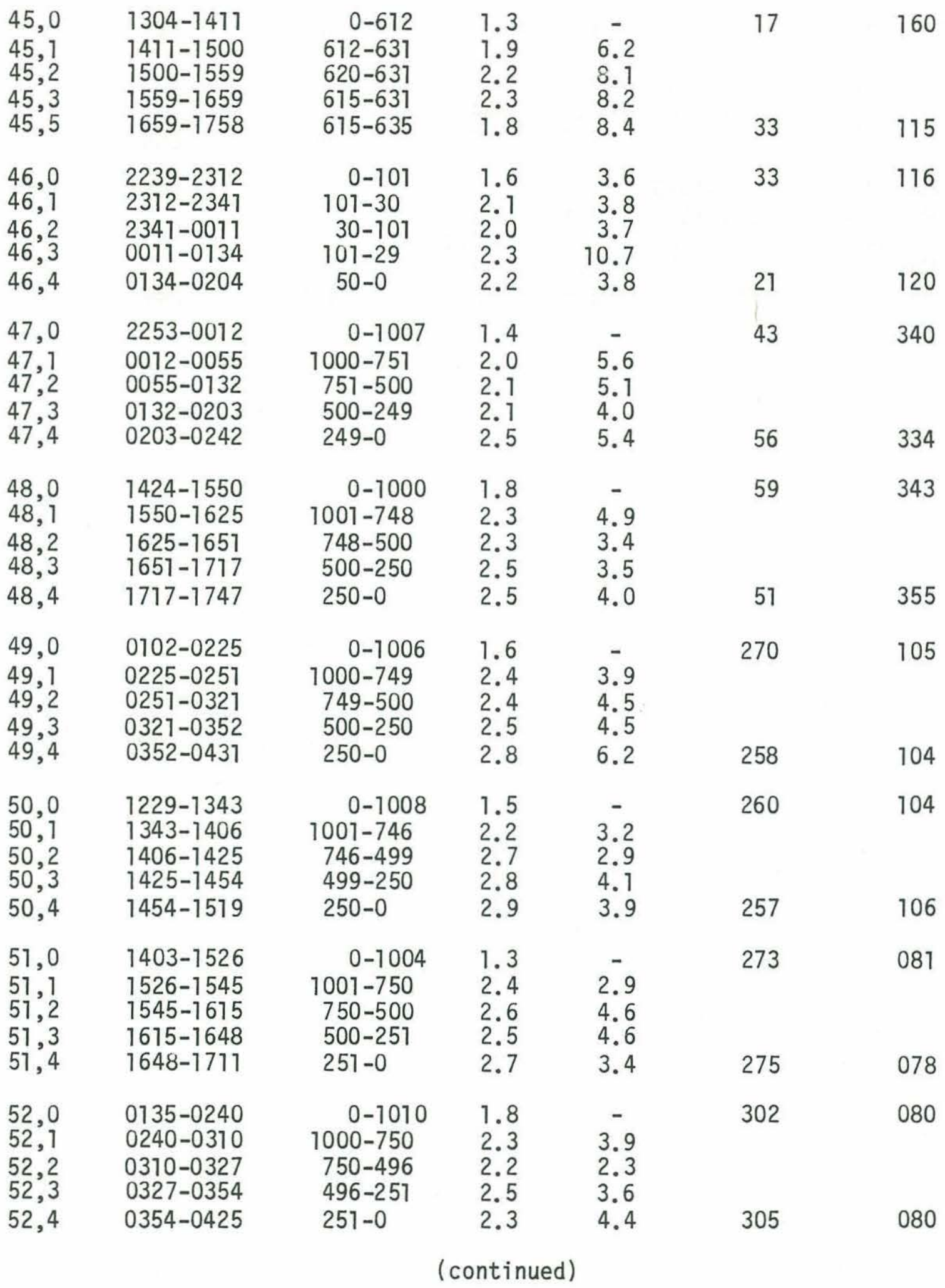




\section{Table 9 (continued)}

$\begin{array}{lcccccc}53,0 & 2346-0104 & 0-1015 & 1.8 & - & 364 & 057 \\ 53,1 & 0104-0134 & 1002-751 & 2.4 & 4.5 & & \\ 53,2 & 0134-0200 & 751-500 & 2.4 & 3.9 & & \\ 53,3 & 0200-0232 & 500-250 & 2.4 & 4.5 & 368 & 055 \\ 53,4 & 0232-0308 & 250-0 & 2.5 & 5.2 & & \\ & & & & & 415 & 046 \\ 54,0 & 1235-1400 & 0-1015 & 1.5 & - & & \\ 54,1 & 1400-1426 & 1001-750 & 2.1 & 3.0 & \\ 54,2 & 1426-1456 & 750-500 & 2.0 & 3.3 & & \\ 54,3 & 1456-1526 & 500-251 & 2.2 & 3.2 & 424 & 044 \\ 54,4 & 1526-1556 & 251-0 & 2.5 & 3.4 & & \end{array}$


Table 10. Summary of MOCNESS-20 collections, Knorr Cruise 98.

\begin{tabular}{|c|c|c|c|c|c|c|}
\hline $\begin{array}{c}\text { MOCNESS } \\
20\end{array}$ & $\begin{array}{l}\text { Time } \\
(+4)\end{array}$ & $\begin{array}{c}\text { Depth } \\
(\mathrm{m})\end{array}$ & $\begin{array}{c}\text { Speed } \\
\text { (knots) }\end{array}$ & $\begin{array}{l}\text { Volume } \\
\left(10,000 \mathrm{~m}^{3}\right)\end{array}$ & $\begin{array}{c}\text { Distance } \\
\text { to Ring } \\
\text { Center } \\
(\mathrm{km})\end{array}$ & $\begin{array}{c}\text { Angle } \\
\text { from Ring } \\
\text { Center }\end{array}$ \\
\hline $\begin{array}{l}55,0 \\
55,1 \\
55,2 \\
55,4 \\
55,5\end{array}$ & $\begin{array}{l}0111-0212 \\
0212-0226 \\
0226-2357 \\
0256-0318 \\
0318-0355\end{array}$ & $\begin{array}{l}\quad 0-1011 \\
997-797 \\
797-400 \\
400-204 \\
204-0\end{array}$ & $\begin{array}{l}1.6 \\
1.6 \\
2.0 \\
2.6 \\
2.1\end{array}$ & $\begin{array}{l}- \\
0.9 \\
2.5 \\
2.2 \\
3.8\end{array}$ & 97 & 252 \\
\hline $\begin{array}{l}56,0 \\
56,1 \\
56,2 \\
56,3 \\
56,4 \\
56,5\end{array}$ & $\begin{array}{l}0012-0131 \\
0131-0202 \\
0202-0222 \\
0222-0242 \\
0242-0301 \\
0301-0333\end{array}$ & $\begin{array}{c}0-1023 \\
1006-802 \\
802-602 \\
602-401 \\
401-201 \\
201-0\end{array}$ & $\begin{array}{l}1.4 \\
2.2 \\
2.2 \\
2.3 \\
2.2 \\
2.2\end{array}$ & $\begin{array}{l}- \\
3.2 \\
1.9 \\
1.9 \\
1.8 \\
3.4\end{array}$ & 88 & 099 \\
\hline $\begin{array}{l}57,0 \\
57,1 \\
57,2 \\
57,3 \\
57,4 \\
57,5\end{array}$ & $\begin{array}{l}2331-0040 \\
0040-0116 \\
0116-0146 \\
0146-0224 \\
0224-0256 \\
0256-0329\end{array}$ & $\begin{array}{c}0-1007 \\
1001-800 \\
800-602 \\
602-401 \\
401-203 \\
203-0\end{array}$ & $\begin{array}{l}1.2 \\
2.4 \\
2.5 \\
2.3 \\
2.0 \\
2.0\end{array}$ & $\begin{array}{c}- \\
3.8 \\
3.2 \\
4.0 \\
3.2 \\
3.2\end{array}$ & 70 & 099 \\
\hline $\begin{array}{l}58,0 \\
58,1 \\
58,2 \\
58,3 \\
58,4 \\
58,5\end{array}$ & $\begin{array}{l}1241-1356 \\
1356-1431 \\
1431-1502 \\
1502-1523 \\
1523-1544 \\
1544-1627\end{array}$ & $\begin{array}{c}0-1004 \\
1000-800 \\
800-599 \\
599-400 \\
400-305 \\
305-40\end{array}$ & $\begin{array}{l}1.5 \\
2.4 \\
2.0 \\
2.4 \\
2.5 \\
1.8\end{array}$ & $\begin{array}{l}- \\
3.8 \\
3.1 \\
2.1 \\
2.4 \\
4.0\end{array}$ & 39 & 098 \\
\hline $\begin{array}{l}59,0 \\
59,1 \\
59,2 \\
59,3 \\
59,4 \\
59,5\end{array}$ & $\begin{array}{l}0056-0202 \\
0202-0242 \\
0242-0308 \\
0308-0340 \\
0340-0404 \\
0404-0431\end{array}$ & $\begin{array}{c}0-1008 \\
1002-800 \\
800-599 \\
599-401 \\
401-200 \\
200-7\end{array}$ & $\begin{array}{l}1.2 \\
2.2 \\
2.3 \\
2.4 \\
2.5 \\
2.3\end{array}$ & $\begin{array}{l}- \\
4.4 \\
2.9 \\
3.4 \\
2.5 \\
3.0\end{array}$ & 150 & 008 \\
\hline $\begin{array}{l}60,0 \\
60,1 \\
60,2 \\
60,3 \\
60,4 \\
60,5\end{array}$ & $\begin{array}{l}1344-1445 \\
1445-1515 \\
1515-1536 \\
1536-1604 \\
1604-1623 \\
1623-1658\end{array}$ & $\begin{array}{c}0-1010 \\
1000-800 \\
800-600 \\
600-399 \\
399-302 \\
302-10\end{array}$ & $\begin{array}{l}1.2 \\
2.3 \\
2.5 \\
2.6 \\
2.8 \\
2.9\end{array}$ & $\begin{array}{l}- \\
3.4 \\
2.3 \\
2.9 \\
2.2 \\
3.8\end{array}$ & 148 & 012 \\
\hline
\end{tabular}


Table 10 (continued)

\begin{tabular}{|c|c|c|c|c|c|c|}
\hline $\begin{array}{l}61,0 \\
61,1 \\
61,2 \\
61,3 \\
61,4 \\
61,5\end{array}$ & $\begin{array}{l}0019-0119 \\
0119-0157 \\
0157-0223 \\
0223-0250 \\
0250-0321 \\
0321-0354\end{array}$ & $\begin{array}{c}0-1005 \\
1002-800 \\
800-600 \\
600-400 \\
400-200 \\
200-12\end{array}$ & $\begin{array}{l}1.3 \\
1.9 \\
2.0 \\
2.1 \\
2.2 \\
2.5\end{array}$ & $\begin{array}{l}- \\
3.8 \\
2.6 \\
2.7 \\
3.3 \\
4.7\end{array}$ & 55 & 025 \\
\hline $\begin{array}{l}62,0 \\
62,1 \\
62,2 \\
62,3 \\
62,4 \\
62,5\end{array}$ & $\begin{array}{l}1121-1223 \\
1223-1252 \\
1252-1326 \\
1326-1416 \\
1416-1448 \\
1448-1533\end{array}$ & $\begin{array}{l}0-1004 \\
999-802 \\
802-602 \\
602-400 \\
400-301 \\
301-5\end{array}$ & $\begin{array}{l}1.1 \\
1.8 \\
2.2 \\
1.9 \\
2.0 \\
2.1\end{array}$ & $\begin{array}{l}- \\
2.8 \\
3.7 \\
4.9 \\
3.4 \\
4.8\end{array}$ & 32 & 052 \\
\hline $\begin{array}{l}63,0 \\
63,1 \\
63,2 \\
63,3 \\
63,4 \\
63,5\end{array}$ & $\begin{array}{l}2313-0020 \\
0020-0054 \\
0054-0120 \\
0120-0142 \\
0142-0225 \\
0225-0325\end{array}$ & $\begin{array}{c}0-1015 \\
1000-800 \\
800-600 \\
600-402 \\
402-370 \\
370-5\end{array}$ & $\begin{array}{l}1.0 \\
1.6 \\
1.9 \\
2.0 \\
2.1 \\
2.1\end{array}$ & $\begin{array}{l}- \\
3.2 \\
2.6 \\
2.1 \\
4.6 \\
6.6\end{array}$ & 32 & 038 \\
\hline $\begin{array}{l}64,0 \\
64,1 \\
64,2 \\
64,3 \\
64,4 \\
64,5\end{array}$ & $\begin{array}{l}1337-1445 \\
1445-1512 \\
1512-1533 \\
1533-1548 \\
1548-1604 \\
1604-1647\end{array}$ & $\begin{array}{l}0-1015 \\
999-800 \\
800-600 \\
600-502 \\
502-400 \\
400-3\end{array}$ & $\begin{array}{l}1.1 \\
1.7 \\
1.9 \\
1.8 \\
1.9 \\
2.3\end{array}$ & $\begin{array}{l}- \\
2.6 \\
2.1 \\
1.4 \\
1.6 \\
4.8\end{array}$ & 8 & 041 \\
\hline $\begin{array}{l}65,0 \\
65,1 \\
65,2 \\
65,3 \\
65,4 \\
65,5\end{array}$ & $\begin{array}{l}0022-0155 \\
0155-0243 \\
0243-0311 \\
0311-0335 \\
0335-0405 \\
0405-0428\end{array}$ & $\begin{array}{c}0-1020 \\
1014-800 \\
800-599 \\
599-400 \\
400-199 \\
199-6\end{array}$ & $\begin{array}{l}1.3 \\
1.9 \\
1.6 \\
1.7 \\
1.7 \\
1.6\end{array}$ & $\begin{array}{l}- \\
5.2 \\
2.5 \\
2.2 \\
2.9 \\
2.3\end{array}$ & & \\
\hline $\begin{array}{l}66,0 \\
66,1 \\
66,2 \\
66,3 \\
66,4 \\
66,5\end{array}$ & $\begin{array}{l}1307-1415 \\
1415-1441 \\
1441-1502 \\
1502-1530 \\
1530-1544 \\
1544-1628\end{array}$ & $\begin{array}{c}0-1018 \\
1000-799 \\
799-601 \\
601-400 \\
400-299 \\
299-7\end{array}$ & $\begin{array}{l}1.0 \\
1.8 \\
1.9 \\
1.8 \\
1.9 \\
1.9\end{array}$ & $\begin{array}{l}- \\
2.6 \\
2.1 \\
2.7 \\
1.4 \\
4.8\end{array}$ & & \\
\hline
\end{tabular}


Table 11. Fishes collected with the MOCNESS-20 during the warm-core Gulf Stream rings cruises. (* Number from up nets only)

\begin{tabular}{|c|c|c|c|c|c|}
\hline \multirow{3}{*}{ Eurypharynx pelecanoides* } & \multicolumn{5}{|c|}{ Number of specimens } \\
\hline & A 110 & Oc 118 & Oc 121 & Oc 125 & Kn 98 \\
\hline & 8 & 3 & 12 & 15 & 11 \\
\hline Saccopharynx ampullaceus* & & & & & 2 \\
\hline $\begin{array}{l}\text { Derichthys serpentinus* } \\
\text { Nessorhamphus ingolfianus* }\end{array}$ & $\begin{array}{l}4 \\
3\end{array}$ & 10 & $\begin{array}{r}22 \\
3\end{array}$ & $\begin{array}{r}22 \\
9\end{array}$ & $\begin{array}{l}6 \\
1\end{array}$ \\
\hline $\begin{array}{l}\text { Serrivomer beanii* } \\
\text { Serrivomer brevidentatus* }\end{array}$ & $\begin{array}{r}16 \\
1\end{array}$ & $\begin{array}{l}6 \\
2\end{array}$ & 24 & $\begin{array}{r}49 \\
3\end{array}$ & $\begin{array}{r}28 \\
3\end{array}$ \\
\hline $\begin{array}{l}\text { Nemichthys curvirostris } \\
\text { Nemichthys scolopaceus }\end{array}$ & $\begin{array}{l}1 \\
1\end{array}$ & 4 & 16 & 27 & 14 \\
\hline Bathylagus spp.* & 16 & 26 & 26 & 27 & 14 \\
\hline $\begin{array}{l}\text { Dolichopteryx binocularis } \\
\text { Opisthoproctus grimaldi i } \\
\text { Rhynchohyal is natalensis }\end{array}$ & & & 1 & & 1 \\
\hline $\begin{array}{l}\text { Bonapartia pedaliota } \\
\text { Cyclothone accljinidens* } \\
\text { Cyclothone alba } \\
\text { Cyclothone braueri } \\
\text { Cyclothone microdon } \\
\text { Cyclothone pallida* } \\
\text { Cyclothone pseudopallida* } \\
\text { Diplophos taenia } \\
\text { Gonostoma atlanticum }\end{array}$ & $\begin{array}{r}51 \\
20 \\
199 \\
3387 \\
5052 \\
158 \\
184 \\
2 \\
3\end{array}$ & $\begin{array}{r}44 \\
21 \\
161 \\
4373 \\
6413 \\
470 \\
263\end{array}$ & $\begin{array}{r}34 \\
44 \\
298 \\
8435 \\
9178 \\
560 \\
371 \\
2\end{array}$ & $\begin{array}{r}101 \\
59 \\
448 \\
7707 \\
15272 \\
1024 \\
491\end{array}$ & $\begin{array}{r}180 \\
28 \\
324 \\
12108 \\
6014 \\
914 \\
388\end{array}$ \\
\hline $\begin{array}{l}\text { Gonostoma atlanticum } \\
\text { Gonostoma bathyphilum }\end{array}$ & 3 & 2 & 3 & $\begin{array}{l}7 \\
3\end{array}$ & $\begin{array}{l}4 \\
2\end{array}$ \\
\hline Gonostoma elongatum & 63 & 46 & 67 & 908 & 207 \\
\hline Margrethia obtusirostra & 6 & 1 & 2 & 9 & 38 \\
\hline $\begin{array}{l}\text { Ichthyococcus ovatus } \\
\text { Pollichthys mauli } \\
\text { Vinciguerria attenuata } \\
\text { Vinciguerria nimbaria } \\
\text { Vinciguerria poweriae } \\
\text { Yarella blackfordi }\end{array}$ & $\begin{array}{r}24 \\
1 \\
34 \\
4 \\
21\end{array}$ & $\begin{array}{r}1 \\
15 \\
6 \\
7 \\
14\end{array}$ & $\begin{array}{r}5 \\
33 \\
214 \\
24 \\
192\end{array}$ & $\begin{array}{r}40 \\
94 \\
176 \\
60 \\
647 \\
1\end{array}$ & $\begin{array}{r}109 \\
111 \\
185 \\
16 \\
49\end{array}$ \\
\hline $\begin{array}{l}\text { Argyropelecus aculeatus } \\
\text { Argyropelecus affinis } \\
\text { Argyropelecus hemigymnus } \\
\text { Argyropelecus sladeni }\end{array}$ & 90 & $\begin{array}{r}92 \\
1 \\
169\end{array}$ & $\begin{array}{r}61 \\
1 \\
220 \\
1\end{array}$ & $\begin{array}{r}62 \\
1 \\
84\end{array}$ & 233 \\
\hline
\end{tabular}


Table 11 (continued

Maurolicus muelleri

Polyipnus asteroides

Sternoptyx diaphana

Sternoptyx pseudobscura

Valenciennellus tripunctulatus

Aristostomias grimaldi $i$

Aristostomias lunifer

Aristostomias polydactylus

Aristostomias tittmanni

Astronesthes gemmifer

Astronesthes macropogon

Astronesthes micropogon

Astrones thes neopogon

Astronesthes niger

Astronesthes similis

Bathophilus altipinnis

Bathophilus brevis

Bathophilus digitatus

Bathophilus longipinnis

Bathophilus pawneei

Bathophilus vaillanti

Borostomias antarcticus

Chauliodus danae*

Chauliodus sloani*

Chirostomias pliopterus

Echiostoma barbatum

Eustomias bibulbosus

Eustomias enbarbatus

Eustomias filifer

Eustomias 1 ipochirus

Eustomias macronema

Eustomias obscurus

Eustomias schiffi

Eustomias schmidti

Eustomias sp. nov.

Flagellostomias boureei

Grammatostomias circularis

Grammatostomias dentatus

Grammatostomias flagellibarba

Idiacanthus fasciola

Leptostomias sp.

Macrostomias longibarbatus*

Malacosteus niger

Melanostomias bartonbeani

Melanostomias biseriatus

Melanostomias melanopogon

Melanostomias sp. nov.

Melanostomias tentaculatus

Melanostomias valdiviae

$\begin{array}{rrrrr}2 & 22 & 131 & 55 & 10 \\ 5 & 1 & 2 & 5 & 16 \\ 166 & 186 & 119 & 590 & 586 \\ & & 5 & 2 & 2 \\ 36 & 86 & 178 & 132 & 113\end{array}$

\section{3}

1

1

1

3

1

2

1

$19 \quad 10$

$87 \quad 23$

$10 \quad 98$

621

1

1

1

$3-1$

$\begin{array}{ll}6 & 2\end{array}$

1

1

13

22

$33 \quad 37$

$303 \quad 126$

13

1

1

1

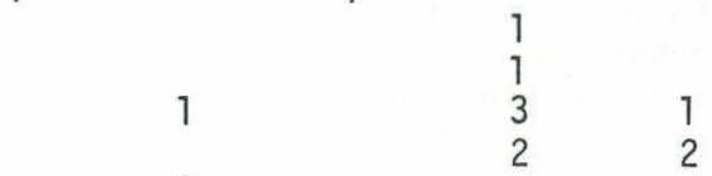

1

$\begin{array}{lllll}13 & 16 & 8 & 19 & 13\end{array}$

$\begin{array}{lllll}7 & 13 & 5 & 9 & 13\end{array}$

$\begin{array}{lllll}3 & 2 & 2 & 18 & 13\end{array}$

13

1

$\begin{array}{lll}1 & 8 & 3\end{array}$

(continued) 
Table 11 (continued)

Neonesthes capensis

Pachystomias microdon

Photonectes braueri

Photonectes margarita

Photonectes mirabil is

Photonectes parvimanus

Photostomias guernei

Photostomias megistus

Stomias affinis

Stomias boa ferox*

Stomias brevibarbatus*

Photostylus pycnopterus

Xenodermichthys copei

$\begin{array}{rrrrr} & 3 & 1 & & 1 \\ & 1 & & & 2 \\ & 10 & 12 & 3 & 1 \\ & & & & 5 \\ & & & & 1 \\ 5 & 10 & 14 & 11 & 18 \\ & 3 & 5 & 4 & 15 \\ 6 & 1 & & 1 & 3 \\ 26 & 16 & 365 & 65 & 12 \\ 1 & 2 & & 1 & 3\end{array}$

Searsia koefoedi

Barbantus sp.

Holtbyrnia sp.

unidentified searsiids

Scopelosaurus argenteus

Scopelosaurus mauli

Scopelosaurus smithii

Alepisaurus spp.

Omosudis lowei

3

16

Rosenblattichthys hubbsi

Scopelarchoides danae

Scopelarchus anal is

Scopelarchus michaelsarsi

Coccorella atlantica

Evermannel la balbo

Evermannella indica

Sudis atrox

Sudis hyalina

unidentified paralepidids

Benthosema glaciale

Benthosema suborbitale

Bol inichthys indicus

Bolinichthys photothorax

Bolinichthys supralateral is

Centrobranchus nigroocell atus

Ceratoscopelus maderensis

Ceratoscopelus warmingi $i$

$\begin{array}{rrrr} & 1 & & \\ & 1 & 1 & \\ 21 & 1 & 1 & \\ & 25 & 21 & 19\end{array}$

1

3

$29 \quad 16$

1

9

7

2

3

Diaphus brachycephalus

$\begin{array}{rrrrr} & & & 1 & \\ 4 & 2 & 3 & 71 & 39 \\ 1 & & 1 & 10 & 1 \\ 1 & 1 & 1 & 13 & 8 \\ & 1 & 1 & 2 & \\ & 1 & & 34 & 36 \\ 1 & & & 6 & 2 \\ 1 & & 1 & & 4 \\ 26 & 54 & 100 & 115 & 111 \\ 577 & 289 & 13966 & 3581 & 506 \\ 36 & 34 & 27 & 75 & 72 \\ 87 & 87 & 49 & 134 & 396 \\ & 1 & 4 & 2 & \\ 9 & 3 & 6 & 24 & 64 \\ 1 & 3 & 3 & 8 & 5 \\ 424 & 1 & 232 & 9808 & 960 \\ 157 & 29 & 75 & 468 & 424 \\ & & & 2 & 5\end{array}$


Table 11 (continued)

Diaphus dumerilii

Diaphus effulgens

Diaphus fragilis

Diaphus garmani

Diaphus lucidus

Diaphus luetkeni

Diaphus metopoclampus

Diaphus mollis

Diaphus perspicillatus

Diaphus problematicus

Diaphus rafinesqui i

Diaphus splendidus

Diaphus termophilus

Diogenichthys atlanticus

Gonichthys cocco

Hygophum benoiti

Hygophum hygomi $i$

Hygophum macrochir

Hygophum reinhardti $i$

Hygophum taaningi

Lampadena anoma1a

Lampadena luminosa

Lampadena speculigera

Lampadena urophaos

Lampanyc tus a latus

Lampanyctus ater

Lampanyctus crocodilus

Lampanyctus cuprarius

Lampanyctus festivus

Lampanyc tus intricarius

Lampanyctus 1 ineatus

Lampanyc tus macdonaldi

Lampanyctus nobilis

Lampanyctus photonotus

Lampanyctus pusil1us

Lampanyc tus tenuiformis

Lepidophanes gaussi

Lepidophanes guentheri

Lobianchia dofleini

Lobianchia gemellarii

Loweina rara

Myctophum affine

Myc tophum asperum

Myc tophum nitidulum

Myc tophum obtusirostre

Myc tophum punctatum

Myctophum selenops

Notolychnus valdiviae

Notoscopelus caudispinosus

$\begin{array}{rrrrr}42 & 64 & 51 & 170 & 33 \\ & 3 & 5 & 20 & 15 \\ & & & 1 & 4 \\ & 1 & & 1 & \\ 2 & 2 & 7 & 1 & 1 \\ 2 & & & & 1 \\ 42 & 22 & 16 & 8 & 67 \\ 11 & 13 & 30 & 76 & 71 \\ 5 & 3 & 2 & 11 & 23 \\ & & 1 & 1 \\ 4 & 211 & 262 & 47 & 5 \\ 5 & 1 & 1 & 8 & 67\end{array}$

$\begin{array}{rrrrr}2 & 1 & 1 & & 1 \\ 95 & 374 & 345 & 345 & 337\end{array}$

$\begin{array}{rrrrr}20 & 6 & 14 & 25 & 10\end{array}$

$\begin{array}{llll}4 & 26 & 281 & 303\end{array}$

$\begin{array}{llll}309 & 126 & 123 & 2\end{array}$

$\begin{array}{rrrrr}2 & & 1 & 1 & \\ 3 & 4 & 2 & 11 & 13\end{array}$

$\begin{array}{lllll}13 & 6 & 7 & 25 & 67\end{array}$

$\begin{array}{lllll}1 & 1 & 4 & 1 & \\ 2 & & 4 & 7 & 5\end{array}$

$\begin{array}{lllll}5 & 26 & 49 & 10 & 5\end{array}$

$\begin{array}{lllll}1 & 1 & 2 & 6 & 14\end{array}$

$\begin{array}{lllll}32 & 15 & 80 & 331 & 24\end{array}$

$\begin{array}{lllll}6 & 11 & 6 & 5 & 16\end{array}$

$\begin{array}{lllll}78 & 2 & 115 & 71 & 23\end{array}$

$\begin{array}{lllll}24 & 92 & 50 & 48 & 165\end{array}$

$\begin{array}{llll}9 & 1 & 26 & 19\end{array}$

123

$\begin{array}{lllll}2 & 3 & 4 & 6 & 22\end{array}$

$\begin{array}{lllll}3 & 5 & 10 & 9 & 1\end{array}$

$\begin{array}{rrrrr}1 & 1 & & 1 & \\ 29 & 13 & 16 & 16 & 125\end{array}$

$\begin{array}{lllll}88 & 36 & 181 & 427 & 234\end{array}$

$\begin{array}{lllll}8 & 2 & 12 & 98 & 104\end{array}$

$\begin{array}{lllll}57 & 33 & 33 & 84 & 114\end{array}$

$\begin{array}{lllll}221 & 8 & 590 & 1139 & 83\end{array}$

$\begin{array}{lllll}8 & 21 & 26 & 17 & 5\end{array}$

$\begin{array}{rrrrr}175 & 27 & 2 & 18 & 7 \\ & 1 & 127 & 21\end{array}$

$\begin{array}{lllll}8 & 1 & 31 & 26 & 13\end{array}$

$491 \quad 17$

$3 \quad 137$

$\begin{array}{lll}344 & 275 & 599\end{array}$

33

.

(1)

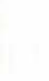

7

.


Table 11 (continued)

Notoscopelus resplendens

Protomyctophum arcticum

Symbolophorus rufinus

Symbolophorus veranyi

Taaningichthys bathyphilus

Taaningichthys minimus

Neoscopelus macrolepidotus

Neoscopelus microchir

Ataxolepis apus

Rondeletia loricata

Barbourisia rufa

Rosaura indica

Chaunax sp.

Caulophryne jordani

Caulophryne spp.

Melanocetus spp.

Himantolophus albinares?

Himantolophus spp.

Chaenophryne Tongiceps

Chaenophryne sp.

Danaphryne nigrifilis

Dolopichthys sp. nov.

Dolopichthys sp.

Leptacanthichthys gracilispinis

Lophodolus acanthognathus

Microlopichthys microlophus

Oneirodes macrosteus

Oneirodes eschrishti i

Oneirodes sp.

Spiniphryne gladisfenae

Ceratias holboelli

Ceratias uranoscopus

Cryptopsaras couesi

Gigantactis gibbsi

Gigantactis vanhoeffeni

Gigantactis sp. nov.

Gigantactis sp.

$\begin{array}{rrrrr}16 & 9 & 69 & 134 & 4 \\ 5 & 1 & & 7 & 2 \\ & & & 2 & 1 \\ 1 & 6 & 69 & 24 & 9 \\ 5 & 17 & 8 & 14 & 12 \\ & 2 & & 1 & \\ & & & 1 & 1\end{array}$

1

$\begin{array}{lll}1 & 1 & 1\end{array}$

1

1

$\begin{array}{lllll}4 & 5 & 7 & 10 & 5\end{array}$

1

1

1

2

1

2
1

1

1

1

5

1
1

1

3

1

1

3

2

2

2

1

19

13

(

2

(1)

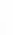

$\begin{array}{ll}1 & \\ 1 & 1 \\ 1 & 1 \\ & 2\end{array}$


Table 11 (continued)

Neoceratias spinifer

Haplophryne mollis

Linophryne arborifera

Linophryne spp.

$\begin{array}{rrrrr} & & & & 1 \\ & & & & 1 \\ & & 1 & & 4 \\ & & & 1 & \\ 1 & 4 & 2 & 3 & \\ 11 & 9 & 12 & 28 & 36 \\ & & & & \\ 71 & 28 & 19 & 363 & 496 \\ 3 & 1 & 6 & 1 & 1 \\ 1 & 3 & 5 & 4 & 6 \\ 5 & 1 & & 1 & 1 \\ 8 & 47 & 41 & 40 & 42 \\ 2 & 3 & 5 & 6 & 24 \\ 4 & 10 & 91 & 17 & 24 \\ 10 & 1 & 7 & 17 & 6\end{array}$

Melanonus zugmayeri

Bregmaceros spp. *

Melamphaes longivelis*

Melamphaes pumilus

Melamphaes suborbital is*

Melamphaes ebeling ${ }^{*}$

Poromitra capito*

Poromitra megalops*

Scopeloberyx opisthopterus*

Scopeloberyx robustus

Scopelogadus beani ${ }^{*}$

Scopelogadus mizolepis*

Diretmus argenteus

Anoplogaster cornuta

$\begin{array}{rrrrr} & 1 & 4 & 7 & 6 \\ 16 & 11 & 11 & 17 & 14\end{array}$

Howella brodei

2

Caristius sp.

4

37

16

10

3

Dysalotus alcocki

Kali normani

1

Pseudoscopelus sp 


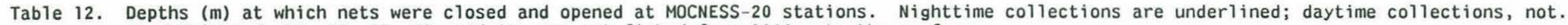
At stations $\underline{6}, 18, \underline{29}, 37, \underline{38}$, and $\underline{39}$, one net fished from $1000 \mathrm{~m}$ to the surface.

\begin{tabular}{|c|c|c|c|c|c|c|c|c|c|c|c|c|c|c|c|c|c|c|c|c|c|c|c|}
\hline & \multicolumn{4}{|c|}{$\begin{array}{c}\text { Oceanus } 118 \\
\text { April }\end{array}$} & \multicolumn{5}{|c|}{$\begin{array}{c}\text { Oceanus } 121 \\
\text { June }\end{array}$} & \multicolumn{5}{|c|}{$\begin{array}{l}\text { Oceanus } 125 \\
\text { August }\end{array}$} & \multicolumn{7}{|c|}{$\begin{array}{l}\text { Knorr } 98 \\
\text { Sept-0ct }\end{array}$} & \multicolumn{2}{|c|}{$\begin{array}{c}\text { A2 } 110 \\
\text { Sept-0ct }\end{array}$} \\
\hline $\begin{array}{l}\text { Slope } \\
\text { Water }\end{array}$ & $\begin{array}{l}\frac{8}{2} \\
\frac{250}{500} \\
\frac{750}{1000} \\
\end{array}$ & $\begin{array}{r}\frac{9}{0} \\
250 \\
500 \\
750 \\
1000\end{array}$ & & & $\begin{array}{c}\frac{19}{\frac{0}{0}} \\
\frac{103}{253} \\
\frac{503}{751} \\
1000 \\
\end{array}$ & $\begin{array}{r}20 \\
0 \\
251 \\
500 \\
751 \\
1001 \\
1250\end{array}$ & 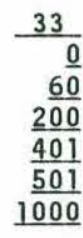 & $\begin{array}{r}\frac{34}{\frac{0}{80}} \\
\frac{400}{1000} \\
\end{array}$ & & $\begin{array}{r}\frac{35}{0} \\
201 \\
402 \\
601 \\
800 \\
992\end{array}$ & $\begin{array}{r}\frac{47}{\frac{0}{9}} \\
\frac{249}{500} \\
\frac{751}{1000} \\
\end{array}$ & $\begin{array}{r}\frac{48}{0} \\
250 \\
500 \\
748 \\
1001\end{array}$ & $\begin{array}{r}\frac{53}{\frac{0}{0}} \\
\frac{250}{500} \\
\frac{751}{1002} \\
\end{array}$ & $\begin{array}{r}54 \\
0 \\
251 \\
500 \\
750 \\
1001\end{array}$ & $\begin{array}{r}\frac{59}{7} \\
\frac{200}{401} \\
\frac{599}{800} \\
\underline{8002} \\
\end{array}$ & $\begin{array}{r}\frac{60}{10} \\
302 \\
399 \\
600 \\
800 \\
1000\end{array}$ & $\begin{array}{r}\frac{65}{\frac{6}{6}} \\
\frac{199}{400} \\
\frac{599}{800} \\
\frac{8014}{1014}\end{array}$ & $\begin{array}{r}66 \\
7 \\
299 \\
400 \\
601 \\
799 \\
1000\end{array}$ & & & & $\begin{array}{r}\frac{4}{0} \\
250 \\
500 \\
750 \\
1000\end{array}$ & $\begin{array}{r}\frac{5}{0} \\
\frac{250}{500} \\
\frac{750}{7500} \\
1000\end{array}$ \\
\hline $\begin{array}{l}\text { Ring } \\
\text { Core }\end{array}$ & $\begin{array}{l}\frac{10}{\frac{0}{0}} \\
\frac{250}{500} \\
\frac{750}{1000} \\
\end{array}$ & $\begin{array}{r}\frac{11}{0} \\
250 \\
500 \\
750 \\
1000\end{array}$ & $\begin{array}{r}\frac{16}{0} \\
400 \\
600 \\
800\end{array}$ & $\begin{array}{r}\frac{17}{\frac{0}{2}} \\
\frac{250}{500} \\
1017 \\
\end{array}$ & $\begin{array}{r}\frac{21}{0 \frac{0}{4}} \\
\frac{401}{\frac{501}{601}} \\
\frac{800}{1000} \\
\end{array}$ & $\begin{array}{l}\frac{23}{\frac{0}{0}} \\
\frac{151}{400} \\
\frac{600}{801} \\
\frac{8000}{1000} \\
\end{array}$ & $\begin{array}{r}24 \\
0 \\
382 \\
400 \\
600 \\
800 \\
1000\end{array}$ & $\begin{array}{r}\frac{28}{0} \\
317 \\
501 \\
749 \\
1001 \\
1250\end{array}$ & & & & & & & $\begin{array}{l}\frac{56}{\frac{0}{0}} \\
\frac{201}{401} \\
\underline{602} \\
\underline{802} \\
\underline{1006} \\
\end{array}$ & $\begin{array}{l}\frac{57}{\frac{0}{3}} \\
\frac{203}{401} \\
\frac{602}{800} \\
\frac{8001}{1001}\end{array}$ & $\begin{array}{r}\frac{58}{40} \\
305 \\
400 \\
599 \\
800 \\
1000\end{array}$ & $\begin{array}{l}\frac{61}{\frac{12}{200}} \\
\frac{200}{\frac{400}{600}} \\
\frac{800}{1002} \\
0\end{array}$ & $\begin{array}{r}\frac{62}{5} \\
301 \\
400 \\
602 \\
802 \\
999\end{array}$ & $\begin{array}{r}\frac{63}{6 \frac{5}{3}} \\
\frac{370}{402} \\
\frac{600}{800} \\
1000 \\
\end{array}$ & $\begin{array}{r}\frac{64}{3} \\
400 \\
502 \\
600 \\
800 \\
999\end{array}$ & $\begin{array}{r}\frac{1}{\frac{0}{0}} \\
\frac{250}{500} \\
\frac{750}{7000} \\
\end{array}$ & \\
\hline $\begin{array}{l}\text { Sargasso } \\
\text { Sea } \\
\& \\
\text { Gulf } \\
\text { Stream }\end{array}$ & & & & & & & & & & $\begin{array}{r}\frac{49}{0} \\
\frac{250}{500} \\
\frac{549}{1000} \\
\end{array}$ & $\begin{array}{r}50 \\
0 \\
250 \\
499 \\
746 \\
1001\end{array}$ & $\begin{array}{r}51 \\
0 \\
251 \\
500 \\
750 \\
1001\end{array}$ & $\begin{array}{r}\frac{52}{0} \\
\frac{251}{496} \\
\frac{750}{1000} \\
\end{array}$ & & & & & & & & & & \\
\hline
\end{tabular}


Table 13. Temperature $\left({ }^{\circ} \mathrm{C}\right)$ at depths where nets were closed and opened.

\begin{tabular}{|c|c|c|c|c|c|c|c|c|c|c|c|c|c|c|c|c|c|c|c|c|c|c|c|}
\hline \multirow[b]{2}{*}{$\begin{array}{l}\text { Slope } \\
\text { Water }\end{array}$} & \multicolumn{4}{|c|}{$\begin{array}{c}\text { Oceanus } 118 \\
\text { April }\end{array}$} & \multicolumn{5}{|c|}{$\begin{array}{c}\text { Oceanus } 121 \\
\text { June }\end{array}$} & \multicolumn{5}{|c|}{$\begin{array}{l}\text { Oceanus } 125 \\
\text { August }\end{array}$} & \multicolumn{7}{|c|}{$\begin{array}{l}\text { Knorr } 98 \\
\text { Sept-0ct }\end{array}$} & \multicolumn{2}{|c|}{$\begin{array}{c}\text { A2 } 110 \\
\text { Sept-0ct }\end{array}$} \\
\hline & $\frac{\frac{8}{12.5}}{\frac{9.5}{5.6}}$ & $\begin{array}{r}\frac{9}{13.9} \\
9.4 \\
5.7 \\
4.5 \\
4.2\end{array}$ & & & $\begin{array}{l}19 \\
= \\
= \\
= \\
= \\
= \\
=\end{array}$ & $\begin{array}{c}20 \\
- \\
- \\
- \\
- \\
-\end{array}$ & $\frac{\frac{33}{21.6}}{\frac{13.7}{10.6}} \frac{6.0}{\frac{5.1}{4.1}}$ & $\frac{\frac{34}{19.4}}{\frac{14.9}{7.2}} \frac{4.3}{4}$ & & $\begin{array}{r}\frac{35}{24.6} \\
11.9 \\
7.0 \\
5.0 \\
4.5 \\
4.3\end{array}$ & $\frac{\frac{47}{25.9}}{\frac{9.4}{5.5}} \frac{4.6}{\underline{4.3}}$ & $\begin{array}{r}\frac{48}{26.0} \\
8.9 \\
5.4 \\
4.5 \\
4.2\end{array}$ & 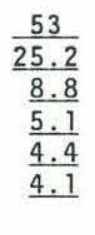 & $\begin{array}{r}\frac{54}{22.8} \\
9.2 \\
5.0 \\
4.5 \\
4.3\end{array}$ & $\frac{\frac{59}{23.1}}{\frac{23.1}{6.9}} \frac{5.1}{\frac{5.6}{4.3}}$ & $\begin{array}{r}\frac{60}{24.3} \\
10.4 \\
8.3 \\
5.2 \\
4.5 \\
4.2\end{array}$ & $\frac{\frac{65}{22.7}}{\frac{11.8}{7.1}} \frac{\frac{5.3}{4.5}}{\frac{4.1}{4.1}}$ & $\begin{array}{r}\frac{66}{23.1} \\
8.9 \\
7.1 \\
5.2 \\
4.5 \\
4.3\end{array}$ & & & & $\begin{array}{r}\frac{4}{20.9} \\
9.6 \\
5.4 \\
5.1 \\
5.1\end{array}$ & $\frac{\frac{5}{19.7}}{\frac{9.7}{5.2}} \frac{5.1}{\frac{5.1}{5.1}}$ \\
\hline $\begin{array}{l}\text { High } \\
\text { Velocity } \\
\text { Rergion }\end{array}$ & $\begin{array}{r}\frac{12}{15.6} \\
14.9 \\
8.9 \\
5.1 \\
4.5\end{array}$ & $\frac{\frac{13}{15.2}}{\frac{14.4}{8.9}} \frac{4.5}{\underline{4.5}}$ & $\frac{\frac{14}{14.6}}{\frac{13.5}{7.2}} \frac{4.9}{\underline{4.3}}$ & $\begin{array}{r}\frac{15}{15.0} \\
13.0 \\
6.9 \\
4.9 \\
4.3\end{array}$ & $\begin{array}{r}\frac{26}{17.6} \\
12.6 \\
9.1 \\
6.4 \\
4.8\end{array}$ & $\begin{array}{r}\frac{27}{14.9} \\
12.7 \\
7.8 \\
5.2 \\
4.5 \\
4.2\end{array}$ & $\begin{array}{r}\frac{30}{20.4} \\
14.4 \\
11.0 \\
6.2 \\
5.0 \\
4.4\end{array}$ & $\begin{array}{r}\frac{31}{21.1} \\
13.1 \\
10.2 \\
5.7 \\
4.8 \\
4.3\end{array}$ & $\frac{\frac{32}{15.5}}{\frac{10.7}{8.1}} \frac{\frac{5.1}{4.4}}{\underline{5}}$ & $\frac{\frac{41}{27.1}}{\frac{6.5}{4.8}} \frac{4.3}{\underline{4.3}}$ & $\frac{\frac{42}{28.1}}{\frac{8.2}{5.2}} \frac{5.5}{\underline{4.2}}$ & $\begin{array}{r}\frac{43}{27.5} \\
15.6 \\
8.0 \\
5.2 \\
4.6 \\
4.3\end{array}$ & $\frac{\frac{44}{28.0}}{\frac{5.2}{4.5}} \frac{4.2}{4}$ & & $\frac{\frac{55}{27.6}}{\frac{18.3}{15.1}} \frac{5.8}{\underline{4.8}}$ & & & & & & & $\frac{\frac{2}{22.9}}{\frac{11.3}{5.8}}$ & $\begin{array}{r}\frac{3}{22.2} \\
11.7 \\
6.3 \\
5.1 \\
5.1\end{array}$ \\
\hline $\begin{array}{l}\text { Ring } \\
\text { Core }\end{array}$ & $\frac{\frac{10}{15.7}}{\frac{15.6}{12.2}} \frac{6.1}{\frac{6.8}{4}}$ & $\begin{array}{r}\frac{11}{15.9} \\
15.7 \\
12.0 \\
6.1 \\
4.7\end{array}$ & $\begin{array}{r}\frac{16}{15.8} \\
15.7 \\
8.8 \\
5.7\end{array}$ & $\frac{\frac{17}{15.8}}{\frac{15.7}{11.9}} \frac{4.7}{4}$ & $\frac{\frac{21}{18.2}}{\frac{15.7}{15.3}} \frac{8.6}{\frac{8.4}{4.7}}$ & $\frac{\frac{23}{18.7}}{\frac{15.7}{14.9}} \frac{8.3}{\frac{8.3}{4.6}}$ & $\begin{array}{r}\frac{24}{18.6} \\
14.9 \\
14.6 \\
7.7 \\
5.2 \\
4.6\end{array}$ & $\begin{array}{r}\frac{28}{18.6} \\
15.6 \\
10.6 \\
5.5 \\
4.6 \\
4.2\end{array}$ & & & & & & & $\frac{\frac{56}{26.3}}{\frac{19.1}{17.7}} \frac{15.2}{\frac{10.5}{6.5}}$ & $\frac{\frac{57}{26.7}}{\frac{18.8}{17.7}} \frac{15.9}{\frac{11.1}{6.7}}$ & $\begin{array}{r}\frac{58}{25.9} \\
18.0 \\
17.6 \\
15.5 \\
10.9 \\
6.7\end{array}$ & $\frac{\frac{61}{25.3}}{\frac{18.5}{17.4}} \frac{14.4}{\frac{8.8}{5.5}}$ & $\begin{array}{r}\frac{62}{25.0} \\
18.1 \\
17.9 \\
15.5 \\
10.2 \\
6.2\end{array}$ & $\frac{\frac{63}{24.9}}{\frac{17.7}{17.6}} \frac{15.1}{\frac{10.6}{6.3}}$ & $\begin{array}{r}\frac{64}{24.3} \\
17.6 \\
17.0 \\
15.3 \\
10.3 \\
6.4\end{array}$ & $\frac{\frac{1}{23.3}}{\frac{17.8}{13.2}} \frac{7.2}{\underline{5.1}}$ & \\
\hline $\begin{array}{l}\text { Sargasso } \\
\text { Sea } \\
\& \\
\text { Gulf } \\
\text { Stream }\end{array}$ & & & & & & & & & & $\frac{\frac{49}{26.8}}{\frac{18.7}{17.5}} \frac{14.1}{\frac{9.4}{19}}$ & $\begin{array}{r}\frac{50}{26.9} \\
18.6 \\
17.5 \\
13.9 \\
8.9\end{array}$ & $\begin{array}{r}\frac{51}{28.7} \\
17.9 \\
13.0 \\
6.8 \\
4.7\end{array}$ & $\frac{\frac{52}{28.4}}{\frac{18.0}{13.7}} \frac{7.4}{\frac{7.8}{4.8}}$ & & & & & & & & & & \\
\hline
\end{tabular}


Table 14. Salinity $(\%)$ at depths where nets were closed and opened.

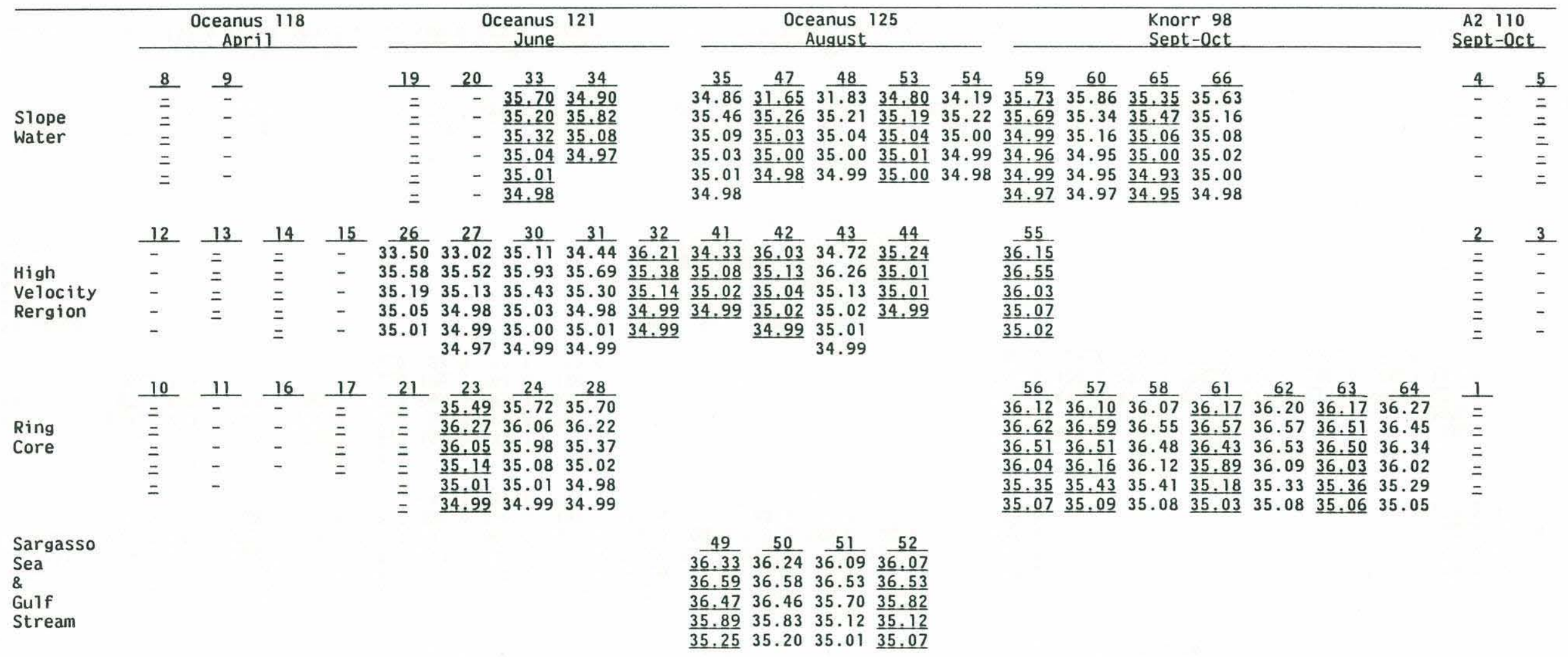


Table 15. Abundance of midwater fishes (specimens $/ 10,000 \mathrm{~m}^{3}$ ).

\begin{tabular}{|c|c|c|c|c|c|c|c|c|c|c|c|c|c|c|c|c|c|c|c|c|c|c|c|c|}
\hline & \multicolumn{4}{|c|}{$\begin{array}{c}\text { Oceanus } 118 \\
\text { April }\end{array}$} & \multicolumn{5}{|c|}{$\begin{array}{c}\text { Oceanus } 121 \\
\text { June }\end{array}$} & \multicolumn{5}{|c|}{$\begin{array}{l}\text { Oceanus } 125 \\
\text { August }\end{array}$} & \multicolumn{7}{|c|}{$\begin{array}{l}\text { Knorr } 98 \\
\text { Sept-0ct }\end{array}$} & \multicolumn{3}{|c|}{$\begin{array}{c}\text { A2 } 110 \\
\text { Sept-0ct }\end{array}$} \\
\hline $\begin{array}{l}\text { Slope } \\
\text { Water }\end{array}$ & 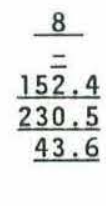 & $\begin{array}{r}\frac{9}{5.3} \\
323.5 \\
350.0 \\
75.3\end{array}$ & & & $\frac{\frac{19}{154.9}}{\frac{37.9}{13.2}} \frac{\frac{252.6}{56.3}}{\underline{56.0}}$ & $\begin{array}{r}\frac{20}{10.5} \\
101.6 \\
293.5 \\
74.1 \\
27.6\end{array}$ & $\frac{\frac{33}{499.0}}{\frac{15.4}{13.5}}$ & $\frac{\frac{34}{136.8}}{\frac{11.5}{210.7}}$ & & \begin{tabular}{r}
\multicolumn{1}{c}{$\frac{35}{5.0}$} \\
46.2 \\
749.7 \\
245.0 \\
68.6
\end{tabular} & $\frac{\frac{47}{36.9}}{\frac{49.0}{176.9}}$ & $\begin{array}{r}\frac{48}{5.8} \\
92.9 \\
219.4 \\
125.0\end{array}$ & $\frac{\frac{53}{76.5}}{\frac{71.3}{186.2}} \frac{36.0}{3}$ & $\begin{array}{r}\frac{54}{7.4} \\
110.0 \\
583.0 \\
225.7\end{array}$ & $\frac{\frac{59}{72.3}}{\frac{51.6}{101.5}} \frac{167.2}{65.7}$ & $\begin{array}{r}\frac{60}{7.6} \\
76.4 \\
237.2 \\
280.9 \\
68.5\end{array}$ & $\frac{\frac{65}{44.3}}{\frac{12.1}{245.0}} \frac{178.0}{79.6}$ & $\begin{array}{r}\frac{66}{5.4} \\
63.6 \\
347.0 \\
214.3 \\
106.5\end{array}$ & & & & $\begin{array}{l}\frac{4}{1.2} \\
196.7 \\
300.6 \\
102.1\end{array}$ & $\begin{array}{r}\frac{5}{\frac{39.7}{87.0}} \\
\frac{244.7}{117.4} \\
\end{array}$ & \\
\hline $\begin{array}{l}\text { High } \\
\text { Velocity } \\
\text { Region }\end{array}$ & $\begin{array}{r}\frac{12}{6.6} \\
234.6 \\
295.8 \\
86.6\end{array}$ & $\frac{\frac{13}{\frac{44.0}{195.0}}}{184.6}$ & $\frac{\frac{14}{46.6}}{\frac{197.0}{291.8}} \frac{65.7}{6}$ & $\begin{array}{r}\frac{15}{13.3} \\
311.8 \\
318.8 \\
71.4\end{array}$ & $\begin{array}{r}\frac{26}{6.8} \\
5.8 \\
358.1 \\
550.7 \\
-\end{array}$ & $\begin{array}{r}\frac{27}{8.9} \\
562.9 \\
815.5 \\
220.4 \\
48.2\end{array}$ & $\begin{array}{r}\frac{30}{6.2} \\
53.2 \\
862.5 \\
156.5 \\
110.0\end{array}$ & $\begin{array}{r}\frac{31}{3.1} \\
3.5 \\
390.7 \\
180.6 \\
50.7\end{array}$ & $\begin{array}{r}\frac{32}{47.6} \\
\frac{232.4}{512.5} \\
59.6\end{array}$ & $\frac{\frac{41}{\frac{32.6}{175.7}}}{\underline{60.0}}$ & 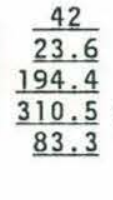 & \begin{tabular}{r}
\multicolumn{1}{c}{$\frac{43}{2.8}$} \\
5.4 \\
279.6 \\
153.5 \\
68.1
\end{tabular} & $\frac{\frac{44}{86.3}}{\frac{193.0}{60.8}}$ & & $\begin{array}{l}\frac{55}{\frac{79.5}{12.3}} \\
\frac{146.8}{150.0} \\
\end{array}$ & & & & & & & $\begin{array}{r}\frac{2}{\frac{39.6}{56.8}} \\
\frac{232.7}{111.9} \\
\end{array}$ & $\begin{array}{r}\frac{3}{10.0} \\
460.5 \\
245.4 \\
145.0\end{array}$ & \\
\hline $\begin{array}{l}\text { Ring } \\
\text { Core }\end{array}$ & $\begin{array}{r}\frac{10}{54.8} \\
\frac{374.7}{262.7} \\
166.5 \\
\end{array}$ & $\begin{array}{r}\frac{11}{2.4} \\
224.4 \\
220.0 \\
174.6\end{array}$ & $\begin{array}{r}\frac{16}{7.5} \\
565.7 \\
214.0\end{array}$ & $\frac{\frac{17}{48.9}}{\frac{137.9}{168.5}}$ & $\frac{\frac{21}{455.0}}{\frac{34.4}{23.3}} \frac{208.8}{155.0}$ & $\frac{\frac{23}{146.5}}{\frac{50.0}{264.5}} \frac{196.4}{93.5}$ & $\begin{array}{r}\frac{24}{22.1} \\
220.0 \\
597.3 \\
303.8 \\
91.1\end{array}$ & $\begin{array}{r}\frac{28}{9.1} \\
308.8 \\
1078.6 \\
130.4 \\
40.0\end{array}$ & & & & & & & $\frac{\frac{56}{117.1}}{\frac{28.3}{408.9}}$ & $\frac{\frac{57}{124.1}}{\frac{39.1}{412.3}} \frac{328.4}{194.2}$ & $\begin{array}{r}\frac{58}{8.3} \\
14.6 \\
438.6 \\
249.7 \\
165.0\end{array}$ & $\begin{array}{r}\frac{61}{83.9} \\
\frac{28.5}{468.5} \\
\frac{205.0}{97.1} \\
\end{array}$ & $\begin{array}{r}\frac{62}{5.2} \\
4.4 \\
437.6 \\
456.5 \\
116.4\end{array}$ & 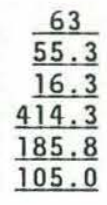 & $\begin{array}{r}\frac{64}{16.3} \\
126.9 \\
591.4 \\
266.7 \\
50.0\end{array}$ & $\frac{\frac{1}{60.4}}{\frac{189.4}{232.3}}$ & & \\
\hline $\begin{array}{l}\text { Sargasso } \\
\text { Sea } \\
\& \\
\text { Guif } \\
\text { Stream }\end{array}$ & & & & & & & & & 49 & $\begin{array}{r}\frac{50}{\frac{91.3}{42.4}} \\
\frac{326.4}{117.4} \\
\end{array}$ & $\begin{array}{r}\frac{51}{10.3} \\
40.2 \\
274.1 \\
110.9\end{array}$ & $\begin{array}{r}\frac{52}{9.4} \\
319.1 \\
330.4 \\
147.6\end{array}$ & $\frac{\frac{73.2}{121.9}}{196.5}$ & & & & & & & & & & & \\
\hline $\begin{array}{l}\text { Slope } \\
\text { Water }\end{array}$ & $\frac{8}{-}$ & $\frac{9}{188.8}$ & $\frac{18}{69.8}$ & & $\frac{19}{101.6}$ & $\frac{20}{120.1}$ & $\frac{33}{129.3}$ & $\frac{34}{139.3}$ & & $\frac{35}{261.0}$ & $\frac{47}{87.3}$ & $\frac{48}{110.4}$ & $\frac{53}{92.9}$ & $\frac{54}{231.7}$ & $\frac{59}{91.6}$ & $\frac{60}{127.5}$ & $\frac{65}{111.9}$ & $\frac{66}{141.8}$ & & & & $\frac{4}{150.2}$ & $\frac{5}{126.6}$ & $\frac{6}{153.1}$ \\
\hline $\begin{array}{l}\text { High } \\
\text { Velocity } \\
\text { Region }\end{array}$ & $\frac{12}{156.1}$ & $\frac{13}{152.4}$ & $\frac{14}{150.7}$ & $\frac{15}{180.2}$ & $3 \frac{27}{16.2}$ & $\frac{29}{149.6}$ & $\frac{30}{233.3}$ & $\frac{31}{125.7}$ & $\frac{32}{-}$ & $\frac{41}{74.0}$ & $\frac{42}{130.1}$ & $\frac{43}{103.2}$ & $\frac{44}{102.4}$ & & $\frac{55}{107.1}$ & & & & & & & $\frac{2}{111.3}$ & $2 \frac{3}{215.3}$ & \\
\hline $\begin{array}{l}\text { Ring } \\
\text { Core }\end{array}$ & $\frac{10}{214.6}$ & $\frac{11}{155.6}$ & $\frac{16}{-}$ & $\frac{17}{130.7}$ & $\frac{21}{192.2}$ & $\frac{23}{145.4}$ & $2 \frac{24}{211.2}$ & $3 \frac{28}{370.6}$ & & $\frac{37}{72.9}$ & $\frac{38}{93.4}$ & $\frac{39}{83.3}$ & & & $\frac{56}{237.4}$ & $\frac{57}{219.4}$ & $\frac{58}{174.9}$ & $\frac{61}{176.7}$ & $\frac{62}{204.1}$ & $\frac{63}{162.2}$ & $\frac{64}{141.9}$ & $\frac{1}{157.2}$ & & \\
\hline SS \& GS & & & & & & & & & & $\frac{49}{144.8}$ & $\frac{50}{108.9}$ & $\frac{51}{201.9}$ & $\frac{52}{140.6}$ & & & & & & & & & & & \\
\hline
\end{tabular}


Table 16. Volume of midwater fishes $\left(\mathrm{ml} / 10,000 \mathrm{~m}^{3}\right)$.

\begin{tabular}{|c|c|c|c|c|c|c|c|c|c|c|c|c|c|c|c|c|c|c|c|c|c|c|c|c|}
\hline & \multicolumn{4}{|c|}{$\begin{array}{c}\text { Oceanus } 118 \\
\text { April }\end{array}$} & \multicolumn{5}{|c|}{$\begin{array}{c}\text { Oceanus } 121 \\
\text { June }\end{array}$} & \multicolumn{5}{|c|}{$\begin{array}{l}\text { Oceanus } 125 \\
\text { August }\end{array}$} & \multicolumn{7}{|c|}{$\begin{array}{l}\text { Knorr } 98 \\
\text { Sept-0ct }\end{array}$} & \multicolumn{3}{|c|}{$\begin{array}{c}\text { A2 } 110 \\
\text { Sept- }-0 c t\end{array}$} \\
\hline $\begin{array}{l}\text { Slope } \\
\text { Water }\end{array}$ & $\begin{array}{l}\frac{8}{7} \\
\frac{29.1}{87.1} \\
\frac{870.8}{30.8}\end{array}$ & $\begin{array}{r}\frac{9}{1.2} \\
67.6 \\
136.7 \\
37.6\end{array}$ & & & $\frac{\frac{19}{31.3}}{\frac{18.0}{\frac{1.2}{39.6}}} \frac{27.4}{27}$ & $\begin{array}{c}\frac{20}{1.9} \\
10.6 \\
57.9 \\
37.2 \\
38.6\end{array}$ & $\frac{\frac{33}{66.4}}{\frac{43.3}{13.9}} \frac{10.9}{\frac{10.9}{30.1}}$ & $\frac{\frac{34}{13.6}}{\frac{16.4}{48.6}}$ & & $\begin{array}{r}\frac{35}{0.4} \\
3.8 \\
41.2 \\
46.8 \\
39.6\end{array}$ & $\frac{\frac{47}{31.1}}{\frac{25.2}{33.1}} \frac{31.1}{31}$ & $\begin{array}{l}\frac{48}{1.3} \\
13.9 \\
75.9 \\
67.8\end{array}$ & $\frac{\frac{53}{\frac{21.6}{10.6}}}{\frac{26.3}{5.6}}$ & $\begin{array}{l}\frac{54}{3.9} \\
11.1 \\
79.5 \\
61.3\end{array}$ & 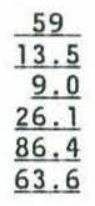 & $\begin{array}{l}\frac{60}{0.6} \\
11.2 \\
22.0 \\
77.8 \\
39.1\end{array}$ & $\frac{\frac{65}{18.0}}{\frac{2.0}{24.1}} \frac{56.8}{14.2}$ & $\begin{array}{r}\frac{66}{0.5} \\
8.4 \\
38.7 \\
84.3 \\
37.4\end{array}$ & & & & $\begin{array}{l}\frac{4}{0.1} \\
18.7 \\
40.1 \\
65.1\end{array}$ & $\frac{\frac{5}{43.0}}{\frac{23.5}{82.5}}$ & \\
\hline $\begin{array}{l}\text { High } \\
\text { Velocity } \\
\text { Region }\end{array}$ & $\begin{array}{r}\frac{12}{0.3} \\
15.7 \\
102.9 \\
69.2\end{array}$ & $\frac{\frac{13}{24.3}}{\frac{52.9}{36.6}}$ & $\frac{\frac{14}{19.7}}{\frac{37.4}{53.9}} \frac{10.4}{10}$ & $\begin{array}{l}\frac{15}{3.1} \\
27.2 \\
57.2 \\
30.0\end{array}$ & $\begin{array}{c}\frac{26}{0.4} \\
1.0 \\
19.4 \\
95.2 \\
-\end{array}$ & $\begin{array}{l}\frac{27}{0.4} \\
17.9 \\
46.2 \\
78.9 \\
28.6\end{array}$ & \begin{tabular}{r}
\multicolumn{1}{c}{30} \\
0.3 \\
9.3 \\
48.7 \\
48.6 \\
27.9
\end{tabular} & $\begin{array}{r}\frac{31}{0.2} \\
0.2 \\
27.9 \\
65.5 \\
14.8\end{array}$ & $\frac{\frac{32}{39.9}}{\frac{16.8}{38.8}} \frac{21.1}{21}$ & $\frac{\frac{41}{6.2}}{\frac{67.2}{29.7}}$ & $\frac{\frac{42}{12.1}}{\frac{14.2}{71.0}}$ & \begin{tabular}{r}
\multicolumn{1}{c}{$\frac{43}{0.3}$} \\
0.5 \\
32.8 \\
52.7 \\
13.4
\end{tabular} & $\frac{\frac{44}{26.1}}{\frac{52.9}{20.2}}$ & & $\begin{array}{l}\frac{55}{6.4} \\
\frac{91.8}{35.1} \\
\underline{82.9}\end{array}$ & & & & & & & $\begin{array}{l}\frac{2}{29.1} \\
\frac{9.2}{85.4} \\
31.0\end{array}$ & $\begin{array}{r}\frac{3}{1.0} \\
39.1 \\
33.0 \\
52.9\end{array}$ & \\
\hline $\begin{array}{l}\text { Ring } \\
\text { Core }\end{array}$ & $\frac{\frac{10}{13.7}}{\frac{24.2}{32.0}} \frac{24.4}{24.0}$ & $\begin{array}{r}\frac{11}{0.1} \\
9.1 \\
25.2 \\
42.4\end{array}$ & $\begin{array}{l}\frac{16}{0.4} \\
27.6 \\
32.0\end{array}$ & $\frac{\frac{17}{11.1}}{\frac{23.1}{28.2}}$ & $\frac{\frac{21}{34.5}}{\frac{37.3}{42.9}} \frac{21.5}{\frac{22.1}{22.1}}$ & $\frac{\frac{23}{36.4}}{\frac{32.1}{75.1}} \frac{22.6}{18.4}$ & $\begin{array}{l}\frac{24}{1.6} \\
23.0 \\
45.4 \\
73.7 \\
43.7\end{array}$ & $\begin{array}{l}\frac{28}{0.8} \\
34.2 \\
74.2 \\
37.7 \\
28.6\end{array}$ & & & & & & & $\begin{array}{l}\frac{56}{8.7} \\
\frac{2.9}{2.2} \\
\frac{22.2}{17.0} \\
10.5 \\
\end{array}$ & $\frac{\frac{57}{8.6}}{\frac{5.8}{5.1}} \frac{17.1}{\frac{15.0}{13.2}}$ & $\begin{array}{r}\frac{58}{0.6} \\
1.0 \\
9.8 \\
15.7 \\
68.9\end{array}$ & $\frac{\frac{61}{6.8}}{\frac{\frac{3.8}{3.3}}{2.9}} \frac{125.8}{\frac{25.0}{25.0}}$ & $\begin{array}{r}\frac{62}{0.3} \\
0.8 \\
13.3 \\
25.2 \\
25.0\end{array}$ & 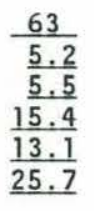 & $\begin{array}{c}\frac{64}{0.6} \\
4.0 \\
16.8 \\
20.6 \\
40.6\end{array}$ & $\frac{\frac{1}{17.7}}{\frac{31.9}{15.3}} \frac{12.1}{1}$ & & \\
\hline $\begin{array}{l}\text { Sargasso } \\
\text { Sea } \\
\& \\
\text { Gulf } \\
\text { Stream }\end{array}$ & & & & & & & & & & $\frac{\frac{49}{10.3}}{\frac{4.1}{13.3}}$ & $\begin{array}{l}\frac{50}{0.5} \\
1.7 \\
17.2 \\
32.7\end{array}$ & $\begin{array}{r}51 \\
0.6 \\
6.4 \\
21.7 \\
8.5\end{array}$ & $\frac{\frac{52}{11.5}}{\frac{7.8}{13.0}} \frac{10.7}{1}$ & & & & & & & & & & & \\
\hline $\begin{array}{l}\text { Slope } \\
\text { Water }\end{array}$ & $\frac{8}{-}$ & $\frac{9}{60.8}$ & $\frac{18}{32.4}$ & & $\frac{19}{22.9}$ & $\frac{20}{26.9}$ & $\frac{33}{26.5}$ & $\frac{34}{35.4}$ & & $\frac{35}{26.3}$ & $\frac{47}{30.1}$ & $\frac{48}{39.8}$ & $\frac{53}{15.9}$ & $\frac{54}{38.9}$ & $\frac{59}{39.7}$ & $\frac{60}{29.1}$ & $\frac{65}{22.6}$ & $\frac{66}{33.1}$ & & & & $\frac{4}{31.0}$ & $5 \frac{5}{53.2}$ & $5 \frac{6}{50.6}$ \\
\hline $\begin{array}{l}\text { High } \\
\text { Velocity } \\
\text { Region }\end{array}$ & $\frac{12}{47.0}$ & $\frac{13}{37.7}$ & $\frac{14}{30.4}$ & $\frac{15}{29.4}$ & $\frac{27}{39.0}$ & $\frac{29}{24.4}$ & $\frac{30}{26.1}$ & $\frac{31}{21.7}$ & $\frac{32}{-}$ & $\frac{41}{27.4}$ & $\frac{42}{26.7}$ & $\frac{43}{20.0}$ & $\frac{44}{29.2}$ & & $\frac{55}{50.3}$ & & & & & & & $\frac{2}{38.8}$ & $3 \frac{3}{1.6}$ & \\
\hline $\begin{array}{l}\text { Ring } \\
\text { Core }\end{array}$ & $\frac{10}{23.6}$ & $\frac{11}{19.2}$ & $\frac{16}{-}$ & $\frac{17}{22.7}$ & $\frac{21}{32.2}$ & $\frac{23}{36.8}$ & $\frac{24}{33.6}$ & $\frac{28}{34.5}$ & & $\frac{37}{16.7}$ & $\frac{38}{19.3}$ & $\frac{39}{14.4}$ & & & $\frac{56}{12.4}$ & $\frac{57}{12.0}$ & $\frac{58}{20.1}$ & $\frac{61}{14.7}$ & $\frac{62}{12.9}$ & $\frac{63}{13.0}$ & $\frac{64}{14.6}$ & $\frac{1}{19.4}$ & & \\
\hline SS \& GS & & & & & & & & & & $\frac{49}{12.9}$ & $\frac{50}{13.0}$ & $\frac{51}{9.3}$ & $\frac{52}{10.8}$ & & & & & & & & & & & \\
\hline
\end{tabular}


Table 17. Abundance of gonostomatids (specimens $/ 10,000 \mathrm{~m}^{3}$ ).

\begin{tabular}{|c|c|c|c|c|c|c|c|c|c|c|c|c|c|c|c|c|c|c|c|c|c|c|c|c|}
\hline & \multicolumn{4}{|c|}{$\begin{array}{c}\text { Oceanus } 118 \\
\text { April }\end{array}$} & \multicolumn{5}{|c|}{$\begin{array}{c}\text { Oceanus } 121 \\
\text { June }\end{array}$} & \multicolumn{5}{|c|}{$\begin{array}{c}\text { Oceanus } 125 \\
\text { August }\end{array}$} & \multicolumn{7}{|c|}{$\begin{array}{l}\text { Knorr } 98 \\
\text { Sept-0ct }\end{array}$} & \multicolumn{3}{|c|}{$\begin{array}{c}\text { A2 } 110 \\
\text { Sept-0ct }\end{array}$} \\
\hline $\begin{array}{l}\text { Slope } \\
\text { Water }\end{array}$ & $\begin{array}{c}\frac{8}{=} \\
\frac{145.9}{222.3} \\
\frac{39.5}{39.5}\end{array}$ & $\begin{array}{r}\frac{9}{2.7} \\
268.8 \\
332.9 \\
71.3\end{array}$ & & & $\begin{array}{r}\frac{19}{\frac{1.0}{4.1}} \\
\frac{8.9}{\frac{8.9}{20.5}} \\
52.1 \\
\end{array}$ & \begin{tabular}{r}
\multicolumn{1}{c}{20} \\
4.7 \\
66.8 \\
246.1 \\
65.5 \\
24.0
\end{tabular} & $\frac{\frac{33}{\frac{1.3}{2.1}}}{\frac{6.1}{\frac{6.1}{2.8}}} \frac{1711.5}{111.5}$ & $\begin{array}{r}\frac{34}{1.8} \\
172.3 \\
\end{array}$ & & $\begin{array}{r}\frac{35}{4.0} \\
11.7 \\
555.3 \\
125.6 \\
62.1\end{array}$ & $\begin{array}{r}\frac{47}{\frac{1.3}{40.2}} \\
\frac{150.0}{51.8} \\
71\end{array}$ & $\begin{array}{r}\frac{48}{2.2} \\
26.0 \\
173.5 \\
105.2\end{array}$ & $\frac{\frac{53}{1.2}}{\frac{32.9}{140.5}}$ & $\begin{array}{r}\frac{54}{3.8} \\
79.7 \\
369.4 \\
210.3\end{array}$ & $\begin{array}{r}\frac{59}{7.3} \\
\frac{67.0}{67.9} \\
\frac{143.1}{48.9}\end{array}$ & $\begin{array}{r}\frac{60}{1.8} \\
0.9 \\
110.7 \\
170.0 \\
50.3\end{array}$ & $\frac{\frac{65}{4.8}}{\frac{1.7}{2.7}}$ & $\begin{array}{r}\frac{66}{2.3} \\
17.9 \\
287.0 \\
147.1 \\
95.0\end{array}$ & & & & $\begin{array}{r}\frac{4}{0.7} \\
145.4 \\
231.6 \\
76.8\end{array}$ & $\begin{array}{r}\frac{5}{\frac{5.7}{77.8}} \\
\frac{210.3}{104.4} \\
\end{array}$ & \\
\hline $\begin{array}{l}\text { High } \\
\text { Velocity } \\
\text { Region }\end{array}$ & $\begin{array}{r}\frac{12}{2.6} \\
200.0 \\
253.3 \\
81.0\end{array}$ & $\begin{array}{r}\frac{13}{\frac{8.5}{180.0}} \\
\underline{173.7}\end{array}$ & $\begin{array}{r}\frac{14}{\frac{3.4}{175.7}} \\
\frac{283.5}{62.9}\end{array}$ & $\begin{array}{r}\frac{15}{5.0} \\
266.5 \\
301.2 \\
62.9\end{array}$ & $\begin{array}{c}\frac{26}{3.2} \\
0 \\
121.4 \\
272.1 \\
-\end{array}$ & $\begin{array}{r}\frac{27}{3.9} \\
0.3 \\
184.5 \\
201.3 \\
41.4\end{array}$ & $\begin{array}{r}\frac{30}{3.3} \\
1.6 \\
549.4 \\
132.4 \\
94.2\end{array}$ & \begin{tabular}{r}
\multicolumn{1}{c}{31} \\
1.6 \\
1.5 \\
234.6 \\
162.9 \\
45.5
\end{tabular} & $\begin{array}{r}\frac{32}{7.6} \\
\frac{204.0}{423.0} \\
53.2\end{array}$ & $\frac{\frac{41}{16.4}}{142.0}$ & $\frac{\frac{42}{1.5}}{\frac{153.5}{240.7}}$ & $\begin{array}{r}\frac{43}{0} \\
1.7 \\
148.3 \\
99.1 \\
62.9\end{array}$ & $\frac{\frac{44}{39.8}}{\frac{127.7}{56.2}}$ & & $\begin{array}{r}\frac{55}{10.0} \\
\frac{0.9}{11.2} \\
133.3 \\
\end{array}$ & & & & & & & $\begin{array}{r}\frac{2}{3.7} \\
\frac{43.8}{196.0} \\
103.1 \\
\end{array}$ & $\begin{array}{r}\frac{3}{3.6} \\
398.1 \\
191.8 \\
127.7\end{array}$ & \\
\hline $\begin{array}{l}\text { Ring } \\
\text { Core }\end{array}$ & $\begin{array}{r}\frac{10}{\frac{4.8}{3.5}} \\
\frac{343.5}{236.4} \\
162.9 \\
\end{array}$ & $\begin{array}{l}\frac{11}{1.2} \\
198.9 \\
204.4 \\
163.9\end{array}$ & $\begin{array}{r}\frac{16}{4.0} \\
540.7 \\
149.3\end{array}$ & $\frac{17}{\frac{1.1}{103.7}}$ & $\begin{array}{r}\frac{21}{\frac{7.0}{3.0}} \\
\frac{283.9}{131.7} \\
148.9 \\
\end{array}$ & $\frac{\frac{23}{\frac{4.1}{7.3}}}{\frac{184.0}{125.5}} \frac{81.2}{\underline{81.2}}$ & $\begin{array}{r}\frac{24}{4.7} \\
105.0 \\
402.7 \\
177.5 \\
82.2\end{array}$ & $\begin{array}{r}\frac{28}{3.2} \\
274.2 \\
415.7 \\
117.3 \\
36.4\end{array}$ & & & & & & & $\begin{array}{r}\frac{56}{\frac{6.8}{4.4}} \\
\frac{14.9}{378.9} \\
\frac{430.0}{157.2} \\
\end{array}$ & $\begin{array}{r}\frac{57}{\frac{9.4}{17.8}} \\
\frac{392.2}{289.4} \\
\frac{281.6}{161.6}\end{array}$ & $\begin{array}{r}\frac{58}{3.2} \\
2.1 \\
391.0 \\
151.6 \\
118.7\end{array}$ & $\begin{array}{r}\frac{61}{\frac{4.1}{6.4}} \\
\frac{421.9}{134.6} \\
76.1 \\
\end{array}$ & \begin{tabular}{r}
\multicolumn{1}{c}{62} \\
\\
3.7 \\
1.8 \\
399.8 \\
368.9 \\
5 \\
57.5
\end{tabular} & $\begin{array}{l}\frac{63}{\frac{6.1}{2.8}} \\
\frac{381.4}{150.0} \\
\underline{70.0}\end{array}$ & $\begin{array}{r}\frac{64}{4.8} \\
106.9 \\
558.6 \\
196.7 \\
26.5\end{array}$ & $\begin{array}{l}\frac{1}{7.8} \\
\frac{171.8}{187.5} \\
116.7\end{array}$ & & \\
\hline $\begin{array}{l}\text { Sargasso } \\
\text { Sea } \\
\& \\
\text { Gulf } \\
\text { Stream }\end{array}$ & & & & & & & & & & $\begin{array}{r}\frac{49}{\frac{7.4}{23.1}} \\
\frac{314.9}{85.9}\end{array}$ & $\begin{array}{r}\frac{50}{3.6} \\
18.0 \\
225.9 \\
71.6\end{array}$ & $\begin{array}{r}\frac{51}{5.9} \\
306.1 \\
244.8 \\
122.1\end{array}$ & $\frac{\frac{52}{11.1}}{\frac{103.1}{150.9}} \frac{147.7}{14}$ & & & & & & & & & & & \\
\hline $\begin{array}{l}\text { Slope } \\
\text { Water }\end{array}$ & $\frac{8}{-}$ & $\frac{9}{168.9}$ & $\frac{18}{61.6}$ & & $\frac{19}{68.3}$ & $\frac{20}{95.8}$ & $\frac{33}{74.6}$ & $\frac{34}{104.3}$ & & $\frac{35}{189.7}$ & $\frac{47}{65.8}$ & $\frac{48}{76.0}$ & $\frac{53}{52.1}$ & $\frac{54}{165.8}$ & $\frac{59}{53.8}$ & $\frac{60}{66.8}$ & $\frac{65}{85.3}$ & $\frac{66}{108.3}$ & & & & $11 \frac{4}{3.6}$ & $\frac{5}{99.6}$ & $\frac{6}{119.0}$ \\
\hline $\begin{array}{l}\text { High } \\
\text { Velocity } \\
\text { Region }\end{array}$ & $\frac{12}{134.2}$ & $\frac{13}{134.0}$ & $\frac{14}{131.4}$ & $\frac{15}{160.2}$ & $\frac{27}{98.3}$ & $\frac{29}{75.2}$ & $\frac{30}{156.4}$ & $\frac{31}{89.2}$ & $\frac{32}{-}$ & $\frac{41}{56.2}$ & $\frac{42}{97.3}$ & $\frac{43}{63.5}$ & $\frac{44}{60.7}$ & & $\frac{55}{121.8}$ & & & & & & & $\frac{2}{86.7}$ & $\frac{3}{180.3}$ & \\
\hline $\begin{array}{l}\text { Ring } \\
\text { Core }\end{array}$ & $\frac{10}{186.9}$ & $\frac{11}{142.1}$ & $\frac{16}{-}$ & $\frac{17}{104.5}$ & $\frac{21}{114.3}$ & $\frac{23}{80.6}$ & $\frac{24}{136.4}$ & $\frac{28}{192.7}$ & & $\frac{37}{42.2}$ & $\frac{38}{56.3}$ & $\frac{39}{58.9}$ & & & $\frac{56}{197.5}$ & $\frac{57}{174.1}$ & $\frac{58}{133.4}$ & $\frac{61}{128.6}$ & $\frac{62}{166.5}$ & $\frac{63}{122.6}$ & $\frac{64}{113.1}$ & $12 \frac{1}{1.0}$ & & \\
\hline SS \& GS & & & & & & & & & & $\frac{49}{107.8}$ & $\frac{50}{79.8}$ & $\frac{51}{169.7}$ & $\frac{52}{103.2}$ & & & & & & & & & & & \\
\hline
\end{tabular}


Table 18. Volume of gonostomatids $\left(\mathrm{ml} / 10,000 \mathrm{~m}^{3}\right)$.

\begin{tabular}{|c|c|c|c|c|c|c|c|c|c|c|c|c|c|c|c|c|c|c|c|c|c|c|c|c|}
\hline & \multicolumn{4}{|c|}{$\begin{aligned} & \text { Oceanus } 118 \\
& \text { April } \\
&\end{aligned}$} & \multicolumn{5}{|c|}{$\begin{array}{l}\text { Oceanus } 121 \\
\text { June }\end{array}$} & \multicolumn{5}{|c|}{$\begin{array}{l}\text { Oceanus } 125 \\
\text { August }\end{array}$} & \multicolumn{7}{|c|}{$\begin{array}{l}\text { Knorr } 98 \\
\text { Sept-0ct }\end{array}$} & \multicolumn{3}{|c|}{$\begin{array}{c}A 2110 \\
\text { Sept-0ct }\end{array}$} \\
\hline $\begin{array}{l}\text { Slope } \\
\text { Water }\end{array}$ & $\begin{array}{c}\frac{8}{=} \\
\frac{19.3}{26.9} \\
\frac{10.0}{10.0}\end{array}$ & $\begin{array}{r}\frac{9}{0.1} \\
12.0 \\
109.5 \\
22.9\end{array}$ & & & $\frac{\frac{19}{\frac{0.2}{8.4}}}{\frac{8.8}{\frac{0.8}{2.0}}} \frac{7.9}{}$ & $\begin{array}{r}\frac{20}{0.6} \\
2.9 \\
41.9 \\
10.4 \\
6.0\end{array}$ & $\begin{array}{l}\frac{33}{0.1} \\
\frac{0.6}{0.2} \\
\underline{\frac{0.9}{0.9}} \\
10.9\end{array}$ & $\frac{\frac{34}{1.5}}{\frac{9.3}{2.5}}$ & & \begin{tabular}{r}
\multicolumn{1}{c}{$\frac{15}{0.3}$} \\
0.4 \\
23.0 \\
10.8 \\
7.6
\end{tabular} & $\frac{\frac{47}{9.8}}{\frac{20.7}{8.6}} \frac{8.3}{\underline{9.3}}$ & $\begin{array}{l}\frac{48}{0.1} \\
1.4 \\
20.6 \\
12.2\end{array}$ & $\frac{\frac{53}{6.6}}{\frac{5.1}{5.5}} \frac{6.4}{4.4}$ & $\begin{array}{r}\frac{54}{3.2} \\
3.1 \\
28.9 \\
22.9\end{array}$ & $\begin{array}{r}\frac{59}{6.8} \\
\frac{0.4}{3.6} \\
\frac{17.8}{7.5} \\
\underline{8}\end{array}$ & $\begin{array}{c}\frac{60}{0.2} \\
0 \\
7.6 \\
18.1 \\
8.6\end{array}$ & $\begin{array}{l}\frac{65}{6.7} \\
\frac{0.1}{5.4} \\
\frac{5.6}{6.3} \\
\underline{6.5}\end{array}$ & $\begin{array}{r}\frac{66}{0.1} \\
0.6 \\
14.1 \\
21.9 \\
10.1\end{array}$ & & & & $\begin{array}{c}\frac{4}{0} \\
7.4 \\
12.1 \\
10.3\end{array}$ & $\frac{\frac{5}{17.0}}{\frac{4.5}{22.5}}$ & \\
\hline $\begin{array}{l}\text { High } \\
\text { Velocity } \\
\text { Region }\end{array}$ & $\begin{array}{l}\frac{12}{0.1} \\
10.7 \\
32.6 \\
11.6\end{array}$ & $\frac{\frac{13}{15.5}}{\frac{42.4}{18.5}}$ & $\frac{\frac{14}{\frac{5.3}{8.1}}}{\frac{18.1}{20.5}} \frac{10.2}{10.2}$ & $\begin{array}{r}\frac{15}{0.4} \\
14.6 \\
40.4 \\
8.8\end{array}$ & $\begin{array}{c}\frac{26}{0.2} \\
0 \\
6.4 \\
30.5 \\
-\end{array}$ & $\begin{array}{c}27 \\
0.3 \\
0 \\
9.2 \\
19.4 \\
8.4\end{array}$ & $\begin{array}{c}\frac{30}{0.2} \\
0 \\
27.7 \\
31.2 \\
11.8\end{array}$ & $\begin{array}{r}\frac{31}{0.1} \\
0.1 \\
10.8 \\
38.6 \\
8.3\end{array}$ & $\frac{\frac{32}{14.9}}{\frac{10.0}{23.4}} \frac{8.4}{\underline{8}}$ & $\frac{\frac{41}{1.5}}{\frac{7.5}{6.9}}$ & $\frac{\frac{42}{\frac{4.0}{6.6}}}{\frac{16.2}{8.3}}$ & $\begin{array}{r}\frac{43}{0} \\
0.3 \\
7.8 \\
21.3 \\
8.9\end{array}$ & $\frac{\frac{44}{3.9}}{\frac{3.8}{7.8}}$ & & 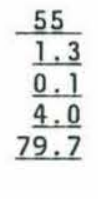 & & & & & & & $\frac{\frac{2}{10.1}}{\frac{6.9}{14.3}} \frac{10.1}{10}$ & $\begin{array}{r}\frac{3}{0.3} \\
21.5 \\
10.4 \\
16.2\end{array}$ & \\
\hline $\begin{array}{l}\text { Ring } \\
\text { Core }\end{array}$ & $\frac{\frac{10}{\frac{6.3}{17.9}}}{\frac{12.3}{21.9}}$ & $\begin{array}{l}\frac{11}{0.1} \\
7.1 \\
19.4 \\
19.2\end{array}$ & $\begin{array}{r}\frac{16}{0.2} \\
24.5 \\
13.7\end{array}$ & $\frac{\frac{17}{0.2}}{\frac{0.9}{3.2}}$ & 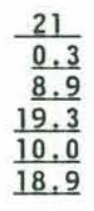 & $\frac{\frac{23}{0.5}}{\frac{1.7}{12.3}} \frac{7.5}{\frac{14.1}{14.1}}$ & $\begin{array}{r}\frac{24}{10.3} \\
6.0 \\
25.2 \\
64.2 \\
38.7\end{array}$ & $\begin{array}{r}\frac{28}{0.4} \\
14.1 \\
38.8 \\
13.5 \\
7.5\end{array}$ & & & & & & & 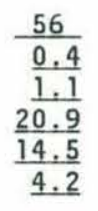 & $\frac{\frac{57}{1.2}}{\frac{1.3}{15.8}} \frac{10.6}{\frac{5.7}{10}}$ & $\begin{array}{l}\frac{58}{0.2} \\
0.2 \\
7.9 \\
8.5 \\
5.6\end{array}$ & $\frac{\frac{61}{0.2}}{\frac{0.5}{0.1}} \frac{11.1}{\frac{19.4}{5.9}}$ & $\begin{array}{c}\frac{62}{0.2} \\
0.2 \\
11.8 \\
19.0 \\
12.8\end{array}$ & 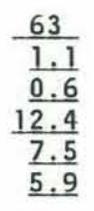 & $\begin{array}{r}\frac{64}{0.1} \\
3.2 \\
14.4 \\
13.4 \\
5.5\end{array}$ & $\frac{\frac{1}{\frac{0.7}{19.3}}}{\frac{11.8}{8.2}}$ & & \\
\hline $\begin{array}{l}\text { Sargasso } \\
\text { Sea } \\
\& \\
\text { Gulf } \\
\text { Stream }\end{array}$ & & & & & & & & & & $\frac{\frac{49}{\frac{0.7}{1.2}}}{\frac{12.3}{3.1}}$ & $\begin{array}{l}\frac{50}{0.2} \\
0.5 \\
8.3 \\
2.8\end{array}$ & $\begin{array}{l}\frac{51}{0.2} \\
6.0 \\
9.8 \\
5.0\end{array}$ & $\frac{\frac{52}{1.1}}{\frac{1.4}{6.7}} \frac{6.7}{6.3}$ & & & & & & & & & & & \\
\hline $\begin{array}{l}\text { Slope } \\
\text { Water }\end{array}$ & $\frac{8}{-}$ & $\frac{9}{36.1}$ & $\frac{18}{17.1}$ & & $\frac{19}{6.5}$ & $\frac{20}{14.0}$ & $\frac{33}{6.8}$ & $\frac{34}{10.6}$ & & $\frac{35}{8.4}$ & $\frac{47}{12.1}$ & $\frac{48}{8.6}$ & $\frac{53}{5.7}$ & $\frac{54}{14.5}$ & $\frac{59}{7.4}$ & $\frac{60}{6.9}$ & $\frac{65}{4.8}$ & $\frac{66}{9.3}$ & & & & $\frac{4}{7.5}$ & $\frac{5}{16.5}$ & $\frac{6}{12.3}$ \\
\hline $\begin{array}{l}\text { High } \\
\text { Velocity } \\
\text { Region }\end{array}$ & $\frac{12}{13.8}$ & $\frac{13}{23.7}$ & $\frac{14}{13.5}$ & $\frac{15}{16.1}$ & $\frac{27}{8.8}$ & $\frac{29}{8.1}$ & $\frac{30}{13.9}$ & $\frac{31}{11.6}$ & $\frac{32}{-}$ & $\frac{41}{4.4}$ & $\frac{42}{7.9}$ & $\frac{43}{7.7}$ & $\frac{44}{5.7}$ & & $\frac{55}{17.8}$ & & & & & & & $\frac{2}{10.4}$ & $\frac{3}{12.1}$ & \\
\hline $\begin{array}{l}\text { Ring } \\
\text { Core }\end{array}$ & $\frac{10}{14.6}$ & $\frac{11}{11.5}$ & $\frac{16}{-}$ & $\frac{17}{9.1}$ & $\frac{21}{12.7}$ & $\frac{23}{7.3}$ & $\frac{24}{25.8}$ & $\frac{28}{15.8}$ & & $\frac{37}{5.6}$ & $\frac{38}{4.4}$ & $\frac{39}{5.2}$ & & & $\frac{56}{8.2}$ & $\frac{57}{6.9}$ & $\frac{58}{4.5}$ & $\frac{61}{7.4}$ & $\frac{62}{8.8}$ & $\frac{63}{5.6}$ & $\frac{64}{5.6}$ & $\frac{1}{10.0}$ & & \\
\hline SS \& GS & & & & & & & & & & $\frac{49}{4.3}$ & $\frac{50}{3.0}$ & $\frac{51}{5.3}$ & $\frac{52}{4.6}$ & & & & & & & & & & & \\
\hline
\end{tabular}


Table 19. Abundance of melamphaids (specimens/10,000 $\mathrm{m}^{3}$ ).

\begin{tabular}{|c|c|c|c|c|c|c|c|c|c|c|c|c|c|c|c|c|c|c|c|c|c|c|c|c|}
\hline & \multicolumn{4}{|c|}{$\begin{array}{c}\text { Oceanus } 118 \\
\text { April }\end{array}$} & \multicolumn{5}{|c|}{$\begin{array}{c}\text { Oceanus } 121 \\
\text { June }\end{array}$} & \multicolumn{5}{|c|}{$\begin{array}{l}\text { Oceanus } 125 \\
\text { August }\end{array}$} & \multicolumn{7}{|c|}{$\begin{array}{l}\text { Knorr } 98 \\
\text { Sept-0ct }\end{array}$} & \multicolumn{3}{|c|}{$\begin{array}{c}\text { A2 } 110 \\
\text { Sept-Oct }\end{array}$} \\
\hline $\begin{array}{l}\text { Slope } \\
\text { Water }\end{array}$ & $\begin{array}{l}\frac{8}{\overline{\overline{0}}} \\
\underline{0} \\
\underline{0.9} \\
\underline{2.7}\end{array}$ & $\begin{array}{c}\frac{9}{0} \\
0 \\
0 \\
2.7\end{array}$ & & & $\begin{array}{l}\frac{19}{0.2} \\
\underline{0.3} \\
\underline{0} \\
\underline{0.5} \\
\underline{0.5}\end{array}$ & $\begin{array}{c}20 \\
0 \\
0 \\
0.4 \\
1.4 \\
0\end{array}$ & $\begin{array}{c}\frac{33}{0} \\
\underline{0.4} \\
\underline{0} \\
\underline{0} \\
\underline{0.9}\end{array}$ & $\begin{array}{c}34 \\
\underline{0} \\
\underline{0} \\
0.9\end{array}$ & & $\begin{array}{c}35 \\
0 \\
0 \\
0 \\
0.6 \\
0.7\end{array}$ & $\begin{array}{l}\frac{47}{0.2} \\
\underline{0} \\
\underline{0.6} \\
\underline{0.4}\end{array}$ & $\begin{array}{c}48 \\
0 \\
0 \\
0.9 \\
0.8\end{array}$ & $\begin{array}{l}\frac{53}{0} \\
\underline{0.2} \\
\underline{0.5} \\
\underline{0.2}\end{array}$ & $\begin{array}{c}54 \\
0 \\
0 \\
0 \\
2.0\end{array}$ & $\begin{array}{l}\frac{59}{5.3} \\
\frac{5.4}{0.2} \\
\frac{1.2}{1.4} \\
1.1\end{array}$ & $\begin{array}{c}60 \\
0.3 \\
0 \\
1.0 \\
0 \\
0.3\end{array}$ & $\begin{array}{l}\frac{65}{1.3} \\
\frac{1.0}{0.9} \\
\frac{1.6}{0.6} \\
\underline{0.6}\end{array}$ & $\begin{array}{c}66 \\
0 \\
0 \\
0.4 \\
2.4 \\
2.3\end{array}$ & & & & $\begin{array}{l}\frac{4}{0} \\
0 \\
0.9 \\
1.5\end{array}$ & $\begin{array}{l}\frac{5}{0.3} \\
\frac{0.4}{1.3} \\
\underline{0.5}\end{array}$ & \\
\hline $\begin{array}{l}\text { High } \\
\text { Velocity } \\
\text { Region }\end{array}$ & $\begin{array}{c}12 \\
0 \\
0.4 \\
0.8 \\
1.4\end{array}$ & $\frac{\frac{13}{1.0}}{\frac{1.1}{1.5}}$ & $\begin{array}{l}\frac{14}{0.3} \\
\frac{0.7}{\underline{0}} \\
\underline{0.7}\end{array}$ & $\begin{array}{c}15 \\
0 \\
0 \\
1.2 \\
4.3\end{array}$ & $\begin{array}{c}26 \\
0 \\
0 \\
0.5 \\
0.7 \\
-\end{array}$ & $\begin{array}{c}27 \\
0 \\
0 \\
0 \\
0.8 \\
1.4\end{array}$ & $\begin{array}{c}30 \\
0 \\
0 \\
0 \\
2.4 \\
1.2\end{array}$ & $\begin{array}{c}31 \\
0 \\
0 \\
0 \\
0.6 \\
2.1\end{array}$ & $\frac{\frac{32}{2.9}}{\frac{3.2}{\frac{0}{0}}}$ & $\begin{array}{l}\frac{41}{0.8} \\
\frac{0.9}{2.3} \\
\underline{0.9}\end{array}$ & $\begin{array}{l}\frac{42}{1.1} \\
\frac{0.3}{0.2} \\
0.5\end{array}$ & $\begin{array}{c}43 \\
0 \\
0 \\
0 \\
0 \\
1.4\end{array}$ & $\begin{array}{l}\frac{44}{0.9} \\
\frac{0.3}{1.5} \\
\underline{1}\end{array}$ & & 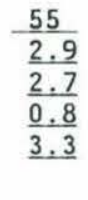 & & & & & & & $\frac{\frac{2}{0.4}}{\frac{0.8}{0.3}} \frac{1.3}{1.3}$ & $\begin{array}{l}\frac{3}{0} \\
1.1 \\
1.4 \\
1.2\end{array}$ & \\
\hline $\begin{array}{l}\text { Ring } \\
\text { Center }\end{array}$ & $\begin{array}{l}\frac{10}{\frac{4.8}{0.6}} \\
\underline{0} \\
\underline{0.6}\end{array}$ & $\begin{array}{c}11 \\
0 \\
0 \\
0.6 \\
1.4\end{array}$ & $\begin{array}{l}16 \\
0 \\
0.7 \\
2.0\end{array}$ & $\begin{array}{l}\frac{17}{\frac{3.3}{3.2}} \\
\frac{3.8}{0.8}\end{array}$ & $\begin{array}{l}\frac{21}{\underline{0}} \\
\underline{0.4} \\
\underline{4.5} \\
\underline{3.3} \\
\underline{0.6}\end{array}$ & $\begin{array}{l}\frac{23}{0} \\
\underline{\underline{0} .7} \\
\underline{2.5} \\
\underline{2.7} \\
\underline{4.1}\end{array}$ & $\begin{array}{c}24 \\
0 \\
0 \\
0 \\
3.7 \\
1.7\end{array}$ & $\begin{array}{c}28 \\
0 \\
0 \\
1.4 \\
1.9 \\
1.5\end{array}$ & & & & & & & $\begin{array}{l}\frac{56}{8.8} \\
\frac{3.3}{\underline{0}} \\
\underline{2.6} \\
\underline{4.1}\end{array}$ & $\frac{\frac{57}{17.2}}{\frac{10.3}{0.8}} \frac{0}{\frac{0.9}{3.9}}$ & $\begin{array}{c}58 \\
0.3 \\
0 \\
1.0 \\
10.6 \\
10.5\end{array}$ & $\frac{\frac{61}{2.7}}{\frac{10.9}{1.1}} \frac{1.2}{\underline{2.6}}$ & $\begin{array}{c}62 \\
0 \\
0 \\
0.2 \\
11.1 \\
9.3\end{array}$ & $\begin{array}{l}\frac{63}{14.4} \\
\frac{2.2}{1.9} \\
\frac{0.4}{1.6} \\
\end{array}$ & $\begin{array}{r}64 \\
0 \\
0 \\
1.4 \\
13.3 \\
6.2\end{array}$ & $\frac{\frac{1}{13.4}}{\frac{2.2}{1.6}}$ & & \\
\hline $\begin{array}{l}\text { Sargasso } \\
\text { Sea } \\
\& \\
\text { Gulf } \\
\text { Stream }\end{array}$ & & & & & & & & & & $\frac{\frac{49}{16.8}}{\frac{1.1}{\frac{0}{0}}}$ & $\begin{array}{c}50 \\
0.8 \\
0 \\
3.4 \\
7.5\end{array}$ & $\begin{array}{r}51 \\
0 \\
0 \\
21.3 \\
6.6\end{array}$ & $\frac{\frac{52}{17.7}}{\frac{1.9}{0.4}} \frac{2.1}{\underline{0.1}}$ & & & & & & & & & & & \\
\hline $\begin{array}{l}\text { Slope } \\
\text { Water }\end{array}$ & $\frac{8}{-}$ & $\frac{9}{0.7}$ & $\frac{18}{0.4}$ & & $\frac{19}{0.3}$ & $\frac{20}{0.5}$ & $\frac{33}{0.5}$ & $\frac{34}{0.5}$ & & $\frac{35}{0.3}$ & $\frac{47}{0.3}$ & $\frac{48}{0.4}$ & $\frac{53}{0.2}$ & $\frac{54}{0.5}$ & $\frac{59}{1.9}$ & $\frac{60}{0.4}$ & $\frac{65}{1.1}$ & $\frac{66}{1.0}$ & & & & $\frac{4}{0.6}$ & $\frac{5}{0.4}$ & $\frac{6}{0.4}$ \\
\hline $\begin{array}{l}\text { High } \\
\text { Velocity } \\
\text { Region }\end{array}$ & $\frac{12}{0.7}$ & $\frac{13}{1.3}$ & $\frac{14}{0.7}$ & $\frac{15}{1.4}$ & $\frac{27}{0.6}$ & $\frac{29}{2.2}$ & $\frac{30}{0.7}$ & $\frac{31}{0.5}$ & $\frac{32}{-}$ & $\frac{41}{1.2}$ & $\frac{42}{0.6}$ & $\frac{43}{0.3}$ & $\frac{44}{0.9}$ & & $\frac{55}{2.3}$ & & & & & & & $\frac{2}{1.0}$ & $\frac{3}{0.9}$ & \\
\hline $\begin{array}{l}\text { Ring } \\
\text { Center }\end{array}$ & $\frac{10}{1.5}$ & $\frac{11}{0.5}$ & $\frac{16}{-}$ & $\frac{17}{1.9}$ & $\frac{21}{1.8}$ & $\frac{23}{2.5}$ & $\frac{24}{1.1}$ & $\frac{28}{1.2}$ & & $\frac{37}{0.4}$ & $\frac{38}{0.5}$ & $\frac{39}{0.6}$ & & & $\frac{56}{3.8}$ & $\frac{57}{6.8}$ & $\frac{58}{4.5}$ & $\frac{61}{3.7}$ & $\frac{62}{4.1}$ & $\frac{63}{6.2}$ & $\frac{64}{4.0}$ & $\frac{1}{4.9}$ & & \\
\hline SS \& GS & & & & & & & & & & $\frac{49}{4.5}$ & $\frac{50}{2.9}$ & $\frac{51}{7.0}$ & $\frac{52}{5.5}$ & & & & & & & & & & & \\
\hline
\end{tabular}


Table 20. Volume of melamphaids $\left(\mathrm{ml} / 10,000 \mathrm{~m}^{3}\right)$.

\begin{tabular}{|c|c|c|c|c|c|c|c|c|c|c|c|c|c|c|c|c|c|c|c|c|c|c|c|c|}
\hline & \multicolumn{4}{|c|}{$\begin{array}{c}\text { Oceanus } 118 \\
\text { April }\end{array}$} & \multicolumn{5}{|c|}{$\begin{array}{c}\text { Oceanus } 121 \\
\text { June }\end{array}$} & \multicolumn{5}{|c|}{$\begin{array}{l}\text { Oceanus } 125 \\
\text { August }\end{array}$} & \multicolumn{7}{|c|}{$\begin{array}{l}\text { Knorr } 98 \\
\text { Sept-Oct }\end{array}$} & \multicolumn{3}{|c|}{$\begin{array}{c}\text { A2 } 110 \\
\text { Sept-0ct }\end{array}$} \\
\hline $\begin{array}{l}\text { Slope } \\
\text { Water }\end{array}$ & $\begin{array}{c}\frac{8}{\overline{\overline{0}}} \\
\underline{0} \\
\frac{11.8}{20.6} \\
\end{array}$ & $\begin{array}{c}9 \\
0 \\
0 \\
0 \\
1.3\end{array}$ & & & $\begin{array}{l}\frac{19}{0.1} \\
\frac{0.9}{\underline{0}} \\
\frac{2.0}{0.1} \\
\underline{0.1}\end{array}$ & $\begin{array}{c}20 \\
0 \\
0 \\
0.1 \\
0.5 \\
0\end{array}$ & $\begin{array}{l}\frac{33}{\underline{0}} \\
\underline{0.1} \\
\underline{0} \\
\underline{0} \\
\underline{0.5}\end{array}$ & $\begin{array}{l}\frac{34}{\underline{0}} \\
\underline{0} \\
11.5 \\
\end{array}$ & & $\begin{array}{c}35 \\
0 \\
0 \\
0 \\
0.1 \\
0.2\end{array}$ & $\begin{array}{c}\frac{47}{0} \\
\underline{0} \\
\underline{3.2} \\
\underline{0.7}\end{array}$ & $\begin{array}{c}48 \\
0 \\
0 \\
0.9 \\
10.8\end{array}$ & $\begin{array}{l}\frac{53}{\underline{0}} \\
\underline{0} \\
\frac{0.1}{\underline{0}}\end{array}$ & $\begin{array}{c}54 \\
0 \\
0 \\
0 \\
1.5\end{array}$ & $\frac{\frac{59}{0.3}}{\frac{0.2}{1.2}} \frac{8.7}{\frac{16.6}{22.4}}$ & $\begin{array}{c}60 \\
0 \\
0 \\
0 \\
0 \\
0.1\end{array}$ & 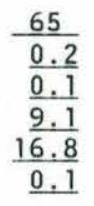 & $\begin{array}{c}66 \\
0 \\
0 \\
0 \\
0.3 \\
\underline{4.2}\end{array}$ & & & & $\begin{array}{l}\frac{4}{0} \\
0 \\
0.1 \\
0.4\end{array}$ & $\begin{array}{r}\frac{5}{0} \\
\underline{\underline{0} .4} \\
\frac{19.7}{0.4}\end{array}$ & \\
\hline $\begin{array}{l}\text { High } \\
\text { Velocity } \\
\text { Region }\end{array}$ & $\begin{array}{c}\frac{12}{0} \\
0.1 \\
0.1 \\
13.8\end{array}$ & $\frac{\frac{13}{0.2}}{\frac{0.2}{0.3}}$ & $\begin{array}{l}\frac{14}{\frac{0.1}{0.9}} \\
\frac{0}{0} \\
0.1\end{array}$ & $\begin{array}{c}15 \\
0 \\
0 \\
0.3 \\
7.4\end{array}$ & $\begin{array}{c}26 \\
0 \\
0 \\
0 \\
0 \\
-\end{array}$ & $\begin{array}{c}27 \\
0 \\
0 \\
0 \\
0 \\
4.2\end{array}$ & $\begin{array}{c}30 \\
0 \\
0 \\
0 \\
0.6 \\
0.5\end{array}$ & $\begin{array}{c}31 \\
0 \\
0 \\
0 \\
0.1 \\
0.9\end{array}$ & $\frac{\frac{32}{0.2}}{\frac{0.1}{\underline{0}}}$ & $\frac{\frac{41}{0.4}}{\frac{0.6}{0.6}}$ & $\begin{array}{l}\frac{42}{\frac{0.4}{1.2}} \\
\frac{0.1}{0.3} \\
\underline{0.3}\end{array}$ & $\begin{array}{c}43 \\
0 \\
0 \\
0 \\
0 \\
0.6\end{array}$ & $\begin{array}{l}\frac{44}{\underline{6.3}} \\
\underline{0} \\
\underline{0.9}\end{array}$ & & $\begin{array}{l}\frac{55}{0.3} \\
\frac{0.5}{0.1} \\
\underline{0.3}\end{array}$ & & & & & & & $\begin{array}{r}\frac{2}{\frac{0.7}{1.2}} \\
\frac{37.3}{0.4} \\
\underline{0.4}\end{array}$ & $\begin{array}{l}\frac{3}{0} \\
0.1 \\
0.4 \\
3.8\end{array}$ & \\
\hline $\begin{array}{l}\text { Ring } \\
\text { Center }\end{array}$ & $\begin{array}{l}\frac{10}{1.2} \\
\frac{0.1}{\underline{0}} \\
0.1\end{array}$ & $\begin{array}{c}11 \\
0 \\
0 \\
0.1 \\
0.5\end{array}$ & $\begin{array}{l}\frac{16}{0} \\
0.6 \\
0.2\end{array}$ & $\frac{\frac{17}{0.1}}{\frac{0.4}{1.2}}$ & $\begin{array}{l}\frac{21}{0} \\
\frac{0.1}{0.2} \\
\underline{0.2} \\
\underline{0.3} \\
\underline{0.2}\end{array}$ & $\begin{array}{l}\frac{23}{0} \\
\underline{0.5} \\
\underline{0.1} \\
\frac{9.7}{1.6} \\
\end{array}$ & $\begin{array}{c}24 \\
0 \\
0 \\
0 \\
0.1 \\
1.7\end{array}$ & $\begin{array}{c}28 \\
0 \\
0 \\
0 \\
2.6 \\
10.5\end{array}$ & & & & & & & $\begin{array}{l}\frac{56}{0.4} \\
\frac{0.4}{0} \\
\underline{0.1} \\
\underline{0.3}\end{array}$ & $\begin{array}{l}\frac{57}{0.9} \\
\frac{0.1}{1.1} \\
\frac{0.1}{\underline{0}} \\
\underline{0.3}\end{array}$ & $\begin{array}{c}58 \\
0 \\
0 \\
0 \\
0.3 \\
9.1\end{array}$ & $\begin{array}{l}\frac{61}{0.2} \\
\frac{0.8}{\underline{0}} \\
\underline{0} \\
\underline{0.3}\end{array}$ & $\begin{array}{c}62 \\
0 \\
0 \\
0 \\
0.4 \\
0.8\end{array}$ & $\begin{array}{l}\frac{63}{1.1} \\
\frac{0.3}{0.2} \\
\underline{0} \underline{0} \\
\underline{0.8}\end{array}$ & $\begin{array}{c}64 \\
0 \\
0 \\
0.3 \\
0.6 \\
1.9\end{array}$ & $\frac{\frac{1}{1.4}}{\frac{0.1}{0.1}}$ & & \\
\hline $\begin{array}{l}\text { Sargasso } \\
\text { Sea } \\
\& \\
\text { Gulf } \\
\text { Stream }\end{array}$ & & & & & & & & & & $\frac{\frac{49}{0.8}}{\frac{0.1}{\underline{0}}}$ & $\begin{array}{c}50 \\
0 \\
0 \\
0.1 \\
1.7\end{array}$ & $\begin{array}{c}51 \\
0 \\
0 \\
1.1 \\
0.6\end{array}$ & $\begin{array}{l}\frac{52}{\frac{1.0}{0.2}} \\
\underline{0.0} \\
\underline{0.6}\end{array}$ & & & & & & & & & & & \\
\hline $\begin{array}{l}\text { Slope } \\
\text { Water }\end{array}$ & $\frac{8}{-}$ & $\frac{9}{0.3}$ & $\frac{18}{0.2}$ & & $\frac{19}{0.3}$ & $\frac{20}{0.2}$ & $\frac{33}{0.3}$ & $\frac{34}{6.9}$ & & $\frac{35}{0.1}$ & $\frac{47}{1.0}$ & $\frac{48}{3.0}$ & $\frac{53}{0.1}$ & $\frac{54}{0.4}$ & $\frac{59}{9.9}$ & $\frac{60}{0.1}$ & $\frac{65}{5.3}$ & $\frac{66}{0.9}$ & & & & $\frac{4}{0.1}$ & $\frac{5}{5.6}$ & $\frac{6}{3.8}$ \\
\hline $\begin{array}{l}\text { High } \\
\text { Ve1ocity } \\
\text { Region }\end{array}$ & $\frac{12}{3.5}$ & $\frac{13}{0.8}$ & $\frac{14}{0.3}$ & $\frac{15}{1.9}$ & $\frac{27}{1.1}$ & $\frac{29}{1.4}$ & $\frac{30}{0.2}$ & $\frac{31}{0.2}$ & $\frac{32}{-}$ & $\frac{41}{7.5}$ & $\frac{42}{0.5}$ & $\frac{43}{0.1}$ & $\frac{44}{4.0}$ & & $\frac{55}{0.3}$ & & & & & & & $\frac{2}{9.9}$ & $\frac{3}{1.1}$ & \\
\hline $\begin{array}{l}\text { Ring } \\
\text { Center }\end{array}$ & $\frac{10}{0.4}$ & $\frac{11}{0.2}$ & $\frac{16}{-}$ & $\frac{17}{0.8}$ & $\frac{21}{0.2}$ & $\frac{23}{2.4}$ & $\frac{24}{0.4}$ & $\frac{28}{0.7}$ & & $\frac{37}{2.4}$ & $\frac{38}{0.1}$ & $\frac{39}{0.2}$ & & & $\frac{56}{0.2}$ & $\frac{57}{0.5}$ & $\frac{58}{1.9}$ & $\frac{61}{0.3}$ & $\frac{62}{0.2}$ & $\frac{63}{0.6}$ & $\frac{64}{0.5}$ & $\frac{1}{0.5}$ & & \\
\hline SS \& GS & & & & & & & & & & $\frac{49}{0.2}$ & $\frac{50}{0.5}$ & $\frac{51}{0.4}$ & $\frac{52}{0.5}$ & & & & & & & & & & & \\
\hline
\end{tabular}


Table 21. Abundance of myctophids (specimens $/ 10,000 \mathrm{~m}^{3}$ ).

\begin{tabular}{|c|c|c|c|c|c|c|c|c|c|c|c|c|c|c|c|c|c|c|c|c|c|c|c|c|}
\hline & \multicolumn{4}{|c|}{$\underset{\text { Apri1 }}{\text { Oceanus }} 118$} & \multicolumn{5}{|c|}{$\begin{array}{c}\text { Oceanus } 121 \\
\text { June }\end{array}$} & \multicolumn{5}{|c|}{$\begin{array}{c}\text { Oceanus } 125 \\
\text { August }\end{array}$} & \multicolumn{7}{|c|}{$\begin{array}{l}\text { Knorr } 98 \\
\text { Sept-0ct }\end{array}$} & \multicolumn{3}{|c|}{$\begin{array}{c}\text { A2 } 110 \\
\text { Sept-0ct }\end{array}$} \\
\hline $\begin{array}{l}\text { Slope } \\
\text { Water }\end{array}$ & $\begin{array}{l}\frac{8}{=} \\
\frac{2.9}{2.7} \\
\frac{0}{2}\end{array}$ & $\begin{array}{r}\frac{9}{0} \\
48.2 \\
10.7 \\
0.7\end{array}$ & & & $\frac{\frac{19}{108.0}}{\frac{3.4}{1.6}} \frac{38.4}{\underline{2.6}}$ & $\begin{array}{r}\frac{20}{1.1} \\
28.9 \\
42.6 \\
5.0 \\
2.4\end{array}$ & $\begin{array}{r}\frac{33}{479.3} \\
\frac{7.9}{5.2} \\
\frac{44.4}{31.0} \\
\end{array}$ & $\frac{\frac{34}{93.6}}{\underline{2.6}}$ & & $\begin{array}{r}\frac{35}{0} \\
27.4 \\
133.7 \\
107.8 \\
2.9\end{array}$ & $\begin{array}{r}\frac{47}{22.0} \\
\frac{7.8}{24.1} \\
\underline{13.4}\end{array}$ & $\begin{array}{l}\frac{48}{1.0} \\
63.1 \\
39.7 \\
17.6\end{array}$ & $\frac{\frac{53}{65.6}}{\frac{35.6}{40.8}} \frac{1.1}{1}$ & $\begin{array}{r}\frac{54}{2.6} \\
27.2 \\
201.8 \\
10.3\end{array}$ & 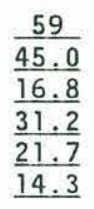 & $\begin{array}{r}\frac{60}{1.6} \\
19.1 \\
119.7 \\
107.4 \\
16.2\end{array}$ & 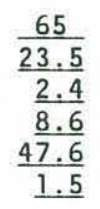 & $\begin{array}{r}\frac{66}{0.4} \\
30.0 \\
47.8 \\
56.7 \\
6.9\end{array}$ & & & & $\begin{array}{r}\frac{4}{0.5} \\
44.1 \\
59.4 \\
20.0\end{array}$ & $\frac{\frac{5}{24.0}}{\frac{4.8}{29.4}}$ & \\
\hline $\begin{array}{l}\text { High } \\
\text { Velocity } \\
\text { Region }\end{array}$ & $\begin{array}{r}\frac{12}{0.3} \\
11.2 \\
31.7 \\
1.4\end{array}$ & $\frac{\frac{13}{25.0}}{\frac{3.3}{2.0}}$ & $\frac{\frac{14}{23.1}}{\frac{6.7}{5.9}} \frac{2.1}{\underline{2}}$ & $\begin{array}{r}\frac{15}{3.3} \\
28.8 \\
11.2 \\
1.4\end{array}$ & $\begin{array}{r}\frac{26}{1.9} \\
4.2 \\
231.9 \\
271.4 \\
-\end{array}$ & $\begin{array}{r}\frac{27}{4.4} \\
561.8 \\
626.4 \\
13.3 \\
3.7\end{array}$ & $\begin{array}{r}\frac{30}{2.6} \\
41.6 \\
272.8 \\
15.4 \\
7.9\end{array}$ & $\begin{array}{r}\frac{31}{1.3} \\
0 \\
143.2 \\
13.7 \\
0.3\end{array}$ & $\frac{\frac{32}{21.1}}{\frac{20.4}{83.5}} \frac{5.4}{\underline{5.4}}$ & $\frac{\frac{41}{10.6}}{\frac{28.6}{6.6}}$ & $\frac{\frac{42}{14.5}}{\frac{31.2}{64.9}} \frac{6.7}{6.7}$ & \begin{tabular}{r}
\multicolumn{1}{c}{43} \\
1.7 \\
3.1 \\
125.4 \\
50.4 \\
3.3
\end{tabular} & $\frac{\frac{44}{39.9}}{\frac{61.3}{1.9}}$ & & $\frac{\frac{55}{57.1}}{\frac{3.6}{1.2}} \frac{6.7}{6}$ & & & & & & & $\frac{\frac{2}{25.8}}{\frac{2.4}{4.7}}$ & $\begin{array}{r}\frac{3}{2.5} \\
45.4 \\
40.0 \\
13.8\end{array}$ & \\
\hline $\begin{array}{l}\text { Ring } \\
\text { Core }\end{array}$ & $\frac{\frac{10}{13.6}}{\frac{12.4}{11.8}} \frac{1.2}{1}$ & $\begin{array}{l}\frac{11}{0} \\
6.1 \\
8.1 \\
5.7\end{array}$ & $\begin{array}{l}\frac{16}{0} \\
15.0 \\
46.7\end{array}$ & $\frac{\frac{17}{37.8}}{\frac{4.2}{2.4}}$ & $\frac{\frac{21}{396.0}}{\frac{17.2}{\frac{35.5}{48.7}}} \frac{3.3}{\underline{3.3}}$ & $\frac{\frac{23}{127.1}}{\frac{23.3}{33.0}} \frac{55.9}{\underline{5.9}}$ & $\begin{array}{r}\frac{24}{6.5} \\
90.0 \\
126.8 \\
112.5 \\
2.2\end{array}$ & $\begin{array}{r}\frac{28}{1.7} \\
21.2 \\
574.6 \\
6.2 \\
1.0\end{array}$ & & & & & & & $\begin{array}{r}\frac{56}{84.1} \\
\frac{3.9}{\underline{0}} \\
\frac{7.4}{13.1} \\
\end{array}$ & $\begin{array}{l}\frac{57}{86.9} \\
\frac{2.5}{2.2} \\
\frac{18.4}{16.6} \\
\end{array}$ & $\begin{array}{c}\frac{58}{0.8} \\
0 \\
14.3 \\
63.2 \\
21.8\end{array}$ & $\begin{array}{l}\frac{61}{63.7} \\
\frac{3.9}{4.1} \\
\frac{48.1}{12.9} \\
\end{array}$ & $\begin{array}{r}\frac{62}{0.4} \\
0.3 \\
22.0 \\
56.5 \\
26.8\end{array}$ & $\begin{array}{r}\frac{63}{25.2} \\
\frac{1.3}{2.4} \\
\frac{19.6}{21.9} \\
\end{array}$ & $\begin{array}{l}\frac{64}{0.2} \\
11.2 \\
21.4 \\
34.3 \\
11.5\end{array}$ & $\frac{\frac{1}{30.8}}{\frac{1.6}{12.8}} \frac{19.2}{19.0}$ & & \\
\hline $\begin{array}{l}\text { Sargasso } \\
\text { Sea } \\
\& \\
\text { Gulf } \\
\text { Stream }\end{array}$ & & & & & & & & & & $\frac{\frac{49}{48.9}}{\frac{3.6}{2.7}}$ & $\begin{array}{r}\frac{50}{0.8} \\
2.9 \\
32.1 \\
10.6\end{array}$ & $\begin{array}{r}\frac{51}{1.5} \\
0.9 \\
46.5 \\
14.5\end{array}$ & $\frac{\frac{52}{33.0}}{\frac{4.7}{23.0}}$ & & & & & & & & & & & \\
\hline . & & & & & & & & & & & & & & & & & & & & & & & & \\
\hline $\begin{array}{l}\text { Slope } \\
\text { Water }\end{array}$ & $\frac{8}{-}$ & $\frac{9}{14.9}$ & $\frac{18}{4.7}$ & & $\frac{19}{22.0}$ & $\frac{20}{19.4}$ & $\frac{33}{50.8}$ & $\frac{34}{27.7}$ & & $\frac{35}{54.4}$ & $\frac{47}{16.8}$ & $\frac{48}{30.4}$ & $\frac{53}{35.8}$ & $\frac{54}{60.5}$ & $\frac{59}{25.8}$ & $\frac{60}{51.1}$ & $\frac{65}{16.7}$ & $\frac{66}{25.4}$ & & & & $3 \frac{4}{1.0}$ & $\frac{5}{17.4}$ & $2 \frac{6}{1.4}$ \\
\hline $\begin{array}{l}\text { High } \\
\text { Velocity } \\
\text { Region }\end{array}$ & $\frac{12}{11.2}$ & $\frac{13}{8.1}$ & $\frac{14}{9.5}$ & $\frac{15}{11.2}$ & $\frac{27}{214.5}$ & $\frac{29}{66.8}$ & $\frac{30}{64.2}$ & $\frac{31}{31.8}$ & $\frac{32}{-}$ & $\frac{41}{14.1}$ & $\frac{42}{26.7}$ & $\frac{43}{36.9}$ & $\frac{44}{36.6}$ & & $\frac{55}{23.0}$ & & & & & & & $\frac{2}{14.8}$ & $\frac{3}{25.4}$ & \\
\hline $\begin{array}{l}\text { Ring } \\
\text { Core }\end{array}$ & $\frac{10}{9.8}$ & $\frac{11}{5.0}$ & $\frac{16}{-}$ & $\frac{17}{11.7}$ & $\frac{21}{43.3}$ & $\frac{23}{43.9}$ & $\frac{24}{52.6}$ & $\frac{28}{149.8}$ & & $\frac{37}{21.1}$ & $\frac{38}{29.9}$ & $\frac{39}{18.0}$ & & & $\frac{56}{21.7}$ & $\frac{57}{25.3}$ & $\frac{58}{20.1}$ & $\frac{61}{26.5}$ & $\frac{62}{21.2}$ & $\frac{63}{18.1}$ & $\frac{64}{12.5}$ & $\frac{1}{16.1}$ & & \\
\hline SS \& GS & & & & & & & & & & $\frac{49}{15.0}$ & $\frac{50}{11.6}$ & $\frac{51}{15.9}$ & $\frac{52}{19.4}$ & & & & & & & & & & & \\
\hline
\end{tabular}


Table 22. Volume of myctophids $\left(\mathrm{m} 1 / 10,000 \mathrm{~m}^{3}\right)$.

\begin{tabular}{|c|c|c|c|c|c|c|c|c|c|c|c|c|c|c|c|c|c|c|c|c|c|c|c|c|}
\hline & \multicolumn{4}{|c|}{$\begin{array}{c}\text { Oceanus } \\
\text { April }\end{array}$} & \multicolumn{5}{|c|}{$\begin{array}{c}\text { Oceanus } 121 \\
\text { June }\end{array}$} & \multicolumn{5}{|c|}{$\begin{array}{l}\text { Oceanus } 125 \\
\text { Auqust }\end{array}$} & \multicolumn{7}{|c|}{$\begin{array}{l}\text { Knorr } 98 \\
\text { Sept-0ct }\end{array}$} & \multicolumn{3}{|c|}{$\begin{array}{c}\text { A2 } 110 \\
\text { Sept-0ct }\end{array}$} \\
\hline $\begin{array}{l}\text { Slope } \\
\text { Water }\end{array}$ & $\begin{array}{l}\frac{8}{2.9} \\
\frac{2.9}{\frac{5.9}{0}}\end{array}$ & $\begin{array}{r}\frac{9}{0} \\
37.5 \\
15.8 \\
0.7\end{array}$ & & & $\frac{\frac{19}{12.0}}{\frac{0.7}{0.1}} \frac{\frac{0}{1.4}}{11.6}$ & $\begin{array}{l}\frac{20}{1.0} \\
2.0 \\
6.1 \\
7.5 \\
2.6\end{array}$ & $\frac{\frac{33}{33.4}}{\frac{3.4}{1.4}} \frac{1.4}{\frac{4.1}{8.4}}$ & $\begin{array}{r}\frac{34}{2.9} \\
\frac{0.5}{10.9} \\
\end{array}$ & & $\begin{array}{r}35 \\
0 \\
2.8 \\
9.4 \\
10.4 \\
1.1\end{array}$ & $\frac{\frac{47}{6.8}}{\frac{6.8}{1.8}} \frac{11.6}{19.7}$ & $\begin{array}{l}\frac{48}{0.1} \\
11.1 \\
24.3 \\
36.9\end{array}$ & $\frac{\frac{53}{6.3}}{\frac{\frac{6}{3.2}}{14.7}} \frac{0.6}{0.6}$ & $\begin{array}{r}\frac{54}{0.1} \\
5.9 \\
22.2 \\
13.0\end{array}$ & 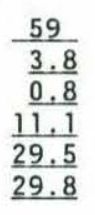 & $\begin{array}{r}\frac{60}{0} \\
7.8 \\
12.8 \\
40.0 \\
25.2\end{array}$ & $\frac{\frac{65}{4.1}}{\frac{4.2}{1.2}}$ & $\begin{array}{r}\frac{66}{10.3} \\
3.7 \\
7.4 \\
18.8 \\
5.3\end{array}$ & & & & $\begin{array}{c}\frac{4}{0} \\
7.2 \\
10.2 \\
29.1\end{array}$ & $\frac{\frac{5}{9.6}}{\frac{1.9}{14.4}}$ & \\
\hline $\begin{array}{l}\text { High } \\
\text { Velocity } \\
\text { Region }\end{array}$ & $\begin{array}{l}\frac{12}{0} \\
2.5 \\
4.2 \\
2.1\end{array}$ & $\frac{\frac{13}{\frac{6.1}{2.2}}}{\frac{2.4}{2.4}}$ & $\begin{array}{l}\frac{14}{\frac{8.6}{2.5}} \\
\frac{8.1}{0.1}\end{array}$ & $\begin{array}{l}\frac{15}{0.2} \\
4.6 \\
15.2 \\
13.4\end{array}$ & $\begin{array}{r}\frac{26}{0.1} \\
0.8 \\
12.5 \\
12.7 \\
-\end{array}$ & \begin{tabular}{r}
\multicolumn{1}{c}{$\frac{27}{0.1}$} \\
17.8 \\
33.0 \\
9.2 \\
4.1
\end{tabular} & $\begin{array}{r}\frac{30}{0.1} \\
8.5 \\
14.7 \\
8.4 \\
7.1\end{array}$ & $\begin{array}{c}31 \\
0.1 \\
0 \\
15.9 \\
10.5 \\
0.1\end{array}$ & $\frac{\frac{32}{7.4}}{\frac{2.7}{2}} \frac{10.3}{\underline{9.8}}$ & $\frac{\frac{41}{1.8}}{\frac{10.4}{13.4}}$ & $\begin{array}{l}\frac{42}{\frac{5.4}{1.9}} \\
\frac{25.5}{10.4} \\
\end{array}$ & \begin{tabular}{r}
\multicolumn{1}{c}{43} \\
0.2 \\
0.1 \\
21.3 \\
23.6 \\
3.9
\end{tabular} & $\frac{\frac{44}{6.2}}{\frac{12.4}{2.9}}$ & & $\frac{\frac{55}{\frac{4.3}{1.0}}}{\frac{6.1}{2.1}}$ & & & & & & & $\frac{\frac{2}{12.0}}{\frac{0.2}{5.9}}$ & $\begin{array}{l}\frac{3}{0.3} \\
14.4 \\
10.6 \\
9.6\end{array}$ & \\
\hline $\begin{array}{l}\text { Ring } \\
\text { Core }\end{array}$ & $\begin{array}{l}\frac{10}{\frac{3.0}{4.1}} \\
\frac{2.8}{0.1}\end{array}$ & $\begin{array}{c}11 \\
0 \\
0.4 \\
4.8 \\
9.8\end{array}$ & $\begin{array}{l}\frac{16}{0} \\
1.1 \\
9.6\end{array}$ & $\begin{array}{l}\frac{17}{\frac{9.4}{2.6}} \\
\frac{2.3}{\underline{2.3}}\end{array}$ & 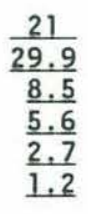 & $\frac{\frac{23}{33.2}}{\frac{4.1}{5.7}} \frac{\frac{4.7}{1.7}}{\frac{1.7}{10}}$ & $\begin{array}{r}\frac{24}{0.6} \\
10.0 \\
14.4 \\
6.1 \\
0.5\end{array}$ & $\begin{array}{r}\frac{28}{0.1} \\
12.7 \\
22.0 \\
4.8 \\
2.6\end{array}$ & & & & & & & $\begin{array}{l}\frac{56}{\frac{5.0}{0.3}} \\
\underline{0} \\
\frac{0.4}{2.2}\end{array}$ & $\begin{array}{l}\frac{57}{5.5} \\
\frac{5.5}{0.5} \\
\frac{3.4}{1.9}\end{array}$ & $\begin{array}{c}\frac{58}{0.1} \\
0 \\
0.6 \\
4.8 \\
2.9\end{array}$ & $\begin{array}{l}\frac{61}{5.1} \\
\underline{5.8} \\
\underline{0.2} \\
\underline{0.7} \\
\underline{4.4}\end{array}$ & $\begin{array}{c}62 \\
0 \\
0 \\
0.7 \\
4.4 \\
7.4\end{array}$ & 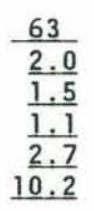 & $\begin{array}{c}64 \\
0 \\
0.3 \\
1.0 \\
2.3 \\
3.2\end{array}$ & $\frac{\frac{1}{6.8}}{\frac{4.2}{1.3}} \frac{1.2}{1.2}$ & & \\
\hline $\begin{array}{l}\text { Sargasso } \\
\text { Sea } \\
\& \\
\text { Gulf } \\
\text { Stream }\end{array}$ & & & & & & & & & & $\begin{array}{l}\frac{49}{7.5} \\
\underline{2.3} \\
\underline{0.2} \\
\underline{0.7}\end{array}$ & $\begin{array}{l}\frac{50}{0.1} \\
0.1 \\
1.2 \\
0.8\end{array}$ & $\begin{array}{l}51 \\
0.1 \\
0 \\
6.3 \\
0.8\end{array}$ & $\frac{52}{\frac{3.0}{1.7}} \frac{0.8}{1.1}$ & & & & & & & & & & & \\
\hline $\begin{array}{l}\text { Slope } \\
\text { Water }\end{array}$ & $\frac{8}{-}$ & $\frac{9}{13.5}$ & $\frac{18}{5.3}$ & & $\frac{19}{4.6}$ & $\frac{20}{4.2}$ & $\frac{33}{7.4}$ & $\frac{34}{6.9}$ & & $\frac{35}{4.7}$ & $\frac{47}{10.0}$ & $\frac{48}{18.1}$ & $\frac{53}{6.2}$ & $\frac{54}{10.3}$ & $\frac{59}{15.0}$ & $\frac{60}{16.4}$ & $\frac{65}{6.2}$ & $\frac{66}{6.8}$ & & & & $\frac{4}{11.6}$ & $\frac{5}{11.1}$ & $\frac{6}{17.4}$ \\
\hline $\begin{array}{l}\text { High } \\
\text { Velocity } \\
\text { Region }\end{array}$ & $\frac{12}{2.2}$ & $\frac{13}{3.3}$ & $\frac{14}{4.8}$ & $\frac{15}{8.4}$ & $\frac{27}{12.6}$ & $\frac{29}{10.2}$ & $\frac{30}{6.9}$ & $\frac{31}{5.3}$ & $\frac{32}{-}$ & $\frac{41}{6.9}$ & $\frac{42}{9.7}$ & $\frac{43}{9.8}$ & $\frac{44}{6.8}$ & & $\frac{55}{3.9}$ & & & & & & & $\frac{2}{6.5}$ & $\frac{3}{8.7}$ & \\
\hline $\begin{array}{l}\text { Ring } \\
\text { Core }\end{array}$ & $\frac{10}{2.5}$ & $\frac{11}{3.8}$ & $\frac{16}{-}$ & $\frac{17}{4.2}$ & $\frac{21}{6.5}$ & $\frac{23}{8.4}$ & $\frac{24}{4.6}$ & $\frac{28}{9.1}$ & & $\frac{37}{6.7}$ & $\frac{38}{5.3}$ & $\frac{39}{4.9}$ & & & $\frac{56}{1.6}$ & $\frac{57}{2.6}$ & $\frac{58}{1.7}$ & $\frac{61}{2.6}$ & $\frac{62}{2.5}$ & $\frac{63}{3.6}$ & $\frac{64}{1.2}$ & $\frac{1}{3.4}$ & & \\
\hline SS \& GS & & & & & & & & & & $\frac{49}{2.7}$ & $\frac{50}{0.6}$ & $\frac{51}{1.8}$ & $\frac{52}{1.7}$ & & & & & & & & & & & \\
\hline
\end{tabular}


Table 23. Abundance of photichthyids (specimens $/ 10,000 \mathrm{~m}^{3}$ ).

\begin{tabular}{|c|c|c|c|c|c|c|c|c|c|c|c|c|c|c|c|c|c|c|c|c|c|c|c|c|}
\hline & \multicolumn{4}{|c|}{$\begin{array}{c}\text { Oceanus } 118 \\
\text { Apri1 }\end{array}$} & \multicolumn{5}{|c|}{$\begin{array}{c}\text { Oceanus } 121 \\
\text { June }\end{array}$} & \multicolumn{5}{|c|}{$\begin{array}{l}\text { Oceanus } 125 \\
\text { August }\end{array}$} & \multicolumn{7}{|c|}{$\begin{array}{l}\text { Knorr } 98 \\
\text { Sept-Oct }\end{array}$} & \multicolumn{3}{|c|}{$\begin{array}{c}\text { A2 } 110 \\
\text { Sept-0ct }\end{array}$} \\
\hline $\begin{array}{l}\text { Slope } \\
\text { Water }\end{array}$ & $\begin{array}{l}8 \\
\overline{\overline{0}} \\
\underline{0} \\
\underline{0}\end{array}$ & $\begin{array}{l}9 \\
0 \\
0 \\
0 \\
0\end{array}$ & & & $\frac{\frac{19}{22.9}}{\frac{0}{0}}$ & $\begin{array}{c}20 \\
0 \\
0.5 \\
0 \\
0 \\
0\end{array}$ & $\frac{33}{\frac{1.7}{0.4}} \frac{\underline{0}}{\underline{0}}$ & $\frac{\frac{34}{17.7}}{\frac{0}{0}}$ & & $\begin{array}{c}\frac{35}{0} \\
1.7 \\
0.7 \\
0 \\
0\end{array}$ & $\frac{\frac{47}{1.9}}{\frac{0}{0}}$ & $\begin{array}{c}48 \\
0 \\
0.6 \\
0 \\
0\end{array}$ & $\frac{\frac{53}{2.1}}{\frac{\underline{2.9}}{\underline{0}}}$ & $\begin{array}{c}54 \\
0 \\
0 \\
0 \\
0\end{array}$ & $\begin{array}{l}\frac{59}{5.0} \\
\frac{4.8}{0.3} \\
\frac{0}{\underline{0}}\end{array}$ & $\begin{array}{c}60 \\
0.8 \\
13.2 \\
0.3 \\
0 \\
0\end{array}$ & $\frac{\frac{65}{2.6}}{\frac{\underline{0} .3}{0}} \frac{\underline{0}}{\underline{0}}$ & $\begin{array}{c}\frac{66}{1.5} \\
4.3 \\
0 \\
0 \\
0\end{array}$ & & & & $\begin{array}{c}\frac{4}{0} \\
0.9 \\
0 \\
0\end{array}$ & $\frac{\frac{5}{1.3}}{\frac{0.7}{\frac{0}{0}}}$ & \\
\hline $\begin{array}{l}\text { High } \\
\text { Velocity } \\
\text { Region }\end{array}$ & $\begin{array}{r}12 \\
0 \\
0 \\
0 \\
0\end{array}$ & $\frac{\frac{13}{1.5}}{\frac{0}{0}}$ & $\frac{\frac{14}{0.3}}{\frac{0.3}{\underline{0}}}$ & $\begin{array}{r}15 \\
0 \\
0 \\
0 \\
0\end{array}$ & $\begin{array}{c}26 \\
0 \\
0.5 \\
1.4 \\
0 \\
-\end{array}$ & $\begin{array}{c}27 \\
0 \\
0.3 \\
0 \\
0 \\
0\end{array}$ & $\begin{array}{c}30 \\
0 \\
4.0 \\
12.2 \\
0 \\
0.2\end{array}$ & $\begin{array}{c}31 \\
0 \\
0.5 \\
2.5 \\
0.3 \\
0\end{array}$ & $\frac{\frac{32}{4.5}}{\frac{1.2}{\frac{0}{0}}}$ & $\frac{\frac{41}{2.7}}{\frac{0}{0}}$ & $\frac{\frac{42}{3.9}}{\frac{3.9}{\underline{0.9}}}$ & $\begin{array}{c}43 \\
0 \\
0.2 \\
0.8 \\
0 \\
0\end{array}$ & $\frac{44}{\frac{1.7}{0}}$ & & $\begin{array}{c}\frac{55}{2.4} \\
\underline{0} \\
\frac{1.6}{\underline{0}}\end{array}$ & & & & & & & $\frac{\frac{2}{2.7}}{\frac{0.5}{\underline{0}}}$ & $\begin{array}{c}\frac{3}{1.8} \\
1.1 \\
0 \\
0\end{array}$ & \\
\hline $\begin{array}{l}\text { Ring } \\
\text { Core }\end{array}$ & $\frac{10}{\frac{0.8}{\underline{0}}} \frac{\underline{0}}{\underline{0}}$ & $\begin{array}{c}11 \\
0 \\
0.6 \\
0 \\
0\end{array}$ & $\begin{array}{r}16 \\
0 \\
0 \\
0\end{array}$ & $\frac{\frac{17}{\frac{1.7}{1.1}}}{\frac{0}{1.1}}$ & $\begin{array}{c}\frac{21}{31.0} \\
\frac{3.2}{8.8} \\
\underline{0} \\
\underline{0.6}\end{array}$ & $\begin{array}{l}\frac{23}{\frac{2.9}{1.3}} \\
\frac{0.5}{\underline{0}} \\
\underline{0.6}\end{array}$ & $\begin{array}{c}\frac{24}{2.1} \\
0 \\
3.2 \\
0 \\
0\end{array}$ & $\begin{array}{c}\frac{28}{0.9} \\
4.6 \\
2.1 \\
0 \\
0\end{array}$ & & & & & & & $\begin{array}{l}\frac{56}{\frac{8.2}{2.2}} \\
\frac{1.6}{0.5} \\
\underline{0}\end{array}$ & $\frac{\frac{57}{4.7}}{\frac{4.7}{3.5}} \frac{\frac{3.5}{2.2}}{\underline{0}}$ & $\begin{array}{c}\frac{58}{0.5} \\
4.6 \\
17.6 \\
0.6 \\
0\end{array}$ & $\begin{array}{l}\frac{61}{3.4} \\
\frac{3.8}{8.9} \\
\frac{8.4}{\underline{0}}\end{array}$ & $\begin{array}{c}\frac{62}{0.2} \\
1.5 \\
5.1 \\
1.1 \\
0\end{array}$ & $\frac{\frac{63}{3.6}}{\frac{5.9}{5.8}}$ & $\begin{array}{c}\frac{64}{3.7} \\
3.1 \\
2.1 \\
0 \\
0\end{array}$ & $\frac{\frac{1}{0.8}}{\frac{0.6}{2.6}} \frac{0.3}{\underline{0}}$ & & \\
\hline $\begin{array}{l}\text { Sargasso } \\
\text { Sea } \\
\& \\
\text { Guiff } \\
\text { Stream }\end{array}$ & & & & & & & & & & $\begin{array}{l}\frac{49}{4.2} \\
\frac{5.8}{0.7} \\
0.3\end{array}$ & $\begin{array}{c}50 \\
0 \\
5.1 \\
0 \\
0.6\end{array}$ & $\begin{array}{c}51 \\
0 \\
8.0 \\
1.1 \\
0\end{array}$ & $\frac{\frac{52}{5.9}}{\frac{5.9}{\frac{0}{0}}}$ & & & & & & & & & & & \\
\hline $\begin{array}{l}\text { Slope } \\
\text { Water }\end{array}$ & $\frac{8}{-}$ & $\frac{9}{0}$ & $\frac{18}{0}$ & & $\frac{19}{2.3}$ & $\frac{20}{0.1}$ & $\frac{33}{0.2}$ & $\frac{34}{1.4}$ & & $\frac{35}{0.5}$ & $\frac{47}{0.5}$ & $\frac{48}{0.2}$ & $\frac{53}{0.8}$ & $\frac{54}{0}$ & $\frac{59}{2.0}$ & $\frac{60}{1.6}$ & $\frac{65}{0.6}$ & $\frac{66}{0.9}$ & & & & $\frac{4}{0.2}$ & $\frac{5}{0.5}$ & $\frac{6}{0.2}$ \\
\hline $\begin{array}{l}\text { High } \\
\text { Velocity } \\
\text { Region }\end{array}$ & $\frac{12}{0}$ & $\frac{13}{0.4}$ & $\frac{14}{0.2}$ & $\frac{15}{0}$ & $\frac{27}{0}$ & $\frac{29}{1.3}$ & $\frac{30}{2.9}$ & $\frac{31}{0.6}$ & $\frac{32}{-}$ & $\frac{41}{0.1}$ & $\frac{42}{1.7}$ & $\frac{43}{0.2}$ & $\frac{44}{1.0}$ & & $\frac{55}{1.1}$ & & & & & & & $\frac{2}{0.8}$ & $\frac{3}{0.7}$ & \\
\hline $\begin{array}{l}\text { Ring } \\
\text { Core }\end{array}$ & $\frac{10}{0.2}$ & $\frac{11}{0.2}$ & $\frac{16}{-}$ & $\frac{17}{0.7}$ & $\frac{21}{4.6}$ & $\frac{23}{1.0}$ & $\frac{24}{1.4}$ & $\frac{28}{1.6}$ & & $\frac{37}{5.2}$ & $\frac{38}{2.3}$ & $\frac{39}{1.8}$ & & & $\frac{56}{2.5}$ & $\frac{57}{2.8}$ & $\frac{58}{4.3}$ & $\frac{61}{2.9}$ & $\frac{62}{1.5}$ & $\frac{63}{2.3}$ & $\frac{64}{2.2}$ & $\frac{1}{0.9}$ & & \\
\hline SS \& GS & & & & & & & & & & $\frac{49}{2.8}$ & $\frac{50}{1.4}$ & $\frac{51}{2.3}$ & $\frac{52}{3.2}$ & & & & & & & & & & & \\
\hline
\end{tabular}


Table 24. Volume of photichthyids $\left(\mathrm{ml} / 10,000 \mathrm{~m}^{3}\right)$.

\begin{tabular}{|c|c|c|c|c|c|c|c|c|c|c|c|c|c|c|c|c|c|c|c|c|c|c|c|c|}
\hline & \multicolumn{4}{|c|}{$\begin{array}{c}\text { Oceanus } 118 \\
\text { April }\end{array}$} & \multicolumn{5}{|c|}{$\begin{array}{c}\text { Oceanus } 121 \\
\text { June }\end{array}$} & \multicolumn{5}{|c|}{$\begin{array}{l}\text { Oceanus } 125 \\
\text { August }\end{array}$} & \multicolumn{7}{|c|}{$\begin{array}{l}\text { Knorr } 98 \\
\text { Sept-0ct }\end{array}$} & \multicolumn{3}{|c|}{$\begin{array}{c}\text { A2 } 110 \\
\text { Sept-0ct }\end{array}$} \\
\hline $\begin{array}{l}\text { Slope } \\
\text { Water }\end{array}$ & $\frac{8}{\overline{\overline{0}}}$ & $\begin{array}{l}9 \\
0 \\
0 \\
0 \\
0\end{array}$ & & & $\frac{19}{\frac{1.1}{\underline{0}}}$ & $\begin{array}{c}20 \\
0 \\
0.3 \\
0 \\
0 \\
0\end{array}$ & $\frac{33}{\frac{0.1}{\underline{0}}}$ & $\frac{\frac{34}{2.4}}{\frac{0}{0}}$ & & $\begin{array}{c}35 \\
0 \\
0.1 \\
0 \\
0 \\
0\end{array}$ & $\frac{47}{\frac{0.1}{\underline{0}}}$ & $\begin{array}{c}48 \\
0 \\
0.1 \\
0 \\
0\end{array}$ & $\frac{\frac{53}{0.2}}{\frac{0}{\underline{0}}}$ & $\begin{array}{l}54 \\
0 \\
0 \\
0 \\
0\end{array}$ & $\frac{\frac{59}{1.1}}{\frac{0.2}{\frac{0}{0}}}$ & $\begin{array}{c}\frac{60}{0.1} \\
0.7 \\
0 \\
0 \\
0\end{array}$ & $\frac{65}{\frac{0.3}{\underline{0}}}$ & $\begin{array}{c}66 \\
0.1 \\
0.4 \\
0 \\
0 \\
0\end{array}$ & & & & $\begin{array}{c}4 \\
0 \\
0.2 \\
0 \\
0\end{array}$ & $\frac{\frac{5}{0.1}}{\frac{0}{0}} \frac{0}{\underline{0}}$ & \\
\hline $\begin{array}{l}\text { High } \\
\text { Velocity } \\
\text { Region }\end{array}$ & $\begin{array}{l}12 \\
0 \\
0 \\
0 \\
0\end{array}$ & $\frac{\frac{13}{0,3}}{\frac{0}{0}}$ & $\frac{\frac{14}{0.1}}{\frac{0}{0}}$ & $\begin{array}{r}15 \\
0 \\
0 \\
0 \\
0\end{array}$ & $\begin{array}{c}26 \\
0 \\
0.1 \\
0 \\
0 \\
-\end{array}$ & $\begin{array}{r}27 \\
0 \\
0 \\
0 \\
0 \\
0\end{array}$ & $\begin{array}{c}30 \\
0 \\
0.1 \\
0.3 \\
0 \\
0\end{array}$ & $\begin{array}{c}31 \\
0 \\
0.1 \\
0.1 \\
0 \\
0\end{array}$ & $\frac{\frac{32}{0.3}}{\frac{0}{0}}$ & $\frac{\frac{41}{0.1}}{\frac{0}{0}}$ & $\frac{\frac{42}{0.6}}{\frac{0.1}{\frac{0}{0}}}$ & $\begin{array}{c}43 \\
0 \\
0 \\
0.1 \\
0 \\
0\end{array}$ & $\frac{44}{\frac{0.1}{\underline{0}}}$ & & $\begin{array}{l}\frac{55}{\frac{0.1}{\underline{0}}} \\
\frac{0.1}{\underline{0}}\end{array}$ & & & & & & & $\frac{\frac{2}{0.4}}{\frac{0}{0}}$ & $\begin{array}{l}\frac{3}{0.2} \\
0.1 \\
0 \\
0\end{array}$ & \\
\hline $\begin{array}{l}\text { Ring } \\
\text { Center }\end{array}$ & $\frac{\frac{10}{0.1}}{\frac{0}{0}} \frac{0}{\underline{0}}$ & $\begin{array}{c}11 \\
0 \\
0.1 \\
0 \\
0\end{array}$ & $\begin{array}{c}16 \\
0 \\
0 \\
0\end{array}$ & $\frac{\frac{17}{0.9}}{\frac{0.3}{0}}$ & $\begin{array}{l}\frac{21}{0.6} \\
\frac{0.9}{0.2} \\
\frac{0}{0} \\
0.1\end{array}$ & $\begin{array}{l}\frac{23}{0.7} \\
\frac{0.2}{0.1} \\
\frac{0.1}{\underline{0}} \\
0.1\end{array}$ & $\begin{array}{c}24 \\
0.1 \\
0 \\
0.1 \\
0 \\
0\end{array}$ & $\begin{array}{c}28 \\
0.2 \\
0.3 \\
0 \\
0 \\
0\end{array}$ & & & & & & & $\begin{array}{l}\frac{56}{0.7} \\
\frac{0.2}{0.2} \\
\frac{0.2}{0.1} \\
\underline{0}\end{array}$ & $\begin{array}{l}\frac{57}{0.3} \\
\frac{0.2}{0.1} \\
\frac{0.1}{\underline{0}}\end{array}$ & $\begin{array}{c}58 \\
0.2 \\
0.5 \\
0.6 \\
0 \\
0\end{array}$ & $\frac{\frac{61}{0.1}}{\frac{0.1}{0.1}} \frac{0.3}{\underline{0}}$ & $\begin{array}{c}62 \\
0 \\
0.5 \\
0.2 \\
0 \\
0\end{array}$ & $\frac{\frac{63}{0.1}}{\frac{0.1}{0.2}} \frac{0.1}{\underline{0}}$ & $\begin{array}{c}\frac{64}{0.2} \\
0.2 \\
0.2 \\
0 \\
0\end{array}$ & $\frac{\frac{1}{0.2}}{\frac{0.1}{0.1}}$ & & \\
\hline $\begin{array}{l}\text { Sargasso } \\
\text { Sea } \\
\& \\
\text { Gulf } \\
\text { Stream }\end{array}$ & & & & & & & & & & $\frac{\frac{49}{0.4}}{\frac{0.2}{\underline{0}}}$ & $\begin{array}{c}50 \\
0 \\
0.5 \\
0 \\
0\end{array}$ & $\begin{array}{c}51 \\
0 \\
0.2 \\
0 \\
0\end{array}$ & $\begin{array}{l}\frac{52}{\underline{0}} \\
\underline{0.2} \\
\underline{0} \\
\underline{0}\end{array}$ & & & & & & & & & & & \\
\hline $\begin{array}{l}\text { Slope } \\
\text { Water }\end{array}$ & $\frac{8}{-}$ & $\frac{9}{0}$ & $\frac{18}{0}$ & & $\frac{19}{0.1}$ & $\frac{20}{0.1}$ & $\frac{33}{0.1}$ & $\frac{34}{0.2}$ & & $\frac{35}{0.1}$ & $\frac{47}{0.1}$ & $\frac{48}{0.1}$ & $\frac{53}{0.1}$ & $\frac{54}{0}$ & $\frac{59}{0.3}$ & $\frac{60}{0.1}$ & $\frac{65}{0.1}$ & $\frac{66}{0.1}$ & & & & $\frac{4}{0}$ & $\frac{5}{0.1}$ & $\frac{6}{0.1}$ \\
\hline $\begin{array}{l}\text { High } \\
\text { Velocity } \\
\text { Region }\end{array}$ & $\frac{12}{0}$ & $\frac{13}{0.1}$ & $\frac{14}{0.1}$ & $\frac{15}{0}$ & $\frac{27}{0}$ & $\frac{29}{0.1}$ & $\frac{30}{0.1}$ & $\frac{31}{0.1}$ & $\frac{32}{-}$ & $\frac{41}{0.1}$ & $\frac{42}{0.3}$ & $\frac{43}{0.1}$ & $\frac{44}{0.1}$ & & $\frac{55}{0.1}$ & & & & & & & $\frac{2}{0.1}$ & $\frac{3}{0.1}$ & \\
\hline $\begin{array}{l}\text { Ring } \\
\text { Center }\end{array}$ & $\frac{10}{0.1}$ & $\frac{11}{0.1}$ & $\frac{16}{-}$ & $\frac{17}{0.1}$ & $\frac{21}{0.4}$ & $\frac{23}{0.5}$ & $\frac{24}{0.1}$ & $\frac{28}{0.1}$ & & $\frac{37}{0.2}$ & $\frac{38}{0.1}$ & $\frac{39}{0.1}$ & & & $\frac{56}{0.2}$ & $\frac{57}{0.1}$ & $\frac{58}{0.2}$ & $\frac{61}{0.1}$ & $\frac{62}{0.1}$ & $\frac{63}{0.1}$ & $\frac{64}{0.1}$ & $\frac{1}{0.1}$ & & \\
\hline SS \& GS & & & & & & & & & & $\frac{49}{0.1}$ & $\frac{50}{0.1}$ & $\frac{51}{0.1}$ & $\frac{52}{0.1}$ & & & & & & & & & & & \\
\hline
\end{tabular}


Table 25. Abundance of sternoptychids (specimens $/ 10,000 \mathrm{~m}^{3}$ ).

\begin{tabular}{|c|c|c|c|c|c|c|c|c|c|c|c|c|c|c|c|c|c|c|c|c|c|c|c|c|}
\hline & \multicolumn{4}{|c|}{$\begin{array}{c}\text { Oceanus } 118 \\
\text { Apri1 }\end{array}$} & \multicolumn{5}{|c|}{$\begin{array}{c}\text { Oceanus } 121 \\
\text { June }\end{array}$} & \multicolumn{5}{|c|}{$\begin{array}{c}\text { Oceanus } 125 \\
\text { August }\end{array}$} & \multicolumn{7}{|c|}{$\begin{array}{l}\text { Knorr } 98 \\
\text { Sept-Oct }\end{array}$} & \multicolumn{3}{|c|}{$\begin{array}{c}\text { A2 } 110 \\
\text { Sept-0ct }\end{array}$} \\
\hline $\begin{array}{l}\text { Slope } \\
\text { Water }\end{array}$ & $\begin{array}{l}\frac{8}{2} \\
\frac{1.2}{\frac{2.3}{0}}\end{array}$ & $\begin{array}{l}\frac{9}{1.3} \\
0.6 \\
2.1 \\
0\end{array}$ & & & $\frac{\frac{19}{19.0}}{\frac{16.2}{2.7}} \frac{2.6}{\underline{0.5}}$ & $\begin{array}{c}\frac{20}{1.6} \\
3.2 \\
0.9 \\
0.5 \\
0\end{array}$ & $\begin{array}{c}\frac{33}{12.3} \\
\frac{2.9}{2.2} \\
\underline{0} \\
\underline{0.9}\end{array}$ & $\frac{\frac{34}{0.5}}{\frac{0.2}{1.1}}$ & & $\begin{array}{l}\frac{35}{1.0} \\
5.1 \\
2.3 \\
5.6 \\
1.4\end{array}$ & $\begin{array}{l}\frac{47}{5.0} \\
\underline{0.5} \\
\underline{0.8} \\
\underline{0.4}\end{array}$ & $\begin{array}{l}\frac{48}{1.5} \\
2.3 \\
1.8 \\
0\end{array}$ & $\begin{array}{l}\frac{53}{3.5} \\
\frac{3.1}{2.8} \\
\underline{0.2}\end{array}$ & $\begin{array}{l}\frac{54}{0.6} \\
0.3 \\
2.4 \\
0.3\end{array}$ & $\frac{\frac{59}{\frac{5.0}{0.0}}}{\frac{26.0}{\underline{0.3}}}$ & $\begin{array}{c}60 \\
2.1 \\
43.2 \\
0.7 \\
0 \\
0\end{array}$ & $\frac{\frac{65}{6.1}}{\frac{6.9}{10.0}} \frac{3.2}{\frac{3.2}{0.2}}$ & $\begin{array}{c}\frac{66}{1.3} \\
9.3 \\
5.6 \\
1.9 \\
0\end{array}$ & & & & $\begin{array}{l}\frac{4}{0} \\
2.0 \\
4.1 \\
0.3\end{array}$ & $\frac{\frac{5}{3.0}}{\frac{1.5}{0.6}}$ & \\
\hline $\begin{array}{l}\text { High } \\
\text { Velocity } \\
\text { Region }\end{array}$ & $\begin{array}{r}\frac{12}{12} \\
0.3 \\
20.0 \\
0.8 \\
0.3\end{array}$ & $\frac{\frac{13}{2.5}}{\frac{2.9}{3.2}}$ & $\frac{\frac{14}{6.0}}{\frac{6.7}{\frac{6.6}{0}}}$ & $\begin{array}{c}\frac{15}{1.7} \\
10.0 \\
1.8 \\
0\end{array}$ & $\begin{array}{c}\frac{26}{0.3} \\
1.1 \\
1.0 \\
1.4 \\
-\end{array}$ & $\begin{array}{c}\frac{27}{0.6} \\
0.6 \\
0 \\
3.3 \\
0.4\end{array}$ & $\begin{array}{c}30 \\
0 \\
6.0 \\
8.8 \\
1.6 \\
2.6\end{array}$ & $\begin{array}{c}31 \\
0 \\
1.5 \\
4.6 \\
0.6 \\
0\end{array}$ & $\begin{array}{l}\frac{32}{\frac{8.9}{1.6}} \\
\frac{1.0}{0.4}\end{array}$ & $\begin{array}{l}\frac{41}{0.6} \\
\frac{0.6}{0.2} \\
\underline{0.2}\end{array}$ & $\frac{\frac{42}{1.7}}{\frac{2.1}{2.7}} \frac{\underline{0}}{\frac{2}{2}}$ & $\begin{array}{c}43 \\
0 \\
0.4 \\
0.4 \\
1.3 \\
0\end{array}$ & $\frac{\frac{44}{1.1}}{\frac{1.7}{\underline{0}}}$ & & $\begin{array}{r}\frac{55}{\underline{0}} \\
\frac{3.2}{8.0} \\
\frac{18}{\underline{0}}\end{array}$ & & & & & & & $\frac{\frac{2}{3.7}}{\frac{7.8}{6.7}} \frac{0}{\underline{0}}$ & $\begin{array}{l}\frac{3}{2.1} \\
8.6 \\
6.8 \\
0\end{array}$ & \\
\hline $\begin{array}{l}\text { Ring } \\
\text { Core }\end{array}$ & $\frac{\frac{10}{\frac{2.8}{17.6}}}{\frac{4.5}{0}}$ & $\begin{array}{l}\frac{11}{0} \\
18.3 \\
3.1 \\
1.4\end{array}$ & $\begin{array}{c}\frac{16}{0} \\
6.4 \\
10.7\end{array}$ & $\begin{array}{l}\frac{17}{\underline{0}} \\
\frac{23.2}{4.8}\end{array}$ & $\begin{array}{l}\frac{21}{4.0} \\
\frac{4.8}{0.9} \\
\underline{1.7} \\
0.6\end{array}$ & $\frac{\frac{23}{4.1}}{\frac{\frac{4.1}{10.7}}{\frac{4.0}{0.9}}}$ & $\begin{array}{r}\frac{24}{2.4} \\
20.0 \\
3.5 \\
2.5 \\
0.6\end{array}$ & $\begin{array}{c}28 \\
0 \\
4.6 \\
1.8 \\
0.4 \\
0\end{array}$ & & & & & & & 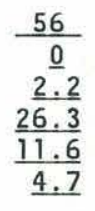 & $\begin{array}{l}\frac{57}{\underline{0}} \\
\frac{3.4}{11.8} \\
\frac{10.9}{7.4}\end{array}$ & $\begin{array}{r}\frac{58}{2.0} \\
7.9 \\
11.0 \\
13.5 \\
6.8\end{array}$ & $\begin{array}{l}\frac{61}{\underline{0}} \\
\frac{1.5}{31.5} \\
\frac{13.1}{1.6}\end{array}$ & $\begin{array}{r}\frac{62}{0.4} \\
0.3 \\
8.2 \\
9.5 \\
16.1\end{array}$ & $\frac{\frac{63}{0.5}}{\frac{0.6}{2.6}} \frac{23.3}{\frac{9.6}{5.0}}$ & $\begin{array}{r}\frac{64}{5.4} \\
4.4 \\
1.4 \\
10.5 \\
0.8\end{array}$ & $\frac{\frac{1}{\underline{0}}}{\frac{10.0}{18.4}} \frac{13.3}{\underline{3}}$ & & \\
\hline $\begin{array}{l}\text { Sargasso } \\
\text { Sea } \\
\& \\
\text { Gulf } \\
\text { Stream }\end{array}$ & & & & & & & & & & $\begin{array}{l}\frac{49}{\frac{0.3}{7.8}} \\
\frac{4.0}{23.1} \\
\end{array}$ & $\begin{array}{r}\frac{50}{0.3} \\
13.7 \\
5.2 \\
15.9\end{array}$ & $\begin{array}{c}51 \\
0 \\
3.9 \\
6.3 \\
0\end{array}$ & $\begin{array}{r}\frac{52}{\underline{0}} \\
\frac{3.9}{16.1} \\
\underline{0.5}\end{array}$ & & & & & & & & & & & \\
\hline $\begin{array}{l}\text { Slope } \\
\text { Water }\end{array}$ & $\frac{8}{-}$ & $\frac{9}{1.0}$ & $\frac{18}{1.4}$ & & $\frac{19}{5.8}$ & $\frac{20}{1.6}$ & $\frac{33}{2.0}$ & $\frac{34}{1.4}$ & & $\frac{35}{3.1}$ & $\frac{47}{1.7}$ & $\frac{48}{1.4}$ & $\frac{53}{1.9}$ & $\frac{54}{0.9}$ & $\frac{59}{6.3}$ & $\frac{60}{5.1}$ & $\frac{65}{5.1}$ & $\frac{66}{2.8}$ & & & & $\frac{4}{1.6}$ & $\frac{5}{1.3}$ & $\frac{6}{0.6}$ \\
\hline $\begin{array}{l}\text { High } \\
\text { Velocity } \\
\text { Region }\end{array}$ & $\frac{12}{5.4}$ & $\frac{13}{3.2}$ & $\frac{14}{3.3}$ & $\frac{15}{3.4}$ & $\frac{27}{1.1}$ & $\frac{29}{3.8}$ & $\frac{30}{3.2}$ & $\frac{31}{1.2}$ & $\frac{32}{-}$ & $\frac{41}{1.0}$ & $\frac{42}{1.6}$ & $\frac{43}{0.5}$ & $\frac{44}{1.0}$ & & $\frac{55}{7.8}$ & & & & & & & $\frac{2}{4.6}$ & $\frac{3}{4.4}$ & \\
\hline $\begin{array}{l}\text { Ring } \\
\text { Core }\end{array}$ & $\frac{10}{6.3}$ & $\frac{11}{5.7}$ & $\frac{16}{-}$ & $\frac{17}{8.2}$ & $\frac{21}{2.5}$ & $\frac{23}{4.3}$ & $\frac{24}{2.6}$ & $\frac{28}{1.4}$ & & $\frac{37}{2.9}$ & $\frac{38}{1.9}$ & $\frac{39}{1.1}$ & & & $\frac{56}{8.9}$ & $\frac{57}{6.7}$ & $\frac{58}{7.7}$ & $\frac{61}{9.5}$ & $\frac{62}{6.9}$ & $\frac{63}{7.8}$ & $\frac{64}{5.0}$ & $\frac{1}{7.9}$ & & \\
\hline$S S \& G S$ & & & & & & & & & & $\frac{49}{8.8}$ & $\frac{50}{8.8}$ & $\frac{51}{2.6}$ & $\frac{52}{5.1}$ & & & & & & & & & & & \\
\hline
\end{tabular}


Table 26. Volume of sternoptychids $\left(\mathrm{ml} / 10,000 \mathrm{~m}^{3}\right)$.

\begin{tabular}{|c|c|c|c|c|c|c|c|c|c|c|c|c|c|c|c|c|c|c|c|c|c|c|c|c|}
\hline & \multicolumn{4}{|c|}{$\begin{array}{c}\text { Oceanus } \\
\text { April }\end{array}$} & \multicolumn{5}{|c|}{$\begin{array}{c}\text { Oceanus } 121 \\
\text { June }\end{array}$} & \multicolumn{5}{|c|}{$\begin{array}{l}\text { Oceanus } 125 \\
\text { August }\end{array}$} & \multicolumn{7}{|c|}{$\begin{array}{l}\text { Knorr } 98 \\
\text { Sept-0ct }\end{array}$} & \multicolumn{3}{|c|}{$\begin{array}{c}\text { A2 } 110 \\
\text { Sept-0ct }\end{array}$} \\
\hline $\begin{array}{l}\text { Slope } \\
\text { Water }\end{array}$ & $\begin{array}{l}\frac{8}{=} \\
\frac{0.1}{0.2} \\
\frac{0}{0}\end{array}$ & $\begin{array}{l}\frac{9}{1.0} \\
0.1 \\
1.1 \\
0\end{array}$ & & & $\begin{array}{l}\frac{19}{7.1} \\
\frac{1.3}{0.2} \\
\frac{0.3}{0.1} \\
\end{array}$ & $\begin{array}{c}20 \\
0.2 \\
0.7 \\
0.5 \\
0 \\
0\end{array}$ & $\begin{array}{l}\frac{33}{1.1} \\
\frac{3.1}{0.3} \\
\underline{0} \underline{0.7} \\
\underline{0.7}\end{array}$ & $\begin{array}{l}\frac{34}{\underline{0}} \\
\frac{1.2}{1.6} \\
\end{array}$ & & $\begin{array}{l}35 \\
0.1 \\
0.4 \\
0.2 \\
1.9 \\
0.6\end{array}$ & $\begin{array}{l}\frac{47}{2.6} \\
\underline{0.1} \\
\underline{0.4} \\
\underline{0.2}\end{array}$ & $\begin{array}{l}\frac{48}{0.1} \\
1.4 \\
0.3 \\
0\end{array}$ & 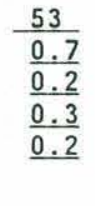 & $\begin{array}{c}\frac{54}{0.1} \\
0 \\
0.6 \\
0.3\end{array}$ & $\frac{\frac{59}{0.8}}{\frac{0.4}{1.4}}$ & $\begin{array}{c}60 \\
0.2 \\
2.6 \\
0.6 \\
0 \\
0\end{array}$ & $\begin{array}{l}\frac{65}{\frac{3.0}{0.4}} \\
\frac{0.1}{1.1} \\
\frac{0.2}{\underline{0}}\end{array}$ & $\begin{array}{l}66 \\
0.1 \\
2.4 \\
0.6 \\
0.2 \\
0\end{array}$ & & & & $\begin{array}{l}\frac{4}{0} \\
1.2 \\
1.2 \\
0.1\end{array}$ & $\frac{\frac{5}{3.0}}{\frac{0.5}{1.0}}$ & \\
\hline $\begin{array}{l}\text { High } \\
\text { Velocity } \\
\text { Region }\end{array}$ & $\begin{array}{c}\frac{12}{0} \\
2.2 \\
0.4 \\
0\end{array}$ & $\frac{\frac{13}{0.9}}{\frac{0.4}{1.0}}$ & $\frac{\frac{14}{\frac{3.6}{1.8}}}{\frac{0.6}{\underline{0}}}$ & $\begin{array}{c}\frac{15}{2.3} \\
7.1 \\
0.3 \\
0\end{array}$ & $\begin{array}{c}\frac{26}{0} \\
0.1 \\
0.3 \\
0.6 \\
-\end{array}$ & $\begin{array}{c}\frac{27}{0.1} \\
0.1 \\
0 \\
2.6 \\
0.7\end{array}$ & $\begin{array}{c}30 \\
0 \\
0.6 \\
4.7 \\
0.8 \\
1.3\end{array}$ & $\begin{array}{c}31 \\
0 \\
0.1 \\
0.4 \\
0.2 \\
0\end{array}$ & $\frac{\frac{32}{10.2}}{\frac{1.0}{0.6}} \frac{0.1}{0.1}$ & $\begin{array}{l}\frac{41}{0.6} \\
\frac{0.5}{0.1}\end{array}$ & $\frac{\frac{42}{\frac{1.5}{0.2}}}{\frac{1.5}{\underline{0}}}$ & $\begin{array}{c}43 \\
0 \\
0 \\
2.1 \\
0.1 \\
0\end{array}$ & $\frac{\frac{44}{0.5}}{\frac{0.2}{\underline{0}}}$ & & $\begin{array}{c}\frac{55}{\underline{0}} \\
\frac{0.3}{0.6} \\
\underline{0}\end{array}$ & & & & & & & $\frac{\frac{2}{4.0}}{\frac{0.7}{1.8}}$ & $\begin{array}{c}\frac{3}{0.2} \\
1.7 \\
1.3 \\
0\end{array}$ & \\
\hline $\begin{array}{l}\text { Ring } \\
\text { Center }\end{array}$ & $\frac{\frac{10}{\frac{1.4}{2.1}}}{\frac{0.6}{\underline{0}}}$ & $\begin{array}{l}11 \\
0 \\
1.5 \\
0.5 \\
0.7\end{array}$ & $\begin{array}{l}16 \\
0 \\
1.1 \\
4.7\end{array}$ & $\frac{17}{\underline{\underline{0}}} \frac{7.5}{\underline{2.6}}$ & $\begin{array}{l}\frac{21}{1.2} \\
\frac{2.9}{0.3} \\
\frac{0.4}{0.1} \\
\underline{0.1}\end{array}$ & $\begin{array}{l}\frac{23}{\underline{0}} \\
\frac{6.5}{0.6} \\
\frac{0.1}{\underline{0}}\end{array}$ & $\begin{array}{l}\frac{24}{0.4} \\
6.5 \\
0.3 \\
0.6 \\
0.2\end{array}$ & $\begin{array}{c}28 \\
0 \\
5.0 \\
0.5 \\
0.3 \\
0\end{array}$ & & & & & & & $\begin{array}{l}\frac{56}{0} \\
\underline{0.7} \\
\underline{1.1} \\
\underline{0.1} \\
\underline{0.9}\end{array}$ & $\begin{array}{l}\frac{57}{\underline{0}} \\
\underline{1.0} \\
\underline{0.5} \\
\underline{0.2} \\
\underline{2.5}\end{array}$ & $\begin{array}{l}\frac{58}{0.1} \\
0.3 \\
0.6 \\
0.2 \\
1.4\end{array}$ & $\begin{array}{l}\frac{61}{\underline{0}} \\
\underline{0.2} \\
\underline{1.1} \\
\underline{1.0} \\
\underline{0.6}\end{array}$ & $\begin{array}{c}62 \\
0 \\
0 \\
0.5 \\
0.3 \\
2.9\end{array}$ & $\begin{array}{l}\frac{63}{\underline{0}} \\
\underline{2.2} \\
\underline{1.5} \\
\underline{0.3} \\
\underline{0.7}\end{array}$ & $\begin{array}{l}\frac{64}{0.1} \\
0.3 \\
0.1 \\
0.2 \\
0.1\end{array}$ & $\begin{array}{l}\frac{1}{0} \\
7.5 \\
\frac{0.7}{2.2}\end{array}$ & & \\
\hline $\begin{array}{l}\text { Sargasso } \\
\text { Sea } \\
\& \\
\text { Gulf } \\
\text { Stream }\end{array}$ & & & & & & & & & & $\begin{array}{l}\frac{49}{0} \\
\frac{0.2}{0.3} \\
\underline{0.2}\end{array}$ & $\begin{array}{l}\frac{50}{0} \\
0.5 \\
0.3 \\
2.0\end{array}$ & $\begin{array}{c}51 \\
0 \\
0.2 \\
1.3 \\
0\end{array}$ & $\begin{array}{l}\frac{52}{0.5} \\
\frac{0.1}{1.0} \\
\underline{0.4}\end{array}$ & & & & & & & & & & & \\
\hline $\begin{array}{l}\text { Slope } \\
\text { Water }\end{array}$ & $\frac{8}{-}$ & $\frac{9}{0.6}$ & $\frac{18}{0.1}$ & & $\frac{19}{1.1}$ & $\frac{20}{0.3}$ & $\frac{33}{0.9}$ & $\frac{34}{1.3}$ & & $\frac{35}{0.6}$ & $\frac{47}{0.8}$ & $\frac{48}{0.5}$ & $\frac{53}{0.4}$ & $\frac{54}{0.3}$ & $\frac{59}{0.4}$ & $\frac{60}{0.4}$ & $\frac{65}{0.9}$ & $\frac{66}{0.4}$ & & & & $\frac{4}{0.6}$ & $\frac{5}{1.1}$ & $\frac{6}{0.4}$ \\
\hline $\begin{array}{l}\text { High } \\
\text { Velocity } \\
\text { Region }\end{array}$ & $\frac{12}{0.7}$ & $\frac{13}{0.8}$ & $\frac{14}{1.5}$ & $\frac{15}{2.4}$ & $\frac{27}{0.9}$ & $\frac{29}{1.7}$ & $\frac{30}{1.4}$ & $\frac{31}{0.1}$ & $\frac{32}{-}$ & $\frac{41}{0.7}$ & $\frac{42}{0.9}$ & $\frac{43}{0.4}$ & $\frac{44}{0.3}$ & & $\frac{55}{0.3}$ & & & & & & & $\frac{2}{1.6}$ & $\frac{3}{0.8}$ & \\
\hline $\begin{array}{l}\text { Ring } \\
\text { Center }\end{array}$ & $\frac{10}{1.0}$ & $\frac{11}{0.7}$ & $\frac{16}{-}$ & $\frac{17}{3.2}$ & $\frac{21}{1.2}$ & $\frac{23}{1.8}$ & $\frac{24}{0.5}$ & $\frac{28}{1.1}$ & & $\frac{37}{1.5}$ & $\frac{38}{0.5}$ & $\frac{39}{0.2}$ & & & $\frac{56}{0.6}$ & $\frac{57}{0.8}$ & $\frac{58}{0.5}$ & $\frac{61}{0.6}$ & $\frac{62}{0.7}$ & $\frac{63}{0.6}$ & $\frac{64}{0.1}$ & $\frac{1}{2.6}$ & & \\
\hline SS \& GS & & & & & & & & & & $\frac{49}{0.4}$ & $\frac{50}{0.7}$ & $\frac{51}{0.4}$ & $\frac{52}{0.5}$ & & & & & & & & & & & \\
\hline
\end{tabular}


Table 27. Abundance of stomiids (specimens $/ 10,000 \mathrm{~m}^{3}$ ).

\begin{tabular}{|c|c|c|c|c|c|c|c|c|c|c|c|c|c|c|c|c|c|c|c|c|c|c|c|c|}
\hline & \multicolumn{4}{|c|}{$\begin{array}{c}\text { Oceanus } 118 \\
\text { April }\end{array}$} & \multicolumn{5}{|c|}{$\begin{array}{c}\text { Oceanus } 121 \\
\text { June }\end{array}$} & \multicolumn{5}{|c|}{$\begin{array}{l}\text { Oceanus } 125 \\
\text { August }\end{array}$} & \multicolumn{7}{|c|}{$\begin{array}{l}\text { Knorr } 98 \\
\text { Sept-Oct }\end{array}$} & \multicolumn{3}{|c|}{$\begin{array}{c}\text { A2 } 110 \\
\text { Sept-0ct }\end{array}$} \\
\hline $\begin{array}{l}\text { Slope } \\
\text { Water }\end{array}$ & $\begin{array}{l}\frac{8}{1=} \\
\frac{1.8}{1.8} \\
\underline{0.5}\end{array}$ & $\begin{array}{l}\frac{9}{0} \\
1.8 \\
2.1 \\
0\end{array}$ & & & $\begin{array}{l}\frac{19}{\frac{1.0}{1.7}} \\
\frac{0}{0.5} \\
\underline{0}\end{array}$ & $\begin{array}{c}20 \\
0 \\
1.1 \\
1.3 \\
0.5 \\
0\end{array}$ & $\begin{array}{l}\frac{33}{1.0} \\
\frac{1.2}{\underline{0}} \\
\underline{0} \\
\underline{0.5}\end{array}$ & $\frac{34}{\frac{3.9}{0.4}} \frac{3.2}{\underline{0.2}}$ & & $\begin{array}{c}35 \\
0 \\
0.2 \\
56.0 \\
2.8 \\
0\end{array}$ & $\begin{array}{l}\frac{47}{\frac{1.1}{0.3}} \\
\frac{0.8}{\underline{0}}\end{array}$ & $\begin{array}{l}\frac{48}{0} \\
0.9 \\
1.5 \\
0.4\end{array}$ & $\begin{array}{l}\frac{53}{\frac{1.2}{\underline{0}}} \\
\frac{1.1}{\underline{0}}\end{array}$ & $\begin{array}{l}54 \\
0 \\
2.2 \\
8.8 \\
0.7\end{array}$ & $\begin{array}{l}\frac{59}{\underline{0.3}} \\
\underline{0.8} \\
\underline{0} \\
\underline{0.3} \\
\underline{0}\end{array}$ & $\begin{array}{c}\frac{60}{0.6} \\
0 \\
3.1 \\
2.5 \\
0.3\end{array}$ & $\begin{array}{l}\frac{65}{2.1} \\
\underline{0} \\
\frac{2.7}{1.2} \\
\underline{0.2}\end{array}$ & $\begin{array}{c}\frac{66}{0} \\
1.4 \\
6.3 \\
2.9 \\
1.2\end{array}$ & & & & $\begin{array}{l}\frac{4}{0} \\
4.1 \\
3.1 \\
1.2\end{array}$ & $\frac{\frac{5}{\frac{3.6}{1.1}}}{\frac{1.9}{\underline{0}}}$ & \\
\hline $\begin{array}{l}\text { High } \\
\text { Velocity } \\
\text { Region }\end{array}$ & $\begin{array}{l}\frac{12}{2.9} \\
1.2 \\
3.3 \\
0.3\end{array}$ & $\frac{\frac{13}{2.0}}{\frac{1.2}{1.9}}$ & $\frac{\frac{14}{2.4}}{\frac{1.7}{1.2}} \frac{0}{\underline{0}}$ & $\begin{array}{c}15 \\
0 \\
4.2 \\
0.6 \\
0\end{array}$ & $\begin{array}{c}\frac{26}{1.2} \\
0 \\
1.9 \\
2.7 \\
-\end{array}$ & $\begin{array}{c}27 \\
0 \\
0 \\
4.1 \\
0.4 \\
0.2\end{array}$ & $\begin{array}{c}30 \\
0.3 \\
0 \\
18.8 \\
1.4 \\
0\end{array}$ & $\begin{array}{c}31 \\
0.2 \\
0 \\
5.1 \\
1.2 \\
0\end{array}$ & $\frac{\frac{32}{1.1}}{\frac{1.6}{\frac{4.0}{0}}}$ & $\frac{\frac{41}{0.1}}{\frac{1.2}{\underline{0}}}$ & $\frac{\frac{42}{0.2}}{\frac{0.0}{1.1}} \frac{\underline{0}}{n}$ & $\begin{array}{c}43 \\
0 \\
0 \\
4.6 \\
0.4 \\
0\end{array}$ & $\frac{\frac{44}{1.2}}{\frac{0.3}{\underline{0}}}$ & & $\frac{\frac{55}{3.0}}{\frac{3.0}{1.6}} \frac{30}{\frac{3.6}{0}}$ & & & & & & & $\begin{array}{l}\frac{2}{1.2} \\
\frac{0.8}{2.6} \\
\underline{0.6}\end{array}$ & $\begin{array}{l}\frac{3}{0} \\
5.7 \\
2.2 \\
0\end{array}$ & \\
\hline $\begin{array}{l}\text { Ring } \\
\text { Center }\end{array}$ & $\frac{\frac{10}{22.8}}{\underline{0}} \frac{4.5}{1.8}$ & $\begin{array}{l}11 \\
0.9 \\
0.6 \\
0.4 \\
0.6\end{array}$ & $\begin{array}{l}\frac{16}{2.5} \\
2.1 \\
1.6\end{array}$ & $\begin{array}{l}\frac{17}{3.3} \\
\frac{3.2}{0.8}\end{array}$ & $\frac{\frac{21}{17.0}}{\frac{4.4}{88.8}}$ & $\frac{\frac{23}{7.1}}{\frac{2.7}{5.0}} \frac{35}{\frac{11.0}{\underline{0}}}$ & $\begin{array}{c}\frac{24}{6.5} \\
5.0 \\
60.0 \\
4.3 \\
0\end{array}$ & \begin{tabular}{r}
\multicolumn{1}{c}{$\frac{18}{3.2}$} \\
4.3 \\
82.2 \\
0.4 \\
0.3
\end{tabular} & & & & & & & $\begin{array}{l}\frac{56}{2.1} \\
\frac{0.6}{1.6} \\
\frac{3.7}{1.5}\end{array}$ & $\frac{\frac{57}{0.3}}{\frac{0.6}{1.3}} \frac{4.3}{\frac{4.1}{1.1}}$ & $\begin{array}{c}58 \\
0 \\
0 \\
3.3 \\
8.0 \\
2.7\end{array}$ & $\begin{array}{l}\frac{61}{1.7} \\
\frac{0.6}{0.7} \\
\underline{5.8} \\
\underline{0.8}\end{array}$ & $\begin{array}{c}\frac{62}{0} \\
0 \\
2.2 \\
7.1 \\
2.1\end{array}$ & $\begin{array}{l}\frac{63}{1.6} \\
\frac{0.2}{1.4} \\
\underline{5.5} \\
\underline{2.5}\end{array}$ & $\begin{array}{r}64 \\
0 \\
1.2 \\
5.7 \\
10.5 \\
0.8\end{array}$ & $\frac{\frac{1}{3.6}}{\frac{1.6}{0.3}} \frac{0.8}{\underline{0.8}}$ & & \\
\hline $\begin{array}{l}\text { Sargasso } \\
\text { Sea } \\
\& \\
\text { Gulf } \\
\text { Stream }\end{array}$ & & & & & & & & & & $\frac{\frac{49}{3.6}}{\frac{0.2}{2.9}} \frac{2.4}{\underline{2.4}}$ & $\begin{array}{l}50 \\
0 \\
0.2 \\
4.8 \\
2.2\end{array}$ & $\begin{array}{l}\frac{51}{0.3} \\
0 \\
7.2 \\
0.6\end{array}$ & $\frac{\frac{52}{1.2}}{\frac{1.1}{4.8}} \frac{1.7}{1.7}$ & & & & & & & & & & & \\
\hline $\begin{array}{l}\text { Slope } \\
\text { Water }\end{array}$ & $\frac{8}{-}$ & $\frac{9}{1.0}$ & $\frac{18}{0.9}$ & & $\frac{19}{0.5}$ & $\frac{20}{0.7}$ & $\frac{33}{0.5}$ & $\frac{34}{2.2}$ & & $\frac{35}{11.8}$ & $\frac{47}{0.6}$ & $\frac{48}{0.7}$ & $\frac{53}{0.6}$ & $\frac{54}{2.9}$ & $\frac{59}{0.3}$ & $\frac{60}{1.4}$ & $\frac{65}{1.2}$ & $\frac{66}{2.2}$ & & & & $\frac{4}{2.1}$ & $\frac{5}{1.7}$ & $\frac{6}{6.9}$ \\
\hline $\begin{array}{l}\text { High } \\
\text { Velocity } \\
\text { Region }\end{array}$ & $\frac{12}{1.9}$ & $\frac{13}{1.8}$ & $\frac{14}{1.3}$ & $\frac{15}{1.2}$ & $\frac{27}{1.0}$ & $\frac{29}{2.7}$ & $\frac{30}{4.1}$ & $\frac{31}{1.3}$ & $\frac{32}{-}$ & $\frac{41}{0.4}$ & $\frac{42}{1.4}$ & $\frac{43}{1.0}$ & $\frac{44}{0.8}$ & & $\frac{55}{2.2}$ & & & & & & & $\frac{2}{1.3}$ & $\frac{3}{2.0}$ & \\
\hline $\begin{array}{l}\text { Ring } \\
\text { Center }\end{array}$ & $\frac{10}{7.3}$ & $\frac{11}{0.6}$ & $\frac{16}{-}$ & $\frac{17}{2.0}$ & $\frac{21}{24.7}$ & $\frac{23}{10.9}$ & $\frac{24}{15.4}$ & $\frac{28}{22.5}$ & & $\frac{37}{0.4}$ & $\frac{38}{1.2}$ & $\frac{39}{1.3}$ & & & $\frac{56}{1.9}$ & $\frac{57}{1.5}$ & $\frac{58}{2.8}$ & $\frac{61}{1.9}$ & $\frac{62}{2.3}$ & $\frac{63}{2.5}$ & $\frac{64}{3.0}$ & $\frac{1}{4.1}$ & & \\
\hline SS \& GS & & & & & & & & & & $\frac{49}{2.3}$ & $\frac{50}{1.8}$ & $\frac{51}{2.0}$ & $\frac{52}{2.2}$ & & & & & & & & & & & \\
\hline
\end{tabular}


Table 28. Volume of stomiids $\left(\mathrm{ml} / 10,000 \mathrm{~m}^{3}\right)$.

\begin{tabular}{|c|c|c|c|c|c|c|c|c|c|c|c|c|c|c|c|c|c|c|c|c|c|c|c|c|}
\hline & \multicolumn{4}{|c|}{$\begin{array}{c}\text { Oceanus } \\
\text { April }\end{array}$} & \multicolumn{5}{|c|}{$\begin{array}{c}\text { Oceanus } 121 \\
\text { June }\end{array}$} & \multicolumn{5}{|c|}{$\begin{array}{l}\text { Oceanus } 125 \\
\text { August }\end{array}$} & \multicolumn{7}{|c|}{$\begin{array}{l}\text { Knorr } 98 \\
\text { Sept-Oct }\end{array}$} & \multicolumn{3}{|c|}{$\begin{array}{c}\text { A2 } 110 \\
\text { Sept-0ct }\end{array}$} \\
\hline $\begin{array}{l}\text { Slope } \\
\text { Water }\end{array}$ & $\begin{array}{l}\frac{8}{\bar{z}} \\
\frac{5.8}{\underline{42.3}} \\
\underline{0}\end{array}$ & $\begin{array}{c}\frac{9}{0} \\
1.2 \\
5.7 \\
0\end{array}$ & & & $\begin{array}{l}\frac{19}{\frac{1.2}{6.3}} \\
\underline{0} \\
\frac{0.2}{\underline{0}}\end{array}$ & $\begin{array}{c}20 \\
0 \\
0.5 \\
8.3 \\
10.0 \\
0\end{array}$ & $\frac{\frac{33}{0.1}}{\frac{30.4}{\underline{0}}}$ & $\frac{\frac{34}{2.2}}{\frac{2.9}{1.9}}$ & & $\begin{array}{c}35 \\
0 \\
0 \\
8.2 \\
20.6 \\
0\end{array}$ & $\frac{\frac{47}{1.2}}{\frac{1.5}{2.2}}$ & $\begin{array}{r}48 \\
0 \\
0 \\
18.8 \\
4.5\end{array}$ & $\begin{array}{l}\frac{53}{\frac{5.2}{0}} \\
\frac{4.6}{\underline{0}}\end{array}$ & $\begin{array}{r}\frac{54}{0} \\
0.7 \\
23.9 \\
19.3\end{array}$ & $\begin{array}{c}\frac{59}{\underline{0}} \\
\frac{1.0}{\underline{0}} \\
\frac{5.1}{\underline{0}}\end{array}$ & $\begin{array}{c}\frac{60}{0.2} \\
0 \\
0.9 \\
18.3 \\
3.5\end{array}$ & $\begin{array}{l}\frac{65}{1.2} \\
\underline{0} \\
\frac{8.2}{9.7} \\
\underline{\underline{4.8}}\end{array}$ & $\begin{array}{r}\frac{66}{0} \\
1.2 \\
16.5 \\
42.2 \\
15.4\end{array}$ & & & & $\begin{array}{c}\frac{4}{0} \\
2.8 \\
15.8 \\
10.0\end{array}$ & $\frac{\frac{5}{13.1}}{\frac{2 \frac{0.1}{3.6}}{\underline{0}}}$ & \\
\hline $\begin{array}{l}\text { High } \\
\text { Velocity } \\
\text { Region }\end{array}$ & $\begin{array}{l}\frac{12}{0} \\
0.2 \\
64.8 \\
31.7\end{array}$ & $\frac{\frac{13}{\frac{1.4}{3.7}}}{\frac{3.0}{12.0}}$ & $\frac{\frac{14}{\frac{1.2}{13.0}}}{\frac{13.5}{\underline{23.5}}}$ & $\begin{array}{c}15 \\
0 \\
0.7 \\
0.2 \\
0\end{array}$ & $\begin{array}{c}26 \\
0 \\
0 \\
0.1 \\
34.5 \\
-\end{array}$ & $\begin{array}{r}27 \\
0 \\
0 \\
4.0 \\
17.5 \\
3.7\end{array}$ & $\begin{array}{c}30 \\
0 \\
0 \\
1.1 \\
3.3 \\
0\end{array}$ & $\begin{array}{c}31 \\
0 \\
0 \\
0.5 \\
11.5 \\
0\end{array}$ & $\begin{array}{c}\frac{32}{\frac{1.7}{0}} \\
\frac{1.2}{\underline{0}}\end{array}$ & $\frac{\frac{41}{\underline{0}}}{\frac{13,5}{\underline{0}}}$ & $\frac{\frac{42}{\frac{0.1}{0.2}}}{\frac{19.8}{\underline{0}}}$ & $\begin{array}{c}43 \\
0 \\
0 \\
1.6 \\
0 \\
0\end{array}$ & $\frac{\frac{44}{\frac{5.0}{1.0}}}{\underline{\underline{0}}}$ & & $\frac{\frac{55}{\frac{0.2}{9.8}}}{\frac{89.2}{\underline{0}}}$ & & & & & & & $\begin{array}{r}\frac{2}{0.4} \\
\frac{0.2}{26.0} \\
\underline{0.1}\end{array}$ & $\begin{array}{l}\frac{3}{0} \\
1.4 \\
6.0 \\
0\end{array}$ & \\
\hline $\begin{array}{l}\text { Ring } \\
\text { Center }\end{array}$ & $\begin{array}{l}\frac{10}{1.3} \\
\underline{0} \\
\frac{2.4}{2.3} \\
\underline{2}\end{array}$ & $\begin{array}{c}11 \\
0 \\
0.1 \\
0.1 \\
10.7\end{array}$ & $\begin{array}{l}\frac{16}{0.1} \\
0.2 \\
3.5\end{array}$ & $\begin{array}{l}\frac{17}{0.1} \\
\frac{0.2}{8.2}\end{array}$ & $\frac{\frac{21}{2.5}}{\frac{\frac{1.2}{1.0}}{17.0}} \frac{7.8}{1.8}$ & $\frac{\frac{23}{\frac{1.2}{2.2}}}{\frac{20.5}{0.6}} \frac{0}{\underline{0}}$ & $\begin{array}{c}\frac{24}{0.2} \\
0.5 \\
5.5 \\
0.2 \\
0\end{array}$ & $\begin{array}{r}\frac{28}{0.1} \\
2.1 \\
12.6 \\
1.5 \\
6.7\end{array}$ & & & & & & & $\begin{array}{l}\frac{56}{1.3} \\
\frac{0.1}{0.1} \\
\frac{1.5}{2.5}\end{array}$ & $\begin{array}{l}\frac{57}{0.1} \\
\frac{0.7}{0.1} \\
\underline{0.5} \\
\underline{1.8}\end{array}$ & $\begin{array}{r}\frac{58}{0} \\
0 \\
0.1 \\
1.7 \\
29.8\end{array}$ & $\begin{array}{r}\frac{61}{0.9} \\
\frac{0.7}{0.1} \\
\frac{2.0}{3.4} \\
\end{array}$ & $\begin{array}{c}\frac{62}{0} \\
0 \\
0.1 \\
0.7 \\
0.5\end{array}$ & $\begin{array}{l}\frac{63}{0.8} \\
\frac{0.3}{\underline{0}} \\
\frac{2.3}{7.0}\end{array}$ & $\begin{array}{c}\frac{64}{0} \\
0.2 \\
0.8 \\
3.1 \\
1.4\end{array}$ & $\frac{\frac{1}{\frac{1.3}{0.1}}}{\frac{0.1}{1.3}}$ & & \\
\hline $\begin{array}{l}\text { Sargasso } \\
\text { Sea } \\
\& \\
\text { Gulf } \\
\text { Stream }\end{array}$ & & & & & & & & & & 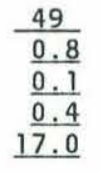 & $\begin{array}{c}50 \\
0 \\
0 \\
1.3 \\
25.0\end{array}$ & $\begin{array}{c}51 \\
0 \\
0 \\
2.5 \\
1.6\end{array}$ & $\begin{array}{l}\frac{52}{\frac{5.0}{1.1}} \\
\underline{0.4} \\
\underline{2.3}\end{array}$ & & & & & & & & & & & \\
\hline $\begin{array}{l}\text { Slope } \\
\text { Water }\end{array}$ & $\frac{8}{-}$ & $\frac{9}{1.7}$ & $\frac{18}{5.7}$ & & $\frac{19}{1.1}$ & $\frac{20}{4.7}$ & $\frac{33}{8.2}$ & $\frac{34}{3.1}$ & & $\frac{35}{5.8}$ & $\frac{47}{1.2}$ & $\frac{48}{5.8}$ & $\frac{53}{2.5}$ & $\frac{54}{11.0}$ & $\frac{59}{1.2}$ & $\frac{60}{4.6}$ & $\frac{65}{4.8}$ & $\frac{66}{14.9}$ & & & & $\frac{4}{7.2}$ & $\frac{5}{9.2}$ & $\frac{6}{16.4}$ \\
\hline $\begin{array}{l}\text { High } \\
\text { Velocity } \\
\text { Region }\end{array}$ & $\frac{12}{24.3}$ & $\frac{13}{7.3}$ & $\frac{14}{9.4}$ & $\frac{15}{0.2}$ & $\frac{27}{5.0}$ & $\frac{29}{1.9}$ & $\frac{30}{0.8}$ & $\frac{31}{2.4}$ & $\frac{32}{-}$ & $\frac{41}{3.4}$ & $\frac{42}{4.0}$ & $\frac{43}{0.3}$ & $\frac{44}{5.2}$ & & $\frac{55}{22.8}$ & & & & & & & $\frac{2}{6.7}$ & $\frac{3}{1.9}$ & \\
\hline $\begin{array}{l}\text { Ring } \\
\text { Center }\end{array}$ & $\frac{10}{1.5}$ & $\frac{11}{2.7}$ & $\frac{16}{-}$ & $\frac{17}{4.7}$ & $\frac{21}{5.9}$ & $\frac{23}{5.0}$ & $\frac{24}{1.2}$ & $\frac{28}{4.1}$ & & $\frac{37}{0.1}$ & $\frac{38}{8.1}$ & $\frac{39}{1.3}$ & & & $\frac{56}{1.1}$ & $\frac{57}{0.4}$ & $\frac{58}{6.2}$ & $\frac{61}{3.4}$ & $\frac{62}{0.3}$ & $\frac{63}{2.2}$ & $\frac{64}{1.1}$ & $\frac{1}{0.7}$ & & \\
\hline SS \& GS & & & & & & & & & & $\frac{49}{4.6}$ & $\frac{50}{6.6}$ & $\frac{51}{1.0}$ & $\frac{52}{2.2}$ & & & & & & & & & & & \\
\hline
\end{tabular}


Table 29. Volume of Anoplogaster cornuta $\left(\mathrm{ml} / 10,000 \mathrm{~m}^{3}\right)$.

\begin{tabular}{|c|c|c|c|c|c|c|c|c|c|c|c|c|c|c|c|c|c|c|c|c|c|c|c|c|}
\hline & \multicolumn{4}{|c|}{$\begin{array}{c}\text { Oceanus } 118 \\
\text { April }\end{array}$} & \multicolumn{5}{|c|}{$\begin{array}{c}\text { Oceanus } 121 \\
\text { June }\end{array}$} & \multicolumn{5}{|c|}{$\begin{array}{l}\text { Oceanus } 125 \\
\text { August }\end{array}$} & \multicolumn{7}{|c|}{$\begin{array}{l}\text { Knorr } 98 \\
\text { Sept-0ct }\end{array}$} & \multicolumn{3}{|c|}{$\begin{array}{c}\text { A2 } 110 \\
\text { Sept-0ct }\end{array}$} \\
\hline $\begin{array}{l}\text { Slope } \\
\text { Water }\end{array}$ & $\begin{array}{l}8 \\
\overline{\overline{0}} \\
\underline{0} \\
\underline{0} \\
\underline{0}\end{array}$ & $\begin{array}{l}9 \\
0 \\
0 \\
0 \\
0\end{array}$ & & & $\begin{array}{l}19 \\
\underline{0} \\
\underline{0} \\
\underline{0} \\
\underline{0} \\
\underline{0}\end{array}$ & $\begin{array}{c}\frac{20}{0} \\
0 \\
0 \\
0 \\
27.30\end{array}$ & $\begin{array}{l}\frac{33}{0} \\
\underline{0} \\
\underline{0} \\
\underline{0} \\
\underline{0}\end{array}$ & $\begin{array}{l}\frac{34}{\underline{0}} \\
\underline{0} \\
\underline{0}\end{array}$ & & $\begin{array}{l}\frac{35}{0} \\
0 \\
0 \\
0 \\
0\end{array}$ & $\begin{array}{l}47 \\
\underline{0} \\
\underline{0} \\
\underline{0} \\
\underline{0}\end{array}$ & $\begin{array}{l}\frac{48}{0} \\
0 \\
0 \\
0\end{array}$ & $\begin{array}{l}\frac{53}{\underline{0}} \\
\frac{0}{0} \\
\underline{0}\end{array}$ & $\begin{array}{l}\frac{54}{0} \\
0 \\
0 \\
0\end{array}$ & $\begin{array}{l}\frac{59}{0} \\
\underline{0} \\
\underline{0} \\
\underline{0} \\
\underline{0} \\
\underline{0}\end{array}$ & $\begin{array}{l}60 \\
0 \\
0 \\
0 \\
0 \\
0\end{array}$ & $\begin{array}{l}\frac{65}{\underline{0}} \\
\underline{0} \\
\underline{0} \\
\underline{0} \\
\underline{0}\end{array}$ & $\begin{array}{l}66 \\
0 \\
0 \\
0 \\
0 \\
0\end{array}$ & & & & $\begin{array}{l}4 \\
0 \\
0 \\
0 \\
0\end{array}$ & $\begin{array}{l}\frac{5}{0} \\
\underline{0} \\
\underline{0} \\
\underline{0} \\
\underline{0}\end{array}$ & \\
\hline $\begin{array}{l}\text { High } \\
\text { Velocity } \\
\text { Region }\end{array}$ & $\begin{array}{l}12 \\
0 \\
0 \\
0 \\
0\end{array}$ & $\begin{array}{l}\frac{13}{\underline{0}} \\
\frac{0}{0}\end{array}$ & $\begin{array}{l}\frac{14}{\underline{0}} \\
\underline{0} \\
\underline{0} \\
\underline{0}\end{array}$ & $\begin{array}{l}\frac{15}{0} \\
0 \\
0 \\
0\end{array}$ & $\begin{array}{l}26 \\
0 \\
0 \\
0 \\
0 \\
-\end{array}$ & $\begin{array}{c}27 \\
0 \\
0 \\
0 \\
30.00 \\
0\end{array}$ & $\begin{array}{l}30 \\
0 \\
0 \\
0 \\
0 \\
0\end{array}$ & $\begin{array}{l}31 \\
0 \\
0 \\
0 \\
0 \\
0\end{array}$ & $\begin{array}{l}32 \\
\underline{0} \\
\underline{0} \\
\underline{0} \\
\underline{0}\end{array}$ & $\begin{array}{l}\frac{41}{\underline{0}} \\
\underline{0} \\
\underline{0}\end{array}$ & $\begin{array}{l}\frac{42}{\underline{0}} \\
\underline{0} \\
\underline{0} \\
\underline{0}\end{array}$ & $\begin{array}{l}43 \\
0 \\
0 \\
0 \\
0 \\
0\end{array}$ & $\begin{array}{l}\frac{44}{0} \\
\underline{0} \underline{0} \\
\underline{0}\end{array}$ & & $\begin{array}{l}\frac{55}{\underline{0}} \\
\underline{0} \\
\underline{0} \\
\underline{0}\end{array}$ & & & & & & & $\begin{array}{l}\frac{2}{0} \\
\underline{0} \\
\underline{0} \\
\underline{0} \\
\underline{0}\end{array}$ & $\begin{array}{l}3 \\
0 \\
0 \\
0 \\
0\end{array}$ & \\
\hline $\begin{array}{l}\text { Ring } \\
\text { Core }\end{array}$ & $\begin{array}{l}10 \\
\frac{0}{0} \\
\underline{0} \\
\underline{0} \\
\underline{0}\end{array}$ & $\begin{array}{l}11 \\
0 \\
0 \\
0 \\
0\end{array}$ & $\begin{array}{l}16 \\
0 \\
0 \\
0\end{array}$ & $\begin{array}{l}17 \\
\underline{0} \\
\underline{0} \\
\underline{0}\end{array}$ & $\begin{array}{l}21 \\
\underline{0} \\
\underline{0} \\
\underline{0} \\
\underline{0} \\
\underline{0}\end{array}$ & $\begin{array}{c}\frac{23}{\underline{0}} \\
\underline{0} \\
\frac{31.50}{\underline{0}} \\
\underline{0}\end{array}$ & $\begin{array}{l}24 \\
0 \\
0 \\
0 \\
0 \\
0\end{array}$ & $\begin{array}{l}28 \\
0 \\
0 \\
0 \\
0 \\
0\end{array}$ & & & & & & & $\begin{array}{l}56 \\
\underline{0} \\
\underline{0} \\
\underline{0} \\
\underline{0} \\
\underline{0}\end{array}$ & $\begin{array}{l}57 \\
\underline{0} \\
\underline{0} \\
\underline{0} \\
\underline{0} \\
\underline{0}\end{array}$ & $\begin{array}{c}58 \\
0 \\
0 \\
0 \\
0 \\
19.21\end{array}$ & $\begin{array}{l}\frac{61}{0} \\
\underline{0} \\
\underline{0} \\
\underline{0} \\
\underline{0} \\
\underline{0}\end{array}$ & $\begin{array}{l}\frac{62}{0} \\
0 \\
0 \\
0 \\
0\end{array}$ & $\begin{array}{l}63 \\
\underline{0} \\
\underline{0} \\
\underline{0} \\
\underline{0} \\
\underline{0}\end{array}$ & $\begin{array}{c}64 \\
0 \\
0 \\
0 \\
0 \\
18.80\end{array}$ & $\begin{array}{l}\frac{1}{0} \\
\underline{0} \\
\underline{0} \\
\underline{0}\end{array}$ & & \\
\hline $\begin{array}{l}\text { Sargasso } \\
\text { Sea } \\
\& \\
\text { Gulf } \\
\text { Stream }\end{array}$ & & & & & & & & & & $\begin{array}{l}\frac{49}{0} \\
\underline{0} \\
\underline{0} \\
\underline{0.02} \\
\end{array}$ & $\begin{array}{l}\frac{50}{0} \\
0 \\
0 \\
0.03\end{array}$ & $\begin{array}{l}\frac{51}{0} \\
0 \\
0 \\
0\end{array}$ & $\begin{array}{l}\frac{52}{0} \\
\underline{0} \\
\underline{0} \\
\underline{0}\end{array}$ & & & & & & & & & & & \\
\hline $\begin{array}{l}\text { Slope } \\
\text { Water }\end{array}$ & $\frac{8}{-}$ & $\frac{9}{0}$ & $\frac{18}{0}$ & & $\frac{19}{0}$ & $\frac{20}{0}$ & $\frac{33}{0}$ & $\frac{34}{0}$ & & $\frac{35}{0}$ & $\frac{47}{0}$ & $\frac{48}{0}$ & $\frac{53}{0}$ & $\frac{54}{0}$ & $\frac{59}{0}$ & $\frac{60}{0}$ & $\frac{65}{0}$ & $\frac{66}{0}$ & & & & $\frac{4}{0}$ & $\frac{5}{0}$ & $\frac{6}{0}$ \\
\hline $\begin{array}{l}\text { High } \\
\text { Velocity } \\
\text { Region }\end{array}$ & $\frac{12}{0}$ & $\frac{13}{0}$ & $\frac{14}{0}$ & $\frac{15}{0}$ & $\frac{27}{7.50}$ & $\frac{29}{0}$ & $\frac{30}{0}$ & $\frac{31}{0}$ & $\frac{32}{-}$ & $\frac{41}{0}$ & $\frac{42}{0}$ & $\frac{43}{0}$ & $\frac{44}{0}$ & & $\frac{55}{0}$ & & & & & & & $\frac{2}{0}$ & $\frac{3}{0}$ & \\
\hline $\begin{array}{l}\text { Ring } \\
\text { Core }\end{array}$ & $\frac{10}{0}$ & $\frac{11}{0}$ & $\frac{16}{-}$ & $\frac{17}{0}$ & $\frac{21}{0}$ & $\frac{23}{6.30}$ & $\frac{24}{0}$ & $\frac{28}{0}$ & & $\frac{37}{0}$ & $\frac{38}{0}$ & $\frac{39}{0}$ & & & $\frac{56}{0}$ & $\frac{57}{0}$ & $\frac{58}{3.84}$ & $\frac{61}{0}$ & $\frac{62}{0}$ & $\frac{63}{0}$ & $\frac{64}{3.62}$ & $\frac{1}{0}$ & & \\
\hline SS \& GS & & & & & & & & & & $\frac{49}{0.01}$ & $\frac{50}{0.01}$ & $\frac{5}{0} !$ & $\frac{52}{0}$ & & & & & & & & & & & \\
\hline
\end{tabular}


Table 30. Abundance of Argyropelecus aculeatus (specimens $/ 10,000 \mathrm{~m}^{3}$ ).

\begin{tabular}{|c|c|c|c|c|c|c|c|c|c|c|c|c|c|c|c|c|c|c|c|c|c|c|c|c|}
\hline & \multicolumn{4}{|c|}{$\begin{array}{c}\text { Oceanus } 118 \\
\text { Apri1 }\end{array}$} & \multicolumn{5}{|c|}{$\begin{array}{c}\text { Oceanus } 121 \\
\text { June }\end{array}$} & \multicolumn{5}{|c|}{$\begin{array}{l}\text { Oceanus } 125 \\
\text { August }\end{array}$} & \multicolumn{7}{|c|}{$\begin{array}{l}\text { Knorr } 98 \\
\text { Sept-0ct }\end{array}$} & \multicolumn{3}{|c|}{$\begin{array}{c}\text { A2 } 110 \\
\text { Sept-0ct }\end{array}$} \\
\hline $\begin{array}{l}\text { Slope } \\
\text { Water }\end{array}$ & $\begin{array}{l}\frac{8}{\overline{\overline{0}}} \\
\underline{0} \\
\underline{0}\end{array}$ & $\begin{array}{l}9 \\
0 \\
0 \\
0 \\
0\end{array}$ & & & $\frac{\frac{19}{0.2}}{\frac{0.3}{0.0}} \frac{\underline{0}}{\underline{0}}$ & $\begin{array}{c}20 \\
0 \\
0.5 \\
0 \\
0 \\
0\end{array}$ & $\begin{array}{c}\frac{33}{\underline{0}} \\
\underline{0.8} \\
\underline{0} \\
\underline{0} \\
\underline{0}\end{array}$ & $\begin{array}{c}\frac{34}{\underline{0}} \\
\frac{0.7}{\underline{0}}\end{array}$ & & $\begin{array}{c}35 \\
0 \\
0.2 \\
0 \\
0 \\
0\end{array}$ & $\frac{47}{\frac{0.6}{\underline{0}}}$ & $\begin{array}{c}48 \\
0 \\
0.6 \\
0 \\
0\end{array}$ & $\frac{\frac{53}{0.4}}{\frac{0}{0}}$ & $\begin{array}{r}54 \\
0 \\
0 \\
0 \\
0\end{array}$ & $\begin{array}{c}\frac{59}{2.0} \\
\underline{0.8} \\
\underline{0} \\
\underline{0} \\
\underline{0}\end{array}$ & $\begin{array}{c}\frac{60}{0.5} \\
6.4 \\
0.7 \\
0 \\
0\end{array}$ & $\begin{array}{l}\frac{65}{\underline{0.9}} \\
\underline{0} \\
\underline{0} \\
\underline{0} \\
\underline{0}\end{array}$ & $\begin{array}{c}\frac{66}{0} \\
0.7 \\
0 \\
0 \\
0\end{array}$ & & & & $\begin{array}{c}\frac{4}{0} \\
0.7 \\
0 \\
0\end{array}$ & $\frac{\frac{5}{1.3}}{\frac{0.4}{\underline{0}}}$ & \\
\hline $\begin{array}{l}\text { High } \\
\text { Velocity } \\
\text { Region }\end{array}$ & $\begin{array}{c}12 \\
0 \\
4.2 \\
0 \\
0\end{array}$ & $\frac{\frac{13}{0.5}}{\frac{0}{0}}$ & $\frac{\frac{14}{3.1}}{\frac{0.3}{\underline{0}}}$ & $\begin{array}{l}15 \\
0.6 \\
2.4 \\
0 \\
0\end{array}$ & $\begin{array}{c}26 \\
0 \\
0 \\
0.5 \\
0 \\
-\end{array}$ & $\begin{array}{r}27 \\
0 \\
0 \\
0 \\
0 \\
0\end{array}$ & $\begin{array}{c}30 \\
0 \\
0.4 \\
0 \\
0 \\
0.5\end{array}$ & $\begin{array}{c}31 \\
0 \\
0 \\
0.4 \\
0 \\
0\end{array}$ & $\frac{\frac{32}{2.1}}{\frac{0}{0}} \frac{\underline{0}}{\underline{0}}$ & $\frac{\frac{41}{0.1}}{\frac{0}{0}}$ & $\frac{\frac{42}{0.9}}{\frac{0}{\underline{0}}} \frac{\underline{0}}{\underline{0}}$ & $\begin{array}{c}43 \\
0 \\
0 \\
0.4 \\
0 \\
0\end{array}$ & $\frac{\frac{44}{0.2}}{\frac{0}{0}}$ & & $\begin{array}{c}\frac{55}{\underline{0}} \\
\frac{0.5}{\underline{0}} \\
\underline{0}\end{array}$ & & & & & & & $\frac{\frac{2}{0.8}}{\frac{0.4}{1.4}} \frac{\underline{0}}{\underline{0}}$ & $\begin{array}{c}\frac{3}{0.4} \\
0.3 \\
0 \\
0\end{array}$ & \\
\hline $\begin{array}{l}\text { Ring } \\
\text { Core }\end{array}$ & $\frac{\frac{10}{1.2}}{\frac{0.6}{0.9}} \frac{\underline{0}}{n}$ & $\begin{array}{c}11 \\
0 \\
1.7 \\
0 \\
0\end{array}$ & $\begin{array}{c}16 \\
0 \\
0 \\
0\end{array}$ & $\frac{\frac{17}{\underline{0}}}{\frac{5.8}{\underline{0}}}$ & $\frac{\frac{21}{2.0}}{\frac{2.6}{\underline{0}}}$ & $\begin{array}{c}\frac{23}{\underline{0}} \\
\underline{3.3} \\
\underline{0} \\
\underline{0} \\
\underline{0}\end{array}$ & $\begin{array}{c}24 \\
0.3 \\
0 \\
0 \\
0 \\
0.6\end{array}$ & $\begin{array}{c}28 \\
0 \\
0.8 \\
0 \\
0 \\
0\end{array}$ & & & & & & & $\begin{array}{c}\frac{56}{\underline{0}} \\
\frac{1.1}{\underline{0}} \\
\underline{0} \\
\underline{0}\end{array}$ & $\begin{array}{c}\frac{57}{\underline{0}} \\
\frac{0.6}{\underline{0}} \\
\underline{0} \underline{0}\end{array}$ & $\begin{array}{c}58 \\
0 \\
0.4 \\
0.5 \\
0 \\
0\end{array}$ & $\begin{array}{c}\frac{61}{\underline{0}} \\
\underline{0.6} \\
\underline{0} \\
\underline{0} \\
\underline{0}\end{array}$ & $\begin{array}{r}62 \\
0 \\
0 \\
0 \\
0 \\
0\end{array}$ & 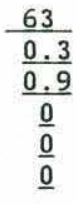 & $\begin{array}{r}64 \\
0 \\
0 \\
0 \\
0 \\
0\end{array}$ & $\frac{\frac{1}{0}}{\underline{\underline{0} .0}}$ & & \\
\hline $\begin{array}{l}\text { Sargasso } \\
\text { Sea } \\
\& \\
\text { Gulf } \\
\text { Stream }\end{array}$ & & & & & & & & & & $\frac{49}{\underline{0}}$ & $\begin{array}{c}50 \\
0 \\
1.0 \\
0 \\
0\end{array}$ & $\begin{array}{c}51 \\
0 \\
0 \\
0.2 \\
0\end{array}$ & $\begin{array}{l}\frac{52}{\underline{0}} \\
\underline{0} \\
\underline{0} \\
\underline{0}\end{array}$ & & & & & & & & & & & \\
\hline $\begin{array}{l}\text { Slope } \\
\text { Water }\end{array}$ & $\frac{8}{-}$ & $\frac{9}{0}$ & $\frac{18}{0}$ & & $\frac{19}{0.1}$ & $\frac{20}{0.1}$ & $\frac{33}{0.1}$ & $\frac{34}{0.2}$ & & $\frac{35}{0}$ & $\frac{47}{0.2}$ & $\frac{48}{0.2}$ & $\frac{53}{0.1}$ & $\frac{54}{0}$ & $\frac{59}{0.6}$ & $\frac{60}{0.9}$ & $\frac{65}{0.2}$ & $\frac{66}{0.1}$ & & & & $\frac{4}{0.2}$ & $\frac{5}{0.4}$ & $\frac{6}{0}$ \\
\hline $\begin{array}{l}\text { High } \\
\text { Velocity } \\
\text { Region }\end{array}$ & $\frac{12}{1.1}$ & $\frac{13}{0.1}$ & $\frac{14}{0.9}$ & $\frac{15}{0.8}$ & $\frac{27}{0}$ & $\frac{29}{0.5}$ & $\frac{30}{0.2}$ & $\frac{31}{0.1}$ & $\frac{32}{-}$ & $\frac{41}{0.1}$ & $\frac{42}{0.3}$ & $\frac{43}{0.1}$ & $\frac{44}{0.1}$ & & $\frac{55}{0.1}$ & & & & & & & $\frac{2}{0.6}$ & $\frac{3}{0.2}$ & \\
\hline $\begin{array}{l}\text { Ring } \\
\text { Core }\end{array}$ & $\frac{10}{0.7}$ & $\frac{11}{0.4}$ & $\frac{16}{-}$ & $\frac{17}{1.5}$ & $\frac{21}{0.7}$ & $\frac{23}{0.8}$ & $\frac{24}{0.2}$ & $\frac{28}{0.2}$ & & $\frac{37}{0.5}$ & $\frac{38}{0.2}$ & $\frac{39}{0.1}$ & & & $\frac{56}{0.2}$ & $\frac{57}{0.1}$ & $\frac{58}{0.1}$ & $\frac{61}{0.1}$ & $\frac{62}{0}$ & $\frac{63}{0.1}$ & $\frac{64}{0}$ & $\frac{1}{0.5}$ & & \\
\hline SS \& GS & & & & & & & & & & $\frac{49}{0.1}$ & $\frac{50}{0.3}$ & $\frac{51}{0.1}$ & $\frac{52}{0}$ & & & & & & & & & & & \\
\hline
\end{tabular}


Table 31. Volume of Argyropelecus aculeatus $\left(\mathrm{ml} / 10,000 \mathrm{~m}^{3}\right)$.

\begin{tabular}{|c|c|c|c|c|c|c|c|c|c|c|c|c|c|c|c|c|c|c|c|c|c|c|c|c|}
\hline & \multicolumn{4}{|c|}{$\begin{array}{c}\text { Oceanus } 118 \\
\text { April }\end{array}$} & \multicolumn{5}{|c|}{$\begin{array}{c}\text { Oceanus } 121 \\
\text { June }\end{array}$} & \multicolumn{5}{|c|}{$\begin{array}{l}\text { Oceanus } 125 \\
\text { August }\end{array}$} & \multicolumn{7}{|c|}{$\begin{array}{l}\text { Knorr } 98 \\
\text { Sept-0ct }\end{array}$} & \multicolumn{3}{|c|}{$\begin{array}{c}\text { A2 } 110 \\
\text { Sept-0ct }\end{array}$} \\
\hline $\begin{array}{l}\text { Slope } \\
\text { Water }\end{array}$ & $\begin{array}{l}\frac{8}{\overline{0}} \\
\underline{0} \underline{0} \\
\underline{0}\end{array}$ & $\begin{array}{l}9 \\
0 \\
0 \\
0 \\
0\end{array}$ & & & $\frac{\frac{19}{1.22}}{\frac{0.14}{\frac{0}{0}}}$ & $\begin{array}{l}\frac{20}{0} \\
0.53 \\
0 \\
0 \\
0\end{array}$ & $\begin{array}{l}\frac{33}{\underline{0}} \\
\frac{2.92}{\underline{0}} \\
\underline{0} \\
\underline{0}\end{array}$ & $\begin{array}{l}\frac{34}{\underline{0}} \\
\frac{0.93}{\underline{0}}\end{array}$ & & $\begin{array}{l}\frac{35}{0} \\
0.21 \\
0 \\
0 \\
0\end{array}$ & $\frac{\frac{47}{2.22}}{\frac{0}{0}}$ & $\begin{array}{l}\frac{48}{0} \\
1.14 \\
0 \\
0\end{array}$ & $\frac{\frac{53}{0.44}}{\frac{0}{0}}$ & $\begin{array}{l}54 \\
0 \\
0 \\
0 \\
0\end{array}$ & $\frac{\frac{59}{0.67}}{\frac{0.08}{\underline{0}}}$ & $\begin{array}{c}\frac{60}{0.07} \\
0.77 \\
0.55 \\
0 \\
0\end{array}$ & $\begin{array}{l}\frac{65}{2.83} \\
\underline{0} \\
\frac{0}{0} \\
\underline{0}\end{array}$ & $\begin{array}{l}\frac{66}{0} \\
1.43 \\
0 \\
0 \\
0\end{array}$ & & & & $\begin{array}{l}\frac{4}{0} \\
1.09 \\
0 \\
0\end{array}$ & $\frac{\frac{5}{2.67}}{\frac{0.04}{\underline{0}}}$ & \\
\hline $\begin{array}{l}\text { High } \\
\text { Velocity } \\
\text { Region }\end{array}$ & $\begin{array}{l}\frac{12}{0} \\
1.15 \\
0 \\
0\end{array}$ & $\frac{\frac{13}{0.75}}{\frac{0}{0}}$ & $\frac{\frac{14}{3.43}}{\frac{0.83}{0}}$ & $\begin{array}{l}\frac{15}{2.22} \\
5.59 \\
0 \\
0\end{array}$ & $\begin{array}{l}\frac{26}{0} \\
0 \\
0.19 \\
0 \\
-\end{array}$ & $\begin{array}{l}27 \\
0 \\
0 \\
0 \\
0 \\
0\end{array}$ & $\begin{array}{c}30 \\
0 \\
0.12 \\
0 \\
0 \\
0.16\end{array}$ & $\begin{array}{l}31 \\
0 \\
0 \\
0 \\
0 \\
0\end{array}$ & $\frac{\frac{32}{8.42}}{\frac{0}{0}}$ & $\frac{\frac{41}{0.52}}{\frac{0}{0}}$ & $\frac{\frac{42}{1.38}}{\frac{0}{0}}$ & $\begin{array}{c}\frac{43}{0} \\
0 \\
2.08 \\
0 \\
0\end{array}$ & $\frac{\frac{44}{0.46}}{\frac{0}{0}}$ & & $\begin{array}{l}\frac{55}{\underline{0}} \\
\underline{0.07} \\
\underline{0} \\
\underline{0}\end{array}$ & & & & & & & $\frac{\frac{2}{3.75}}{\frac{0.08}{\frac{0}{0}}}$ & $\begin{array}{l}\frac{3}{0.04} \\
1.35 \\
0 \\
0\end{array}$ & \\
\hline $\begin{array}{l}\text { Ring } \\
\text { Core }\end{array}$ & $\frac{\frac{10}{1.20}}{\frac{0.12}{0.18}}$ & $\begin{array}{l}\frac{11}{0} \\
0.56 \\
0 \\
0\end{array}$ & $\begin{array}{l}\frac{16}{0} \\
0 \\
0\end{array}$ & $\frac{\frac{17}{0}}{\underline{\underline{0} .11}} \frac{0}{\underline{0}}$ & $\frac{\frac{21}{0.60}}{\frac{0.20}{1.20}}$ & $\begin{array}{l}\frac{23}{\underline{0}} \\
\underline{4.67} \\
\underline{0} \\
\underline{0} \\
\underline{0}\end{array}$ & $\begin{array}{c}\frac{24}{0.04} \\
0 \\
0 \\
0 \\
0.17\end{array}$ & $\begin{array}{c}\frac{28}{0} \\
2.42 \\
0 \\
0 \\
0\end{array}$ & & & & & & & $\begin{array}{l}\frac{56}{\underline{0}} \\
\underline{0.56} \\
\underline{0} \\
\underline{0} \\
\underline{0}\end{array}$ & $\begin{array}{l}\frac{57}{\underline{0}} \\
\underline{0.94} \\
\underline{0} \\
\underline{0} \\
\underline{0}\end{array}$ & $\begin{array}{l}\frac{58}{0} \\
0.04 \\
0.05 \\
0 \\
0\end{array}$ & $\begin{array}{l}\frac{61}{\underline{0}} \\
\underline{0.12} \\
\underline{0} \\
\underline{0} \\
\underline{0}\end{array}$ & $\begin{array}{l}\frac{62}{0} \\
0 \\
0 \\
0 \\
0\end{array}$ & $\frac{\frac{63}{0.02}}{\frac{2.17}{\underline{0}}}$ & $\begin{array}{l}64 \\
0 \\
0 \\
0 \\
0 \\
0\end{array}$ & $\frac{\frac{1}{\underline{0}}}{\underline{6.52}}$ & & \\
\hline $\begin{array}{l}\text { Sargasso } \\
\text { Sea } \\
\& \\
\text { Gulf } \\
\text { Stream }\end{array}$ & & & & & & & & & & $\begin{array}{l}\frac{49}{\underline{0}} \\
\frac{0.07}{\underline{0}} \\
\underline{0}\end{array}$ & $\begin{array}{l}\frac{50}{0} \\
0.05 \\
0 \\
0\end{array}$ & $\begin{array}{c}51 \\
0 \\
0 \\
0.43 \\
0\end{array}$ & $\begin{array}{l}\frac{52}{\underline{0}} \\
\underline{0} \\
\underline{0} \\
\underline{0}\end{array}$ & & & & & & & & & & & \\
\hline $\begin{array}{l}\text { Slope } \\
\text { Water }\end{array}$ & $\frac{8}{-}$ & $\frac{9}{0}$ & $\frac{18}{0}$ & & $\frac{19}{0.14}$ & $\frac{20}{0.13}$ & $\frac{33}{0.41}$ & $\frac{34}{0.30}$ & & $\frac{35}{0.04}$ & $\frac{47}{0.56}$ & $\frac{48}{0.29}$ & $\frac{53}{0.11}$ & $\frac{54}{0}$ & $\frac{59}{0.15}$ & $\frac{60}{0.21}$ & $\frac{65}{0.57}$ & $\frac{66}{0.14}$ & & & & $\frac{4}{0.27}$ & $\frac{5}{0.68}$ & $\frac{6}{0}$ \\
\hline $\begin{array}{l}\text { High } \\
\text { Velocity } \\
\text { Region }\end{array}$ & $\frac{12}{0.29}$ & $\frac{13}{0.19}$ & $\frac{14}{1.07}$ & $\frac{15}{1.95}$ & $\frac{27}{0}$ & $\frac{29}{0.90}$ & $\frac{30}{0.04}$ & $\frac{31}{0}$ & $\frac{32}{-}$ & $\frac{41}{0.26}$ & $\frac{42}{0.52}$ & $\frac{43}{0.42}$ & $\frac{44}{0.28}$ & & $\frac{55}{0.01}$ & & & & & & & $\frac{2}{0.96}$ & $\frac{3}{0.35}$ & \\
\hline $\begin{array}{l}\text { Ring } \\
\text { Core }\end{array}$ & $\frac{10}{0.38}$ & $\frac{11}{0.14}$ & $\frac{16}{-}$ & $\frac{17}{0.53}$ & $\frac{21}{0.45}$ & $\frac{23}{1.17}$ & $\frac{24}{0.05}$ & $\frac{28}{0.44}$ & & $\frac{37}{1.12}$ & $\frac{38}{0.38}$ & $\frac{39}{0.04}$ & & & $\frac{56}{0.11}$ & $\frac{57}{0.19}$ & $\frac{58}{0.01}$ & $\frac{61}{0.02}$ & $\frac{62}{0}$ & $\frac{63}{0.07}$ & $\frac{64}{0}$ & $\frac{1}{1.63}$ & & \\
\hline SS \& GS & & & & & & & & & & $\frac{49}{0.02}$ & $\frac{50}{0.01}$ & $\frac{51}{0.11}$ & $\frac{52}{0}$ & & & & & & & & & & & \\
\hline
\end{tabular}


Table 32. Abundance of Argyropelecus hemigymnus (specimens $/ 10,000 \mathrm{~m}^{3}$ ).

\begin{tabular}{|c|c|c|c|c|c|c|c|c|c|c|c|c|c|c|c|c|c|c|c|c|c|c|c|c|}
\hline & \multicolumn{4}{|c|}{$\begin{array}{c}\text { Oceanus } 118 \\
\text { Apri1 }\end{array}$} & \multicolumn{5}{|c|}{$\begin{array}{c}\text { Oceanus } 121 \\
\text { June }\end{array}$} & \multicolumn{5}{|c|}{$\begin{array}{l}\text { Oceanus } 125 \\
\text { August }\end{array}$} & \multicolumn{7}{|c|}{$\begin{array}{l}\text { Knorr } 98 \\
\text { Sept-0ct }\end{array}$} & \multicolumn{3}{|c|}{$\begin{array}{c}\text { A2 } 110 \\
\text { Sept-0ct }\end{array}$} \\
\hline $\begin{array}{l}\text { Slope } \\
\text { Water }\end{array}$ & $\begin{array}{l}8 \\
\overline{0} \\
\underline{0} \\
\underline{0} \\
\underline{0}\end{array}$ & $\begin{array}{c}9 \\
0 \\
0.6 \\
0 \\
0\end{array}$ & & & $\begin{array}{l}\frac{19}{\underline{0}} \\
\frac{0}{0.7} \\
\frac{0}{0}\end{array}$ & $\begin{array}{r}20 \\
0 \\
0 \\
0 \\
0 \\
0\end{array}$ & $\begin{array}{c}\frac{33}{\underline{0}} \\
\underline{0} \\
\underline{0} \\
\underline{0} \\
\underline{0}\end{array}$ & $\begin{array}{l}\frac{34}{\underline{0}} \\
\underline{0} \\
\underline{0}\end{array}$ & & $\begin{array}{c}35 \\
0 \\
0.4 \\
0 \\
0 \\
0\end{array}$ & $\begin{array}{l}\frac{47}{\underline{0}} \\
\frac{0.3}{\underline{0}} \\
\underline{0}\end{array}$ & $\begin{array}{r}48 \\
0 \\
0 \\
0 \\
0\end{array}$ & $\frac{53}{\frac{0.1}{\underline{0}}}$ & $\begin{array}{r}54 \\
0 \\
0 \\
0 \\
0\end{array}$ & $\begin{array}{c}\frac{59}{\underline{0}} \\
\frac{8.8}{\underline{0}} \\
\underline{0} \\
\underline{0}\end{array}$ & $\begin{array}{c}\frac{60}{0} \\
10.5 \\
0 \\
0 \\
0\end{array}$ & $\begin{array}{c}\frac{65}{\underline{0}} \\
\underline{2.8} \\
\underline{0} \\
\underline{0} \\
\underline{0}\end{array}$ & $\begin{array}{c}\frac{66}{0} \\
3.6 \\
0 \\
0 \\
0\end{array}$ & & & & $\begin{array}{c}\frac{4}{0} \\
0.4 \\
0 \\
0\end{array}$ & $\begin{array}{l}\frac{5}{0} \\
\underline{0} \\
\underline{0} \\
\underline{0}\end{array}$ & \\
\hline $\begin{array}{l}\text { High } \\
\text { Velocity } \\
\text { Region }\end{array}$ & $\begin{array}{c}12 \\
0 \\
6.2 \\
0 \\
0\end{array}$ & $\frac{\frac{13}{0}}{\frac{\underline{0}}{\underline{0} .7}}$ & $\frac{\frac{14}{0.3}}{\frac{\underline{3.0}}{\underline{0}} \underline{\underline{0}}}$ & $\begin{array}{l}\frac{15}{0.6} \\
2.9 \\
0 \\
0\end{array}$ & $\begin{array}{c}26 \\
0 \\
0 \\
0 \\
0 \\
-\end{array}$ & $\begin{array}{r}27 \\
0 \\
0 \\
0 \\
0 \\
0\end{array}$ & $\begin{array}{c}30 \\
0 \\
2.8 \\
8.8 \\
0 \\
1.6\end{array}$ & $\begin{array}{c}31 \\
0 \\
0 \\
1.4 \\
0 \\
0\end{array}$ & $\frac{\frac{32}{4.5}}{\frac{1.6}{\underline{0}}}$ & $\frac{\frac{41}{0.1}}{\frac{0}{0}}$ & $\frac{\frac{42}{0.4}}{\frac{0}{0}}$ & $\begin{array}{r}43 \\
0 \\
0 \\
0 \\
0 \\
0\end{array}$ & $\begin{array}{c}\frac{44}{\underline{0}} \\
\underline{0} \\
\underline{0}\end{array}$ & & 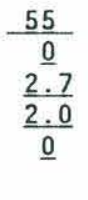 & & & & & & & $\frac{\frac{2}{0.4}}{\frac{5.4}{\frac{0}{0}}}$ & $\begin{array}{l}\frac{3}{0.4} \\
5.9 \\
0 \\
0\end{array}$ & \\
\hline $\begin{array}{l}\text { Ring } \\
\text { Core }\end{array}$ & $\frac{\frac{10}{0.8}}{\frac{0.4}{\underline{0}}}$ & $\begin{array}{c}\frac{11}{0} \\
13.3 \\
0 \\
0\end{array}$ & $\begin{array}{c}\frac{16}{0} \\
5.0 \\
0\end{array}$ & 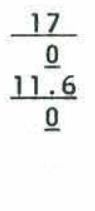 & $\frac{\frac{21}{1.0}}{\frac{1.8}{1.6}} \frac{0.6}{\underline{0}} \underline{\underline{0}}$ & $\begin{array}{c}\frac{23}{\underline{0}} \\
\frac{5.3}{3.0} \\
\frac{0}{0}\end{array}$ & $\begin{array}{c}24 \\
0.9 \\
15.0 \\
2.7 \\
0 \\
0\end{array}$ & $\begin{array}{c}28 \\
0 \\
3.5 \\
0 \\
0 \\
0\end{array}$ & & & & & & & $\begin{array}{c}\frac{56}{\underline{0}} \\
\underline{0} \\
\underline{8.4} \\
\underline{0} \\
\underline{0}\end{array}$ & $\begin{array}{c}\frac{57}{\underline{0}} \\
\frac{0.3}{3.0} \\
\frac{0}{0}\end{array}$ & $\begin{array}{c}\frac{58}{0} \\
1.3 \\
2.9 \\
0 \\
0\end{array}$ & 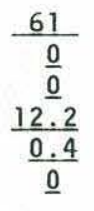 & $\begin{array}{c}62 \\
0 \\
0 \\
3.3 \\
0 \\
0\end{array}$ & 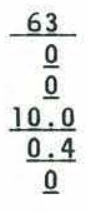 & $\begin{array}{c}64 \\
0.8 \\
0.6 \\
0.7 \\
0 \\
0\end{array}$ & $\frac{\frac{1}{\underline{0}}}{\frac{6.6}{\underline{0}}}$ & & \\
\hline $\begin{array}{l}\text { Sargasso } \\
\text { Sea } \\
\& \\
\text { Gulf } \\
\text { Stream }\end{array}$ & & & & & & & & & & $\begin{array}{l}\frac{49}{0} \\
\frac{0.4}{1.3} \\
\underline{0}\end{array}$ & $\begin{array}{c}\frac{50}{0} \\
2.9 \\
1.7 \\
0\end{array}$ & $\begin{array}{c}51 \\
0 \\
0.4 \\
0 \\
0\end{array}$ & $\begin{array}{c}\frac{52}{\underline{0}} \\
\frac{0.3}{\underline{0}} \\
\underline{0}\end{array}$ & & & & & & v & & & & & \\
\hline $\begin{array}{l}\text { Slope } \\
\text { Water }\end{array}$ & $\frac{8}{-}$ & $\frac{9}{0.2}$ & $\frac{18}{0}$ & & $\frac{19}{0.2}$ & $\frac{20}{0}$ & $\frac{33}{0}$ & $\frac{34}{0}$ & & $\frac{35}{0.1}$ & $\frac{47}{0.1}$ & $\frac{48}{0}$ & $\frac{53}{0}$ & $\frac{54}{0}$ & $\frac{59}{1.8}$ & $\frac{60}{1.1}$ & $\frac{65}{0.6}$ & $\frac{66}{0.4}$ & & & & $\frac{4}{0.1}$ & $\frac{5}{0}$ & $\frac{6}{0}$ \\
\hline $\begin{array}{l}\text { High } \\
\text { Velocity } \\
\text { Region }\end{array}$ & $\frac{12}{1.6}$ & $\frac{13}{0.4}$ & $\frac{14}{0.8}$ & $\frac{15}{0.9}$ & $\frac{27}{0}$ & $\frac{29}{1.8}$ & $\frac{30}{2.4}$ & $\frac{31}{0.3}$ & $\frac{32}{-}$ & $\frac{41}{0.1}$ & $\frac{42}{0.2}$ & $\frac{43}{0}$ & $\frac{44}{0}$ & & $\frac{55}{1.3}$ & & & & & & & $\frac{2}{1.5}$ & $\frac{3}{1.6}$ & \\
\hline $\begin{array}{l}\text { Ring } \\
\text { Core }\end{array}$ & $\frac{10}{2.6}$ & $\frac{11}{3.3}$ & $\frac{16}{-}$ & $\frac{17}{2.9}$ & $\frac{21}{0.8}$ & $\frac{23}{1.9}$ & $\frac{24}{1.2}$ & $\frac{28}{0.6}$ & & $\frac{37}{0.3}$ & $\frac{38}{0.5}$ & $\frac{39}{0.2}$ & & & $\frac{56}{1.7}$ & $\frac{57}{0.7}$ & $\frac{58}{0.7}$ & $\frac{61}{0.9}$ & $\frac{62}{0.7}$ & $\frac{63}{2.1}$ & $\frac{64}{0.5}$ & $\frac{1}{1.7}$ & & \\
\hline SS \& GS & & & & & & & & & & $\frac{49}{0.4}$ & $\frac{50}{1.2}$ & $\frac{51}{0.1}$ & $\frac{52}{0.1}$ & & & & & & & & & & & \\
\hline
\end{tabular}


Table 33. Volume of Argyropelecus hemigymnus $\left(\mathrm{ml} / 10,000 \mathrm{~m}^{3}\right)$.

\begin{tabular}{|c|c|c|c|c|c|c|c|c|c|c|c|c|c|c|c|c|c|c|c|c|c|c|c|c|}
\hline & \multicolumn{4}{|c|}{$\begin{array}{c}\text { Oceanus } \\
\text { April }\end{array}$} & \multicolumn{5}{|c|}{$\begin{array}{c}\text { Oceanus } 121 \\
\text { June }\end{array}$} & \multicolumn{5}{|c|}{$\begin{array}{l}\text { Oceanus } 125 \\
\text { August }\end{array}$} & \multicolumn{7}{|c|}{$\begin{array}{l}\text { Knorr } 98 \\
\text { Sept-0ct }\end{array}$} & \multicolumn{3}{|c|}{$\begin{array}{c}\text { A2 } 110 \\
\text { Sept-0ct }\end{array}$} \\
\hline $\begin{array}{l}\text { Slope } \\
\text { Water }\end{array}$ & $\begin{array}{l}\frac{8}{\overline{0}} \\
\underline{0} \\
\underline{0} \\
\underline{0}\end{array}$ & $\begin{array}{c}\frac{9}{0} \\
0.06 \\
0 \\
0\end{array}$ & & & $\begin{array}{l}\frac{19}{\underline{0}} \\
\underline{0} \\
\frac{0.03}{\underline{0}} \\
\underline{0}\end{array}$ & $\begin{array}{l}20 \\
0 \\
0 \\
0 \\
0 \\
0\end{array}$ & $\begin{array}{l}33 \\
\underline{0} \\
\underline{0} \\
\underline{0} \\
\underline{0} \\
\underline{0}\end{array}$ & $\begin{array}{l}\frac{34}{\underline{0}} \\
\underline{0} \\
\underline{0}\end{array}$ & & $\begin{array}{l}\frac{35}{0} \\
0.04 \\
0 \\
0 \\
0\end{array}$ & $\frac{\frac{47}{\underline{0}}}{\frac{0.05}{\underline{0}}}$ & $\begin{array}{l}\frac{48}{0} \\
0 \\
0 \\
0\end{array}$ & $\begin{array}{l}\frac{53}{\underline{0}} \\
\frac{0.18}{\underline{0}} \\
\underline{0}\end{array}$ & $\begin{array}{l}54 \\
0 \\
0 \\
0 \\
0\end{array}$ & $\begin{array}{l}\frac{59}{\underline{0}} \\
\underline{0.64} \\
\underline{0} \\
\underline{0} \\
\underline{0}\end{array}$ & $\begin{array}{l}\frac{60}{0} \\
0.55 \\
0 \\
0 \\
0\end{array}$ & $\begin{array}{l}\frac{65}{\underline{0}} \\
\frac{0.48}{\underline{0}} \\
\underline{0} \\
\underline{0}\end{array}$ & $\begin{array}{c}\frac{66}{0} \\
0.64 \\
0 \\
0 \\
0\end{array}$ & & & & $\begin{array}{c}\frac{4}{0} \\
0.02 \\
0 \\
0\end{array}$ & $\begin{array}{l}\frac{5}{0} \\
\underline{0} \\
\underline{0} \\
\underline{0}\end{array}$ & \\
\hline $\begin{array}{l}\text { High } \\
\text { Velocity } \\
\text { Region }\end{array}$ & $\begin{array}{l}\frac{12}{0} \\
0.85 \\
0 \\
0\end{array}$ & $\begin{array}{l}\frac{13}{\underline{0}} \\
\frac{0.28}{\underline{0}}\end{array}$ & $\frac{\frac{14}{0.06}}{\frac{0.73}{\frac{0}{0}}}$ & $\begin{array}{l}\frac{15}{0.06} \\
1.18 \\
0 \\
0\end{array}$ & $\begin{array}{l}26 \\
0 \\
0 \\
0 \\
0 \\
-\end{array}$ & $\begin{array}{l}27 \\
0 \\
0 \\
0 \\
0 \\
0\end{array}$ & $\begin{array}{c}\frac{30}{0} \\
0.28 \\
4.69 \\
0 \\
0.51\end{array}$ & $\begin{array}{l}31 \\
0 \\
0 \\
0 \\
0 \\
0\end{array}$ & $\frac{\frac{32}{1.58}}{\frac{1.00}{\frac{0}{0}}}$ & $\frac{\frac{41}{0.03}}{\frac{0}{0}}$ & $\frac{\frac{42}{0.13}}{\frac{0}{0}}$ & $\begin{array}{l}43 \\
0 \\
0 \\
0 \\
0 \\
0\end{array}$ & $\begin{array}{l}\frac{44}{0} \\
\frac{0}{0} \\
\underline{0}\end{array}$ & & $\begin{array}{l}\frac{55}{\underline{0}} \\
\frac{0.23}{\underline{0.24}} \\
\underline{0}\end{array}$ & & & & & & & $\frac{\frac{2}{0.04}}{\frac{0.54}{\underline{0}}}$ & $\begin{array}{l}\frac{3}{0.04} \\
0.27 \\
0 \\
0\end{array}$ & \\
\hline $\begin{array}{l}\text { Ring } \\
\text { Core }\end{array}$ & $\frac{\frac{10}{0.12}}{\frac{1.76}{\frac{0}{0}}}$ & $\begin{array}{l}\frac{11}{0} \\
0.72 \\
0 \\
0\end{array}$ & $\begin{array}{l}\frac{16}{0} \\
1.07 \\
0\end{array}$ & $\begin{array}{l}\frac{17}{\underline{0}} \\
\frac{4.74}{\underline{0}}\end{array}$ & $\frac{\frac{21}{0.50}}{\frac{0.52}{1.52}}$ & $\begin{array}{l}\frac{23}{\underline{0}} \\
\frac{1.67}{0.55} \\
\frac{0}{0}\end{array}$ & $\begin{array}{l}\frac{24}{0.21} \\
5.00 \\
0.27 \\
0 \\
0\end{array}$ & $\begin{array}{l}\frac{28}{0} \\
2.50 \\
0 \\
0 \\
0\end{array}$ & & & & & & & $\begin{array}{l}\frac{56}{\underline{0}} \\
\underline{0} \\
\underline{0.53} \\
\underline{0} \\
\underline{0}\end{array}$ & $\begin{array}{l}\frac{57}{\underline{0}} \\
\underline{0.03} \\
\underline{0.38} \\
\underline{0} \\
\underline{0}\end{array}$ & $\begin{array}{l}\frac{58}{0} \\
0.04 \\
0.29 \\
0 \\
0\end{array}$ & $\begin{array}{l}\frac{61}{\underline{0}} \\
\underline{0} \\
\frac{0.63}{0.15} \\
\underline{0}\end{array}$ & $\begin{array}{l}\frac{62}{0} \\
0 \\
0.39 \\
0 \\
0\end{array}$ & $\begin{array}{l}\frac{63}{\underline{0}} \\
\underline{0} \\
\frac{1.23}{0.06} \\
\underline{0}\end{array}$ & $\begin{array}{l}\frac{64}{0.04} \\
0.06 \\
0.07 \\
0 \\
0\end{array}$ & $\frac{\frac{1}{\underline{0}}}{\underline{0.80}}$ & & \\
\hline $\begin{array}{l}\text { Sargasso } \\
\text { Sea } \\
\& \\
\text { Gulf } \\
\text { Stream }\end{array}$ & & & & & & & & & & $\begin{array}{l}\frac{49}{\underline{0}} \\
\frac{0.02}{0.22} \\
\underline{0}\end{array}$ & $\begin{array}{l}\frac{50}{0} \\
0.22 \\
0.28 \\
0\end{array}$ & $\begin{array}{l}\frac{51}{0} \\
0.09 \\
0 \\
0\end{array}$ & $\begin{array}{l}\frac{52}{\underline{0}} \\
\underline{0.03} \\
\underline{0} \\
\underline{0}\end{array}$ & & & & & & & & & & & \\
\hline $\begin{array}{l}\text { Slope } \\
\text { Water }\end{array}$ & $\frac{8}{-}$ & $\frac{9}{0.02}$ & $\frac{18}{0}$ & & $\frac{19}{0.01}$ & $\frac{20}{0}$ & $\frac{33}{0}$ & $\frac{34}{0}$ & & $\frac{35}{0.01}$ & $\frac{47}{0.01}$ & $\frac{48}{0}$ & $\frac{53}{0.05}$ & $\frac{54}{0}$ & $\frac{59}{0.13}$ & $\frac{60}{0.06}$ & $\frac{65}{0.10}$ & $\frac{66}{0.06}$ & & & & $\frac{4}{0.01}$ & $\frac{5}{0}$ & $\frac{6}{0}$ \\
\hline $\begin{array}{l}\text { High } \\
\text { Velocity } \\
\text { Region }\end{array}$ & $\frac{12}{0.21}$ & $\frac{13}{0.07}$ & $\frac{14}{0.20}$ & $\frac{15}{0.31}$ & $\frac{27}{0}$ & $\frac{29}{0.41}$ & $\frac{30}{1.07}$ & $\frac{31}{0}$ & $\frac{32}{-}$ & $\frac{41}{0.02}$ & $\frac{42}{0.05}$ & $\frac{43}{0}$ & $\frac{44}{0}$ & & $\frac{55}{0.14}$ & & & & & & & $\frac{2}{0.15}$ & $\frac{3}{0.08}$ & \\
\hline $\begin{array}{l}\text { Ring } \\
\text { Core }\end{array}$ & $\frac{10}{0.47}$ & $\frac{11}{0.18}$ & $\frac{16}{-}$ & $\frac{17}{1.19}$ & $\frac{21}{0.56}$ & $\frac{23}{0.53}$ & $\frac{24}{0.23}$ & $\frac{28}{0.45}$ & & $\frac{37}{0.08}$ & $\frac{38}{0}$ & $\frac{39}{0.09}$ & & & $\frac{56}{0.10}$ & $\frac{57}{0.08}$ & $\frac{58}{0.06}$ & $\frac{61}{0.15}$ & $\frac{62}{0.08}$ & $\frac{63}{0.26}$ & $\frac{64}{0.03}$ & $\frac{1}{0.20}$ & & \\
\hline SS \& GS & & & & & & & & & & $\frac{49}{0.06}$ & $\frac{50}{0.13}$ & $\frac{51}{0.02}$ & $\frac{52}{0.01}$ & & & & & & & & & & & \\
\hline
\end{tabular}


Table 34. Volume of Aristostomias grimaldii $\left(\mathrm{ml} / 10,000 \mathrm{~m}^{3}\right)$.

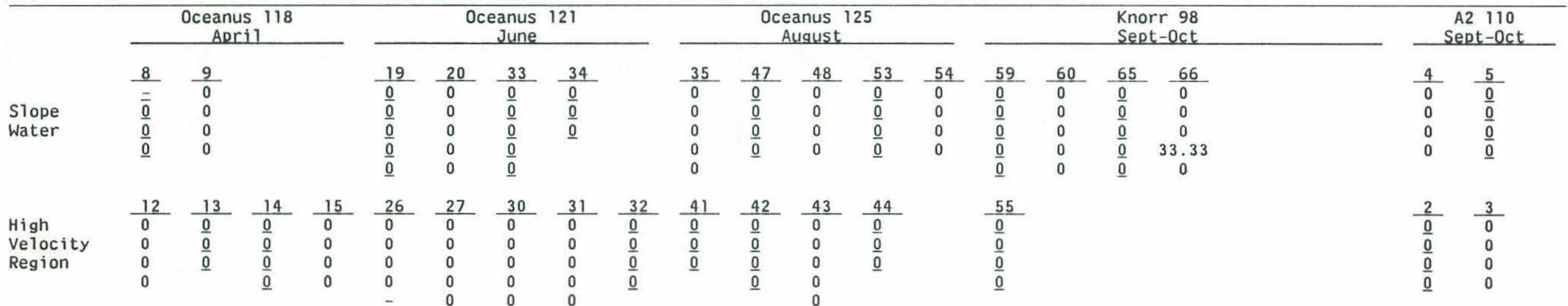

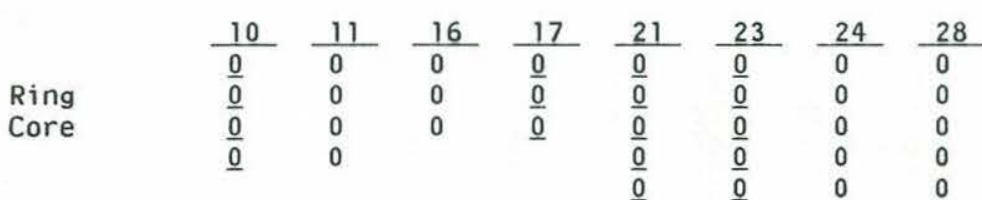


Table 35. Abundance of Benthosema glaciale (specimens $/ 10,000 \mathrm{~m}^{3}$ ).

\begin{tabular}{|c|c|c|c|c|c|c|c|c|c|c|c|c|c|c|c|c|c|c|c|c|c|c|c|c|}
\hline & \multicolumn{4}{|c|}{$\begin{array}{c}\text { Oceanus } 118 \\
\text { Aprif }\end{array}$} & \multicolumn{5}{|c|}{$\begin{array}{c}\text { Oceanus } 121 \\
\text { June }\end{array}$} & \multicolumn{5}{|c|}{$\begin{array}{l}\text { Oceanus } 125 \\
\text { August }\end{array}$} & \multicolumn{7}{|c|}{$\begin{array}{l}\text { Knorr } 98 \\
\text { Sept-0ct }\end{array}$} & \multicolumn{3}{|c|}{$\begin{array}{c}\text { A2 } 110 \\
\text { Sept-0ct }\end{array}$} \\
\hline $\begin{array}{l}\text { Slope } \\
\text { Water }\end{array}$ & $\begin{array}{l}\frac{8}{=} \\
\frac{2.9}{2.7} \\
\underline{0}\end{array}$ & $\begin{array}{c}\frac{9}{0} \\
38.8 \\
10.0 \\
0\end{array}$ & & & $\frac{\frac{19}{23.9}}{\frac{1.4}{1.1}} \frac{32.6}{1.6}$ & \begin{tabular}{r}
\multicolumn{1}{c}{20} \\
0.5 \\
23.7 \\
39.1 \\
3.6 \\
1.2
\end{tabular} & $\frac{\frac{33}{400.0}}{\frac{1.3}{5.2}} \frac{4 \frac{1.7}{29.4}}{\underline{29.4}}$ & $\begin{array}{r}\frac{34}{56.4} \\
\underline{0.7} \\
\underline{20.5} \\
\end{array}$ & & $\begin{array}{c}\frac{35}{0} \\
17.9 \\
77.7 \\
4.4 \\
0\end{array}$ & $\frac{\frac{47}{6.1}}{\frac{6.8}{11.8}} \frac{12.3}{12}$ & $\begin{array}{l}\frac{48}{0} \\
43.7 \\
16.8 \\
15.3\end{array}$ & $\frac{\frac{53}{18.7}}{\frac{5.3}{3.1}} \frac{0.2}{\underline{0.2}}$ & $\begin{array}{c}\frac{54}{0} \\
7.8 \\
7.3 \\
4.0\end{array}$ & $\begin{array}{r}\frac{59}{\underline{0}} \\
\underline{0} \\
\frac{3.8}{19.7} \\
13.4\end{array}$ & $\begin{array}{r}60 \\
0 \\
0 \\
3.8 \\
26.5 \\
13.2\end{array}$ & $\begin{array}{r}\frac{65}{\underline{1.3}} \\
\underline{0} \\
\underline{2.3} \\
\underline{24.8} \\
\underline{0.6}\end{array}$ & $\begin{array}{r}\frac{66}{0.2} \\
10.7 \\
11.9 \\
21.9 \\
0.8\end{array}$ & & & & $\begin{array}{r}\frac{4}{0.2} \\
21.3 \\
10.6 \\
12.4\end{array}$ & $\frac{\frac{5}{1.7}}{\frac{1.1}{13.8}}$ & \\
\hline $\begin{array}{l}\text { High } \\
\text { Velocity } \\
\text { Region }\end{array}$ & $\begin{array}{c}\frac{12}{0} \\
0 \\
1.7 \\
0\end{array}$ & $\begin{array}{l}\frac{13}{\underline{0.5}} \\
\underline{0.7}\end{array}$ & $\begin{array}{l}\frac{14}{1.1} \\
\frac{1.0}{3.5} \\
0.7\end{array}$ & $\begin{array}{c}15 \\
0 \\
1.2 \\
7.1 \\
0\end{array}$ & $\begin{array}{r}\frac{26}{1.6} \\
1.1 \\
214.3 \\
244.5 \\
-\end{array}$ & \begin{tabular}{r}
\multicolumn{1}{c}{$\frac{7}{4.4}$} \\
558.8 \\
618.2 \\
8.8 \\
1.8
\end{tabular} & $\begin{array}{r}\frac{30}{1.3} \\
4.4 \\
250.6 \\
6.2 \\
3.3\end{array}$ & $\begin{array}{c}\frac{31}{0.4} \\
0 \\
126.1 \\
4.0 \\
0\end{array}$ & $\frac{\frac{32}{1.3}}{\frac{19.6}{79.0}} \frac{5.0}{\underline{5.0}}$ & $\begin{array}{l}\frac{41}{0.4} \\
\frac{0.4}{6.0}\end{array}$ & $\frac{\frac{42}{0}}{\underline{\underline{5} .3}}$ & $\begin{array}{r}\frac{43}{0} \\
2.1 \\
65.8 \\
18.7 \\
1.9\end{array}$ & $\begin{array}{l}\frac{44}{\frac{5.5}{6.3}} \\
\frac{6.2}{1.2}\end{array}$ & & $\begin{array}{c}\frac{55}{\underline{0}} \\
\underline{0} \underline{0} \\
\underline{1.1} \\
\end{array}$ & & & & & & & $\frac{\frac{2}{0.8}}{\frac{0.8}{6.0}} \frac{3.1}{\underline{3.1}}$ & $\begin{array}{l}\frac{3}{0.4} \\
0.5 \\
9.3 \\
9.6\end{array}$ & \\
\hline $\begin{array}{l}\text { Ring } \\
\text { Core }\end{array}$ & $\begin{array}{l}\frac{10}{0} \\
\underline{0} \\
\frac{0.9}{0}\end{array}$ & $\begin{array}{c}11 \\
0 \\
0 \\
0 \\
0.4\end{array}$ & $\begin{array}{l}\frac{16}{0} \\
0 \\
0.7\end{array}$ & $\begin{array}{l}\frac{17}{\underline{0}} \\
\underline{0.5} \\
\underline{0.6}\end{array}$ & $\frac{\frac{21}{263.0}}{\frac{6.2}{21.8}} \frac{27.5}{\frac{1.7}{1.7}}$ & $\frac{\frac{23}{44.7}}{\frac{10.7}{\frac{30.5}{27.3}}} \frac{1.2}{1}$ & $\begin{array}{c}\frac{24}{1.8} \\
20.0 \\
107.0 \\
80.0 \\
0\end{array}$ & $\begin{array}{r}\frac{28}{10.4} \\
2.3 \\
535.7 \\
0.4 \\
0.5\end{array}$ & & & & & & & $\begin{array}{l}\frac{56}{0.3} \\
\frac{0}{0} \\
\underline{0} \underline{0} \\
\underline{0.3}\end{array}$ & $\begin{array}{c}57 \\
\underline{0} \\
\underline{0} \\
\underline{0} \\
\underline{0} \\
\underline{0.5}\end{array}$ & $\begin{array}{r}58 \\
0 \\
0 \\
0 \\
0 \\
0\end{array}$ & $\begin{array}{r}61 \\
\underline{0} \\
\underline{0} \\
\underline{0} \\
\underline{0} \\
\underline{0}\end{array}$ & $\begin{array}{r}62 \\
0 \\
0 \\
0 \\
0 \\
0\end{array}$ & $\begin{array}{r}\frac{63}{0} \\
\frac{0}{0} \\
\underline{0} \\
\underline{0} \\
\underline{0}\end{array}$ & $\begin{array}{r}64 \\
0 \\
0 \\
0 \\
0 \\
0\end{array}$ & $\begin{array}{l}\frac{1}{\underline{0}} \\
\frac{0.2}{\underline{0}} \\
\underline{0}\end{array}$ & & \\
\hline $\begin{array}{l}\text { Sargasso } \\
\text { Sea } \\
\& \\
\text { Gulf } \\
\text { Stream }\end{array}$ & & & & & & & & & & $\begin{array}{c}\frac{49}{\underline{0}} \\
\underline{0} \\
\underline{0} \\
\underline{0.3}\end{array}$ & $\begin{array}{r}50 \\
0 \\
0 \\
0 \\
0\end{array}$ & $\begin{array}{c}51 \\
0 \\
0 \\
0 \\
0.3\end{array}$ & $\begin{array}{r}52 \\
\underline{0} \\
\underline{0} \\
\underline{0} \\
\underline{0}\end{array}$ & & & & & & & & & & & \\
\hline $\begin{array}{l}\text { Slope } \\
\text { Water }\end{array}$ & $\frac{8}{-}$ & $\frac{9}{12.2}$ & $\frac{18}{3.6}$ & & $\frac{19}{11.4}$ & $\frac{20}{16.7}$ & $\frac{33}{44.1}$ & $\frac{34}{17.0}$ & & $\frac{35}{19.9}$ & $\frac{47}{9.3}$ & $\frac{48}{19.0}$ & $\frac{53}{6.8}$ & $\frac{54}{4.8}$ & $\frac{59}{7.4}$ & $\frac{60}{8.7}$ & $\frac{65}{5.8}$ & $\frac{66}{8.1}$ & & & & $\frac{4}{11.1}$ & $\frac{5}{6.9}$ & $\frac{6}{9.6}$ \\
\hline $\begin{array}{l}\text { High } \\
\text { Velocity } \\
\text { Region }\end{array}$ & $\frac{12}{0.4}$ & $\frac{13}{0.5}$ & $\frac{14}{1.6}$ & $\frac{15}{2.1}$ & $\frac{27}{210.8}$ & $\frac{29}{49.0}$ & $\frac{30}{52.7}$ & $\frac{31}{26.8}$ & $\frac{32}{-}$ & $\frac{41}{4.1}$ & $\frac{42}{5.5}$ & $\frac{43}{17.7}$ & $\frac{44}{4.8}$ & & $\frac{55}{0.2}$ & & & & & & & $\frac{2}{2.7}$ & $\frac{3}{5.0}$ & \\
\hline $\begin{array}{l}\text { Ring } \\
\text { Core }\end{array}$ & $\frac{10}{0.2}$ & $\frac{11}{0.1}$ & $\frac{16}{-}$ & $\frac{17}{0.4}$ & $\frac{21}{26.5}$ & $\frac{23}{21.2}$ & $\frac{24}{38.5}$ & $\frac{28}{134.6}$ & & $\frac{37}{1.2}$ & $\frac{38}{13.3}$ & $\frac{39}{1.2}$ & & & $\frac{56}{0.1}$ & $\frac{57}{0.1}$ & $\frac{58}{0}$ & $\frac{61}{0}$ & $\frac{62}{0}$ & $\frac{63}{0}$ & $\frac{64}{0}$ & $\frac{1}{0.1}$ & & \\
\hline SS \& GS & & & & & & & & & & $\frac{49}{0.1}$ & $\frac{50}{0}$ & $\frac{51}{0.1}$ & $\frac{52}{0}$ & & & & & & & & & & & \\
\hline
\end{tabular}


Table 36. Volume of Benthosema glaciale $\left(\mathrm{ml} / 10,000 \mathrm{~m}^{3}\right)$.

\begin{tabular}{|c|c|c|c|c|c|c|c|c|c|c|c|c|c|c|c|c|c|c|c|c|c|c|c|c|}
\hline & \multicolumn{4}{|c|}{$\begin{array}{c}\text { Oceanus } 118 \\
\text { April }\end{array}$} & \multicolumn{5}{|c|}{$\begin{array}{c}\text { Oceanus } 121 \\
\text { June }\end{array}$} & \multicolumn{5}{|c|}{$\begin{array}{c}\text { Oceanus } 125 \\
\text { August }\end{array}$} & \multicolumn{7}{|c|}{$\begin{array}{l}\text { Knorr } 98 \\
\text { Sept-0ct }\end{array}$} & \multicolumn{3}{|c|}{$\begin{array}{c}\text { A2 } 110 \\
\text { Sept-0ct }\end{array}$} \\
\hline $\begin{array}{l}\text { Slope } \\
\text { Water }\end{array}$ & $\begin{array}{l}\frac{8}{=} \\
\frac{2.94}{5.91} \\
\frac{0}{0}\end{array}$ & $\begin{array}{c}\frac{9}{0} \\
36.47 \\
15.71 \\
0\end{array}$ & & & $\begin{array}{l}\frac{19}{0.73} \\
\frac{0.07}{0.07} \\
\frac{0.05}{1.05} \\
\underline{3.68}\end{array}$ & $\begin{array}{l}\frac{20}{0.95} \\
1.05 \\
5.65 \\
5.91 \\
1.80\end{array}$ & $\frac{\frac{33}{14.33}}{\frac{0.04}{1.39}} \frac{\frac{3.89}{6.17}}{\underline{6.1}}$ & $\frac{\frac{34}{1.59}}{\frac{0.04}{9.55}}$ & & $\begin{array}{l}\frac{35}{0} \\
0.85 \\
5.67 \\
4.44 \\
0\end{array}$ & 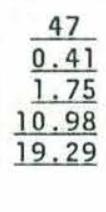 & $\begin{array}{c}\frac{48}{0} \\
4.86 \\
22.65 \\
27.55\end{array}$ & $\begin{array}{l}\frac{53}{0.85} \\
\frac{0.78}{3.85} \\
\underline{0.02}\end{array}$ & $\begin{array}{c}\frac{54}{0} \\
1.63 \\
10.90 \\
11.00\end{array}$ & $\begin{array}{l}\frac{59}{\underline{0}} \\
\underline{0} \\
\frac{2.65}{25.86} \\
21.14 \\
\end{array}$ & $\begin{array}{c}\frac{60}{0} \\
0 \\
3.86 \\
34.78 \\
22.06\end{array}$ & $\begin{array}{l}\frac{65}{0.39} \\
\underline{0} \\
\underline{0.09} \\
\underline{23.20} \\
\underline{0.60}\end{array}$ & $\begin{array}{r}\frac{66}{0.25} \\
0.50 \\
1.19 \\
14.76 \\
1.35\end{array}$ & & & & $\begin{array}{l}\frac{4}{0.02} \\
2.28 \\
5.63 \\
12.65\end{array}$ & $\frac{\frac{5}{0.77}}{\frac{0.67}{12.50}} \frac{12.95}{17.9}$ & \\
\hline $\begin{array}{l}\text { High } \\
\text { Velocity } \\
\text { Region }\end{array}$ & $\begin{array}{l}\frac{12}{0} \\
0 \\
1.54 \\
0\end{array}$ & $\begin{array}{l}\frac{13}{0.45} \\
\underline{0} \\
\underline{1.22}\end{array}$ & $\frac{\frac{14}{1.77}}{\frac{1.67}{4.41}} \frac{0.07}{0}$ & $\begin{array}{l}\frac{15}{0} \\
0.06 \\
6.47 \\
0\end{array}$ & $\begin{array}{c}\frac{26}{0.03} \\
0.05 \\
6.67 \\
10.00 \\
-\end{array}$ & $\begin{array}{r}\frac{27}{3.97} \\
6.18 \\
30.45 \\
24.17 \\
0.04\end{array}$ & $\begin{array}{l}\frac{30}{0.03} \\
0.56 \\
8.13 \\
6.49 \\
5.58\end{array}$ & $\begin{array}{c}\frac{31}{0.02} \\
0 \\
6.61 \\
4.00 \\
0\end{array}$ & $\frac{\frac{32}{0.03}}{\frac{0.48}{10.00}} \frac{11.30}{\underline{11.30}}$ & $\begin{array}{r}\frac{41}{\frac{0.01}{9.43}} \\
\underline{\frac{12.77}{2.73}}\end{array}$ & $\begin{array}{r}\frac{42}{0} \\
\frac{0.74}{17.56} \\
10.00 \\
\end{array}$ & \begin{tabular}{r}
\multicolumn{1}{c}{$\frac{43}{0}$} \\
0.08 \\
4.17 \\
21.30 \\
2.86
\end{tabular} & $\frac{\frac{44}{0.28}}{\frac{0.33}{2.31}}$ & & $\begin{array}{l}\frac{55}{\underline{0}} \\
\underline{0} \\
\underline{0} \\
1.77\end{array}$ & & & & & & $\underline{2.81}$ & $\frac{\frac{2}{0.10}}{\frac{0.08}{1.87}} \frac{9.04}{9.04}$ & $\begin{array}{l}\frac{3}{0.04} \\
0.05 \\
7.14\end{array}$ & \\
\hline $\begin{array}{l}\text { Ring } \\
\text { Core }\end{array}$ & $\begin{array}{l}\frac{10}{\underline{0}} \\
\underline{0} \\
\frac{0.64}{\underline{0}}\end{array}$ & $\begin{array}{l}\frac{11}{0} \\
0 \\
0 \\
1.36\end{array}$ & $\begin{array}{l}\frac{16}{0} \\
0 \\
0.53\end{array}$ & $\begin{array}{l}\frac{17}{\underline{0}} \\
\frac{0.05}{1.12} \\
\end{array}$ & $\frac{\frac{21}{10.00}}{\frac{0.20}{0.45}} \frac{0.63}{0.06}$ & $\begin{array}{l}\frac{23}{1.35} \\
\frac{0.33}{0.75} \\
\frac{2.27}{1.18} \\
\end{array}$ & $\begin{array}{c}\frac{24}{0.03} \\
0.50 \\
3.24 \\
1.25 \\
0\end{array}$ & $\begin{array}{r}\frac{28}{0.02} \\
0.08 \\
16.61 \\
0.96 \\
0.03\end{array}$ & & & & & & & $\begin{array}{l}\frac{56}{0.03} \\
\frac{0}{0} \\
\underline{0} \\
0.31\end{array}$ & $\begin{array}{l}\frac{57}{0} \\
\underline{0} \\
\underline{0} \\
\underline{0} \\
\underline{0.97}\end{array}$ & $\begin{array}{l}58 \\
0 \\
0 \\
0 \\
0 \\
0\end{array}$ & $\begin{array}{l}\frac{61}{0} \\
\underline{0} \\
\underline{0} \\
\underline{0} \\
\underline{0}\end{array}$ & $\begin{array}{l}62 \\
0 \\
0 \\
0 \\
0 \\
0\end{array}$ & $\begin{array}{l}\frac{63}{0} \\
\frac{0}{0} \\
\underline{0} \\
\underline{0} \\
\underline{0}\end{array}$ & $\begin{array}{l}\frac{64}{0} \\
0 \\
0 \\
0 \\
0\end{array}$ & $\begin{array}{l}\frac{1}{\underline{0}} \\
\frac{0.50}{\underline{0}} \\
\underline{0}\end{array}$ & & \\
\hline $\begin{array}{l}\text { Sargasso } \\
\text { Sea } \\
\& \\
\text { Gulf } \\
\text { Stream }\end{array}$ & & & & & & & & & & $\begin{array}{l}\frac{49}{0} \\
\underline{0} \\
\underline{0} \\
\underline{0.13}\end{array}$ & $\begin{array}{l}50 \\
0 \\
0 \\
0 \\
0\end{array}$ & $\begin{array}{l}\frac{51}{0} \\
0 \\
0 \\
0.17\end{array}$ & $\begin{array}{l}\frac{52}{0} \\
\underline{0} \\
\underline{0} \\
\underline{0}\end{array}$ & & & & & & & . & & & & \\
\hline $\begin{array}{l}\text { Slope } \\
\text { Water }\end{array}$ & $\frac{8}{-}$ & $\frac{9}{13.05}$ & $\frac{18}{1.84}$ & & $\frac{19}{1.28}$ & $\frac{20}{3.39}$ & $\frac{33}{4.62}$ & $\frac{34}{5.87}$ & & $\frac{35}{2.19}$ & $\frac{47}{8.11}$ & $\frac{48}{13.77}$ & $\frac{53}{1.63}$ & $\frac{54}{5.88}$ & $\frac{59}{9.93}$ & $\frac{60}{12.14}$ & $\frac{65}{4.57}$ & $\frac{66}{3.59}$ & & & & $\frac{4}{5.15}$ & $\frac{5}{8.22}$ & $\frac{6}{9.37}$ \\
\hline $\begin{array}{l}\text { High } \\
\text { Velocity } \\
\text { Region }\end{array}$ & $\frac{12}{0.39}$ & $\frac{13}{0.72}$ & $\frac{14}{1.98}$ & $\frac{15}{1.63}$ & $\frac{27}{13.67}$ & $\frac{29}{2.97}$ & $\frac{30}{4.11}$ & $\frac{31}{2.13}$ & $\frac{32}{-}$ & $\frac{41}{5.56}$ & $\frac{42}{5.67}$ & $\frac{43}{5.68}$ & $\frac{44}{2.50}$ & & $\frac{55}{0.35}$ & & & & & & & $\frac{2}{1.22}$ & $\frac{3}{4.07}$ & \\
\hline $\begin{array}{l}\text { Ring } \\
\text { Core }\end{array}$ & $\frac{10}{0.16}$ & $\frac{11}{0.34}$ & $\frac{16}{-}$ & $\frac{17}{0.57}$ & $\frac{21}{0.80}$ & $\frac{23}{1.13}$ & $\frac{24}{0.92}$ & $\frac{28}{4.41}$ & & $\frac{37}{0.96}$ & $\frac{38}{4.02}$ & $\frac{39}{1.35}$ & & & $\frac{56}{0.07}$ & $\frac{57}{0.19}$ & $\frac{58}{0}$ & $\frac{61}{0}$ & $\frac{62}{0}$ & $\frac{63}{0}$ & $\frac{64}{0}$ & $\frac{1}{0.13}$ & & \\
\hline SS \& GS & & & & & & & & & & $\frac{49}{0.03}$ & $\frac{50}{0}$ & $\frac{51}{0.04}$ & $\frac{52}{0}$ & & & & & & & & & & & \\
\hline
\end{tabular}


Table 37. Abundance of Benthosema suborbitale (specimens $/ 10,000 \mathrm{~m}^{3}$ ).

\begin{tabular}{|c|c|c|c|c|c|c|c|c|c|c|c|c|c|c|c|c|c|c|c|c|c|c|c|c|}
\hline & \multicolumn{4}{|c|}{$\begin{array}{c}\text { Oceanus } \\
\text { Apri1 }\end{array}$} & \multicolumn{5}{|c|}{$\begin{array}{c}\text { Oceanus } 121 \\
\text { June }\end{array}$} & \multicolumn{5}{|c|}{$\begin{array}{l}\text { Oceanus } 125 \\
\text { Auqust }\end{array}$} & \multicolumn{7}{|c|}{$\begin{array}{l}\text { Knorr } 98 \\
\text { Sept-0ct }\end{array}$} & \multicolumn{3}{|c|}{$\begin{array}{c}\text { A2 } 110 \\
\text { Sept-Oct }\end{array}$} \\
\hline $\begin{array}{l}\text { Slope } \\
\text { Water }\end{array}$ & $\begin{array}{l}\frac{8}{\overline{0}} \\
\underline{0} \\
\underline{0} \\
\underline{0}\end{array}$ & $\begin{array}{l}\frac{9}{0} \\
0.6 \\
0 \\
0\end{array}$ & & & $\frac{\frac{19}{1.0}}{\frac{0}{0}} \frac{\underline{0}}{\underline{0}}$ & $\begin{array}{c}20 \\
0 \\
0.5 \\
0 \\
0 \\
0\end{array}$ & $\frac{33}{\frac{1.0}{\underline{0}}}$ & $\frac{34}{\frac{1.4}{\frac{0}{0}}}$ & & $\begin{array}{c}35 \\
0 \\
0 \\
0.7 \\
0 \\
0\end{array}$ & $\begin{array}{l}\frac{47}{0.4} \\
\frac{0}{0} \\
\underline{0} \\
\underline{0}\end{array}$ & $\begin{array}{c}48 \\
0 \\
0.9 \\
0 \\
0\end{array}$ & $\frac{53}{\frac{0.4}{\underline{0}}}$ & $\begin{array}{r}54 \\
0 \\
0 \\
0 \\
0\end{array}$ & $\begin{array}{l}5 \underline{5} \\
\underline{0} \\
\underline{0} \\
\underline{0} \\
\underline{0} \\
\underline{0}\end{array}$ & $\begin{array}{c}60 \\
0 \\
0 \\
2.1 \\
0 \\
0\end{array}$ & $\frac{65}{\frac{1.7}{\underline{0}}}$ & $\begin{array}{c}\frac{66}{0} \\
0 \\
1.5 \\
0 \\
0\end{array}$ & & & & $\begin{array}{l}4 \\
0 \\
0 \\
0 \\
0\end{array}$ & $\frac{\frac{5}{0.7}}{\frac{0}{0}} \frac{0}{\underline{0}}$ & \\
\hline $\begin{array}{l}\text { High } \\
\text { Velocity } \\
\text { Region }\end{array}$ & $\begin{array}{c}\frac{12}{0} \\
0.8 \\
0 \\
0\end{array}$ & $\frac{\frac{13}{1.0}}{\frac{0}{0}}$ & $\frac{\frac{14}{0.9}}{\frac{0.7}{\frac{0}{0}}}$ & $\begin{array}{c}15 \\
0 \\
0.6 \\
0 \\
0\end{array}$ & $\begin{array}{c}26 \\
0 \\
0 \\
0 \\
0 \\
-\end{array}$ & $\begin{array}{c}27 \\
0 \\
0 \\
0 \\
0 \\
0\end{array}$ & $\begin{array}{r}30 \\
0 \\
0 \\
0 \\
0 \\
0\end{array}$ & $\begin{array}{r}31 \\
0 \\
0 \\
0 \\
0 \\
0\end{array}$ & $\begin{array}{r}\frac{32}{\underline{0}} \\
\underline{0} \\
\underline{0} \\
\underline{0}\end{array}$ & $\frac{41}{\frac{0.4}{\underline{0}}}$ & $\frac{42}{\frac{0.1}{\frac{0}{0}}}$ & $\begin{array}{c}\frac{43}{0.6} \\
0 \\
0 \\
0 \\
0\end{array}$ & $\frac{44}{\frac{0.1}{\underline{0}}}$ & & $\frac{55}{\frac{1.6}{\underline{0}}}$ & & & & & & & $\begin{array}{l}\frac{2}{\underline{0}} \\
\underline{0} \\
\underline{0} \\
\underline{0}\end{array}$ & $\begin{array}{c}\frac{3}{0} \\
5.1 \\
0 \\
0\end{array}$ & \\
\hline $\begin{array}{l}\text { Ring } \\
\text { Core }\end{array}$ & $\frac{10}{\frac{0.8}{\frac{0}{0}}}$ & $\begin{array}{c}11 \\
0 \\
1.1 \\
0 \\
0\end{array}$ & $\begin{array}{c}16 \\
0 \\
0.7 \\
0\end{array}$ & $\frac{17}{\frac{0.6}{0}}$ & $\begin{array}{r}21 \\
\underline{0} \\
\underline{0} \\
\underline{0} \\
\underline{0} \\
\underline{0}\end{array}$ & $\begin{array}{l}\frac{23}{\underline{0}} \\
\underline{0.7} \\
\underline{0 .} \\
\underline{0.5}\end{array}$ & $\begin{array}{r}24 \\
0 \\
0 \\
0 \\
0 \\
0\end{array}$ & $\begin{array}{r}28 \\
0 \\
0 \\
0 \\
0 \\
0\end{array}$ & & & & & & & $\frac{56}{\frac{2.6}{\underline{0}}}$ & $\begin{array}{l}\frac{57}{\frac{0.9}{0}} \\
\underline{0} \\
\frac{0.6}{\underline{0}}\end{array}$ & $\begin{array}{c}58 \\
0 \\
0 \\
1.4 \\
0 \\
0\end{array}$ & $\frac{61}{\frac{1.5}{\underline{0}}}$ & $\begin{array}{c}62 \\
0 \\
0 \\
0.6 \\
0.3 \\
0\end{array}$ & $\begin{array}{r}63 \\
\underline{0} \\
\underline{0} \\
\underline{0} \\
\underline{0} \\
\underline{0}\end{array}$ & $\begin{array}{r}64 \\
0 \\
0 \\
0 \\
0 \\
0\end{array}$ & $\begin{array}{l}\frac{1}{\underline{0}} \\
\underline{0} \\
\underline{0.3} \\
\underline{0}\end{array}$ & & \\
\hline $\begin{array}{l}\text { Sargasso } \\
\text { Sea } \\
\& \\
\text { Gulf } \\
\text { Stream }\end{array}$ & & & & & & & & & & $\begin{array}{l}\frac{49}{\frac{0.3}{0}} \\
\frac{0.2}{\underline{0}}\end{array}$ & $\begin{array}{c}50 \\
0 \\
0 \\
0 \\
0\end{array}$ & $\begin{array}{r}51 \\
0 \\
0 \\
0 \\
0\end{array}$ & $\begin{array}{l}52 \\
\frac{0}{0} \\
\underline{0} \\
\underline{0}\end{array}$ & & & & & & & & & & & \\
\hline $\begin{array}{l}\text { Slope } \\
\text { Water }\end{array}$ & $\frac{8}{=}$ & $\frac{9}{0.2}$ & $\frac{18}{0}$ & & $\frac{19}{0.1}$ & $\frac{20}{0.1}$ & $\frac{33}{0.1}$ & $\frac{34}{0.1}$ & & $\frac{35}{0.1}$ & $\frac{47}{0.1}$ & $\frac{48}{0.2}$ & $\frac{53}{0.1}$ & $\frac{54}{0}$ & $\frac{59}{0}$ & $\frac{60}{0.4}$ & $\frac{65}{0.3}$ & $\frac{66}{0.3}$ & & & & $\frac{4}{0}$ & $\frac{5}{0.2}$ & $\frac{6}{0}$ \\
\hline $\begin{array}{l}\text { High } \\
\text { Velocity } \\
\text { Region }\end{array}$ & $\frac{12}{0.2}$ & $\frac{13}{0.3}$ & $\frac{14}{0.4}$ & $\frac{15}{0.2}$ & $\frac{27}{0}$ & $\frac{29}{0}$ & $\frac{30}{0}$ & $\frac{31}{0}$ & $\frac{32}{-}$ & $\frac{41}{0.2}$ & $\frac{42}{0.1}$ & $\frac{43}{0.1}$ & $\frac{44}{0.1}$ & & $\frac{55}{0.3}$ & & & & & & & $\frac{2}{0}$ & $\frac{3}{1.3}$ & \\
\hline $\begin{array}{l}\text { Ring } \\
\text { Core }\end{array}$ & $\frac{10}{0.2}$ & $\frac{11}{0.3}$ & $\frac{16}{-}$ & $\frac{17}{0.2}$ & $\frac{21}{0}$ & $\frac{23}{0.3}$ & $\frac{24}{0}$ & $\frac{28}{0}$ & & $\frac{37}{0}$ & $\frac{38}{0}$ & $\frac{39}{0}$ & & & $\frac{56}{0.5}$ & $\frac{57}{0.3}$ & $\frac{58}{0.3}$ & $\frac{61}{0.3}$ & $\frac{62}{0.2}$ & $\frac{63}{0}$ & $\frac{64}{0}$ & $\frac{1}{0.1}$ & & \\
\hline SS \& GG & & & & & & & & & & $\frac{49}{0.1}$ & $\frac{50}{0}$ & $\frac{51}{0}$ & $\frac{52}{0}$ & & & & & & & & & & & \\
\hline
\end{tabular}


Table 38. Abundance of Bolinichthys indicus (specimens $/ 10,000 \mathrm{~m}^{3}$ ).

\begin{tabular}{|c|c|c|c|c|c|c|c|c|c|c|c|c|c|c|c|c|c|c|c|c|c|c|c|c|}
\hline & \multicolumn{4}{|c|}{$\begin{array}{c}\text { Oceanus } \\
\text { Apri1 }\end{array}$} & \multicolumn{5}{|c|}{$\begin{array}{c}\text { Oceanus } 121 \\
\text { June }\end{array}$} & \multicolumn{5}{|c|}{$\begin{array}{l}\text { Oceanus } 125 \\
\text { August }\end{array}$} & \multicolumn{7}{|c|}{$\begin{array}{l}\text { Knorr } 98 \\
\text { Sept-0ct }\end{array}$} & \multicolumn{3}{|c|}{$\begin{array}{c}\text { A2 } 110 \\
\text { Sept-0ct }\end{array}$} \\
\hline $\begin{array}{l}\text { Slope } \\
\text { Water }\end{array}$ & $\begin{array}{l}\frac{8}{\overline{0}} \\
\underline{0} \\
\underline{0} \\
\underline{0}\end{array}$ & $\begin{array}{c}9 \\
0 \\
1.2 \\
0 \\
0\end{array}$ & & & $\begin{array}{r}\frac{19}{\underline{0}} \\
\underline{0} \\
\underline{0} \\
\underline{0} \\
\underline{0}\end{array}$ & $\begin{array}{r}20 \\
0 \\
0 \\
0 \\
0 \\
0\end{array}$ & $\begin{array}{r}\frac{33}{\underline{0}} \\
\underline{0} \\
\underline{0} \\
\underline{0} \\
\underline{0}\end{array}$ & $\frac{34}{\frac{0.9}{\underline{0}}}$ & & $\begin{array}{r}35 \\
0 \\
0 \\
0 \\
0 \\
0\end{array}$ & $\begin{array}{r}47 \\
\underline{0} \\
\underline{0} \\
\underline{0} \\
\underline{0}\end{array}$ & $\begin{array}{r}48 \\
0 \\
0 \\
0 \\
0\end{array}$ & $\begin{array}{r}53 \\
\underline{0} \\
\underline{0} \\
\underline{0} \\
\underline{0}\end{array}$ & $\begin{array}{r}54 \\
0 \\
0 \\
0 \\
0\end{array}$ & $\begin{array}{l}\frac{59}{2.0} \\
\underline{0} \\
\underline{0} \\
\underline{0} \\
\underline{0}\end{array}$ & $\begin{array}{c}60 \\
0 \\
0 \\
2.4 \\
0 \\
0\end{array}$ & $\begin{array}{l}\frac{65}{0.9} \\
\frac{0}{0} \\
\underline{0} \\
\underline{0}\end{array}$ & $\begin{array}{c}66 \\
0 \\
0 \\
1.1 \\
0 \\
0\end{array}$ & & & & $\begin{array}{c}\frac{4}{0} \\
2.0 \\
0 \\
0\end{array}$ & $\begin{array}{l}\frac{5}{0} \\
\underline{0} \\
\underline{0} \\
\underline{0}\end{array}$ & \\
\hline $\begin{array}{l}\text { High } \\
\text { Velocity } \\
\text { Region }\end{array}$ & $\begin{array}{c}12 \\
0 \\
0 \\
0.8 \\
0\end{array}$ & $\frac{\frac{13}{1.5}}{\frac{0}{0}}$ & $\frac{14}{\frac{1.7}{\underline{0}}}$ & $\begin{array}{c}15 \\
0.6 \\
1.2 \\
0 \\
0\end{array}$ & $\begin{array}{c}26 \\
0 \\
0 \\
0 \\
0 \\
-\end{array}$ & $\begin{array}{c}27 \\
0 \\
0 \\
0.9 \\
0 \\
0\end{array}$ & $\begin{array}{c}30 \\
0 \\
0 \\
0.3 \\
0.5 \\
0\end{array}$ & $\begin{array}{c}31 \\
0 \\
0 \\
0 \\
0.3 \\
0\end{array}$ & $\begin{array}{r}32 \\
\underline{0} \\
\underline{0} \\
\underline{0} \\
\underline{0}\end{array}$ & $\frac{\frac{41}{0.6}}{\frac{0}{0}}$ & $\frac{\frac{42}{0.1}}{\frac{0}{\underline{0}}}$ & $\begin{array}{r}43 \\
0 \\
0 \\
0 \\
0 \\
0\end{array}$ & $\frac{\frac{44}{1.0}}{\frac{0}{0}}$ & & $\frac{55}{\frac{4.5}{\underline{0}}}$ & & & & & & & $\frac{\frac{2}{0.4}}{\frac{0}{0}}$ & $\begin{array}{c}\frac{3}{0} \\
2.7 \\
0 \\
0\end{array}$ & \\
\hline $\begin{array}{l}\text { Ring } \\
\text { Core }\end{array}$ & $\frac{10}{\frac{0.8}{\underline{0}}} \frac{\underline{0}}{\underline{0}}$ & $\begin{array}{r}11 \\
0 \\
0 \\
0 \\
0\end{array}$ & $\begin{array}{c}\frac{16}{0} \\
0.7 \\
10.7\end{array}$ & $\frac{\frac{17}{6.1}}{\frac{6.6}{0.1}}$ & $\begin{array}{l}\frac{21}{1.0} \\
\frac{0.8}{\underline{0}} \\
\underline{0} \\
\underline{0}\end{array}$ & $\begin{array}{l}\frac{23}{\underline{5.3}} \\
\underline{0} \\
\underline{0} \\
\underline{0} \\
\underline{0}\end{array}$ & $\begin{array}{c}24 \\
0 \\
0 \\
1.4 \\
0.6 \\
0\end{array}$ & $\begin{array}{c}28 \\
0 \\
0 \\
3.6 \\
0 \\
0\end{array}$ & & & & & & & $\begin{array}{c}\frac{56}{7.6} \\
\frac{0}{0} \\
\underline{0} \\
\underline{0} \\
\underline{0}\end{array}$ & $\begin{array}{c}\frac{57}{16.9} \\
\frac{0.3}{\underline{0}} \\
\underline{0}\end{array}$ & $\begin{array}{c}58 \\
0 \\
0 \\
1.4 \\
9.4 \\
0.3\end{array}$ & $\begin{array}{c}\frac{61}{19.5} \\
\frac{0}{0.4} \\
\frac{1.2}{\underline{0}}\end{array}$ & $\begin{array}{c}62 \\
0 \\
0 \\
0 \\
9.7 \\
0\end{array}$ & $\begin{array}{c}\frac{63}{2.1} \\
\frac{0}{0} \\
\underline{0} \\
\underline{0.4} \\
\underline{0}\end{array}$ & $\begin{array}{c}64 \\
0 \\
0 \\
5.0 \\
3.8 \\
0\end{array}$ & $\frac{\frac{1}{6.0}}{\frac{0}{0}}$ & & \\
\hline $\begin{array}{l}\text { Sargasso } \\
\text { Sea } \\
\& \\
\text { Gulf } \\
\text { Stream }\end{array}$ & & & & & & & & & & $\frac{49}{\frac{3.1}{\underline{0}}}$ & $\begin{array}{c}50 \\
0 \\
0.2 \\
1.0 \\
0\end{array}$ & $\begin{array}{c}51 \\
0 \\
0 \\
4.3 \\
0\end{array}$ & $\frac{\frac{52}{6.6}}{\frac{0}{0}}$ & & & & & & & & & & & \\
\hline $\begin{array}{l}\text { Slope } \\
\text { Water }\end{array}$ & $\frac{8}{-}$ & $\frac{9}{0.3}$ & $\frac{18}{0}$ & & $\frac{19}{0}$ & $\frac{20}{0}$ & $\frac{33}{0}$ & $\frac{34}{0.1}$ & & $\frac{35}{0}$ & $\frac{47}{0}$ & $\frac{48}{0}$ & $\frac{53}{0}$ & $\frac{54}{0}$ & $\frac{59}{0.4}$ & $\frac{60}{0.5}$ & $\frac{65}{0.2}$ & $\frac{66}{0.2}$ & & & & $\frac{4}{0.5}$ & $\frac{5}{0}$ & $\frac{6}{0}$ \\
\hline $\begin{array}{l}\text { High } \\
\text { Velocity } \\
\text { Region }\end{array}$ & $\frac{12}{0.2}$ & $\frac{13}{0.4}$ & $\frac{14}{0.4}$ & $\frac{15}{0.5}$ & $\frac{27}{0.2}$ & $\frac{29}{0.1}$ & $\frac{30}{0.2}$ & $\frac{31}{0.1}$ & $\frac{32}{-}$ & $\frac{41}{0.3}$ & $\frac{42}{0.1}$ & $\frac{43}{0}$ & $\frac{44}{0.6}$ & & $\frac{55}{0.9}$ & & & & & & & $\frac{2}{0.1}$ & $\frac{3}{0.7}$ & \\
\hline $\begin{array}{l}\text { Ring } \\
\text { Core }\end{array}$ & $\frac{10}{0.2}$ & $\frac{11}{0}$ & $\frac{16}{-}$ & $\frac{17}{2.0}$ & $\frac{21}{0.3}$ & $\frac{23}{0.8}$ & $\frac{24}{0.4}$ & $\frac{28}{0.9}$ & & $\frac{37}{0.2}$ & $\frac{38}{0}$ & $\frac{39}{0}$ & & & $\frac{56}{1.5}$ & $\frac{57}{3.4}$ & $\frac{58}{2.2}$ & $\frac{61}{4.2}$ & $\frac{62}{1.9}$ & $\frac{63}{0.9}$ & $\frac{64}{1.3}$ & $\frac{1}{1.5}$ & & \\
\hline SS \& GS & & & & & & & & & & $\frac{49}{0.8}$ & $\frac{50}{0.3}$ & $\frac{51}{1.1}$ & $\frac{52}{1.7}$ & & & & & & & & & & & \\
\hline
\end{tabular}


Table 39. Volume of Bolinichthys indicus $\left(\mathrm{ml} / 10,000 \mathrm{~m}^{3}\right)$.

\begin{tabular}{|c|c|c|c|c|c|c|c|c|c|c|c|c|c|c|c|c|c|c|c|c|c|c|c|c|}
\hline & \multicolumn{4}{|c|}{$\underset{\substack{\text { Oceanus } \\
\text { Apri1 }}}{118}$} & \multicolumn{5}{|c|}{$\begin{array}{c}\text { Oceanus } 121 \\
\text { June }\end{array}$} & \multicolumn{5}{|c|}{$\begin{array}{l}\text { Oceanus } 125 \\
\text { Auqust }\end{array}$} & \multicolumn{7}{|c|}{$\begin{array}{l}\text { Knorr } 98 \\
\text { Sept-Oct }\end{array}$} & \multicolumn{3}{|c|}{$\begin{array}{c}\text { A2 } 110 \\
\text { Sept-0ct }\end{array}$} \\
\hline $\begin{array}{l}\text { Slope } \\
\text { Water }\end{array}$ & $\begin{array}{l}\frac{8}{\overline{\overline{0}}} \\
\underline{0} \\
\underline{0} \\
\underline{0}\end{array}$ & $\begin{array}{l}\frac{9}{0} \\
0.12 \\
0 \\
0\end{array}$ & & & $\frac{\frac{19}{0.02}}{\frac{0}{\frac{0}{0}}}$ & $\begin{array}{l}\frac{20}{0} \\
0 \\
0 \\
0 \\
0\end{array}$ & $\frac{\frac{33}{0.03}}{\frac{0}{\frac{0}{0}}}$ & $\frac{34}{\underline{0}}$ & & $\begin{array}{l}35 \\
0 \\
0 \\
0 \\
0 \\
0\end{array}$ & $\begin{array}{l}47 \\
\underline{0} \\
\underline{0} \\
\underline{0} \\
\underline{0}\end{array}$ & $\begin{array}{l}48 \\
0 \\
0 \\
0 \\
0\end{array}$ & $\begin{array}{l}\frac{53}{0} \\
\underline{0} \\
\underline{0} \\
\underline{0}\end{array}$ & $\begin{array}{l}54 \\
0 \\
0 \\
0 \\
0\end{array}$ & $\frac{\frac{59}{0.07}}{\frac{0}{0}}$ & $\begin{array}{l}\frac{60}{0} \\
0 \\
0.14 \\
0 \\
0\end{array}$ & $\begin{array}{l}\frac{65}{0.04} \\
\frac{0}{0} \\
\frac{0}{0} \\
\underline{0}\end{array}$ & $\begin{array}{l}\frac{66}{0} \\
0 \\
0.04 \\
0 \\
0\end{array}$ & & & & $\begin{array}{l}\frac{4}{0} \\
0.09 \\
0 \\
0\end{array}$ & $\begin{array}{l}\frac{5}{\underline{0}} \\
\underline{0} \\
\underline{0} \\
\underline{0}\end{array}$ & \\
\hline $\begin{array}{l}\text { High } \\
\text { Velocity } \\
\text { Region }\end{array}$ & $\begin{array}{l}\frac{12}{0} \\
0 \\
0.13 \\
0\end{array}$ & $\frac{\frac{13}{0.10}}{\frac{0}{0}}$ & $\frac{\frac{14}{0.43}}{\frac{0}{0}}$ & $\begin{array}{l}\frac{15}{0.06} \\
0.35 \\
0 \\
0\end{array}$ & $\begin{array}{l}26 \\
0 \\
0 \\
0 \\
0 \\
-\end{array}$ & $\begin{array}{l}27 \\
0 \\
0 \\
0.41 \\
0 \\
0\end{array}$ & $\begin{array}{l}\frac{30}{0} \\
0 \\
0.09 \\
0.22 \\
0\end{array}$ & $\begin{array}{l}31 \\
0 \\
0 \\
0 \\
0.11 \\
0\end{array}$ & $\begin{array}{l}\frac{32}{\underline{0}} \\
\underline{0} \\
\underline{0} \\
\underline{0}\end{array}$ & $\frac{\frac{41}{0.36}}{\frac{0}{0}}$ & $\frac{\frac{42}{0.06}}{\frac{0}{0}}$ & $\begin{array}{l}43 \\
0 \\
0 \\
0 \\
0 \\
0\end{array}$ & $\frac{\frac{44}{0.46}}{\underline{0}}$ & & $\frac{\frac{55}{0.39}}{\frac{0}{0}}$ & & & & & & & $\frac{\frac{2}{0.02}}{\frac{0}{0}} \frac{\underline{0}}{\underline{0}}$ & $\begin{array}{l}\frac{3}{0} \\
0.08 \\
0 \\
0\end{array}$ & \\
\hline $\begin{array}{l}\text { Ring } \\
\text { Core }\end{array}$ & $\frac{\frac{10}{0.12}}{\frac{0}{\underline{0}}}$ & $\begin{array}{l}11 \\
0 \\
0 \\
0 \\
0\end{array}$ & $\begin{array}{l}\frac{16}{0} \\
0.79 \\
2.33\end{array}$ & $\begin{array}{l}\frac{17}{1.22} \\
\frac{0.42}{0.04} \\
\end{array}$ & $\frac{\frac{21}{0.40}}{\frac{0.44}{\underline{0}}}$ & $\frac{\frac{23}{0.29}}{\frac{0}{0}}$ & $\begin{array}{l}\frac{24}{0} \\
0 \\
0.62 \\
0.25 \\
0\end{array}$ & $\begin{array}{c}\frac{28}{0} \\
0 \\
2.14 \\
0 \\
0\end{array}$ & & & & & & & $\frac{\frac{56}{0.35}}{\frac{0}{0}}$ & $\begin{array}{l}\frac{57}{0.97} \\
\underline{0} \\
\frac{0.03}{\underline{0}} \\
\underline{0}\end{array}$ & $\begin{array}{l}\frac{58}{0} \\
0 \\
0.05 \\
0.77 \\
0.03\end{array}$ & $\begin{array}{l}\frac{61}{0.85} \\
\frac{0.85}{0.04} \\
\frac{0.04}{\underline{0}}\end{array}$ & $\begin{array}{l}\frac{62}{0} \\
0 \\
0 \\
0.43 \\
0\end{array}$ & $\begin{array}{l}\frac{63}{0.14} \\
\frac{0}{0} \\
\underline{0.04} \\
\underline{0}\end{array}$ & $\begin{array}{l}\frac{64}{0} \\
0 \\
0.14 \\
0.43 \\
0\end{array}$ & $\frac{\frac{1}{0.76}}{\frac{0}{\underline{0}}}$ & & \\
\hline $\begin{array}{l}\text { Sargasso } \\
\text { Sea } \\
\& \\
\text { Gulf } \\
\text { Stream }\end{array}$ & & & & & & & & & & $\frac{\frac{49}{0.26}}{\underline{0}} \frac{\underline{0}}{\underline{0}}$ & $\begin{array}{l}\frac{50}{0} \\
0 \\
0 \\
0\end{array}$ & $\begin{array}{l}\frac{51}{0} \\
0 \\
1.63 \\
0\end{array}$ & $\frac{\frac{52}{0.50}}{\underline{0}} \frac{\underline{0}}{\underline{0}}$ & & & & & & & & & & & \\
\hline $\begin{array}{l}\text { Slope } \\
\text { Water }\end{array}$ & $\frac{8}{-}$ & $\frac{9}{0.03}$ & $\frac{18}{0}$ & & $\frac{19}{0.01}$ & $\frac{20}{0}$ & $\frac{33}{0.01}$ & $\frac{34}{0}$ & & $\frac{35}{0}$ & $\frac{47}{0}$ & $\frac{48}{0}$ & $\frac{53}{0}$ & $\frac{54}{0}$ & $\frac{59}{0.01}$ & $\frac{60}{0.03}$ & $\frac{65}{0.01}$ & $\frac{66}{0.01}$ & & & & $\frac{4}{0.02}$ & $\frac{5}{0}$ & $\frac{6}{0}$ \\
\hline $\begin{array}{l}\text { High } \\
\text { Velocity } \\
\text { Region }\end{array}$ & $\frac{12}{0.03}$ & $\frac{13}{0.03}$ & $\frac{14}{0.11}$ & $\frac{15}{0.09}$ & $\frac{27}{0.08}$ & $\frac{29}{0.02}$ & $\frac{30}{0.06}$ & $\frac{31}{0.02}$ & $\frac{32}{-}$ & $\frac{41}{0.18}$ & $\frac{42}{0.02}$ & $\frac{43}{0}$ & $\frac{44}{0.28}$ & & $\frac{55}{0.08}$ & & & & & & & $\frac{2}{0.01}$ & $\frac{3}{0.02}$ & \\
\hline $\begin{array}{l}\text { Ring } \\
\text { Core }\end{array}$ & $\frac{10}{0.03}$ & $\frac{11}{0}$ & $\frac{16}{-}$ & $\frac{17}{0.43}$ & $\frac{21}{0.17}$ & $\frac{23}{0.04}$ & $\frac{24}{0.17}$ & $\frac{28}{0.54}$ & & $\frac{37}{0.06}$ & $\frac{38}{0}$ & $\frac{39}{0}$ & & & $\frac{56}{0.07}$ & $\frac{57}{0.20}$ & $\frac{58}{0.17}$ & $\frac{61}{0.19}$ & $\frac{62}{0.09}$ & $\frac{63}{0.06}$ & $\frac{64}{0.10}$ & $\frac{1}{0.19}$ & & \\
\hline SS \& GS & & & & & & & & & & $\frac{49}{0.07}$ & $\frac{50}{0}$ & $\frac{51}{0.41}$ & $\frac{52}{0.13}$ & & & & & & & & & & & \\
\hline
\end{tabular}


Table 40. Abundance of Bolinichthys supralateralis (specimens $/ 10,000 \mathrm{~m}^{3}$ ).

\begin{tabular}{|c|c|c|c|c|c|c|c|c|c|c|c|c|c|c|c|c|c|c|c|c|c|c|c|c|}
\hline & \multicolumn{4}{|c|}{$\begin{array}{c}\text { Oceanus } 118 \\
\text { April }\end{array}$} & \multicolumn{5}{|c|}{$\begin{array}{c}\text { Oceanus } 121 \\
\text { June }\end{array}$} & \multicolumn{5}{|c|}{$\begin{array}{l}\text { Oceanus } 125 \\
\text { August }\end{array}$} & \multicolumn{7}{|c|}{$\begin{array}{l}\text { Knorr } 98 \\
\text { Sept-Oct }\end{array}$} & \multicolumn{3}{|c|}{$\begin{array}{c}\text { A2 } 110 \\
\text { Sept-Oct }\end{array}$} \\
\hline $\begin{array}{l}\text { Slope } \\
\text { Water }\end{array}$ & $\begin{array}{l}\frac{8}{\overline{0}} \\
\underline{0} \\
\underline{0} \\
\underline{0}\end{array}$ & $\begin{array}{l}9 \\
0 \\
0 \\
0 \\
0\end{array}$ & & & $\begin{array}{r}19 \\
\underline{0} \\
\underline{0} \\
\underline{0} \\
\underline{0} \\
\underline{0}\end{array}$ & $\begin{array}{r}20 \\
0 \\
0 \\
0 \\
0 \\
0\end{array}$ & $\begin{array}{c}\frac{33}{\underline{0}} \\
\underline{0} \\
\underline{0} \\
\underline{0} \\
\underline{0}\end{array}$ & $\begin{array}{l}\frac{34}{\underline{0}} \\
\underline{0} \\
\underline{0}\end{array}$ & & $\begin{array}{r}35 \\
0 \\
0 \\
0 \\
0 \\
0\end{array}$ & $\begin{array}{l}47 \\
\underline{0} \\
\underline{0} \\
\underline{0}\end{array}$ & $\begin{array}{r}48 \\
0 \\
0 \\
0 \\
0\end{array}$ & $\begin{array}{l}\frac{53}{\underline{0}} \\
\underline{0} \\
\underline{0.5}\end{array}$ & $\begin{array}{c}54 \\
0 \\
0 \\
0.3 \\
0\end{array}$ & $\frac{\frac{59}{0.3}}{\frac{0.2}{1.2}} \frac{\underline{0}}{\underline{0}} \underline{\underline{0}}$ & $\begin{array}{c}60 \\
0 \\
0 \\
\frac{1.4}{0} \\
0\end{array}$ & $\begin{array}{r}65 \\
\underline{0} \\
\underline{0} \\
\underline{0} \\
\underline{0} \\
\underline{0}\end{array}$ & $\begin{array}{c}66 \\
0 \\
0 \\
0.7 \\
0 \\
0\end{array}$ & & & & $\begin{array}{l}4 \\
0 \\
0 \\
0 \\
0\end{array}$ & $\begin{array}{l}\frac{5}{\underline{0}} \\
\underline{0} \\
\underline{0} \\
\underline{0}\end{array}$ & \\
\hline $\begin{array}{l}\text { High } \\
\text { Velocity } \\
\text { Region }\end{array}$ & $\begin{array}{r}12 \\
0 \\
0 \\
0 \\
0\end{array}$ & $\frac{13}{\underline{0}}$ & $\begin{array}{l}\frac{14}{\underline{0}} \\
\frac{0.3}{\underline{0}} \\
\underline{0}\end{array}$ & $\begin{array}{r}15 \\
0 \\
0 \\
0 \\
0\end{array}$ & $\begin{array}{c}26 \\
0 \\
0 \\
0 \\
0 \\
-\end{array}$ & $\begin{array}{c}27 \\
0 \\
0 \\
0 \\
0 \\
0\end{array}$ & $\begin{array}{r}30 \\
0 \\
0 \\
0 \\
0 \\
0\end{array}$ & $\begin{array}{r}31 \\
0 \\
0 \\
0 \\
0 \\
0\end{array}$ & $\begin{array}{r}\frac{32}{\underline{0}} \\
\underline{0} \\
\underline{0} \\
\underline{0}\end{array}$ & $\frac{41}{\underline{0}}$ & $\begin{array}{l}\frac{42}{\underline{0}} \\
\underline{0} \\
\underline{0} \\
\underline{0}\end{array}$ & $\begin{array}{r}43 \\
0 \\
0 \\
0 \\
0 \\
0\end{array}$ & $\frac{44}{\underline{0}}$ & & $\begin{array}{l}\frac{55}{0.8} \\
\frac{0}{0} \\
\underline{0} \\
1.7\end{array}$ & & & & & & & $\frac{\frac{2}{0.4}}{\frac{0}{0}}$ & $\begin{array}{l}\frac{3}{0} \\
1.4 \\
0 \\
0\end{array}$ & \\
\hline $\begin{array}{l}\text { Ring } \\
\text { Core }\end{array}$ & $\begin{array}{l}10 \\
\frac{0}{0} \\
\underline{0} \\
\underline{0}\end{array}$ & $\begin{array}{c}11 \\
0 \\
0 \\
0.6 \\
0\end{array}$ & $\begin{array}{c}\frac{16}{0} \\
0 \\
0\end{array}$ & $\frac{17}{\frac{0}{0}}$ & $\begin{array}{l}\frac{21}{\underline{0}} \\
\frac{0.2}{0.6} \\
\frac{0}{\underline{0}}\end{array}$ & $\begin{array}{l}\frac{23}{\underline{0}} \\
\underline{0} \\
\underline{0} \\
\underline{0} \\
\underline{0}\end{array}$ & $\begin{array}{c}24 \\
0 \\
0 \\
0.3 \\
0 \\
0\end{array}$ & $\begin{array}{r}28 \\
0 \\
0 \\
0 \\
0 \\
0\end{array}$ & & & & & & & $\begin{array}{c}\frac{56}{\underline{0}} \\
\frac{0.6}{\underline{0}} \\
\underline{0} \\
\underline{0}\end{array}$ & $\begin{array}{c}57 \\
\underline{0} \\
\frac{0.3}{\underline{0}} \\
\underline{0} \\
\underline{0}\end{array}$ & $\begin{array}{c}58 \\
0 \\
0 \\
0 \\
1.3 \\
0\end{array}$ & $\begin{array}{l}\frac{61}{\underline{0}} \\
\frac{1.8}{0.4} \\
\frac{0.8}{\underline{0}}\end{array}$ & $\begin{array}{c}62 \\
0 \\
0.3 \\
0 \\
3.5 \\
0\end{array}$ & $\begin{array}{l}\frac{63}{0.8} \\
\frac{0.7}{\underline{0}} \\
\frac{0.8}{\underline{0}}\end{array}$ & $\begin{array}{c}64 \\
0 \\
0 \\
1.4 \\
1.0 \\
0\end{array}$ & $\begin{array}{l}\frac{1}{\underline{0}} \\
\underline{0} \\
\underline{0} \\
\underline{0}\end{array}$ & & \\
\hline $\begin{array}{l}\text { Sargasso } \\
\text { Sea } \\
\& \\
\text { Gulf } \\
\text { Stream }\end{array}$ & & & & & & & & & & $\frac{\frac{49}{0.3}}{\frac{0.4}{\underline{0}}}$ & $\begin{array}{c}50 \\
0 \\
0 \\
0.3 \\
0\end{array}$ & $\begin{array}{c}51 \\
0 \\
0 \\
0.2 \\
0\end{array}$ & $\begin{array}{l}\frac{52}{\underline{0}} \\
\frac{0.3}{\underline{0}} \\
\underline{0}\end{array}$ & & & & & & & & & & & \\
\hline $\begin{array}{l}\text { Slope } \\
\text { Water }\end{array}$ & $\frac{8}{-}$ & $\frac{9}{0}$ & $\frac{18}{0}$ & & $\frac{19}{0}$ & $\frac{20}{0}$ & $\frac{33}{0}$ & $\frac{34}{0}$ & & $\frac{35}{0}$ & $\frac{47}{0}$ & $\frac{48}{0}$ & $\frac{53}{0.1}$ & $\frac{54}{0.1}$ & $\frac{59}{0.3}$ & $\frac{60}{0.3}$ & $\frac{65}{0}$ & $\frac{66}{0.2}$ & & & & $\frac{4}{0}$ & $\frac{5}{0}$ & $\frac{6}{0}$ \\
\hline $\begin{array}{l}\text { High } \\
\text { Velocity } \\
\text { Region }\end{array}$ & $\frac{12}{0}$ & $\frac{13}{0}$ & $\frac{14}{0.1}$ & $\frac{15}{0}$ & $\frac{27}{0}$ & $\frac{29}{0.1}$ & $\frac{30}{0}$ & $\frac{31}{0}$ & $\frac{32}{-}$ & $\frac{41}{0}$ & $\frac{42}{0}$ & $\frac{43}{0}$ & $\frac{44}{0}$ & & $\frac{55}{0.5}$ & & & & & & & $\frac{2}{0.1}$ & $\frac{3}{0.3}$ & \\
\hline $\begin{array}{l}\text { Ring } \\
\text { Core }\end{array}$ & $\frac{10}{0}$ & $\frac{11}{0.2}$ & $\frac{16}{-}$ & $\frac{17}{0}$ & $\frac{21}{0.2}$ & $\frac{23}{0}$ & $\frac{24}{0.1}$ & $\frac{28}{0}$ & & $\frac{37}{0}$ & $\frac{38}{0}$ & $\frac{39}{0}$ & & & $\frac{56}{0.1}$ & $\frac{57}{0.1}$ & $\frac{58}{0.3}$ & $\frac{61}{0.6}$ & $\frac{62}{0.7}$ & $\frac{63}{0.5}$ & $\frac{64}{0.3}$ & $\frac{1}{0}$ & & \\
\hline SS \& GS & & & & & & & & & & $\frac{49}{0.2}$ & $\frac{50}{0.1}$ & $\frac{51}{0.1}$ & $\frac{52}{0.1}$ & & & & & & & & 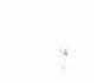 & & & \\
\hline
\end{tabular}


Table 41. Abundance of Bonapartia pedaliota (specimens $/ 10,000 \mathrm{~m}^{3}$ ).

\begin{tabular}{|c|c|c|c|c|c|c|c|c|c|c|c|c|c|c|c|c|c|c|c|c|c|c|c|c|}
\hline & \multicolumn{4}{|c|}{$\begin{array}{c}\text { Oceanus } 118 \\
\text { April }\end{array}$} & \multicolumn{5}{|c|}{$\begin{array}{c}\text { Oceanus } 121 \\
\text { June }\end{array}$} & \multicolumn{5}{|c|}{$\begin{array}{l}\text { Oceanus } 125 \\
\text { August }\end{array}$} & \multicolumn{7}{|c|}{$\begin{array}{l}\text { Knorr } 98 \\
\text { Sept-0ct }\end{array}$} & \multicolumn{3}{|c|}{$\begin{array}{c}\text { A2 } 110 \\
\text { Sept-0ct }\end{array}$} \\
\hline $\begin{array}{l}\text { Slope } \\
\text { Water }\end{array}$ & $\begin{array}{l}\frac{8}{\bar{a}} \\
\underline{0} \\
\underline{0} \\
\underline{0}\end{array}$ & $\begin{array}{l}9 \\
0 \\
0 \\
0 \\
0\end{array}$ & & & $\begin{array}{l}\frac{19}{0.5} \\
\frac{0}{0.2} \\
\frac{0}{0}\end{array}$ & $\begin{array}{r}20 \\
0 \\
0 \\
0 \\
0 \\
0\end{array}$ & $\begin{array}{l}\frac{33}{\underline{0}} \\
\underline{0} \\
\underline{0} \\
\underline{0} \\
\underline{0}\end{array}$ & $\begin{array}{r}\frac{34}{\underline{0}} \\
\underline{0} \\
\underline{0}\end{array}$ & & $\begin{array}{c}35 \\
0 \\
0.2 \\
0 \\
0 \\
0\end{array}$ & $\frac{47}{\frac{1.3}{\underline{0}}}$ & $\begin{array}{r}48 \\
0 \\
0 \\
0 \\
0\end{array}$ & $\frac{\frac{53}{0.2}}{\frac{0}{\underline{0}}}$ & $\begin{array}{r}54 \\
0 \\
0 \\
0 \\
0\end{array}$ & 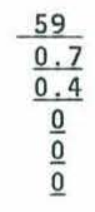 & $\begin{array}{r}60 \\
0 \\
0 \\
0 \\
0 \\
0\end{array}$ & $\begin{array}{l}\frac{65}{\underline{0}} \\
\underline{0} \\
\underline{0} \\
\underline{0} \\
\underline{0}\end{array}$ & $\begin{array}{c}\frac{66}{0.2} \\
0 \\
0 \\
0 \\
0\end{array}$ & & & & $\begin{array}{l}4 \\
0 \\
0 \\
0 \\
0\end{array}$ & $\begin{array}{l}5 \\
\underline{0} \\
\underline{0} \\
\underline{0} \\
\underline{0}\end{array}$ & \\
\hline $\begin{array}{l}\text { High } \\
\text { Velocity } \\
\text { Region }\end{array}$ & $\begin{array}{r}12 \\
0 \\
0 \\
0 \\
0\end{array}$ & $\frac{13}{\underline{0}}$ & $\frac{\frac{14}{0.3}}{\frac{0.3}{\underline{0}}} \frac{0}{\underline{0}}$ & $\begin{array}{r}15 \\
0 \\
0 \\
0 \\
0\end{array}$ & $\begin{array}{c}\frac{26}{0.3} \\
0 \\
0 \\
0 \\
-\end{array}$ & $\begin{array}{r}27 \\
0 \\
0 \\
0 \\
0 \\
0\end{array}$ & $\begin{array}{r}30 \\
0 \\
0 \\
0 \\
0 \\
0\end{array}$ & $\begin{array}{c}31 \\
0.2 \\
0 \\
0 \\
0 \\
0\end{array}$ & $\frac{32}{\frac{1.1}{\frac{0}{0}}}$ & $\frac{\frac{41}{0.4}}{\frac{0}{0}}$ & $\frac{\frac{42}{0.3}}{\frac{0}{0}} \frac{\underline{0}}{\underline{0}}$ & $\begin{array}{r}43 \\
0 \\
0 \\
0 \\
0 \\
0\end{array}$ & $\frac{\frac{44}{0.4}}{\frac{0}{0}}$ & & $\frac{\frac{55}{0.8}}{\frac{0.5}{\underline{0}}}$ & & & & & & & $\begin{array}{l}\frac{2}{0} \\
\underline{0} \\
\underline{0} \\
\underline{0}\end{array}$ & $\begin{array}{l}3 \\
0 \\
0 \\
0 \\
0\end{array}$ & \\
\hline $\begin{array}{l}\text { Ring } \\
\text { Core }\end{array}$ & $\begin{array}{l}\frac{10}{0.4} \\
\frac{0.7}{\underline{0}} \\
\underline{0.6}\end{array}$ & $\begin{array}{c}11 \\
0 \\
0.6 \\
0 \\
0\end{array}$ & $\begin{array}{r}16 \\
0 \\
0 \\
0\end{array}$ & $\frac{17}{\frac{0}{6.3}} \frac{\underline{0}}{\underline{6}}$ & $\begin{array}{c}\frac{21}{\underline{0}} \\
\frac{1.8}{2.1} \\
\frac{0}{\underline{0}} \\
\underline{0}\end{array}$ & $\begin{array}{c}\frac{23}{\underline{0}} \\
\underline{0.7} \\
\underline{0.5} \\
\underline{0} \underline{0}\end{array}$ & $\begin{array}{r}24 \\
0 \\
0 \\
0 \\
0 \\
0\end{array}$ & $\begin{array}{r}28 \\
0 \\
0 \\
0 \\
0 \\
0\end{array}$ & & & & & & & $\frac{\frac{56}{2.6}}{\frac{10.6}{10.6}}$ & 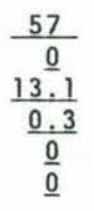 & $\begin{array}{c}\frac{58}{0.8} \\
0.8 \\
0 \\
0 \\
0\end{array}$ & $\frac{\frac{61}{\frac{0.2}{3.6}}}{\frac{0.7}{0.7}}$ & $\begin{array}{c}62 \\
0.6 \\
0.6 \\
0 \\
0 \\
0\end{array}$ & $\frac{\frac{63}{3.2}}{\frac{3.7}{0.7}} \frac{1.0}{\frac{0}{0}}$ & $\begin{array}{c}64 \\
1.0 \\
0 \\
0 \\
0 \\
0\end{array}$ & $\frac{\frac{1}{2.4}}{\frac{0}{0}} \frac{\underline{0}}{\underline{0}}$ & & \\
\hline $\begin{array}{l}\text { Sargasso } \\
\text { Sea } \\
\& \\
\text { Gulf } \\
\text { Stream }\end{array}$ & & & & & & & & & & $\frac{\frac{49}{\frac{2.1}{2.2}}}{\frac{2.2}{0.2}}$ & $\begin{array}{c}50 \\
1.5 \\
0.2 \\
0 \\
0\end{array}$ & $\begin{array}{l}\frac{51}{1.8} \\
0.2 \\
0 \\
0.3\end{array}$ & $\frac{\frac{52}{\frac{3.2}{2.2}}}{\frac{0}{\underline{0}}}$ & & & & & & & & & & & \\
\hline $\begin{array}{l}\text { Slope } \\
\text { Water }\end{array}$ & $\frac{8}{-}$ & $\frac{9}{0}$ & $\frac{18}{0}$ & & $\frac{19}{0.1}$ & $\frac{20}{0}$ & $\frac{33}{0}$ & $\frac{34}{0}$ & & $\frac{35}{0}$ & $\frac{47}{0.3}$ & $\frac{48}{0}$ & $\frac{53}{0.1}$ & $\frac{54}{0}$ & $\frac{59}{0.2}$ & $\frac{60}{0}$ & $\frac{65}{0}$ & $\frac{66}{0.1}$ & & & & $\frac{4}{0}$ & $\frac{5}{0}$ & $\frac{6}{0}$ \\
\hline $\begin{array}{l}\text { High } \\
\text { Velocity } \\
\text { Region }\end{array}$ & $\frac{12}{0}$ & $\frac{13}{0}$ & $\frac{14}{0.1}$ & $\frac{15}{0}$ & $\frac{27}{0}$ & $\frac{29}{0.2}$ & $\frac{30}{0}$ & $\frac{31}{0.1}$ & $\frac{32}{-}$ & $\frac{41}{0.2}$ & $\frac{42}{0.1}$ & $\frac{43}{0}$ & $\frac{44}{0.2}$ & & $\frac{55}{0.3}$ & & & & & & & $\frac{2}{0}$ & $\frac{3}{0}$ & \\
\hline $\begin{array}{l}\text { Ring } \\
\text { Core }\end{array}$ & $\frac{10}{1.4}$ & $\frac{11}{0.2}$ & $\frac{16}{-}$ & $\frac{17}{1.6}$ & $\frac{21}{1.0}$ & $\frac{23}{0.3}$ & $\frac{24}{0}$ & $\frac{28}{0}$ & & $\frac{37}{0}$ & $\frac{38}{0.2}$ & $\frac{39}{0.3}$ & & & $\frac{56}{2.6}$ & $\frac{57}{2.7}$ & $\frac{58}{0.6}$ & $\frac{61}{0.5}$ & $\frac{62}{0.2}$ & $\frac{63}{1.2}$ & $\frac{64}{0.4}$ & $\frac{1}{0.6}$ & & \\
\hline SS \& GS & & & & & & & & & & $\frac{49}{1.1}$ & $\frac{50}{0.4}$ & $\frac{51}{0.6}$ & $\frac{52}{1.3}$ & & & & & & & & & & & \\
\hline
\end{tabular}


Table 42. Volume of Bonapartia pedaliota $\left(\mathrm{ml} / 10,000 \mathrm{~m}^{3}\right)$.

\begin{tabular}{|c|c|c|c|c|c|c|c|c|c|c|c|c|c|c|c|c|c|c|c|c|c|c|c|c|}
\hline & \multicolumn{4}{|c|}{$\begin{array}{c}\text { Oceanus } 118 \\
\text { Apri1 }\end{array}$} & \multicolumn{5}{|c|}{$\begin{array}{c}\text { Oceanus } 121 \\
\text { June }\end{array}$} & \multicolumn{5}{|c|}{$\begin{array}{l}\text { Oceanus } 125 \\
\text { August }\end{array}$} & \multicolumn{7}{|c|}{$\begin{array}{l}\text { Knorr } 98 \\
\text { Sept-Oct }\end{array}$} & \multicolumn{3}{|c|}{$\begin{array}{c}\text { A2 } 110 \\
\text { Sept-0ct }\end{array}$} \\
\hline $\begin{array}{l}\text { Slope } \\
\text { Water }\end{array}$ & $\begin{array}{l}\frac{8}{\overline{0}} \\
\underline{0} \\
\underline{0} \\
\underline{0}\end{array}$ & $\begin{array}{l}9 \\
0 \\
0 \\
0 \\
0\end{array}$ & & & $\begin{array}{l}\frac{19}{\underline{0}} \\
\underline{\underline{0}} \\
\frac{0.18}{\underline{0}} \\
\underline{0}\end{array}$ & $\begin{array}{l}20 \\
0 \\
0 \\
0 \\
0 \\
0\end{array}$ & $\begin{array}{l}33 \\
\underline{0} \\
\underline{0} \\
\underline{0} \\
\underline{0} \\
\underline{0}\end{array}$ & $\begin{array}{l}\frac{34}{\underline{0}} \\
\frac{0}{0}\end{array}$ & & $\begin{array}{l}\frac{35}{0} \\
0.11 \\
0 \\
0 \\
0\end{array}$ & $\frac{47}{\frac{0}{0}}$ & $\begin{array}{l}\frac{48}{0} \\
0 \\
0 \\
0\end{array}$ & $\frac{\frac{53}{0.02}}{\frac{0}{0}}$ & $\begin{array}{l}\frac{54}{0} \\
0 \\
0 \\
0\end{array}$ & $\begin{array}{l}\frac{59}{0.03} \\
\frac{0.36}{\underline{0}} \\
\frac{0}{0}\end{array}$ & $\begin{array}{l}\frac{60}{0} \\
0 \\
0 \\
0 \\
0\end{array}$ & $\begin{array}{l}\frac{65}{\underline{0}} \\
\underline{0} \\
\underline{0} \\
\underline{0} \\
\underline{0}\end{array}$ & $\begin{array}{c}\frac{66}{0.02} \\
0 \\
0 \\
0 \\
0\end{array}$ & & & & $\begin{array}{l}4 \\
0 \\
0 \\
0 \\
0\end{array}$ & $\begin{array}{l}5 \\
\underline{0} \\
\underline{0} \\
\underline{0} \\
\underline{0}\end{array}$ & \\
\hline $\begin{array}{l}\text { High } \\
\text { Velocity } \\
\text { Region }\end{array}$ & $\begin{array}{l}12 \\
0 \\
0 \\
0 \\
0\end{array}$ & $\frac{13}{\frac{0}{0}}$ & $\frac{\frac{14}{0.03}}{\frac{0}{0}}$ & $\begin{array}{l}\frac{15}{0} \\
0 \\
0 \\
0\end{array}$ & $\begin{array}{c}\frac{26}{0.03} \\
0 \\
0 \\
0 \\
-\end{array}$ & $\begin{array}{l}27 \\
0 \\
0 \\
0 \\
0 \\
0\end{array}$ & $\begin{array}{l}30 \\
0 \\
0 \\
0 \\
0 \\
0\end{array}$ & $\begin{array}{c}\frac{31}{0.02} \\
0 \\
0 \\
0 \\
0\end{array}$ & $\frac{\frac{32}{0.66}}{\frac{0}{0}}$ & $\frac{\frac{41}{0.39}}{\frac{0}{0}}$ & $\frac{\frac{42}{0.01}}{\frac{0}{0}}$ & $\begin{array}{l}43 \\
0 \\
0 \\
0 \\
0 \\
0\end{array}$ & $\frac{\frac{44}{0.21}}{\frac{0}{0}}$ & & $\frac{\frac{55}{0.04}}{\frac{0.05}{\underline{0}}}$ & & & & & & & $\begin{array}{l}\frac{2}{0} \\
\underline{0} \\
\underline{0} \\
\underline{0}\end{array}$ & $\begin{array}{l}3 \\
0 \\
0 \\
0 \\
0\end{array}$ & \\
\hline $\begin{array}{l}\text { Ring } \\
\text { Core }\end{array}$ & $\begin{array}{l}\frac{10}{\frac{0.08}{3.53}} \\
\underline{0} \\
\underline{1.12}\end{array}$ & $\begin{array}{c}11 \\
0 \\
0.83 \\
0 \\
0\end{array}$ & $\begin{array}{l}\frac{16}{0} \\
0 \\
0\end{array}$ & $\frac{\frac{17}{\underline{0}}}{\frac{6.05}{\underline{0}}}$ & $\frac{\frac{21}{\underline{0}}}{\frac{1.80}{3.03}} \frac{\underline{0}}{\underline{0}}$ & $\begin{array}{l}\frac{23}{\underline{0}} \\
\frac{0.53}{0.75} \\
\frac{0}{0} \\
\underline{0}\end{array}$ & $\begin{array}{l}24 \\
0 \\
0 \\
0 \\
0 \\
0\end{array}$ & $\begin{array}{l}28 \\
0 \\
0 \\
0 \\
0 \\
0\end{array}$ & & & & & & & $\begin{array}{l}\frac{56}{0.06} \\
\frac{0.78}{\underline{0}} \\
\underline{0} \\
\underline{0}\end{array}$ & $\begin{array}{l}\frac{57}{\underline{0}} \\
\underline{1.03} \\
\underline{0.18} \\
\underline{0} \\
\underline{0}\end{array}$ & $\begin{array}{l}\frac{58}{0.05} \\
0.13 \\
0 \\
0 \\
0\end{array}$ & $\begin{array}{l}\frac{61}{0.02} \\
\frac{0.39}{0.52} \\
\frac{0}{0}\end{array}$ & $\begin{array}{l}\frac{62}{0.04} \\
0.10 \\
0 \\
0 \\
0\end{array}$ & $\frac{\frac{63}{0.38}}{\frac{0.50}{0.50}} \frac{0.81}{\frac{0}{0}}$ & $\begin{array}{c}\frac{64}{0.04} \\
0 \\
0 \\
0 \\
0\end{array}$ & $\frac{\frac{1}{0.08}}{\frac{0}{0}}$ & & \\
\hline $\begin{array}{l}\text { Sargasso } \\
\text { Sea } \\
\& \\
\text { Gulf } \\
\text { Stream }\end{array}$ & & & & & & & & & & $\frac{\frac{49}{0.10}}{\frac{0.67}{0.18}} \frac{0}{\underline{0}}$ & $\begin{array}{l}\frac{50}{0.05} \\
0.12 \\
0 \\
0\end{array}$ & $\begin{array}{c}\frac{51}{0.06} \\
0.04 \\
0 \\
0.07\end{array}$ & $\frac{\frac{52}{0.14}}{\frac{0.69}{\underline{0}}}$ & & & & & & & & & & & \\
\hline $\begin{array}{l}\text { Slope } \\
\text { Water }\end{array}$ & $\frac{8}{-}$ & $\frac{9}{0}$ & $\frac{18}{0}$ & & $\frac{19}{0.05}$ & $\frac{20}{0}$ & $\frac{33}{0}$ & $\frac{34}{0}$ & & $\frac{35}{0.02}$ & $\frac{47}{0}$ & $\frac{48}{0}$ & $\frac{53}{0.01}$ & $\frac{54}{0}$ & $\frac{59}{0.08}$ & $\frac{60}{0}$ & $\frac{65}{0}$ & $\frac{66}{0.01}$ & & & & $\frac{4}{0}$ & $\frac{5}{0}$ & $\frac{6}{0}$ \\
\hline $\begin{array}{l}\text { High } \\
\text { Velocity } \\
\text { Region }\end{array}$ & $\frac{12}{0}$ & $\frac{13}{0}$ & $\frac{14}{0.01}$ & $\frac{15}{0}$ & $\frac{27}{0}$ & $\frac{29}{0.07}$ & $\frac{30}{0}$ & $\frac{31}{0.01}$ & $\frac{32}{-}$ & $\frac{41}{0.20}$ & $\frac{42}{0.01}$ & $\frac{43}{0}$ & $\frac{44}{0.13}$ & & $\frac{55}{0.02}$ & & & & & & & $\frac{2}{0}$ & $\frac{3}{0}$ & \\
\hline $\begin{array}{l}\text { Ring } \\
\text { Core }\end{array}$ & $\frac{10}{1.18}$ & $\frac{11}{0.21}$ & $\frac{16}{-}$ & $\frac{17}{1.51}$ & $\frac{21}{1.24}$ & $\frac{23}{0.28}$ & $\frac{24}{0}$ & $\frac{28}{0}$ & & $\frac{37}{0}$ & $\frac{38}{0.18}$ & $\frac{39}{0.05}$ & & & $\frac{56}{0.17}$ & $\frac{57}{0.24}$ & $\frac{58}{0.03}$ & $\frac{61}{0.19}$ & $\frac{62}{0.02}$ & $\frac{63}{0.32}$ & $\frac{64}{0.02}$ & $\frac{1}{0.02}$ & & \\
\hline SS \& GS & & & & & & & & & & $\frac{49}{0.24}$ & $\frac{50}{0.04}$ & $\frac{51}{0.04}$ & $\frac{52}{0.21}$ & & & & & & & & & & & \\
\hline
\end{tabular}


Table 43. Abundance of Ceratoscopelus maderensis (specimens $/ 10,000 \mathrm{~m}^{3}$ ).

\begin{tabular}{|c|c|c|c|c|c|c|c|c|c|c|c|c|c|c|c|c|c|c|c|c|c|c|c|c|}
\hline & \multicolumn{4}{|c|}{$\begin{array}{c}\text { Oceanus } \\
\text { April }\end{array}$} & \multicolumn{5}{|c|}{$\begin{array}{c}\text { Oceanus } 121 \\
\text { June }\end{array}$} & \multicolumn{5}{|c|}{$\begin{array}{l}\text { Oceanus } 125 \\
\text { Auqust }\end{array}$} & \multicolumn{7}{|c|}{$\begin{array}{l}\text { Knorr } 98 \\
\text { Sept-0ct }\end{array}$} & \multicolumn{3}{|c|}{$\begin{array}{c}\text { A2 } 110 \\
\text { Sept-0ct }\end{array}$} \\
\hline $\begin{array}{l}\text { Slope } \\
\text { Water }\end{array}$ & $\begin{array}{l}8 \\
\overline{\overline{0}} \\
\underline{0} \\
\underline{0} \\
\underline{0}\end{array}$ & $\begin{array}{l}9 \\
0 \\
0 \\
0 \\
0\end{array}$ & & & $\begin{array}{l}\frac{19}{\frac{1.5}{0.7}} \\
\frac{0.4}{\underline{0.2}} \\
\underline{0}\end{array}$ & $\begin{array}{c}20 \\
0 \\
0 \\
0 \\
0 \\
0\end{array}$ & $\begin{array}{l}\frac{33}{31.0} \\
\frac{2.9}{\underline{0}} \\
\underline{2.2} \\
\underline{0.4}\end{array}$ & $\begin{array}{l}\frac{34}{1.8} \\
\frac{0.4}{6.8}\end{array}$ & & $\begin{array}{r}35 \\
0 \\
0.2 \\
37.7 \\
98.9 \\
0.7\end{array}$ & $\frac{\frac{47}{1.5}}{\frac{0.8}{0.8}} \frac{10}{\underline{0}}$ & \begin{tabular}{r}
\multicolumn{1}{c}{48} \\
0.3 \\
0.9 \\
20.3 \\
0.2
\end{tabular} & $\frac{\frac{53}{26.2}}{\frac{29.8}{30.3}} \frac{0.2}{\underline{0.2}}$ & $\begin{array}{r}54 \\
2.6 \\
6.9 \\
182.1 \\
3.7\end{array}$ & $\frac{\frac{59}{30.0}}{\frac{\frac{30.6}{11.6}}{\frac{24.4}{0}}}$ & $\begin{array}{c}60 \\
1.6 \\
0 \\
74.8 \\
76.5 \\
0\end{array}$ & 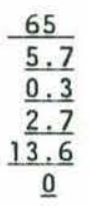 & $\begin{array}{c}66 \\
0 \\
0 \\
14.4 \\
12.4 \\
1.2\end{array}$ & & & & $\begin{array}{c}\frac{4}{0.2} \\
1.7 \\
21.2 \\
0\end{array}$ & $\frac{\frac{5}{3.0}}{\frac{3.3}{3.1}} \frac{\underline{0}}{\underline{3}}$ & \\
\hline $\begin{array}{l}\text { High } \\
\text { Velocity } \\
\text { Region }\end{array}$ & $\begin{array}{r}12 \\
0 \\
0 \\
0 \\
0\end{array}$ & $\frac{\frac{13}{0.5}}{\frac{0}{0}}$ & $\begin{array}{l}\frac{14}{\underline{0}} \\
\underline{0} \\
\underline{0} \\
\underline{0}\end{array}$ & $\begin{array}{r}15 \\
0 \\
0 \\
0 \\
0\end{array}$ & $\begin{array}{c}26 \\
0 \\
0 \\
0 \\
0 \\
-\end{array}$ & $\begin{array}{r}27 \\
0 \\
0 \\
0 \\
0 \\
0\end{array}$ & $\begin{array}{c}30 \\
0.3 \\
0 \\
0.9 \\
0 \\
0\end{array}$ & $\begin{array}{c}31 \\
0 \\
0 \\
0.4 \\
0 \\
0\end{array}$ & $\begin{array}{l}\frac{32}{\frac{0.5}{0}} \\
\frac{2.5}{\underline{0}}\end{array}$ & $\frac{\frac{41}{\frac{1.4}{4.0}}}{\frac{0}{0}}$ & $\frac{\frac{42}{\frac{3.4}{20.9}}}{\frac{44.6}{0.5}}$ & $\begin{array}{r}43 \\
0 \\
0 \\
15.0 \\
27.0 \\
0.5\end{array}$ & $\frac{\frac{44}{21.7}}{\frac{53.0}{\underline{0}}}$ & & $\begin{array}{l}\frac{55}{1.1} \\
\underline{\underline{0}} \\
\frac{2.8}{2.2}\end{array}$ & & & & & & & $\frac{\frac{2}{4.8}}{\frac{0.3}{12.0}}$ & $\begin{array}{c}\frac{3}{0.4} \\
7.3 \\
8.2 \\
0\end{array}$ & \\
\hline $\begin{array}{l}\text { Ring } \\
\text { Core }\end{array}$ & $\begin{array}{r}10 \\
\underline{0} \\
\underline{0} \\
\underline{0} \\
\underline{0}\end{array}$ & $\begin{array}{r}11 \\
0 \\
0 \\
0 \\
0\end{array}$ & $\begin{array}{r}16 \\
0 \\
0 \\
0\end{array}$ & $\frac{17}{\underline{0}} \frac{\underline{0}}{\underline{0}}$ & $\begin{array}{l}\frac{21}{1.0} \\
\frac{0}{0.6} \\
\frac{2.1}{\underline{0}}\end{array}$ & $\frac{23}{\frac{0.6}{\underline{0}}}$ & $\begin{array}{c}24 \\
0 \\
0 \\
0.5 \\
0.6 \\
0\end{array}$ & $\begin{array}{c}28 \\
0 \\
0 \\
3.6 \\
0 \\
0\end{array}$ & & & & & & & $\begin{array}{c}\frac{56}{0.6} \\
\underline{0} \\
\underline{0} \\
\frac{1.6}{\underline{0}}\end{array}$ & $\begin{array}{l}\frac{57}{\frac{0.3}{0}} \\
\underline{0} \\
\frac{0.3}{\underline{0}}\end{array}$ & $\begin{array}{c}58 \\
0 \\
0 \\
0 \\
5.5 \\
0.8\end{array}$ & $\begin{array}{l}\frac{61}{\underline{0}} \\
\underline{0.3} \\
\frac{1.9}{0.4} \\
\underline{0.3}\end{array}$ & $\begin{array}{c}62 \\
0 \\
0 \\
0.2 \\
1.1 \\
0.7\end{array}$ & $\frac{63}{\frac{0.3}{\underline{0}}}$ & $\begin{array}{c}64 \\
0 \\
0 \\
0 \\
1.0 \\
0.4\end{array}$ & $\frac{\frac{1}{1.0}}{\frac{0.2}{2.5}}$ & & \\
\hline $\begin{array}{l}\text { Sargasso } \\
\text { Sea } \\
\& \\
\text { Gulf } \\
\text { Stream }\end{array}$ & & & & & & & & & & $\begin{array}{l}\frac{49}{\underline{0}} \\
\underline{0} \\
\frac{0.4}{1.5} \\
\end{array}$ & $\begin{array}{c}50 \\
0 \\
0 \\
0 \\
0\end{array}$ & $\begin{array}{c}51 \\
0 \\
0 \\
0.4 \\
0\end{array}$ & $\begin{array}{l}\frac{52}{\underline{0}} \\
\underline{0} \\
\underline{0} \\
\underline{0}\end{array}$ & & & & & & & & & & & \\
\hline $\begin{array}{l}\text { Slope } \\
\text { Water }\end{array}$ & $\frac{8}{-}$ & $\frac{9}{0}$ & $\frac{18}{0}$ & & $\frac{19}{1.2}$ & $\frac{20}{0}$ & $\frac{33}{2.9}$ & $\frac{34}{4.4}$ & & $\frac{35}{27.3}$ & $\frac{47}{3.3}$ & $\frac{48}{5.4}$ & $\frac{53}{21.6}$ & $\frac{54}{48.8}$ & $\frac{59}{13.3}$ & $\frac{60}{30.7}$ & $\frac{65}{4.5}$ & $\frac{66}{5.6}$ & & & & $\frac{4}{5.8}$ & $\frac{5}{4.9}$ & $\frac{6}{3.3}$ \\
\hline $\begin{array}{l}\text { High } \\
\text { Velocity } \\
\text { Region }\end{array}$ & $\frac{12}{0}$ & $\frac{13}{0.1}$ & $\frac{14}{0}$ & $\frac{15}{0}$ & $\frac{27}{0}$ & $\frac{29}{0.2}$ & $\frac{30}{0.3}$ & $\frac{31}{0.1}$ & $\frac{32}{-}$ & $\frac{41}{4.2}$ & $\frac{42}{14.8}$ & $\frac{43}{8.5}$ & $\frac{44}{23.5}$ & & $\frac{55}{1.8}$ & & & & & & & $\frac{2}{4.3}$ & $\frac{3}{4.0}$ & \\
\hline $\begin{array}{l}\text { Ring } \\
\text { Core }\end{array}$ & $\frac{10}{0}$ & $\frac{11}{0}$ & $\frac{16}{-}$ & $\frac{17}{0}$ & $\frac{21}{0.6}$ & $\frac{23}{0.1}$ & $\frac{24}{0.2}$ & $\frac{28}{0.9}$ & & $\frac{37}{2.4}$ & $\frac{38}{4.0}$ & $\frac{39}{11.5}$ & & & $\frac{56}{0.4}$ & $\frac{57}{0.1}$ & $\frac{58}{1.3}$ & $\frac{61}{0.8}$ & $\frac{62}{0.4}$ & $\frac{63}{0.1}$ & $\frac{64}{0.3}$ & $\frac{1}{1.1}$ & & \\
\hline SS \& GS & & & & & & & & & & $\frac{49}{0.5}$ & $\frac{50}{0}$ & $\frac{51}{0.1}$ & $\frac{52}{0}$ & & & & & & & & & & & \\
\hline
\end{tabular}


Table 44. Volume of Ceratoscopelus maderensis $\left(\mathrm{ml} / 10,000 \mathrm{~m}^{3}\right)$.

\begin{tabular}{|c|c|c|c|c|c|c|c|c|c|c|c|c|c|c|c|c|c|c|c|c|c|c|c|c|}
\hline & \multicolumn{4}{|c|}{$\begin{array}{c}\text { Oceanus } 118 \\
\text { Apri1 }\end{array}$} & \multicolumn{5}{|c|}{$\begin{array}{c}\text { Oceanus } 121 \\
\text { June }\end{array}$} & \multicolumn{5}{|c|}{$\begin{array}{l}\text { Oceanus } 125 \\
\text { August }\end{array}$} & \multicolumn{7}{|c|}{$\begin{array}{l}\text { Knorr } 98 \\
\text { Sept-0ct }\end{array}$} & \multicolumn{3}{|c|}{$\begin{array}{c}\text { A2 } 110 \\
\text { Sept-0ct }\end{array}$} \\
\hline $\begin{array}{l}\text { Slope } \\
\text { Water }\end{array}$ & $\begin{array}{l}8 \\
\overline{\overline{0}} \\
\underline{0} \\
\underline{0} \\
\underline{0}\end{array}$ & $\begin{array}{l}\frac{9}{0} \\
0 \\
0 \\
0\end{array}$ & & & $\frac{\frac{19}{0.02}}{\frac{0.03}{0.04}} \frac{0.04}{\frac{0.05}{0}}$ & $\begin{array}{l}20 \\
0 \\
0 \\
0 \\
0 \\
0\end{array}$ & $\begin{array}{l}\frac{33}{2.16} \\
\frac{0.04}{\underline{0}} \\
\frac{0.11}{0.01} \\
\underline{0.01}\end{array}$ & $\frac{\frac{34}{0.09}}{\frac{0.04}{0.36}}$ & & $\begin{array}{l}\frac{35}{0} \\
0.02 \\
1.83 \\
5.00 \\
0.07\end{array}$ & $\frac{\frac{47}{0.07}}{\frac{0.03}{0.49}} \frac{\underline{0}}{\underline{0.03}}$ & $\begin{array}{l}\frac{48}{0.03} \\
0.06 \\
0.97 \\
0.02\end{array}$ & $\begin{array}{l}\frac{53}{1.06} \\
\frac{1.33}{1.54} \\
\underline{0.02}\end{array}$ & $\begin{array}{r}\frac{54}{0.13} \\
0.47 \\
10.00 \\
0.27\end{array}$ & $\frac{\frac{59}{0.83}}{\frac{0.48}{1.06}} \frac{0.03}{\frac{0.03}{\underline{0}}}$ & $\begin{array}{c}\frac{60}{0.03} \\
0 \\
3.45 \\
3.48 \\
0\end{array}$ & $\begin{array}{l}\frac{65}{0.26} \\
\frac{0.03}{0.05} \\
\frac{0.05}{0.48} \\
\underline{0}\end{array}$ & $\begin{array}{l}\frac{66}{0} \\
0 \\
0.67 \\
0.52 \\
0.04\end{array}$ & & & & $\begin{array}{l}\frac{4}{0.02} \\
0.13 \\
1.34 \\
0\end{array}$ & $\frac{\frac{5}{0.20}}{\frac{0.19}{0.75}} \frac{\underline{0}}{\underline{0}}$ & \\
\hline $\begin{array}{l}\text { High } \\
\text { Velocity } \\
\text { Region }\end{array}$ & $\begin{array}{l}12 \\
0 \\
0 \\
0 \\
0\end{array}$ & $\frac{\frac{13}{0.85}}{\frac{0}{0}}$ & $\begin{array}{l}14 \\
\underline{0} \\
\underline{0} \\
\underline{0} \\
\underline{0}\end{array}$ & $\begin{array}{l}15 \\
0 \\
0 \\
0 \\
0\end{array}$ & $\begin{array}{l}26 \\
0 \\
0 \\
0 \\
0 \\
-\end{array}$ & $\begin{array}{l}\frac{27}{0} \\
0 \\
0 \\
0 \\
0\end{array}$ & $\begin{array}{c}30 \\
0 \\
0 \\
0.03 \\
0 \\
0.02\end{array}$ & $\begin{array}{l}31 \\
0 \\
0 \\
0.04 \\
0 \\
0\end{array}$ & $\begin{array}{l}\frac{32}{\underline{0.03}} \\
\underline{0} \\
\underline{0.05} \\
\underline{0}\end{array}$ & $\frac{\frac{41}{0.08}}{\frac{0.77}{0}}$ & $\frac{\frac{42}{0.15}}{\frac{0.03}{1.95}} \frac{1.05}{\underline{0.05}}$ & $\begin{array}{l}\frac{43}{0} \\
0 \\
0.46 \\
1.52 \\
0\end{array}$ & $\frac{\frac{44}{0.97}}{\frac{3.00}{0}}$ & & $\begin{array}{l}\frac{55}{0.05} \\
\underline{0} \\
\underline{0.12} \\
\underline{0.11}\end{array}$ & & & & & & & $\frac{\frac{2}{\frac{0.31}{0.03}}}{\frac{0.60}{\underline{0}}}$ & $\begin{array}{c}\frac{3}{0.04} \\
0.54 \\
0.36 \\
0\end{array}$ & \\
\hline $\begin{array}{l}\text { Ring } \\
\text { Core }\end{array}$ & $\begin{array}{l}10 \\
\underline{0} \\
\underline{0} \\
\underline{0} \\
\underline{0}\end{array}$ & $\begin{array}{l}11 \\
0 \\
0 \\
0 \\
0\end{array}$ & $\begin{array}{l}\frac{16}{0} \\
0 \\
0\end{array}$ & $\begin{array}{l}17 \\
\underline{0} \\
\underline{0} \\
\underline{0}\end{array}$ & $\begin{array}{l}\frac{21}{0.10} \\
\frac{0}{0.03} \\
\frac{0.04}{\underline{0}}\end{array}$ & $\frac{\frac{23}{0.06}}{\frac{0}{0}}$ & $\begin{array}{l}24 \\
0 \\
0 \\
0.03 \\
0.06 \\
0\end{array}$ & $\begin{array}{l}\frac{28}{0} \\
0 \\
0.18 \\
0 \\
0\end{array}$ & & & & & & & $\begin{array}{l}\frac{56}{0.03} \\
\underline{0} \\
\underline{0} \\
\underline{0.05} \\
\underline{0}\end{array}$ & $\begin{array}{l}\frac{57}{0.03} \\
\frac{0}{0} \\
\underline{0.03} \\
\underline{0}\end{array}$ & $\begin{array}{l}\frac{58}{0} \\
0 \\
0 \\
0.29 \\
0.03\end{array}$ & $\begin{array}{l}\frac{61}{\underline{0}} \\
\underline{0.03} \\
\underline{0.04} \\
\underline{0.04} \\
\underline{0.05}\end{array}$ & $\begin{array}{l}\frac{62}{0} \\
0 \\
0.02 \\
0.03 \\
0.04\end{array}$ & $\begin{array}{l}\frac{63}{0.02} \\
\underline{0} \\
\underline{0} \\
\underline{0} \\
\underline{0}\end{array}$ & $\begin{array}{l}\frac{64}{0} \\
0 \\
0 \\
0.05 \\
0.04\end{array}$ & $\frac{\frac{1}{0.08}}{\frac{0.02}{0.02}} \frac{0.16}{\underline{0.08}}$ & & \\
\hline $\begin{array}{l}\text { Sargasso } \\
\text { Sea } \\
\& \\
\text { Gulf } \\
\text { Stream }\end{array}$ & & & & & & & & & & $\begin{array}{l}\frac{49}{\underline{0}} \\
\underline{0} \\
0.02 \\
\underline{0.05} \\
\end{array}$ & $\begin{array}{l}50 \\
0 \\
0 \\
0 \\
0\end{array}$ & $\begin{array}{c}\frac{51}{0} \\
0 \\
0.02 \\
0\end{array}$ & $\begin{array}{l}\frac{52}{\underline{0}} \\
\underline{0} \\
\underline{0} \\
\underline{0}\end{array}$ & & & & & & & & & & & \\
\hline $\begin{array}{l}\text { Slope } \\
\text { Water }\end{array}$ & $\frac{8}{-}$ & $\frac{9}{0}$ & $\frac{18}{0}$ & & $\frac{19}{0.03}$ & $\frac{20}{0}$ & $\frac{33}{0.15}$ & $\frac{34}{0.24}$ & & $\frac{35}{1.38}$ & $\frac{47}{0.15}$ & $\frac{48}{0.27}$ & $\frac{53}{0.99}$ & $\frac{54}{2.72}$ & $\frac{59}{0.48}$ & $\frac{60}{1.40}$ & $\frac{65}{0.16}$ & $\frac{66}{0.25}$ & & & & $\frac{4}{0.37}$ & $\frac{5}{0.29}$ & $\frac{6}{0.48}$ \\
\hline $\begin{array}{l}\text { High } \\
\text { Velocity } \\
\text { Region }\end{array}$ & $\frac{12}{0}$ & $\frac{13}{0.21}$ & $\frac{14}{0}$ & $\frac{15}{0}$ & $\frac{27}{0}$ & $\frac{29}{0.01}$ & $\frac{30}{0.01}$ & $\frac{31}{0.01}$ & $\frac{32}{-}$ & $\frac{41}{0.23}$ & $\frac{42}{0.68}$ & $\frac{43}{0.40}$ & $\frac{44}{1.18}$ & & $\frac{55}{0.08}$ & & & & & & & $\frac{2}{0.24}$ & $\frac{3}{0.24}$ & \\
\hline $\begin{array}{l}\text { Ring } \\
\text { Core }\end{array}$ & $\frac{10}{0}$ & $\frac{11}{0}$ & $\frac{16}{-}$ & $\frac{17}{0}$ & $\frac{21}{0.02}$ & $\frac{23}{0.01}$ & $\frac{24}{0.02}$ & $\frac{28}{0.05}$ & & $\frac{37}{0.12}$ & $\frac{38}{0.19}$ & $\frac{39}{0.59}$ & & & $\frac{56}{0.02}$ & $\frac{57}{0.01}$ & $\frac{58}{0.06}$ & $\frac{61}{0.03}$ & $\frac{62}{0.02}$ & $\frac{63}{0.01}$ & $\frac{64}{0.02}$ & $\frac{1}{0.09}$ & & \\
\hline SS \& GS & & & & & & & & & & $\frac{49}{0.02}$ & $\frac{50}{0}$ & $\frac{51}{0.01}$ & $\frac{52}{0}$ & & & & & & & & & & & \\
\hline
\end{tabular}


Table 45. Abundance of Ceratoscopelus warmingii (specimens $/ 10,000 \mathrm{~m}^{3}$ ).

\begin{tabular}{|c|c|c|c|c|c|c|c|c|c|c|c|c|c|c|c|c|c|c|c|c|c|c|c|c|}
\hline & \multicolumn{4}{|c|}{$\begin{array}{c}\text { Oceanus } 118 \\
\text { April }\end{array}$} & \multicolumn{5}{|c|}{$\begin{array}{c}\text { Oceanus } 121 \\
\text { June }\end{array}$} & \multicolumn{5}{|c|}{$\begin{array}{c}\text { Oceanus } 125 \\
\text { Auqust }\end{array}$} & \multicolumn{7}{|c|}{$\begin{array}{l}\text { Knorr } 98 \\
\text { Sept-Oct }\end{array}$} & \multicolumn{3}{|c|}{$\begin{array}{c}\text { A2 } 110 \\
\text { Sept-0ct }\end{array}$} \\
\hline $\begin{array}{l}\text { Slope } \\
\text { Water }\end{array}$ & $\begin{array}{l}\frac{8}{\overline{\overline{0}}} \\
\underline{0} \\
\underline{0}\end{array}$ & $\begin{array}{l}9 \\
0 \\
0 \\
0 \\
0\end{array}$ & & & $\begin{array}{l}\frac{19}{\frac{2.0}{0}} \\
\frac{0}{0} \\
\frac{0.5}{0}\end{array}$ & $\begin{array}{c}20 \\
0 \\
0 \\
0 \\
0 \\
0.8\end{array}$ & 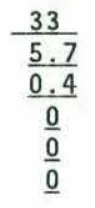 & $\begin{array}{l}\frac{34}{2.3} \\
\underline{0} \\
\underline{1.4}\end{array}$ & & $\begin{array}{c}35 \\
0 \\
0 \\
0 \\
0 \\
0.7\end{array}$ & $\begin{array}{l}\frac{47}{0.4} \\
\frac{0}{0} \\
\underline{0} \\
\underline{0}\end{array}$ & $\begin{array}{c}48 \\
0 \\
0 \\
0 \\
0.6\end{array}$ & $\frac{\frac{53}{8.8}}{\frac{0}{0}}$ & $\begin{array}{c}54 \\
0 \\
0 \\
0 \\
0.7\end{array}$ & $\begin{array}{l}\frac{59}{\underline{0}} \\
\underline{0.4} \\
\underline{0} \\
\underline{0.3} \\
\underline{0.2}\end{array}$ & $\begin{array}{c}60 \\
0 \\
0 \\
0 \\
0.4 \\
0.6\end{array}$ & $\begin{array}{l}\frac{65}{\underline{3.0}} \\
\underline{0} \\
\underline{0} \\
\frac{0.4}{\underline{0}}\end{array}$ & $\begin{array}{c}66 \\
0 \\
0 \\
0 \\
3.8 \\
1.9\end{array}$ & & & & $\begin{array}{c}\frac{4}{0} \\
0 \\
1.9 \\
1.8\end{array}$ & $\frac{\frac{5}{6.7}}{\frac{0}{0}}$ & \\
\hline $\begin{array}{l}\text { High } \\
\text { Velocity } \\
\text { Region }\end{array}$ & $\begin{array}{c}12 \\
0 \\
0 \\
0.4 \\
0.3\end{array}$ & $\frac{\frac{13}{1.0}}{\frac{0}{\underline{0}}}$ & $\frac{\frac{14}{0.9}}{\frac{0}{0}} \frac{0}{\underline{0}}$ & $\begin{array}{c}15 \\
0 \\
0 \\
0.6 \\
0.5\end{array}$ & $\begin{array}{l}26 \\
0 \\
0 \\
0 \\
0 \\
-\end{array}$ & $\begin{array}{r}27 \\
0 \\
0 \\
0 \\
0 \\
0\end{array}$ & $\begin{array}{c}30 \\
0 \\
0 \\
0 \\
0 \\
0.5\end{array}$ & $\begin{array}{l}31 \\
0 \\
0 \\
0 \\
0 \\
0\end{array}$ & $\frac{32}{\frac{0.3}{\underline{0}}}$ & $\frac{41}{\frac{0.8}{\underline{0}}}$ & $\frac{\frac{42}{1.0}}{\frac{0}{0}} \frac{0}{\underline{0}}$ & $\begin{array}{l}43 \\
0 \\
0 \\
0 \\
0 \\
0\end{array}$ & $\frac{\frac{44}{0.6}}{\frac{0}{0}}$ & & $\begin{array}{l}\frac{55}{7.1} \\
\underline{0} \\
\frac{1.6}{1.1}\end{array}$ & & & & & & & $\frac{\frac{2}{4.2}}{\frac{0}{0}}$ & $\begin{array}{c}\frac{3}{0} \\
0 \\
3.2 \\
1.2\end{array}$ & \\
\hline $\begin{array}{l}\text { Ring } \\
\text { Core }\end{array}$ & $\frac{\frac{10}{0.4}}{\frac{0}{0}} \frac{0}{\underline{0}}$ & $\begin{array}{l}11 \\
0 \\
0 \\
0.6 \\
1.1\end{array}$ & $\begin{array}{l}16 \\
0 \\
0 \\
0.7\end{array}$ & $\frac{17}{\frac{0}{0}}$ & $\begin{array}{l}21 \\
\underline{0} \\
\underline{0} \\
\underline{0} \\
\underline{0} \\
\underline{0}\end{array}$ & $\begin{array}{l}\frac{23}{1.8} \\
\frac{0}{0} \\
\frac{0}{0} \\
\underline{0}\end{array}$ & $\begin{array}{c}24 \\
0 \\
0 \\
0 \\
0 \\
0\end{array}$ & $\begin{array}{c}28 \\
0 \\
0 \\
0 \\
0.4 \\
0\end{array}$ & & & & & & & $\begin{array}{l}\frac{56}{\frac{4.1}{0}} \\
\underline{0} \\
\frac{0.5}{\underline{0}}\end{array}$ & $\begin{array}{l}\frac{57}{\underline{6.2}} \\
\underline{0} \\
\frac{0.3}{2.2} \\
\underline{5.0}\end{array}$ & $\begin{array}{c}58 \\
0 \\
0 \\
0.5 \\
0.6 \\
6.1\end{array}$ & $\begin{array}{c}\frac{61}{\frac{0}{0}} \\
\underline{0} \\
\frac{15.4}{\underline{0}}\end{array}$ & $\begin{array}{r}62 \\
0 \\
0 \\
0 \\
10.3 \\
7.5\end{array}$ & $\begin{array}{r}\frac{63}{\frac{3.0}{0}} \\
\underline{0} \\
\underline{5.0} \\
\underline{4.1}\end{array}$ & $\begin{array}{c}\frac{64}{0} \\
0 \\
0.7 \\
7.1 \\
1.9\end{array}$ & $\frac{\frac{1}{4.6}}{\frac{0}{0}} \frac{0}{\underline{0}}$ & & \\
\hline $\begin{array}{l}\text { Sargasso } \\
\text { Sea } \\
\& \\
\text { Gulf } \\
\text { Stream }\end{array}$ & & & & & & & & & & $\frac{\frac{49}{11.5}}{\frac{0}{0}}$ & $\begin{array}{c}50 \\
0 \\
0 \\
0 \\
2.8\end{array}$ & $\begin{array}{l}51 \\
0 \\
0 \\
1.5 \\
6.6\end{array}$ & $\frac{\frac{52}{10.5}}{\frac{0.8}{0.4}} \frac{\underline{4.9}}{\underline{0.8}}$ & & & & & & & & & & & \\
\hline $\begin{array}{l}\text { Slope } \\
\text { Water }\end{array}$ & $\frac{8}{-}$ & $\frac{9}{0}$ & $\frac{18}{0}$ & & $\frac{19}{0.3}$ & $\frac{20}{0}$ & $\frac{33}{0.4}$ & $\frac{34}{1.0}$ & & $\frac{35}{0.1}$ & $\frac{47}{0.1}$ & $\frac{48}{0.2}$ & $\frac{53}{2.2}$ & $\frac{54}{0.2}$ & $\frac{59}{0.2}$ & $\frac{60}{0.2}$ & $\frac{65}{0.7}$ & $\frac{66}{1.1}$ & & & & $\frac{4}{0.9}$ & $\frac{5}{1.7}$ & $\frac{6}{0}$ \\
\hline $\begin{array}{l}\text { High } \\
\text { Velocity } \\
\text { Region }\end{array}$ & $\frac{12}{0.2}$ & $\frac{13}{0.3}$ & $\frac{14}{0.2}$ & $\frac{15}{0.3}$ & $\frac{27}{0}$ & $\frac{29}{0.3}$ & $\frac{30}{0.1}$ & $\frac{31}{0}$ & $\frac{32}{-}$ & $\frac{41}{0.4}$ & $\frac{42}{0.4}$ & $\frac{43}{0}$ & $\frac{44}{0.4}$ & & $\frac{55}{2.3}$ & & & & & & & $\frac{2}{1.1}$ & $\frac{3}{1.1}$ & \\
\hline $\begin{array}{l}\text { Ring } \\
\text { Core }\end{array}$ & $\frac{10}{0.1}$ & $\frac{11}{0.4}$ & $\frac{16}{-}$ & $\frac{17}{0}$ & $\frac{21}{0}$ & $\frac{23}{0.3}$ & $\frac{24}{0}$ & $\frac{28}{0.1}$ & & $\frac{37}{0}$ & $\frac{38}{0.7}$ & $\frac{39}{0.4}$ & & & $\frac{56}{0.9}$ & $\frac{57}{2.7}$ & $\frac{58}{1.4}$ & $\frac{61}{3.1}$ & $\frac{62}{3.6}$ & $\frac{63}{2.9}$ & $\frac{64}{1.9}$ & $\frac{1}{1.2}$ & & \\
\hline SS \& GS & & & & & & & & & & $\frac{49}{2.9}$ & $\frac{50}{0.7}$ & $\frac{51}{2.0}$ & $\frac{52}{4.2}$ & & & & & & & & & & & \\
\hline
\end{tabular}


Table 46. Volume of Ceratoscopelus warmingii $\left(\mathrm{ml} / 10,000 \mathrm{~m}^{3}\right)$.

\begin{tabular}{|c|c|c|c|c|c|c|c|c|c|c|c|c|c|c|c|c|c|c|c|c|c|c|c|c|}
\hline & \multicolumn{4}{|c|}{$\begin{array}{c}\text { Oceanus } 118 \\
\text { Apri1 }\end{array}$} & \multicolumn{5}{|c|}{$\begin{array}{c}\text { Oceanus } 121 \\
\text { June }\end{array}$} & \multicolumn{5}{|c|}{$\begin{array}{l}\text { Oceanus } 125 \\
\text { August }\end{array}$} & \multicolumn{7}{|c|}{$\begin{array}{l}\text { Knorr } 98 \\
\text { Sept-Oct }\end{array}$} & \multicolumn{3}{|c|}{$\begin{array}{c}\text { A2 } 110 \\
\text { Sept-0ct }\end{array}$} \\
\hline $\begin{array}{l}\text { Slope } \\
\text { Water }\end{array}$ & $\begin{array}{l}\frac{8}{\overline{0}} \\
\underline{0} \\
\underline{0} \\
\underline{0}\end{array}$ & $\begin{array}{l}9 \\
0 \\
0 \\
0 \\
0\end{array}$ & & & $\begin{array}{l}\frac{19}{2.93} \\
\frac{0}{0} \\
\frac{0.05}{\underline{0}}\end{array}$ & $\begin{array}{l}20 \\
0 \\
0 \\
0 \\
0 \\
0.52\end{array}$ & $\frac{\frac{33}{3.83}}{\frac{1.00}{\frac{0}{0}}}$ & $\begin{array}{l}\frac{34}{0.09} \\
\underline{0} \\
\underline{0.09}\end{array}$ & & $\begin{array}{l}\frac{35}{0} \\
0 \\
0 \\
0 \\
0.07\end{array}$ & $\frac{\frac{47}{0.06}}{\frac{0}{0}}$ & $\begin{array}{l}\frac{48}{0} \\
0 \\
0 \\
0.08\end{array}$ & $\frac{\frac{53}{1.44}}{\frac{0}{0}}$ & $\begin{array}{l}54 \\
0 \\
0 \\
0 \\
0.10\end{array}$ & $\begin{array}{l}\frac{59}{\underline{0}} \\
\underline{0.04} \\
\underline{0} \\
\underline{0.03} \\
\underline{0.18}\end{array}$ & $\begin{array}{l}\frac{60}{0} \\
0 \\
0 \\
0.04 \\
0.06\end{array}$ & $\begin{array}{l}\frac{65}{\frac{0.43}{0}} \\
\underline{0} \\
\underline{0.04} \\
\underline{0}\end{array}$ & $\begin{array}{l}\frac{66}{0} \\
0 \\
0 \\
0.10 \\
0.77\end{array}$ & & & & $\begin{array}{l}\frac{4}{0} \\
0 \\
0.06 \\
0.32\end{array}$ & $\frac{\frac{5}{3.33}}{\frac{0}{0}}$ & \\
\hline $\begin{array}{l}\text { High } \\
\text { Velocity } \\
\text { Region }\end{array}$ & $\begin{array}{l}\frac{12}{0} \\
0 \\
0.04 \\
0.03\end{array}$ & $\frac{\frac{13}{0.50}}{\frac{0}{0}}$ & $\frac{\frac{14}{1.29}}{\underline{0}} \frac{\underline{0}}{\underline{0}}$ & $\begin{array}{l}\frac{15}{0} \\
0 \\
0.06 \\
0.05\end{array}$ & $\begin{array}{l}26 \\
0 \\
0 \\
0 \\
0 \\
-\end{array}$ & $\begin{array}{l}27 \\
0 \\
0 \\
0 \\
0 \\
0\end{array}$ & $\begin{array}{l}30 \\
0 \\
0 \\
0 \\
0 \\
0.05\end{array}$ & $\begin{array}{l}31 \\
0 \\
0 \\
0 \\
0 \\
0\end{array}$ & $\frac{\frac{32}{0.92}}{\frac{0}{0}}$ & $\frac{\frac{41}{0.17}}{\frac{0}{0}}$ & $\frac{\frac{42}{1.00}}{\frac{0}{0}}$ & $\begin{array}{l}43 \\
0 \\
0 \\
0 \\
0 \\
0.05\end{array}$ & $\frac{\frac{44}{0.09}}{\frac{0}{0}}$ & & $\begin{array}{l}\frac{55}{0.97} \\
\underline{0} \\
0.08 \\
\underline{0.11}\end{array}$ & & & & & & & $\frac{\frac{2}{1.67}}{\frac{0}{0}} \frac{\underline{0}}{\underline{0}}$ & $\begin{array}{l}\frac{3}{0} \\
0 \\
0.14 \\
0.08\end{array}$ & \\
\hline $\begin{array}{l}\text { Ring } \\
\text { Core }\end{array}$ & $\frac{\frac{10}{0.28}}{\frac{0}{0}}$ & $\begin{array}{l}11 \\
0 \\
0 \\
0.06 \\
0.14\end{array}$ & $\begin{array}{l}\frac{16}{0} \\
0 \\
0.07\end{array}$ & $\begin{array}{l}17 \\
\underline{0} \\
\underline{0} \\
\underline{0}\end{array}$ & $\begin{array}{l}21 \\
\underline{0} \\
\underline{0} \\
\underline{0} \\
\underline{0} \\
\underline{0}\end{array}$ & $\begin{array}{l}\frac{23}{4.12} \\
\frac{0}{0} \\
\underline{0} \\
\underline{0}\end{array}$ & $\begin{array}{l}24 \\
0 \\
0 \\
0 \\
0 \\
0\end{array}$ & $\begin{array}{l}28 \\
0 \\
0 \\
0 \\
0.35 \\
0\end{array}$ & & & & & & & $\begin{array}{l}\frac{56}{0.68} \\
\frac{0}{0} \\
\frac{0.05}{\underline{0}}\end{array}$ & $\begin{array}{l}\frac{57}{1.25} \\
\underline{0} \\
\underline{0.33} \\
\underline{0.06} \\
\underline{0.21}\end{array}$ & $\begin{array}{l}\frac{58}{0} \\
0 \\
0.05 \\
0.16 \\
0.39\end{array}$ & $\begin{array}{l}\frac{61}{1.83} \\
\frac{0}{0} \\
\frac{0.77}{\underline{0}}\end{array}$ & $\begin{array}{l}\frac{62}{0} \\
0 \\
0 \\
0.38 \\
0.39\end{array}$ & $\begin{array}{l}\frac{63}{0.53} \\
\frac{0}{0} \\
\underline{0.23} \\
\underline{0.25}\end{array}$ & $\begin{array}{l}\frac{64}{0} \\
0 \\
0.07 \\
0.29 \\
0.19\end{array}$ & $\frac{\frac{1}{2.20}}{\frac{0}{0}}$ & & \\
\hline $\begin{array}{l}\text { Sargasso } \\
\text { Sea } \\
\& \\
\text { Gulf } \\
\text { Stream }\end{array}$ & & & & & & & & & & $\frac{\frac{49}{4.68}}{\frac{0}{0}}$ & $\begin{array}{l}\frac{50}{0} \\
0 \\
0 \\
0.19\end{array}$ & $\begin{array}{l}51 \\
0 \\
0 \\
0.06 \\
0.31\end{array}$ & $\begin{array}{l}\frac{52}{1.00} \\
\frac{0.19}{0.04} \\
\frac{0.21}{0.21}\end{array}$ & & & & & & & & & & & \\
\hline $\begin{array}{l}\text { Slope } \\
\text { Water }\end{array}$ & $\frac{8}{-}$ & $\frac{9}{0}$ & $\frac{18}{0}$ & & $\frac{19}{0.31}$ & $\frac{20}{0}$ & $\frac{33}{0.37}$ & $\frac{34}{0.06}$ & & $\frac{35}{0.01}$ & $\frac{47}{0.02}$ & $\frac{48}{0.02}$ & $\frac{53}{0.36}$ & $\frac{54}{0.03}$ & $\frac{59}{0.05}$ & $\frac{60}{0.02}$ & $\frac{65}{0.09}$ & $\frac{66}{0.17}$ & & & & $\frac{4}{0.10}$ & $\frac{5}{0.83}$ & $\frac{6}{0}$ \\
\hline $\begin{array}{l}\text { High } \\
\text { Velocity } \\
\text { Region }\end{array}$ & $\frac{12}{0.02}$ & $\frac{13}{0.13}$ & $\frac{14}{0.32}$ & $\frac{15}{0.03}$ & $\frac{27}{0}$ & $\frac{29}{0.48}$ & $\frac{30}{0.01}$ & $\frac{31}{0}$ & $\frac{32}{-}$ & $\frac{41}{0.09}$ & $\frac{42}{0.38}$ & $\frac{43}{0.01}$ & $\frac{44}{0.05}$ & & $\frac{55}{0.25}$ & & & & & & & $\frac{2}{0.42}$ & $\frac{3}{0.06}$ & \\
\hline $\begin{array}{l}\text { Ring } \\
\text { Core }\end{array}$ & $\frac{10}{0.07}$ & $\frac{11}{0.05}$ & $\frac{16}{-}$ & $\frac{17}{0}$ & $\frac{21}{0}$ & $\frac{23}{0.62}$ & $\frac{24}{0}$ & $\frac{28}{0.09}$ & & $\frac{37}{0}$ & $\frac{38}{0.12}$ & $\frac{39}{0.15}$ & & & $\frac{56}{0.15}$ & $\frac{57}{0.37}$ & $\frac{58}{0.12}$ & $\frac{61}{0.52}$ & $\frac{62}{0.15}$ & $\frac{63}{0.29}$ & $\frac{64}{0.10}$ & $\frac{1}{0.55}$ & & \\
\hline SS \& GS & & & & & & & & & & $\frac{49}{1.17}$ & $\frac{50}{0.05}$ & $\frac{51}{0.09}$ & $\frac{52}{0.36}$ & & & & & & . & & & & & \\
\hline
\end{tabular}


Table 47. Abundance of Chauliodus danae (specimens $/ 10,000 \mathrm{~m}^{3}$ ).

\begin{tabular}{|c|c|c|c|c|c|c|c|c|c|c|c|c|c|c|c|c|c|c|c|c|c|c|c|c|}
\hline & \multicolumn{4}{|c|}{$\begin{array}{c}\text { Oceanus } 118 \\
\text { April }\end{array}$} & \multicolumn{5}{|c|}{$\begin{array}{c}\text { Oceanus } \\
\text { June }\end{array}$} & \multicolumn{5}{|c|}{$\begin{array}{l}\text { Oceanus } 125 \\
\text { August }\end{array}$} & \multicolumn{7}{|c|}{$\begin{array}{l}\text { Knorr } 98 \\
\text { Sept-Oct }\end{array}$} & \multicolumn{3}{|c|}{$\begin{array}{c}\text { A2 } 110 \\
\text { Sept-Oct }\end{array}$} \\
\hline $\begin{array}{l}\text { Slope } \\
\text { Water }\end{array}$ & $\begin{array}{l}\frac{8}{=} \\
\frac{0.6}{\underline{0}} \\
\underline{0}\end{array}$ & $\begin{array}{l}\frac{9}{0} \\
0 \\
0 \\
0\end{array}$ & & & $\frac{19}{\underline{0}}$ & $\begin{array}{r}20 \\
0 \\
0 \\
0 \\
0 \\
0\end{array}$ & $\begin{array}{l}\frac{33}{\underline{0}} \\
\underline{0} \\
\underline{0} \\
\underline{0} \\
\underline{0}\end{array}$ & $\begin{array}{r}\frac{34}{\underline{0}} \\
\underline{0} \\
\underline{0}\end{array}$ & & $\begin{array}{c}35 \\
0 \\
0 \\
0 \\
0 \\
0\end{array}$ & $\begin{array}{r}47 \\
\underline{0} \\
\underline{0} \\
\underline{0} \\
\underline{0}\end{array}$ & $\begin{array}{r}48 \\
0 \\
0 \\
0 \\
0\end{array}$ & $\begin{array}{l}53 \\
\underline{0} \\
\underline{0} \\
\underline{0} \\
\underline{0}\end{array}$ & $\begin{array}{r}54 \\
0 \\
0 \\
0 \\
0\end{array}$ & $\begin{array}{l}\frac{59}{0.3} \\
\frac{0}{0} \\
\underline{0} \\
\underline{0}\end{array}$ & $\begin{array}{r}60 \\
0 \\
0 \\
0 \\
0 \\
0\end{array}$ & $\begin{array}{l}65 \\
\underline{0} \\
\underline{0} \\
\underline{0} \\
\underline{0} \\
\underline{0}\end{array}$ & $\begin{array}{c}66 \\
0 \\
0 \\
0 \\
0 \\
0\end{array}$ & & & & $\begin{array}{l}4 \\
0 \\
0 \\
0 \\
0\end{array}$ & $\begin{array}{l}\frac{5}{0} \\
\underline{0} \\
\underline{0} \\
\underline{0} \\
\underline{0}\end{array}$ & \\
\hline $\begin{array}{l}\text { High } \\
\text { Velocity } \\
\text { Region }\end{array}$ & $\begin{array}{c}12 \\
0 \\
0 \\
0.4 \\
0\end{array}$ & $\begin{array}{l}\frac{13}{0.5} \\
\underline{0} \underline{0.2}\end{array}$ & $\begin{array}{r}14 \\
\underline{0} \\
\underline{0} \\
\underline{0} \\
\underline{0}\end{array}$ & $\begin{array}{r}15 \\
0 \\
0 \\
0 \\
0\end{array}$ & $\begin{array}{r}26 \\
0 \\
0 \\
0 \\
0 \\
-\end{array}$ & $\begin{array}{r}27 \\
0 \\
0 \\
0 \\
0 \\
0\end{array}$ & $\begin{array}{c}30 \\
0 \\
0 \\
0.6 \\
0 \\
0\end{array}$ & $\begin{array}{r}31 \\
0 \\
0 \\
0 \\
0 \\
0\end{array}$ & $\begin{array}{c}\frac{32}{\underline{0}} \\
\underline{0} \underline{0.5} \\
\underline{0}\end{array}$ & $\frac{41}{\frac{0}{0}}$ & $\begin{array}{r}\frac{42}{\underline{0}} \\
\underline{0} \\
\underline{0} \\
\underline{0}\end{array}$ & $\begin{array}{r}43 \\
0 \\
0 \\
0 \\
0 \\
0\end{array}$ & $\begin{array}{r}44 \\
\underline{0} \\
\underline{0} \\
\underline{0}\end{array}$ & & $\frac{55}{\frac{1.1}{\underline{0}}}$ & & & & & & & $\begin{array}{l}\frac{2}{0} \\
\frac{0.7}{\underline{0}} \\
\underline{0}\end{array}$ & $\begin{array}{c}\frac{3}{0} \\
1.1 \\
0 \\
0\end{array}$ & \\
\hline $\begin{array}{l}\text { Ring } \\
\text { Core }\end{array}$ & $\begin{array}{c}\frac{10}{\underline{0}} \\
\underline{0} \\
\frac{1.8}{\underline{0}}\end{array}$ & $\begin{array}{r}11 \\
0 \\
0 \\
0 \\
0\end{array}$ & $\begin{array}{r}16 \\
0 \\
0 \\
0\end{array}$ & $\begin{array}{c}\frac{17}{\underline{0}} \\
\underline{1.6} \\
\underline{0.2}\end{array}$ & $\begin{array}{l}\frac{21}{0} \\
\frac{0.2}{7.6} \\
\underline{9.6} \\
\underline{0.6}\end{array}$ & $\begin{array}{l}\frac{23}{0.6} \\
\underline{0} \\
\frac{2.0}{0.5} \\
\frac{0}{\underline{0}}\end{array}$ & $\begin{array}{c}24 \\
0.3 \\
0 \\
3.5 \\
0.6 \\
0\end{array}$ & $\begin{array}{c}28 \\
0 \\
0 \\
4.6 \\
0 \\
0\end{array}$ & & & & & & & $\begin{array}{l}\frac{56}{\underline{0}} \\
\underline{0} \\
\underline{0} \\
\frac{0.5}{\underline{0}}\end{array}$ & $\begin{array}{l}\frac{57}{\frac{0.3}{\underline{0}}} \\
\underline{0} \\
\frac{1.3}{\underline{0}}\end{array}$ & $\begin{array}{c}58 \\
0 \\
0 \\
0 \\
0.7 \\
0\end{array}$ & $\begin{array}{l}\frac{61}{0.5} \\
\underline{0} \\
\frac{0.7}{0.8} \\
\underline{0}\end{array}$ & $\begin{array}{c}62 \\
0 \\
0 \\
0.6 \\
1.4 \\
0\end{array}$ & $\begin{array}{l}\frac{63}{\underline{0.3}} \\
\frac{0}{\underline{0}} \\
\frac{1.9}{\underline{0}}\end{array}$ & $\begin{array}{c}64 \\
0 \\
0.6 \\
0.7 \\
0.5 \\
0\end{array}$ & $\begin{array}{l}\frac{1}{1.6} \\
\frac{0}{1.9} \\
\underline{0}\end{array}$ & & \\
\hline $\begin{array}{l}\text { Sargasso } \\
\text { Sea } \\
\& \\
\text { Gulf } \\
\text { Stream }\end{array}$ & & & & & & & & & & $\begin{array}{l}\frac{49}{0.5} \\
\underline{0} \\
\underline{0.9} \\
\underline{0.5}\end{array}$ & $\begin{array}{c}50 \\
0 \\
0 \\
2.4 \\
0.3\end{array}$ & $\begin{array}{c}51 \\
0 \\
0 \\
2.4 \\
0\end{array}$ & $\begin{array}{c}\frac{52}{\underline{0}} \\
\underline{0} \\
\underline{0.9} \\
\underline{0}\end{array}$ & & & & & & & & & & & \\
\hline $\begin{array}{l}\text { Slope } \\
\text { Water }\end{array}$ & $\frac{8}{-}$ & $\frac{9}{0}$ & $\frac{18}{0}$ & & $\frac{19}{0}$ & $\frac{20}{0}$ & $\frac{33}{0}$ & $\frac{34}{0}$ & & $\frac{35}{0}$ & $\frac{47}{0}$ & $\frac{48}{0}$ & $\frac{53}{0}$ & $\frac{54}{0}$ & $\frac{59}{0.1}$ & $\frac{60}{0}$ & $\frac{65}{0}$ & $\frac{66}{0}$ & & & & $\frac{4}{0}$ & $\frac{5}{0}$ & $\frac{6}{0}$ \\
\hline $\begin{array}{l}\text { High } \\
\text { Velocity } \\
\text { Region }\end{array}$ & $\frac{12}{0.1}$ & $\frac{13}{0.3}$ & $\frac{14}{0}$ & $\frac{15}{0}$ & $\frac{27}{0}$ & $\frac{29}{0.6}$ & $\frac{30}{0.1}$ & $\frac{31}{0}$ & $\frac{32}{-}$ & $\frac{41}{0}$ & $\frac{42}{0}$ & $\frac{43}{0}$ & $\frac{44}{0}$ & & $\frac{55}{0.2}$ & & & & & & & $\frac{2}{0.2}$ & $\frac{3}{0.3}$ & \\
\hline $\begin{array}{l}\text { Ring } \\
\text { Core }\end{array}$ & $\frac{10}{0.5}$ & $\frac{11}{0}$ & $\frac{16}{-}$ & $\frac{17}{0.5}$ & $\frac{21}{3.6}$ & $\frac{23}{0.6}$ & $\frac{24}{0.9}$ & $\frac{28}{1.2}$ & & $\frac{37}{0.1}$ & $\frac{38}{0.2}$ & $\frac{39}{0}$ & & & $\frac{56}{0.1}$ & $\frac{57}{0.3}$ & $\frac{58}{0.1}$ & $\frac{61}{0.4}$ & $\frac{62}{0.4}$ & $\frac{63}{0.5}$ & $\frac{64}{0.2}$ & $\frac{1}{0.9}$ & & \\
\hline SS \& GS & & & & & & & & & & $\frac{49}{0.5}$ & $\frac{50}{0.7}$ & $\frac{51}{0.6}$ & $\frac{52}{0.2}$ & & & & & & & & & & & \\
\hline
\end{tabular}


Table 48. Volume of Chauliodus danae $\left(\mathrm{ml} / 10,000 \mathrm{~m}^{3}\right)$.

\begin{tabular}{|c|c|c|c|c|c|c|c|c|c|c|c|c|c|c|c|c|c|c|c|c|c|c|c|c|}
\hline & \multicolumn{4}{|c|}{$\begin{array}{c}\text { Oceanus } 118 \\
\text { April }\end{array}$} & \multicolumn{5}{|c|}{$\begin{array}{c}\text { Oceanus } 121 \\
\text { June }\end{array}$} & \multicolumn{5}{|c|}{$\begin{array}{l}\text { Oceanus } 125 \\
\text { August }\end{array}$} & \multicolumn{7}{|c|}{$\begin{array}{l}\text { Knorr } 98 \\
\text { Sept-0ct }\end{array}$} & \multicolumn{3}{|c|}{$\begin{array}{c}\text { A2 } 110 \\
\text { Sept-0ct }\end{array}$} \\
\hline $\begin{array}{l}\text { Slope } \\
\text { Water }\end{array}$ & $\begin{array}{l}\frac{8}{1=} \\
\frac{1.06}{\underline{0}} \\
\underline{0}\end{array}$ & $\begin{array}{l}9 \\
0 \\
0 \\
0 \\
0\end{array}$ & & & $\begin{array}{l}\frac{19}{0} \\
\frac{0}{0} \\
\underline{0} \\
\underline{0} \\
\underline{0}\end{array}$ & $\begin{array}{l}20 \\
0 \\
0 \\
0 \\
0 \\
0\end{array}$ & $\begin{array}{l}33 \\
\underline{0} \\
\underline{0} \\
\underline{0} \\
\underline{0} \\
\underline{0}\end{array}$ & $\begin{array}{l}\frac{34}{\underline{0}} \\
\underline{0} \\
\underline{0}\end{array}$ & & $\begin{array}{l}35 \\
0 \\
0 \\
0 \\
0 \\
0\end{array}$ & $\begin{array}{l}47 \\
\underline{0} \\
\underline{0} \\
\underline{0} \\
\underline{0}\end{array}$ & $\begin{array}{l}\frac{48}{0} \\
0 \\
0 \\
0.71\end{array}$ & $\begin{array}{l}\frac{53}{0} \\
\underline{0} \\
\underline{0} \\
\underline{0} \\
\underline{0}\end{array}$ & $\begin{array}{l}\frac{54}{0} \\
0 \\
0 \\
0\end{array}$ & $\begin{array}{l}\frac{59}{0.03} \\
\frac{0}{0} \\
\underline{0} \\
\underline{0}\end{array}$ & $\begin{array}{l}60 \\
0 \\
0 \\
0 \\
0 \\
0\end{array}$ & $\begin{array}{l}\frac{65}{\underline{0}} \\
\underline{0} \\
\underline{0} \\
\underline{0} \\
\underline{0}\end{array}$ & $\begin{array}{l}66 \\
0 \\
0 \\
0 \\
0 \\
0\end{array}$ & & & & $\begin{array}{l}\frac{4}{0} \\
0 \\
0 \\
0\end{array}$ & $\begin{array}{l}\frac{5}{0} \\
\underline{0} \\
\underline{0} \\
\underline{0}\end{array}$ & \\
\hline $\begin{array}{l}\text { High } \\
\text { Velocity } \\
\text { Region }\end{array}$ & $\begin{array}{l}\frac{12}{0} \\
0 \\
0.42 \\
0\end{array}$ & $\begin{array}{l}\frac{13}{0.75} \\
\underline{0} \\
\underline{0.02}\end{array}$ & $\begin{array}{l}14 \\
\underline{0} \\
\underline{0} \\
\underline{0} \\
\underline{0}\end{array}$ & $\begin{array}{l}\frac{15}{0} \\
0 \\
0 \\
0\end{array}$ & $\begin{array}{l}26 \\
0 \\
0 \\
0 \\
0 \\
-\end{array}$ & $\begin{array}{l}27 \\
0 \\
0 \\
0 \\
0 \\
0\end{array}$ & $\begin{array}{l}30 \\
0 \\
0 \\
0 \\
0 \\
0\end{array}$ & $\begin{array}{c}31 \\
0 \\
0 \\
0.04 \\
0 \\
0\end{array}$ & $\begin{array}{l}\frac{32}{\underline{0}} \\
\underline{0} \\
\underline{0} \\
\underline{0.87}\end{array}$ & $\begin{array}{l}41 \\
\underline{0} \\
\underline{0} \\
\underline{0}\end{array}$ & $\begin{array}{l}\frac{42}{\underline{0}} \\
\underline{0} \\
\underline{0} \\
\underline{0}\end{array}$ & $\begin{array}{l}43 \\
0 \\
0 \\
0 \\
0 \\
0\end{array}$ & $\begin{array}{l}\frac{44}{\underline{0}} \\
\underline{0} \\
\underline{0}\end{array}$ & & $\frac{\frac{55}{0.05}}{\frac{0}{\underline{0}}}$ & & & & & & & $\begin{array}{l}\frac{2}{\underline{0}} \\
\frac{0.03}{\underline{0}} \\
\underline{0}\end{array}$ & $\begin{array}{l}\frac{3}{0} \\
0.95 \\
0 \\
0\end{array}$ & \\
\hline $\begin{array}{l}\text { Ring } \\
\text { Core }\end{array}$ & $\begin{array}{l}\frac{10}{\underline{0}} \\
\underline{0} \\
\frac{1.82}{\underline{0}}\end{array}$ & $\begin{array}{l}\frac{11}{0} \\
0 \\
0 \\
0\end{array}$ & $\begin{array}{l}16 \\
0 \\
0 \\
0\end{array}$ & $\begin{array}{l}\frac{17}{0} \\
\underline{\underline{2.11}} \\
\underline{1.08}\end{array}$ & $\begin{array}{l}\frac{21}{\underline{0}} \\
\frac{0.10}{2.73} \\
\underline{\underline{2.92}} \\
\underline{0.06}\end{array}$ & $\begin{array}{l}\frac{23}{0.06} \\
\underline{0} \\
\frac{2.25}{0.05} \\
\underline{0}\end{array}$ & $\begin{array}{c}\frac{24}{0.03} \\
0 \\
0.19 \\
0.06 \\
0\end{array}$ & $\begin{array}{l}28 \\
0 \\
0 \\
3.57 \\
0 \\
0\end{array}$ & & & & & & & $\begin{array}{l}\frac{56}{\underline{0}} \\
\underline{0} \\
\underline{0} \\
\frac{0.11}{\underline{0}}\end{array}$ & $\begin{array}{l}\frac{57}{\underline{0.03}} \\
\underline{0} \\
\underline{0} \\
\frac{0.16}{\underline{0}}\end{array}$ & $\begin{array}{l}\frac{58}{0} \\
0 \\
0 \\
0.10 \\
0\end{array}$ & $\begin{array}{l}\frac{61}{0.02} \\
0 \\
\frac{0.07}{1.15} \\
\underline{0}\end{array}$ & $\begin{array}{c}\frac{62}{0} \\
0 \\
0.02 \\
0.05 \\
0\end{array}$ & $\begin{array}{l}\frac{63}{0.02} \\
\frac{0}{0} \\
\frac{1.92}{\underline{0}}\end{array}$ & $\begin{array}{c}\frac{6 t_{r}}{0} \\
0.06 \\
0.07 \\
0.05 \\
0\end{array}$ & $\begin{array}{l}\frac{1}{0.16} \\
\underline{0} \\
\underline{0.47} \\
\underline{0}\end{array}$ & & \\
\hline $\begin{array}{l}\text { Sargasso } \\
\text { Sea } \\
\& \\
\text { Gulf } \\
\text { Stream }\end{array}$ & & & & & & & & & & $\begin{array}{l}\frac{49}{0.02} \\
\underline{0} \\
\underline{0.04}\end{array}$ & $\begin{array}{l}\frac{50}{0} \\
0 \\
0.52 \\
0.47\end{array}$ & $\begin{array}{c}\frac{51}{0} \\
0 \\
1.08 \\
0\end{array}$ & $\begin{array}{l}\frac{52}{\underline{0}} \\
\underline{0} \\
\underline{0.22} \\
\underline{0}\end{array}$ & & & & & & & & & & & \\
\hline $\begin{array}{l}\text { Slope } \\
\text { Water }\end{array}$ & $\frac{8}{-}$ & $\frac{9}{0}$ & $\frac{18}{0}$ & & $\frac{19}{0}$ & $\frac{20}{0}$ & $\frac{33}{0}$ & $\frac{34}{0}$ & & $\frac{35}{0}$ & $\frac{47}{0}$ & $\frac{48}{0.18}$ & $\frac{53}{0}$ & $\frac{54}{0}$ & $\frac{59}{0.01}$ & $\frac{60}{0}$ & $\frac{65}{0}$ & $\frac{66}{0}$ & & & & $\frac{4}{0}$ & $\frac{5}{0}$ & $\frac{6}{0}$ \\
\hline $\begin{array}{l}\text { High } \\
\text { Velocity } \\
\text { Region }\end{array}$ & $\frac{12}{0.11}$ & $\frac{13}{0.20}$ & $\frac{14}{0}$ & $\frac{15}{0}$ & $\frac{27}{0}$ & $\frac{29}{0.83}$ & $\frac{30}{0.01}$ & $\frac{31}{0}$ & $\frac{32}{-}$ & $\frac{41}{0}$ & $\frac{42}{0}$ & $\frac{43}{0}$ & $\frac{44}{0}$ & & $\frac{55}{0.01}$ & & & & & & & $\frac{2}{0.01}$ & $\frac{3}{0.24}$ & \\
\hline $\begin{array}{l}\text { Ring } \\
\text { Core }\end{array}$ & $\frac{10}{0.46}$ & $\frac{11}{0}$ & $\frac{16}{-}$ & $\frac{17}{0.80}$ & $\frac{21}{1.18}$ & $\frac{23}{0.47}$ & $\frac{24}{0.06}$ & $\frac{28}{0.89}$ & & $\frac{37}{0.03}$ & $\frac{38}{0.01}$ & $\frac{39}{0}$ & & & $\frac{56}{0.02}$ & $\frac{57}{0.04}$ & $\frac{58}{0.02}$ & $\frac{61}{0.39}$ & $\frac{62}{0.01}$ & $\frac{63}{0.39}$ & $\frac{64}{0.02}$ & $\frac{1}{0.16}$ & & \\
\hline SS \& GS & & & & & & & & & & $\frac{49}{0.02}$ & $\frac{50}{0.25}$ & $\frac{51}{0.27}$ & $\frac{52}{0.06}$ & & & & & & & & & & & \\
\hline
\end{tabular}


Table 49. Abundance of Chauliodus sloani (specimens $/ 10,000 \mathrm{~m}^{3}$ ).

\begin{tabular}{|c|c|c|c|c|c|c|c|c|c|c|c|c|c|c|c|c|c|c|c|c|c|c|c|c|}
\hline & \multicolumn{4}{|c|}{$\begin{array}{c}\text { Oceanus } 118 \\
\text { April }\end{array}$} & \multicolumn{5}{|c|}{$\begin{array}{c}\text { Oceanus } 121 \\
\text { June }\end{array}$} & \multicolumn{5}{|c|}{$\begin{array}{l}\text { Oceanus } 125 \\
\text { Auqust }\end{array}$} & \multicolumn{7}{|c|}{$\begin{array}{l}\text { Knorr } 98 \\
\text { Sept-0ct }\end{array}$} & \multicolumn{3}{|c|}{$\begin{array}{c}\text { A2 } 110 \\
\text { Sept-0ct }\end{array}$} \\
\hline $\begin{array}{l}\text { Slope } \\
\text { Water }\end{array}$ & $\begin{array}{l}\frac{8}{=} \\
\frac{0.6}{\underline{0}} \\
\underline{0}\end{array}$ & $\begin{array}{c}\frac{9}{0} \\
1.8 \\
0 \\
0\end{array}$ & & & $\begin{array}{c}\frac{19}{\underline{0}} \\
\underline{0} \\
\underline{0} \\
\underline{0.5}\end{array}$ & $\begin{array}{c}\frac{20}{0} \\
1.1 \\
1.3 \\
0.5 \\
0\end{array}$ & $\begin{array}{l}\frac{33}{0.3} \\
\frac{0}{\underline{0}} \\
\underline{0} \\
\underline{0.3} \\
\underline{0.3}\end{array}$ & $\begin{array}{l}\frac{34}{\underline{0.9}} \\
\underline{0} \\
\underline{0.9}\end{array}$ & & $\begin{array}{c}35 \\
0 \\
0.2 \\
52.0 \\
1.1 \\
0\end{array}$ & $\frac{\frac{47}{0.2}}{\frac{0.3}{0.4}} \frac{0}{\underline{0}}$ & $\begin{array}{c}48 \\
0 \\
0 \\
0.3 \\
0.2\end{array}$ & $\begin{array}{l}\frac{53}{0.2} \\
\underline{0} \\
\frac{0.8}{\underline{0}}\end{array}$ & $\begin{array}{c}54 \\
0 \\
2.2 \\
5.5 \\
0\end{array}$ & $\begin{array}{c}\frac{59}{\underline{0}} \\
\underline{0.8} \\
\underline{0} \\
\underline{0} \\
\underline{0}\end{array}$ & $\begin{array}{c}60 \\
0 \\
0 \\
1.4 \\
0.4 \\
0.3\end{array}$ & $\begin{array}{l}\frac{65}{1.3} \\
\underline{0} \\
\frac{1.8}{0.4} \\
\underline{0.2}\end{array}$ & $\begin{array}{c}\frac{66}{0} \\
0.7 \\
3.3 \\
0 \\
0.4\end{array}$ & & & & $\begin{array}{l}\frac{4}{0} \\
1.7 \\
0.9 \\
0\end{array}$ & $\frac{\frac{5}{1.0}}{\frac{1.1}{\frac{1.3}{0}}}$ & \\
\hline $\begin{array}{l}\text { High } \\
\text { Velocity } \\
\text { Region }\end{array}$ & $\begin{array}{c}12 \\
0 \\
1.2 \\
0 \\
0\end{array}$ & $\begin{array}{l}\frac{13}{\underline{0}} \\
\underline{0.6} \\
\underline{0.7}\end{array}$ & $\begin{array}{l}\frac{14}{\underline{0}} \\
\frac{0.7}{0.6} \\
\underline{0}\end{array}$ & $\begin{array}{c}15 \\
0 \\
1.2 \\
0 \\
0\end{array}$ & $\begin{array}{c}26 \\
0 \\
0 \\
1.9 \\
1.7 \\
-\end{array}$ & $\begin{array}{c}27 \\
0 \\
0 \\
1.4 \\
0.4 \\
0\end{array}$ & $\begin{array}{c}30 \\
0 \\
0 \\
5.9 \\
0 \\
0\end{array}$ & $\begin{array}{c}31 \\
0 \\
0 \\
1.1 \\
0.3 \\
0\end{array}$ & $\frac{\frac{32}{0.3}}{\frac{0.8}{2.5}} \frac{\underline{0}}{\underline{0}}$ & $\frac{41}{\underline{0}} \frac{0.3}{\underline{0}}$ & $\frac{\frac{42}{0}}{\frac{\underline{0}}{2.9}} \frac{0.7}{\underline{0}}$ & $\begin{array}{c}43 \\
0 \\
0 \\
1.7 \\
0 \\
0\end{array}$ & $\frac{\frac{44}{0.5}}{\frac{0.3}{\underline{0}}}$ & & $\begin{array}{c}\frac{55}{\underline{0}} \\
\underline{\underline{0}} \\
\underline{2.8} \\
\underline{0}\end{array}$ & & & & & & & $\frac{\frac{2}{0.8}}{\frac{0.7}{1.3}}$ & $\begin{array}{c}\frac{3}{0} \\
3.2 \\
1.1 \\
0\end{array}$ & \\
\hline $\begin{array}{l}\text { Ring } \\
\text { Core }\end{array}$ & $\frac{10}{\frac{0.4}{\underline{0}}} \frac{0}{\underline{0}}$ & $\begin{array}{c}11 \\
0 \\
0 \\
0 \\
0.4\end{array}$ & $\begin{array}{c}16 \\
0 \\
0.7 \\
0\end{array}$ & $\begin{array}{c}\frac{17}{0} \\
\underline{0} \\
\underline{0.2}\end{array}$ & $\frac{\frac{21}{1.0}}{\frac{\frac{0.8}{2.4}}{\frac{8.3}{0}}}$ & $\frac{\frac{23}{4.1}}{\frac{\frac{0.7}{0.0}}{\frac{206}{\underline{0}}}}$ & $\begin{array}{c}24 \\
5.9 \\
0 \\
17.8 \\
3.1 \\
0\end{array}$ & $\begin{array}{c}\frac{28}{0.4} \\
3.1 \\
52.9 \\
0 \\
0\end{array}$ & & & & & & & $\begin{array}{l}\frac{56}{\underline{0.6}} \\
\underline{0} \\
\frac{1.6}{2.6} \\
\underline{0}\end{array}$ & $\begin{array}{c}\frac{57}{\underline{0}} \\
\underline{0} \\
\frac{1.3}{2.5} \\
\underline{0}\end{array}$ & $\begin{array}{c}58 \\
0 \\
0 \\
3.3 \\
2.9 \\
0\end{array}$ & $\begin{array}{l}\frac{61}{0.5} \\
\underline{0} \\
\underline{0} \\
\underline{2.3} \\
\underline{0.5}\end{array}$ & $\begin{array}{c}62 \\
0 \\
0 \\
1.4 \\
2.4 \\
0.7\end{array}$ & $\begin{array}{l}\frac{63}{0.2} \\
\underline{0} \\
\frac{1.4}{1.5} \\
\underline{0}\end{array}$ & $\begin{array}{c}64 \\
0 \\
0 \\
2.1 \\
5.7 \\
0.4\end{array}$ & $\frac{\frac{1}{0.6}}{\frac{0.6}{7.8}}$ & & \\
\hline $\begin{array}{l}\text { Sargasso } \\
\text { Sea } \\
\& \\
\text { Gulf } \\
\text { Stream }\end{array}$ & & & & & & & & & & $\begin{array}{l}\frac{49}{0.8} \\
\frac{0.2}{2.0} \\
\underline{1.5}\end{array}$ & $\begin{array}{c}50 \\
0 \\
0.2 \\
1.4 \\
1.3\end{array}$ & $\begin{array}{c}51 \\
0 \\
0 \\
3.3 \\
0\end{array}$ & $\begin{array}{l}\frac{52}{0.7} \\
\frac{0.8}{0.8} \\
\underline{3.9} \\
\underline{0.3}\end{array}$ & & & & & & & & & & & \\
\hline $\begin{array}{l}\text { Slope } \\
\text { Water }\end{array}$ & $\frac{8}{-}$ & $\frac{9}{0.4}$ & $\frac{18}{0.3}$ & & $\frac{19}{0.1}$ & $\frac{20}{0.7}$ & $\frac{33}{0.1}$ & $\frac{34}{0.6}$ & & $\frac{35}{10.7}$ & $\frac{47}{0.2}$ & $\frac{48}{0.1}$ & $\frac{53}{0.2}$ & $\frac{54}{1.9}$ & $\frac{59}{0.2}$ & $\frac{60}{0.4}$ & $\frac{65}{0.7}$ & $\frac{66}{0.8}$ & & & & $\frac{4}{0.7}$ & $\frac{5}{0.8}$ & $\frac{6}{0.6}$ \\
\hline $\begin{array}{l}\text { High } \\
\text { Velocity } \\
\text { Region }\end{array}$ & $\frac{12}{0.3}$ & $\frac{13}{0.5}$ & $\frac{14}{0.3}$ & $\frac{15}{0.3}$ & $\frac{27}{0.4}$ & $\frac{29}{1.0}$ & $\frac{30}{1.2}$ & $\frac{31}{0.3}$ & $\frac{32}{-}$ & $\frac{41}{0.1}$ & $\frac{42}{0.8}$ & $\frac{43}{0.3}$ & $\frac{44}{0.3}$ & & $\frac{55}{1.1}$ & & & & & & & $\frac{2}{0.7}$ & $\frac{3}{1.1}$ & \\
\hline $\begin{array}{l}\text { Ring } \\
\text { Core }\end{array}$ & $\frac{10}{0.1}$ & $\frac{11}{0.1}$ & $\frac{16}{-}$ & $\frac{17}{0.1}$ & $\frac{21}{14.5}$ & $\frac{23}{6.5}$ & $\frac{24}{6.4}$ & $\frac{28}{13.9}$ & & $\frac{37}{0.2}$ & $\frac{38}{0.5}$ & $\frac{39}{0.9}$ & & & $\frac{56}{1.0}$ & $\frac{57}{0.8}$ & $\frac{58}{1.3}$ & $\frac{61}{0.7}$ & $\frac{62}{0.9}$ & $\frac{63}{0.7}$ & $\frac{64}{1.4}$ & $\frac{1}{2.5}$ & & \\
\hline SS \& GS & & & & & & & & & & $\frac{49}{1.1}$ & $\frac{50}{0.7}$ & $\frac{51}{0.8}$ & $\frac{52}{1.4}$ & & & & & & & & & & & \\
\hline
\end{tabular}


Table 50. Volume of Chauliodus sloani $\left(\mathrm{ml} / 10,000 \mathrm{~m}^{3}\right)$.

\begin{tabular}{|c|c|c|c|c|c|c|c|c|c|c|c|c|c|c|c|c|c|c|c|c|c|c|c|c|}
\hline & \multicolumn{4}{|c|}{$\begin{array}{c}\text { Oceanus } 118 \\
\text { April }\end{array}$} & \multicolumn{5}{|c|}{$\begin{array}{c}\text { Oceanus } 121 \\
\text { June }\end{array}$} & \multicolumn{5}{|c|}{$\begin{array}{c}\text { Oceanus } 125 \\
\text { August }\end{array}$} & \multicolumn{7}{|c|}{$\begin{array}{l}\text { Knorr } 98 \\
\text { Sept-0ct }\end{array}$} & \multicolumn{3}{|c|}{$\begin{array}{c}\text { A2 } 110 \\
\text { Sept-0ct }\end{array}$} \\
\hline $\begin{array}{l}\text { Slope } \\
\text { Water }\end{array}$ & $\begin{array}{l}\frac{8}{=} \\
\frac{4.12}{\underline{0}}\end{array}$ & $\begin{array}{l}\frac{9}{0} \\
1.18 \\
0 \\
0\end{array}$ & & & $\begin{array}{l}\frac{19}{\underline{0}} \\
\frac{0}{0} \\
\underline{0.16} \\
\underline{0}\end{array}$ & $\begin{array}{c}\frac{20}{0} \\
0.53 \\
8.26 \\
10.00 \\
0\end{array}$ & $\begin{array}{l}\frac{33}{0.03} \\
\frac{0}{0} \\
\underline{0} \\
\underline{3.09}\end{array}$ & $\begin{array}{l}\frac{34}{1.36} \\
\underline{0} \\
\underline{0.05}\end{array}$ & & $\begin{array}{c}\frac{35}{0} \\
0.02 \\
6.67 \\
18.33 \\
0\end{array}$ & $\frac{\frac{47}{0.02}}{\frac{1.50}{2.16}} \frac{0}{\underline{n}}$ & $\begin{array}{l}\frac{48}{0} \\
0 \\
4.71 \\
3.98\end{array}$ & $\begin{array}{l}\frac{53}{0.02} \\
\underline{0} \\
\frac{4.62}{\underline{0}}\end{array}$ & $\begin{array}{c}\frac{54}{0} \\
0.72 \\
12.12 \\
0\end{array}$ & $\frac{\frac{59}{\underline{0}}}{\frac{1.00}{\underline{0}}}$ & $\begin{array}{l}\frac{60}{0} \\
0 \\
0.41 \\
2.17 \\
3.53\end{array}$ & $\begin{array}{l}\frac{65}{0.96} \\
\underline{0} \\
\frac{8.18}{9.60} \\
\underline{4.81}\end{array}$ & $\begin{array}{c}\frac{66}{0} \\
0.07 \\
2.04 \\
0 \\
10.00\end{array}$ & & & & $\begin{array}{c}\frac{4}{0} \\
2.39 \\
3.37 \\
0\end{array}$ & $\frac{\frac{5}{1.07}}{\frac{0.07}{7.19}} \frac{0}{\underline{0}}$ & \\
\hline $\begin{array}{l}\text { High } \\
\text { Velocity } \\
\text { Region }\end{array}$ & $\begin{array}{l}\frac{12}{0} \\
0.19 \\
0 \\
0\end{array}$ & $\begin{array}{l}\frac{13}{\underline{0}} \\
\underline{0.11} \\
\underline{9.27}\end{array}$ & $\begin{array}{c}\frac{14}{\underline{0}} \\
\frac{0.17}{17.65} \\
\underline{0}\end{array}$ & $\begin{array}{l}\frac{15}{0} \\
0.24 \\
0 \\
0\end{array}$ & $\begin{array}{c}26 \\
0 \\
0 \\
0.14 \\
34.48 \\
-\end{array}$ & $\begin{array}{c}27 \\
0 \\
0 \\
3.18 \\
17.50 \\
0\end{array}$ & $\begin{array}{l}\frac{30}{0} \\
0 \\
0.28 \\
0 \\
0\end{array}$ & $\begin{array}{l}\frac{31}{0} \\
0 \\
0.29 \\
0.03 \\
0\end{array}$ & $\frac{\frac{32}{0.53}}{\frac{0.04}{0.04}}$ & $\begin{array}{c}\frac{41}{\underline{0}} \\
\frac{13.43}{\underline{0}}\end{array}$ & $\begin{array}{c}\frac{42}{0} \\
\frac{0.15}{18.29} \\
\underline{0}\end{array}$ & $\begin{array}{l}\frac{43}{0} \\
0 \\
1.33 \\
0 \\
0\end{array}$ & $\frac{\frac{44}{1.11}}{\frac{11.00}{0}}$ & & $\begin{array}{c}\frac{55}{\underline{0}} \\
\frac{\underline{0}}{24.00} \\
\underline{0}\end{array}$ & & & & & & & $\frac{\frac{2}{0.42}}{\frac{0.03}{4.67}} \frac{0}{\underline{4}}$ & $\begin{array}{c}\frac{3}{0} \\
0.27 \\
0.14 \\
0\end{array}$ & \\
\hline $\begin{array}{l}\text { Ring } \\
\text { Core }\end{array}$ & $\frac{\frac{10}{0.04}}{\frac{0}{0}}$ & $\begin{array}{c}\frac{11}{0} \\
0 \\
0 \\
10.71\end{array}$ & $\begin{array}{c}\frac{16}{0} \\
0.07 \\
0\end{array}$ & $\begin{array}{l}\frac{17}{\underline{0}} \\
\underline{0} \\
\underline{3.28}\end{array}$ & $\frac{\frac{21}{0.10}}{\frac{0.02}{3.06}} \frac{\frac{0.38}{0}}{\frac{0}{0}}$ & $\begin{array}{l}\frac{23}{\underline{0}} \\
\frac{0.07}{1.05} \\
\frac{0.36}{\underline{0}}\end{array}$ & $\begin{array}{l}\frac{24}{0} \\
0 \\
1.57 \\
0.06 \\
0\end{array}$ & $\begin{array}{l}\frac{28}{0.02} \\
0.15 \\
2.86 \\
0 \\
0\end{array}$ & & & & & & & $\begin{array}{l}\frac{56}{0.74} \\
\underline{0} \\
\frac{0.05}{1.32} \\
\underline{0}\end{array}$ & $\begin{array}{l}\frac{57}{\underline{0}} \\
\underline{0} \\
\frac{0.05}{0.28} \\
\underline{0}\end{array}$ & $\begin{array}{l}\frac{58}{0} \\
0 \\
0.10 \\
0.29 \\
0\end{array}$ & $\begin{array}{r}\frac{61}{0.02} \\
\frac{0}{0} \\
\underline{0.31} \\
\underline{13.42} \\
\end{array}$ & $\begin{array}{l}\frac{62}{0} \\
0 \\
0.06 \\
0.19 \\
0.07\end{array}$ & $\begin{array}{c}\frac{63}{0.02} \\
\underline{0} \\
\frac{0.05}{0.19} \\
\underline{0}\end{array}$ & $\begin{array}{l}\frac{64}{0} \\
0 \\
0.29 \\
0.76 \\
0.19\end{array}$ & $\frac{\frac{1}{0.10}}{\frac{0.08}{0.84}} \frac{\underline{0}}{\frac{0.0}{0.0}}$ & & \\
\hline $\begin{array}{l}\text { Sargasso } \\
\text { Sea } \\
\& \\
\text { Gulf } \\
\text { Stream }\end{array}$ & & & & & & & & & & $\begin{array}{r}\frac{49}{0.16} \\
\frac{0.09}{0.38} \\
\frac{13.33}{13} \\
\end{array}$ & $\begin{array}{c}\frac{50}{0} \\
0.02 \\
0.69 \\
17.19\end{array}$ & $\begin{array}{c}\frac{51}{0} \\
0 \\
0.13 \\
0\end{array}$ & $\begin{array}{l}\frac{52}{5.00} \\
\frac{0.03}{0.22} \\
\underline{0.38}\end{array}$ & & & & & & & & & & & \\
\hline $\begin{array}{l}\text { Slope } \\
\text { Water }\end{array}$ & $\frac{8}{-}$ & $\frac{9}{0.30}$ & $\frac{18}{0.04}$ & & $\frac{19}{0.04}$ & $\frac{20}{4.70}$ & $\frac{33}{1.55}$ & $\frac{34}{0.14}$ & & $\frac{35}{6.26}$ & $\frac{47}{0.92}$ & $\frac{48}{2.17}$ & $\frac{53}{1.16}$ & $\frac{54}{3.21}$ & $\frac{59}{0.25}$ & $\frac{60}{1.22}$ & $\frac{65}{4.71}$ & $\frac{66}{2.42}$ & & & & $\frac{4}{1.44}$ & $\frac{5}{4.58}$ & $\frac{6}{7.46}$ \\
\hline $\begin{array}{l}\text { High } \\
\text { Velocity } \\
\text { Region }\end{array}$ & $\frac{12}{0.05}$ & $\frac{13}{4.66}$ & $\frac{14}{4.46}$ & $\frac{15}{0.06}$ & $\frac{27}{5.01}$ & $\frac{29}{0.03}$ & $\frac{30}{0.06}$ & $\frac{31}{0.06}$ & $\frac{32}{-}$ & $\frac{41}{3.36}$ & $\frac{42}{3.69}$ & $\frac{43}{0.27}$ & $\frac{44}{2.87}$ & & $\frac{55}{9.60}$ & & & & & & & $\frac{2}{1.28}$ & $\frac{3}{0.10}$ & \\
\hline $\begin{array}{l}\text { Ring } \\
\text { Core }\end{array}$ & $\frac{10}{0.01}$ & $\frac{11}{2.68}$ & $\frac{16}{-}$ & $\frac{17}{1.64}$ & $\frac{21}{0.70}$ & $\frac{23}{0.30}$ & $\frac{24}{0.33}$ & $\frac{28}{0.75}$ & & $\frac{37}{0.01}$ & $\frac{38}{1.89}$ & $\frac{39}{0.82}$ & & & $\frac{56}{0.42}$ & $\frac{57}{0.07}$ & $\frac{58}{0.08}$ & $\frac{61}{2.75}$ & $\frac{62}{0.06}$ & $\frac{63}{0.06}$ & $\frac{64}{0.25}$ & $\frac{1}{0.26}$ & & \\
\hline SS \& GS & & & & & & & & & & $\frac{49}{3.49}$ & $\frac{50}{4.48}$ & $\frac{51}{0.03}$ & $\frac{52}{1.41}$ & & & & & & & & & & & \\
\hline
\end{tabular}


Table 51. Abundance of Cyclothone acclinidens (specimens $/ 10,000 \mathrm{~m}^{3}$ ).

\begin{tabular}{|c|c|c|c|c|c|c|c|c|c|c|c|c|c|c|c|c|c|c|c|c|c|c|c|c|}
\hline & & $\begin{array}{r}\text { Oceanu } \\
\text { Apr }\end{array}$ & $1^{118}$ & & & & $\begin{array}{l}\text { eanus } \\
\text { June }\end{array}$ & 121 & & & $\begin{array}{r}O c E \\
A\end{array}$ & $\begin{array}{l}\text { anus } 1 \\
\text { ugust }\end{array}$ & & & & & $\begin{array}{l}\text { Knor } \\
\text { Sept }\end{array}$ & $\begin{array}{r}98 \\
-0 c t \\
\end{array}$ & & & & $\begin{array}{r}\mathrm{A} \\
\mathrm{Se}\end{array}$ & $\begin{array}{c}110 \\
t-0 c t\end{array}$ & \\
\hline $\begin{array}{l}\text { Slope } \\
\text { Water }\end{array}$ & $\begin{array}{c}\frac{8}{\overline{0}} \\
\underline{0} \\
\underline{0} \\
1.4\end{array}$ & $\begin{array}{l}9 \\
0 \\
0 \\
0 \\
0\end{array}$ & & & $\begin{array}{r}19 \\
\underline{0} \\
\underline{0} \\
\underline{0} \\
\underline{0} \\
\underline{0}\end{array}$ & $\begin{array}{c}20 \\
0 \\
0 \\
1.3 \\
0.5 \\
0.4\end{array}$ & $\begin{array}{c}\frac{33}{\underline{0}} \\
\frac{0}{0} \\
\underline{0} \\
\underline{0} \\
\underline{0.5}\end{array}$ & $\begin{array}{l}\frac{34}{\underline{0}} \\
\underline{0} \\
\underline{0.2}\end{array}$ & & $\begin{array}{c}35 \\
0 \\
0 \\
0 \\
2.2 \\
0\end{array}$ & $\begin{array}{l}\frac{47}{\underline{0}} \\
\underline{0} \\
\underline{0} \\
\underline{0.5}\end{array}$ & $\begin{array}{c}48 \\
0 \\
0 \\
0 \\
0.7\end{array}$ & $\begin{array}{l}\frac{53}{\underline{0}} \\
\underline{0} \\
\underline{0.5} \\
\underline{0.2}\end{array}$ & $\begin{array}{c}54 \\
0 \\
0 \\
1.5 \\
1.3\end{array}$ & $\begin{array}{r}59 \\
\underline{0} \\
\underline{0} \\
\underline{0} \\
\underline{0} \\
\underline{0}\end{array}$ & $\begin{array}{c}60 \\
0 \\
0 \\
0 \\
0.4 \\
0.3\end{array}$ & $\begin{array}{c}\frac{65}{\underline{0}} \\
\underline{0} \\
\underline{0} \\
\underline{0.8} \\
\underline{0}\end{array}$ & $\begin{array}{c}66 \\
0 \\
0 \\
0 \\
0.5 \\
0\end{array}$ & & & & $\begin{array}{c}4 \\
0 \\
0 \\
0.6 \\
0\end{array}$ & $\begin{array}{l}\frac{5}{\underline{0}} \\
\underline{0} \\
\underline{0.6} \\
\underline{1.3}\end{array}$ & \\
\hline $\begin{array}{l}\text { High } \\
\text { Velocity } \\
\text { Region }\end{array}$ & $\begin{array}{c}12 \\
0 \\
0 \\
0 \\
0.7\end{array}$ & $\begin{array}{l}\frac{13}{\underline{0}} \\
\underline{0} \\
\underline{1.5}\end{array}$ & $\begin{array}{l}14 \\
\underline{0} \\
\underline{0} \\
\underline{0} \\
\underline{0}\end{array}$ & $\begin{array}{c}15 \\
0 \\
0 \\
1.2 \\
0.5\end{array}$ & $\begin{array}{c}26 \\
0.3 \\
0 \\
0 \\
0 \\
-\end{array}$ & $\begin{array}{c}27 \\
0 \\
0 \\
0 \\
0.8 \\
0.4\end{array}$ & $\begin{array}{c}30 \\
0 \\
0 \\
0 \\
0.8 \\
0.5\end{array}$ & $\begin{array}{c}31 \\
0 \\
0 \\
0 \\
0 \\
1.7\end{array}$ & $\begin{array}{l}32 \\
\underline{0} \\
\underline{0} \\
\underline{0} \\
\underline{0}\end{array}$ & $\begin{array}{l}\frac{41}{\underline{0}} \\
\underline{0.6} \\
\underline{1.1}\end{array}$ & $\begin{array}{l}\frac{42}{\underline{0}} \\
\underline{0} \\
\underline{0.2} \\
\underline{0}\end{array}$ & $\begin{array}{r}43 \\
0 \\
0 \\
0 \\
0 \\
0\end{array}$ & $\begin{array}{l}\frac{44}{0} \\
\underline{0.7} \\
\underline{0.4}\end{array}$ & & $\begin{array}{l}\frac{55}{\underline{0}} \\
\underline{0} \\
\frac{0.4}{1.1} \\
\end{array}$ & & & & & & & $\begin{array}{l}\frac{2}{0} \\
\underline{0} \\
\underline{0} \\
\underline{0.6}\end{array}$ & $\begin{array}{c}3 \\
0 \\
0 \\
1.8 \\
1.2\end{array}$ & \\
\hline $\begin{array}{l}\text { Ring } \\
\text { Core }\end{array}$ & $\begin{array}{c}\frac{10}{\underline{0}} \\
\underline{0} \\
\underline{0} \\
\underline{0.6}\end{array}$ & $\begin{array}{c}11 \\
0 \\
0 \\
0 \\
0.4\end{array}$ & $\begin{array}{c}16 \\
0 \\
0 \\
0.7\end{array}$ & $\frac{17}{\frac{0}{0}}$ & $\begin{array}{c}21 \\
\underline{0} \\
\underline{0} \\
\underline{0} \\
\underline{0.8} \\
0.6 \\
\end{array}$ & $\begin{array}{l}\frac{23}{0.6} \\
\frac{0}{0} \\
\underline{0.9} \\
\underline{0.2}\end{array}$ & $\begin{array}{c}\frac{24}{0.3} \\
0 \\
0 \\
2.5 \\
1.7\end{array}$ & $\begin{array}{c}28 \\
0 \\
0 \\
0 \\
0.8 \\
0\end{array}$ & & & & & & & $\begin{array}{c}\frac{56}{0} \\
\underline{0} \underline{0} \\
\underline{0} \\
\underline{0} \\
\underline{0.3}\end{array}$ & $\begin{array}{c}\frac{57}{0} \\
\underline{0} \\
\underline{0} \\
\underline{0} \\
\underline{0.5} \\
\underline{0.5}\end{array}$ & $\begin{array}{c}58 \\
0 \\
0 \\
0 \\
0 \\
0\end{array}$ & $\begin{array}{c}\frac{61}{\underline{0}} \\
\underline{0} \\
\underline{0} \\
\underline{0.4} \\
\underline{0.8}\end{array}$ & $\begin{array}{c}62 \\
0 \\
0 \\
0 \\
0 \\
1.1\end{array}$ & $\begin{array}{c}\frac{63}{0} \\
\underline{0} \\
\underline{0} \\
\underline{0} \\
\underline{0} \\
\underline{2.5}\end{array}$ & $\begin{array}{c}64 \\
0 \\
0 \\
0 \\
0 \\
1.2\end{array}$ & $\begin{array}{l}\frac{1}{0} \\
\underline{0} \\
\underline{0} \\
1.7 \\
\end{array}$ & & \\
\hline $\begin{array}{l}\text { Sargasso } \\
\text { Sea } \\
\& \\
\text { Gulf } \\
\text { Stream }\end{array}$ & & & & & & & & & & $\begin{array}{l}\frac{49}{\underline{0}} \\
\underline{0} \\
\underline{0} \\
\underline{0}\end{array}$ & $\begin{array}{r}50 \\
0 \\
0 \\
0 \\
0\end{array}$ & $\begin{array}{c}51 \\
0 \\
0 \\
0 \\
0.3\end{array}$ & $\begin{array}{l}\frac{52}{0} \\
\underline{0} \\
\underline{0} \\
\underline{0.8}\end{array}$ & & & & & & & & & & & \\
\hline $\begin{array}{l}\text { Slope } \\
\text { Water }\end{array}$ & $\frac{8}{0.4}$ & $\frac{9}{0}$ & $\frac{18}{0.4}$ & & $\frac{19}{0}$ & $\frac{20}{0.5}$ & $\frac{33}{0.3}$ & $\frac{34}{0.1}$ & & $\frac{35}{0.4}$ & $\frac{47}{0.1}$ & $\frac{48}{0.2}$ & $\frac{53}{0.2}$ & $\frac{54}{0.7}$ & $\frac{59}{0}$ & $\frac{60}{0.1}$ & $\frac{65}{0.2}$ & $\frac{66}{0.1}$ & & & & $\frac{4}{0.2}$ & $\frac{5}{0.5}$ & $\frac{6}{0}$ \\
\hline $\begin{array}{l}\text { High } \\
\text { Velocity } \\
\text { Region }\end{array}$ & $\frac{12}{0.2}$ & $\frac{13}{0.8}$ & $\frac{14}{0}$ & $\frac{15}{0.4}$ & $\frac{27}{0.3}$ & $\frac{29}{0.1}$ & $\frac{30}{0.3}$ & $\frac{31}{0.3}$ & $\frac{32}{0}$ & $\frac{41}{0.4}$ & $\frac{42}{0}$ & $\frac{43}{0}$ & $\frac{44}{0.2}$ & & $\frac{55}{0.6}$ & & & & & & & $\frac{2}{0.2}$ & $\frac{3}{0.8}$ & \\
\hline $\begin{array}{l}\text { Ring } \\
\text { Core }\end{array}$ & $\frac{10}{0.2}$ & $\frac{11}{0.1}$ & $\frac{16}{-}$ & $\frac{17}{0}$ & $\frac{21}{0.3}$ & $\frac{23}{0.5}$ & $\frac{24}{1.0}$ & $\frac{28}{0.2}$ & & $\frac{37}{0.1}$ & $\frac{38}{0}$ & $\frac{39}{0}$ & & & $\frac{56}{0.1}$ & $\frac{57}{0.1}$ & $\frac{58}{0}$ & $\frac{61}{0.2}$ & $\frac{62}{0.2}$ & $\frac{63}{0.5}$ & $\frac{64}{0.2}$ & $\frac{1}{0.4}$ & & \\
\hline SS \& GS & & & & & & & & & & $\frac{49}{0}$ & $\frac{50}{0}$ & $\frac{51}{0.1}$ & $\frac{52}{0.2}$ & & & & & & & & & & & \\
\hline
\end{tabular}


Table 52. Abundance of Cyclothone alba (specimens $/ 10,000 \mathrm{~m}^{3}$ ).

\begin{tabular}{|c|c|c|c|c|c|c|c|c|c|c|c|c|c|c|c|c|c|c|c|c|c|c|c|c|}
\hline & \multicolumn{4}{|c|}{$\begin{array}{c}\text { Oceanus } \\
\text { April }\end{array}$} & \multicolumn{5}{|c|}{$\begin{array}{c}\text { Oceanus } 121 \\
\text { June }\end{array}$} & \multicolumn{5}{|c|}{$\begin{array}{l}\text { Oceanus } 125 \\
\text { Auqust }\end{array}$} & \multicolumn{7}{|c|}{$\begin{array}{l}\text { Knorr } 98 \\
\text { Sept-0ct }\end{array}$} & \multicolumn{3}{|c|}{$\begin{array}{c}\text { A2 } 110 \\
\text { Sept-0ct }\end{array}$} \\
\hline $\begin{array}{l}\text { Slope } \\
\text { Water }\end{array}$ & $\begin{array}{l}\frac{8}{=} \\
\frac{8.8}{0.5} \\
\frac{0}{0}\end{array}$ & $\begin{array}{c}\frac{9}{0} \\
12.9 \\
0 \\
0\end{array}$ & & & $\begin{array}{c}\frac{19}{0} \\
\frac{1.0}{3.6} \\
\frac{0}{0}\end{array}$ & $\begin{array}{c}20 \\
0 \\
8.4 \\
0 \\
0 \\
0\end{array}$ & $\begin{array}{c}\frac{33}{\underline{0}} \\
\underline{0} \\
\frac{1.3}{10.0} \\
\frac{1.1}{1.1}\end{array}$ & $\begin{array}{l}\frac{34}{0} \\
\underline{0} \\
1.6 \\
\end{array}$ & & $\begin{array}{c}\frac{35}{\underline{0}} \\
\frac{3 \underline{8} .8}{10.3} \\
\frac{0}{0}\end{array}$ & $\begin{array}{c}47 \\
0 \\
15.7 \\
1.2 \\
0\end{array}$ & $\begin{array}{c}\frac{48}{0} \\
7.4 \\
1.2 \\
0\end{array}$ & $\frac{\frac{53}{0.2}}{\frac{0.3}{\frac{5.3}{0}}}$ & $\begin{array}{c}\frac{54}{0} \\
10.6 \\
0.6 \\
0\end{array}$ & $\begin{array}{c}\frac{59}{\underline{0}} \\
\underline{0} \\
\underline{5.3} \\
\underline{0} \\
\underline{0}\end{array}$ & $\begin{array}{c}60 \\
0 \\
0 \\
2.8 \\
0 \\
0\end{array}$ & $\begin{array}{c}\frac{65}{\underline{0}} \\
\frac{1.4}{4.5} \\
\underline{0} \\
\underline{0}\end{array}$ & $\begin{array}{c}\frac{66}{0} \\
2.9 \\
6.3 \\
0 \\
0\end{array}$ & & & & $\begin{array}{l}\frac{4}{0} \\
6.1 \\
0.9 \\
0\end{array}$ & $\begin{array}{l}\frac{5}{\underline{0}} \\
\underline{8.1} \\
\frac{0.3}{\underline{0}}\end{array}$ & \\
\hline $\begin{array}{l}\text { High } \\
\text { Velocity } \\
\text { Region }\end{array}$ & $\begin{array}{c}\frac{12}{0} \\
4.2 \\
4.6 \\
0\end{array}$ & $\frac{13}{\underline{0}} \frac{7.8}{\underline{0.7}}$ & $\begin{array}{l}\frac{14}{0} \\
\frac{8,3}{\underline{0}} \\
\underline{0}\end{array}$ & $\begin{array}{c}15 \\
0 \\
6.5 \\
0.6 \\
0\end{array}$ & $\begin{array}{c}26 \\
0 \\
0 \\
10.5 \\
0 \\
-\end{array}$ & $\begin{array}{c}27 \\
0 \\
0 \\
5.9 \\
0.4 \\
0\end{array}$ & $\begin{array}{r}30 \\
0 \\
0 \\
12.5 \\
0.5 \\
0.2\end{array}$ & $\begin{array}{c}31 \\
0 \\
0 \\
10.0 \\
0 \\
0\end{array}$ & $\frac{\frac{32}{0.3}}{\frac{0.4}{8.0}}$ & $\frac{\frac{41}{4.9}}{\frac{0.6}{\underline{0}}}$ & $\begin{array}{l}\frac{42}{0} \\
\frac{7.6}{\underline{0}} \\
\underline{0}\end{array}$ & $\begin{array}{c}43 \\
0 \\
0 \\
7.1 \\
0 \\
0\end{array}$ & $\frac{\frac{44}{2.0}}{\frac{0}{0}}$ & & $\begin{array}{c}\frac{55}{\underline{0}} \\
\underline{\underline{0}} \\
\underline{6} .8\end{array}$ & & & & & & & $\frac{\frac{2}{0.2}}{\frac{0.9}{0.7}} \frac{\underline{0}}{\underline{0}}$ & $\begin{array}{c}\frac{3}{0.7} \\
12.7 \\
0 \\
0\end{array}$ & \\
\hline $\begin{array}{l}\text { Ring } \\
\text { Core }\end{array}$ & $\frac{\frac{10}{0}}{\frac{4.1}{\frac{5.5}{0}}}$ & $\begin{array}{c}11 \\
0 \\
1.7 \\
1.9 \\
0\end{array}$ & $\begin{array}{l}\frac{16}{0.5} \\
5.7 \\
0.7\end{array}$ & $\frac{17}{\frac{0}{0.5}} \frac{1.2}{1.2}$ & $\begin{array}{c}\frac{21}{\underline{0}} \\
\underline{0} \\
\frac{2.1}{1.7} \\
\underline{0}\end{array}$ & $\begin{array}{l}\frac{23}{0.6} \\
\underline{0} \\
\frac{3.0}{5.0} \\
\underline{0}\end{array}$ & $\begin{array}{c}24 \\
0 \\
0 \\
4.9 \\
1.9 \\
0\end{array}$ & $\begin{array}{c}28 \\
0 \\
3.5 \\
3.6 \\
0 \\
0\end{array}$ & & & & & & & $\begin{array}{c}\frac{56}{\underline{0}} \\
\underline{0} \\
\underline{0} \\
15.3 \\
\underline{5.0}\end{array}$ & $\begin{array}{l}\frac{57}{\underline{0}} \\
\underline{0} \\
\frac{4.0}{7.5} \\
\underline{3.4}\end{array}$ & $\begin{array}{c}58 \\
0 \\
0 \\
3.3 \\
5.8 \\
0.8\end{array}$ & $\begin{array}{c}\frac{61}{\underline{0}} \\
\underline{0} \\
\frac{4.1}{5.0} \\
\underline{0}\end{array}$ & $\begin{array}{c}\frac{62}{0.2} \\
0 \\
5.5 \\
8.1 \\
1.1\end{array}$ & $\begin{array}{c}\frac{63}{\underline{0}} \\
\underline{0} \\
\underline{2.9} \\
\underline{4.6} \\
\underline{0.3}\end{array}$ & $\begin{array}{c}64 \\
0 \\
0 \\
3.6 \\
5.2 \\
0\end{array}$ & $\begin{array}{l}\frac{1}{0} \\
\frac{1.0}{9.4} \\
\underline{2.5}\end{array}$ & & \\
\hline $\begin{array}{l}\text { Sargasso } \\
\text { Sea } \\
\& \\
\text { Gulf } \\
\text { Stream }\end{array}$ & & & & & & & & & & $\begin{array}{l}\frac{49}{0.2} \\
\underline{0.2} \\
\underline{0.9} \\
\underline{2.8}\end{array}$ & $\begin{array}{c}50 \\
0 \\
0 \\
2.8 \\
3.4\end{array}$ & $\begin{array}{c}51 \\
0 \\
0.9 \\
5.7 \\
0\end{array}$ & $\begin{array}{c}\frac{52}{\underline{0}} \\
\frac{0.8}{2.2} \\
\underline{0}\end{array}$ & & & & & & & & & & & \\
\hline $\begin{array}{l}\text { Slope } \\
\text { Water }\end{array}$ & $\frac{8}{2.3}$ & $\frac{9}{3.2}$ & $\frac{18}{0.9}$ & & $\frac{19}{1.1}$ & $\frac{20}{2.1}$ & $\frac{33}{1.8}$ & $\frac{34}{1.0}$ & & $\frac{35}{2.8}$ & $\frac{47}{4.2}$ & $\frac{48}{2.1}$ & $\frac{53}{1.4}$ & $\frac{54}{2.8}$ & $\frac{59}{1.1}$ & $\frac{60}{0.6}$ & $\frac{65}{1.2}$ & $\frac{66}{1.6}$ & & & & $\frac{4}{1.8}$ & $\frac{5}{2.1}$ & $\frac{6}{1.8}$ \\
\hline $\begin{array}{l}\text { High } \\
\text { Velocity } \\
\text { Region }\end{array}$ & $\frac{12}{2.2}$ & $\frac{13}{2.3}$ & $\frac{14}{2.1}$ & $\frac{15}{1.8}$ & $\frac{27}{1.3}$ & $\frac{29}{0.9}$ & $\frac{30}{2.6}$ & $\frac{31}{2.0}$ & $\frac{32}{2.6}$ & $\frac{41}{2.6}$ & $\frac{42}{1.7}$ & $\frac{43}{1.4}$ & $\frac{44}{1.2}$ & & $\frac{55}{2.7}$ & & & & & & & $\frac{2}{2.5}$ & $\frac{3}{3.4}$ & \\
\hline $\begin{array}{l}\text { Ring } \\
\text { Core }\end{array}$ & $\frac{10}{2.4}$ & $\frac{11}{0.9}$ & $\frac{16}{-}$ & $\frac{17}{0.7}$ & $\frac{21}{0.8}$ & $\frac{23}{1.7}$ & $\frac{24}{1.4}$ & $\frac{28}{1.5}$ & & $\frac{37}{1.2}$ & $\frac{38}{1.4}$ & $\frac{39}{1.5}$ & & & $\frac{56}{4.1}$ & $\frac{57}{3.0}$ & $\frac{58}{2.0}$ & $\frac{61}{1.8}$ & $\frac{62}{3.0}$ & $\frac{63}{1.6}$ & $\frac{64}{1.4}$ & $\frac{1}{3.2}$ & & \\
\hline SS \& GS & & & & & & & & & & $\frac{49}{1.0}$ & $\frac{50}{1.6}$ & $\frac{51}{1.7}$ & $\frac{52}{0.8}$ & & & & & & & & & & & \\
\hline
\end{tabular}


Table 53. Volume of Cyclothone alba $\left(\mathrm{ml} / 10,000 \mathrm{~m}^{3}\right)$.

\begin{tabular}{|c|c|c|c|c|c|c|c|c|c|c|c|c|c|c|c|c|c|c|c|c|c|c|c|c|}
\hline & & $\begin{array}{r}\text { Oceanu } \\
\text { Apr }\end{array}$ & $\begin{array}{l}5118 \\
i 1\end{array}$ & & & & $\begin{array}{l}\text { eanus } \\
\text { June }\end{array}$ & 121 & & & $\begin{array}{r}\text { Oce } \\
\mathrm{A}\end{array}$ & $\begin{array}{l}\text { anus } 1 \\
\text { Igust }\end{array}$ & & & & & $\begin{array}{l}\text { Knor } \\
\text { Sept }\end{array}$ & $\begin{array}{r}98 \\
-0 c t\end{array}$ & & & & & $\begin{array}{l}2 \quad 110 \\
\text { pt-0ct }\end{array}$ & \\
\hline $\begin{array}{l}\text { Slope } \\
\text { Water }\end{array}$ & $\begin{array}{l}\frac{8}{=} \\
\frac{0.53}{0.05} \\
\frac{0}{0}\end{array}$ & $\begin{array}{l}\frac{9}{0} \\
0.88 \\
0 \\
0\end{array}$ & & & 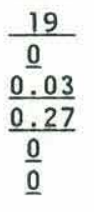 & $\begin{array}{l}\frac{20}{0} \\
0.53 \\
0 \\
0 \\
0\end{array}$ & $\begin{array}{l}\frac{33}{\underline{0}} \\
\underline{0} \\
0.04 \\
0.67 \\
\underline{0.06}\end{array}$ & $\begin{array}{l}\frac{34}{\underline{0}} \\
\underline{0} \\
\underline{0.18}\end{array}$ & & $\begin{array}{c}\frac{35}{0} \\
0.15 \\
0.50 \\
0 \\
0\end{array}$ & $\begin{array}{l}\frac{47}{\underline{0}} \\
\frac{0.88}{0.12} \\
\underline{0}\end{array}$ & $\begin{array}{c}\frac{48}{0} \\
0.49 \\
0.10 \\
0\end{array}$ & $\frac{\frac{53}{0.02}}{\frac{0.33}{0.0}}$ & $\begin{array}{l}\frac{54}{0} \\
0.63 \\
0.06 \\
0\end{array}$ & $\begin{array}{l}\frac{59}{\underline{0}} \\
\underline{0} \\
\frac{0.18}{\underline{0}} \\
\underline{0}\end{array}$ & $\begin{array}{l}\frac{60}{0} \\
0 \\
0.17 \\
0 \\
0\end{array}$ & $\begin{array}{l}\frac{65}{\underline{0}} \\
\frac{0.03}{0.18} \\
\frac{0}{0}\end{array}$ & $\begin{array}{l}\frac{66}{0} \\
0.07 \\
0.26 \\
0 \\
0\end{array}$ & & & & $\begin{array}{c}\frac{4}{0} \\
0.43 \\
0.06 \\
0\end{array}$ & $\begin{array}{l}\frac{5}{\underline{0}} \\
\frac{0.52}{0.03} \\
\underline{0}\end{array}$ & \\
\hline $\begin{array}{l}\text { High } \\
\text { Velocity } \\
\text { Region }\end{array}$ & $\begin{array}{c}\frac{12}{0} \\
0.31 \\
0.33 \\
0\end{array}$ & $\begin{array}{l}\frac{13}{\underline{0}} \\
\underline{0.33} \\
\underline{0.02}\end{array}$ & $\begin{array}{l}\frac{14}{\underline{0}} \\
\underline{0.40} \\
\underline{0} \\
\underline{0}\end{array}$ & $\begin{array}{l}\frac{15}{0} \\
0.59 \\
0.06 \\
0\end{array}$ & $\begin{array}{l}26 \\
0 \\
0 \\
0.57 \\
0.07 \\
-\end{array}$ & $\begin{array}{l}27 \\
0 \\
0 \\
0.45 \\
0.04 \\
0\end{array}$ & $\begin{array}{l}\frac{30}{0} \\
0 \\
0.63 \\
0.05 \\
0.02\end{array}$ & $\begin{array}{l}31 \\
0 \\
0 \\
0.54 \\
0 \\
0\end{array}$ & $\frac{\frac{32}{0.04}}{\frac{0.36}{0.50}}$ & $\frac{\frac{41}{0.26}}{\frac{0.06}{\underline{0}}}$ & $\begin{array}{l}\frac{42}{\underline{0}} \\
\frac{0.47}{\underline{0}} \\
\underline{0}\end{array}$ & $\begin{array}{l}\frac{43}{0} \\
0 \\
0.42 \\
0 \\
0\end{array}$ & $\frac{\frac{44}{0.11}}{\frac{0}{0}}$ & & $\frac{\frac{55}{\underline{0}}}{\underline{0}} \frac{1.16}{\underline{0}}$ & & & & & & & $\frac{\frac{2}{0.02}}{\frac{0.14}{1.14}} \frac{0.07}{\underline{0}}$ & $\begin{array}{l}\frac{3}{0.07} \\
0.65 \\
0 \\
0\end{array}$ & \\
\hline $\begin{array}{l}\text { Ring } \\
\text { Core }\end{array}$ & $\begin{array}{l}\frac{10}{\underline{0}} \\
\underline{0.18} \\
\underline{0.27} \\
\underline{0.12}\end{array}$ & $\begin{array}{l}11 \\
0 \\
0.06 \\
0.13 \\
0\end{array}$ & $\begin{array}{l}\frac{16}{0.05} \\
0.14 \\
0.13\end{array}$ & $\begin{array}{l}\frac{17}{0} \\
\underline{0.05} \\
\underline{0.06}\end{array}$ & $\begin{array}{l}\frac{21}{\underline{0}} \\
\frac{0.09}{0.03} \\
\frac{0}{0}\end{array}$ & $\begin{array}{l}\frac{23}{0.06} \\
\frac{0}{0.20} \\
\frac{0.36}{\underline{0}}\end{array}$ & $\begin{array}{l}24 \\
0 \\
0 \\
0.35 \\
0.13 \\
0\end{array}$ & $\begin{array}{l}\frac{28}{0} \\
0.19 \\
0.25 \\
0 \\
0\end{array}$ & & & & & & & $\begin{array}{l}\frac{56}{0} \\
\underline{0} \\
\underline{0} \\
0.47 \\
\underline{0.25}\end{array}$ & $\begin{array}{l}\frac{57}{\underline{0}} \\
\underline{0} \\
\underline{0.10} \\
\underline{0.25} \\
\underline{0.18}\end{array}$ & $\begin{array}{l}\frac{58}{0} \\
0 \\
0.05 \\
0.29 \\
0.08\end{array}$ & $\begin{array}{l}\frac{61}{\frac{0}{0}} \\
\frac{0.07}{0.19} \\
\underline{0}\end{array}$ & $\begin{array}{c}\frac{62}{0.02} \\
0 \\
0.06 \\
0.24 \\
0.04\end{array}$ & $\begin{array}{l}\frac{63}{\underline{0}} \\
\underline{0} \\
\underline{0.10} \\
0.19 \\
\underline{0.03}\end{array}$ & $\begin{array}{l}\frac{64}{0} \\
0 \\
0.07 \\
0.19 \\
0\end{array}$ & $\begin{array}{l}\frac{1}{\underline{0}} \\
\underline{0.06} \\
\underline{0.50} \\
\underline{0.25}\end{array}$ & & \\
\hline $\begin{array}{l}\text { Sargasso } \\
\text { Sea } \\
\& \\
\text { Guif } \\
\text { Stream }\end{array}$ & & & & & & & & & & $\begin{array}{l}\frac{49}{0.02} \\
\underline{0} \\
\underline{0.02} \\
\underline{0.13}\end{array}$ & $\begin{array}{l}50 \\
0 \\
0 \\
0.03 \\
0.16\end{array}$ & $\begin{array}{c}51 \\
0 \\
0.02 \\
0.24 \\
0\end{array}$ & $\begin{array}{l}\frac{52}{\underline{0}} \\
\underline{0.03} \\
\frac{0.09}{\underline{0}}\end{array}$ & & & & & & & & & & & \\
\hline $\begin{array}{l}\text { Slope } \\
\text { Water }\end{array}$ & $\frac{8}{-}$ & $\frac{9}{0.22}$ & $\frac{18}{0.03}$ & & $\frac{19}{0.07}$ & $\frac{20}{0.13}$ & $\frac{33}{0.11}$ & $\frac{34}{0.11}$ & & $\frac{35}{0.13}$ & $\frac{47}{0.25}$ & $\frac{48}{0.15}$ & $\frac{53}{0.09}$ & $\frac{54}{0.17}$ & $-\frac{59}{0.04}$ & $\frac{60}{0.03}$ & $\frac{65}{0.04}$ & $\frac{66}{0.06}$ & & & & $\frac{4}{0.12}$ & $\frac{5}{0.14}$ & $\frac{6}{0.13}$ \\
\hline $\begin{array}{l}\text { High } \\
\text { Velocity } \\
\text { Region }\end{array}$ & $\frac{12}{0.16}$ & $\frac{13}{0.09}$ & $\frac{14}{0.10}$ & $\frac{15}{0.14}$ & $\frac{27}{0.10}$ & $\frac{29}{0.03}$ & $\frac{30}{0.14}$ & $\frac{31}{0.11}$ & $\frac{32}{0.17}$ & $\frac{41}{0.15}$ & $\frac{42}{0.10}$ & $\frac{43}{0.08}$ & $\frac{44}{0.07}$ & & $\frac{55}{0.45}$ & & & & & & & $\frac{2}{0.31}$ & $\frac{3}{0.18}$ & \\
\hline $\begin{array}{l}\text { Ring } \\
\text { Core }\end{array}$ & $\frac{10}{0.14}$ & $\frac{11}{0}$ & $\frac{16}{-}$ & $\frac{17}{0.03}$ & $\frac{21}{0.02}$ & $\frac{23}{0.12}$ & $\frac{24}{0.10}$ & $\frac{28}{0}$ & & $\frac{37}{0.05}$ & $\frac{38}{0.07}$ & $\frac{39}{0.07}$ & & & $\frac{56}{0.14}$ & $\frac{57}{0.11}$ & $\frac{58}{0.08}$ & $\frac{61}{0.05}$ & $\frac{62}{0.07}$ & $\frac{63}{0.06}$ & $\frac{64}{0.05}$ & $\frac{1}{0.20}$ & & \\
\hline SS \& GS & & & & & & & & & & $\frac{49}{0.03}$ & $\frac{50}{0.05}$ & $\frac{51}{0.07}$ & $\frac{52}{0.03}$ & & & & & & & & & & & \\
\hline
\end{tabular}


Table 54. Abundance of Cyclothone braueri (specimens $/ 10,000 \mathrm{~m}^{3}$ ).

\begin{tabular}{|c|c|c|c|c|c|c|c|c|c|c|c|c|c|c|c|c|c|c|c|c|c|c|c|c|}
\hline & \multicolumn{4}{|c|}{$\begin{array}{c}\text { Oceanus } 118 \\
\text { April }\end{array}$} & \multicolumn{5}{|c|}{$\begin{array}{c}\text { Oceanus } 121 \\
\text { June }\end{array}$} & \multicolumn{5}{|c|}{$\begin{array}{l}\text { Oceanus } 125 \\
\text { August }\end{array}$} & \multicolumn{7}{|c|}{$\begin{array}{l}\text { Knorr } 98 \\
\text { Sept-Oct }\end{array}$} & \multicolumn{3}{|c|}{$\begin{array}{c}\text { A2 } 110 \\
\text { Sept-0ct }\end{array}$} \\
\hline $\begin{array}{l}\text { Slope } \\
\text { Water }\end{array}$ & $\frac{8}{\frac{8}{74.7}} \frac{\underline{0}}{\underline{0}}$ & $\begin{array}{r}\frac{9}{0} \\
47.1 \\
0.7 \\
0.7\end{array}$ & & & $\begin{array}{l}\frac{19}{\underline{0}} \\
\underline{\underline{0}} \\
\frac{3.9}{\underline{4.2}} \\
\underline{0}\end{array}$ & $\begin{array}{c}\frac{20}{0.5} \\
8.9 \\
0.9 \\
0 \\
0\end{array}$ & $\begin{array}{l}\frac{33}{\underline{0}} \\
\frac{1.7}{\frac{4.3}{6.1}} \\
\frac{6.9}{6}\end{array}$ & $\begin{array}{l}\frac{34}{\underline{0.5}} \\
\underline{0} \\
\underline{54.8}\end{array}$ & & $\begin{array}{c}\frac{35}{3.0} \\
7.0 \\
377.0 \\
0 \\
.1 .4\end{array}$ & $\begin{array}{l}\frac{47}{\underline{0}} \\
\frac{20.5}{17.1} \\
\underline{0.2}\end{array}$ & $\begin{array}{c}48 \\
0.5 \\
13.4 \\
6.2 \\
0\end{array}$ & $\frac{53}{\underline{0}} \frac{\underline{4.2}}{\underline{0}} \underline{\underline{0}}$ & $\begin{array}{c}\frac{54}{0.3} \\
33.1 \\
15.2 \\
0\end{array}$ & $\begin{array}{l}\frac{59}{\frac{0.3}{0}} \\
\underline{\underline{0} .5} \\
\underline{0.7} \\
\underline{0.2}\end{array}$ & $\begin{array}{c}60 \\
0 \\
0 \\
29.0 \\
0 \\
0\end{array}$ & $\begin{array}{c}\frac{65}{\underline{0}} \\
\frac{0.3}{27.3} \\
\underline{0} \\
\underline{0.2}\end{array}$ & $\begin{array}{c}\frac{66}{0.4} \\
9.3 \\
49.6 \\
0 \\
0.4\end{array}$ & & & & $\begin{array}{c}\frac{4}{0} \\
42.2 \\
14.1 \\
0\end{array}$ & $\frac{\frac{5}{\underline{0}}}{\frac{50.4}{\underline{8} .8}}$ & \\
\hline $\begin{array}{l}\text { High } \\
\text { Velocity } \\
\text { Region }\end{array}$ & $\begin{array}{r}\frac{12}{1.4} \\
183.1 \\
60.8 \\
1.4\end{array}$ & $\frac{\frac{13}{\frac{4.5}{3.9}}}{16.3}$ & $\frac{\frac{14}{1.7}}{\frac{127.3}{21.8}} \frac{\underline{0}}{\frac{21.8}{10}}$ & $\begin{array}{r}\frac{15}{1.1} \\
135.9 \\
7.6 \\
1.4\end{array}$ & $\begin{array}{c}26 \\
0.3 \\
0 \\
45.7 \\
3.4 \\
-\end{array}$ & $\begin{array}{r}27 \\
0 \\
0.3 \\
65.0 \\
0.8 \\
0.2\end{array}$ & $\begin{array}{r}\frac{30}{2.3} \\
1.6 \\
492.2 \\
2.4 \\
3.0\end{array}$ & $\begin{array}{c}\frac{31}{0.7} \\
0 \\
29.3 \\
28.6 \\
1.0\end{array}$ & $\frac{\frac{32}{4.5}}{\frac{197.2}{251.5}}$ & $\frac{\frac{41}{2.5}}{\frac{15.7}{5.2}}$ & $\begin{array}{c}\frac{42}{\underline{0}} \\
\frac{50.6}{\frac{3.9}{\underline{0}}}\end{array}$ & $\begin{array}{r}43 \\
0 \\
0 \\
35.4 \\
3.9 \\
0.5\end{array}$ & $\frac{\frac{44}{18.5}}{\underline{2.3}}$ & & $\begin{array}{c}\frac{55}{\underline{0}} \\
\underline{0} \\
\frac{54.8}{\underline{0}}\end{array}$ & & & & & & & $\frac{\frac{2}{\underline{0}}}{\frac{17.0}{\underline{7.3}}}$ & $\begin{array}{r}\frac{3}{2.5} \\
364.6 \\
18.6 \\
0\end{array}$ & \\
\hline $\begin{array}{l}\text { Ring } \\
\text { Core }\end{array}$ & $\frac{\frac{10}{1.2}}{\frac{33 \frac{1.1}{129.1}}{1.8}}$ & $\begin{array}{r}\frac{11}{0.6} \\
195.0 \\
127.5 \\
1.4\end{array}$ & $\begin{array}{r}\frac{16}{2.0} \\
478.6 \\
12.0\end{array}$ & $\frac{\frac{17}{0.6}}{\underline{\frac{0.6}{3.7}}} \underline{\underline{50.6}}$ & $\begin{array}{r}\frac{21}{\frac{3.0}{0.8}} \\
\frac{268.5}{\underline{0.8}} \\
\underline{3.3}\end{array}$ & $\begin{array}{r}\frac{23}{\underline{0}} \\
\frac{5.3}{174.0} \\
\frac{4.5}{\underline{0}}\end{array}$ & $\begin{array}{r}\frac{24}{3.2} \\
105.0 \\
388.6 \\
5.6 \\
1.7\end{array}$ & $\begin{array}{c}\frac{28}{1.7} \\
270.0 \\
299.3 \\
0 \\
0.5\end{array}$ & & & & & & & $\begin{array}{r}\frac{56}{\frac{0.6}{1.7}} \\
\frac{376.3}{362.6} \\
\underline{7.5}\end{array}$ & $\begin{array}{r}\frac{57}{1.9} \\
\frac{2.8}{386.5} \\
\frac{214.1}{12.9} \\
\end{array}$ & $\begin{array}{r}\frac{58}{0.8} \\
0.8 \\
385.2 \\
104.5 \\
3.7\end{array}$ & $\frac{\frac{61}{\frac{0.2}{1.5}}}{\frac{\frac{415.2}{5.2}}{\underline{44.6}}}$ & $\begin{array}{r}\frac{62}{1.9} \\
0.9 \\
392.9 \\
311.1 \\
2.1\end{array}$ & $\begin{array}{r}\frac{63}{1.1} \\
\frac{2.2}{375.7} \\
\frac{110.8}{1.6}\end{array}$ & $\begin{array}{r}\frac{64}{2.1} \\
106.3 \\
539.3 \\
153.3 \\
1.9\end{array}$ & $\frac{\frac{1}{1.2}}{\frac{170.0}{148.4}}$ & & \\
\hline $\begin{array}{l}\text { Sargasso } \\
\text { Sea } \\
\& \\
\text { Gulf } \\
\text { Stream }\end{array}$ & & & & & & & & & & $\begin{array}{r}\frac{49}{\frac{1.1}{0.4}} \\
\frac{313.1}{26.9} \\
\underline{26.1}\end{array}$ & $\begin{array}{r}\frac{50}{0.8} \\
17.8 \\
219.0 \\
16.2\end{array}$ & $\begin{array}{r}\frac{51}{2.1} \\
304.3 \\
167.6 \\
1.7\end{array}$ & 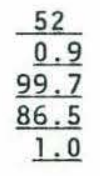 & & & & & & & & & & & \\
\hline $\begin{array}{l}\text { Slope } \\
\text { Water }\end{array}$ & $\frac{8}{18.7}$ & $\frac{9}{12.1}$ & $\frac{18}{2.9}$ & & $\frac{19}{2.0}$ & $\frac{20}{2.6}$ & $\frac{33}{10.2}$ & $\frac{34}{32.8}$ & & $\frac{35}{77.7}$ & $\frac{47}{9.5}$ & $\frac{48}{5.0}$ & $\frac{53}{1.1}$ & $\frac{54}{12.2}$ & $\frac{59}{5.5}$ & $\frac{60}{5.8}$ & $\frac{65}{5.6}$ & $\frac{66}{11.1}$ & & & & $\frac{4}{14.1}$ & $\frac{5}{14.8}$ & $\frac{6}{13.3}$ \\
\hline $\begin{array}{l}\text { High } \\
\text { Velocity } \\
\text { Region }\end{array}$ & $\frac{12}{61.7}$ & $\frac{13}{46.8}$ & $\frac{14}{37.7}$ & $\frac{15}{37.2}$ & $\frac{27}{13.3}$ & $\frac{29}{39.8}$ & $\frac{30}{100.3}$ & $\frac{31}{12.0}$ & $\frac{32}{79.2}$ & $\frac{41}{5.2}$ & $\frac{42}{11.8}$ & $\frac{43}{8.2}$ & $\frac{44}{11.6}$ & & $\frac{55}{21.9}$ & & & & & & & $\frac{2}{6.1}$ & $\frac{3}{96.4}$ & \\
\hline $\begin{array}{l}\text { Ring } \\
\text { Core }\end{array}$ & $\frac{10}{116.6}$ & $\frac{11}{81.1}$ & $\frac{16}{-}$ & $\frac{17}{48.9}$ & $\frac{21}{56.6}$ & $\frac{23}{37.0}$ & $\frac{24}{82.3}$ & $\frac{28}{125.0}$ & & $\frac{37}{6.2}$ & $\frac{38}{9.9}$ & $\frac{39}{12.6}$ & & & $\frac{56}{149.7}$ & $\frac{57}{123.6}$ & $\frac{58}{99.0}$ & $\frac{61}{92.4}$ & $\frac{62}{141.9}$ & $\frac{63}{98.1}$ & $\frac{64}{96.4}$ & $\frac{1}{80.7}$ & & \\
\hline SS \& GS & & & & & & & & & & $\frac{49}{90.4}$ & $\frac{50}{63.5}$ & $\frac{51}{118.9}$ & $\frac{52}{47.0}$ & & & & & & & & & & & \\
\hline
\end{tabular}


Table 55. Volume of Cyclothone braueri $\left(\mathrm{ml} / 10,000 \mathrm{~m}^{3}\right)$.

\begin{tabular}{|c|c|c|c|c|c|c|c|c|c|c|c|c|c|c|c|c|c|c|c|c|c|c|c|c|}
\hline & \multicolumn{4}{|c|}{$\begin{array}{c}\text { Oceanus } 118 \\
\text { Apri1 }\end{array}$} & \multicolumn{5}{|c|}{$\begin{array}{c}\text { Oceanus } 121 \\
\text { June }\end{array}$} & \multicolumn{5}{|c|}{$\begin{array}{l}\text { Oceanus } 125 \\
\text { August }\end{array}$} & \multicolumn{7}{|c|}{$\begin{array}{l}\text { Knorr } 98 \\
\text { Sept-0ct }\end{array}$} & \multicolumn{3}{|c|}{$\begin{array}{c}\text { A2 } 110 \\
\text { Sept-0ct }\end{array}$} \\
\hline $\begin{array}{l}\text { Slope } \\
\text { Water }\end{array}$ & $\begin{array}{l}\frac{8}{\bar{a}} \\
\frac{4.88}{\underline{0}} \\
\underline{0}\end{array}$ & $\begin{array}{l}\frac{9}{0} \\
2.94 \\
0.07 \\
0.07\end{array}$ & & & $\begin{array}{l}\frac{19}{\underline{0}} \\
\frac{0}{0.27} \\
\frac{0.42}{\underline{0}}\end{array}$ & $\begin{array}{c}\frac{20}{0.05} \\
0.53 \\
0.04 \\
0 \\
0\end{array}$ & $\begin{array}{l}\frac{33}{\underline{0}} \\
\underline{0.04} \\
\underline{0.13} \\
\underline{3.06} \\
\underline{0.56}\end{array}$ & $\begin{array}{l}\frac{34}{0.05} \\
\underline{0} \\
\underline{3.18}\end{array}$ & & $\begin{array}{c}\frac{35}{0.20} \\
0.13 \\
16.67 \\
0 \\
0.14\end{array}$ & $\begin{array}{l}\frac{47}{0} \\
\underline{0.88} \\
\underline{1.27} \\
\underline{0.02}\end{array}$ & $\begin{array}{l}\frac{48}{0.03} \\
0.71 \\
0.59 \\
0\end{array}$ & $\begin{array}{l}\frac{53}{\underline{0}} \\
\underline{0.13} \\
\underline{0} \\
\underline{0}\end{array}$ & $\begin{array}{l}\frac{54}{0.03} \\
1.41 \\
1.36 \\
0\end{array}$ & $\begin{array}{l}\frac{59}{0.03} \\
\underline{0} \\
\frac{1.03}{0.07} \\
\underline{0.02}\end{array}$ & $\begin{array}{l}\frac{60}{0} \\
0 \\
1.72 \\
0 \\
0\end{array}$ & $\begin{array}{c}\frac{65}{\underline{0}} \\
\underline{0.03} \\
\frac{1.23}{\underline{0}} \\
\underline{0.02}\end{array}$ & $\begin{array}{c}\frac{66}{0.02} \\
0.21 \\
2.22 \\
0 \\
0.04\end{array}$ & & & & $\begin{array}{l}\frac{4}{0} \\
3.04 \\
1.19 \\
0\end{array}$ & $\begin{array}{l}\frac{5}{\underline{0}} \\
\frac{2.96}{0.88} \\
\underline{0}\end{array}$ & \\
\hline $\begin{array}{l}\text { High } \\
\text { Velocity } \\
\text { Region }\end{array}$ & $\begin{array}{r}\frac{12}{0.09} \\
10.00 \\
4.79 \\
0.07\end{array}$ & $\frac{\frac{13}{0.30}}{\frac{0.56}{0.85}}$ & $\frac{\frac{14}{0.06}}{\frac{0.67}{1.76}} \frac{0}{\underline{0}}$ & $\begin{array}{l}\frac{15}{0.06} \\
8.23 \\
0.71 \\
0.05\end{array}$ & $\begin{array}{c}\frac{26}{0.03} \\
0 \\
2.86 \\
0.45 \\
-\end{array}$ & $\begin{array}{l}\frac{27}{0} \\
0.03 \\
4.09 \\
0.08 \\
0.02\end{array}$ & $\begin{array}{r}\frac{30}{0.13} \\
0.04 \\
25.00 \\
0.16 \\
0.23\end{array}$ & $\begin{array}{c}\frac{31}{0.04} \\
0 \\
1.61 \\
2.00 \\
0.07\end{array}$ & $\frac{\frac{32}{0.24}}{\frac{9.60}{13.00}} \frac{0.87}{\underline{0.87}}$ & $\begin{array}{l}\frac{41}{0.09} \\
\frac{0.77}{0.02} \\
\end{array}$ & $\frac{\frac{42}{\underline{0}}}{\frac{2.65}{2.20}} \frac{\underline{0}}{\underline{2}}$ & $\begin{array}{l}\frac{43}{0} \\
0 \\
2.29 \\
0.26 \\
0.05\end{array}$ & $\frac{\frac{44}{1.11}}{\frac{0.20}{0.04}}$ & & $\begin{array}{l}\frac{55}{\underline{0}} \\
\frac{\underline{0} .40}{\underline{0}}\end{array}$ & & & & & & & $\begin{array}{l}\frac{2}{\underline{0}} \\
\underline{0.78} \\
\frac{0.60}{\underline{0}}\end{array}$ & $\begin{array}{c}\frac{3}{0.18} \\
18.92 \\
1.25 \\
0\end{array}$ & \\
\hline $\begin{array}{l}\text { Ring } \\
\text { Core }\end{array}$ & $\frac{\frac{10}{0.04}}{\frac{14.12}{7.27}} \frac{0.12}{\underline{0.12}}$ & $\begin{array}{r}\frac{11}{0.03} \\
6.11 \\
15.00 \\
0.07\end{array}$ & $\begin{array}{r}\frac{16}{0.10} \\
22.14 \\
0.80\end{array}$ & $\frac{\frac{17}{0.06}}{\frac{3.68}{2.99}}$ & 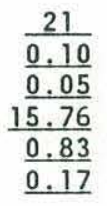 & $\frac{\frac{23}{\underline{0}}}{\frac{0.27}{11.00}} \frac{0.41}{\underline{0}}$ & $\begin{array}{r}\frac{24}{0.18} \\
6.00 \\
24.32 \\
0.56 \\
0.11\end{array}$ & $\begin{array}{c}\frac{28}{0.09} \\
13.85 \\
19.64 \\
0 \\
0.03\end{array}$ & & & & & & & 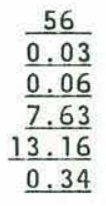 & $\begin{array}{l}\frac{57}{0.09} \\
\underline{0.09} \\
\underline{8.00} \\
\underline{9.38} \\
\underline{0.79}\end{array}$ & $\begin{array}{l}\frac{58}{0.03} \\
0.04 \\
7.62 \\
6.13 \\
0.26\end{array}$ & $\frac{\frac{61}{0.02}}{\frac{0.06}{0.37}} \frac{10.08}{\underline{3.08}}$ & $\begin{array}{r}\frac{62}{0.08} \\
0.03 \\
11.63 \\
16.76 \\
0.11\end{array}$ & $\frac{\frac{63}{0.02}}{\frac{0.13}{0.11}} \frac{11.43}{\frac{6.15}{0.09}}$ & $\begin{array}{r}\frac{64}{0.04} \\
3.13 \\
14.29 \\
8.10 \\
0.12\end{array}$ & $\frac{\frac{1}{\frac{0.04}{6.60}}}{\frac{10.00}{0.17}}$ & & \\
\hline $\begin{array}{l}\text { Sargasso } \\
\text { Sea } \\
\& \\
\text { Gulf } \\
\text { Stream }\end{array}$ & & & & & & & & & & $\frac{\frac{49}{0.03}}{\frac{0.47}{12.00}} \frac{1.67}{1}$ & $\begin{array}{l}\frac{50}{0.05} \\
0.41 \\
7.93 \\
0.94\end{array}$ & $\begin{array}{l}\frac{51}{0.06} \\
5.87 \\
7.61 \\
0.07\end{array}$ & $\frac{\frac{52}{0.05}}{\frac{0.61}{3.56}} \frac{0}{0.03}$ & & & & & & & & & & & \\
\hline $\begin{array}{l}\text { Slope } \\
\text { Water }\end{array}$ & $\frac{8}{1.22}$ & $\frac{9}{0.77}$ & $\frac{18}{0.15}$ & & $\frac{19}{0.17}$ & $\frac{20}{0.16}$ & $\frac{33}{0.62}$ & $\frac{34}{1.91}$ & & $\frac{35}{3.43}$ & $\frac{47}{0.54}$ & $\frac{48}{0.33}$ & $\frac{53}{0.03}$ & $\frac{54}{0.70}$ & $\frac{59}{0.23}$ & $\frac{60}{0.34}$ & $\frac{65}{0.26}$ & $\frac{66}{0.48}$ & & & & $\frac{4}{1.06}$ & $\frac{5}{0.96}$ & $\frac{6}{0.90}$ \\
\hline $\begin{array}{l}\text { High } \\
\text { Velocity } \\
\text { Region }\end{array}$ & $\frac{12}{3.74}$ & $\frac{13}{2.57}$ & $\frac{14}{2.12}$ & $\frac{15}{2.26}$ & $\frac{27}{0.85}$ & $\frac{29}{2.00}$ & $\frac{30}{5.12}$ & $\frac{31}{0.76}$ & $\frac{32}{4.22}$ & $\frac{41}{0.24}$ & $\frac{42}{1.02}$ & $\frac{43}{0.52}$ & $\frac{44}{0.71}$ & & $\frac{55}{0.96}$ & & & & & & & $\frac{2}{0.35}$ & $\frac{3}{5.09}$ & \\
\hline $\begin{array}{l}\text { Ring } \\
\text { Core }\end{array}$ & $\frac{10}{5.39}$ & $\frac{11}{5.30}$ & $\frac{16}{-}$ & $\frac{17}{2.43}$ & $\frac{21}{3.37}$ & $\frac{23}{2.35}$ & $\frac{24}{5.17}$ & $\frac{28}{7.47}$ & & $\frac{37}{0.31}$ & $\frac{38}{0.49}$ & $\frac{39}{0.53}$ & & & $\frac{56}{4.24}$ & $\frac{57}{3.67}$ & $\frac{58}{2.82}$ & $\frac{61}{2.72}$ & $\frac{62}{5.73}$ & $\frac{63}{3.55}$ & $\frac{64}{3.40}$ & $\frac{1}{4.22}$ & & \\
\hline SS \& GS & & & & & & & & & & $\frac{49}{3.54}$ & $\frac{50}{2.33}$ & $\frac{51}{3.40}$ & $\frac{52}{2.06}$ & & & & & & & & & & & \\
\hline
\end{tabular}


Table 56. Abundance of cyclothone microdon (specimens $/ 10,000 \mathrm{~m}^{3}$ ).

\begin{tabular}{|c|c|c|c|c|c|c|c|c|c|c|c|c|c|c|c|c|c|c|c|c|c|c|c|c|}
\hline & \multicolumn{4}{|c|}{$\begin{array}{c}\text { Oceanus } \\
\text { Apri1 }\end{array}$} & \multicolumn{5}{|c|}{$\begin{array}{c}\text { Oceanus } 121 \\
\text { June }\end{array}$} & \multicolumn{5}{|c|}{$\begin{array}{l}\text { Oceanus } 125 \\
\text { August }\end{array}$} & \multicolumn{7}{|c|}{$\begin{array}{l}\text { Knorr } 98 \\
\text { Sept-Oct }\end{array}$} & \multicolumn{3}{|c|}{$\begin{array}{c}\text { A2 } 110 \\
\text { Sept-0ct }\end{array}$} \\
\hline $\begin{array}{l}\text { Slope } \\
\text { Water }\end{array}$ & $\begin{array}{l}\frac{8}{=} \\
\frac{105.9}{209.5} \\
\frac{34.5}{}\end{array}$ & $\begin{array}{r}\frac{9}{2.7} \\
190.0 \\
313.6 \\
70.0\end{array}$ & & & 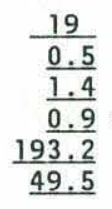 & $\begin{array}{r}\frac{20}{4.2} \\
44.7 \\
232.2 \\
63.6 \\
23.6\end{array}$ & $\begin{array}{l}\frac{33}{\frac{1.3}{0}} \\
\underline{0.4} \\
\frac{99.4}{98.0} \\
\end{array}$ & $\begin{array}{r}\frac{34}{\frac{0.9}{1.1}} \\
106.4 \\
\end{array}$ & & $\begin{array}{r}\frac{35}{0} \\
0.4 \\
143.0 \\
111.7 \\
60.7\end{array}$ & $\begin{array}{r}\frac{47}{0} \\
\frac{1.7}{122.7} \\
63.9\end{array}$ & \begin{tabular}{r}
\multicolumn{1}{c}{48} \\
1.7 \\
2.9 \\
158.8 \\
98.8
\end{tabular} & $\begin{array}{r}\frac{53}{\frac{0.6}{21.6}} \\
\frac{129.2}{129.7} \\
32.7\end{array}$ & $\begin{array}{r}\frac{54}{2.9} \\
31.9 \\
310.9 \\
199.7\end{array}$ & $\frac{\frac{59}{1.3}}{\frac{1.6}{35.0}} \frac{\underline{138.3}}{\underline{45.0}}$ & \begin{tabular}{r}
\multicolumn{1}{c}{60} \\
1.6 \\
0.9 \\
72.1 \\
163.5 \\
47.1
\end{tabular} & $\begin{array}{c}\frac{65}{\underline{3.0}} \\
\underline{0} \\
\frac{162.3}{112.0} \\
\underline{74.0}\end{array}$ & \begin{tabular}{r}
\multicolumn{6}{c}{$\frac{16}{1.5}$} \\
5.7 \\
213.0 \\
136.7 \\
92.7
\end{tabular} & & & & $\begin{array}{r}\frac{4}{0.7} \\
85.9 \\
204.7 \\
74.1\end{array}$ & $\frac{\frac{5}{\frac{2.3}{8.5}}}{\frac{184.7}{194.5}}$ & \\
\hline $\begin{array}{l}\text { High } \\
\text { Velocity } \\
\text { Region }\end{array}$ & $\begin{array}{r}\frac{12}{1.1} \\
8.5 \\
164.6 \\
73.4\end{array}$ & $\begin{array}{r}\frac{13}{\frac{1.0}{5.0}} \\
\underline{148.8} \\
\end{array}$ & $\begin{array}{r}\frac{14}{\frac{1.1}{33.3}} \\
\frac{251.2}{51.2} \\
52.9\end{array}$ & $\begin{array}{r}\frac{15}{3.3} \\
115.3 \\
282.4 \\
51.4\end{array}$ & $\begin{array}{c}\frac{26}{2.3} \\
0 \\
52.4 \\
256.9 \\
-\end{array}$ & $\begin{array}{r}\frac{27}{3.9} \\
0 \\
94.1 \\
194.2 \\
37.0\end{array}$ & $\begin{array}{r}\frac{30}{1.0} \\
0 \\
32.5 \\
120.5 \\
87.4\end{array}$ & \begin{tabular}{r}
\multicolumn{1}{c}{31} \\
0.7 \\
1.5 \\
180.7 \\
126.6 \\
35.5
\end{tabular} & $\begin{array}{r}\frac{32}{\underline{0}} \\
\frac{0.4}{146.5} \\
\underline{43.6}\end{array}$ & $\frac{\frac{41}{\frac{5.8}{5.1}}}{11.9}$ & $\begin{array}{r}\frac{42}{\frac{0.1}{83.2}} \\
\frac{227.8}{70.0} \\
\end{array}$ & $\begin{array}{r}\frac{43}{0} \\
0.4 \\
94.6 \\
84.8 \\
60.0\end{array}$ & $\frac{\frac{44}{15.7}}{115.7}$ & & $\begin{array}{r}\frac{55}{\underline{0}} \\
\underline{0.5} \\
\frac{44.8}{127.8} \\
\end{array}$ & & & & & & & $\frac{\frac{2}{\frac{0.2}{7.6}}}{\frac{184.0}{96.9}}$ & $\begin{array}{r}\frac{3}{0.4} \\
10.3 \\
161.4 \\
121.2\end{array}$ & \\
\hline $\begin{array}{l}\text { Ring } \\
\text { Core }\end{array}$ & $\begin{array}{r}\frac{10}{\frac{2.0}{0.6}} \\
\frac{82.7}{151.8} \\
\end{array}$ & $\begin{array}{r}11 \\
0.6 \\
1.7 \\
51.2 \\
152.5\end{array}$ & $\begin{array}{l}\frac{16}{1.5} \\
30.0 \\
81.3\end{array}$ & $\frac{17}{\underline{0}}$ & $\begin{array}{r}\frac{21}{2.0} \\
\underline{0} \\
\frac{4.8}{85.8} \\
141.1 \\
\end{array}$ & 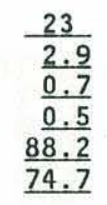 & $\begin{array}{r}24 \\
0.9 \\
0 \\
2.7 \\
140.6 \\
77.2\end{array}$ & $\begin{array}{r}\frac{28}{1.5} \\
0 \\
83.6 \\
110.8 \\
35.4\end{array}$ & & & & & & & $\begin{array}{r}\frac{56}{\frac{0.9}{0.6}} \\
\frac{30.0}{1.1} \\
\frac{30.31 .3}{13}\end{array}$ & $\begin{array}{r}\frac{57}{\frac{0.3}{0.9}} \\
\frac{1.3}{\frac{34.4}{130.0}} \\
\end{array}$ & \begin{tabular}{r}
\multicolumn{1}{c}{58} \\
1.0 \\
0.4 \\
0.5 \\
12.9 \\
85.5
\end{tabular} & 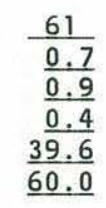 & $\begin{array}{c}\frac{62}{0.4} \\
0 \\
0.2 \\
13.2 \\
29.3\end{array}$ & $\begin{array}{c}\frac{63}{\frac{0.3}{0}} \\
\underline{0} .4 \\
\frac{8.1}{8.1} \\
\end{array}$ & $\begin{array}{c}\frac{64}{0.6} \\
0.6 \\
0 \\
10.5 \\
5.0\end{array}$ & $\begin{array}{r}\frac{1}{0.8} \\
\underline{0} \\
\underline{2.2} \\
\underline{98.3} \\
\end{array}$ & & \\
\hline $\begin{array}{l}\text { Sargasso } \\
\text { Sea } \\
\& \\
\text { Gulf } \\
\text { Stream }\end{array}$ & & & & & & & & & & $\begin{array}{l}\frac{49}{\underline{0}} \\
\underline{0} \\
\underline{0.2} \\
\underline{32.3}\end{array}$ & $\begin{array}{c}50 \\
0 \\
0 \\
0 \\
20.0\end{array}$ & $\begin{array}{r}\frac{51}{1.5} \\
0.7 \\
58.0 \\
115.9\end{array}$ & $\begin{array}{r}\frac{52}{\frac{0.2}{0.3}} \\
\frac{43.5}{139.7} \\
\end{array}$ & & & & & & & & & & & \\
\hline $\begin{array}{l}\text { Slope } \\
\text { Water }\end{array}$ & $\frac{8}{87.5}$ & $\frac{9}{144.1}$ & $\frac{18}{50.8}$ & & $\frac{19}{61.2}$ & $\frac{20}{86.2}$ & $\frac{33}{59.1}$ & $\frac{34}{64.3}$ & & $\frac{35}{63.2}$ & $\frac{47}{47.1}$ & $\frac{48}{65.6}$ & $\frac{53}{46.0}$ & $\frac{54}{136.4}$ & $\frac{59}{44.2}$ & $\frac{60}{57.1}$ & $\frac{65}{70.3}$ & $\frac{66}{89.5}$ & & & & $\frac{4}{91.4}$ & $\frac{5}{78.5}$ & $\frac{6}{99.3}$ \\
\hline $\begin{array}{l}\text { High } \\
\text { Velocity } \\
\text { Region }\end{array}$ & $\frac{12}{61.9}$ & $\frac{13}{75.9}$ & $\frac{14}{84.6}$ & $\frac{15}{113.7}$ & $\frac{27}{77.2}$ & $\frac{29}{29.1}$ & $\frac{30}{48.4}$ & $\frac{31}{68.9}$ & $\frac{32}{45.3}$ & $\frac{41}{42.2}$ & $\frac{42}{77.7}$ & $\frac{43}{48.0}$ & $\frac{44}{43.3}$ & & $\frac{55}{43.6}$ & & & & & & & $\frac{2}{74.7}$ & $\frac{3}{73.3}$ & \\
\hline $\begin{array}{l}\text { Ring } \\
\text { Core }\end{array}$ & $\frac{10}{59.3}$ & $\frac{11}{51.5}$ & $\frac{16}{-}$ & $\frac{17}{46.2}$ & $\frac{21}{46.5}$ & $\frac{23}{33.3}$ & $\frac{24}{44.4}$ & $\frac{28}{49.1}$ & & $\frac{37}{31.5}$ & $\frac{38}{35.6}$ & $\frac{39}{40.2}$ & & & $\frac{56}{32.8}$ & $\frac{57}{33.4}$ & $\frac{58}{20.1}$ & $\frac{61}{20.3}$ & $\frac{62}{8.7}$ & $\frac{63}{11.6}$ & $\frac{64}{3.4}$ & $\frac{1}{25.3}$ & & \\
\hline SS \& GS & & & & & & & & & & $\frac{49}{8.1}$ & $\frac{50}{5.0}$ & $\frac{51}{44.0}$ & $\frac{52}{45.9}$ & & & & & & & & & & & \\
\hline
\end{tabular}


Table 57. Volume of Cyclothone microdon $\left(\mathrm{ml} / 10,000 \mathrm{~m}^{3}\right)$.

\begin{tabular}{|c|c|c|c|c|c|c|c|c|c|c|c|c|c|c|c|c|c|c|c|c|c|c|}
\hline & \multicolumn{4}{|c|}{$\begin{array}{c}\text { Oceanus } 118 \\
\text { April }\end{array}$} & \multicolumn{5}{|c|}{$\begin{array}{c}\text { Oceanus } \\
\text { June }\end{array}$} & \multicolumn{5}{|c|}{$\begin{array}{c}\text { Oceanus } 125 \\
\text { Auqust }\end{array}$} & \multicolumn{7}{|c|}{$\begin{array}{l}\text { Knorr } 98 \\
\text { Sept-Oct }\end{array}$} & $\begin{array}{r}\text { A2 } 110 \\
\text { Sept-0ct }\end{array}$ \\
\hline $\begin{array}{l}\text { Slope } \\
\text { Water }\end{array}$ & $\begin{array}{l}\frac{8}{=} \\
\frac{4.00}{25.91} \\
\frac{9.18}{9}\end{array}$ & \begin{tabular}{r}
\multicolumn{1}{c}{$\frac{9}{0.13}$} \\
7.06 \\
32.86 \\
22.67
\end{tabular} & & & $\frac{\frac{19}{0.02}}{\frac{0.07}{0.09}} \frac{\frac{0.09}{10.53}}{7.37}$ & $\begin{array}{r}\frac{20}{0.53} \\
1.58 \\
16.52 \\
10.00 \\
6.00\end{array}$ & $\begin{array}{l}\frac{33}{0.13} \\
\underline{0} \\
\frac{0.04}{2.78} \\
\underline{9.63}\end{array}$ & $\frac{\frac{34}{0.05}}{\frac{0.04}{7.95}}$ & & $\begin{array}{l}\frac{35}{0} \\
0.02 \\
4.00 \\
8.33 \\
7.50\end{array}$ & $\begin{array}{l}\frac{47}{0} \\
\frac{0.13}{6.27} \\
\underline{7.50} \\
\end{array}$ & $\begin{array}{r}\frac{48}{0.10} \\
0.09 \\
9.41 \\
10.82\end{array}$ & $\frac{\frac{53}{0.04}}{\frac{0.33}{0.33}} \frac{5.64}{4.22}$ & $\begin{array}{c}\frac{54}{0.18} \\
0.94 \\
15.15 \\
20.67\end{array}$ & $\begin{array}{l}\frac{59}{\frac{0.07}{0.08}} \\
\frac{2.21}{17.24} \\
7.73\end{array}$ & $\begin{array}{r}\frac{60}{0.13} \\
0.05 \\
3.79 \\
12.61 \\
7.94\end{array}$ & $\begin{array}{l}\frac{65}{0.09} \\
\underline{0} \\
\frac{3.18}{5.00} \\
\frac{5.96}{5.96}\end{array}$ & $\begin{array}{l}\frac{66}{0.04} \\
0.29 \\
4.44 \\
5.24 \\
9.62\end{array}$ & & & & $\begin{array}{ll}\frac{4}{0.05} & \frac{5}{0.27} \\
3.07 & \underline{0.93} \\
9.41 & \frac{20.31}{20.77} \\
9.71 & \underline{20.77}\end{array}$ \\
\hline $\begin{array}{l}\text { High } \\
\text { Velocity } \\
\text { Region }\end{array}$ & $\begin{array}{l}\frac{12}{0.06} \\
0.23 \\
10.00 \\
10.34\end{array}$ & $\frac{\frac{13}{0.10}}{\frac{0.11}{9.27}}$ & 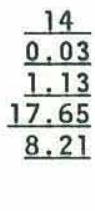 & $\begin{array}{r}\frac{15}{0.22} \\
4.82 \\
22.35 \\
7.62\end{array}$ & $\begin{array}{c}\frac{26}{0.16} \\
0 \\
1.43 \\
21.38 \\
-\end{array}$ & $\begin{array}{c}\frac{27}{0.28} \\
0 \\
3.32 \\
18.33 \\
7.37\end{array}$ & $\begin{array}{c}\frac{30}{0.08} \\
0 \\
1.09 \\
11.62 \\
10.93\end{array}$ & $\begin{array}{r}\frac{31}{0.07} \\
0.05 \\
7.86 \\
12.86 \\
6.21\end{array}$ & $\begin{array}{l}\frac{32}{\underline{0}} \\
\underline{0.04} \\
\underline{9.00} \\
\underline{8.26}\end{array}$ & $\frac{\frac{41}{0.19}}{\underline{\frac{5.43}{5.11}}}$ & $\begin{array}{r}\frac{42}{0.01} \\
\frac{2.65}{12.68} \\
8.10 \\
\end{array}$ & $\begin{array}{l}\frac{43}{0} \\
0.04 \\
4.17 \\
7.17 \\
8.10\end{array}$ & $\frac{\frac{44}{0.42}}{\frac{7.33}{7.31}}$ & & $\begin{array}{l}\frac{55}{0} \\
\frac{0.05}{1.00} \\
\underline{6.67}\end{array}$ & & & & & & & $\begin{array}{cc}\frac{2}{0.02} & \frac{3}{0.04} \\
\frac{0.62}{0.62} & 0.22 \\
\frac{13.33}{9.86} & 7.86 \\
9.38 & 14.62\end{array}$ \\
\hline $\begin{array}{l}\text { Ring } \\
\text { Core }\end{array}$ & $\begin{array}{r}\frac{10}{0.16} \\
\frac{0.12}{3.18} \\
\frac{18.23}{8.23} \\
\end{array}$ & $\begin{array}{c}\frac{11}{0.06} \\
0.11 \\
3.13 \\
16.43\end{array}$ & $\begin{array}{l}\frac{16}{0.05} \\
1.07 \\
4.00\end{array}$ & $\begin{array}{l}\frac{17}{\underline{0}} \\
\underline{0.11} \\
\underline{8.36}\end{array}$ & $\begin{array}{l}\frac{21}{0.10} \\
\underline{0} \\
\frac{0.12}{4.17} \\
\underline{17.78}\end{array}$ & 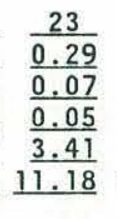 & $\begin{array}{c}\frac{24}{0.03} \\
0 \\
0.08 \\
8.13 \\
10.56\end{array}$ & $\begin{array}{c}\frac{28}{0.32} \\
0 \\
3.04 \\
12.31 \\
6.92\end{array}$ & & & & & & & $\begin{array}{l}\frac{56}{0.03} \\
\frac{0.06}{0.06} \\
\frac{0.05}{0.37} \\
\underline{\underline{2.50}}\end{array}$ & $\begin{array}{l}\frac{57}{0.03} \\
\frac{0.03}{0.03} \\
\frac{0.38}{0.38} \\
\underline{3.68}\end{array}$ & $\begin{array}{l}\frac{58}{0.03} \\
0 \\
0.05 \\
0.16 \\
2.11\end{array}$ & $\begin{array}{l}\frac{61}{0.02} \\
\frac{0.03}{0.03} \\
\frac{0.04}{0.46} \\
\underline{\underline{2.63}}\end{array}$ & $\begin{array}{l}\frac{62}{0.04} \\
0 \\
0.02 \\
0.16 \\
0.43\end{array}$ & $\begin{array}{l}\frac{63}{0.02} \\
\underline{0} \\
\frac{0.05}{0.12} \\
\frac{1.47}{1.47}\end{array}$ & $\begin{array}{l}\frac{64}{0.02} \\
0.06 \\
0 \\
0.14 \\
0.19\end{array}$ & $\begin{array}{l}\frac{1}{0.08} \\
\underline{0.13} \\
\underline{0.67}\end{array}$ \\
\hline $\begin{array}{l}\text { Sargasso } \\
\text { Sea } \\
\& \\
\text { Gulf } \\
\text { Stream }\end{array}$ & & & & & & & & & & $\begin{array}{l}\frac{49}{\underline{0}} \\
\underline{0} \\
\underline{0.02} \\
\underline{0.38} \\
\end{array}$ & $\begin{array}{l}50 \\
0 \\
0 \\
0 \\
0.63\end{array}$ & $\begin{array}{l}\frac{51}{0.06} \\
0.02 \\
1.00 \\
3.45\end{array}$ & $\begin{array}{l}\frac{52}{0.02} \\
\frac{0.03}{0.78} \\
\frac{0.10}{4.10}\end{array}$ & & & & & & & & & \\
\hline $\begin{array}{l}\text { Slope } \\
\text { Water }\end{array}$ & $\frac{8}{9.77}$ & $\frac{9}{15.68}$ & $\frac{18}{3.69}$ & & $\frac{19}{4.51}$ & $\frac{20}{7.16}$ & $\frac{33}{5.11}$ & $\frac{34}{4.79}$ & & $\frac{35}{3.97}$ & $\frac{47}{3.48}$ & $\frac{48}{5.11}$ & $\frac{53}{2.56}$ & $\frac{54}{9.24}$ & $\frac{59}{5.47}$ & $\frac{60}{4.91}$ & $\frac{65}{2.85}$ & $\frac{66}{3.90}$ & & & & $\frac{4}{5.56} \quad \frac{5}{10.57} \quad \frac{6}{10.40}$ \\
\hline $\begin{array}{l}\text { High } \\
\text { Velocity } \\
\text { Region }\end{array}$ & $\frac{12}{5.16}$ & $\frac{13}{4.69}$ & $\frac{14}{6.76}$ & $\frac{15}{8.75}$ & $\frac{27}{7.13}$ & $\frac{29}{2.28}$ & $\frac{30}{4.74}$ & $\frac{31}{5.45}$ & $\frac{32}{4.31}$ & $\frac{41}{2.73}$ & $\frac{42}{4.73}$ & $\frac{43}{3.90}$ & $\frac{44}{3.27}$ & & $\frac{55}{1.74}$ & & & & & & & $\frac{2}{5.84} \quad \frac{3}{5.69}$ \\
\hline $\begin{array}{l}\text { Ring } \\
\text { Core }\end{array}$ & $\frac{10}{5.42}$ & $\frac{11}{4.93}$ & $\frac{16}{-}$ & $\frac{17}{4.21}$ & $\frac{21}{4.42}$ & $\frac{23}{2.99}$ & $\frac{24}{3.77}$ & $\frac{28}{3.94}$ & & $\frac{37}{2.62}$ & $\frac{38}{2.42}$ & $\frac{39}{2.00}$ & & & $\frac{56}{0.60}$ & $\frac{57}{0.83}$ & $\frac{58}{0.47}$ & $\frac{61}{0.64}$ & $\frac{62}{0.13}$ & $\frac{63}{0.34}$ & $\frac{64}{0.08}$ & $\frac{1}{1.47}$ \\
\hline SS \& GS & & & & & & & & & & $\frac{49}{0.10}$ & $\frac{50}{0.16}$ & $\frac{51}{1.13}$ & $\frac{52}{1.23}$ & & & & & & & & & \\
\hline
\end{tabular}


Table 58. Abundance of Cyclothone pallida (specimens $/ 10,000 \mathrm{~m}^{3}$ ).

\begin{tabular}{|c|c|c|c|c|c|c|c|c|c|c|c|c|c|c|c|c|c|c|c|c|c|c|c|c|}
\hline & \multicolumn{4}{|c|}{$\begin{array}{c}\text { Oceanus } 118 \\
\text { April }\end{array}$} & \multicolumn{5}{|c|}{$\begin{array}{c}\text { Oceanus } 121 \\
\text { June }\end{array}$} & \multicolumn{5}{|c|}{$\begin{array}{l}\text { Oceanus } 125 \\
\text { August }\end{array}$} & \multicolumn{7}{|c|}{$\begin{array}{l}\text { Knorr } 98 \\
\text { Sept-0ct }\end{array}$} & \multicolumn{3}{|c|}{$\begin{array}{c}\text { A2 } 110 \\
\text { Sept-Oct }\end{array}$} \\
\hline $\begin{array}{l}\text { Slope } \\
\text { Water }\end{array}$ & $\begin{array}{c}\frac{8}{\overline{\overline{0}}} \\
\underline{\underline{0}} \\
10.5 \\
3.6\end{array}$ & $\begin{array}{l}9 \\
0 \\
1.2 \\
7.9 \\
0\end{array}$ & & & $\begin{array}{c}\frac{19}{\underline{0}} \\
\underline{0} \\
\underline{0} \\
\underline{7.9} \\
\underline{2.1}\end{array}$ & $\begin{array}{c}20 \\
0 \\
0.5 \\
7.4 \\
1.4 \\
0\end{array}$ & $\begin{array}{c}\frac{33}{\underline{0}} \\
\underline{0} \\
\underline{0} \\
\underline{0.6} \\
\underline{4.4}\end{array}$ & $\begin{array}{l}\frac{34}{0} \\
\underline{0.4} \\
\underline{6.4}\end{array}$ & & $\begin{array}{c}35 \\
0 \\
0 \\
7.0 \\
11.7 \\
0\end{array}$ & $\begin{array}{l}\frac{47}{\underline{0}} \\
\underline{0} \\
\underline{2.5} \\
\underline{7.0}\end{array}$ & $\begin{array}{c}48 \\
0 \\
0 \\
3.5 \\
5.5\end{array}$ & $\begin{array}{l}\frac{53}{\underline{0}} \\
\underline{0} \\
\underline{6.9} \\
\underline{0.4}\end{array}$ & $\begin{array}{c}54 \\
0 \\
0 \\
19.7 \\
9.0\end{array}$ & $\begin{array}{c}\frac{59}{\underline{0}} \\
\underline{0} \\
\underline{0} \\
\underline{3.4} \\
\underline{3.6}\end{array}$ & $\begin{array}{c}60 \\
0 \\
0 \\
0.3 \\
5.7 \\
2.9\end{array}$ & $\begin{array}{l}\frac{65}{\frac{0.9}{0}} \\
\frac{12.7}{8.4} \\
\underline{8.5}\end{array}$ & $\begin{array}{c}\frac{66}{0.2} \\
0 \\
7.4 \\
7.6 \\
1.9\end{array}$ & & & & $\begin{array}{l}\frac{4}{0} \\
0.2 \\
1.6 \\
2.6\end{array}$ & $\begin{array}{l}\frac{5}{0.3} \\
\underline{0} \\
\frac{4.7}{4.6}\end{array}$ & \\
\hline $\begin{array}{l}\text { High } \\
\text { Velocity } \\
\text { Region }\end{array}$ & $\begin{array}{c}12 \\
0 \\
0.8 \\
9.6 \\
5.5\end{array}$ & $\begin{array}{l}\frac{13}{\underline{0}} \\
\underline{0.6} \\
\underline{9.5}\end{array}$ & $\begin{array}{r}\frac{14}{0} \\
\frac{3.0}{4.7} \\
10.0 \\
\end{array}$ & $\begin{array}{c}15 \\
0 \\
1.8 \\
6.5 \\
9.5\end{array}$ & $\begin{array}{c}26 \\
0 \\
0 \\
0.5 \\
6.2 \\
-\end{array}$ & $\begin{array}{c}27 \\
0 \\
0 \\
2.3 \\
4.6 \\
3.9\end{array}$ & $\begin{array}{c}30 \\
0 \\
0 \\
2.8 \\
4.9 \\
3.0\end{array}$ & $\begin{array}{c}31 \\
0 \\
0 \\
0.7 \\
4.9 \\
7.2\end{array}$ & $\begin{array}{l}\frac{32}{\underline{0}} \\
\underline{0} \\
\underline{7.0} \\
\underline{5.4}\end{array}$ & $\begin{array}{l}\frac{41}{0} \\
\underline{7.4} \\
\underline{6.6}\end{array}$ & $\begin{array}{l}\frac{42}{\underline{0}} \\
\underline{3.8} \\
\underline{8.3} \\
\underline{4.3}\end{array}$ & $\begin{array}{c}43 \\
0 \\
0 \\
3.7 \\
9.6 \\
2.4\end{array}$ & $\frac{\frac{44}{0.2}}{\frac{0.3}{8.3}}$ & & $\begin{array}{l}\frac{55}{\underline{0}} \\
\underline{0} \\
\underline{2.4} \\
\underline{3.3} \\
\end{array}$ & & & & & & & $\begin{array}{l}\frac{2}{0.2} \\
\underline{0} \\
\underline{\underline{0.7}} \\
\underline{5.6}\end{array}$ & $\begin{array}{c}3 \\
0 \\
0 \\
4.6 \\
5.4\end{array}$ & \\
\hline $\begin{array}{l}\text { Ring } \\
\text { Core }\end{array}$ & $\begin{array}{c}\frac{10}{\underline{0}} \\
\underline{0} \\
10.0 \\
\underline{8.2}\end{array}$ & $\begin{array}{c}11 \\
0 \\
0 \\
15.0 \\
9.6\end{array}$ & $\begin{array}{c}\frac{16}{0} \\
15.7 \\
42.0\end{array}$ & $\frac{17}{\underline{0}} \frac{1.1}{10.0}$ & $\begin{array}{r}\frac{21}{\frac{2.0}{0}} \\
\frac{4.5}{30.0} \\
\underline{3.3}\end{array}$ & $\begin{array}{r}\frac{23}{\underline{0}} \\
\underline{0} \\
\frac{2.5}{27.8} \\
\underline{5.3}\end{array}$ & $\begin{array}{c}24 \\
0.3 \\
0 \\
1.4 \\
19.4 \\
0.6\end{array}$ & $\begin{array}{r}28 \\
0 \\
0 \\
12.5 \\
5.8 \\
0.5\end{array}$ & & & & & & & $\begin{array}{c}\frac{56}{\frac{0}{0}} \\
\underline{1.1} \\
\frac{17.9}{7.1} \\
\underline{3.1}\end{array}$ & $\begin{array}{c}\frac{57}{\frac{0}{0}} \\
\frac{0}{0} \\
23.7 \\
\underline{7.1}\end{array}$ & $\begin{array}{c}58 \\
0.5 \\
0 \\
1.0 \\
21.0 \\
22.1\end{array}$ & $\begin{array}{c}\frac{61}{\frac{0}{0}} \\
\underline{1.1} \\
\frac{31.5}{14.7} \\
\end{array}$ & $\begin{array}{c}\frac{62}{0.2} \\
0 \\
0.8 \\
21.9 \\
18.6\end{array}$ & $\begin{array}{r}\frac{63}{\underline{0}} \\
\underline{0} \\
\underline{0.5} \\
18.1 \\
13.1 \\
\end{array}$ & $\begin{array}{c}64 \\
0 \\
0 \\
0 \\
18.6 \\
16.9\end{array}$ & $\begin{array}{l}\frac{1}{0.2} \\
\underline{0} \\
\underline{8.1} \\
\underline{8.3}\end{array}$ & & \\
\hline $\begin{array}{l}\text { Sargasso } \\
\text { Sea } \\
\& \\
\text { Gulf } \\
\text { Stream }\end{array}$ & & & & & & & & & & $\begin{array}{r}49 \\
\frac{0}{0} \\
\underline{0.2} \\
15.6 \\
\end{array}$ & $\begin{array}{c}50 \\
0 \\
0 \\
0.3 \\
19.1\end{array}$ & $\begin{array}{c}51 \\
0.3 \\
0 \\
5.9 \\
3.8\end{array}$ & $\begin{array}{r}\frac{52}{0} \\
\underline{0} \\
11.3 \\
\underline{5.6}\end{array}$ & & & & & & & & & & & \\
\hline $\begin{array}{l}\text { Slope } \\
\text { Water }\end{array}$ & $\frac{8}{3.5}$ & $\frac{9}{2.3}$ & $\frac{18}{4.9}$ & & $\frac{19}{2.5}$ & $\frac{20}{2.3}$ & $\frac{33}{2.3}$ & $\frac{34}{4.0}$ & & $\frac{35}{3.7}$ & $\frac{47}{2.4}$ & $\frac{48}{2.3}$ & $\frac{53}{1.8}$ & $\frac{54}{7.2}$ & $\frac{59}{1.4}$ & $\frac{60}{1.8}$ & $\frac{65}{4.7}$ & $\frac{66}{3.4}$ & & & & $\frac{4}{1.1}$ & $\frac{5}{2.4}$ & $\frac{6}{2.5}$ \\
\hline $\begin{array}{l}\text { High } \\
\text { Velocity } \\
\text { Region }\end{array}$ & $\frac{12}{4.0}$ & $\frac{13}{4.9}$ & $\frac{14}{4.4}$ & $\frac{15}{4.5}$ & $\frac{27}{2.6}$ & $\frac{29}{2.8}$ & $\frac{30}{2.1}$ & $\frac{31}{2.6}$ & $\frac{32}{3.1}$ & $\frac{41}{3.5}$ & $\frac{42}{3.4}$ & $\frac{43}{3.1}$ & $\frac{44}{2.2}$ & & $\frac{55}{1.6}$ & & & & & & & $\frac{2}{2.1}$ & $\frac{3}{2.5}$ & \\
\hline $\begin{array}{l}\text { Ring } \\
\text { Core }\end{array}$ & $\frac{10}{4.6}$ & $\frac{11}{6.2}$ & $\frac{16}{-}$ & $\frac{17}{5.3}$ & $\frac{21}{7.7}$ & $\frac{23}{5.9}$ & $\frac{24}{4.4}$ & $\frac{28}{4.6}$ & & $\frac{37}{1.8}$ & $\frac{38}{1.4}$ & $\frac{39}{0.9}$ & & & $\frac{56}{4.4}$ & $\frac{57}{6.2}$ & $\frac{58}{9.0}$ & $\frac{61}{9.5}$ & $\frac{62}{8.3}$ & $\frac{63}{6.3}$ & $\frac{64}{7.1}$ & $\frac{1}{4.2}$ & & \\
\hline SS \& GS & & & & & & & & & & $\frac{49}{4.0}$ & $\frac{50}{4.9}$ & $\frac{51}{2.5}$ & $\frac{52}{4.2}$ & & & & & & & & & & & \\
\hline
\end{tabular}


Table 59. Volume of Cyclothone pallida $\left(\mathrm{ml} / 10,000 \mathrm{~m}^{3}\right)$.

\begin{tabular}{|c|c|c|c|c|c|c|c|c|c|c|c|c|c|c|c|c|c|c|c|c|c|c|c|c|}
\hline & \multicolumn{4}{|c|}{$\begin{array}{c}\text { Oceanus } 118 \\
\text { Apri1 }\end{array}$} & \multicolumn{5}{|c|}{$\begin{array}{c}\text { Oceanus } 121 \\
\text { June }\end{array}$} & \multicolumn{5}{|c|}{$\begin{array}{l}\text { Oceanus } 125 \\
\text { August }\end{array}$} & \multicolumn{7}{|c|}{$\begin{array}{l}\text { Knorr } 98 \\
\text { Sept-0ct }\end{array}$} & \multicolumn{3}{|c|}{$\begin{array}{c}\text { A2 } 110 \\
\text { Sept-0ct }\end{array}$} \\
\hline $\begin{array}{l}\text { Slope } \\
\text { Water }\end{array}$ & $\begin{array}{c}\frac{8}{\bar{z}} \\
\underline{0} \\
0.68 \\
\underline{0.73}\end{array}$ & $\begin{array}{c}\frac{9}{0} \\
0.06 \\
0.43 \\
0\end{array}$ & & & $\begin{array}{l}\frac{19}{\underline{0}} \\
\underline{0} \\
\underline{0} \\
\underline{0.47} \\
\underline{0.53}\end{array}$ & $\begin{array}{c}\frac{20}{0} \\
0.05 \\
1.09 \\
0.32 \\
0\end{array}$ & $\begin{array}{l}\frac{33}{\underline{0}} \\
\underline{0} \\
\underline{0} \\
\underline{0.06} \\
\underline{0.56}\end{array}$ & $\begin{array}{l}\frac{34}{\underline{0}} \\
\underline{0} \\
\underline{0.80}\end{array}$ & & $\begin{array}{l}35 \\
0 \\
0 \\
0.50 \\
2.22 \\
0\end{array}$ & $\begin{array}{l}\frac{47}{\underline{0}} \\
\underline{0} \\
0.18 \\
1.70 \\
\end{array}$ & $\begin{array}{l}\frac{48}{0} \\
0 \\
0.18 \\
1.25\end{array}$ & $\begin{array}{l}\frac{53}{\underline{0}} \\
\underline{0} \\
\underline{0.36} \\
\underline{0.06}\end{array}$ & $\begin{array}{l}\frac{54}{0} \\
0 \\
3.79 \\
2.00\end{array}$ & $\begin{array}{l}\frac{59}{\underline{0}} \\
\underline{0} \\
\underline{0} \\
\underline{0.38} \\
\underline{0.80}\end{array}$ & $\begin{array}{l}\frac{60}{0} \\
0 \\
0.03 \\
1.09 \\
0.59\end{array}$ & $\begin{array}{l}\frac{65}{0.04} \\
\underline{0} \\
\underline{0.23} \\
0.48 \\
0.37\end{array}$ & $\begin{array}{c}\frac{66}{0.02} \\
0 \\
0.37 \\
0.71 \\
0.46\end{array}$ & & & & $\begin{array}{l}\frac{4}{0} \\
0.02 \\
0.13 \\
0.59\end{array}$ & $\begin{array}{l}\frac{5}{0.03} \\
\underline{0} \\
\frac{1.13}{1.03} \\
\end{array}$ & \\
\hline $\begin{array}{l}\text { High } \\
\text { Velocity } \\
\text { Region }\end{array}$ & $\begin{array}{l}\frac{12}{0} \\
0.04 \\
0.50 \\
1.10\end{array}$ & $\begin{array}{l}\frac{13}{\underline{0}} \\
\underline{0.06} \\
\underline{0.78}\end{array}$ & $\begin{array}{l}\frac{14}{\underline{0}} \\
\underline{0.03} \\
\underline{0.24} \\
\underline{2.00}\end{array}$ & $\begin{array}{l}\frac{15}{0} \\
0.06 \\
0.29 \\
1.05\end{array}$ & $\begin{array}{l}\frac{26}{0} \\
0 \\
0.05 \\
0.69 \\
-\end{array}$ & $\begin{array}{l}\frac{27}{0} \\
0 \\
0.09 \\
0.75 \\
0.96\end{array}$ & $\begin{array}{l}\frac{30}{0} \\
0 \\
0.06 \\
1.22 \\
0.58\end{array}$ & $\begin{array}{l}\frac{31}{0} \\
0 \\
0.04 \\
0.49 \\
1.72\end{array}$ & $\begin{array}{l}\frac{32}{\underline{0}} \\
\underline{0} \\
\underline{0.20} \\
\underline{1.09} \\
\end{array}$ & $\frac{\frac{41}{0}}{\frac{0}{1.00}} \frac{1.70}{1.70}$ & $\begin{array}{l}\frac{42}{\underline{0}} \\
\underline{0.09} \\
\underline{1.22} \\
\underline{0.71}\end{array}$ & $\begin{array}{l}\frac{43}{0} \\
0 \\
0.08 \\
0.78 \\
0.71\end{array}$ & $\frac{\frac{44}{0.01}}{\frac{0.00}{0.42}}$ & & $\begin{array}{l}\frac{55}{\underline{0}} \\
\underline{0} \\
\underline{0.08} \\
\underline{0}\end{array}$ & & & & & & & $\begin{array}{l}\frac{2}{0.02} \\
\underline{0} \\
\underline{0.13} \\
\underline{0.63}\end{array}$ & $\begin{array}{l}\frac{3}{0} \\
0 \\
0.50 \\
1.38\end{array}$ & \\
\hline $\begin{array}{l}\text { Ring } \\
\text { Core }\end{array}$ & $\begin{array}{l}\frac{10}{\underline{0}} \\
\underline{0} \\
\underline{0.36} \\
\underline{2.35}\end{array}$ & $\begin{array}{l}\frac{11}{0} \\
0 \\
0.75 \\
2.68\end{array}$ & $\begin{array}{l}\frac{16}{0} \\
0.71 \\
7.33\end{array}$ & $\begin{array}{l}\frac{17}{0} \\
\underline{0.05} \\
\underline{1.57}\end{array}$ & $\begin{array}{l}\frac{21}{0.10} \\
\underline{0} \\
\frac{0.12}{3.03} \\
\underline{0.83}\end{array}$ & $\begin{array}{l}\frac{23}{\underline{0}} \\
\frac{\underline{0}}{0.10} \\
\frac{2.50}{\underline{0}}\end{array}$ & $\begin{array}{c}\frac{24}{0.03} \\
0 \\
0.05 \\
4.38 \\
0.06\end{array}$ & $\begin{array}{l}\frac{28}{0} \\
0 \\
1.07 \\
1.04 \\
0.51\end{array}$ & & & & & & & $\begin{array}{l}\frac{56}{\underline{0}} \\
\underline{0} \\
\underline{0.05} \\
\underline{0.32} \\
\underline{0.25}\end{array}$ & $\begin{array}{l}\frac{57}{\underline{0}} \\
\underline{0} \\
\underline{0} \\
\underline{0.34} \\
\underline{0.39} \\
\end{array}$ & $\begin{array}{c}\frac{58}{0.03} \\
0 \\
0.05 \\
0.71 \\
2.50\end{array}$ & $\begin{array}{l}\frac{61}{\underline{0}} \\
\underline{0} \\
\underline{0.04} \\
\frac{1.92}{3.16} \\
\end{array}$ & $\begin{array}{c}\frac{62}{0.02} \\
0 \\
0.02 \\
1.35 \\
4.29\end{array}$ & $\begin{array}{l}\frac{63}{\underline{0}} \\
\underline{0} \\
\underline{0.05} \\
\underline{0.77} \\
\underline{3.75}\end{array}$ & $\begin{array}{l}\frac{64}{0} \\
0 \\
0 \\
0.67 \\
5.00\end{array}$ & $\begin{array}{l}\frac{1}{0.02} \\
\underline{0} \\
\underline{0.41} \\
\underline{1.67}\end{array}$ & & \\
\hline $\begin{array}{l}\text { Sargasso } \\
\text { Sea } \\
\& \\
\text { Gulf } \\
\text { Stream }\end{array}$ & & & & & & & & & & $\begin{array}{l}\frac{49}{\underline{0}} \\
\underline{0} \\
\underline{0.02} \\
\underline{0.51}\end{array}$ & $\begin{array}{l}\frac{50}{0} \\
0 \\
0 \\
0.78\end{array}$ & $\begin{array}{c}\frac{51}{0.03} \\
0 \\
0.41 \\
1.38\end{array}$ & $\begin{array}{l}\frac{52}{\underline{0}} \\
\underline{0} \\
\underline{0.52} \\
\underline{2.05}\end{array}$ & & & & & & & & & & & \\
\hline $\begin{array}{l}\text { Slope } \\
\text { Water }\end{array}$ & $\frac{8}{-}$ & $\frac{9}{0.12}$ & $\frac{18}{0.63}$ & & $\frac{19}{0.25}$ & $\frac{20}{0.37}$ & $\frac{33}{0.29}$ & $\frac{34}{0.48}$ & & $\frac{35}{0.54}$ & $\frac{47}{0.47}$ & $\frac{48}{0.36}$ & $\frac{53}{0.11}$ & $\frac{54}{1.45}$ & $\frac{59}{0.24}$ & $\frac{60}{0.34}$ & $\frac{65}{0.22}$ & $\frac{66}{0.31}$ & & & & $\frac{4}{0.19}$ & $\frac{5}{0.55}$ & $\frac{6}{0.56}$ \\
\hline $\begin{array}{l}\text { High } \\
\text { Velocity } \\
\text { Region }\end{array}$ & $\frac{12}{0.41}$ & $\frac{13}{0.21}$ & $\frac{14}{0.57}$ & $\frac{15}{0.35}$ & $\frac{27}{0.45}$ & $\frac{29}{0.17}$ & $\frac{30}{0.37}$ & $\frac{31}{0.45}$ & $\frac{32}{0.34}$ & $\frac{41}{0.68}$ & $\frac{42}{0.41}$ & $\frac{43}{0.31}$ & $\frac{44}{0.29}$ & & $\frac{55}{0.03}$ & & & & & & & $\frac{2}{0.20}$ & $\frac{3}{0.47}$ & \\
\hline $\begin{array}{l}\text { Ring } \\
\text { Core }\end{array}$ & $\frac{10}{0.68}$ & $\frac{11}{0.86}$ & $\frac{16}{-}$ & $\frac{17}{0.41}$ & $\frac{21}{0.80}$ & $\frac{23}{0.52}$ & $\frac{24}{0.91}$ & $\frac{28}{0.53}$ & & $\frac{37}{0.19}$ & $\frac{38}{0.16}$ & $\frac{39}{0.06}$ & & & $\frac{56}{0.12}$ & $\frac{57}{0.15}$ & $\frac{58}{0.66}$ & $\frac{61}{1.02}$ & $\frac{62}{1.14}$ & $\frac{63}{0.91}$ & $\frac{64}{1.13}$ & $\frac{1}{0.53}$ & & \\
\hline SS \& GS & & & & & & & & & & $\frac{49}{0.13}$ & $\frac{50}{0.20}$ & $\frac{51}{0.46}$ & $\frac{52}{0.64}$ & & & & & & & & & & & \\
\hline
\end{tabular}


Table 60. Abundance of Cyclothone pseudopallida (specimens $/ 10,000 \mathrm{~m}^{3}$ ).

\begin{tabular}{|c|c|c|c|c|c|c|c|c|c|c|c|c|c|c|c|c|c|c|c|c|c|c|c|c|}
\hline & & $\begin{array}{r}\text { Ocean } \\
\text { Apr }\end{array}$ & $\begin{array}{l}s 118 \\
i 1\end{array}$ & & & & $\begin{array}{l}\text { eanus } \\
\text { June }\end{array}$ & 121 & & & $\begin{array}{r}\text { Oce } \\
A\end{array}$ & $\begin{array}{l}\text { anus } 1 \\
\text { dqust }\end{array}$ & & & & & $\begin{array}{l}\text { Knor } \\
\text { Sept }\end{array}$ & $\begin{array}{r}r 98 \\
-0 c t \\
\end{array}$ & & & & SE & $\begin{array}{c}110 \\
t-0 c t\end{array}$ & \\
\hline $\begin{array}{l}\text { Slope } \\
\text { Water }\end{array}$ & $\frac{\frac{8}{\bar{a}}}{\frac{14.7}{\frac{1.8}{0}}}$ & $\begin{array}{c}\frac{9}{0} \\
17.6 \\
7.9 \\
0\end{array}$ & & & $\begin{array}{l}\frac{19}{\underline{0}} \\
\underline{0} \\
\underline{0.4} \\
\underline{4.2} \\
\underline{0.5}\end{array}$ & $\begin{array}{c}20 \\
0 \\
4.2 \\
2.6 \\
0 \\
0\end{array}$ & $\begin{array}{c}\frac{33}{\underline{0}} \\
\underline{0} \\
\underline{0} \\
\underline{6.7} \\
\underline{0.5}\end{array}$ & $\begin{array}{c}\frac{34}{\underline{0}} \\
\underline{0} \\
\underline{3.0}\end{array}$ & & $\begin{array}{c}35 \\
1.0 \\
0 \\
18.0 \\
0 \\
0\end{array}$ & $\begin{array}{c}\frac{47}{\underline{0}} \\
\underline{1.5} \\
\underline{6.5} \\
\underline{0.2}\end{array}$ & $\begin{array}{c}48 \\
0 \\
2.0 \\
3.2 \\
0.2\end{array}$ & $\begin{array}{l}\frac{53}{\underline{0}} \\
\frac{1.6}{3.8} \\
\underline{0.2}\end{array}$ & \begin{tabular}{r}
\multicolumn{1}{c}{54} \\
0.3 \\
4.1 \\
20.3 \\
0.3
\end{tabular} & $\begin{array}{c}\frac{59}{\underline{0}} \\
\underline{0} \\
\frac{1.2}{0.7} \\
\underline{0} \underline{0}\end{array}$ & $\begin{array}{c}60 \\
0 \\
0 \\
2.8 \\
0 \\
0\end{array}$ & $\begin{array}{r}\frac{65}{\frac{0}{0}} \\
\frac{15.9}{0.4} \\
\frac{0.4}{\underline{0}}\end{array}$ & $\begin{array}{c}66 \\
0 \\
0 \\
9.3 \\
0.5 \\
0\end{array}$ & & & & $\begin{array}{c}\frac{4}{0} \\
11.1 \\
9.7 \\
0\end{array}$ & $\frac{\frac{5}{0.3}}{\frac{0.7}{1.3}}$ & \\
\hline $\begin{array}{l}\text { High } \\
\text { Velocity } \\
\text { Region }\end{array}$ & $\begin{array}{c}\frac{12}{0} \\
3.5 \\
11.7 \\
0\end{array}$ & $\begin{array}{l}\frac{13}{\underline{0}} \\
\frac{1.1}{3.7}\end{array}$ & $\begin{array}{l}\frac{14}{\underline{0}} \\
\frac{3.0}{5.9} \\
\underline{0}\end{array}$ & $\begin{array}{c}15 \\
0.6 \\
12.9 \\
2.4 \\
0\end{array}$ & $\begin{array}{c}26 \\
0 \\
0 \\
11.9 \\
4.5 \\
-\end{array}$ & $\begin{array}{c}27 \\
0 \\
0 \\
16.8 \\
0.4 \\
0\end{array}$ & $\begin{array}{c}30 \\
0 \\
0 \\
9.4 \\
2.4 \\
0\end{array}$ & $\begin{array}{c}31 \\
0 \\
0 \\
13.9 \\
0.6 \\
0\end{array}$ & $\begin{array}{c}\frac{32}{\frac{0.3}{0}} \\
\frac{10.0}{\underline{0}}\end{array}$ & $\frac{\frac{41}{1.2}}{\frac{2.3}{0}}$ & $\frac{\frac{42}{0}}{\frac{\underline{0} .2}{0.5}} \frac{\underline{0}}{\frac{0}{0}}$ & $\begin{array}{c}43 \\
0 \\
0 \\
7.5 \\
0 \\
0\end{array}$ & $\frac{\frac{44}{1.3}}{\frac{0.7}{\underline{0}}}$ & & $\begin{array}{c}\frac{55}{\underline{0}} \\
\underline{0} \\
\underline{2.0} \\
\underline{0}\end{array}$ & & & & , & & & $\begin{array}{c}\frac{2}{\underline{0}} \\
\underline{0} \\
\frac{1.3}{\underline{0}}\end{array}$ & $\begin{array}{c}3 \\
0 \\
8.4 \\
5.4 \\
0\end{array}$ & \\
\hline $\begin{array}{l}\text { Ring } \\
\text { Core }\end{array}$ & $\begin{array}{c}\frac{10}{\underline{0}} \\
\underline{0} \\
\frac{9.1}{\underline{0}}\end{array}$ & $\begin{array}{c}11 \\
0 \\
0 \\
8.8 \\
0\end{array}$ & $\begin{array}{l}\frac{16}{0} \\
10.7 \\
12.7\end{array}$ & $\begin{array}{l}\frac{17}{\underline{0}} \\
\underline{0} \\
\underline{3.4}\end{array}$ & $\begin{array}{c}\frac{21}{\underline{0}} \\
\underline{0} \\
\underline{1.8} \\
\underline{4.6} \\
\underline{0.6}\end{array}$ & $\begin{array}{l}\frac{23}{\underline{0}} \\
\frac{3.5}{0} \\
\frac{5.0}{\underline{0}}\end{array}$ & $\begin{array}{c}24 \\
0 \\
0 \\
5.1 \\
5.6 \\
0.6\end{array}$ & $\begin{array}{c}28 \\
0 \\
0.8 \\
16.4 \\
0 \\
0\end{array}$ & & & & & & & $\begin{array}{c}\frac{56}{\frac{0}{0}} \\
\underline{0} \\
\underline{0} \\
\underline{4.2} \\
10.0 \\
\end{array}$ & $\begin{array}{c}\frac{57}{\underline{0}} \\
\underline{0} \\
\underline{0} \\
\underline{9.7} \\
\underline{7.4}\end{array}$ & $\begin{array}{c}58 \\
0 \\
0 \\
0.5 \\
5.8 \\
6.3\end{array}$ & $\begin{array}{c}\frac{61}{\frac{0.2}{0}} \\
\frac{0.4}{3.1} \\
\frac{0}{\underline{0}}\end{array}$ & $\begin{array}{r}62 \\
0 \\
0.3 \\
0.4 \\
14.1 \\
5.0\end{array}$ & $\begin{array}{c}\frac{63}{\underline{0}} \\
\underline{0} \\
\underline{0} \\
\underline{8.5} \\
\underline{4.4}\end{array}$ & $\begin{array}{c}64 \\
0 \\
0 \\
1.4 \\
8.6 \\
1.5\end{array}$ & $\begin{array}{c}\frac{1}{\underline{0}} \\
\underline{0} \\
\underline{5.0} \\
\underline{2.5}\end{array}$ & & \\
\hline $\begin{array}{l}\text { Sargasso } \\
\text { Sea } \\
\& \\
\text { Gu1f } \\
\text { Stream }\end{array}$ & & & & & & & & & & $\begin{array}{l}\frac{49}{\underline{0}} \\
\underline{0} \\
\underline{0.2} \\
\underline{8.2}\end{array}$ & $\begin{array}{c}50 \\
0 \\
0 \\
0.3 \\
12.8\end{array}$ & $\begin{array}{c}51 \\
0 \\
0 \\
5.9 \\
0\end{array}$ & $\begin{array}{l}\frac{52}{\underline{0}} \\
\underline{0} \\
\underline{7.0} \\
\underline{0.5}\end{array}$ & & & & & & & & & & & \\
\hline $\begin{array}{l}\text { Slope } \\
\text { Water }\end{array}$ & $\frac{8}{4.1}$ & $\frac{9}{6.4}$ & $\frac{18}{1.2}$ & & $\frac{19}{1.3}$ & $\frac{20}{1.7}$ & $\frac{33}{0.9}$ & $\frac{34}{1.8}$ & & $\frac{35}{3.8}$ & $\frac{47}{2.1}$ & $\frac{48}{0.9}$ & $\frac{53}{1.4}$ & $\frac{54}{6.3}$ & $\frac{59}{0.4}$ & $\frac{60}{0.6}$ & $\frac{65}{3.3}$ & $\frac{66}{2.0}$ & & & & $\frac{4}{5.2}$ & $\frac{5}{0.6}$ & $\frac{6}{2.2}$ \\
\hline $\begin{array}{l}\text { High } \\
\text { Velocity } \\
\text { Region }\end{array}$ & $\frac{12}{3.8}$ & $\frac{13}{2.1}$ & $\frac{14}{2.2}$ & $\frac{15}{4.0}$ & $\frac{27}{3.5}$ & $\frac{29}{1.9}$ & $\frac{30}{2.4}$ & $\frac{31}{2.9}$ & $\frac{32}{2.4}$ & $\frac{41}{1.2}$ & $\frac{42}{1.9}$ & $\frac{43}{1.5}$ & $\frac{44}{0.9}$ & & $\frac{55}{0.8}$ & & & & & & & $\frac{2}{0.3}$ & $\frac{3}{3.5}$ & \\
\hline $\begin{array}{l}\text { Ring } \\
\text { Core }\end{array}$ & $\frac{10}{2.3}$ & $\frac{11}{2.2}$ & $\frac{16}{-}$ & $\frac{17}{1.7}$ & $\frac{21}{1.4}$ & $\frac{23}{1.7}$ & $\frac{24}{2.3}$ & $\frac{28}{4.3}$ & & $\frac{37}{0.9}$ & $\frac{38}{1.0}$ & $\frac{39}{2.6}$ & & & $-\frac{56}{2.8}$ & $\frac{57}{3.4}$ & $\frac{58}{2.5}$ & $\frac{61}{2.7}$ & $\frac{62}{3.9}$ & $\frac{63}{2.6}$ & $\frac{64}{2.2}$ & $\frac{1}{1.9}$ & & \\
\hline SS \& GS & & & & & & & & & & $\frac{49}{2.1}$ & $\frac{50}{3.3}$ & $\frac{51}{1.5}$ & $\frac{52}{1.9}$ & & & & & & & & & & & \\
\hline
\end{tabular}


Table 61. Volume of Cyclothone pseudopal1ida $\left(\mathrm{ml} / 10,000 \mathrm{~m}^{3}\right)$.

\begin{tabular}{|c|c|c|c|c|c|c|c|c|c|c|c|c|c|c|c|c|c|c|c|c|c|c|c|c|}
\hline & \multicolumn{4}{|c|}{$\begin{array}{c}\text { Oceanus } 118 \\
\text { April }\end{array}$} & \multicolumn{5}{|c|}{$\begin{array}{c}\text { Oceanus } 121 \\
\text { June }\end{array}$} & \multicolumn{5}{|c|}{$\begin{array}{c}\text { Oceanus } 125 \\
\text { August }\end{array}$} & \multicolumn{7}{|c|}{$\begin{array}{l}\text { Knorr } 98 \\
\text { Sept-0ct }\end{array}$} & \multicolumn{3}{|c|}{$\begin{array}{c}\text { A2 } 110 \\
\text { Sept-0ct }\end{array}$} \\
\hline $\begin{array}{l}\text { Slope } \\
\text { Water }\end{array}$ & $\begin{array}{c}\frac{8}{=} \\
\frac{1.06}{0.23} \\
\frac{0}{\underline{0}}\end{array}$ & $\begin{array}{l}-\frac{9}{0} \\
1.06 \\
1.14 \\
0\end{array}$ & & & $\begin{array}{l}\frac{19}{\underline{0}} \\
\underline{0} \\
\underline{0.04} \\
\underline{0.58} \\
\underline{0.05}\end{array}$ & $\begin{array}{c}20 \\
0 \\
0.26 \\
0.30 \\
0 \\
0\end{array}$ & $\begin{array}{l}\frac{33}{\underline{0}} \\
\underline{0} \\
\underline{0} \\
\underline{0.33} \\
\underline{0.05} \\
\end{array}$ & $\begin{array}{l}\frac{34}{\underline{0}} \\
\underline{0} \\
0.34 \\
\end{array}$ & & $\begin{array}{c}\frac{35}{0.10} \\
0 \\
1.33 \\
0 \\
0\end{array}$ & $\begin{array}{l}\frac{47}{\underline{0}} \\
\underline{0.06} \\
\underline{0.78} \\
\underline{0.04}\end{array}$ & $\begin{array}{l}\frac{48}{0} \\
0.09 \\
0.29 \\
0.04\end{array}$ & $\begin{array}{l}\frac{53}{0} \\
\underline{0.07} \\
0.46 \\
0.04\end{array}$ & $\begin{array}{l}\frac{54}{0.03} \\
0.16 \\
3.03 \\
0.03\end{array}$ & $\begin{array}{l}\frac{59}{\underline{0}} \\
\underline{0} \\
\frac{0.15}{0.14} \\
\underline{0}\end{array}$ & $\begin{array}{l}\frac{60}{0} \\
0 \\
0.31 \\
0 \\
0\end{array}$ & $\begin{array}{l}\frac{65}{\underline{0}} \\
\underline{0} \\
\frac{0.59}{0.04} \\
\underline{0}\end{array}$ & $\begin{array}{c}\frac{66}{0} \\
0 \\
0.56 \\
0.14 \\
0\end{array}$ & & & & $\begin{array}{l}\frac{4}{0} \\
0.80 \\
1.22 \\
0\end{array}$ & $\frac{\frac{5}{0.03}}{\frac{0.07}{0.13}} \frac{\underline{0}}{\underline{0}}$ & \\
\hline $\begin{array}{l}\text { High } \\
\text { Velocity } \\
\text { Region }\end{array}$ & $\begin{array}{c}\frac{12}{0} \\
0.15 \\
1.54 \\
0\end{array}$ & $\begin{array}{l}\frac{13}{\underline{0}} \\
\underline{0.06} \\
\underline{0.61}\end{array}$ & $\begin{array}{l}\frac{14}{\underline{0}} \\
\frac{0.17}{0.88} \\
\underline{0}\end{array}$ & $\begin{array}{c}\frac{15}{0.11} \\
0.94 \\
0.41 \\
0\end{array}$ & $\begin{array}{l}26 \\
0 \\
0 \\
0.67 \\
0.69 \\
-\end{array}$ & $\begin{array}{l}\frac{27}{0} \\
0 \\
1.14 \\
0.08 \\
0\end{array}$ & $\begin{array}{l}30 \\
0 \\
0 \\
0.94 \\
0.49 \\
0\end{array}$ & $\begin{array}{c}31 \\
0 \\
0 \\
0.79 \\
0.09 \\
0\end{array}$ & $\begin{array}{c}\frac{32}{0.04} \\
\underline{0} \\
\underline{0.70} \\
\underline{0}\end{array}$ & $\frac{\frac{41}{0.05}}{\frac{0.20}{\underline{0}}}$ & $\begin{array}{l}\frac{42}{\underline{0}} \\
\frac{0.74}{0.07} \\
\underline{0}\end{array}$ & $\begin{array}{c}\frac{43}{0} \\
0 \\
0.83 \\
0 \\
0\end{array}$ & $\frac{\frac{44}{0.09}}{\frac{0.23}{\underline{0}}}$ & & $\begin{array}{l}\frac{55}{\underline{0}} \\
\underline{0} \\
\underline{0.16} \\
\underline{0}\end{array}$ & & & & & & & $\begin{array}{l}\frac{2}{\underline{0}} \\
\underline{0} \\
\underline{0.13} \\
\underline{0}\end{array}$ & $\begin{array}{c}\frac{3}{0} \\
0.57 \\
0.57 \\
0\end{array}$ & \\
\hline $\begin{array}{l}\text { Ring } \\
\text { Core }\end{array}$ & $\begin{array}{l}\frac{10}{\underline{0}} \\
\underline{0} \\
\frac{1.18}{\underline{0}}\end{array}$ & $\begin{array}{l}\frac{11}{0} \\
0 \\
0.44 \\
0.07\end{array}$ & $\begin{array}{l}\frac{16}{0} \\
0.43 \\
1.33\end{array}$ & $\begin{array}{l}\frac{17}{\underline{0}} \\
\underline{0} \\
\underline{0.22} \\
\end{array}$ & $\begin{array}{l}\frac{21}{\underline{0}} \\
\underline{0} \\
\underline{0.15} \\
\underline{0.55} \\
\underline{0.11}\end{array}$ & $\begin{array}{l}\frac{23}{\underline{0}} \\
\underline{0} \\
\frac{0.20}{0.64} \\
\underline{0}\end{array}$ & $\begin{array}{l}\frac{24}{0} \\
0 \\
0.41 \\
0.75 \\
0.06\end{array}$ & $\begin{array}{l}\frac{28}{0} \\
0.04 \\
1.61 \\
0 \\
0\end{array}$ & & & & & & & $\begin{array}{l}\frac{56}{\underline{0}} \\
\underline{0} \\
\underline{0} \\
\underline{0.21} \\
\underline{0.78} \\
\end{array}$ & $\begin{array}{l}\frac{57}{\underline{0}} \\
\underline{0} \\
\underline{0} \\
\underline{0.25} \\
\underline{0.53} \\
\end{array}$ & $\begin{array}{l}\frac{58}{0} \\
0.04 \\
0.05 \\
0.19 \\
0.66\end{array}$ & $\begin{array}{c}\frac{61}{0.02} \\
\underline{0} \\
\frac{0.04}{0.65} \\
\underline{0}\end{array}$ & $\begin{array}{l}\frac{62}{0} \\
0.03 \\
0.02 \\
0.43 \\
0.36\end{array}$ & $\begin{array}{l}\frac{63}{\underline{0}} \\
\underline{0} \\
\underline{0} \\
\underline{0.27} \\
\underline{0.41}\end{array}$ & $\begin{array}{l}\frac{64}{0} \\
0 \\
0 \\
0.48 \\
0.12\end{array}$ & $\begin{array}{l}\frac{1}{\underline{0}} \\
\underline{0} \\
\underline{0.47} \\
\underline{0.25}\end{array}$ & & \\
\hline $\begin{array}{l}\text { Sargasso } \\
\text { Sea } \\
\& \\
\text { Gulf } \\
\text { Stream }\end{array}$ & & & & & & & & & & $\begin{array}{l}\frac{49}{\underline{0}} \\
\underline{0} \\
\underline{0.02} \\
\underline{0.38}\end{array}$ & $\begin{array}{c}50 \\
0 \\
0 \\
0.03 \\
0.63\end{array}$ & $\begin{array}{l}\frac{51}{0} \\
0 \\
0.43 \\
0\end{array}$ & $\begin{array}{l}\frac{52}{\underline{0}} \\
\underline{0} \\
\underline{0.70} \\
\underline{0.03}\end{array}$ & & & & & & & & & & & \\
\hline $\begin{array}{l}\text { Slope } \\
\text { Water }\end{array}$ & $\frac{8}{-}$ & $\frac{9}{0.58}$ & $\frac{18}{0.17}$ & & $\frac{19}{0.17}$ & $\frac{20}{0.14}$ & $\frac{33}{0.06}$ & $\frac{34}{0.20}$ & & $\frac{35}{0.29}$ & $\frac{47}{0.22}$ & $\frac{48}{0.11}$ & $\frac{53}{0.14}$ & $\frac{54}{0.81}$ & $\frac{59}{0.06}$ & $\frac{60}{0.06}$ & $\frac{65}{0.13}$ & $\frac{66}{0.14}$ & & & & $\frac{4}{0.51}$ & $\frac{5}{0.06}$ & $\frac{6}{0.27}$ \\
\hline $\begin{array}{l}\text { High } \\
\text { Velocity } \\
\text { Region }\end{array}$ & $\frac{12}{0.42}$ & $\frac{13}{0.17}$ & $\frac{14}{0.26}$ & $\frac{15}{0.37}$ & $\frac{27}{0.25}$ & $\frac{29}{0.14}$ & $\frac{30}{0.29}$ & $\frac{31}{0.18}$ & $\frac{32}{0.18}$ & $\frac{41}{0.08}$ & $\frac{42}{0.18}$ & $\frac{43}{0.17}$ & $\frac{44}{0.10}$ & & $\frac{55}{0.06}$ & & & & & & & $\frac{2}{0.03}$ & $\frac{3}{0.29}$ & \\
\hline $\begin{array}{l}\text { Ring } \\
\text { Core }\end{array}$ & $\frac{10}{0.30}$ & $\frac{11}{0.13}$ & $\frac{16}{-}$ & $\frac{17}{0.06}$ & $\frac{21}{0.16}$ & $\frac{23}{0.17}$ & $\frac{24}{0.24}$ & $\frac{28}{0.41}$ & & $\frac{37}{0.07}$ & $\frac{38}{0.07}$ & $\frac{39}{0.16}$ & & & $\frac{56}{0.20}$ & $\frac{57}{0.16}$ & $\frac{58}{0.18}$ & $\frac{61}{0.14}$ & $\frac{62}{0.17}$ & $\frac{63}{0.14}$ & $\frac{64}{0.12}$ & $\frac{1}{0.18}$ & & \\
\hline SS \& GS & & & & & & & & & & $\frac{49}{0.10}$ & $\frac{50}{0.17}$ & $\frac{51}{0.11}$ & $\frac{52}{0.18}$ & & & & & & & & & & & \\
\hline
\end{tabular}


Table 62. Abundance of Derichthys serpentinus (specimens $/ 10,000 \mathrm{~m}^{3}$ ).

\begin{tabular}{|c|c|c|c|c|c|c|c|c|c|c|c|c|c|c|c|c|c|c|c|c|c|c|c|c|}
\hline & \multicolumn{4}{|c|}{$\begin{array}{c}\text { Oceanus } 118 \\
\text { April }\end{array}$} & \multicolumn{5}{|c|}{$\begin{array}{c}\text { Oceanus } 121 \\
\text { June }\end{array}$} & \multicolumn{5}{|c|}{$\begin{array}{c}\text { Oceanus } 125 \\
\text { August }\end{array}$} & \multicolumn{7}{|c|}{$\begin{array}{l}\text { Knorr } 98 \\
\text { Sept-Oct }\end{array}$} & \multicolumn{3}{|c|}{$\begin{array}{c}\text { A2 } 110 \\
\text { Sept-0ct }\end{array}$} \\
\hline $\begin{array}{l}\text { Slope } \\
\text { Water }\end{array}$ & $\begin{array}{l}\frac{8}{0} \\
\frac{0}{0} \\
\frac{0}{0}\end{array}$ & $\begin{array}{c}9 \\
0 \\
0 \\
0.7 \\
0.7\end{array}$ & & & $\begin{array}{l}\frac{19}{\underline{0}} \\
\frac{0}{0} \\
\underline{0} \\
\underline{0}\end{array}$ & $\begin{array}{r}20 \\
0 \\
0 \\
0 \\
0 \\
0\end{array}$ & $\begin{array}{c}33 \\
\underline{0} \\
\underline{0} \\
\underline{0} \\
\underline{0} \\
\underline{0.1}\end{array}$ & $\begin{array}{c}\frac{34}{\underline{0}} \\
\underline{0} \\
\underline{0.5}\end{array}$ & & $\begin{array}{c}35 \\
0 \\
0 \\
0 \\
0.6 \\
0\end{array}$ & $\frac{\frac{47}{1.5}}{\frac{0.3}{\underline{0}}}$ & $\begin{array}{c}48 \\
0 \\
0 \\
0.3 \\
0.2\end{array}$ & $\frac{53}{\frac{0.2}{\underline{0}}}$ & $\begin{array}{c}54 \\
0 \\
0.3 \\
0 \\
0\end{array}$ & $\begin{array}{l}\frac{59}{\underline{0}} \\
\frac{0.4}{0.6} \\
\frac{0}{0}\end{array}$ & $\begin{array}{c}60 \\
0 \\
0 \\
0 \\
0.4 \\
0\end{array}$ & $\begin{array}{l}\frac{65}{0} \\
\underline{0} \\
\underline{0} \\
\underline{0} \\
\underline{0}\end{array}$ & $\begin{array}{c}66 \\
0 \\
0 \\
0 \\
0.5 \\
0\end{array}$ & & & & $\begin{array}{l}4 \\
0 \\
0 \\
0 \\
0.3\end{array}$ & $\begin{array}{l}\frac{5}{0} \\
\frac{0.4}{\underline{0}} \\
\underline{0}\end{array}$ & \\
\hline $\begin{array}{l}\text { High } \\
\text { Velocity } \\
\text { Region }\end{array}$ & $\begin{array}{c}12 \\
0 \\
0 \\
0 \\
1.0\end{array}$ & $\frac{13}{\underline{0}} \frac{0.6}{\underline{0}}$ & $\begin{array}{c}\frac{14}{\underline{0}} \\
\frac{0.3}{0.6} \\
\underline{0}\end{array}$ & $\begin{array}{r}15 \\
0 \\
0 \\
0 \\
0\end{array}$ & $\begin{array}{c}26 \\
0 \\
0 \\
0 \\
0.7 \\
-\end{array}$ & $\begin{array}{r}27 \\
0 \\
0 \\
0 \\
0 \\
0\end{array}$ & $\begin{array}{c}30 \\
0 \\
0 \\
0 \\
0.3 \\
0.9\end{array}$ & $\begin{array}{r}31 \\
0 \\
0 \\
0 \\
0 \\
0\end{array}$ & $\frac{\frac{32}{0.5}}{\frac{0.4}{\underline{0}}}$ & $\begin{array}{l}\frac{41}{\underline{0}} \\
\underline{0.3} \\
\underline{0}\end{array}$ & $\frac{42}{\frac{0}{0}} \frac{0.3}{\underline{0}}$ & $\begin{array}{r}43 \\
0 \\
0 \\
0 \\
0 \\
0\end{array}$ & $\frac{\frac{44}{0.1}}{\frac{0.0}{\underline{0}}}$ & & $\begin{array}{l}55 \\
\underline{0} \\
\underline{0} \\
\underline{0}\end{array}$ & & & & & & & $\begin{array}{l}\frac{2}{0} \\
\underline{0} \\
\underline{0} \\
\underline{0}\end{array}$ & $\begin{array}{l}3 \\
0 \\
0 \\
0 \\
0\end{array}$ & \\
\hline $\begin{array}{l}\text { Ring } \\
\text { Core }\end{array}$ & $\begin{array}{r}10 \\
\frac{0}{0} \\
\underline{0} \\
\underline{0} \\
\underline{0}\end{array}$ & $\begin{array}{c}11 \\
0 \\
0 \\
0 \\
0.4\end{array}$ & $\begin{array}{r}16 \\
0 \\
0 \\
0\end{array}$ & $\begin{array}{r}17 \\
\underline{0} \\
\underline{0} \\
\underline{0}\end{array}$ & $\begin{array}{l}\frac{21}{\underline{0}} \\
\frac{0.2}{\underline{0}} \\
\underline{0} \\
\underline{0}\end{array}$ & $\begin{array}{c}\frac{23}{\underline{0}} \\
\underline{0} \\
\frac{1.5}{\underline{0}} \\
\underline{0}\end{array}$ & $\begin{array}{c}24 \\
0 \\
0 \\
0 \\
0.6 \\
0\end{array}$ & $\begin{array}{r}28 \\
0 \\
0 \\
0 \\
0 \\
0\end{array}$ & & & & & & & $\begin{array}{l}56 \\
\underline{0} \\
\underline{0} \\
\underline{0} \\
\underline{0} \\
\underline{0}\end{array}$ & $\begin{array}{l}57 \\
\underline{0} \\
\underline{0} \\
\underline{0} \\
\underline{0} \\
\underline{0}\end{array}$ & $\begin{array}{r}58 \\
0 \\
0 \\
0 \\
0 \\
0\end{array}$ & $\begin{array}{l}61 \\
\underline{0} \\
\underline{0} \\
\underline{0} \\
\underline{0} \\
\underline{0}\end{array}$ & $\begin{array}{r}62 \\
0 \\
0 \\
0 \\
0 \\
0\end{array}$ & $\begin{array}{l}\frac{63}{0.2} \\
\frac{0}{0} \\
\underline{0} \\
\underline{0}\end{array}$ & $\begin{array}{r}64 \\
0 \\
0 \\
0 \\
0 \\
0\end{array}$ & $\begin{array}{l}\frac{1}{\underline{0}} \\
\frac{0.2}{\underline{0}} \\
\underline{0}\end{array}$ & & \\
\hline $\begin{array}{l}\text { Sargasso } \\
\text { Sea } \\
\& \\
\text { Gulf } \\
\text { Stream }\end{array}$ & & & & & & & & & & $\frac{49}{\underline{0}}$ & $\begin{array}{r}50 \\
0 \\
0 \\
0 \\
0\end{array}$ & $\begin{array}{c}51 \\
0 \\
0 \\
0.2 \\
0\end{array}$ & $\begin{array}{c}\frac{52}{\underline{0}} \\
\underline{0} \\
\frac{0.4}{\underline{0}}\end{array}$ & & & & & & & & & & & \\
\hline $\begin{array}{l}\text { Slope } \\
\text { Water }\end{array}$ & $\frac{8}{-}$ & $\frac{9}{0.4}$ & $\frac{18}{0.1}$ & & $\frac{19}{0}$ & $\frac{20}{0}$ & $\frac{33}{0.1}$ & $\frac{34}{0.3}$ & & $\frac{35}{0.1}$ & $\frac{47}{0.4}$ & $\frac{48}{0.1}$ & $\frac{53}{0.1}$ & $\frac{54}{0.1}$ & $\frac{59}{0.2}$ & $\frac{60}{0.1}$ & $\frac{65}{0}$ & $\frac{66}{0.1}$ & & & & $\frac{4}{0.1}$ & $\frac{5}{0.1}$ & $\frac{6}{0.1}$ \\
\hline $\begin{array}{l}\text { High } \\
\text { Velocity } \\
\text { Region }\end{array}$ & $\frac{12}{0.3}$ & $\frac{13}{0.1}$ & $\frac{14}{0.2}$ & $\frac{15}{0}$ & $\frac{27}{0}$ & $\frac{29}{0.1}$ & $\frac{30}{0.3}$ & $\frac{31}{0}$ & $\frac{32}{-}$ & $\frac{41}{0.1}$ & $\frac{42}{0.1}$ & $\frac{43}{0}$ & $\frac{44}{0.1}$ & & $\frac{55}{0}$ & & & & & & & $\frac{2}{0}$ & $\frac{3}{0}$ & \\
\hline $\begin{array}{l}\text { Ring } \\
\text { Core }\end{array}$ & $\frac{10}{0}$ & $\frac{11}{0.1}$ & $\frac{16}{-}$ & $\frac{17}{0}$ & $\frac{21}{0.1}$ & $\frac{23}{0.3}$ & $\frac{24}{0.1}$ & $\frac{28}{0}$ & & $\frac{37}{0.1}$ & $\frac{38}{0}$ & $\frac{39}{0.1}$ & & & $\frac{56}{0}$ & $\begin{array}{r}57 \\
0\end{array}$ & $\frac{58}{0}$ & $\frac{61}{0}$ & $\frac{62}{0}$ & $\frac{63}{0.1}$ & $\frac{64}{n}$ & $\frac{1}{0.1}$ & & \\
\hline SS \& GS & & & & & & & & & & $\frac{49}{0}$ & $\frac{50}{0}$ & $\frac{51}{0.1}$ & $\frac{52}{0.1}$ & & & & & & & & & & & \\
\hline
\end{tabular}


Table 63. Volume of Derichthys serpentinus $\left(\mathrm{ml} / 10,000 \mathrm{~m}^{3}\right)$.

\begin{tabular}{|c|c|c|c|c|c|c|c|c|c|c|c|c|c|c|c|c|c|c|c|c|c|c|c|c|}
\hline & \multicolumn{4}{|c|}{$\begin{array}{c}\text { Oceanus } 118 \\
\text { April }\end{array}$} & \multicolumn{5}{|c|}{$\begin{array}{c}\text { Oceanus } 121 \\
\text { June }\end{array}$} & \multicolumn{5}{|c|}{$\begin{array}{l}\text { Oceanus } 125 \\
\text { August }\end{array}$} & \multicolumn{7}{|c|}{$\begin{array}{l}\text { Knorr } 98 \\
\text { Sept-0ct }\end{array}$} & \multicolumn{3}{|c|}{$\begin{array}{c}\text { A2 } 110 \\
\text { Sept-0ct }\end{array}$} \\
\hline $\begin{array}{l}\text { Slope } \\
\text { Water }\end{array}$ & $\begin{array}{l}\frac{8}{\overline{\overline{0}}} \\
\underline{0} \\
\underline{0}\end{array}$ & $\begin{array}{c}\frac{9}{0} \\
0 \\
4.29 \\
12.67\end{array}$ & & & $\begin{array}{l}\frac{19}{0} \\
\underline{0} \\
\underline{0} \\
\underline{0} \\
\underline{0}\end{array}$ & $\begin{array}{l}20 \\
0 \\
0 \\
0 \\
0 \\
0\end{array}$ & $\begin{array}{l}\frac{33}{0} \\
\frac{0}{0} \\
\underline{0} \\
\underline{0} \\
\underline{0.74}\end{array}$ & $\begin{array}{l}\frac{34}{\underline{0}} \\
\underline{0} \\
\underline{2.73} \\
\end{array}$ & & $\begin{array}{l}\frac{35}{0} \\
0 \\
0 \\
1.67 \\
0\end{array}$ & $\frac{\frac{47}{5.56}}{\frac{1.00}{\frac{0}{0}}}$ & $\begin{array}{l}\frac{48}{0} \\
0 \\
2.06 \\
1.02\end{array}$ & $\frac{\frac{53}{0.42}}{\frac{0}{0}}$ & $\begin{array}{l}\frac{54}{0} \\
1.25 \\
0 \\
0\end{array}$ & $\begin{array}{l}\frac{59}{\underline{0}} \\
\frac{1.52}{2.65} \\
\frac{0}{0}\end{array}$ & $\begin{array}{l}60 \\
0 \\
0 \\
0 \\
1.26 \\
0\end{array}$ & $\begin{array}{l}\frac{65}{0} \\
\underline{0} \\
\underline{0} \\
\underline{0} \\
\underline{0}\end{array}$ & $\begin{array}{l}\frac{66}{0} \\
0 \\
0 \\
0.48 \\
0\end{array}$ & & & & $\begin{array}{l}\frac{4}{0} \\
0 \\
0 \\
0.88\end{array}$ & $\frac{\frac{5}{\underline{0}}}{\frac{1.11}{\underline{0}}}$ & \\
\hline $\begin{array}{l}\text { High } \\
\text { Velocity } \\
\text { Region }\end{array}$ & $\begin{array}{l}\frac{12}{0} \\
0 \\
0 \\
8.62\end{array}$ & $\frac{\frac{13}{\underline{0}}}{\frac{3.06}{\underline{0}}}$ & $\frac{\frac{14}{\underline{0}}}{\frac{0.67}{1.18}} \frac{\underline{0}}{\underline{1}}$ & $\begin{array}{l}\frac{15}{0} \\
0 \\
0 \\
0\end{array}$ & $\begin{array}{l}\frac{26}{0} \\
0 \\
0 \\
9.66 \\
-\end{array}$ & $\begin{array}{l}27 \\
0 \\
0 \\
0 \\
0 \\
0\end{array}$ & $\begin{array}{l}\frac{30}{0} \\
0 \\
0 \\
0.30 \\
2.79\end{array}$ & $\begin{array}{l}31 \\
0 \\
0 \\
0 \\
0 \\
0\end{array}$ & $\frac{\frac{32}{3.68}}{\frac{2.80}{\underline{0}}}$ & $\frac{\frac{41}{\underline{0}}}{\frac{2.57}{\underline{0}}}$ & $\frac{\frac{42}{\underline{0}}}{\frac{3.53}{\underline{0}}}$ & $\begin{array}{l}\frac{43}{0} \\
0 \\
0 \\
0 \\
0\end{array}$ & $\frac{\frac{44}{0.14}}{\frac{0}{0}}$ & & $\begin{array}{l}\frac{55}{0} \\
\underline{0} \\
\underline{0} \\
\underline{0}\end{array}$ & & & & & & & $\begin{array}{l}\frac{2}{0} \\
\underline{0} \\
\underline{0} \\
\underline{0}\end{array}$ & $\begin{array}{l}3 \\
0 \\
0 \\
0 \\
0\end{array}$ & \\
\hline $\begin{array}{l}\text { Ring } \\
\text { Core }\end{array}$ & $\begin{array}{l}\frac{10}{0} \\
\frac{0}{0} \\
\frac{0}{0}\end{array}$ & $\begin{array}{l}11 \\
0 \\
0 \\
0 \\
0.93\end{array}$ & $\begin{array}{l}\frac{16}{0} \\
0 \\
0\end{array}$ & $\begin{array}{l}\frac{17}{\underline{0}} \\
\frac{0}{0}\end{array}$ & $\begin{array}{l}\frac{21}{\underline{0}} \\
\underline{0.08} \\
\underline{0} \\
\underline{0} \\
\underline{0}\end{array}$ & $\begin{array}{l}\frac{23}{\underline{0}} \\
\underline{\underline{0}} \\
\frac{3.50}{\underline{0}} \\
\underline{0}\end{array}$ & $\begin{array}{c}\frac{24}{0} \\
0 \\
0 \\
2.06 \\
0\end{array}$ & $\begin{array}{l}28 \\
0 \\
0 \\
0 \\
0 \\
0\end{array}$ & & . & & & & & $\begin{array}{l}\frac{56}{\underline{0}} \\
\underline{0} \\
\underline{0} \\
\underline{0} \\
\underline{0}\end{array}$ & $\begin{array}{l}57 \\
\underline{0} \\
\underline{0} \\
\underline{0} \\
\underline{0} \\
\underline{0}\end{array}$ & $\begin{array}{l}58 \\
0 \\
0 \\
0 \\
0 \\
0\end{array}$ & $\begin{array}{l}\frac{61}{0} \\
\underline{0} \\
\underline{0} \\
\underline{0} \\
\underline{0}\end{array}$ & $\begin{array}{l}\frac{62}{0} \\
0 \\
0 \\
0 \\
0\end{array}$ & $\begin{array}{l}\frac{63}{0.02} \\
\underline{0} \\
\underline{0} \\
\underline{0} \\
\underline{0}\end{array}$ & $\begin{array}{l}\frac{64}{0} \\
0 \\
0 \\
0 \\
0\end{array}$ & $\frac{\frac{1}{\underline{0}}}{\frac{0.30}{\underline{0}}}$ & & \\
\hline $\begin{array}{l}\text { Sargasso } \\
\text { Sea } \\
\& \\
\text { Gulf } \\
\text { Stream }\end{array}$ & & & & & & & & & & $\begin{array}{l}\frac{49}{\underline{0}} \\
\underline{0} \\
\underline{0} \\
\underline{0}\end{array}$ & $\begin{array}{l}50 \\
0 \\
0 \\
0 \\
0\end{array}$ & $\begin{array}{l}\frac{51}{0} \\
0 \\
0.02 \\
0\end{array}$ & $\begin{array}{l}\frac{52}{\underline{0}} \\
\underline{0} \\
\underline{3.91} \\
\underline{0}\end{array}$ & & & & & & & & & & & \\
\hline $\begin{array}{l}\text { Slope } \\
\text { Water }\end{array}$ & $\frac{8}{-}$ & $\frac{9}{4.24}$ & $\frac{18}{0.24}$ & & $\frac{19}{0}$ & $\frac{20}{0}$ & $\frac{33}{0.37}$ & $\frac{34}{1.64}$ & & $\frac{35}{0.33}$ & $\frac{47}{1.64}$ & $\frac{48}{0.77}$ & $\frac{53}{0.11}$ & $\frac{54}{0.31}$ & $\frac{59}{0.83}$ & $\frac{60}{0.25}$ & $\frac{65}{0}$ & $\frac{66}{0.10}$ & & & & $\frac{4}{0.22}$ & $\frac{5}{0.28}$ & $\frac{6}{0.03}$ \\
\hline $\begin{array}{l}\text { High } \\
\text { Velocity } \\
\text { Region }\end{array}$ & $\frac{12}{2.16}$ & $\frac{13}{0.77}$ & $\frac{14}{0.46}$ & $\frac{15}{0}$ & $\frac{27}{0}$ & $\frac{29}{0.04}$ & $\frac{30}{0.62}$ & $\frac{31}{0}$ & $\frac{32}{-}$ & $\frac{41}{0.64}$ & $\frac{42}{0.78}$ & $\frac{43}{0}$ & $\frac{44}{0.08}$ & & $\frac{55}{0}$ & & & & & & & $\frac{2}{0}$ & $\frac{3}{0}$ & \\
\hline $\begin{array}{l}\text { Ring } \\
\text { Core }\end{array}$ & $\frac{10}{0}$ & $\frac{11}{0.23}$ & $\frac{16}{-}$ & $\frac{17}{0}$ & $\frac{21}{0.03}$ & $\frac{23}{0.70}$ & $\frac{24}{0.41}$ & $\frac{28}{0}$ & & $\frac{37}{0.19}$ & $\frac{38}{0}$ & $\frac{39}{0.14}$ & & & $\frac{56}{0}$ & $\frac{57}{0}$ & $\frac{58}{0}$ & $\frac{61}{0}$ & $\frac{62}{0}$ & $\frac{63}{0.01}$ & $\frac{64}{0}$ & $\frac{1}{0.10}$ & & \\
\hline SS \& GS & & & & & & & & & & $\frac{49}{0}$ & $\frac{50}{0}$ & $\frac{51}{0.01}$ & $\frac{52}{0.98}$ & & & & & & & & & & & \\
\hline
\end{tabular}


Table 64. Abundance of Diaphus dumerilii (specimens $/ 10,000 \mathrm{~m}^{3}$ ).

\begin{tabular}{|c|c|c|c|c|c|c|c|c|c|c|c|c|c|c|c|c|c|c|c|c|c|c|c|c|}
\hline & \multicolumn{4}{|c|}{$\begin{array}{c}\text { Oceanus } 118 \\
\text { Apri1 }\end{array}$} & \multicolumn{5}{|c|}{$\begin{array}{c}\text { Oceanus } 121 \\
\text { June }\end{array}$} & \multicolumn{5}{|c|}{$\begin{array}{l}\text { Oceanus } 125 \\
\text { August }\end{array}$} & \multicolumn{7}{|c|}{$\begin{array}{l}\text { Knorr } 98 \\
\text { Sept-0ct }\end{array}$} & \multicolumn{3}{|c|}{$\begin{array}{c}\text { A2 } 110 \\
\text { Sept-0ct }\end{array}$} \\
\hline $\begin{array}{l}\text { Slope } \\
\text { Water }\end{array}$ & $\begin{array}{l}\frac{8}{\overline{0}} \\
\underline{0} \\
\underline{0} \\
\underline{0}\end{array}$ & $\begin{array}{c}\frac{9}{0} \\
1.2 \\
0 \\
0\end{array}$ & & & 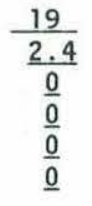 & $\begin{array}{c}20 \\
0 \\
0.5 \\
0.4 \\
0 \\
0\end{array}$ & $\frac{\frac{33}{6.3}}{\frac{0}{0}} \frac{\underline{0}}{\underline{0}}$ & $\frac{34}{\frac{1.8}{\underline{0}}}$ & & $\begin{array}{c}35 \\
0 \\
0 \\
1.3 \\
0 \\
0\end{array}$ & $\frac{47}{\frac{0.2}{\underline{0}}}$ & $\begin{array}{c}48 \\
0 \\
0.9 \\
0 \\
0\end{array}$ & $\frac{53}{\frac{1.7}{\frac{0}{0}}}$ & $\begin{array}{c}54 \\
0 \\
0.3 \\
0 \\
0\end{array}$ & $\begin{array}{l}\frac{59}{\underline{0.3}} \\
\underline{0} \\
\underline{0} \\
\underline{0} \\
\underline{0}\end{array}$ & $\begin{array}{r}60 \\
0 \\
0 \\
0 \\
0 \\
0\end{array}$ & $\frac{\frac{65}{2.2}}{\frac{\underline{0}}{\underline{0}}}$ & $\begin{array}{c}66 \\
0 \\
1.4 \\
0.7 \\
0 \\
0\end{array}$ & & & & $\begin{array}{c}\frac{4}{0} \\
0.4 \\
0 \\
0\end{array}$ & $\frac{\frac{5}{0.7}}{\frac{0}{\underline{0}}}$ & \\
\hline $\begin{array}{l}\text { High } \\
\text { Velocity } \\
\text { Region }\end{array}$ & $\begin{array}{c}\frac{12}{0} \\
0.4 \\
0 \\
0\end{array}$ & $\frac{\frac{13}{0.5}}{\frac{0}{0}}$ & $\frac{14}{\frac{0.9}{\underline{0}}} \frac{\underline{0}}{\underline{0}}$ & $\begin{array}{c}15 \\
0 \\
1.8 \\
0 \\
0\end{array}$ & $\begin{array}{c}26 \\
0 \\
0 \\
1.0 \\
0 \\
-\end{array}$ & $\begin{array}{c}27 \\
0 \\
0 \\
0.5 \\
0 \\
0\end{array}$ & $\begin{array}{c}30 \\
0 \\
0 \\
0 \\
0 \\
0\end{array}$ & $\begin{array}{r}31 \\
0 \\
0 \\
0 \\
0 \\
0\end{array}$ & $\begin{array}{r}32 \\
\underline{0} \\
\underline{0} \\
\underline{0} \\
\underline{0}\end{array}$ & $\frac{41}{\frac{1.0}{\underline{0}}}$ & $\frac{\frac{42}{0.6}}{\frac{0}{0}}$ & $\begin{array}{c}43 \\
0 \\
0 \\
0.4 \\
0 \\
0\end{array}$ & $\frac{\frac{44}{3.2}}{\frac{0}{0}}$ & & $\frac{\frac{55}{3.4}}{\frac{0}{0}}$ & & & & & & & $\frac{\frac{2}{2.5}}{\frac{0}{0}} \frac{0}{\underline{0}}$ & $\begin{array}{c}3 \\
0 \\
0.8 \\
0 \\
0\end{array}$ & \\
\hline $\begin{array}{l}\text { Ring } \\
\text { Core }\end{array}$ & $\frac{10}{\frac{0}{0}}$ & $\begin{array}{r}11 \\
0 \\
0 \\
0 \\
0\end{array}$ & $\begin{array}{c}16 \\
0 \\
0 \\
0\end{array}$ & $\begin{array}{r}17 \\
0 \\
0 \\
0\end{array}$ & $\begin{array}{r}21 \\
\underline{0} \\
\underline{0} \\
\underline{0} \\
\underline{0} \\
\underline{0}\end{array}$ & $\begin{array}{l}2 \underline{23} \\
\underline{0} \\
\underline{0} \\
\underline{0} \\
\underline{0} \\
\underline{0}\end{array}$ & $\begin{array}{r}24 \\
0 \\
0 \\
0 \\
0 \\
0\end{array}$ & $\begin{array}{r}28 \\
0 \\
0 \\
0 \\
0 \\
0\end{array}$ & & & & & & & $\frac{56}{\frac{5.2}{\underline{0}}}$ & $\begin{array}{c}57 \\
\underline{0} \\
\underline{0} \\
\underline{0} \\
\underline{0} \\
\underline{0}\end{array}$ & $\begin{array}{c}58 \\
0 \\
0 \\
0 \\
0 \\
0\end{array}$ & $\begin{array}{l}61 \\
\underline{0} \\
\underline{0} \\
\underline{0} \\
\underline{0} \\
\underline{0}\end{array}$ & $\begin{array}{c}62 \\
0 \\
0 \\
0 \\
0.3 \\
0\end{array}$ & $\begin{array}{r}63 \\
\underline{0} \\
\underline{0} \\
\underline{0} \\
\underline{0} \\
\underline{0}\end{array}$ & $\begin{array}{r}64 \\
0 \\
0 \\
0 \\
0 \\
0\end{array}$ & $\begin{array}{l}\frac{1}{0} \\
\underline{0} \\
\underline{0} \\
\underline{0}\end{array}$ & & \\
\hline $\begin{array}{l}\text { Sargasso } \\
\text { Sea } \\
\& \\
\text { Gulf } \\
\text { Stream }\end{array}$ & & & & & & & & & & $\begin{array}{l}\frac{49}{\underline{0}} \\
\underline{0} \\
\underline{0} \\
\underline{0}\end{array}$ & $\begin{array}{r}50 \\
0 \\
0 \\
0 \\
0\end{array}$ & $\begin{array}{c}51 \\
0 \\
0 \\
0.7 \\
0\end{array}$ & $\frac{52}{\frac{0.2}{\underline{0}}} \frac{\underline{0}}{\underline{0}}$ & & & & & & & & & & & \\
\hline $\begin{array}{l}\text { Slope } \\
\text { Water }\end{array}$ & $\frac{8}{-}$ & $\frac{9}{0.3}$ & $\frac{18}{0.1}$ & & $\frac{19}{0.3}$ & $\frac{20}{0.2}$ & $\frac{33}{0.4}$ & $\frac{34}{0.1}$ & & $\frac{35}{0.3}$ & $\frac{47}{0.1}$ & $\frac{48}{0.2}$ & $\frac{53}{0.4}$ & $\frac{54}{0.1}$ & $\frac{59}{0.1}$ & $\frac{60}{0}$ & $\frac{65}{0.4}$ & $\frac{66}{0.3}$ & & & & $\frac{4}{0.1}$ & $\frac{5}{0.2}$ & $\frac{6}{0.2}$ \\
\hline $\begin{array}{l}\text { High } \\
\text { Velocity } \\
\text { Region }\end{array}$ & $\frac{12}{0.1}$ & $\frac{13}{0.1}$ & $\frac{14}{0.2}$ & $\frac{15}{0.5}$ & $\frac{27}{0.1}$ & $\frac{29}{0}$ & $\frac{30}{0}$ & $\frac{31}{0}$ & $\frac{32}{-}$ & $\frac{41}{0.5}$ & $\frac{42}{0.2}$ & $\frac{43}{0.1}$ & $\frac{44}{1.9}$ & & $\frac{55}{0.7}$ & & & & & & & $\frac{2}{0.6}$ & $\frac{3}{0.2}$ & \\
\hline $\begin{array}{l}\text { Ring } \\
\text { Core }\end{array}$ & $\frac{10}{0}$ & $\frac{11}{0}$ & $\frac{16}{-}$ & $\frac{17}{0}$ & $\frac{21}{0}$ & $\frac{23}{0}$ & $\frac{24}{0}$ & $\frac{28}{0}$ & & $\frac{37}{0.7}$ & $\frac{38}{0.2}$ & $\frac{39}{0.5}$ & & & $\frac{56}{0.2}$ & $\begin{array}{r}57 \\
0\end{array}$ & $\frac{58}{0}$ & $\frac{61}{0}$ & $\frac{62}{0.1}$ & $\frac{63}{0}$ & $\frac{64}{0}$ & $\frac{1}{0}$ & & \\
\hline SS \& GS & & & & & & & & & & $\frac{49}{0}$ & $\frac{50}{0}$ & $\frac{51}{0.2}$ & $\frac{52}{0.1}$ & & & & & & & & & & & \\
\hline
\end{tabular}


Table 65. Volume of Diaphus effulgens $\left(\mathrm{ml} / 10,000 \mathrm{~m}^{3}\right)$.

\begin{tabular}{|c|c|c|c|c|c|c|c|c|c|c|c|c|c|c|c|c|c|c|c|c|c|c|c|c|}
\hline & \multicolumn{4}{|c|}{$\begin{array}{c}\text { Oceanus } \\
\text { April }\end{array}$} & \multicolumn{5}{|c|}{$\begin{array}{c}\text { Oceanus } 121 \\
\text { June }\end{array}$} & \multicolumn{5}{|c|}{$\begin{array}{l}\text { Oceanus } 125 \\
\text { August }\end{array}$} & \multicolumn{7}{|c|}{$\begin{array}{l}\text { Knorr } 98 \\
\text { Sept-Oct }\end{array}$} & \multicolumn{3}{|c|}{$\begin{array}{c}\text { A2 } 110 \\
\text { Sept-0ct }\end{array}$} \\
\hline $\begin{array}{l}\text { Slope } \\
\text { Water }\end{array}$ & $\begin{array}{l}\frac{8}{\overline{\overline{0}}} \\
\underline{0} \\
\underline{0} \\
\underline{0}\end{array}$ & $\begin{array}{l}9 \\
0 \\
0 \\
0 \\
0\end{array}$ & & & $\begin{array}{l}19 \\
\frac{0}{0} \\
\underline{0} \\
\frac{0}{0} \\
\underline{0}\end{array}$ & $\begin{array}{l}20 \\
0 \\
0 \\
0 \\
0 \\
0\end{array}$ & $\begin{array}{l}33 \\
\underline{0} \\
\underline{0} \\
\underline{0} \\
\underline{0} \\
\underline{0}\end{array}$ & $\begin{array}{l}34 \\
\underline{0} \\
\underline{0} \\
\underline{0}\end{array}$ & & $\begin{array}{l}35 \\
0 \\
0 \\
0 \\
0 \\
0\end{array}$ & $\begin{array}{l}47 \\
\underline{0} \\
\underline{0} \\
\underline{0} \\
\underline{0}\end{array}$ & $\begin{array}{l}\frac{48}{0} \\
0 \\
0 \\
0\end{array}$ & $\begin{array}{l}\frac{53}{0} \\
\frac{0}{0} \\
\underline{0}\end{array}$ & $\begin{array}{l}54 \\
0 \\
0 \\
0 \\
0\end{array}$ & $\begin{array}{l}\frac{59}{0} \\
\underline{0} \\
\underline{0} \\
\underline{0} \\
\underline{0}\end{array}$ & $\begin{array}{l}60 \\
0 \\
0 \\
0 \\
0 \\
0\end{array}$ & $\begin{array}{l}\frac{65}{\underline{0}} \\
\underline{0} \\
\underline{0} \\
\underline{0} \\
\underline{0}\end{array}$ & $\begin{array}{l}\frac{66}{0} \\
0 \\
2.67 \\
0 \\
0\end{array}$ & & & & $\begin{array}{l}4 \\
0 \\
0 \\
0 \\
0\end{array}$ & $\begin{array}{l}\frac{5}{\underline{0}} \\
\underline{0} \\
\underline{0} \\
\underline{0}\end{array}$ & \\
\hline $\begin{array}{l}\text { High } \\
\text { Velocity } \\
\text { Region }\end{array}$ & $\begin{array}{l}12 \\
0 \\
0 \\
0 \\
0\end{array}$ & $\begin{array}{l}\frac{13}{\underline{0}} \\
\underline{0} \underline{0}\end{array}$ & $\begin{array}{l}\frac{14}{0} \\
\underline{0} \\
\underline{0} \\
\underline{0}\end{array}$ & $\begin{array}{l}\frac{15}{0} \\
0 \\
0 \\
0\end{array}$ & $\begin{array}{l}26 \\
0 \\
0 \\
0 \\
0 \\
-\end{array}$ & $\begin{array}{l}27 \\
0 \\
0 \\
0 \\
0 \\
0\end{array}$ & $\begin{array}{l}30 \\
0 \\
0 \\
0 \\
0 \\
0\end{array}$ & $\begin{array}{l}31 \\
0 \\
0 \\
0 \\
0 \\
0\end{array}$ & $\begin{array}{l}\frac{32}{\underline{0}} \\
\underline{0} \\
\underline{0} \\
\underline{0}\end{array}$ & $\begin{array}{l}\frac{41}{\underline{0}} \\
\underline{0} \\
\underline{0}\end{array}$ & $\frac{\frac{42}{0.75}}{\frac{0}{0}}$ & $\begin{array}{l}\frac{43}{0} \\
0 \\
2.92 \\
0 \\
0\end{array}$ & $\frac{\frac{44}{0.27}}{\frac{0}{0}}$ & & $\frac{\frac{55}{0.16}}{\frac{0}{0}}$ & & & & & & & $\begin{array}{l}\frac{2}{0} \\
\underline{0} \\
\underline{0} \\
\underline{0}\end{array}$ & $\begin{array}{l}\frac{3}{0} \\
3.38 \\
0 \\
0\end{array}$ & \\
\hline $\begin{array}{l}\text { Ring } \\
\text { Core }\end{array}$ & $\begin{array}{l}10 \\
\underline{0} \\
\underline{0} \\
\underline{0} \\
\underline{0}\end{array}$ & $\begin{array}{l}11 \\
0 \\
0 \\
0 \\
0\end{array}$ & $\begin{array}{l}\frac{16}{0} \\
0 \\
0\end{array}$ & $\begin{array}{l}17 \\
\underline{0} \\
\underline{0} \\
\underline{0}\end{array}$ & $\begin{array}{l}\frac{21}{\underline{0}} \\
\frac{1.00}{\underline{0}} \\
\underline{0} \\
\underline{0}\end{array}$ & $\begin{array}{l}\frac{23}{\underline{0}} \\
\frac{1.27}{\underline{0}} \\
\underline{0} \\
\underline{0}\end{array}$ & $\begin{array}{l}24 \\
0 \\
0 \\
0.49 \\
0 \\
0\end{array}$ & $\begin{array}{l}28 \\
0 \\
0 \\
0 \\
0 \\
0\end{array}$ & & & & & & & $\begin{array}{l}\frac{56}{0.03} \\
\frac{0}{0} \\
\underline{0} \\
\underline{0}\end{array}$ & $\begin{array}{l}\frac{57}{0} \\
\underline{0} \\
\underline{0} \\
\underline{0} \\
\underline{0}\end{array}$ & $\begin{array}{l}\frac{58}{0} \\
0 \\
0.05 \\
0 \\
0\end{array}$ & $\begin{array}{l}\frac{61}{0.02} \\
\frac{0}{0} \\
\underline{0} \\
\underline{0}\end{array}$ & $\begin{array}{l}\frac{62}{0} \\
0 \\
0.02 \\
0 \\
0\end{array}$ & $\frac{\frac{63}{0.03}}{\frac{0}{0}}$ & $\begin{array}{l}\frac{64}{0} \\
0 \\
0.07 \\
0 \\
0\end{array}$ & $\begin{array}{l}\frac{1}{0} \\
\underline{0} \\
\underline{0} \\
\underline{0}\end{array}$ & & \\
\hline $\begin{array}{l}\text { Sargasso } \\
\text { Sea } \\
\& \\
\text { Gulf } \\
\text { Stream }\end{array}$ & & & & & & & & & & $\frac{\frac{49}{0.24}}{\frac{0}{0}} \frac{\underline{0}}{\underline{0}}$ & $\begin{array}{l}\frac{50}{0} \\
0 \\
0.07 \\
0\end{array}$ & $\begin{array}{c}\frac{51}{0} \\
0 \\
0.02 \\
0\end{array}$ & $\begin{array}{l}\frac{52}{\underline{0}} \\
\underline{0} \\
\underline{0} \\
\underline{0}\end{array}$ & & & & & & & & & & & \\
\hline $\begin{array}{l}\text { Slope } \\
\text { Water }\end{array}$ & $\frac{8}{-}$ & $\frac{9}{0}$ & $\frac{18}{0}$ & & $\frac{19}{0}$ & $\frac{20}{0}$ & $\frac{33}{0}$ & $\frac{34}{0}$ & & $\frac{35}{0}$ & $\frac{47}{0}$ & $\frac{48}{0}$ & $\frac{53}{0}$ & $\frac{54}{0}$ & $\frac{59}{0}$ & $\frac{60}{0}$ & $\frac{65}{0}$ & $\frac{66}{0.53}$ & & & & $\frac{4}{0}$ & $\frac{5}{0}$ & $\frac{6}{0}$ \\
\hline $\begin{array}{l}\text { High } \\
\text { Velocity } \\
\text { Region }\end{array}$ & $\frac{12}{0}$ & $\frac{13}{0}$ & $\frac{14}{0}$ & $\frac{15}{0}$ & $\frac{27}{0}$ & $\frac{29}{0}$ & $\frac{30}{0}$ & $\frac{31}{0}$ & $\frac{32}{-}$ & $\frac{41}{0}$ & $\frac{42}{0.29}$ & $\frac{43}{0.58}$ & $\frac{44}{0.16}$ & & $\frac{55}{0.03}$ & & & & & & & $\frac{2}{0}$ & $\frac{3}{0.85}$ & \\
\hline $\begin{array}{l}\text { Ring } \\
\text { Core }\end{array}$ & $\frac{10}{0}$ & $\frac{11}{0}$ & $\frac{16}{-}$ & $\frac{17}{0}$ & $\frac{21}{0.35}$ & $\frac{23}{0.32}$ & $\frac{24}{0.10}$ & $\frac{28}{0}$ & & $\frac{37}{0}$ & $\frac{38}{0}$ & $\frac{39}{0.32}$ & & & $\frac{56}{0.01}$ & $\frac{57}{0}$ & $\frac{58}{0.01}$ & $\frac{61}{0.01}$ & $\frac{62}{0.01}$ & $\frac{63}{0.01}$ & $\frac{64}{0.01}$ & $\frac{1}{0}$ & & \\
\hline SS \& GS & & & & & & & & & & $\frac{49}{0.06}$ & $\frac{50}{0.02}$ & $\frac{51}{0.01}$ & $\frac{52}{0}$ & & & & & & & & & & & \\
\hline
\end{tabular}


Table 66. Abundance of Diaphus metopoclampus (specimens $/ 10,000 \mathrm{~m}^{3}$ ).

\begin{tabular}{|c|c|c|c|c|c|c|c|c|c|c|c|c|c|c|c|c|c|c|c|c|c|c|c|c|}
\hline & \multicolumn{4}{|c|}{$\begin{array}{c}\text { Oceanus } \\
\text { April }\end{array}$} & \multicolumn{5}{|c|}{$\begin{array}{c}\text { Oceanus } 121 \\
\text { June }\end{array}$} & \multicolumn{5}{|c|}{$\begin{array}{l}\text { Oceanus } 125 \\
\text { Auqust }\end{array}$} & \multicolumn{7}{|c|}{$\begin{array}{l}\text { Knorr } 98 \\
\text { Sept-0ct }\end{array}$} & \multicolumn{3}{|c|}{$\begin{array}{c}\text { A2 } 110 \\
\text { Sept-0ct }\end{array}$} \\
\hline $\begin{array}{l}\text { Slope } \\
\text { Water }\end{array}$ & $\begin{array}{l}\frac{8}{\overline{\overline{0}}} \\
\underline{0} \\
\underline{0}\end{array}$ & $\begin{array}{l}9 \\
0 \\
0 \\
0 \\
0\end{array}$ & & & $\begin{array}{l}\frac{19}{0.2} \\
\frac{0}{0.2} \\
\frac{0}{0} \\
\underline{0}\end{array}$ & $\begin{array}{r}20 \\
0 \\
0 \\
0 \\
0 \\
0\end{array}$ & $\begin{array}{r}33 \\
\underline{0} \\
\underline{0} \\
\underline{0} \\
\underline{0} \\
\underline{0}\end{array}$ & $\begin{array}{r}\frac{34}{0} \\
\underline{0} \\
\underline{0}\end{array}$ & & $\begin{array}{r}35 \\
0 \\
0 \\
0 \\
0 \\
0\end{array}$ & $\begin{array}{r}47 \\
\underline{0} \\
\underline{0} \\
\underline{0} \\
\underline{0}\end{array}$ & $\begin{array}{r}48 \\
0 \\
0 \\
0 \\
0\end{array}$ & $\frac{53}{\frac{0}{0}}$ & $\begin{array}{c}54 \\
0 \\
0.9 \\
0 \\
0\end{array}$ & $\begin{array}{c}\frac{59}{\underline{0}} \\
\frac{0.9}{0} \\
\frac{0}{0}\end{array}$ & $\begin{array}{c}60 \\
0 \\
0 \\
1.4 \\
0 \\
0\end{array}$ & $\begin{array}{r}65 \\
\underline{0} \\
\underline{0} \\
\underline{0} \\
\underline{0} \\
\underline{0}\end{array}$ & $\begin{array}{c}66 \\
0 \\
0 \\
0.7 \\
0 \\
0\end{array}$ & & & & $\begin{array}{l}4 \\
0 \\
0 \\
0 \\
0\end{array}$ & $\begin{array}{l}\frac{5}{0} \\
\underline{0} \\
\underline{0} \\
\underline{0}\end{array}$ & \\
\hline $\begin{array}{l}\text { High } \\
\text { Velocity } \\
\text { Region }\end{array}$ & $\begin{array}{l}12 \\
0.3 \\
0.4 \\
0 \\
0\end{array}$ & $\frac{13}{\frac{0}{0}}$ & $\frac{\frac{14}{0.6}}{\frac{0.3}{\underline{0}}}$ & $\begin{array}{r}15 \\
0 \\
0 \\
0 \\
0\end{array}$ & $\begin{array}{c}26 \\
0 \\
0 \\
0 \\
0.3 \\
-\end{array}$ & $\begin{array}{r}27 \\
0 \\
0 \\
0 \\
0 \\
0\end{array}$ & $\begin{array}{r}30 \\
0 \\
0 \\
0 \\
0 \\
0\end{array}$ & $\begin{array}{r}31 \\
0 \\
0 \\
0 \\
0 \\
0\end{array}$ & $\frac{32}{\frac{0.5}{\underline{0}}}$ & $\begin{array}{r}41 \\
\underline{0} \\
\underline{0} \\
\underline{0}\end{array}$ & $\frac{\frac{42}{0.1}}{\frac{0}{\underline{0}}}$ & $\begin{array}{r}43 \\
0 \\
0 \\
0 \\
0 \\
0\end{array}$ & $\frac{\frac{44}{0.1}}{\frac{0}{0}}$ & & $\begin{array}{c}\frac{55}{\underline{0}} \\
\underline{0} \\
\underline{0.8} \\
\underline{0}\end{array}$ & & & & & & & $\frac{\frac{2}{0.8}}{\frac{0.4}{\underline{0}}}$ & $\begin{array}{c}\frac{3}{0} \\
2.4 \\
0.7 \\
0\end{array}$ & \\
\hline $\begin{array}{l}\text { Ring } \\
\text { Core }\end{array}$ & $\frac{10}{\frac{0}{1.8}} \frac{\underline{0}}{\underline{0}}$ & $\begin{array}{c}11 \\
0 \\
0 \\
0.6 \\
0\end{array}$ & $\begin{array}{c}\frac{16}{0} \\
2.1 \\
0\end{array}$ & $\frac{17}{\frac{0}{0}}$ & $\begin{array}{c}\frac{21}{\underline{0}} \\
\underline{0.4} \\
\frac{0.6}{\underline{0}} \\
\underline{0}\end{array}$ & $\begin{array}{r}2 \underline{23} \\
\underline{0} \\
\underline{0} \\
\underline{0} \\
\underline{0} \\
\underline{0}\end{array}$ & $\begin{array}{c}24 \\
0 \\
0 \\
0.3 \\
0 \\
0\end{array}$ & $\begin{array}{c}28 \\
0 \\
0 \\
0.4 \\
0 \\
0\end{array}$ & & & & & & & $\begin{array}{c}\frac{56}{\underline{0}} \\
\frac{0}{0} \\
\frac{1.1}{\underline{0}}\end{array}$ & $\begin{array}{c}\frac{57}{\underline{0}} \\
\underline{0} \\
\underline{0} \\
\underline{3.4} \\
\underline{0.8}\end{array}$ & $\begin{array}{c}58 \\
0 \\
0 \\
0 \\
2.3 \\
0.3\end{array}$ & $\begin{array}{c}\frac{61}{\underline{0}} \\
\underline{0} \\
\underline{0.7} \\
\underline{3.9} \\
\underline{0.3}\end{array}$ & $\begin{array}{c}62 \\
0 \\
0 \\
0 \\
1.6 \\
0.4\end{array}$ & $\begin{array}{c}\frac{63}{\underline{0}} \\
\underline{0} \\
\underline{0} \\
\underline{1.2} \\
\underline{0.9}\end{array}$ & $\begin{array}{c}\frac{64}{0} \\
0.6 \\
0.7 \\
1.4 \\
0\end{array}$ & $\begin{array}{l}\frac{1}{\underline{0}} \\
\frac{0.2}{3.4} \\
\underline{0}\end{array}$ & & \\
\hline $\begin{array}{l}\text { Sargasso } \\
\text { Sea } \\
\& \\
\text { Gulf } \\
\text { Stream }\end{array}$ & & & & & & & & & & $\begin{array}{c}\frac{49}{\underline{0}} \\
\underline{0} \\
\underline{0} \\
\underline{0.5}\end{array}$ & $\begin{array}{r}50 \\
0 \\
0 \\
0 \\
0\end{array}$ & $\begin{array}{c}51 \\
0 \\
0 \\
0.2 \\
0\end{array}$ & $\begin{array}{r}52 \\
\underline{0} \\
\underline{0} \\
\underline{0} \\
\underline{0}\end{array}$ & & & & & & & & & & & \\
\hline $\begin{array}{l}\text { Slope } \\
\text { Water }\end{array}$ & $\frac{8}{-}$ & $\frac{9}{0}$ & $\frac{18}{0}$ & & $\frac{19}{0.1}$ & $\frac{20}{0}$ & $\frac{33}{0}$ & $\frac{34}{0}$ & & $\frac{35}{0}$ & $\frac{47}{0}$ & $\frac{48}{0}$ & $\frac{53}{0}$ & $\frac{54}{0.2}$ & $\frac{59}{0.2}$ & $\frac{60}{0.3}$ & $\frac{65}{0}$ & $\frac{66}{0.2}$ & & & & $\frac{4}{0}$ & $\frac{5}{0}$ & $\frac{6}{0}$ \\
\hline $\begin{array}{l}\text { High } \\
\text { Velocity } \\
\text { Region }\end{array}$ & $\frac{12}{0.2}$ & $\frac{13}{0}$ & $\frac{14}{0.2}$ & $\frac{15}{0}$ & $\frac{27}{0}$ & $\frac{29}{0.1}$ & $\frac{30}{0}$ & $\frac{31}{0}$ & $\frac{32}{-}$ & $\frac{41}{0}$ & $\frac{42}{0.1}$ & $\frac{43}{0}$ & $\frac{44}{0.1}$ & & $\frac{55}{0.4}$ & & & & & & & $\frac{2}{0.6}$ & $\frac{3}{0.8}$ & \\
\hline $\begin{array}{l}\text { Ring } \\
\text { Core }\end{array}$ & $\frac{10}{0.4}$ & $\frac{11}{0.2}$ & $\frac{16}{-}$ & $\frac{17}{0}$ & $\frac{21}{0.3}$ & $\frac{23}{0}$ & $\frac{24}{0.1}$ & $\frac{28}{0.1}$ & & $\frac{37}{0}$ & $\frac{38}{0}$ & $\frac{39}{0.1}$ & & & $\frac{56}{0.2}$ & $\frac{57}{0.8}$ & $\frac{58}{0.5}$ & $\frac{61}{1.0}$ & $\frac{62}{0.4}$ & $\frac{63}{0.4}$ & $\frac{64}{0.4}$ & $\frac{1}{0.9}$ & & \\
\hline SS \& GS & & & & & & & & & & $\frac{49}{0.1}$ & $\frac{50}{0}$ & $\frac{51}{0.1}$ & $\frac{52}{0}$ & & & & & & & & & & & \\
\hline
\end{tabular}


Tab1e 67. Volume of Diaphus metopoclampus $\left(\mathrm{ml} / 10,000 \mathrm{~m}^{3}\right)$.

\begin{tabular}{|c|c|c|c|c|c|c|c|c|c|c|c|c|c|c|c|c|c|c|c|c|c|c|c|c|}
\hline & \multicolumn{4}{|c|}{$\begin{array}{c}\text { Oceanus } \\
\text { April }\end{array}$} & \multicolumn{5}{|c|}{$\begin{array}{l}\text { Oceanus } 121 \\
\text { June }\end{array}$} & \multicolumn{5}{|c|}{$\begin{array}{l}\text { Oceanus } 125 \\
\text { August }\end{array}$} & \multicolumn{7}{|c|}{$\begin{array}{l}\text { Knorr } 98 \\
\text { Sept-0ct }\end{array}$} & \multicolumn{3}{|c|}{$\begin{array}{c}\text { A2 } 110 \\
\text { Sept-0ct }\end{array}$} \\
\hline $\begin{array}{l}\text { Slope } \\
\text { Water }\end{array}$ & $\begin{array}{l}\frac{8}{\overline{0}} \\
\underline{0} \\
\underline{0} \\
\underline{0}\end{array}$ & $\begin{array}{l}9 \\
0 \\
0 \\
0 \\
0\end{array}$ & & & $\begin{array}{l}\frac{19}{0.02} \\
\frac{0}{0.02} \\
\frac{0}{0}\end{array}$ & $\begin{array}{l}20 \\
0 \\
0 \\
0 \\
0 \\
0\end{array}$ & $\begin{array}{l}33 \\
\underline{0} \\
\underline{0} \\
\underline{0} \\
\underline{0} \\
\underline{0}\end{array}$ & $\begin{array}{l}34 \\
\frac{0}{0} \\
\underline{0} \\
\underline{0}\end{array}$ & & $\begin{array}{l}35 \\
0 \\
0 \\
0 \\
0 \\
0\end{array}$ & $\begin{array}{l}47 \\
\underline{0} \\
\underline{0} \\
\underline{0} \\
\underline{0}\end{array}$ & $\begin{array}{l}\frac{48}{0} \\
0 \\
0 \\
0\end{array}$ & $\begin{array}{l}\frac{53}{0} \\
\underline{0} \\
\underline{0} \\
\underline{0}\end{array}$ & $\begin{array}{l}\frac{54}{0} \\
0.50 \\
0 \\
0\end{array}$ & $\begin{array}{l}\frac{59}{\underline{0}} \\
\underline{\underline{0}} \\
\frac{7.35}{\underline{0}} \\
\underline{0}\end{array}$ & $\begin{array}{c}\frac{60}{0} \\
0 \\
2.10 \\
0 \\
0\end{array}$ & $\begin{array}{l}\frac{65}{0} \\
\underline{0} \\
\underline{0} \\
\underline{0} \\
\underline{0} \\
\underline{0}\end{array}$ & $\begin{array}{l}\frac{66}{0} \\
0 \\
0.11 \\
0 \\
0\end{array}$ & & & & $\begin{array}{l}4 \\
0 \\
0 \\
0 \\
0\end{array}$ & $\begin{array}{l}\frac{5}{0} \\
\underline{0} \\
\underline{0} \\
\underline{0}\end{array}$ & \\
\hline $\begin{array}{l}\text { High } \\
\text { Velocity } \\
\text { Region }\end{array}$ & $\begin{array}{l}\frac{12}{0.03} \\
0.04 \\
0 \\
0\end{array}$ & $\begin{array}{l}\frac{13}{0} \\
\frac{0}{0} \\
\underline{0}\end{array}$ & $\frac{\frac{14}{0.09}}{\frac{0.03}{\underline{0}}}$ & $\begin{array}{l}\frac{15}{0} \\
0 \\
0 \\
0\end{array}$ & $\begin{array}{l}26 \\
0 \\
0 \\
0 \\
0.07 \\
-\end{array}$ & $\begin{array}{l}\frac{27}{0} \\
0 \\
0 \\
0 \\
0\end{array}$ & $\begin{array}{l}30 \\
0 \\
0 \\
0 \\
0 \\
0\end{array}$ & $\begin{array}{l}31 \\
0 \\
0 \\
0 \\
0 \\
0\end{array}$ & $\frac{\frac{32}{0.18}}{\frac{0}{0}}$ & $\begin{array}{l}41 \\
\frac{0}{0} \\
\underline{0} \\
\underline{0}\end{array}$ & $\frac{\frac{42}{0.01}}{\frac{0}{0}}$ & $\begin{array}{l}43 \\
0 \\
0 \\
0 \\
0 \\
0\end{array}$ & $\frac{\frac{44}{0.08}}{\frac{0}{0}}$ & & $\begin{array}{l}\frac{55}{\underline{0}} \\
\underline{\underline{0}} \\
\frac{4.40}{\underline{0}}\end{array}$ & & & & & & & $\frac{\frac{2}{0.29}}{\frac{0.05}{\underline{0}}}$ & $\begin{array}{c}\frac{3}{0} \\
4.46 \\
0.04 \\
0\end{array}$ & \\
\hline $\begin{array}{l}\text { Ring } \\
\text { Core }\end{array}$ & $\frac{\frac{10}{\underline{0}}}{\frac{0.06}{\underline{0}}}$ & $\begin{array}{c}11 \\
0 \\
0 \\
0.06 \\
0\end{array}$ & $\begin{array}{c}\frac{16}{0} \\
0.07 \\
0\end{array}$ & $\begin{array}{l}\frac{17}{\underline{0}} \\
\underline{0} \\
\underline{0}\end{array}$ & $\begin{array}{l}\frac{21}{\underline{0}} \\
\frac{0.12}{0.15} \\
\frac{0}{\underline{0}}\end{array}$ & $\begin{array}{l}23 \\
\underline{0} \\
\underline{0} \\
\underline{0} \\
\underline{0} \\
\underline{0}\end{array}$ & $\begin{array}{l}24 \\
0 \\
0 \\
0.03 \\
0 \\
0\end{array}$ & $\begin{array}{l}\frac{28}{0} \\
0 \\
0.18 \\
0 \\
0\end{array}$ & & & & & & & $\begin{array}{l}\frac{56}{\underline{0}} \\
\underline{0} \\
\underline{0} \\
\frac{0.05}{\underline{0}}\end{array}$ & $\begin{array}{l}\frac{57}{\underline{0}} \\
\underline{0} \\
\underline{0} \\
\underline{2.56} \\
\underline{0.03} \\
\end{array}$ & $\begin{array}{l}\frac{58}{0} \\
0 \\
0 \\
0.05 \\
0.03\end{array}$ & $\begin{array}{l}\frac{61}{\underline{0}} \\
\underline{0} \\
\underline{0.04} \\
\underline{0.04} \\
\underline{0.03}\end{array}$ & $\begin{array}{l}\frac{62}{0} \\
0 \\
0 \\
0.03 \\
0.04\end{array}$ & $\begin{array}{l}\frac{63}{\underline{0}} \\
\underline{0} \\
\underline{0} \\
\underline{0.04} \\
\underline{0.03}\end{array}$ & $\begin{array}{c}\frac{64}{0} \\
0.06 \\
0.07 \\
0.45 \\
0\end{array}$ & $\frac{\frac{1}{\underline{0}}}{\frac{0.02}{0.09}} \frac{\underline{0}}{\frac{0}{0}}$ & & \\
\hline $\begin{array}{l}\text { Sargasso } \\
\text { Sea } \\
\& \\
\text { Gulf } \\
\text { Stream }\end{array}$ & & & & & & & & & & $\begin{array}{l}\frac{49}{\underline{0}} \\
\underline{0} \\
\underline{0} \\
\underline{0.03}\end{array}$ & $\begin{array}{l}50 \\
0 \\
0 \\
0 \\
0\end{array}$ & $\begin{array}{c}\frac{51}{0} \\
0 \\
0.09 \\
0\end{array}$ & $\begin{array}{l}\frac{52}{\underline{0}} \\
\underline{0} \\
\underline{0} \\
\underline{0}\end{array}$ & & & & & & & & & & & \\
\hline $\begin{array}{l}\text { Slope } \\
\text { Water }\end{array}$ & $\frac{8}{-}$ & $\frac{9}{0}$ & $\frac{18}{0}$ & & $\frac{19}{0.01}$ & $\frac{20}{0}$ & $\frac{33}{0}$ & $\frac{34}{0}$ & & $\frac{35}{0}$ & $\frac{47}{0}$ & $\frac{48}{0}$ & $\frac{53}{0}$ & $\frac{54}{0.13}$ & $\frac{59}{1.47}$ & $\frac{60}{0.42}$ & $\frac{65}{0}$ & $\frac{66}{0.02}$ & & & & $\frac{4}{0}$ & $\frac{5}{0}$ & $\frac{6}{0}$ \\
\hline $\begin{array}{l}\text { High } \\
\text { Velocity } \\
\text { Region }\end{array}$ & $\frac{12}{0.02}$ & $\frac{13}{0}$ & $\frac{14}{0.03}$ & $\frac{15}{0}$ & $\frac{27}{0}$ & $\frac{29}{0.04}$ & $\frac{30}{0}$ & $\frac{31}{0}$ & $\frac{32}{-}$ & $\frac{41}{0}$ & $\frac{42}{0.01}$ & $\frac{43}{0}$ & $\frac{44}{0.05}$ & & $\frac{55}{1.76}$ & & & & & & & $\frac{2}{0.07}$ & $\frac{3}{1.13}$ & \\
\hline $\begin{array}{l}\text { Ring } \\
\text { Core }\end{array}$ & $\frac{10}{0.02}$ & $\frac{11}{0.02}$ & $\frac{16}{-}$ & $\frac{17}{0}$ & $\frac{21}{0.07}$ & $\frac{23}{0}$ & $\frac{24}{0.01}$ & $\frac{28}{0.05}$ & & $\frac{37}{0}$ & $\frac{38}{0}$ & $\frac{39}{0.03}$ & & & $\frac{56}{0.01}$ & $\frac{57}{0.52}$ & $\frac{58}{0.02}$ & $\frac{61}{0.02}$ & $\frac{62}{0.01}$ & $\frac{63}{0.01}$ & $\frac{64}{0.03}$ & $\frac{1}{0.03}$ & & \\
\hline SS \& GS & & & & & & & & & & $\frac{49}{0.01}$ & $\frac{50}{0}$ & $\frac{51}{0.02}$ & $\frac{52}{0}$ & & & & & & & & & & & \\
\hline
\end{tabular}


Table 68. Abundance of Diaphus mollis (specimens $/ 10,000 \mathrm{~m}^{3}$ ).

\begin{tabular}{|c|c|c|c|c|c|c|c|c|c|c|c|c|c|c|c|c|c|c|c|c|c|c|c|c|}
\hline & \multicolumn{4}{|c|}{$\begin{array}{c}\text { Oceanus } 118 \\
\text { April }\end{array}$} & \multicolumn{5}{|c|}{$\begin{array}{c}\text { Oceanus } 121 \\
\text { June }\end{array}$} & \multicolumn{5}{|c|}{$\begin{array}{l}\text { Oceanus } 125 \\
\text { August }\end{array}$} & \multicolumn{7}{|c|}{$\begin{array}{l}\text { Knorr } 98 \\
\text { Sept-0ct }\end{array}$} & \multicolumn{3}{|c|}{$\begin{array}{c}\text { A2 } 110 \\
\text { Sept-0ct }\end{array}$} \\
\hline $\begin{array}{l}\text { Slope } \\
\text { Water }\end{array}$ & $\begin{array}{l}\frac{8}{\bar{z}} \\
\underline{0} \\
\underline{0} \\
\underline{0}\end{array}$ & $\begin{array}{c}\frac{9}{0} \\
1.8 \\
0 \\
0\end{array}$ & & & $\frac{\frac{19}{0.7}}{\frac{0}{0}}$ & $\begin{array}{c}20 \\
0 \\
1.1 \\
0 \\
0 \\
0\end{array}$ & $\frac{33}{\frac{1.0}{\underline{0}}}$ & $\frac{\frac{34}{0.5}}{\frac{0}{0}}$ & & $\begin{array}{c}35 \\
0 \\
0.2 \\
0 \\
0 \\
0\end{array}$ & $\begin{array}{l}47 \\
\underline{0} \\
\underline{0} \\
\underline{0} \\
\underline{0}\end{array}$ & $\begin{array}{c}48 \\
0 \\
0.6 \\
0 \\
0\end{array}$ & $\begin{array}{l}53 \\
\underline{0} \\
\underline{0} \\
\underline{0}\end{array}$ & $\begin{array}{r}54 \\
0 \\
0 \\
0 \\
0\end{array}$ & $\begin{array}{c}5 \underline{59} \\
\underline{0} \\
\underline{0} \\
\underline{0} \\
\underline{0} \\
\underline{0}\end{array}$ & $\begin{array}{c}60 \\
0 \\
0 \\
0.3 \\
0 \\
0\end{array}$ & $\begin{array}{r}65 \\
\underline{0} \\
\underline{0} \\
\underline{0} \\
\underline{0} \\
\underline{0}\end{array}$ & $\begin{array}{c}66 \\
0 \\
0.7 \\
0 \\
0 \\
0\end{array}$ & & & & $\begin{array}{c}\frac{4}{0} \\
0.2 \\
0 \\
0\end{array}$ & $\begin{array}{l}\frac{5}{0} \\
\underline{0} \\
\underline{0} \\
\underline{0} \\
\underline{0}\end{array}$ & \\
\hline $\begin{array}{l}\text { High } \\
\text { Velocity } \\
\text { Region }\end{array}$ & $\begin{array}{c}12 \\
0 \\
0.8 \\
0 \\
0\end{array}$ & $\frac{13}{\underline{0}}$ & $\begin{array}{l}\frac{14}{\underline{0}} \\
\underline{0} \\
\underline{0} \\
\underline{0}\end{array}$ & $\begin{array}{r}15 \\
0 \\
0 \\
0 \\
0\end{array}$ & $\begin{array}{c}26 \\
0 \\
0 \\
0 \\
0 \\
-\end{array}$ & $\begin{array}{r}27 \\
0 \\
0 \\
0 \\
0 \\
0\end{array}$ & $\begin{array}{r}30 \\
0 \\
0 \\
0 \\
0 \\
0\end{array}$ & $\begin{array}{c}31 \\
0 \\
0 \\
0 \\
0 \\
0\end{array}$ & $\frac{\frac{32}{0.3}}{\frac{0}{0}} \frac{\underline{0}}{\underline{0}}$ & $\begin{array}{r}41 \\
\underline{0} \\
\underline{0} \\
\underline{0}\end{array}$ & $\begin{array}{l}\frac{42}{0.9} \\
\underline{0} \\
\frac{0.2}{\underline{0}}\end{array}$ & $\begin{array}{r}43 \\
0 \\
0 \\
0 \\
0 \\
0\end{array}$ & $\frac{44}{\underline{0}}$ & & $\frac{\frac{55}{2.4}}{\frac{0}{\underline{0}}}$ & & & & & & & $\frac{\frac{2}{0.2}}{\frac{0}{0}} \frac{\underline{0}}{\underline{0}}$ & $\begin{array}{c}\frac{3}{0.7} \\
0.3 \\
0 \\
0\end{array}$ & \\
\hline $\begin{array}{l}\text { Ring } \\
\text { Core }\end{array}$ & $\frac{\frac{10}{0.8}}{\frac{0}{0}} \frac{0}{\underline{0}}$ & $\begin{array}{r}11 \\
0 \\
0 \\
0 \\
0\end{array}$ & $\begin{array}{r}16 \\
0 \\
0 \\
0\end{array}$ & $\frac{\frac{17}{2.8}}{\frac{0}{0}}$ & $\frac{21}{\underline{0}}$ & $\frac{23}{\frac{1.8}{0.7}} \frac{\underline{0}}{\underline{0}}$ & $\begin{array}{c}24 \\
0 \\
10.0 \\
0 \\
0 \\
0\end{array}$ & $\begin{array}{r}28 \\
0 \\
0 \\
0 \\
0 \\
0\end{array}$ & & & & & & & $\begin{array}{l}\frac{56}{1.8} \\
\frac{0}{0} \\
\underline{0} \\
\underline{0}\end{array}$ & $\frac{57}{\frac{1.9}{\underline{0}}}$ & $\begin{array}{c}58 \\
0 \\
0 \\
2.4 \\
0 \\
0\end{array}$ & 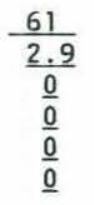 & $\begin{array}{c}62 \\
0 \\
0 \\
1.4 \\
0 \\
0\end{array}$ & $\frac{63}{\frac{1.1}{\underline{0}}} \frac{\underline{0}}{\underline{0}}$ & $\begin{array}{c}\frac{64}{0} \\
1.9 \\
0.7 \\
0 \\
0\end{array}$ & $\frac{\frac{1}{0.2}}{\frac{0}{0}} \frac{\underline{0}}{\underline{0}}$ & & \\
\hline $\begin{array}{l}\text { Sargasso } \\
\text { Sea } \\
\& \\
\text { Gulf } \\
\text { Stream }\end{array}$ & & & & & & & & & & $\frac{\frac{49}{\frac{1.9}{0.2}}}{\frac{0}{0}}$ & $\begin{array}{c}50 \\
0 \\
0.2 \\
2.1 \\
0\end{array}$ & $\begin{array}{c}51 \\
0 \\
0.4 \\
2.2 \\
0\end{array}$ & $\frac{52}{\frac{0.5}{\underline{0}}}$ & & & & & & & & & & & \\
\hline $\begin{array}{l}\text { Slope } \\
\text { Water }\end{array}$ & $\frac{8}{-}$ & $\frac{9}{0.5}$ & $\frac{18}{0}$ & & $\frac{19}{0.1}$ & $\frac{20}{0.3}$ & $\frac{33}{0.1}$ & $\frac{34}{0.1}$ & & $\frac{35}{0.1}$ & $\frac{47}{0}$ & $\frac{48}{0.2}$ & $\frac{53}{0}$ & $\frac{54}{0}$ & $\frac{59}{0}$ & $\frac{60}{0.1}$ & $\frac{65}{0}$ & $\frac{66}{0.1}$ & & & & $\frac{4}{0.1}$ & $\frac{5}{0}$ & $\frac{6}{0}$ \\
\hline $\begin{array}{l}\text { High } \\
\text { Velocity } \\
\text { Region }\end{array}$ & $\frac{12}{0.2}$ & $\frac{13}{0}$ & $\frac{14}{0}$ & $\frac{15}{0}$ & $\frac{27}{0}$ & $\frac{29}{0.1}$ & $\frac{30}{0}$ & $\frac{31}{0}$ & $\frac{32}{-}$ & $\frac{41}{0}$ & $\frac{42}{0.4}$ & $\frac{43}{0}$ & $\frac{44}{0}$ & & $\frac{55}{0.5}$ & & & & & & & $\frac{2}{0.1}$ & $\frac{3}{0.3}$ & \\
\hline $\begin{array}{l}\text { Ring } \\
\text { Core }\end{array}$ & $\frac{10}{0.2}$ & $\frac{11}{0}$ & $\frac{16}{-}$ & $\frac{17}{0.7}$ & $\frac{21}{0.4}$ & $\frac{23}{0.5}$ & $\frac{24}{0.2}$ & $\frac{28}{0}$ & & $\frac{37}{0}$ & $\frac{38}{0.2}$ & $\frac{39}{0}$ & & & $\frac{56}{0.4}$ & $\frac{57}{0.4}$ & $\frac{58}{0.5}$ & $\frac{61}{0.6}$ & $\frac{62}{0.3}$ & $\frac{63}{0.4}$ & $\frac{64}{0.3}$ & $\frac{1}{0.1}$ & & \\
\hline SS \& GS & & & & & & & & & & $\frac{49}{0.5}$ & $\frac{50}{0.6}$ & $\frac{51}{0.7}$ & $\frac{52}{0.1}$ & & & & & & & & & & & \\
\hline
\end{tabular}


Table 69. Abundance of Diaphus rafinesquii (specimens $/ 10,000 \mathrm{~m}^{3}$ ).

\begin{tabular}{|c|c|c|c|c|c|c|c|c|c|c|c|c|c|c|c|c|c|c|c|c|c|c|c|c|}
\hline & \multicolumn{4}{|c|}{$\begin{array}{c}\text { Oceanus } 118 \\
\text { Apri1 }\end{array}$} & \multicolumn{5}{|c|}{$\begin{array}{c}\text { Oceanus } 121 \\
\text { June }\end{array}$} & \multicolumn{5}{|c|}{$\begin{array}{l}\text { Oceanus } 125 \\
\text { Auqust }\end{array}$} & \multicolumn{7}{|c|}{$\begin{array}{l}\text { Knorr } 98 \\
\text { Sept-0ct }\end{array}$} & \multicolumn{3}{|c|}{$\begin{array}{c}\text { A2 } 110 \\
\text { Sept-0ct }\end{array}$} \\
\hline $\begin{array}{l}\text { Slope } \\
\text { Water }\end{array}$ & $\begin{array}{l}\frac{8}{\overline{\overline{0}}} \\
\underline{0} \\
\underline{0} \\
\underline{0}\end{array}$ & $\begin{array}{l}9 \\
0 \\
0 \\
0 \\
0\end{array}$ & & & 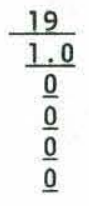 & $\begin{array}{r}20 \\
0 \\
0 \\
0 \\
0 \\
0\end{array}$ & $\begin{array}{r}3 \underline{3} \\
\underline{0} \\
\underline{0} \\
\underline{0} \\
\underline{0} \\
\underline{0}\end{array}$ & $\begin{array}{r}\frac{34}{\underline{0}} \\
\underline{0} \\
\underline{0}\end{array}$ & & $\begin{array}{r}35 \\
0 \\
0 \\
0 \\
0 \\
0\end{array}$ & $\begin{array}{r}47 \\
\underline{0} \\
\underline{0} \\
\underline{0} \\
\underline{0}\end{array}$ & $\begin{array}{r}48 \\
0 \\
0 \\
0 \\
0\end{array}$ & $\frac{53}{\frac{0.2}{\underline{0}}} \frac{\underline{0}}{\underline{0}}$ & $\begin{array}{r}54 \\
0 \\
0 \\
0 \\
0\end{array}$ & $\frac{59}{\frac{0.3}{\underline{0}}}$ & $\begin{array}{c}60 \\
0 \\
0.5 \\
0 \\
0 \\
0\end{array}$ & $\begin{array}{l}\frac{65}{0.4} \\
\frac{0}{0} \\
\underline{0} \\
\underline{0} \\
\underline{0}\end{array}$ & $\begin{array}{r}66 \\
0 \\
0 \\
0 \\
0 \\
0\end{array}$ & & & & $\begin{array}{l}4 \\
0 \\
0 \\
0 \\
0\end{array}$ & $\begin{array}{l}\frac{5}{0} \\
\underline{0} \\
\underline{0} \\
\underline{0}\end{array}$ & \\
\hline $\begin{array}{l}\text { High } \\
\text { Velocity } \\
\text { Region }\end{array}$ & $\begin{array}{c}12 \\
0 \\
4.2 \\
0.4 \\
0\end{array}$ & $\frac{\frac{13}{0.5}}{\frac{0}{0}}$ & $\frac{14}{\frac{1.4}{\underline{0}}}$ & $\begin{array}{c}\frac{15}{0} \\
11.8 \\
0 \\
0\end{array}$ & $\begin{array}{c}26 \\
0 \\
0 \\
10.0 \\
0 \\
-\end{array}$ & $\begin{array}{c}27 \\
0 \\
0.3 \\
0 \\
0 \\
0\end{array}$ & $\begin{array}{c}30 \\
0 \\
6.0 \\
5.0 \\
0 \\
0\end{array}$ & $\begin{array}{c}31 \\
0 \\
0 \\
1.4 \\
0 \\
0\end{array}$ & $\frac{\frac{32}{\frac{3.7}{0.4}}}{\frac{0}{\underline{0}}}$ & $\begin{array}{l}41 \\
\underline{0} \\
\underline{0} \\
\underline{0}\end{array}$ & $\begin{array}{l}\frac{42}{\underline{0}} \\
\underline{0} \\
\underline{0} \\
\underline{0}\end{array}$ & $\begin{array}{r}43 \\
0 \\
0 \\
0 \\
0 \\
0\end{array}$ & $\frac{44}{\frac{0.5}{\underline{0}}}$ & & $\begin{array}{l}55 \\
\underline{0} \\
\underline{0} \\
\underline{0}\end{array}$ & & & & & & & $\begin{array}{l}\frac{2}{\underline{0}} \\
\underline{0} \\
\underline{0} \\
\underline{0}\end{array}$ & $\begin{array}{c}3 \\
0 \\
0.5 \\
0 \\
0\end{array}$ & \\
\hline $\begin{array}{l}\text { Ring } \\
\text { Core }\end{array}$ & $\frac{\frac{10}{0.4}}{\frac{0}{0}} \frac{0}{\underline{0}}$ & $\begin{array}{r}11 \\
0 \\
0 \\
0 \\
0\end{array}$ & $\begin{array}{r}16 \\
0 \\
0 \\
0\end{array}$ & $\frac{\frac{17}{0.6}}{\frac{0}{0}}$ & $\frac{\frac{21}{\underline{0}}}{\frac{1.4}{0.3}} \frac{\underline{0}}{\underline{0}}$ & $\frac{\frac{23}{16.5}}{\frac{1.3}{0.5}} \frac{\underline{0}}{\underline{0}}$ & $\begin{array}{c}24 \\
0 \\
0 \\
2.4 \\
0 \\
0\end{array}$ & $\begin{array}{c}28 \\
0 \\
4.6 \\
0 \\
0 \\
0\end{array}$ & & & & & & & $\begin{array}{l}\frac{56}{\underline{0}} \\
\underline{0} \\
\underline{0} \\
\underline{0} \\
\underline{0}\end{array}$ & $\begin{array}{c}\frac{57}{\underline{0}} \\
\underline{0.3} \\
\underline{0} \\
\underline{0} \\
\underline{0}\end{array}$ & $\begin{array}{r}58 \\
0 \\
0 \\
0 \\
0 \\
0\end{array}$ & $\begin{array}{r}61 \\
\underline{0} \\
\underline{0} \\
\underline{0} \\
\underline{0} \\
\underline{0}\end{array}$ & $\begin{array}{c}62 \\
0 \\
0 \\
0 \\
0.3 \\
0\end{array}$ & $\begin{array}{c}\frac{63}{\underline{0}} \\
\frac{0.2}{\underline{0}} \\
\underline{0} \\
\underline{0}\end{array}$ & $\begin{array}{r}64 \\
0 \\
0 \\
0 \\
0 \\
0\end{array}$ & $\begin{array}{l}\frac{1}{\underline{0}} \\
\frac{0.4}{\underline{0}} \\
\underline{0}\end{array}$ & & \\
\hline $\begin{array}{l}\text { Sargasso } \\
\text { Sea } \\
\& \\
\text { Gulf } \\
\text { Stream }\end{array}$ & & & & & & & & & & $\frac{49}{\frac{0}{0}} \frac{0.2}{\underline{0}} \underline{\underline{0}}$ & $\begin{array}{l}50 \\
0 \\
0 \\
0 \\
0\end{array}$ & $\begin{array}{c}51 \\
0 \\
0 \\
0.2 \\
0\end{array}$ & $\begin{array}{l}\frac{52}{\underline{0}} \\
\underline{0} \\
\underline{0} \\
\underline{0}\end{array}$ & & & & & & & & & & & \\
\hline $\begin{array}{l}\text { Slope } \\
\text { Water }\end{array}$ & $\frac{8}{-}$ & $\frac{9}{0}$ & $\frac{18}{0}$ & & $\frac{19}{0.1}$ & $\frac{20}{0}$ & $\frac{33}{0}$ & $\frac{34}{0}$ & & $\frac{35}{0}$ & $\frac{47}{0}$ & $\frac{48}{0}$ & $\frac{53}{0.1}$ & $\frac{54}{0}$ & $\frac{59}{0.1}$ & $\frac{60}{0.1}$ & $\frac{65}{0.1}$ & $\frac{66}{0}$ & & & & $\frac{4}{0}$ & $\frac{5}{0}$ & $\frac{6}{0}$ \\
\hline $\begin{array}{l}\text { High } \\
\text { Velocity } \\
\text { Region }\end{array}$ & $\frac{12}{1.1}$ & $\frac{13}{0.1}$ & $\frac{14}{0.4}$ & $\frac{15}{3.0}$ & $\frac{27}{0.1}$ & $\frac{29}{2.8}$ & $\frac{30}{1.6}$ & $\frac{31}{0.3}$ & $\frac{32}{-}$ & $\frac{41}{0}$ & $\frac{42}{0}$ & $\frac{43}{0}$ & $\frac{44}{0.3}$ & & $\frac{55}{0}$ & & & & & & & $\frac{2}{0}$ & $\frac{3}{0.1}$ & \\
\hline $\begin{array}{l}\text { Ring } \\
\text { Core }\end{array}$ & $\frac{10}{0.1}$ & $\frac{11}{0}$ & $\frac{16}{-}$ & $\frac{17}{0.2}$ & $\frac{21}{0.6}$ & $\frac{23}{2.9}$ & $\frac{24}{0.5}$ & $\frac{28}{0.9}$ & & $\frac{37}{0.5}$ & $\frac{38}{0}$ & $\frac{39}{0.2}$ & & & $\frac{56}{0}$ & $\frac{57}{0.1}$ & $\frac{58}{0}$ & $\frac{61}{0}$ & $\frac{62}{0.1}$ & $\frac{63}{0}$ & $\frac{64}{0}$ & $\frac{1}{0.1}$ & & \\
\hline SS \& GS & & & & & & & & & & $\frac{49}{0.1}$ & $\frac{50}{0}$ & $\frac{51}{0.1}$ & $\frac{52}{0}$ & & & & & & & & & & & \\
\hline
\end{tabular}


Table 70. Volume of Diaphus rafinesquii $\left(\mathrm{ml} / 10,000 \mathrm{~m}^{3}\right)$.

\begin{tabular}{|c|c|c|c|c|c|c|c|c|c|c|c|c|c|c|c|c|c|c|c|c|c|c|c|c|}
\hline & \multicolumn{4}{|c|}{$\begin{array}{c}\text { Oceanus } \\
\text { Apri1 }\end{array}$} & \multicolumn{5}{|c|}{$\begin{array}{c}\text { Oceanus } 121 \\
\text { June }\end{array}$} & \multicolumn{5}{|c|}{$\begin{array}{c}\text { Oceanus } 125 \\
\text { August }\end{array}$} & \multicolumn{7}{|c|}{$\begin{array}{l}\text { Knorr } 98 \\
\text { Sept-0ct }\end{array}$} & \multicolumn{3}{|c|}{$\begin{array}{c}A 2 \quad 110 \\
\text { Sept-0ct }\end{array}$} \\
\hline $\begin{array}{l}\text { Slope } \\
\text { Water }\end{array}$ & $\frac{8}{\overline{\overline{0}}}$ & $\begin{array}{l}9 \\
0 \\
0 \\
0 \\
0\end{array}$ & & & $\begin{array}{l}\frac{19}{0.29} \\
\frac{0}{0} \\
\frac{0}{0} \\
\underline{0}\end{array}$ & $\begin{array}{l}20 \\
0 \\
0 \\
0 \\
0 \\
0\end{array}$ & $\begin{array}{l}\frac{33}{0} \\
\underline{0} \\
\underline{0} \\
\underline{0} \\
\underline{0}\end{array}$ & $\begin{array}{l}34 \\
\underline{0} \\
\underline{0} \\
\underline{0}\end{array}$ & & $\begin{array}{l}35 \\
0 \\
0 \\
0 \\
0 \\
0\end{array}$ & $\begin{array}{l}\frac{47}{\underline{0}} \\
\frac{0}{0} \\
\frac{0}{0}\end{array}$ & $\begin{array}{l}\frac{48}{0} \\
0 \\
0 \\
0\end{array}$ & $\frac{\frac{53}{0.08}}{\underline{0}}$ & $\begin{array}{l}54 \\
0 \\
0 \\
0 \\
0\end{array}$ & $\begin{array}{l}\frac{59}{0.50} \\
\frac{0}{0} \\
\frac{0}{0} \\
\underline{0}\end{array}$ & $\begin{array}{l}\frac{60}{0} \\
0.23 \\
0 \\
0 \\
0\end{array}$ & $\begin{array}{l}\frac{65}{1.43} \\
\underline{0} \\
\frac{0}{0} \\
\underline{0}\end{array}$ & $\begin{array}{l}\frac{66}{0} \\
0 \\
0 \\
0 \\
0\end{array}$ & & & & $\begin{array}{l}4 \\
0 \\
0 \\
0 \\
0\end{array}$ & $\begin{array}{l}\frac{5}{0} \\
\underline{0} \\
\underline{0} \\
\underline{0}\end{array}$ & \\
\hline $\begin{array}{l}\text { High } \\
\text { Velocity } \\
\text { Region }\end{array}$ & $\begin{array}{c}\frac{12}{0} \\
0.27 \\
0.08 \\
0\end{array}$ & $\frac{\frac{13}{0.05}}{\frac{0}{0}}$ & $\frac{\frac{14}{0.20}}{\frac{0}{0}}$ & $\begin{array}{l}\frac{15}{0} \\
0 \\
0 \\
0\end{array}$ & $\begin{array}{l}26 \\
0 \\
0 \\
3.81 \\
0 \\
-\end{array}$ & $\begin{array}{l}\frac{27}{0} \\
0.18 \\
0 \\
0 \\
0\end{array}$ & $\begin{array}{l}\frac{30}{0} \\
1.92 \\
1.56 \\
0 \\
0\end{array}$ & $\begin{array}{c}31 \\
0 \\
0 \\
5.71 \\
0 \\
0\end{array}$ & $\frac{\frac{32}{1.84}}{\frac{2.20}{\underline{0}}}$ & $\frac{\frac{41}{0.19}}{\frac{0}{0}}$ & $\begin{array}{l}\frac{42}{\underline{0}} \\
\underline{0} \\
\underline{0} \\
\underline{0}\end{array}$ & $\begin{array}{l}43 \\
0 \\
0 \\
0 \\
0 \\
0\end{array}$ & $\frac{\frac{44}{0.39}}{\underline{0}}$ & & $\begin{array}{l}\frac{55}{0} \\
\underline{0} \\
\underline{0} \\
\underline{0}\end{array}$ & & & & & & & $\begin{array}{l}\frac{2}{0} \\
\underline{0} \\
\underline{0} \\
\underline{0}\end{array}$ & $\begin{array}{c}\frac{3}{0} \\
2.70 \\
0 \\
0\end{array}$ & \\
\hline $\begin{array}{l}\text { Ring } \\
\text { Core }\end{array}$ & $\frac{\frac{10}{0.04}}{\frac{0}{0}}$ & $\begin{array}{l}11 \\
0 \\
0 \\
0 \\
0\end{array}$ & $\begin{array}{l}16 \\
0 \\
0 \\
0\end{array}$ & $\frac{\frac{17}{0.28}}{\frac{0}{0}}$ & $\frac{\frac{21}{\underline{0}}}{\frac{1.50}{1.91}} \frac{\underline{0}}{\underline{0}}$ & $\frac{\frac{23}{9.41}}{\frac{0.80}{4.25}} \frac{\underline{0}}{\underline{0}}$ & $\begin{array}{c}\frac{24}{0} \\
0 \\
7.57 \\
0 \\
0\end{array}$ & $\begin{array}{c}\frac{28}{0} \\
10.00 \\
0 \\
0 \\
0\end{array}$ & & & & & & & $\begin{array}{l}\frac{56}{0} \\
\underline{0} \\
\underline{0} \\
\underline{0} \\
\underline{0}\end{array}$ & $\begin{array}{l}\frac{57}{\underline{0}} \\
\underline{1.34} \\
\underline{0} \\
\underline{0} \\
\underline{0}\end{array}$ & $\begin{array}{l}\frac{58}{0} \\
0 \\
0 \\
0 \\
0\end{array}$ & $\begin{array}{l}\frac{61}{0} \\
\underline{0} \\
\underline{0} \\
\underline{0} \\
\underline{0}\end{array}$ & $\begin{array}{l}\frac{62}{0} \\
0 \\
0 \\
1.62 \\
0\end{array}$ & $\begin{array}{l}\frac{63}{\underline{0}} \\
\frac{0.98}{\underline{0}} \\
\underline{0} \\
\underline{0}\end{array}$ & $\begin{array}{l}64 \\
0 \\
0 \\
0 \\
0 \\
0\end{array}$ & $\frac{\frac{1}{\underline{0}}}{\frac{3.00}{\underline{0}}}$ & & \\
\hline $\begin{array}{l}\text { Sargasso } \\
\text { Sea } \\
\& \\
\text { Gulf } \\
\text { Stream }\end{array}$ & & & & & & & & & & $\begin{array}{l}\frac{49}{\underline{0}} \\
\frac{1.44}{\underline{0}} \\
\underline{0}\end{array}$ & $\begin{array}{l}50 \\
0 \\
0 \\
0 \\
0\end{array}$ & $\begin{array}{c}\frac{51}{0} \\
0 \\
1.24 \\
0\end{array}$ & $\begin{array}{l}\frac{52}{\underline{0}} \\
\underline{0} \\
\underline{0} \\
\underline{0}\end{array}$ & & & & & & & & & & & \\
\hline $\begin{array}{l}\text { Slope } \\
\text { Water }\end{array}$ & $\frac{8}{-}$ & $\frac{9}{0}$ & $\frac{18}{0}$ & & $\frac{19}{0.03}$ & $\frac{20}{0}$ & $\frac{33}{0}$ & $\frac{34}{0}$ & & $\frac{35}{0}$ & $\frac{47}{0}$ & $\frac{48}{0}$ & $\frac{53}{0.02}$ & $\frac{54}{0}$ & $\frac{59}{0.10}$ & $\frac{60}{0.02}$ & $\frac{65}{0.29}$ & $\frac{66}{0}$ & & & & $\frac{4}{0}$ & $\frac{5}{0}$ & $\frac{6}{0}$ \\
\hline $\begin{array}{l}\text { High } \\
\text { Velocity } \\
\text { Region }\end{array}$ & $\frac{12}{0.09}$ & $\frac{13}{0.01}$ & $\frac{14}{0.05}$ & $\frac{15}{0}$ & $\frac{27}{0.03}$ & $\frac{29}{1.59}$ & $\frac{30}{0.50}$ & $\frac{31}{1.14}$ & $\frac{32}{-}$ & $\frac{41}{0.10}$ & $\frac{42}{0}$ & $\frac{43}{0}$ & $\frac{44}{0.23}$ & & $\frac{55}{0}$ & & & & & & & $\frac{2}{0}$ & $\frac{3}{0.68}$ & \\
\hline $\begin{array}{l}\text { Ring } \\
\text { Core }\end{array}$ & $\frac{10}{0.01}$ & $\frac{11}{0}$ & $\frac{16}{-}$ & $\frac{17}{0.07}$ & $\frac{21}{0.91}$ & $\frac{23}{2.46}$ & $\frac{24}{1.51}$ & $\frac{28}{1.80}$ & & $\frac{37}{0.42}$ & $\frac{38}{0}$ & $\frac{39}{0.50}$ & & & $\frac{56}{0}$ & $\frac{57}{0.27}$ & $\frac{58}{0}$ & $\frac{61}{0}$ & $\frac{62}{0.32}$ & $\frac{63}{0.03}$ & $\frac{64}{0}$ & $\frac{1}{0.75}$ & & \\
\hline SS \& GS & & & & & & & & & & $\frac{49}{0.36}$ & $\frac{50}{0}$ & $\frac{51}{0.31}$ & $\frac{52}{0}$ & & & & & & & & & & & \\
\hline
\end{tabular}


Table 71. Abundance of Diegenichthys atlanticus (specimens $/ 10,000 \mathrm{~m}^{3}$ ).

\begin{tabular}{|c|c|c|c|c|c|c|c|c|c|c|c|c|c|c|c|c|c|c|c|c|c|c|c|c|}
\hline & \multicolumn{4}{|c|}{$\begin{array}{c}\text { Oceanus } 118 \\
\text { April }\end{array}$} & \multicolumn{5}{|c|}{$\begin{array}{l}\text { Oceanus } 121 \\
\text { June }\end{array}$} & \multicolumn{5}{|c|}{$\begin{array}{l}\text { Oceanus } 125 \\
\text { August }\end{array}$} & \multicolumn{7}{|c|}{$\begin{array}{l}\text { Knorr } 98 \\
\text { Sept-Oct }\end{array}$} & \multicolumn{3}{|c|}{$\begin{array}{c}\text { A2 } 110 \\
\text { Sept-Oct }\end{array}$} \\
\hline $\begin{array}{l}\text { Slope } \\
\text { Water }\end{array}$ & $\begin{array}{l}\frac{8}{\overline{\overline{0}}} \\
\underline{0} \\
\underline{0} \\
\underline{0}\end{array}$ & $\begin{array}{l}9 \\
0 \\
0 \\
0 \\
0\end{array}$ & & & $\begin{array}{l}\frac{19}{\frac{2.4}{0}} \\
\frac{0}{0} \\
\frac{0.5}{\underline{0}}\end{array}$ & $\begin{array}{c}20 \\
0 \\
0 \\
0.4 \\
0 \\
0\end{array}$ & $\begin{array}{l}\frac{33}{2.7} \\
\frac{0}{0} \\
\underline{0} \\
\underline{0} \\
\underline{0.1}\end{array}$ & $\begin{array}{l}\frac{34}{\underline{0}} \\
\underline{0} \\
\underline{0.2}\end{array}$ & & $\begin{array}{c}35 \\
0 \\
0 \\
0.3 \\
0 \\
0\end{array}$ & $\begin{array}{c}\frac{47}{\underline{0}} \\
\underline{0} \\
\underline{0.4} \\
\underline{0}\end{array}$ & $\begin{array}{c}48 \\
0 \\
0.3 \\
0.3 \\
0\end{array}$ & $\frac{\frac{53}{1.0}}{\frac{0.2}{1.0}} \frac{\underline{0}}{\frac{0}{1.0}}$ & $\begin{array}{c}54 \\
0 \\
0 \\
1.5 \\
0\end{array}$ & $\frac{\frac{59}{0.3}}{\frac{0.4}{0.4}} \frac{\underline{0}}{\frac{0}{0}}$ & $\begin{array}{c}60 \\
0 \\
0 \\
2.8 \\
0 \\
0\end{array}$ & $\frac{\frac{65}{0.4}}{\frac{0.4}{\underline{0}}}$ & $\begin{array}{c}66 \\
0 \\
0 \\
2.6 \\
1.9 \\
0\end{array}$ & & & & $\begin{array}{l}\frac{4}{0} \\
0.4 \\
1.6 \\
0.6\end{array}$ & $\frac{\frac{5}{0.7}}{\frac{0}{0}}$ & \\
\hline $\begin{array}{l}\text { High } \\
\text { Velocity } \\
\text { Region }\end{array}$ & $\begin{array}{c}\frac{12}{0} \\
1.2 \\
22.5 \\
0\end{array}$ & $\frac{\frac{13}{3.0}}{\frac{0}{0}}$ & $\frac{\frac{14}{2.3}}{\frac{0}{0}}$ & $\begin{array}{c}15 \\
0 \\
0.6 \\
1.2 \\
0\end{array}$ & $\begin{array}{c}26 \\
0 \\
0 \\
0 \\
0 \\
-\end{array}$ & $\begin{array}{c}27 \\
0 \\
0 \\
0.5 \\
0 \\
0\end{array}$ & $\begin{array}{c}30 \\
0 \\
0 \\
3.4 \\
1.9 \\
0.2\end{array}$ & $\begin{array}{c}31 \\
0 \\
0 \\
0.7 \\
0.3 \\
0\end{array}$ & $\begin{array}{r}32 \\
\underline{0} \\
\underline{0} \\
\underline{0} \\
\underline{0}\end{array}$ & $\frac{\frac{41}{0.6}}{\frac{0.6}{\underline{0}}}$ & $\frac{\frac{42}{0.8}}{\frac{0.3}{\underline{0}}}$ & $\begin{array}{c}43 \\
0 \\
0 \\
1.7 \\
0 \\
0\end{array}$ & $\frac{44}{\frac{0.1}{\underline{0}}}$ & & $\frac{\frac{55}{4.5}}{\frac{\underline{0.9}}{\underline{0}}}$ & & & & & & & $\begin{array}{l}\frac{2}{0} \\
\underline{0} \\
\underline{0} \\
\underline{0}\end{array}$ & $\begin{array}{c}\frac{3}{0} \\
7.6 \\
0.4 \\
0\end{array}$ & \\
\hline $\begin{array}{l}\text { Ring } \\
\text { Core }\end{array}$ & $\frac{10}{\frac{4.8}{\underline{0}}} \frac{0}{\underline{0}}$ & $\begin{array}{c}11 \\
0 \\
0 \\
2.5 \\
0\end{array}$ & $\begin{array}{r}\frac{16}{0} \\
4.3 \\
22.7\end{array}$ & $\begin{array}{c}\frac{17}{14.4} \\
\underline{0} \\
0.3\end{array}$ & $\frac{\frac{21}{38.0}}{\frac{0}{0}}$ & $\begin{array}{c}\frac{23}{14.7} \\
\frac{0}{0} \\
\frac{0.5}{\underline{0}}\end{array}$ & $\begin{array}{c}24 \\
0 \\
0 \\
5.1 \\
4.4 \\
0\end{array}$ & $\begin{array}{c}28 \\
0 \\
0.8 \\
2.9 \\
0 \\
0\end{array}$ & & & & & & & 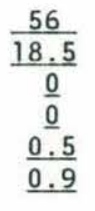 & $\begin{array}{c}\frac{57}{16.6} \\
\frac{0}{0} \\
\underline{1.3} \\
\underline{0.5}\end{array}$ & $\begin{array}{c}\frac{58}{0.5} \\
0 \\
1.0 \\
8.7 \\
0.8\end{array}$ & $\begin{array}{l}\frac{61}{\frac{6.1}{0}} \\
\underline{0} \\
\underline{0.8} \\
\underline{0.3}\end{array}$ & $\begin{array}{c}62 \\
0 \\
0 \\
0.2 \\
3.8 \\
0\end{array}$ & $\begin{array}{c}\frac{63}{2.0} \\
\frac{0}{0} \\
\underline{1.9} \\
\underline{2.2}\end{array}$ & $\begin{array}{c}64 \\
0 \\
0 \\
2.1 \\
1.4 \\
0\end{array}$ & $\begin{array}{l}\frac{1}{\frac{5.8}{0}} \\
\frac{1.6}{\underline{0}}\end{array}$ & & \\
\hline $\begin{array}{l}\text { Sargasso } \\
\text { Sea } \\
\& \\
\text { Gulf } \\
\text { Stream }\end{array}$ & & & & & & & & & & $\begin{array}{c}\frac{49}{10.8} \\
\frac{0}{0.7} \\
\underline{0}\end{array}$ & $\begin{array}{c}50 \\
0 \\
0 \\
7.2 \\
1.3\end{array}$ & $\begin{array}{c}51 \\
0 \\
0 \\
2.8 \\
0\end{array}$ & $\begin{array}{l}\frac{52}{\frac{6.6}{0}} \\
\frac{1.3}{0.3}\end{array}$ & & & & & & & & & & & \\
\hline $\begin{array}{l}\text { Slope } \\
\text { Water }\end{array}$ & $\frac{8}{-}$ & $\frac{9}{0}$ & $\frac{18}{0.1}$ & & $\frac{19}{0.4}$ & $\frac{20}{0.1}$ & $\frac{33}{0.2}$ & $\frac{34}{0.1}$ & & $\frac{35}{0.1}$ & $\frac{47}{0.1}$ & $\frac{48}{0.2}$ & $\frac{53}{0.6}$ & $\frac{54}{0.4}$ & $\frac{59}{0.1}$ & $\frac{60}{0.6}$ & $\frac{65}{0.1}$ & $\frac{66}{0.9}$ & & & & $\frac{4}{0.5}$ & $\frac{5}{0.2}$ & $\frac{6}{0}$ \\
\hline $\begin{array}{l}\text { High } \\
\text { Velocity } \\
\text { Region }\end{array}$ & $\frac{12}{5.8}$ & $\frac{13}{0.8}$ & $\frac{14}{0.6}$ & $\frac{15}{0.5}$ & $\frac{27}{0.1}$ & $\frac{29}{3.5}$ & $\frac{30}{1.1}$ & $\frac{31}{0.2}$ & $\frac{32}{-}$ & $\frac{41}{0.5}$ & $\frac{42}{0.4}$ & $\frac{43}{0.3}$ & $\frac{44}{0.1}$ & & $\frac{55}{1.1}$ & & & & & & & $\frac{2}{0}$ & $\frac{3}{2.0}$ & \\
\hline $\begin{array}{l}\text { Ring } \\
\text { Core }\end{array}$ & $\frac{10}{1.2}$ & $\frac{11}{0.6}$ & $\frac{16}{-}$ & $\frac{17}{3.8}$ & $\frac{21}{2.2}$ & $\frac{23}{2.3}$ & $\frac{24}{1.9}$ & $\frac{28}{0.9}$ & & $\frac{37}{0.4}$ & $\frac{38}{0}$ & $\frac{39}{0.3}$ & & & $\frac{56}{4.0}$ & $\frac{57}{3.7}$ & $\frac{58}{2.3}$ & $\frac{61}{1.4}$ & $\frac{62}{0.8}$ & $\frac{63}{1.6}$ & $\frac{64}{0.5}$ & $\frac{1}{1.9}$ & & \\
\hline SS \& GS & & & & & & & & & & $\frac{49}{2.9}$ & $\frac{50}{2.1}$ & $\frac{51}{0.7}$ & $\frac{52}{2.1}$ & & & & & & & & & & & \\
\hline
\end{tabular}


Table 72. Volume of Eurypharynx pelecanoides $\left(\mathrm{ml} / 10,000 \mathrm{~m}^{3}\right)$.

\begin{tabular}{|c|c|c|c|c|c|c|c|c|c|c|c|c|c|c|c|c|c|c|c|c|c|c|c|c|}
\hline & \multicolumn{4}{|c|}{$\begin{array}{c}\text { Oceanus } \\
\text { April }\end{array}$} & \multicolumn{5}{|c|}{$\begin{array}{c}\text { Oceanus } 121 \\
\text { June }\end{array}$} & \multicolumn{5}{|c|}{$\begin{array}{c}\text { Oceanus } 125 \\
\text { August }\end{array}$} & \multicolumn{7}{|c|}{$\begin{array}{l}\text { Knorr } 98 \\
\text { Sept-0ct }\end{array}$} & \multicolumn{3}{|c|}{$\begin{array}{c}\text { A2 } 110 \\
\text { Sept-0ct }\end{array}$} \\
\hline $\begin{array}{l}\text { Slope } \\
\text { Water }\end{array}$ & $\begin{array}{l}\frac{8}{\overline{\overline{0}}} \\
\underline{0} \\
\underline{0} \\
\underline{0.09}\end{array}$ & $\begin{array}{l}9 \\
0 \\
0 \\
0 \\
0\end{array}$ & & & $\begin{array}{l}19 \\
\frac{0}{0} \\
\underline{0} \\
\frac{0}{0} \\
\underline{0}\end{array}$ & $\begin{array}{l}\frac{20}{0} \\
0 \\
0 \\
3.64 \\
2.80\end{array}$ & $\begin{array}{l}\frac{33}{0} \\
\underline{0} \\
\underline{0} \\
\underline{0} \\
0.86\end{array}$ & $\begin{array}{l}\frac{34}{\underline{0}} \\
\underline{0} \\
\underline{0}\end{array}$ & & $\begin{array}{l}35 \\
0 \\
0 \\
0 \\
0.28 \\
0\end{array}$ & $\begin{array}{l}\frac{47}{\underline{0}} \\
\underline{0} \\
\underline{0} \\
\underline{0.18}\end{array}$ & $\begin{array}{c}\frac{48}{0} \\
0 \\
0 \\
0.16 \\
\end{array}$ & $\begin{array}{l}\frac{53}{\underline{0}} \\
\frac{0}{0} \\
\underline{0.24} \\
\end{array}$ & $\begin{array}{c}\frac{54}{0} \\
0 \\
0 \\
4.33\end{array}$ & $\begin{array}{l}\frac{59}{\underline{0}} \\
\underline{0} \\
\underline{0} \\
\underline{0} \\
1.34\end{array}$ & $\begin{array}{l}60 \\
0 \\
0 \\
0 \\
0 \\
0.15\end{array}$ & $\begin{array}{l}\frac{65}{0} \\
\underline{0} \\
\underline{0} \\
\underline{0} \\
\underline{1.44}\end{array}$ & $\begin{array}{l}66 \\
0 \\
0 \\
0 \\
0 \\
2.31\end{array}$ & & & & $\begin{array}{c}4 \\
0 \\
0 \\
0 \\
5.29\end{array}$ & $\begin{array}{l}\frac{5}{\underline{0}} \\
\underline{0} \\
0.28 \\
1.54\end{array}$ & \\
\hline $\begin{array}{l}\text { High } \\
\text { Velocity } \\
\text { Region }\end{array}$ & $\begin{array}{l}12 \\
0 \\
0 \\
0 \\
0\end{array}$ & $\begin{array}{l}\frac{13}{\underline{0}} \\
\underline{0} \\
\underline{0.49}\end{array}$ & $\begin{array}{l}14 \\
\underline{0} \\
\underline{0} \\
\underline{0} \\
\underline{0}\end{array}$ & $\begin{array}{l}15 \\
0 \\
0 \\
0 \\
0\end{array}$ & $\begin{array}{l}26 \\
0 \\
0 \\
0 \\
0 \\
-\end{array}$ & $\begin{array}{l}27 \\
0 \\
0 \\
0 \\
0 \\
2.28\end{array}$ & $\begin{array}{l}30 \\
0 \\
0 \\
0 \\
0 \\
0.05\end{array}$ & $\begin{array}{c}31 \\
0 \\
0 \\
0.05 \\
0 \\
0.52\end{array}$ & $\begin{array}{l}\frac{32}{\underline{0}} \\
\underline{0} \\
\underline{0} \\
1.74 \\
\end{array}$ & $\begin{array}{l}\frac{41}{\underline{0}} \\
\underline{0} \\
\underline{0.43} \\
\end{array}$ & $\begin{array}{l}\frac{42}{\underline{0}} \\
\underline{0} \\
\underline{0} \\
\underline{0.95}\end{array}$ & $\begin{array}{l}\frac{43}{0} \\
0 \\
0 \\
0 \\
0\end{array}$ & $\begin{array}{l}\frac{44}{\underline{0}} \\
\underline{0} \\
\underline{1.15}\end{array}$ & & $\begin{array}{l}\frac{55}{\underline{0}} \\
\underline{0} \\
\underline{0} \\
\underline{0}\end{array}$ & & & & & & & $\begin{array}{c}\frac{2}{\underline{0}} \\
\frac{0}{0} \\
12.50 \\
\end{array}$ & $\begin{array}{c}3 \\
0 \\
0 \\
0 \\
6.54\end{array}$ & \\
\hline $\begin{array}{l}\text { Ring } \\
\text { Core }\end{array}$ & $\begin{array}{l}10 \\
\frac{0}{0} \\
\underline{0} \\
\underline{0}\end{array}$ & $\begin{array}{l}11 \\
0 \\
0 \\
0 \\
0\end{array}$ & $\begin{array}{l}16 \\
0 \\
0 \\
0\end{array}$ & $\begin{array}{l}17 \\
\underline{0} \\
\underline{0} \\
\underline{0}\end{array}$ & $\begin{array}{l}21 \\
\underline{0} \\
\underline{0} \\
\underline{0} \\
\underline{0} \\
\underline{0}\end{array}$ & $\begin{array}{l}23 \\
\underline{0} \\
\underline{0} \\
\underline{0} \\
\underline{0} \\
\underline{0}\end{array}$ & $\begin{array}{l}24 \\
0 \\
0 \\
0 \\
0 \\
0\end{array}$ & $\begin{array}{l}28 \\
0 \\
0 \\
0 \\
0 \\
0\end{array}$ & & & & & & & $\begin{array}{l}\frac{56}{0} \\
\underline{0} \\
\underline{0} \\
\underline{0} \\
\underline{0}\end{array}$ & $\begin{array}{l}\frac{57}{0} \\
\underline{0} 0 \\
\underline{0} \\
\underline{0} \\
\underline{0.03} \\
\end{array}$ & $\begin{array}{l}58 \\
0 \\
0 \\
0 \\
0 \\
0\end{array}$ & $\begin{array}{l}\frac{61}{0} \\
\underline{0} \\
\underline{0} \\
\underline{0} \\
\underline{0.05}\end{array}$ & $\begin{array}{l}62 \\
0 \\
0 \\
0 \\
0 \\
0\end{array}$ & $\begin{array}{l}\frac{63}{\underline{0}} \\
\underline{0} \\
\underline{0} \\
\underline{0} \\
\underline{0.16}\end{array}$ & $\begin{array}{l}\frac{64}{0} \\
0 \\
0 \\
0 \\
0.12\end{array}$ & $\begin{array}{l}\frac{1}{0} \\
\underline{0} \\
\underline{0} \\
\underline{0}\end{array}$ & & \\
\hline $\begin{array}{l}\text { Sargasso } \\
\text { Sea } \\
\& \\
\text { Gulf } \\
\text { Stream }\end{array}$ & & & & & & & & & & $\begin{array}{l}49 \\
\underline{0} \\
\underline{0} \\
\underline{0} \\
\underline{0}\end{array}$ & $\begin{array}{l}50 \\
0 \\
0 \\
0 \\
0\end{array}$ & $\begin{array}{l}51 \\
0 \\
0 \\
0 \\
0\end{array}$ & $\begin{array}{l}\frac{52}{\underline{0}} \\
\underline{0} \\
\underline{0} \\
\underline{0.06}\end{array}$ & & & & & & & & & & & \\
\hline $\begin{array}{l}\text { Slope } \\
\text { Water }\end{array}$ & $\frac{8}{-}$ & $\frac{9}{0}$ & $\frac{18}{0}$ & & $\frac{19}{0}$ & $\frac{20}{0.91}$ & $\frac{33}{0.43}$ & $\frac{34}{0}$ & & $\frac{35}{0.06}$ & $\frac{47}{0.05}$ & $\frac{48}{0.04}$ & $\frac{53}{0.06}$ & $\frac{54}{1.08}$ & $\frac{59}{0.27}$ & $\frac{60}{0.03}$ & $\frac{65}{0.29}$ & $\frac{66}{0.46}$ & & & & $\frac{4}{1.32}$ & $\frac{5}{0.46}$ & $\frac{6}{0}$ \\
\hline $\begin{array}{l}\text { High } \\
\text { Velocity } \\
\text { Region }\end{array}$ & $\frac{12}{0}$ & $\frac{13}{0.25}$ & $\frac{14}{0}$ & $\frac{15}{0}$ & $\frac{27}{0.57}$ & $\frac{29}{0}$ & $\frac{30}{0.01}$ & $\frac{31}{0.11}$ & $\frac{32}{-}$ & $\frac{41}{0.11}$ & $\frac{42}{0.19}$ & $\frac{43}{0}$ & $\frac{44}{0.23}$ & & $\frac{55}{0}$ & & & & & & & $\frac{2}{3.13}$ & $\frac{3}{1.64}$ & \\
\hline $\begin{array}{l}\text { Ring } \\
\text { Core }\end{array}$ & $\frac{10}{0}$ & $\frac{11}{0}$ & $\frac{16}{-}$ & $\frac{17}{0}$ & $\frac{21}{0}$ & $\frac{23}{0}$ & $\frac{24}{0}$ & $\frac{28}{0}$ & & $\frac{37}{0}$ & $\frac{38}{0.04}$ & $\frac{39}{0}$ & & & $\frac{56}{0}$ & $\frac{57}{0.01}$ & $\frac{58}{0}$ & $\frac{61}{0.01}$ & $\frac{62}{0}$ & $\frac{63}{0.03}$ & $\frac{64}{0.02}$ & $\frac{1}{0}$ & & \\
\hline SS \& GS & & & & & & & & & & $\frac{49}{0}$ & $\frac{50}{0}$ & $\frac{51}{0}$ & $\frac{52}{0.02}$ & & & & & & & & & & & \\
\hline
\end{tabular}


Table 73. Abundance of Gonostoma elongatum (specimens $/ 10,000 \mathrm{~m}^{3}$ )

\begin{tabular}{|c|c|c|c|c|c|c|c|c|c|c|c|c|c|c|c|c|c|c|c|c|c|c|c|c|}
\hline & \multicolumn{4}{|c|}{$\begin{array}{c}\text { Oceanus } 118 \\
\text { April }\end{array}$} & \multicolumn{5}{|c|}{$\begin{array}{c}\text { Oceanus } 121 \\
\text { June }\end{array}$} & \multicolumn{5}{|c|}{$\begin{array}{l}\text { Oceanus } 125 \\
\text { August }\end{array}$} & \multicolumn{7}{|c|}{$\begin{array}{l}\text { Knorr } 98 \\
\text { Sept-0ct }\end{array}$} & \multicolumn{3}{|c|}{$\begin{array}{c}\text { A2 } 110 \\
\text { Sept-0ct }\end{array}$} \\
\hline $\begin{array}{l}\text { Slope } \\
\text { Water }\end{array}$ & $\begin{array}{l}\frac{8}{=} \\
\frac{0.6}{\underline{0}} \\
\underline{0}\end{array}$ & $\begin{array}{c}9 \\
0 \\
0 \\
2.9 \\
0\end{array}$ & & & $\frac{19}{\underline{0}}$ & $\begin{array}{c}20 \\
0 \\
0 \\
1.7 \\
0 \\
0\end{array}$ & $\begin{array}{l}\frac{33}{\underline{0}} \\
\underline{0.4} \\
\underline{0} \\
\underline{0} \\
\underline{0}\end{array}$ & $\frac{34}{\underline{0}} \frac{1.1}{\underline{0}}$ & & $\begin{array}{r}35 \\
0 \\
0 \\
0 \\
0 \\
0\end{array}$ & $\begin{array}{c}\frac{47}{\underline{0}} \\
\frac{0.8}{\underline{0}} \\
\underline{0}\end{array}$ & $\begin{array}{c}\frac{48}{0} \\
0.3 \\
0.6 \\
0\end{array}$ & $\frac{\frac{53}{0.2}}{\frac{0.2}{0.2}} \frac{\underline{0}}{\underline{0}}$ & $\begin{array}{c}54 \\
0.3 \\
0 \\
1.2 \\
0\end{array}$ & $\begin{array}{l}\frac{59}{5.0} \\
\frac{0}{0} \\
\frac{0}{0} \\
\underline{0}\end{array}$ & $\begin{array}{c}60 \\
0.3 \\
0 \\
3.8 \\
0.4 \\
0\end{array}$ & $\frac{\frac{65}{0.9}}{\frac{0.9}{\underline{0}}}$ & $\begin{array}{c}66 \\
0 \\
0 \\
1.5 \\
1.9 \\
0\end{array}$ & & & & $\begin{array}{l}4 \\
0 \\
0 \\
0 \\
0\end{array}$ & $\frac{\frac{5}{2.7}}{\frac{0}{0}}$ & \\
\hline $\begin{array}{l}\text { High } \\
\text { Velocity } \\
\text { Region }\end{array}$ & $\begin{array}{c}12 \\
0 \\
0 \\
2.1 \\
0\end{array}$ & $\begin{array}{c}\frac{13}{\underline{0}} \\
\underline{1.7} \\
\underline{0.2}\end{array}$ & $\frac{\frac{14}{0.6}}{\frac{0.7}{\underline{0}}}$ & $\begin{array}{c}15 \\
0 \\
0 \\
0.6 \\
0\end{array}$ & $\begin{array}{c}26 \\
0 \\
0 \\
0 \\
0.7 \\
-\end{array}$ & $\begin{array}{c}27 \\
0 \\
0 \\
0.5 \\
0 \\
0\end{array}$ & $\begin{array}{c}30 \\
0 \\
0 \\
0 \\
0.8 \\
0\end{array}$ & $\begin{array}{c}31 \\
0 \\
0 \\
0 \\
2.3 \\
0\end{array}$ & $\frac{32}{\frac{0.8}{\underline{0}}}$ & $\frac{\frac{41}{1.6}}{\frac{0}{0}}$ & $\frac{42}{\frac{1.1}{\underline{0}}}$ & $\begin{array}{c}43 \\
0 \\
1.0 \\
0 \\
0.9 \\
0\end{array}$ & $\frac{44}{\frac{1.7}{\underline{0}}}$ & & $\begin{array}{c}\frac{55}{\frac{8.7}{0}} \\
\underline{0} \\
\underline{1.1}\end{array}$ & & & & & & & $\frac{\frac{2}{3.1}}{\frac{0.3}{0}}$ & $\begin{array}{c}\frac{3}{0} \\
1.9 \\
0 \\
0\end{array}$ & \\
\hline $\begin{array}{l}\text { Ring } \\
\text { Core }\end{array}$ & $\frac{10}{\frac{1.2}{\underline{0}}} \frac{\underline{0}}{\underline{0}}$ & $\begin{array}{c}11 \\
0 \\
0 \\
0 \\
0\end{array}$ & $\begin{array}{r}16 \\
0 \\
0 \\
0\end{array}$ & $\frac{\frac{17}{0.6}}{\frac{0}{\underline{0}}}$ & $\begin{array}{l}\frac{21}{\underline{0}} \\
\underline{0.4} \\
\underline{0} \\
\underline{0} \\
\underline{0}\end{array}$ & $\begin{array}{c}\frac{23}{\underline{0}} \\
\frac{0.7}{\underline{0}} \\
\underline{0} \\
\underline{0}\end{array}$ & $\begin{array}{c}24 \\
0 \\
0 \\
0 \\
1.9 \\
\underline{0.6} \\
\end{array}$ & $\begin{array}{c}28 \\
0 \\
0 \\
0.4 \\
0 \\
0\end{array}$ & & & & & & & $\begin{array}{l}\frac{56}{2.4} \\
\frac{0}{0.5} \\
\frac{0}{0}\end{array}$ & $\begin{array}{l}\frac{57}{\frac{5.3}{\underline{0}}} \\
\frac{0.3}{\underline{0}} \\
\underline{0}\end{array}$ & $\begin{array}{c}58 \\
0 \\
0 \\
0.5 \\
1.6 \\
0\end{array}$ & $\begin{array}{c}\frac{61}{2.2} \\
\frac{0}{0} \\
\frac{0.4}{\underline{0}}\end{array}$ & $\begin{array}{c}\frac{62}{0.4} \\
0 \\
0 \\
0.5 \\
0.4\end{array}$ & $\frac{63}{\frac{1.1}{\underline{0}}}$ & $\begin{array}{c}\frac{64}{0.6} \\
0 \\
0 \\
0.5 \\
0\end{array}$ & $\frac{\frac{1}{2.2}}{\frac{0.8}{\underline{0}}}$ & & \\
\hline $\begin{array}{l}\text { Sargasso } \\
\text { Sea } \\
\& \\
\text { Gulf } \\
\text { Stream }\end{array}$ & & & & & & & & & & $\frac{49}{\frac{3.5}{\frac{0}{0}}}$ & $\begin{array}{c}\frac{50}{1.3} \\
0 \\
3.4 \\
0\end{array}$ & $\begin{array}{c}\frac{51}{0.3} \\
0 \\
1.7 \\
0\end{array}$ & $\begin{array}{l}\frac{52}{\frac{6.8}{0}} \\
\frac{0.4}{\underline{0}}\end{array}$ & & & & & & & & & & & \\
\hline $\begin{array}{l}\text { Slope } \\
\text { Water }\end{array}$ & $\frac{8}{-}$ & $\frac{9}{0.7}$ & $\frac{18}{0.6}$ & & $\frac{19}{0.3}$ & $\frac{20}{0.4}$ & $\frac{33}{0.1}$ & $\frac{34}{0.4}$ & & $\frac{35}{0}$ & $\frac{47}{0.2}$ & $\frac{48}{0.2}$ & $\frac{53}{0.1}$ & $\frac{54}{0.4}$ & $\frac{59}{1.0}$ & $\frac{60}{0.9}$ & $\frac{65}{0.2}$ & $\frac{66}{0.7}$ & & & & $\frac{4}{0}$ & $\frac{5}{0.7}$ & $\frac{6}{0}$ \\
\hline $\begin{array}{l}\text { High } \\
\text { Velocity } \\
\text { Region }\end{array}$ & $\frac{12}{0.5}$ & $\frac{13}{0.5}$ & $\frac{14}{0.3}$ & $\frac{15}{0.2}$ & $\frac{27}{0.1}$ & $\frac{29}{0.3}$ & $\frac{30}{0.2}$ & $\frac{31}{0.5}$ & $\frac{32}{-}$ & $\frac{41}{0.8}$ & $\frac{42}{0.4}$ & $\frac{43}{0.5}$ & $\frac{44}{1.0}$ & & $\frac{55}{2.0}$ & & & & & & & $\frac{2}{0.9}$ & $\frac{3}{0.5}$ & \\
\hline $\begin{array}{l}\text { Ring } \\
\text { Core }\end{array}$ & $\frac{10}{0.3}$ & $\frac{11}{0}$ & $\frac{16}{-}$ & $\frac{17}{0.2}$ & $\frac{21}{0.1}$ & $\frac{23}{0.2}$ & $\frac{24}{0.5}$ & $\frac{28}{0.1}$ & & $\frac{37}{0.7}$ & $\frac{38}{6.9}$ & $\frac{39}{0.8}$ & & & $\frac{56}{0.6}$ & $\frac{57}{1.1}$ & $\frac{58}{0.4}$ & $\frac{61}{0.3}$ & $\frac{62}{0.3}$ & $\frac{63}{0.4}$ & $\frac{64}{0.3}$ & $\frac{1}{0.8}$ & & \\
\hline SS \& GS & & & & & & & & & & $\frac{49}{0.9}$ & $\frac{50}{1.2}$ & $\frac{51}{0.5}$ & $\frac{52}{1.8}$ & & & & & & & & & & & \\
\hline
\end{tabular}


Table 74. Volume of Gonostoma elengatum $\left(\mathrm{ml} / 10,000 \mathrm{~m}^{3}\right)$.

\begin{tabular}{|c|c|c|c|c|c|c|c|c|c|c|c|c|c|c|c|c|c|c|c|c|c|c|c|c|}
\hline & . & $\begin{array}{r}\text { Oceanu } \\
\text { Apr }\end{array}$ & $\begin{array}{l}\text { us } 118 \\
\text { ril }\end{array}$ & & & & $\begin{array}{r}\text { ceanus } \\
\text { June }\end{array}$ & 121 & & & & $\begin{array}{l}\text { eanus } \\
\text { August }\end{array}$ & & & & & $\begin{array}{l}\text { Knor } \\
\text { Sept }\end{array}$ & $\begin{array}{l}r r 98 \\
t-0 c t\end{array}$ & & & & & $\begin{array}{l}\text { A2 } 110 \\
\text { ept-0ct }\end{array}$ & \\
\hline $\begin{array}{l}\text { Slope } \\
\text { Water }\end{array}$ & $\begin{array}{l}\frac{8}{\bar{a}} \\
\frac{8.82}{\underline{0}} \\
\underline{0}\end{array}$ & $\begin{array}{c}\frac{9}{0} \\
0 \\
75.00 \\
0\end{array}$ & & & $\frac{\frac{19}{0.17}}{\frac{8.28}{\frac{0}{0}}}$ & $\begin{array}{c}\frac{20}{0} \\
0 \\
23.91 \\
0 \\
0\end{array}$ & $\begin{array}{l}\frac{33}{\underline{0}} \\
\underline{4.58} \\
\underline{0} \\
\underline{0} \\
\underline{0}\end{array}$ & $\frac{\frac{34}{0}}{\frac{\underline{0} .26}{\underline{0}}}$ & & $\begin{array}{l}35 \\
0 \\
0 \\
0 \\
0 \\
0\end{array}$ & $\frac{\frac{47}{\frac{9.81}{8.75}}}{\underline{0}}$ & $\begin{array}{c}\frac{48}{0} \\
0.03 \\
10.00 \\
0\end{array}$ & $\frac{\frac{53}{6.54}}{\frac{4.22}{\underline{0}}}$ & $\begin{array}{c}\frac{54}{2.94} \\
0 \\
5.45 \\
0\end{array}$ & $\begin{array}{l}\frac{59}{6.67} \\
\underline{0} \\
\underline{0} \\
\underline{0} \\
\underline{0}\end{array}$ & $\begin{array}{c}\frac{60}{0.05} \\
0 \\
1.55 \\
4.35 \\
0\end{array}$ & $\frac{\frac{65}{6.52}}{\frac{0}{0}}$ & $\begin{array}{c}\frac{66}{0} \\
12.14 \\
0 \\
15.71 \\
0\end{array}$ & & & & $\begin{array}{l}4 \\
0 \\
0 \\
0 \\
0\end{array}$ & $\frac{\frac{5}{16.67}}{\frac{0}{\underline{0}}}$ & \\
\hline $\begin{array}{l}\text { High } \\
\text { Velocity } \\
\text { Region }\end{array}$ & $\begin{array}{c}\frac{12}{0} \\
0 \\
15.42 \\
0\end{array}$ & $\frac{\frac{13}{15.10}}{\frac{33.33}{6.83}}$ & $\frac{\frac{14}{5.14}}{\frac{9.67}{\underline{0}}}$ & $\begin{array}{c}\frac{15}{0} \\
0 \\
16.47 \\
0\end{array}$ & $\begin{array}{c}26 \\
0 \\
0 \\
0 \\
7.24 \\
-\end{array}$ & $\begin{array}{l}\frac{27}{0} \\
0 \\
0.07 \\
0 \\
0\end{array}$ & $\begin{array}{c}30 \\
0 \\
0 \\
0 \\
17.57 \\
0\end{array}$ & $\begin{array}{c}31 \\
0 \\
0 \\
0 \\
23.14 \\
0\end{array}$ & $\frac{\frac{32}{13.94}}{\frac{0}{\underline{0}}}$ & $\frac{\frac{41}{0.51}}{\frac{0}{0}}$ & $\frac{\frac{42}{4.00}}{\frac{0}{0}}$ & $\begin{array}{c}43 \\
0 \\
0.04 \\
0 \\
13.04 \\
0\end{array}$ & $\frac{\frac{44}{1.94}}{\frac{0}{0}}$ & & $\begin{array}{l}\frac{55}{1.24} \\
\frac{0}{0} \\
71.11\end{array}$ & & & & & & & $\frac{\frac{2}{10.04}}{\frac{4.32}{\underline{0}}}$ & $\begin{array}{l}\frac{3}{0} \\
1.08 \\
0 \\
0\end{array}$ & \\
\hline $\begin{array}{l}\text { Ring } \\
\text { Core }\end{array}$ & $\frac{\frac{10}{6.00}}{\frac{0}{0}}$ & $\begin{array}{l}11 \\
0 \\
0 \\
0 \\
0\end{array}$ & $\begin{array}{l}-16 \\
0 \\
0 \\
0\end{array}$ & $\frac{\frac{17}{0.11}}{\frac{0}{0}}$ & $\begin{array}{l}\frac{21}{\underline{0}} \\
\frac{7.00}{\underline{0}} \\
\underline{0} \\
\underline{0}\end{array}$ & $\begin{array}{l}\frac{23}{\underline{0}} \\
\frac{0.80}{\underline{0}} \\
\underline{0} \\
\underline{0}\end{array}$ & $\begin{array}{c}24 \\
0 \\
0 \\
0 \\
50.00 \\
27.78\end{array}$ & $\begin{array}{c}\frac{28}{0} \\
0 \\
13.21 \\
0 \\
0\end{array}$ & & & & & & & $\begin{array}{c}\frac{56}{0.24} \\
\frac{13.16}{\underline{0}} \\
\underline{0}\end{array}$ & $\begin{array}{l}\frac{57}{0.94} \\
\underline{0} \\
\underline{7.50} \\
\underline{0} \\
\underline{0}\end{array}$ & $\begin{array}{l}\frac{58}{0} \\
0 \\
0.05 \\
1.06 \\
0\end{array}$ & $\begin{array}{c}\frac{61}{\frac{0.07}{\underline{0}}} \\
\frac{13.08}{\underline{0}}\end{array}$ & $\begin{array}{c}\frac{62}{0.02} \\
0 \\
0 \\
0.05 \\
7.50\end{array}$ & $\begin{array}{l}\frac{63}{0.23} \\
\frac{0}{0} \\
\underline{0} \\
\underline{0}\end{array}$ & $\begin{array}{c}\frac{64}{0.02} \\
0 \\
0 \\
2.86 \\
0\end{array}$ & $\frac{\frac{1}{0.50}}{\frac{0.60}{\underline{0}}}$ & & \\
\hline $\begin{array}{l}\text { Sargasso } \\
\text { Sea } \\
\& \\
\text { Gulf } \\
\text { Stream }\end{array}$ & & & & & & & & & & $\frac{\frac{49}{0.56}}{\frac{0}{0}}$ & $\begin{array}{c}\frac{50}{0.05} \\
0 \\
0.28 \\
0\end{array}$ & $\begin{array}{c}\frac{51}{0.03} \\
0 \\
0.15 \\
0\end{array}$ & $\begin{array}{l}\frac{52}{0.91} \\
\underline{0} \\
\frac{0.04}{\underline{0}}\end{array}$ & & & & & & & & & & & \\
\hline $\begin{array}{l}\text { Slope } \\
\text { Water }\end{array}$ & $\frac{8}{-}$ & $\frac{9}{18.75}$ & $\frac{18}{11.94}$ & & $\frac{19}{1.26}$ & $\frac{20}{5.98}$ & $\frac{33}{0.64}$ & $\frac{34}{2.96}$ & & $\frac{35}{0}$ & $\frac{47}{7.14}$ & $\frac{48}{2.51}$ & $\frac{53}{2.69}$ & $\frac{54}{2.10}$ & $\frac{59}{1.33}$ & $\frac{60}{0.42}$ & $\frac{65}{1.30}$ & $\frac{66}{4.40}$ & & & & $\frac{4}{0}$ & $\frac{5}{4.17}$ & $\frac{6}{0}$ \\
\hline $\begin{array}{l}\text { High } \\
\text { Velocity } \\
\text { Region }\end{array}$ & $\frac{12}{3.86}$ & $\frac{13}{13.82}$ & $\frac{14}{3.70}$ & $\frac{15}{4.12}$ & $\frac{27}{0.01}$ & $\frac{29}{3.31}$ & $\frac{30}{3.51}$ & $\frac{31}{4.63}$ & $\frac{32}{-}$ & $\frac{41}{0.26}$ & $\frac{42}{1.52}$ & $\frac{43}{2.61}$ & $\frac{44}{1.16}$ & & $\frac{55}{14.47}$ & & & & & & & $\frac{2}{3.59}$ & $\frac{3}{0.27}$ & \\
\hline $\begin{array}{l}\text { Ring } \\
\text { Core }\end{array}$ & $\frac{10}{1.50}$ & $\frac{11}{0}$ & $\frac{16}{-}$ & $\frac{17}{0.03}$ & $\frac{21}{2.45}$ & $\frac{23}{0.20}$ & $\frac{24}{15.56}$ & $\frac{28}{3.30}$ & & $\frac{37}{2.31}$ & $\frac{38}{0.98}$ & $\frac{39}{2.35}$ & & & $\frac{56}{2.68}$ & $\frac{57}{1.69}$ & $\frac{58}{0.22}$ & $\frac{61}{2.63}$ & $\frac{62}{1.52}$ & $\frac{63}{0.09}$ & $\frac{64}{0.58}$ & $\frac{1}{3.28}$ & & \\
\hline SS \& GS & & & & & & & & & & $\frac{49}{0.14}$ & $\frac{50}{0.08}$ & $\frac{51}{0.05}$ & $\frac{52}{0.24}$ & & & & & & & & & & & \\
\hline
\end{tabular}


Table 75. Abundance of Hygophum benoiti (specimens $/ 10,000 \mathrm{~m}^{3}$ ).

\begin{tabular}{|c|c|c|c|c|c|c|c|c|c|c|c|c|c|c|c|c|c|c|c|c|c|c|c|c|}
\hline & \multicolumn{4}{|c|}{$\begin{array}{c}\text { Oceanus } 118 \\
\text { Apri1 }\end{array}$} & \multicolumn{5}{|c|}{$\begin{array}{c}\text { Oceanus } 121 \\
\text { June }\end{array}$} & \multicolumn{5}{|c|}{$\begin{array}{l}\text { Oceanus } 125 \\
\text { August }\end{array}$} & \multicolumn{7}{|c|}{$\begin{array}{l}\text { Knorr } 98 \\
\text { Sept-Oct }\end{array}$} & \multicolumn{3}{|c|}{$\begin{array}{c}\text { A2 } 110 \\
\text { Sept-0ct }\end{array}$} \\
\hline $\begin{array}{l}\text { Slope } \\
\text { Water }\end{array}$ & $\begin{array}{l}\frac{8}{\overline{0}} \\
\underline{0} \\
\underline{0} \\
\underline{0}\end{array}$ & $\begin{array}{l}9 \\
0 \\
0 \\
0 \\
0\end{array}$ & & & $\begin{array}{r}19 \\
\underline{0} \\
\underline{0} \\
\underline{0} \\
\underline{0}\end{array}$ & $\begin{array}{r}20 \\
0 \\
0 \\
0 \\
0 \\
0\end{array}$ & $\begin{array}{l}33 \\
\underline{0} \\
\underline{0} \\
\underline{0} \\
\underline{0} \\
\underline{0}\end{array}$ & $\frac{34}{\underline{0}}$ & & $\begin{array}{c}35 \\
0 \\
0 \\
2.7 \\
0.6 \\
0\end{array}$ & $\begin{array}{r}47 \\
\frac{0}{0} \\
\underline{0} \\
\underline{0}\end{array}$ & $\begin{array}{c}48 \\
0 \\
0 \\
0.3 \\
0\end{array}$ & $\begin{array}{c}\frac{53}{\underline{0}} \\
\frac{1.8}{\underline{0}}\end{array}$ & $\begin{array}{c}54 \\
0 \\
0 \\
3.9 \\
0\end{array}$ & $\begin{array}{l}\frac{59}{4.7} \\
\frac{0}{0} \\
\frac{0}{0} \\
\underline{0}\end{array}$ & $\begin{array}{c}60 \\
0 \\
0.5 \\
14.5 \\
0 \\
0\end{array}$ & $\begin{array}{l}\frac{65}{\underline{0}} \\
\underline{0} \\
\frac{0.9}{4.0} \\
\underline{0}\end{array}$ & $\begin{array}{c}66 \\
0 \\
0 \\
0.4 \\
4.3 \\
0\end{array}$ & & & & $\begin{array}{c}\frac{4}{0} \\
0 \\
10.3 \\
0\end{array}$ & $\begin{array}{l}\frac{5}{0.3} \\
\underline{0} \\
\frac{0.6}{\underline{0}}\end{array}$ & \\
\hline $\begin{array}{l}\text { High } \\
\text { Velocity } \\
\text { Region }\end{array}$ & $\begin{array}{r}12 \\
0 \\
0 \\
0 \\
0\end{array}$ & $\frac{13}{\underline{0}}$ & $\frac{\frac{14}{0.3}}{\frac{0}{0}}$ & $\begin{array}{r}15 \\
0 \\
0 \\
0 \\
0\end{array}$ & $\begin{array}{r}26 \\
0 \\
0 \\
0 \\
0 \\
-\end{array}$ & $\begin{array}{r}27 \\
0 \\
0 \\
0 \\
0 \\
0\end{array}$ & $\begin{array}{c}30 \\
0 \\
0 \\
0.9 \\
0 \\
0\end{array}$ & $\begin{array}{c}31 \\
0 \\
0 \\
0.4 \\
0.3 \\
0\end{array}$ & $\begin{array}{l}\frac{32}{\underline{0.3}} \\
\frac{0.5}{\underline{0}}\end{array}$ & $\begin{array}{c}\frac{41}{\underline{0}} \\
\frac{3.4}{\underline{0}}\end{array}$ & $\begin{array}{l}\frac{42}{\underline{0}} \\
\frac{2.9}{0.7} \\
\frac{0}{\underline{0}}\end{array}$ & $\begin{array}{c}43 \\
0 \\
0 \\
1.3 \\
0.4 \\
0\end{array}$ & $\frac{\frac{44}{0.3}}{\frac{1.7}{\underline{0}}}$ & & $\begin{array}{c}\frac{55}{\underline{0}} \\
\underline{0} \\
\frac{2.0}{\underline{0}}\end{array}$ & & & & & & & $\begin{array}{l}\frac{2}{\underline{0}} \\
\underline{0} \\
\frac{3.3}{\underline{0}}\end{array}$ & $\begin{array}{r}\frac{3}{0} \\
0.5 \\
10.4 \\
0.4\end{array}$ & \\
\hline $\begin{array}{l}\text { Ring } \\
\text { Core }\end{array}$ & $\begin{array}{r}10 \\
\underline{0} \\
\underline{0} \\
\underline{0} \\
\underline{0}\end{array}$ & $\begin{array}{r}11 \\
0 \\
0 \\
0 \\
0\end{array}$ & $\begin{array}{r}16 \\
0 \\
0 \\
0\end{array}$ & $\frac{17}{\underline{0}}$ & $\begin{array}{c}\frac{21}{\underline{0}} \\
\underline{0} \\
\underline{0} \\
\underline{1.7}\end{array}$ & $\begin{array}{l}\frac{23}{\underline{0}} \\
\underline{0} \\
\underline{0} \\
\underline{0.5} \\
\underline{0}\end{array}$ & $\begin{array}{c}24 \\
0 \\
0 \\
0.3 \\
0 \\
0\end{array}$ & $\begin{array}{c}28 \\
0 \\
0 \\
2.5 \\
0 \\
0\end{array}$ & & & & & & & $\frac{\frac{56}{3.2}}{\frac{\frac{3.1}{1.1}}{\frac{0}{0}}}$ & $\begin{array}{l}\frac{57}{8.4} \\
\underline{\underline{0.6}} \\
\frac{0.8}{\underline{0}} \\
\underline{0}\end{array}$ & $\begin{array}{c}\frac{58}{0.3} \\
0 \\
0 \\
7.1 \\
0.3\end{array}$ & 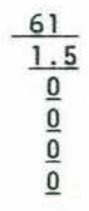 & $\begin{array}{c}62 \\
0 \\
0 \\
0 \\
1.9 \\
0.7\end{array}$ & $\begin{array}{l}\frac{63}{\underline{0}} \\
\underline{0} \\
\frac{0.5}{1.2} \\
\underline{0}\end{array}$ & $\begin{array}{c}64 \\
0 \\
0 \\
0 \\
0 \\
3.8\end{array}$ & $\begin{array}{c}\frac{1}{\underline{0}} \\
\underline{0} \\
1 \frac{1.9}{0.0} \\
\end{array}$ & & \\
\hline $\begin{array}{l}\text { Sargasso } \\
\text { Sea } \\
\& \\
\text { Gulf } \\
\text { Stream }\end{array}$ & & & & & & & & & & $\begin{array}{c}\frac{49}{0} \\
\frac{0}{0} \\
\underline{0} \\
\underline{0.5}\end{array}$ & $\begin{array}{c}50 \\
0 \\
0 \\
0 \\
1.3\end{array}$ & $\begin{array}{c}51 \\
0 \\
0 \\
8.7 \\
3.1\end{array}$ & $\begin{array}{c}\frac{52}{\frac{0}{0}} \\
\underline{12.2} \\
\underline{2.1}\end{array}$ & & & & & & & & & & & \\
\hline $\begin{array}{l}\text { Slope } \\
\text { Water }\end{array}$ & $\frac{8}{-}$ & $\frac{9}{0}$ & $\frac{18}{0}$ & & $\frac{19}{0}$ & $\frac{20}{0}$ & $\frac{33}{0}$ & $\frac{34}{0}$ & & $\frac{35}{0.7}$ & $\frac{47}{0}$ & $\frac{48}{0.1}$ & $\frac{53}{0.5}$ & $\frac{54}{1.0}$ & $\frac{59}{0.9}$ & $\frac{60}{2.9}$ & $\frac{65}{1.0}$ & $\frac{66}{0.9}$ & & & & $\frac{4}{2.6}$ & $\frac{5}{0.2}$ & $\frac{6}{0.7}$ \\
\hline $\begin{array}{l}\text { High } \\
\text { Velocity } \\
\text { Region }\end{array}$ & $\frac{12}{0}$ & $\frac{13}{0}$ & $\frac{14}{0.1}$ & $\frac{15}{0}$ & $\frac{27}{0}$ & $\frac{29}{0.3}$ & $\frac{30}{0.2}$ & $\frac{31}{0.1}$ & $\frac{32}{-}$ & $\frac{41}{0.9}$ & $\frac{42}{0.8}$ & $\frac{43}{0.3}$ & $\frac{44}{0.2}$ & & $\frac{55}{0.8}$ & & & & & & & $\frac{2}{0.8}$ & $\frac{3}{2.8}$ & \\
\hline $\begin{array}{l}\text { Ring } \\
\text { Core }\end{array}$ & $\frac{10}{0}$ & $\frac{11}{0}$ & $\frac{16}{-}$ & $\frac{17}{0}$ & $\frac{21}{0.3}$ & $\frac{23}{0.1}$ & $\frac{24}{0.1}$ & $\frac{28}{0.6}$ & & $\frac{37}{0.5}$ & $\frac{38}{0.5}$ & $\frac{39}{0.5}$ & & & $\frac{56}{0.9}$ & $\frac{57}{2.0}$ & $\frac{58}{1.6}$ & $\frac{61}{0.3}$ & $\frac{62}{0.5}$ & $\frac{63}{0.3}$ & $\frac{64}{0.8}$ & $\frac{1}{3.0}$ & & \\
\hline SS \& GS & & & & & & & & & & $\frac{49}{0.1}$ & $\frac{50}{0.3}$ & $\frac{51}{3.0}$ & $\frac{52}{3.6}$ & & & & & & & & & & & \\
\hline
\end{tabular}


Table 76. Abundance of Hygophum hygomii (specimens $/ 10,000 \mathrm{~m}^{3}$ ).

\begin{tabular}{|c|c|c|c|c|c|c|c|c|c|c|c|c|c|c|c|c|c|c|c|c|c|c|c|c|}
\hline & & $\begin{array}{r}\text { Apr } \\
\text { Apanu }\end{array}$ & $\begin{array}{l}5118 \\
i 1\end{array}$ & & & & $\begin{array}{l}\text { eanus } \\
\text { June }\end{array}$ & 121 & & & $\begin{array}{r}\text { Oce } \\
A\end{array}$ & $\begin{array}{l}\text { anus } \\
\text { ugust }\end{array}$ & 25 & & & & $\begin{array}{l}\text { Knor } \\
\text { Sept }\end{array}$ & $\begin{array}{r}98 \\
-0 c t \\
\end{array}$ & & & & $S_{S}$ & $\begin{array}{c}110 \\
t-0 c t\end{array}$ & \\
\hline $\begin{array}{l}\text { Slope } \\
\text { Water }\end{array}$ & $\begin{array}{l}\frac{8}{\overline{\overline{0}}} \\
\underline{0} \\
\underline{0}\end{array}$ & $\begin{array}{c}\frac{9}{0} \\
1.2 \\
0.7 \\
0\end{array}$ & & & $\frac{\frac{19}{0.7}}{\frac{0}{0}} \frac{\underline{0}}{\frac{0}{0}}$ & $\begin{array}{c}20 \\
0 \\
0.5 \\
0 \\
0 \\
0\end{array}$ & $\begin{array}{l}\frac{33}{\underline{0}} \\
\underline{0} \\
\underline{0} \\
\underline{0} \\
\underline{0}\end{array}$ & $\frac{34}{\underline{0}} \frac{0.4}{\underline{0}}$ & & $\begin{array}{c}35 \\
0 \\
0 \\
0.3 \\
0 \\
0\end{array}$ & $\begin{array}{l}\frac{47}{0.2} \\
\underline{0} \\
\underline{0} \\
0.2\end{array}$ & $\begin{array}{c}48 \\
0 \\
0.9 \\
0 \\
0\end{array}$ & $\begin{array}{l}\frac{53}{0.4} \\
\frac{0}{0} \\
\underline{0} \\
\underline{0}\end{array}$ & $\begin{array}{c}54 \\
0 \\
0.3 \\
0.6 \\
0\end{array}$ & $\begin{array}{r}59 \\
\underline{0} \\
\underline{0} \\
\underline{0} \\
\underline{0} \\
\underline{0}\end{array}$ & $\begin{array}{r}60 \\
0 \\
0 \\
0 \\
0 \\
0\end{array}$ & $\begin{array}{r}\frac{65}{0} \\
\underline{0} \\
\underline{0} \\
\underline{0} \\
\underline{0} \\
\underline{0}\end{array}$ & $\begin{array}{c}66 \\
0 \\
0 \\
0.4 \\
0 \\
0\end{array}$ & & & & $\begin{array}{c}\frac{4}{0} \\
0.4 \\
0.3 \\
0\end{array}$ & $\begin{array}{l}\frac{5}{0} \\
\frac{0}{0} \\
\underline{0} \\
\underline{0}\end{array}$ & \\
\hline $\begin{array}{l}\text { High } \\
\text { Velocity } \\
\text { Region }\end{array}$ & $\begin{array}{c}12 \\
0 \\
2.3 \\
1.7 \\
0\end{array}$ & $\frac{\frac{13}{1.5}}{\frac{1.1}{0.2}}$ & $\frac{\frac{14}{\frac{3.7}{1.0}}}{\frac{0.6}{0}}$ & $\begin{array}{c}15 \\
1.1 \\
2.9 \\
0 \\
0\end{array}$ & $\begin{array}{c}26 \\
0 \\
0 \\
0.5 \\
0.7 \\
-\end{array}$ & $\begin{array}{c}27 \\
0 \\
0.6 \\
1.8 \\
0 \\
0\end{array}$ & $\begin{array}{c}30 \\
0 \\
0 \\
1.6 \\
0 \\
0\end{array}$ & $\begin{array}{c}31 \\
0 \\
0 \\
1.4 \\
1.4 \\
0\end{array}$ & $\begin{array}{l}\frac{32}{\underline{0}} \\
\underline{0} \\
\underline{0} \\
\underline{0}\end{array}$ & $\begin{array}{r}\frac{41}{\underline{0}} \\
\underline{0} \\
\underline{0}\end{array}$ & $\frac{\frac{42}{0.6}}{\frac{0}{\underline{0}}} \frac{\underline{0}}{\underline{0}}$ & $\begin{array}{r}43 \\
0 \\
0 \\
0 \\
0 \\
0\end{array}$ & $\frac{\frac{44}{0.3}}{\frac{0}{0}}$ & & $\begin{array}{r}55 \\
\underline{0} \\
\underline{0} \\
\underline{0} \\
\underline{0}\end{array}$ & & & & & & & $\frac{\frac{2}{0.8}}{\frac{0}{0}}$ & $\begin{array}{l}3 \\
0 \\
0 \\
0 \\
0\end{array}$ & \\
\hline $\begin{array}{l}\text { Ring } \\
\text { Core }\end{array}$ & $\frac{\frac{10}{\frac{0.4}{5.3}}}{\frac{7.3}{\underline{0}}}$ & $\begin{array}{c}11 \\
0 \\
0.6 \\
2.5 \\
0\end{array}$ & $\begin{array}{c}16 \\
0 \\
0.7 \\
0\end{array}$ & $\frac{\frac{17}{5.0}}{\frac{0}{0}}$ & $\begin{array}{c}\frac{21}{24.0} \\
\frac{0}{0.6} \\
\underline{0} \\
\underline{0}\end{array}$ & 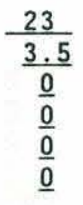 & $\begin{array}{c}24 \\
0 \\
0 \\
1.1 \\
0.6 \\
0\end{array}$ & $\begin{array}{c}28 \\
0 \\
1.2 \\
0 \\
0 \\
0\end{array}$ & & & & & & & $\begin{array}{l}\frac{56}{\underline{0}} \\
\underline{0} \\
\underline{0} \\
\underline{0} \\
\underline{0}\end{array}$ & $\begin{array}{c}57 \\
\underline{0} \\
\underline{0} \\
\underline{0} \\
\underline{0} \\
\underline{0}\end{array}$ & $\begin{array}{c}58 \\
0 \\
0 \\
0 \\
0.3 \\
0\end{array}$ & $\begin{array}{r}61 \\
\underline{0} \\
\underline{0} \\
\underline{0} \\
\underline{0} \\
\underline{0}\end{array}$ & $\begin{array}{r}62 \\
0 \\
0 \\
0 \\
0 \\
0\end{array}$ & $\begin{array}{r}63 \\
\underline{0} \\
\underline{0} \\
\underline{0} \\
\underline{0} \\
\underline{0}\end{array}$ & $\begin{array}{r}64 \\
0 \\
0 \\
0 \\
0 \\
0\end{array}$ & $\begin{array}{l}\frac{1}{\underline{0}} \\
\underline{0} \\
\underline{0} \\
\underline{0}\end{array}$ & & \\
\hline $\begin{array}{l}\text { Sargasso } \\
\text { Sea } \\
\& \\
\text { Gulf } \\
\text { Stream }\end{array}$ & & & & & & & & & & $\frac{49}{\frac{0.2}{\underline{0}}}$ & $\begin{array}{r}50 \\
0 \\
0 \\
0 \\
0\end{array}$ & $\begin{array}{r}51 \\
0 \\
0 \\
0 \\
0\end{array}$ & $\begin{array}{l}52 \\
\underline{0} \\
\underline{0} \\
\underline{0} \\
\underline{0}\end{array}$ & & & & & & & & & & & \\
\hline $\begin{array}{l}\text { Slope } \\
\text { Water }\end{array}$ & $\frac{8}{-}$ & $\frac{9}{0.5}$ & $\frac{18}{0}$ & & $\frac{19}{0.1}$ & $\frac{20}{0.1}$ & $\frac{33}{0}$ & $\frac{34}{0.1}$ & & $\frac{35}{0.1}$ & $\frac{47}{0.1}$ & $\frac{48}{0.2}$ & $\frac{53}{0.1}$ & $\frac{54}{0.2}$ & $\frac{59}{0}$ & $\frac{60}{0}$ & $\frac{65}{0}$ & $\frac{66}{0.1}$ & & & & $\frac{4}{0.2}$ & $\frac{5}{0}$ & $\frac{6}{0}$ \\
\hline $\begin{array}{l}\text { High } \\
\text { Velocity } \\
\text { Region }\end{array}$ & $\frac{12}{1.0}$ & $\frac{13}{0.7}$ & $\frac{14}{1.3}$ & $\frac{15}{1.0}$ & $\frac{27}{0.5}$ & $\frac{29}{0.4}$ & $\frac{30}{0.3}$ & $\frac{31}{0.6}$ & $\frac{32}{-}$ & $\frac{41}{0}$ & $\frac{42}{0.2}$ & $\frac{43}{0}$ & $\frac{44}{0.2}$ & & $\frac{55}{0}$ & & & & & & & $\frac{2}{0.2}$ & $\frac{3}{0}$ & \\
\hline $\begin{array}{l}\text { Ring } \\
\text { Core }\end{array}$ & $\frac{10}{3.3}$ & $\frac{11}{0.6}$ & $\frac{16}{-}$ & $\frac{17}{1.3}$ & $\frac{21}{1.4}$ & $\frac{23}{0.5}$ & $\frac{24}{0.3}$ & $\frac{28}{0.2}$ & & $\frac{37}{0}$ & $\frac{38}{0}$ & $\frac{39}{0.2}$ & & & $\frac{56}{0}$ & $\begin{array}{r}57 \\
0\end{array}$ & $\frac{58}{0.1}$ & $\frac{61}{0}$ & $\frac{62}{0}$ & $\frac{63}{0}$ & $\frac{64}{0}$ & $\frac{1}{0}$ & & \\
\hline SS \& GS & & & & & & & & & & $\frac{49}{0.1}$ & $\frac{50}{0}$ & $\frac{51}{0}$ & $\frac{52}{0}$ & & & & & & & & & & & \\
\hline
\end{tabular}


Table 77. Volume of Hygophum hygomii $\left(\mathrm{ml} / 10,000 \mathrm{~m}^{3}\right)$.

\begin{tabular}{|c|c|c|c|c|c|c|c|c|c|c|c|c|c|c|c|c|c|c|c|c|c|c|c|c|}
\hline & \multicolumn{4}{|c|}{$\begin{array}{c}\text { Oceanus } \\
\text { Apri1 }\end{array}$} & \multicolumn{5}{|c|}{$\begin{array}{c}\text { Oceanus } 121 \\
\text { June }\end{array}$} & \multicolumn{5}{|c|}{$\begin{array}{l}\text { Oceanus } 125 \\
\text { August }\end{array}$} & \multicolumn{7}{|c|}{$\begin{array}{l}\text { Knorr } 98 \\
\text { Sept-0ct }\end{array}$} & \multicolumn{3}{|c|}{$\begin{array}{c}\text { A2 } 110 \\
\text { Sept-0ct }\end{array}$} \\
\hline $\begin{array}{l}\text { Slope } \\
\text { Water }\end{array}$ & $\begin{array}{l}\frac{8}{\overline{0}} \\
\underline{0} \\
\underline{0} \\
\underline{0}\end{array}$ & $\begin{array}{l}\frac{9}{0} \\
0.06 \\
0.07 \\
0\end{array}$ & & & $\frac{\frac{19}{0.02}}{\frac{0}{0}}$ & $\begin{array}{l}\frac{20}{0} \\
0.37 \\
0 \\
0 \\
0\end{array}$ & $\begin{array}{l}\frac{33}{0} \\
\underline{0} \\
\underline{0} \\
\underline{0} \\
\underline{0}\end{array}$ & $\frac{\frac{34}{0.05}}{\frac{0.04}{\underline{0}}}$ & & $\begin{array}{l}\frac{35}{0} \\
0 \\
0.10 \\
0 \\
0\end{array}$ & $\begin{array}{l}\frac{47}{0.30} \\
\frac{0}{0} \\
\underline{0.11}\end{array}$ & $\begin{array}{l}\frac{48}{0} \\
1.43 \\
0 \\
0\end{array}$ & $\frac{\frac{53}{0.73}}{\frac{0}{0}}$ & $\begin{array}{c}\frac{54}{0} \\
0.22 \\
0.36 \\
0\end{array}$ & $\begin{array}{l}\frac{59}{\underline{0}} \\
\underline{0} \\
\underline{0} \\
\underline{0} \\
\underline{0}\end{array}$ & $\begin{array}{l}60 \\
0 \\
0 \\
0 \\
0 \\
0\end{array}$ & $\begin{array}{l}\frac{65}{\underline{0}} \\
\underline{0} \\
\underline{0} \\
\underline{0} \\
\underline{0}\end{array}$ & $\begin{array}{l}\frac{66}{0} \\
0 \\
0.56 \\
0 \\
0\end{array}$ & & & & $\begin{array}{c}\frac{4}{0} \\
0.80 \\
0.53 \\
0\end{array}$ & $\begin{array}{l}\frac{5}{0} \\
\underline{0} \\
\underline{0} \\
\underline{0} \\
\underline{0}\end{array}$ & \\
\hline $\begin{array}{l}\text { High } \\
\text { Velocity } \\
\text { Region }\end{array}$ & $\begin{array}{c}\frac{12}{0} \\
0.77 \\
0.29 \\
0\end{array}$ & $\frac{\frac{13}{0.40}}{\frac{0.06}{0.02}}$ & $\frac{\frac{14}{0.86}}{\frac{0.03}{0.06}} \frac{0}{\underline{0}}$ & $\begin{array}{c}\frac{15}{0.06} \\
0.76 \\
0 \\
0\end{array}$ & $\begin{array}{l}26 \\
0 \\
0 \\
0.81 \\
0.62 \\
-\end{array}$ & $\begin{array}{c}\frac{27}{0} \\
0.29 \\
1.45 \\
0 \\
0\end{array}$ & $\begin{array}{l}30 \\
0 \\
0 \\
0.34 \\
0 \\
0\end{array}$ & $\begin{array}{l}31 \\
0 \\
0 \\
1.61 \\
1.29 \\
0\end{array}$ & $\begin{array}{l}32 \\
\underline{0} \\
\underline{0} \\
\underline{0} \\
\underline{0}\end{array}$ & $\begin{array}{l}\frac{41}{\underline{0}} \\
\underline{0} \\
\underline{0}\end{array}$ & $\begin{array}{c}\frac{42}{1.31} \\
\frac{0}{0} \\
\underline{0}\end{array}$ & $\begin{array}{l}43 \\
0 \\
0 \\
0 \\
0 \\
0\end{array}$ & $\begin{array}{l}\frac{44}{0.60} \\
\underline{0} \\
\underline{0}\end{array}$ & & $\begin{array}{l}\frac{55}{\underline{0}} \\
\underline{0} \\
\underline{0} \\
\underline{0}\end{array}$ & & & & & & & $\frac{\frac{2}{1.77}}{\frac{0}{0}}$ & $\begin{array}{l}3 \\
0 \\
0 \\
0 \\
0\end{array}$ & \\
\hline $\begin{array}{l}\text { Ring } \\
\text { Core }\end{array}$ & $\frac{\frac{10}{0.04}}{\frac{0.12}{0.09}}$ & $\begin{array}{l}\frac{11}{0} \\
0.06 \\
0.06 \\
0\end{array}$ & $\begin{array}{c}\frac{16}{0} \\
0.07 \\
0\end{array}$ & $\frac{\frac{17}{0.22}}{\underline{0}}$ & $\begin{array}{l}\frac{21}{8.50} \\
\underline{0} \\
\frac{0.03}{\underline{0}} \\
\underline{0}\end{array}$ & $\begin{array}{l}\frac{23}{5.88} \\
\frac{0}{0} \\
\frac{0}{0} \\
\underline{0}\end{array}$ & $\begin{array}{l}\frac{24}{0} \\
0 \\
0.81 \\
0.06 \\
0\end{array}$ & $\begin{array}{l}\frac{28}{0} \\
0.96 \\
0 \\
0 \\
0\end{array}$ & & & & & & & $\begin{array}{l}\frac{56}{0} \\
\underline{0} \\
\underline{0} \\
\underline{0} \\
\underline{0}\end{array}$ & $\begin{array}{l}57 \\
\underline{0} \\
\underline{0} \\
\underline{0} \\
\underline{0} \\
\underline{0}\end{array}$ & $\begin{array}{c}58 \\
0 \\
0 \\
0 \\
0.87 \\
0\end{array}$ & $\begin{array}{l}\frac{61}{0} \\
\underline{0} \\
\underline{0} \\
\underline{0} \\
\underline{0}\end{array}$ & $\begin{array}{l}62 \\
0 \\
0 \\
0 \\
0 \\
0\end{array}$ & $\begin{array}{l}\frac{63}{\underline{0}} \\
\underline{0} \\
\underline{0} \\
\underline{0} \\
\underline{0}\end{array}$ & $\begin{array}{l}64 \\
0 \\
0 \\
0 \\
0 \\
0\end{array}$ & $\frac{\frac{1}{0.38}}{\frac{0}{0}}$ & & \\
\hline $\begin{array}{l}\text { Sargasso } \\
\text { Sea } \\
\& \\
\text { Gulf } \\
\text { Stream }\end{array}$ & & & & & & & & & & $\frac{\frac{49}{0.31}}{\frac{0}{0}}$ & $\begin{array}{l}50 \\
0 \\
0 \\
0 \\
0\end{array}$ & $\begin{array}{l}\frac{51}{0} \\
0 \\
0 \\
0\end{array}$ & $\begin{array}{l}\frac{52}{\underline{0}} \\
\frac{0}{0} \\
\underline{0}\end{array}$ & & & & & & & & & & & \\
\hline $\begin{array}{l}\text { Slope } \\
\text { Water }\end{array}$ & $\frac{8}{-}$ & $\frac{9}{0.03}$ & $\frac{18}{0}$ & & $\frac{19}{0.01}$ & $\frac{20}{0.09}$ & $\frac{33}{0}$ & $\frac{34}{0.02}$ & & $\frac{35}{0.02}$ & $\frac{47}{0.10}$ & $\frac{48}{0.36}$ & $\frac{53}{0.18}$ & $\frac{54}{0.15}$ & $\frac{59}{0}$ & $\frac{60}{0}$ & $\frac{65}{0}$ & $\frac{66}{0.11}$ & & & & $\frac{4}{0.33}$ & $\frac{5}{0}$ & $\frac{6}{0}$ \\
\hline $\begin{array}{l}\text { High } \\
\text { Ve1ocity } \\
\text { Region }\end{array}$ & $\frac{12}{0.27}$ & $\frac{13}{0.13}$ & $\frac{14}{0.24}$ & $\frac{15}{0.21}$ & $\frac{27}{0.33}$ & $\frac{29}{0.15}$ & $\frac{30}{0.07}$ & $\frac{31}{0.58}$ & $\frac{32}{-}$ & $\frac{41}{0}$ & $\frac{42}{0.50}$ & $\frac{43}{0}$ & $\frac{44}{0.36}$ & & $\frac{55}{0}$ & & & & & & & $\frac{2}{0.44}$ & $\frac{3}{0}$ & \\
\hline $\begin{array}{l}\text { Ring } \\
\text { Core }\end{array}$ & $\frac{10}{0.06}$ & $\frac{11}{0.03}$ & $\frac{16}{-}$ & $\frac{17}{0.06}$ & $\frac{21}{0.43}$ & $\frac{23}{0.88}$ & $\frac{24}{0.17}$ & $\frac{28}{0.17}$ & & $\frac{37}{0}$ & $\frac{38}{0}$ & $\frac{39}{0.41}$ & & & $\frac{56}{0}$ & $\frac{57}{0}$ & $\frac{58}{0.17}$ & $\frac{61}{0}$ & $\frac{62}{0}$ & $\frac{63}{0}$ & $\frac{64}{0}$ & $\frac{1}{0.10}$ & & \\
\hline SS \& GS & & & & & & & & & & $\frac{49}{0.08}$ & $\frac{50}{0}$ & $\frac{51}{0}$ & $\frac{52}{0}$ & & & & & & & & & & & \\
\hline
\end{tabular}


Table 78 Abundance of Hygophum taaningi (specimens $/ 10,000 \mathrm{~m}^{3}$ ).

\begin{tabular}{|c|c|c|c|c|c|c|c|c|c|c|c|c|c|c|c|c|c|c|c|c|c|c|c|c|}
\hline & & $\begin{array}{r}\text { Ocean } \\
\text { Ap }\end{array}$ & 1118 & & & & $\begin{array}{l}\text { eanus } \\
\text { June }\end{array}$ & 121 & & & $\begin{array}{r}\text { Oce } \\
\mathrm{A}\end{array}$ & $\begin{array}{l}\text { nus } \\
\text { Igust }\end{array}$ & & & & & $\begin{array}{l}\text { Knor } \\
\text { Sept }\end{array}$ & $\begin{array}{r}r 98 \\
-0 c t \\
\end{array}$ & & & & $\begin{array}{r}\mathrm{A}^{2} \\
\mathrm{Se} \\
\end{array}$ & $\begin{array}{c}110 \\
t-0 c t\end{array}$ & \\
\hline $\begin{array}{l}\text { Slope } \\
\text { Water }\end{array}$ & $\begin{array}{l}\frac{8}{\overline{0}} \\
\underline{0} \\
\underline{0} \\
\underline{0}\end{array}$ & $\begin{array}{l}\frac{9}{0} \\
0 \\
0 \\
0\end{array}$ & & & $\begin{array}{l}\frac{19}{\frac{0.2}{0}} \\
\frac{0}{0} \\
\frac{0.5}{\underline{0}}\end{array}$ & $\begin{array}{r}20 \\
0 \\
0 \\
0 \\
0 \\
0\end{array}$ & $\begin{array}{l}33 \\
\underline{0} \\
\underline{0} \\
\underline{0} \\
\underline{0} \\
\underline{0}\end{array}$ & $\frac{34}{\frac{1,4}{\underline{0}}}$ & & $\begin{array}{c}35 \\
0 \\
0 \\
0 \\
0.6 \\
0\end{array}$ & $\begin{array}{l}\frac{47}{\underline{0}} \\
\underline{0} \\
\underline{0} \\
\underline{0}\end{array}$ & $\begin{array}{r}48 \\
0 \\
0 \\
0 \\
0\end{array}$ & $\begin{array}{c}\frac{53}{\underline{0}} \\
\underline{0} \\
\frac{0.8}{\underline{0}}\end{array}$ & $\begin{array}{r}54 \\
0 \\
0 \\
0 \\
0\end{array}$ & $\begin{array}{l}59 \\
\underline{0} \\
\underline{0} \\
\underline{0} \\
\underline{0} \\
\underline{0}\end{array}$ & $\begin{array}{c}60 \\
0 \\
0 \\
0.7 \\
0 \\
0\end{array}$ & $\begin{array}{c}\frac{65}{\underline{0}} \\
\underline{0} \\
\frac{1.4}{1.2} \\
\underline{0}\end{array}$ & $\begin{array}{c}66 \\
0 \\
0 \\
1.1 \\
0.5 \\
0\end{array}$ & & & & $\begin{array}{l}\frac{4}{0} \\
0 \\
0.9 \\
0\end{array}$ & $\frac{\frac{5}{0.3}}{\frac{0}{0}} \frac{\underline{0}}{\underline{0}}$ & \\
\hline $\begin{array}{l}\text { High } \\
\text { Velocity } \\
\text { Region }\end{array}$ & $\begin{array}{c}12 \\
0 \\
0 \\
0.4 \\
0\end{array}$ & $\frac{13}{\underline{0}}$ & $\frac{14}{\frac{0}{0}}$ & $\begin{array}{r}15 \\
0 \\
0 \\
0 \\
0\end{array}$ & $\begin{array}{c}26 \\
0 \\
0 \\
0 \\
0 \\
-\end{array}$ & $\begin{array}{r}27 \\
0 \\
0 \\
0 \\
0 \\
0\end{array}$ & $\begin{array}{c}30 \\
0 \\
0 \\
0 \\
0 \\
0\end{array}$ & $\begin{array}{c}31 \\
0 \\
0 \\
0 \\
0 \\
0\end{array}$ & $\begin{array}{r}\frac{32}{0} \\
\underline{0} \\
\underline{0} \\
\underline{0}\end{array}$ & $\frac{41}{\frac{0}{0}}$ & 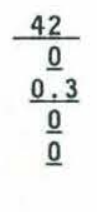 & $\begin{array}{r}43 \\
0 \\
0 \\
0 \\
0 \\
0\end{array}$ & $\begin{array}{r}44 \\
\underline{0} \\
\underline{0} \\
\underline{0}\end{array}$ & & $\frac{\frac{55}{0.5}}{\frac{0.5}{\underline{0}}}$ & & & & & & & $\begin{array}{l}\frac{2}{0} \\
\underline{0} \\
\frac{1.3}{\underline{0}}\end{array}$ & $\begin{array}{l}3 \\
0 \\
0 \\
0.7 \\
0\end{array}$ & \\
\hline $\begin{array}{l}\text { Ring } \\
\text { Core }\end{array}$ & $\begin{array}{l}10 \\
\frac{0}{0} \\
\underline{0.9} \\
\underline{0.6}\end{array}$ & $\begin{array}{r}11 \\
0 \\
0 \\
0 \\
0\end{array}$ & $\begin{array}{r}16 \\
0 \\
0 \\
0\end{array}$ & $\frac{17}{\underline{0}}$ & $\begin{array}{r}21 \\
\underline{0} \\
\underline{0} \\
\underline{0} \\
\underline{0} \\
\underline{0}\end{array}$ & $\begin{array}{l}\frac{23}{\underline{0}} \\
\underline{0} \\
\underline{0} \\
\underline{0} \\
\underline{0}\end{array}$ & $\begin{array}{r}24 \\
0 \\
0 \\
0 \\
0 \\
0\end{array}$ & $\begin{array}{r}28 \\
0 \\
0 \\
0 \\
0 \\
0\end{array}$ & & & & & & & $\begin{array}{l}\frac{56}{3.8} \\
\frac{\underline{1.1}}{\underline{0}} \\
\underline{0} \\
\underline{0.3}\end{array}$ & $\begin{array}{l}\frac{57}{1.9} \\
\underline{0} \\
\frac{0.3}{\underline{0}} \\
\underline{0}\end{array}$ & $\begin{array}{c}58 \\
0 \\
0 \\
0 \\
1.6 \\
0.5\end{array}$ & $\begin{array}{l}\frac{61}{0.5} \\
\frac{0}{0} \\
\underline{0} \\
\underline{0}\end{array}$ & $\begin{array}{c}62 \\
0 \\
0 \\
0 \\
0.3 \\
0\end{array}$ & $\begin{array}{c}\frac{63}{\underline{0.3}} \\
\underline{0} \\
\underline{0} \\
\underline{0} \\
\underline{0.3}\end{array}$ & $\begin{array}{r}64 \\
0 \\
0 \\
0 \\
0 \\
0\end{array}$ & $\begin{array}{l}\frac{1}{0} \\
\underline{0} \\
\underline{0.3} \\
1.7\end{array}$ & & \\
\hline $\begin{array}{l}\text { Sargasso } \\
\text { Sea } \\
\& \\
\text { Gu1f } \\
\text { Stream }\end{array}$ & & & & & & & & & & $\begin{array}{r}49 \\
\underline{0} \\
\underline{0} \\
\underline{0} \\
\underline{0}\end{array}$ & $\begin{array}{r}50 \\
0 \\
0 \\
0 \\
0\end{array}$ & $\begin{array}{c}51 \\
0 \\
0 \\
0.2 \\
0\end{array}$ & $\begin{array}{c}\frac{52}{\underline{0}} \\
\underline{0} \\
1.7 \\
\underline{0.8}\end{array}$ & & & & & & & & & & & \\
\hline $\begin{array}{l}\text { Slope } \\
\text { Water }\end{array}$ & $\frac{8}{-}$ & $\frac{9}{0}$ & $\frac{18}{0}$ & & $\frac{19}{0.2}$ & $\frac{20}{0}$ & $\frac{33}{0}$ & $\frac{34}{0.1}$ & & $\frac{35}{0.1}$ & $\frac{47}{0}$ & $\frac{48}{0}$ & $\frac{53}{0.2}$ & $\frac{54}{0}$ & $\frac{59}{0}$ & $\frac{60}{0.1}$ & $\frac{65}{0.5}$ & $\frac{66}{0.3}$ & & & & $\frac{4}{0.2}$ & $\frac{5}{0.1}$ & $\frac{6}{0.1}$ \\
\hline $\begin{array}{l}\text { High } \\
\text { Velocity } \\
\text { Region }\end{array}$ & $\frac{12}{0.1}$ & $\frac{13}{0}$ & $\frac{14}{0}$ & $\frac{15}{0}$ & $\frac{27}{0}$ & $\frac{29}{0}$ & $\frac{30}{0}$ & $\frac{31}{0}$ & $\frac{32}{-}$ & $\frac{41}{0}$ & $\frac{42}{0.1}$ & $\frac{43}{0}$ & $\frac{44}{0}$ & & $\frac{55}{0.2}$ & & & & & & & $\frac{2}{0.3}$ & $\frac{3}{0.2}$ & \\
\hline $\begin{array}{l}\text { Ring } \\
\text { Core }\end{array}$ & $\frac{10}{0.4}$ & $\frac{11}{0}$ & $\frac{16}{-}$ & $\frac{17}{0}$ & $\frac{21}{0}$ & $\frac{23}{0}$ & $\frac{24}{0}$ & $\frac{28}{0}$ & & $\frac{37}{0}$ & $\frac{38}{0}$ & $\frac{39}{0}$ & & & $\frac{56}{1.1}$ & $\frac{57}{0.4}$ & $\frac{58}{0.4}$ & $\frac{61}{0.1}$ & $\frac{62}{0.1}$ & $\frac{63}{0.2}$ & $\frac{64}{0}$ & $\frac{1}{0.5}$ & & \\
\hline SS \& GS & & & & & & & & & & $\frac{49}{0}$ & $\frac{50}{0}$ & $\frac{51}{0.1}$ & $\frac{52}{0.6}$ & & & & & & & & & & & \\
\hline
\end{tabular}


Table 79. Abundance of Ichthyococcus ovatus (specimens $/ 10,000 \mathrm{~m}^{3}$ ).

\begin{tabular}{|c|c|c|c|c|c|c|c|c|c|c|c|c|c|c|c|c|c|c|c|c|c|c|c|c|}
\hline & \multicolumn{4}{|c|}{$\begin{array}{c}\text { Oceanus } 118 \\
\text { April }\end{array}$} & \multicolumn{5}{|c|}{$\begin{array}{c}\text { Oceanus } 121 \\
\text { June }\end{array}$} & \multicolumn{5}{|c|}{$\begin{array}{l}\text { Oceanus } 125 \\
\text { August }\end{array}$} & \multicolumn{7}{|c|}{$\begin{array}{l}\text { Knorr } 98 \\
\text { Sept-0ct }\end{array}$} & \multicolumn{3}{|c|}{$\begin{array}{c}\text { A2 } 110 \\
\text { Sept-Oct }\end{array}$} \\
\hline $\begin{array}{l}\text { Slope } \\
\text { Water }\end{array}$ & $\begin{array}{l}\frac{8}{\overline{0}} \\
\underline{0} \\
\underline{0} \\
\underline{0}\end{array}$ & $\begin{array}{l}9 \\
0 \\
0 \\
0 \\
0\end{array}$ & & & $\begin{array}{r}\frac{19}{\underline{0}} \\
\underline{0} \\
\underline{0} \\
\underline{0} \\
\underline{0}\end{array}$ & $\begin{array}{r}20 \\
0 \\
0 \\
0 \\
0 \\
0\end{array}$ & $\begin{array}{r}\frac{33}{\underline{0}} \\
\underline{0} \\
\underline{0} \\
\underline{0} \\
\underline{0}\end{array}$ & $\begin{array}{l}\frac{34}{0} \\
\underline{0} \\
\underline{0}\end{array}$ & & $\begin{array}{c}35 \\
0 \\
0.2 \\
0 \\
0 \\
0\end{array}$ & $\begin{array}{r}47 \\
\underline{0} \\
\underline{0} \\
\underline{0} \\
\underline{0}\end{array}$ & $\begin{array}{r}48 \\
0 \\
0 \\
0 \\
0\end{array}$ & $\begin{array}{r}53 \\
\underline{0} \\
\underline{0} \\
\underline{0} \\
\underline{0}\end{array}$ & $\begin{array}{r}54 \\
0 \\
0 \\
0 \\
0\end{array}$ & $\begin{array}{c}\frac{59}{\underline{0}} \\
\underline{2.4} \\
\underline{0} \\
\underline{0} \\
\underline{0}\end{array}$ & $\begin{array}{c}60 \\
0 \\
3.2 \\
0 \\
0 \\
0\end{array}$ & $\begin{array}{c}\frac{65}{\underline{0}} \\
\underline{0.3} \\
\underline{0} \\
\underline{0} \\
\underline{0}\end{array}$ & $\begin{array}{r}66 \\
0 \\
0 \\
0 \\
0 \\
0\end{array}$ & & & & $\begin{array}{l}4 \\
0 \\
0 \\
0 \\
0\end{array}$ & $\frac{5}{\underline{0}}$ & \\
\hline $\begin{array}{l}\text { High } \\
\text { Velocity } \\
\text { Region }\end{array}$ & $\begin{array}{r}12 \\
0 \\
0 \\
0 \\
0\end{array}$ & $\frac{13}{\frac{0}{0}}$ & $\begin{array}{r}\frac{14}{0} \\
\underline{0} \\
\underline{0} \\
\underline{0}\end{array}$ & $\begin{array}{r}15 \\
0 \\
0 \\
0 \\
0\end{array}$ & $\begin{array}{r}26 \\
0 \\
0 \\
0 \\
0 \\
-\end{array}$ & $\begin{array}{r}27 \\
0 \\
0 \\
0 \\
0 \\
0\end{array}$ & $\begin{array}{r}30 \\
0 \\
0 \\
0 \\
0 \\
0\end{array}$ & $\begin{array}{r}31 \\
0 \\
0 \\
0 \\
0 \\
0\end{array}$ & $\begin{array}{r}\frac{32}{0} \\
\underline{0} \\
\underline{0} \\
\underline{0}\end{array}$ & $\frac{41}{\underline{0}}$ & $\begin{array}{r}42 \\
\underline{0} \\
\underline{0} \\
\underline{0} \\
\underline{0}\end{array}$ & $\begin{array}{r}43 \\
0 \\
0 \\
0 \\
0 \\
0\end{array}$ & $\frac{44}{\frac{0.1}{\underline{0}}}$ & & $\begin{array}{c}\frac{55}{\underline{0}} \\
\underline{0} \\
\frac{1.2}{\underline{0}}\end{array}$ & & & & & & & $\begin{array}{l}\frac{2}{\underline{0}} \\
\frac{0.5}{\underline{0}} \\
\underline{0}\end{array}$ & $\begin{array}{c}\frac{3}{0} \\
0.5 \\
0 \\
0\end{array}$ & \\
\hline $\begin{array}{l}\text { Ring } \\
\text { Core }\end{array}$ & $\begin{array}{l}\frac{10}{\underline{0}} \\
\underline{0} \\
\underline{0} \\
\underline{0}\end{array}$ & $\begin{array}{l}11 \\
0 \\
0 \\
0 \\
0\end{array}$ & $\begin{array}{r}16 \\
0 \\
0 \\
0\end{array}$ & $\frac{17}{\frac{0}{0}}$ & $\begin{array}{c}21 \\
\underline{0} \\
\underline{0.2} \\
\underline{0} \\
\underline{0} \\
\underline{0}\end{array}$ & $\begin{array}{c}\frac{23}{\underline{0}} \\
\frac{1}{1.3} \\
\underline{0} \\
\underline{0} \\
\underline{0}\end{array}$ & $\begin{array}{r}24 \\
0 \\
0 \\
0 \\
0 \\
0\end{array}$ & $\begin{array}{c}28 \\
0.2 \\
0 \\
0 \\
0 \\
0\end{array}$ & & & & & & & $\begin{array}{l}\frac{56}{0.9} \\
\underline{0} \\
\frac{0.5}{0.5} \\
\frac{0}{\underline{0}}\end{array}$ & $\begin{array}{l}\frac{57}{0.9} \\
\frac{0.9}{2.5} \\
\frac{2.2}{\underline{0}}\end{array}$ & $\begin{array}{c}\frac{58}{0.3} \\
1.7 \\
0.5 \\
0.6 \\
0\end{array}$ & $\begin{array}{c}\frac{61}{\underline{0}} \\
\underline{0} \\
\frac{3.3}{0.4} \\
\underline{0}\end{array}$ & $\begin{array}{c}62 \\
0 \\
1.2 \\
2.9 \\
1.1 \\
0\end{array}$ & $\frac{\frac{63}{\underline{0}}}{\frac{1.5}{1.9}}$ & $\begin{array}{c}\frac{64}{0.2} \\
1.9 \\
0.7 \\
0 \\
0\end{array}$ & $\begin{array}{l}\frac{1}{\underline{0}} \\
\frac{1.6}{1.6} \\
\underline{0}\end{array}$ & & \\
\hline $\begin{array}{l}\text { Sargasso } \\
\text { Sea } \\
\& \\
\text { Gu1f } \\
\text { Stream }\end{array}$ & & & & & & & & & & $\begin{array}{l}\frac{49}{0} \\
\underline{0.4} \\
\underline{0.4} \\
\underline{0.3}\end{array}$ & $\begin{array}{c}50 \\
0 \\
0.5 \\
0 \\
0.6\end{array}$ & $\begin{array}{c}51 \\
0 \\
2.0 \\
1.1 \\
0\end{array}$ & $\frac{\frac{52}{\frac{0.2}{0.3}}}{\frac{0}{0}}$ & & & & & & & & & & & \\
\hline $\begin{array}{l}\text { Slope } \\
\text { Water }\end{array}$ & $\frac{8}{-}$ & $\frac{9}{0}$ & $\frac{18}{0}$ & & $\frac{19}{0}$ & $\frac{20}{0}$ & $\frac{33}{0}$ & $\frac{34}{0}$ & & $\frac{35}{0.1}$ & $\frac{47}{0}$ & $\frac{48}{0}$ & $\frac{53}{0}$ & $\frac{54}{0}$ & $\frac{59}{0.5}$ & $\frac{60}{0.3}$ & $\frac{65}{0.1}$ & $\frac{66}{0}$ & & & & $\frac{4}{0}$ & $\frac{5}{0.2}$ & $\frac{6}{0}$ \\
\hline $\begin{array}{l}\text { High } \\
\text { Velocity } \\
\text { Region }\end{array}$ & $\frac{12}{0}$ & $\frac{13}{0}$ & $\frac{14}{0}$ & $\frac{15}{0}$ & $\frac{27}{0}$ & $\frac{29}{0}$ & $\frac{30}{0}$ & $\frac{31}{0}$ & $\frac{32}{-}$ & $\frac{41}{0}$ & $\frac{42}{0}$ & $\frac{43}{0}$ & $\frac{44}{0.1}$ & & $\frac{55}{0.5}$ & & & & . & & & $\frac{2}{0.1}$ & $\frac{3}{0.1}$ & \\
\hline $\begin{array}{l}\text { Ring } \\
\text { Core }\end{array}$ & $\frac{10}{0}$ & $\frac{11}{0}$ & $\frac{16}{-}$ & $\frac{17}{0}$ & $\frac{21}{0.1}$ & $\frac{23}{0.3}$ & $\frac{24}{0}$ & $\frac{28}{0.1}$ & & $\frac{37}{0}$ & $\frac{38}{0}$ & $\frac{39}{0.2}$ & & & $\frac{56}{0.4}$ & $\frac{57}{1.3}$ & $\frac{58}{0.5}$ & $\frac{61}{0.3}$ & $\frac{62}{0.9}$ & $\frac{63}{0.4}$ & $\frac{64}{0.4}$ & $\frac{1}{0.8}$ & & \\
\hline SS \& GS & & & & & & & & & & $\frac{49}{0.3}$ & $\frac{50}{0.3}$ & $\frac{51}{0.8}$ & $\frac{52}{0.1}$ & & & & & & & & & & & \\
\hline
\end{tabular}


Table 80. Abundance of Lampadena speculigera (specimens $/ 10,000 \mathrm{~m}^{3}$ ).

\begin{tabular}{|c|c|c|c|c|c|c|c|c|c|c|c|c|c|c|c|c|c|c|c|c|c|c|c|c|}
\hline & \multicolumn{4}{|c|}{$\begin{array}{c}\text { Oceanus } 118 \\
\text { April }\end{array}$} & \multicolumn{5}{|c|}{$\begin{array}{c}\text { Oceanus } 121 \\
\text { June }\end{array}$} & \multicolumn{5}{|c|}{$\begin{array}{l}\text { Oceanus } 125 \\
\text { August }\end{array}$} & \multicolumn{7}{|c|}{$\begin{array}{l}\text { Knorr } 98 \\
\text { Sept-0ct }\end{array}$} & \multicolumn{3}{|c|}{$\begin{array}{c}\text { A2 } 110 \\
\text { Sept-0ct }\end{array}$} \\
\hline $\begin{array}{l}\text { Slope } \\
\text { Water }\end{array}$ & $\begin{array}{l}\frac{8}{\overline{\overline{0}}} \\
\underline{0} \\
\underline{0}\end{array}$ & $\begin{array}{l}9 \\
0 \\
0 \\
0 \\
0\end{array}$ & & & $\frac{\frac{19}{0.2}}{\frac{0}{\underline{0}}} \frac{\underline{0}}{\underline{0}}$ & $\begin{array}{r}20 \\
0 \\
0 \\
0 \\
0 \\
0\end{array}$ & $\begin{array}{l}\frac{33}{0.3} \\
\underline{0} \\
\underline{0} \\
\underline{0} \\
\underline{0}\end{array}$ & $\begin{array}{c}\frac{34}{\underline{0}} \\
\underline{0} \\
\underline{0.2}\end{array}$ & & $\begin{array}{r}35 \\
0 \\
0 \\
0 \\
0 \\
0\end{array}$ & $\begin{array}{l}\frac{47}{\underline{0}} \\
\underline{0} \\
\frac{0.2}{\underline{0}}\end{array}$ & $\begin{array}{c}48 \\
0 \\
0 \\
0 \\
0.2\end{array}$ & $\begin{array}{l}\frac{53}{\underline{0}} \\
\underline{0} \\
\underline{0.3} \\
\underline{0}\end{array}$ & $\begin{array}{c}54 \\
0 \\
0 \\
0 \\
0\end{array}$ & $\begin{array}{c}59 \\
\frac{59}{0} \\
\underline{0} \\
\underline{0.3} \\
\underline{0.2}\end{array}$ & $\begin{array}{r}60 \\
0 \\
0 \\
0 \\
0 \\
0\end{array}$ & $\begin{array}{r}65 \\
\underline{0} \\
\underline{0} \\
\underline{0} \\
\underline{0} \\
\underline{0}\end{array}$ & $\begin{array}{c}66 \\
0 \\
0 \\
0 \\
0.5 \\
0\end{array}$ & & & & $\begin{array}{l}4 \\
0 \\
0 \\
0 \\
0.3\end{array}$ & $\begin{array}{l}\frac{5}{0} \\
\underline{0} \\
\underline{0} \\
\underline{0}\end{array}$ & \\
\hline $\begin{array}{l}\text { High } \\
\text { Velocity } \\
\text { Region }\end{array}$ & $\begin{array}{c}12 \\
0 \\
0 \\
0.8 \\
0\end{array}$ & $\frac{\frac{13}{0.5}}{\frac{0.6}{0}}$ & $\frac{\frac{14}{0.6}}{\frac{0}{0}} \frac{0}{\underline{0}}$ & $\begin{array}{r}15 \\
0 \\
0 \\
0 \\
0\end{array}$ & $\begin{array}{c}26 \\
0 \\
0 \\
0 \\
0.7 \\
-\end{array}$ & $\begin{array}{c}27 \\
0 \\
0 \\
0.5 \\
0 \\
0\end{array}$ & $\begin{array}{c}30 \\
0 \\
0 \\
0 \\
0.5 \\
0.2\end{array}$ & $\begin{array}{c}31 \\
0 \\
0 \\
0 \\
0.9 \\
0\end{array}$ & $\frac{\frac{32}{0.5}}{\frac{0}{0}}$ & $\frac{41}{\underline{0}}$ & $\frac{\frac{42}{0.1}}{\frac{0}{0}}$ & $\begin{array}{r}43 \\
0 \\
0 \\
0 \\
0 \\
0\end{array}$ & $\frac{44}{\underline{0}}$ & & $\begin{array}{l}\frac{55}{\underline{0}} \\
\underline{0} \\
\underline{0} \\
\underline{0}\end{array}$ & & & & & & & $\begin{array}{l}\frac{2}{\underline{0}} \\
\underline{0} \\
\underline{0.7} \\
\underline{0}\end{array}$ & $\begin{array}{l}3 \\
0 \\
0 \\
0 \\
0\end{array}$ & \\
\hline $\begin{array}{l}\text { Ring } \\
\text { Core }\end{array}$ & $\begin{array}{r}10 \\
\underline{0} \\
\underline{0} \\
\underline{0} \\
\underline{0}\end{array}$ & $\begin{array}{c}11 \\
0 \\
0 \\
0.6 \\
0\end{array}$ & $\begin{array}{c}16 \\
0 \\
0 \\
2.0\end{array}$ & $\begin{array}{l}\frac{17}{1.7} \\
\underline{0} \\
0.2\end{array}$ & $\begin{array}{l}\frac{21}{\underline{0}} \\
\frac{0.4}{0.3} \\
\frac{1.3}{\underline{0}}\end{array}$ & $\begin{array}{c}\frac{23}{\underline{0}} \\
\frac{1.3}{\underline{0}} \\
\frac{0.5}{\underline{0}}\end{array}$ & $\begin{array}{c}24 \\
0 \\
0 \\
0 \\
\frac{5.0}{0}\end{array}$ & $\begin{array}{c}28 \\
0 \\
0 \\
0.7 \\
0 \\
0\end{array}$ & & $\cdot$ & & & & & $\begin{array}{r}56 \\
\underline{0} \\
\underline{0} \\
\underline{0} \\
\underline{0} \\
\underline{0}\end{array}$ & $\begin{array}{c}57 \\
\underline{0} \\
\underline{0} \\
\underline{0} \\
\underline{0} \\
\underline{0.3}\end{array}$ & $\begin{array}{r}58 \\
0 \\
0 \\
0 \\
0 \\
0\end{array}$ & $\begin{array}{r}61 \\
\underline{0} \\
\underline{0} \\
\underline{0} \\
\underline{0} \\
\underline{0}\end{array}$ & $\begin{array}{r}62 \\
0 \\
0 \\
0 \\
0 \\
0\end{array}$ & $\begin{array}{r}63 \\
\underline{0} \\
\underline{0} \\
\underline{0} \\
\underline{0} \\
\underline{0}\end{array}$ & $\begin{array}{r}64 \\
0 \\
0 \\
0 \\
n \\
0\end{array}$ & $\begin{array}{l}\frac{1}{\underline{0}} \\
\underline{0} \\
\underline{0} \\
\underline{0}\end{array}$ & & \\
\hline $\begin{array}{l}\text { Sargasso } \\
\text { Sea } \\
\& \\
\text { Gu1f } \\
\text { Stream }\end{array}$ & & & & & & & & & & $\begin{array}{r}\frac{49}{\underline{0}} \\
\underline{0} \\
\underline{0} \\
\underline{0}\end{array}$ & $\begin{array}{r}50 \\
0 \\
0 \\
0 \\
0\end{array}$ & $\begin{array}{l}51 \\
0 \\
0 \\
0 \\
0\end{array}$ & $\begin{array}{l}\frac{52}{\underline{0}} \\
\underline{0} \\
\underline{0} \\
\underline{0}\end{array}$ & & & & & & & & & & & \\
\hline $\begin{array}{l}\text { Slope } \\
\text { Water }\end{array}$ & $\frac{8}{-}$ & $\frac{9}{0}$ & $\frac{18}{0.4}$ & & $\frac{19}{0.1}$ & $\frac{20}{0}$ & $\frac{33}{0.1}$ & $\frac{34}{0.1}$ & & $\frac{35}{0}$ & $\frac{47}{0.1}$ & $\frac{48}{0.1}$ & $\frac{53}{0.1}$ & $\frac{54}{0}$ & $\frac{59}{0.1}$ & $\frac{60}{0}$ & $\frac{65}{0}$ & $\frac{66}{0.1}$ & & & & $\frac{4}{0.1}$ & $\frac{5}{0}$ & $\frac{6}{0.1}$ \\
\hline $\begin{array}{l}\text { High } \\
\text { Velocity } \\
\text { Region }\end{array}$ & $\frac{12}{0.2}$ & $\frac{13}{0.3}$ & $\frac{14}{0.1}$ & $\frac{15}{0}$ & $\frac{27}{0.1}$ & $\frac{29}{0.1}$ & $\frac{30}{0.2}$ & $\frac{31}{0.2}$ & $\frac{32}{-}$ & $\frac{41}{0}$ & $\frac{42}{0.1}$ & $\frac{43}{0}$ & $\frac{44}{0}$ & & $\frac{55}{0}$ & & & & & & & $\frac{2}{0.2}$ & $\frac{3}{0}$ & \\
\hline $\begin{array}{l}\text { Ring } \\
\text { Core }\end{array}$ & $\frac{10}{0}$ & $\frac{11}{0.2}$ & $\frac{16}{-}$ & $\frac{17}{0.5}$ & $\frac{21}{0.5}$ & $\frac{23}{0.4}$ & $\frac{24}{1.0}$ & $\frac{28}{0.2}$ & & $\frac{37}{0}$ & $\frac{38}{0}$ & $\frac{39}{0.1}$ & & & $\frac{56}{0}$ & $\frac{57}{0.1}$ & $\frac{58}{0}$ & $\frac{61}{0}$ & $\frac{62}{0}$ & $\frac{63}{0}$ & $\frac{64}{0}$ & $\frac{1}{0}$ & & \\
\hline SS \& GS & & & & & & & & & & $\frac{49}{0}$ & $\frac{50}{0}$ & $\frac{51}{0}$ & $\frac{52}{0}$ & & & & & & & & & & & \\
\hline
\end{tabular}


Table 81. Volume of Lampadena speculigera $\left(\mathrm{ml} / 10,000 \mathrm{~m}^{3}\right)$.

\begin{tabular}{|c|c|c|c|c|c|c|c|c|c|c|c|c|c|c|c|c|c|c|c|c|c|c|c|c|}
\hline & \multicolumn{4}{|c|}{$\begin{array}{c}\text { Oceanus } 118 \\
\text { Aprit }\end{array}$} & \multicolumn{5}{|c|}{$\begin{array}{c}\text { Oceanus } 121 \\
\text { June }\end{array}$} & \multicolumn{5}{|c|}{$\begin{array}{l}\text { Oceanus } 125 \\
\text { August }\end{array}$} & \multicolumn{7}{|c|}{$\begin{array}{l}\text { Knorr } 98 \\
\text { Sept-0ct }\end{array}$} & \multicolumn{3}{|c|}{$\begin{array}{c}\text { A2 } 110 \\
\text { Sept-0ct }\end{array}$} \\
\hline $\begin{array}{l}\text { Slope } \\
\text { Water }\end{array}$ & $\begin{array}{l}\frac{8}{\overline{0}} \\
\underline{0} \\
\underline{0} \\
\underline{0}\end{array}$ & $\begin{array}{l}9 \\
0 \\
0 \\
0 \\
0\end{array}$ & & & $\frac{\frac{19}{0.05}}{\frac{0}{0}}$ & $\begin{array}{l}20 \\
0 \\
0 \\
0 \\
0 \\
0\end{array}$ & $\begin{array}{l}\frac{33}{0.07} \\
\frac{0}{0} \\
\underline{0} \\
\underline{0}\end{array}$ & $\begin{array}{l}\frac{34}{\underline{0}} \\
\underline{0} \\
\underline{0.68}\end{array}$ & & $\begin{array}{l}35 \\
0 \\
0 \\
0 \\
0 \\
0\end{array}$ & $\begin{array}{c}\frac{47}{\underline{0}} \\
\underline{0} \\
\frac{0.06}{\underline{0}}\end{array}$ & $\begin{array}{l}\frac{48}{0} \\
0 \\
0 \\
7.14\end{array}$ & $\begin{array}{c}\frac{53}{\underline{0}} \\
\underline{0} \\
\frac{8.97}{\underline{0}}\end{array}$ & $\begin{array}{l}54 \\
0 \\
0 \\
0 \\
0\end{array}$ & $\begin{array}{l}\frac{59}{\underline{0}} \\
\underline{0} \\
\underline{0} \\
\frac{3.45}{7.05} \\
\underline{\underline{n}}\end{array}$ & $\begin{array}{l}\frac{60}{0} \\
0 \\
0 \\
0 \\
0\end{array}$ & $\begin{array}{l}\frac{65}{\underline{0}} \\
\underline{0} \\
\underline{0} \\
\underline{0} \\
\underline{0}\end{array}$ & $\begin{array}{l}\frac{66}{0} \\
0 \\
0 \\
0.38 \\
0\end{array}$ & & & & $\begin{array}{c}\frac{4}{0} \\
0 \\
0 \\
10.29\end{array}$ & $\begin{array}{l}\frac{5}{0} \\
\underline{0} \\
\underline{0} \\
\underline{0}\end{array}$ & \\
\hline $\begin{array}{l}\text { High } \\
\text { Velocity } \\
\text { Region }\end{array}$ & $\begin{array}{c}\frac{12}{0} \\
0 \\
0.04 \\
0\end{array}$ & $\frac{\frac{13}{0.05}}{\frac{0.06}{\underline{0}}}$ & $\frac{\frac{14}{0.03}}{\frac{0}{0}}$ & $\begin{array}{l}15 \\
0 \\
0 \\
0 \\
0\end{array}$ & $\begin{array}{l}26 \\
0 \\
0 \\
0 \\
0.14 \\
-\end{array}$ & $\begin{array}{c}27 \\
0 \\
0 \\
0.05 \\
0 \\
0\end{array}$ & $\begin{array}{l}30 \\
0 \\
0 \\
0 \\
0.05 \\
0.23\end{array}$ & $\begin{array}{l}31 \\
0 \\
0 \\
0 \\
0.09 \\
0\end{array}$ & $\frac{\frac{32}{0.05}}{\underline{0}}$ & $\begin{array}{l}\frac{41}{0} \\
\underline{0} \\
\underline{0}\end{array}$ & $\frac{\frac{42}{0.06}}{\underline{0}}$ & $\begin{array}{l}43 \\
0 \\
0 \\
0 \\
0 \\
0\end{array}$ & $\begin{array}{l}\frac{44}{\underline{0}} \\
\underline{0} \\
\underline{0}\end{array}$ & & $\begin{array}{l}\frac{55}{\underline{0}} \\
\underline{0} \\
\underline{0} \\
\underline{0}\end{array}$ & & & & & & & $\begin{array}{c}\frac{2}{\underline{0}} \\
\underline{\underline{0}} \\
\underline{3.00}\end{array}$ & $\begin{array}{l}\frac{3}{0} \\
0 \\
0.11 \\
0\end{array}$ & \\
\hline $\begin{array}{l}\text { Ring } \\
\text { Core }\end{array}$ & $\begin{array}{l}\frac{10}{\underline{0}} \\
\frac{0}{0} \\
\underline{0}\end{array}$ & $\begin{array}{l}\frac{11}{0} \\
0 \\
0.06 \\
0\end{array}$ & $\begin{array}{l}\frac{16}{0} \\
0 \\
0.07\end{array}$ & $\begin{array}{l}\frac{17}{0.06} \\
\underline{0} \\
\underline{0.01}\end{array}$ & $\begin{array}{l}\frac{21}{\underline{0}} \\
\frac{0.06}{0.03} \\
\frac{0.04}{\underline{0}}\end{array}$ & $\begin{array}{l}\frac{23}{\underline{0}} \\
\underline{0.15} \\
\underline{0} \\
\underline{0.05} \\
\underline{0}\end{array}$ & $\begin{array}{l}24 \\
0 \\
0 \\
0 \\
0.56 \\
0\end{array}$ & $\begin{array}{l}\frac{28}{0} \\
0 \\
0.04 \\
0 \\
0\end{array}$ & & & & & & & $\begin{array}{l}\frac{56}{\underline{0}} \\
\underline{0} \\
\underline{0} \\
\underline{0} \\
\underline{0}\end{array}$ & $\begin{array}{l}\frac{57}{0} \\
\underline{0} \\
\underline{0} \\
\underline{0} \\
\underline{0.11}\end{array}$ & $\begin{array}{l}58 \\
0 \\
0 \\
0 \\
0 \\
0\end{array}$ & $\begin{array}{l}\frac{61}{0} \\
\underline{0} \\
\underline{0} \\
\underline{0} \\
\underline{0} \\
\underline{0}\end{array}$ & $\begin{array}{l}62 \\
0 \\
0 \\
0 \\
0 \\
0\end{array}$ & $\begin{array}{l}\frac{63}{0} \\
\underline{0} \\
\underline{0} \\
\underline{0} \\
\underline{0}\end{array}$ & $\begin{array}{l}64 \\
0 \\
0 \\
0 \\
0 \\
0\end{array}$ & $\begin{array}{l}\frac{1}{0} \\
\underline{0} \\
\underline{0} \\
\underline{0} \\
\underline{0}\end{array}$ & & \\
\hline $\begin{array}{l}\text { Sargasso } \\
\text { Sea } \\
\& \\
\text { Gulf } \\
\text { Stream }\end{array}$ & & & & & & & & & & $\begin{array}{l}\frac{49}{0} \\
\frac{0}{0} \\
\underline{0} \\
\underline{0}\end{array}$ & $\begin{array}{l}50 \\
0 \\
0 \\
0 \\
0\end{array}$ & $\begin{array}{l}51 \\
0 \\
0 \\
0 \\
0\end{array}$ & $\begin{array}{l}\frac{52}{\underline{0}} \\
\underline{0} \\
\underline{0} \\
\underline{0}\end{array}$ & & & & & & & & & & & \\
\hline $\begin{array}{l}\text { Slope } \\
\text { Water }\end{array}$ & $\frac{8}{-}$ & $\frac{9}{0}$ & $\frac{18}{3.32}$ & & $\frac{19}{0.01}$ & $\frac{20}{0}$ & $\frac{33}{0.01}$ & $\frac{34}{0.41}$ & & $\frac{35}{0}$ & $\frac{47}{0.02}$ & $\frac{48}{1.79}$ & $\frac{53}{2.24}$ & $\frac{54}{0}$ & $\frac{59}{2.10}$ & $\frac{60}{0}$ & $\frac{65}{0}$ & $\frac{66}{0.08}$ & & & & $\frac{4}{2.57}$ & $\frac{5}{0}$ & $\frac{6}{3.17}$ \\
\hline $\begin{array}{l}\text { High } \\
\text { Velocity } \\
\text { Region }\end{array}$ & $\frac{12}{0.01}$ & $\frac{13}{0.03}$ & $\frac{14}{0.01}$ & $\frac{15}{0}$ & $\frac{27}{0.01}$ & $\frac{29}{0.01}$ & $\frac{30}{0.06}$ & $\frac{31}{0.02}$ & $\frac{32}{-}$ & $\frac{41}{0}$ & $\frac{42}{0.02}$ & $\frac{43}{0}$ & $\frac{44}{0}$ & & $\frac{55}{0}$ & & & & & & & $\frac{2}{0.75}$ & $\frac{3}{0.03}$ & \\
\hline $\begin{array}{l}\text { Ring } \\
\text { Core }\end{array}$ & $\frac{10}{0}$ & $\frac{11}{0.02}$ & $\frac{16}{-}$ & $\frac{17}{0.02}$ & $\frac{21}{0.04}$ & $\frac{23}{0.05}$ & $\frac{24}{0.11}$ & $\frac{28}{0.01}$ & & $\frac{37}{0}$ & $\frac{38}{0}$ & $\frac{39}{0}$ & & & $\frac{56}{0}$ & $\frac{57}{0.02}$ & $\frac{58}{0}$ & $\frac{61}{0}$ & $\frac{62}{0}$ & $\frac{63}{0}$ & $\frac{64}{0}$ & $\frac{1}{0}$ & & \\
\hline SS \& GS & & & & & & & & & & $\frac{49}{0}$ & $\frac{50}{0}$ & $\frac{51}{0}$ & $\frac{52}{0}$ & & & & & & & & & & & \\
\hline
\end{tabular}


Table 82. Abundance of Lampanyctus alatus (specimens $/ 10,000 \mathrm{~m}^{3}$ ).

\begin{tabular}{|c|c|c|c|c|c|c|c|c|c|c|c|c|c|c|c|c|c|c|c|c|c|c|c|c|}
\hline & \multicolumn{4}{|c|}{$\begin{array}{c}\text { Oceanus } 118 \\
\text { April }\end{array}$} & \multicolumn{5}{|c|}{$\begin{array}{c}\text { Oceanus } 121 \\
\text { June }\end{array}$} & \multicolumn{5}{|c|}{$\begin{array}{c}\text { Oceanus } 125 \\
\text { August }\end{array}$} & \multicolumn{7}{|c|}{$\begin{array}{l}\text { Knorr } 98 \\
\text { Sept-0ct }\end{array}$} & \multicolumn{3}{|c|}{$\begin{array}{c}\text { A2 } 110 \\
\text { Sept-0ct }\end{array}$} \\
\hline $\begin{array}{l}\text { Slope } \\
\text { Water }\end{array}$ & $\begin{array}{l}\frac{8}{\overline{0}} \\
\underline{0} \\
\underline{0} \\
\underline{0}\end{array}$ & $\begin{array}{l}9 \\
0 \\
0 \\
0 \\
0\end{array}$ & & & $\frac{19}{\frac{6.8}{0}}$ & $\begin{array}{c}20 \\
0 \\
0 \\
0.9 \\
0 \\
0\end{array}$ & $\begin{array}{c}\frac{33}{7.0} \\
\frac{0.4}{0} \\
\underline{0} \\
\underline{0}\end{array}$ & $\frac{34}{\frac{0.9}{\underline{0}}}$ & & $\begin{array}{c}35 \\
0 \\
0 \\
3.3 \\
0 \\
0\end{array}$ & $\begin{array}{l}\frac{47}{1.1} \\
\frac{0}{0.2} \\
\underline{0}\end{array}$ & $\begin{array}{c}48 \\
0 \\
0 \\
0.9 \\
0.4\end{array}$ & $\frac{53}{\frac{1.2}{\underline{0}}}$ & $\begin{array}{c}54 \\
0 \\
0 \\
0.9 \\
0\end{array}$ & $\begin{array}{c}59 \\
\underline{0} \\
\underline{0} \\
\underline{0} \\
\underline{0} \\
\underline{0}\end{array}$ & $\begin{array}{c}60 \\
0 \\
0 \\
0 \\
0.9 \\
0\end{array}$ & $\begin{array}{c}\frac{65}{0.9} \\
\frac{0}{0} \\
\underline{0} \\
\underline{0}\end{array}$ & $\begin{array}{c}66 \\
0 \\
0 \\
1.9 \\
0.5 \\
0\end{array}$ & & & & $\begin{array}{c}4 \\
0 \\
0.2 \\
0 \\
0\end{array}$ & $\frac{\frac{5}{0.3}}{\frac{0}{0}}$ & \\
\hline $\begin{array}{l}\text { High } \\
\text { Velocity } \\
\text { Region }\end{array}$ & $\begin{array}{r}12 \\
0 \\
0 \\
0 \\
0\end{array}$ & $\frac{\frac{13}{0.5}}{\frac{0}{0}}$ & $\frac{\frac{14}{0.3}}{\frac{0.3}{\underline{0}}}$ & $\begin{array}{c}15 \\
0 \\
0.6 \\
0.6 \\
0\end{array}$ & $\begin{array}{c}26 \\
0 \\
0 \\
0 \\
0.3 \\
-\end{array}$ & $\begin{array}{r}27 \\
0 \\
0 \\
0 \\
0 \\
0\end{array}$ & $\begin{array}{c}30 \\
0 \\
0 \\
0 \\
0.8 \\
0.2\end{array}$ & $\begin{array}{c}31 \\
0 \\
0 \\
0 \\
0.9 \\
0\end{array}$ & $\frac{\frac{32}{0.3}}{\frac{0}{\underline{0}}}$ & $\frac{41}{\frac{0}{0}}$ & $\frac{\frac{42}{0.3}}{\frac{0.0}{\underline{0}}}$ & $\begin{array}{r}43 \\
0 \\
0 \\
0 \\
0 \\
0\end{array}$ & $\frac{\frac{44}{0.3}}{\frac{0}{\underline{0}}}$ & & $\frac{\frac{55}{0.3}}{\frac{0}{0}}$ & & & & & & & $\frac{\frac{2}{0.6}}{\underline{0}} \frac{0}{\underline{0}}$ & $\begin{array}{c}\frac{3}{0} \\
0.8 \\
0 \\
0\end{array}$ & \\
\hline $\begin{array}{l}\text { Ring } \\
\text { Core }\end{array}$ & $\frac{\frac{10}{0.4}}{\frac{0.4}{\underline{0}}} \frac{\underline{0}}{\underline{0}}$ & $\begin{array}{c}11 \\
0 \\
0 \\
0 \\
0\end{array}$ & $\begin{array}{r}16 \\
0 \\
0 \\
0\end{array}$ & $\frac{17}{\underline{0}}$ & $\begin{array}{l}21 \\
\underline{0} \\
\underline{0} \\
\underline{0} \\
\underline{0} \\
\underline{0}\end{array}$ & $\begin{array}{r}23 \\
\underline{0} \\
\underline{0} \\
\underline{0} \\
\underline{0} \\
\underline{0}\end{array}$ & $\begin{array}{r}24 \\
0 \\
0 \\
0 \\
0 \\
0\end{array}$ & $\begin{array}{c}28 \\
0 \\
0 \\
0.4 \\
0 \\
0\end{array}$ & & & & & & & $\begin{array}{r}56 \\
\underline{0} \\
\underline{0} \\
\underline{0} \\
\underline{0} \\
\underline{0}\end{array}$ & $\begin{array}{c}\frac{57}{\underline{0}} \\
\frac{0.3}{\underline{0}} \\
\underline{0} \\
\underline{0}\end{array}$ & $\begin{array}{r}58 \\
0 \\
0 \\
0 \\
0 \\
0\end{array}$ & $\begin{array}{c}\frac{61}{\underline{0.2}} \\
\underline{0} \\
\underline{0} \\
\underline{0.4} \\
\underline{0}\end{array}$ & $\begin{array}{r}62 \\
0 \\
0 \\
0 \\
0 \\
0\end{array}$ & $\frac{63}{\frac{0.5}{\underline{0}}}$ & $\begin{array}{r}64 \\
0 \\
0 \\
0 \\
0 \\
0\end{array}$ & $\frac{\frac{1}{2.0}}{\underline{0}}$ & & \\
\hline $\begin{array}{l}\text { Sargasso } \\
\text { Sea } \\
\& \\
\text { Gulf } \\
\text { Stream }\end{array}$ & & & & & & & & & & $\begin{array}{l}\frac{49}{\underline{0.6}} \\
\underline{0} \\
\frac{0.2}{\underline{0}}\end{array}$ & $\begin{array}{c}50 \\
0 \\
0 \\
0.7 \\
0\end{array}$ & $\begin{array}{c}51 \\
0 \\
0 \\
0.2 \\
0\end{array}$ & $\begin{array}{l}\frac{52}{\underline{0}} \\
\underline{0} \\
\underline{0.4}\end{array}$ & & & & & & & & & & & \\
\hline $\begin{array}{l}\text { Slope } \\
\text { Water }\end{array}$ & $\frac{8}{-}$ & $\frac{9}{0}$ & $\frac{18}{0.1}$ & & $\frac{19}{0.7}$ & $\frac{20}{0.2}$ & $\frac{33}{0.5}$ & $\frac{34}{0.1}$ & & $\frac{35}{0.7}$ & $\frac{47}{0.3}$ & $\frac{48}{0.3}$ & $\frac{53}{0.3}$ & $\frac{54}{0.2}$ & $\frac{59}{0}$ & $\frac{60}{0.2}$ & $\frac{65}{0.2}$ & $\frac{66}{0.5}$ & & & & $\frac{4}{0.1}$ & $\frac{5}{0.1}$ & $\frac{6}{0.1}$ \\
\hline $\begin{array}{l}\text { High } \\
\text { Velocity } \\
\text { Region }\end{array}$ & $\frac{12}{0}$ & $\frac{13}{0.1}$ & $\frac{14}{0.2}$ & $\frac{15}{0.3}$ & $\frac{27}{0}$ & $\frac{29}{0.2}$ & $\frac{30}{0.2}$ & $\frac{31}{0.2}$ & $\frac{32}{-}$ & $\frac{41}{0}$ & $\frac{42}{0.1}$ & $\frac{43}{0}$ & $\frac{44}{0.2}$ & & $\frac{55}{0.1}$ & & & & & & & $\frac{2}{0.2}$ & $\frac{3}{0.2}$ & \\
\hline $\begin{array}{l}\text { Ring } \\
\text { Core }\end{array}$ & $\frac{10}{0.1}$ & $\frac{11}{0}$ & $\frac{16}{-}$ & $\frac{17}{0}$ & $\frac{21}{0}$ & $\frac{23}{0}$ & $\frac{24}{0}$ & $\frac{28}{0.1}$ & & $\frac{37}{0.3}$ & $\frac{38}{0.1}$ & $\frac{39}{0}$ & & & $\frac{56}{0}$ & $\frac{57}{0.1}$ & $\frac{58}{0}$ & $\frac{61}{0.1}$ & $\frac{62}{0}$ & $\frac{63}{0.2}$ & $\frac{64}{0}$ & $\frac{1}{0.5}$ & & \\
\hline SS \& GS & & & & & & & & & & $\frac{49}{0.2}$ & $\frac{50}{0.2}$ & $\frac{51}{0.1}$ & $\frac{52}{0.1}$ & & & & & & & & & & & \\
\hline
\end{tabular}


Table 83. Volume of Lampanyctus ater $\left(\mathrm{ml} / 10,000 \mathrm{~m}^{3}\right)$.

\begin{tabular}{|c|c|c|c|c|c|c|c|c|c|c|c|c|c|c|c|c|c|c|c|c|c|c|c|c|}
\hline & \multicolumn{4}{|c|}{$\begin{array}{c}\text { Oceanus } 118 \\
\text { April }\end{array}$} & \multicolumn{5}{|c|}{$\begin{array}{c}\text { Oceanus } 121 \\
\text { June }\end{array}$} & \multicolumn{5}{|c|}{$\begin{array}{l}\text { Oceanus } 125 \\
\text { August }\end{array}$} & \multicolumn{7}{|c|}{$\begin{array}{l}\text { Knorr } 98 \\
\text { Sept-0ct }\end{array}$} & \multicolumn{3}{|c|}{$\begin{array}{c}A 2 \quad 110 \\
\text { Sept-0ct }\end{array}$} \\
\hline $\begin{array}{l}\text { Slope } \\
\text { Water }\end{array}$ & 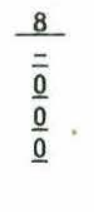 & $\begin{array}{l}9 \\
0 \\
0 \\
0 \\
0\end{array}$ & & & $\begin{array}{l}19 \\
\underline{0} \\
\underline{0} \\
\underline{0} \\
\underline{0} \\
\underline{0}\end{array}$ & $\begin{array}{l}20 \\
0 \\
0 \\
0 \\
0 \\
0\end{array}$ & $\begin{array}{l}\frac{33}{0} \\
\underline{0} \\
\underline{0} \\
\underline{0} \\
\underline{1.23}\end{array}$ & $\begin{array}{l}\frac{34}{\underline{0}} \\
\underline{0} \\
\underline{0}\end{array}$ & & $\begin{array}{l}35 \\
0 \\
0 \\
0 \\
0 \\
0\end{array}$ & $\begin{array}{l}47 \\
\underline{0} \\
\underline{0} \\
\underline{0} \\
\underline{0}\end{array}$ & $\begin{array}{l}\frac{48}{0} \\
0 \\
0 \\
0\end{array}$ & $\begin{array}{l}\frac{53}{0} \\
\underline{0} \\
\underline{0} \\
\underline{0}\end{array}$ & $\begin{array}{l}54 \\
0 \\
0 \\
0 \\
0.80\end{array}$ & $\begin{array}{l}59 \\
\underline{0} \\
\underline{0} \\
\underline{0} \\
\underline{0} \\
\underline{0}\end{array}$ & $\begin{array}{l}60 \\
0 \\
0 \\
0 \\
0 \\
0\end{array}$ & $\begin{array}{l}\frac{65}{0.17} \\
\frac{0.62}{\underline{0}} \\
\underline{0} \\
\underline{0.10}\end{array}$ & $\begin{array}{l}\frac{66}{0} \\
0 \\
0 \\
0.86 \\
1.35\end{array}$ & & & & $\begin{array}{l}4 \\
0 \\
0 \\
0 \\
0\end{array}$ & $\begin{array}{l}\frac{5}{\underline{0}} \\
\underline{\underline{0}} \\
\frac{0.41}{\underline{0}}\end{array}$ & \\
\hline $\begin{array}{l}\text { High } \\
\text { Velocity } \\
\text { Region }\end{array}$ & $\begin{array}{l}\frac{12}{0} \\
0 \\
0 \\
0\end{array}$ & $\begin{array}{l}\frac{13}{\underline{0}} \\
\frac{1.39}{\underline{0}}\end{array}$ & $\begin{array}{c}\frac{14}{\underline{0}} \\
\underline{0} \\
\underline{3.53} \\
\underline{0}\end{array}$ & $\begin{array}{l}\frac{15}{0} \\
0 \\
5.88 \\
0\end{array}$ & $\begin{array}{l}26 \\
0 \\
0 \\
0 \\
0 \\
-\end{array}$ & $\begin{array}{l}\frac{27}{0} \\
0 \\
0 \\
0 \\
0\end{array}$ & $\begin{array}{l}30 \\
0 \\
0 \\
0 \\
0 \\
0\end{array}$ & $\begin{array}{l}31 \\
0 \\
0 \\
0 \\
3.14 \\
0\end{array}$ & $\begin{array}{l}\frac{32}{\underline{0}} \\
\underline{0} \\
\underline{0} \\
\underline{0}\end{array}$ & $\begin{array}{l}41 \\
\underline{0} \\
\underline{0} \\
\underline{0}\end{array}$ & $\begin{array}{l}42 \\
\underline{0} \\
\underline{0} \\
\underline{0} \\
\underline{0}\end{array}$ & $\begin{array}{l}43 \\
0 \\
0 \\
0 \\
0.35 \\
0\end{array}$ & $\begin{array}{l}\frac{44}{0} \\
\underline{0} \\
\underline{0}\end{array}$ & & $\begin{array}{l}\frac{55}{0} \\
\underline{0} \\
\underline{0} \\
\underline{0}\end{array}$ & & & & & & & $\frac{\frac{2}{1.00}}{\frac{0}{0}}$ & $\begin{array}{l}3 \\
0 \\
0 \\
0 \\
0\end{array}$ & \\
\hline $\begin{array}{l}\text { Ring } \\
\text { Core }\end{array}$ & $\frac{\frac{10}{\underline{0}}}{\frac{2.94}{\underline{0}}}$ & $\begin{array}{l}\frac{11}{0} \\
0 \\
0 \\
0\end{array}$ & $\begin{array}{l}16 \\
0 \\
0 \\
0\end{array}$ & $\begin{array}{l}\frac{17}{\underline{0}} \\
\underline{0} \\
\underline{0}\end{array}$ & $\begin{array}{l}\frac{21}{\underline{0}} \\
\underline{0.02} \\
\underline{0} \\
\underline{0} \\
\underline{0}\end{array}$ & $\begin{array}{l}23 \\
\underline{0} \\
\underline{0} \\
\underline{0} \\
\underline{0} \\
\underline{0}\end{array}$ & $\begin{array}{l}24 \\
0 \\
0 \\
0 \\
0 \\
0\end{array}$ & $\begin{array}{l}28 \\
0 \\
0 \\
0 \\
0 \\
0\end{array}$ & & & & & & & $\begin{array}{l}56 \\
\underline{0} \\
\underline{0} \\
\underline{0} \\
\underline{0} \\
\underline{0}\end{array}$ & $\begin{array}{l}57 \\
\underline{0} \\
\underline{0} \\
\underline{0} \\
\underline{0} \\
\underline{0}\end{array}$ & $\begin{array}{l}\frac{58}{0} \\
0 \\
0 \\
0 \\
0.17\end{array}$ & $\begin{array}{l}\frac{61}{0} \\
\underline{0} \\
\underline{0} \\
\underline{0} \\
\underline{0.07}\end{array}$ & $\begin{array}{l}\frac{62}{0} \\
0 \\
0 \\
0 \\
0.14\end{array}$ & $\begin{array}{l}\frac{63}{\underline{0}} \\
\underline{0} \\
\underline{0.95} \\
\underline{0} \\
\underline{6.25}\end{array}$ & $\begin{array}{l}64 \\
0 \\
0 \\
0 \\
0 \\
0\end{array}$ & $\begin{array}{l}\frac{1}{0} \\
\underline{0} \\
\underline{0} \\
\underline{0}\end{array}$ & & \\
\hline $\begin{array}{l}\text { Sargasso } \\
\text { Sea } \\
\& \\
\text { Gulf } \\
\text { Stream }\end{array}$ & & & & & & & & & & $\begin{array}{l}49 \\
\underline{0} \\
\underline{0} \\
\underline{0} \\
\underline{0}\end{array}$ & $\begin{array}{l}50 \\
0 \\
0 \\
0 \\
0\end{array}$ & $\begin{array}{l}51 \\
0 \\
0 \\
0 \\
0\end{array}$ & $\begin{array}{l}\frac{52}{\underline{0}} \\
\underline{0} \\
\underline{0} \\
\underline{0.21} \\
\end{array}$ & & & & & & & & & & & \\
\hline $\begin{array}{l}\text { Slope } \\
\text { Water }\end{array}$ & $\frac{8}{-}$ & $\frac{9}{0}$ & $\frac{18}{0}$ & & $\frac{19}{0}$ & $\frac{20}{0}$ & $\frac{33}{0.62}$ & $\frac{34}{0}$ & & $\frac{35}{0}$ & $\frac{47}{0}$ & $\frac{48}{0}$ & $\frac{53}{0}$ & $\frac{54}{0.20}$ & $\frac{59}{0}$ & $\frac{60}{0}$ & $\frac{65}{0.18}$ & $\frac{66}{0.44}$ & & & & $\frac{4}{0}$ & $\frac{5}{0.10}$ & $\frac{6}{0.79}$ \\
\hline $\begin{array}{l}\text { High } \\
\text { Velocity } \\
\text { Region }\end{array}$ & $\frac{12}{0}$ & $\frac{13}{0.35}$ & $\frac{14}{0.88}$ & $\frac{15}{1.47}$ & $\frac{27}{0}$ & $\frac{29}{0}$ & $\frac{30}{0}$ & $\frac{31}{0.63}$ & $\frac{32}{-}$ & $\frac{41}{0}$ & $\frac{42}{0}$ & $\frac{43}{0.07}$ & $\frac{44}{0}$ & & $\frac{55}{0}$ & & & & & & & $\frac{2}{0.25}$ & $\frac{3}{0}$ & \\
\hline $\begin{array}{l}\text { Ring } \\
\text { Core }\end{array}$ & $\frac{10}{0.74}$ & $\frac{11}{0}$ & $\frac{16}{-}$ & $\frac{17}{0}$ & $\frac{21}{0.01}$ & $\frac{23}{0}$ & $\frac{24}{0}$ & $\frac{28}{0}$ & & $\frac{37}{0}$ & $\frac{38}{0}$ & $\frac{39}{0}$ & & & $\frac{56}{0}$ & $\frac{57}{0}$ & $\frac{58}{0.03}$ & $\frac{61}{0.01}$ & $\frac{62}{0.03}$ & $\frac{63}{1.44}$ & $\frac{64}{0}$ & $\frac{1}{0}$ & & \\
\hline SS \& GS & & & & & & & & & & $\frac{49}{0}$ & $\frac{50}{0}$ & $\frac{51}{0}$ & $\frac{52}{0.05}$ & & & & & & & & & & & \\
\hline
\end{tabular}


Table 84. Abundance of Lampanyctus crocodilus (specimens $/ 10,000 \mathrm{~m}^{3}$ ).

\begin{tabular}{|c|c|c|c|c|c|c|c|c|c|c|c|c|c|c|c|c|c|c|c|c|c|c|c|c|}
\hline & \multicolumn{4}{|c|}{$\begin{array}{c}\text { Oceanus } \\
\text { April }\end{array}$} & \multicolumn{5}{|c|}{$\begin{array}{c}\text { Oceanus } \\
\text { June }\end{array}$} & \multicolumn{5}{|c|}{$\begin{array}{c}\text { Oceanus } 125 \\
\text { August }\end{array}$} & \multicolumn{7}{|c|}{$\begin{array}{l}\text { Knorr } 98 \\
\text { Sept-Oct }\end{array}$} & \multicolumn{3}{|c|}{$\begin{array}{c}\text { A2 } 110 \\
\text { Sept-0ct }\end{array}$} \\
\hline $\begin{array}{l}\text { Slope } \\
\text { Water }\end{array}$ & $\begin{array}{l}8 \\
\overline{\overline{0}} \\
\underline{0} \\
\underline{0}\end{array}$ & $\begin{array}{l}9 \\
0 \\
0 \\
0 \\
0\end{array}$ & & & $\begin{array}{l}\frac{19}{0} \\
\frac{0}{0} \\
\underline{0} \\
\frac{0}{0}\end{array}$ & $\begin{array}{r}20 \\
0 \\
0 \\
0 \\
0 \\
0\end{array}$ & $\begin{array}{l}\frac{33}{\frac{1.0}{0.4}} \\
\frac{0.4}{0} \\
\underline{0} \\
\underline{0.1}\end{array}$ & $\begin{array}{l}\frac{34}{\underline{0}} \\
\underline{0.5} \\
\underline{\underline{0}}\end{array}$ & & $\begin{array}{l}35 \\
0 \\
0 \\
0 \\
0 \\
0\end{array}$ & $\begin{array}{l}\frac{47}{0.4} \\
\frac{0}{0} \\
0.2\end{array}$ & $\begin{array}{l}48 \\
0 \\
0 \\
0 \\
0\end{array}$ & $\frac{53}{\frac{1.0}{\frac{0}{0}}} \frac{0}{\underline{0}}$ & $\begin{array}{c}54 \\
0 \\
0 \\
0 \\
1.0\end{array}$ & $\begin{array}{l}\frac{59}{0} \\
\underline{0} \\
\underline{0} \\
\underline{0.3} \\
\underline{0.5} \\
\end{array}$ & $\begin{array}{c}60 \\
0 \\
0 \\
0 \\
1.3 \\
0.6\end{array}$ & $\begin{array}{c}\frac{65}{0} \\
\underline{0} 0 \\
\underline{0} \\
\underline{0} \\
\underline{0.2} \\
\end{array}$ & $\begin{array}{l}\frac{66}{0.2} \\
0 \\
0 \\
0.5 \\
1.5\end{array}$ & & & & $\begin{array}{c}4 \\
0 \\
0 \\
0 \\
3.8\end{array}$ & $\frac{\frac{5}{1.0}}{\frac{0}{0}}$ & \\
\hline $\begin{array}{l}\text { High } \\
\text { Velocity } \\
\text { Region }\end{array}$ & $\begin{array}{r}12 \\
0 \\
0 \\
0 \\
0\end{array}$ & $\frac{13}{\frac{0}{0}}$ & $\begin{array}{l}\frac{14}{0} \\
\frac{0}{0} \\
\underline{0} \\
\underline{0}\end{array}$ & $\begin{array}{l}15 \\
0 \\
0 \\
0 \\
0\end{array}$ & $\begin{array}{l}26 \\
0 \\
0 \\
0 \\
0 \\
-\end{array}$ & $\begin{array}{c}27 \\
0 \\
0 \\
0 \\
2.1 \\
0\end{array}$ & $\begin{array}{c}30 \\
0 \\
0 \\
0 \\
1.9 \\
1.2\end{array}$ & $\begin{array}{c}31 \\
0 \\
0 \\
0 \\
1.1 \\
0\end{array}$ & $\begin{array}{l}\frac{32}{\underline{0}} \\
\frac{\underline{0}}{0.5} \\
\underline{0}\end{array}$ & $\frac{\frac{41}{0.1}}{\frac{0}{0}}$ & $\begin{array}{r}\frac{42}{\underline{0}} \\
\underline{0} \\
\underline{0} \\
\underline{0}\end{array}$ & $\begin{array}{r}43 \\
0 \\
0 \\
0 \\
0 \\
0\end{array}$ & $\begin{array}{r}\frac{44}{0} \\
\frac{0}{0} \\
\underline{0}\end{array}$ & & $\begin{array}{l}\frac{55}{\underline{0}} \\
\underline{0} \\
\underline{0} \\
\underline{0}\end{array}$ & & & & & & & $\frac{\frac{2}{0.2}}{\frac{0}{0}} \frac{0}{\underline{0}}$ & $\begin{array}{c}\frac{3}{0} \\
0 \\
1.4 \\
0\end{array}$ & \\
\hline $\begin{array}{l}\text { Ring } \\
\text { Core }\end{array}$ & $\frac{10}{\frac{0}{0}}$ & $\begin{array}{c}11 \\
0 \\
0 \\
0 \\
0.4\end{array}$ & $\begin{array}{l}16 \\
0 \\
0 \\
0\end{array}$ & $\frac{17}{\frac{0}{0}}$ & $\begin{array}{c}\frac{21}{\underline{0}} \\
\frac{0}{0} \\
\underline{0} \\
\frac{1.7}{\underline{0}}\end{array}$ & $\begin{array}{l}\frac{23}{\underline{0}} \\
\underline{0} \\
\frac{0.5}{19.1} \\
\frac{0}{0}\end{array}$ & $\begin{array}{c}\frac{24}{0.3} \\
0 \\
0 \\
1.3 \\
0\end{array}$ & $\begin{array}{c}28 \\
0 \\
0.4 \\
2.5 \\
0 \\
0\end{array}$ & & & & & & & $\begin{array}{l}\frac{56}{0} \\
\frac{0}{0} \\
\frac{0}{0} \\
\underline{0}\end{array}$ & $\begin{array}{l}\frac{57}{\underline{0}} \\
\underline{0} \\
\underline{0} \\
\underline{0.3} \\
\underline{0.3} \\
\end{array}$ & $\begin{array}{r}58 \\
0 \\
0 \\
0 \\
0 \\
0\end{array}$ & $\begin{array}{l}\frac{61}{\underline{0}} \\
\underline{0} \\
\underline{0} \\
\underline{0} \\
\underline{0}\end{array}$ & $\begin{array}{r}62 \\
0 \\
0 \\
0 \\
0 \\
0\end{array}$ & $\begin{array}{c}\frac{63}{0.2} \\
\frac{0}{0} \\
\underline{0} \\
\underline{0} \\
\underline{0.3}\end{array}$ & $\begin{array}{l}64 \\
0 \\
0 \\
0 \\
0 \\
0\end{array}$ & $\frac{\frac{1}{1.6}}{\frac{0}{0}} \frac{0}{0}$ & & \\
\hline $\begin{array}{l}\text { Sargasso } \\
\text { Sea } \\
\& \\
\text { Gulf } \\
\text { Stream }\end{array}$ & & & & & & & & & & $\begin{array}{l}\frac{49}{\underline{0.2}} \\
\underline{0} \\
\frac{0.2}{\underline{0}}\end{array}$ & $\begin{array}{c}50 \\
0 \\
0 \\
0 \\
0.3\end{array}$ & $\begin{array}{c}51 \\
0 \\
0 \\
0 \\
0\end{array}$ & $\begin{array}{c}\frac{52}{0} \\
\underline{0} \\
\underline{0} \\
\underline{0.3}\end{array}$ & & & & & & & & & & & \\
\hline $\begin{array}{l}\text { Slope } \\
\text { Water }\end{array}$ & $\frac{8}{-}$ & $\frac{9}{0}$ & $\frac{18}{0}$ & & $\frac{19}{0}$ & $\frac{20}{0}$ & $\frac{33}{0.2}$ & $\frac{34}{0.3}$ & & $\frac{35}{0}$ & $\frac{47}{0.2}$ & $\frac{48}{0}$ & $\frac{53}{0.3}$ & $\frac{54}{0.3}$ & $\frac{59}{0.2}$ & $\frac{60}{0.4}$ & $\frac{65}{0}$ & $\frac{66}{0.5}$ & & & & $\frac{4}{1.0}$ & $\frac{5}{0.3}$ & $\frac{6}{0.2}$ \\
\hline $\begin{array}{l}\text { High } \\
\text { Velocity } \\
\text { Region }\end{array}$ & $\frac{12}{0}$ & $\frac{13}{0}$ & $\frac{14}{0}$ & $\frac{15}{0}$ & $\frac{27}{0.5}$ & $\frac{29}{0.2}$ & $\frac{30}{0.7}$ & $\frac{31}{0.2}$ & $\frac{32}{-}$ & $\frac{41}{0.1}$ & $\frac{42}{0}$ & $\frac{43}{0}$ & $\frac{44}{0}$ & & $\frac{55}{0}$ & & & & & & & $\frac{2}{0.1}$ & $\frac{3}{0.4}$ & \\
\hline $\begin{array}{l}\text { Ring } \\
\text { Core }\end{array}$ & $\frac{10}{0}$ & $\frac{11}{0.1}$ & $\frac{16}{-}$ & $\frac{17}{0}$ & $\frac{21}{0.3}$ & $\frac{23}{3.9}$ & $\frac{24}{0.4}$ & $\frac{28}{0.7}$ & & $\frac{37}{0}$ & $\frac{38}{0.5}$ & $\frac{39}{0.1}$ & & & $\frac{56}{0}$ & $\frac{57}{0.1}$ & $\frac{58}{0}$ & $\frac{61}{0}$ & $\frac{62}{0}$ & $\frac{63}{0.1}$ & $\frac{64}{0}$ & $\frac{1}{0.4}$ & & \\
\hline SS \& GS & & & & & & & & & & $\frac{49}{0.1}$ & $\frac{50}{0.1}$ & $\frac{51}{0}$ & $\frac{52}{0.1}$ & & & & & & & & & & & \\
\hline
\end{tabular}


Table 85. Abundance of Lampanyctus cuprarius (specimens $/ 10,000 \mathrm{~m}^{3}$ ).

\begin{tabular}{|c|c|c|c|c|c|c|c|c|c|c|c|c|c|c|c|c|c|c|c|c|c|c|c|c|}
\hline & \multicolumn{4}{|c|}{$\begin{array}{c}\text { Oceanus } \\
\text { April }\end{array}$} & \multicolumn{5}{|c|}{$\begin{array}{c}\text { Oceanus } 121 \\
\text { June }\end{array}$} & \multicolumn{5}{|c|}{$\begin{array}{c}\text { Oceanus } 125 \\
\text { August }\end{array}$} & \multicolumn{7}{|c|}{$\begin{array}{l}\text { Knorr } 98 \\
\text { Sept-0ct }\end{array}$} & \multicolumn{3}{|c|}{$\begin{array}{c}\text { A2 } 110 \\
\text { Sept-0ct }\end{array}$} \\
\hline $\begin{array}{l}\text { Slope } \\
\text { Water }\end{array}$ & $\begin{array}{l}8 \\
\overline{\overline{0}} \\
\underline{0} \\
\underline{0}\end{array}$ & $\begin{array}{l}\frac{9}{0} \\
0 \\
0 \\
0.7\end{array}$ & & & $\begin{array}{l}\frac{19}{\underline{0}} \\
\frac{0}{0} \\
\frac{0}{0} \\
\underline{0}\end{array}$ & $\begin{array}{r}20 \\
0 \\
0 \\
0 \\
0 \\
0\end{array}$ & $\begin{array}{c}\frac{33}{0} \\
\underline{0} \\
\underline{0} \\
\underline{0} \\
\underline{0.1}\end{array}$ & $\begin{array}{r}\frac{34}{\underline{0}} \\
\underline{0} \\
\underline{0}\end{array}$ & & $\begin{array}{c}35 \\
0 \\
0 \\
0 \\
0.6 \\
0.7\end{array}$ & $\begin{array}{l}47 \\
\underline{0} \\
\underline{0} \\
\underline{0} \\
\underline{0}\end{array}$ & $\begin{array}{c}48 \\
0 \\
0 \\
0.3 \\
0\end{array}$ & $\begin{array}{l}\frac{53}{\frac{0.2}{\underline{0}}} \\
\underline{0} \underline{0.2}\end{array}$ & $\begin{array}{c}54 \\
0 \\
0 \\
0 \\
0.3\end{array}$ & $\begin{array}{l}\frac{59}{0} \\
\underline{0} \\
\underline{0} \\
\underline{0} \\
\underline{0}\end{array}$ & $\begin{array}{r}60 \\
0 \\
0 \\
0 \\
0 \\
0\end{array}$ & $\begin{array}{l}\frac{65}{\underline{0}} \\
\underline{1.4} \\
\underline{0} \\
0.8 \\
\underline{0.2}\end{array}$ & $\begin{array}{l}\frac{66}{0} \\
0.7 \\
0.4 \\
1.0 \\
0.4\end{array}$ & & & & $\begin{array}{l}4 \\
0 \\
0 \\
0.3 \\
0\end{array}$ & $\begin{array}{l}\frac{5}{\underline{0}} \\
\underline{0} \\
\frac{0.3}{\underline{0}}\end{array}$ & \\
\hline $\begin{array}{l}\text { High } \\
\text { Velocity } \\
\text { Region }\end{array}$ & $\begin{array}{c}12 \\
0 \\
0 \\
1.7 \\
0.3\end{array}$ & $\frac{\frac{13}{1.5}}{\frac{0.6}{1.0}}$ & $\begin{array}{l}\frac{14}{\underline{0}} \\
\frac{0.7}{0.6} \\
\underline{0}\end{array}$ & $\begin{array}{c}15 \\
0 \\
0 \\
1.2 \\
0\end{array}$ & $\begin{array}{c}26 \\
0 \\
0 \\
0 \\
0 \\
-\end{array}$ & $\begin{array}{c}27 \\
0 \\
0 \\
0 \\
0.4 \\
0.4\end{array}$ & $\begin{array}{c}30 \\
0 \\
0 \\
0 \\
1.4 \\
0\end{array}$ & $\begin{array}{c}31 \\
0 \\
0 \\
0 \\
0.3 \\
0.3\end{array}$ & $\begin{array}{c}\frac{32}{\underline{0}} \\
\underline{0} \\
\underline{0.4} \\
\underline{0.4}\end{array}$ & $\begin{array}{c}\frac{41}{\underline{0}} \\
\underline{0} \\
\underline{0.2}\end{array}$ & $\frac{\frac{42}{0.3}}{\frac{0}{0}}$ & $\begin{array}{c}43 \\
0 \\
0 \\
0 \\
0 \\
0\end{array}$ & $\frac{44}{\underline{0}}$ & & $\frac{\frac{55}{0.5}}{\frac{0.9}{0.8}} \frac{0}{\underline{0}}$ & & & & & & & $\begin{array}{c}\frac{2}{0.4} \\
\frac{0}{0} \\
\underline{1.9}\end{array}$ & $\begin{array}{c}\frac{3}{0} \\
0.3 \\
0.4 \\
0\end{array}$ & \\
\hline $\begin{array}{l}\text { Ring } \\
\text { Core }\end{array}$ & $\begin{array}{l}\frac{10}{\underline{0}} \\
\frac{0.6}{\underline{2.7}} \\
\underline{0}\end{array}$ & $\begin{array}{c}11 \\
0 \\
0 \\
0 \\
3.2\end{array}$ & $\begin{array}{c}16 \\
0 \\
0 \\
4.0\end{array}$ & $\begin{array}{l}\frac{17}{\underline{0}} \\
\frac{1.6}{0.7}\end{array}$ & $\begin{array}{l}\frac{21}{\underline{0}} \\
\frac{0.6}{0.3} \\
\underline{1.7} \\
\underline{0.6}\end{array}$ & $\begin{array}{l}\frac{23}{\underline{0}} \\
\frac{2.7}{0.5} \\
\frac{0.5}{\underline{0}}\end{array}$ & $\begin{array}{c}24 \\
0 \\
0 \\
0 \\
1.3 \\
0\end{array}$ & $\begin{array}{c}28 \\
0 \\
0 \\
0.4 \\
0.8 \\
0\end{array}$ & & & & & & & $\begin{array}{c}56 \\
\underline{0} \\
\underline{0} \\
\underline{0} \\
\underline{0} \\
\underline{1.3}\end{array}$ & $\begin{array}{c}57 \\
\underline{0} \\
\underline{0} \\
\underline{0} \\
\underline{0} \\
\underline{0.3}\end{array}$ & $\begin{array}{c}58 \\
0 \\
0 \\
0 \\
0 \\
2.9\end{array}$ & $\begin{array}{l}\frac{61}{\underline{0}} \\
\underline{0.3} \\
\underline{0} \\
\underline{0.8} \\
\underline{3.2}\end{array}$ & $\begin{array}{c}62 \\
0 \\
0 \\
0 \\
5.4 \\
4.6\end{array}$ & $\begin{array}{l}\frac{63}{0.8} \\
\frac{0.4}{\underline{0}} \\
\frac{1.5}{\underline{2.8}}\end{array}$ & $\begin{array}{c}64 \\
0 \\
0 \\
0 \\
3.3 \\
1.9\end{array}$ & $\frac{\frac{1}{\frac{0.2}{0.2}}}{\frac{0.2}{0.3}}$ & & \\
\hline $\begin{array}{l}\text { Sargasso } \\
\text { Sea } \\
\& \\
\text { Gulf } \\
\text { Stream }\end{array}$ & & & & & & & & & & $\begin{array}{l}\frac{49}{0.5} \\
\frac{0.2}{\underline{0}} \\
\underline{0.3}\end{array}$ & $\begin{array}{r}50 \\
0 \\
0 \\
0 \\
0\end{array}$ & $\begin{array}{r}51 \\
0 \\
0 \\
0 \\
0\end{array}$ & $\frac{\frac{52}{0.2}}{\frac{0.1}{1.1}} \frac{0.4}{\underline{0}}$ & & & & & & & & & & & \\
\hline $\begin{array}{l}\text { Slope } \\
\text { Water }\end{array}$ & $\frac{8}{-}$ & $\frac{9}{0.2}$ & $\frac{18}{0}$ & & $\frac{19}{0}$ & $\frac{20}{0}$ & $\frac{33}{0.1}$ & $\frac{34}{0}$ & & $\frac{35}{0.3}$ & $\frac{47}{0}$ & $\frac{48}{0.1}$ & $\frac{53}{0.1}$ & $\frac{54}{0.1}$ & $\frac{59}{0}$ & $\frac{60}{0}$ & $\frac{65}{0.5}$ & $\frac{66}{0.4}$ & & & & $\frac{4}{0.1}$ & $\frac{5}{0.1}$ & $\frac{6}{0}$ \\
\hline $\begin{array}{l}\text { High } \\
\text { Velocity } \\
\text { Region }\end{array}$ & $\frac{12}{0.5}$ & $\frac{13}{0.8}$ & $\frac{14}{0.3}$ & $\frac{15}{0.3}$ & $\frac{27}{0.2}$ & $\frac{29}{0.1}$ & $\frac{30}{0.3}$ & $\frac{31}{0.1}$ & $\frac{32}{-}$ & $\frac{41}{0.1}$ & $\frac{42}{0.1}$ & $\frac{43}{0}$ & $\frac{44}{0}$ & & $\frac{55}{0.6}$ & & & & & & & $\frac{2}{0.6}$ & $\frac{3}{0.2}$ & \\
\hline $\begin{array}{l}\text { Ring } \\
\text { Core }\end{array}$ & $\frac{10}{0.8}$ & $\frac{11}{0.8}$ & $\frac{16}{-}$ & $\frac{17}{0.8}$ & $\frac{21}{0.7}$ & $\frac{23}{0.9}$ & $\frac{24}{0.3}$ & $\frac{28}{0.3}$ & & $\frac{37}{0.1}$ & $\frac{38}{0.1}$ & $\frac{39}{0.2}$ & & & $\frac{56}{0.3}$ & $\frac{57}{0.1}$ & $\frac{58}{0.6}$ & $\frac{61}{0.9}$ & $\frac{62}{2.0}$ & $\frac{63}{1.2}$ & $\frac{64}{1.0}$ & $\frac{1}{0.2}$ & & \\
\hline SS \& GS & & & & & & & & & & $\frac{49}{0.3}$ & $\frac{50}{0}$ & $\frac{51}{0}$ & $\frac{52}{0.4}$ & & & & & & & & & & & \\
\hline
\end{tabular}


Tab1e 86. Volume of Lampanyctus cuprarius $\left(\mathrm{ml} / 10,000 \mathrm{~m}^{3}\right)$.

\begin{tabular}{|c|c|c|c|c|c|c|c|c|c|c|c|c|c|c|c|c|c|c|c|c|c|c|c|c|}
\hline & \multicolumn{4}{|c|}{$\begin{array}{c}\text { Oceanus } \\
\text { Apri } 118\end{array}$} & \multicolumn{5}{|c|}{$\begin{array}{c}\text { Oceanus } 121 \\
\text { June }\end{array}$} & \multicolumn{5}{|c|}{$\begin{array}{l}\text { Oceanus } 125 \\
\text { August }\end{array}$} & \multicolumn{7}{|c|}{$\begin{array}{l}\text { Knorr } 98 \\
\text { Sept-Oct }\end{array}$} & \multicolumn{3}{|c|}{$\begin{array}{c}\text { A2 } 110 \\
\text { Sept-0ct }\end{array}$} \\
\hline $\begin{array}{l}\text { Slope } \\
\text { Water }\end{array}$ & $\begin{array}{l}\frac{8}{\overline{\overline{0}}} \\
\underline{0} \\
\underline{0}\end{array}$ & $\begin{array}{l}\frac{9}{0} \\
0 \\
0 \\
0.67\end{array}$ & & & $\begin{array}{l}19 \\
\frac{0}{0} \\
\underline{0} \\
\underline{0} \\
\frac{0}{0}\end{array}$ & $\begin{array}{l}20 \\
0 \\
0 \\
0 \\
0 \\
0\end{array}$ & $\begin{array}{l}\frac{33}{0} \\
\underline{0} \\
\underline{0} \\
\underline{0} \\
\underline{0.23}\end{array}$ & $\begin{array}{l}\frac{34}{\underline{0}} \\
\underline{0} \\
\underline{0}\end{array}$ & & $\begin{array}{l}\frac{35}{0} \\
0 \\
0 \\
0.67 \\
1.00\end{array}$ & $\begin{array}{l}47 \\
\frac{0}{0} \\
\underline{0} \\
\underline{0} \\
\underline{0}\end{array}$ & $\begin{array}{l}\frac{48}{0} \\
0 \\
0.09 \\
0\end{array}$ & $\begin{array}{l}\frac{53}{0.29} \\
\underline{0} \\
\underline{0} \\
\underline{0.56}\end{array}$ & $\begin{array}{l}54 \\
0 \\
0 \\
0 \\
0.40\end{array}$ & $\begin{array}{l}\frac{59}{0} \\
\underline{0} \\
\underline{0} \\
\underline{0} \\
\underline{0}\end{array}$ & $\begin{array}{l}60 \\
0 \\
0 \\
0 \\
0 \\
0\end{array}$ & $\begin{array}{l}\frac{65}{\underline{0}} \\
\underline{0.52} \\
\underline{0} \\
\underline{0.04} \\
\underline{0.23}\end{array}$ & $\begin{array}{l}\frac{66}{0} \\
0.14 \\
0.04 \\
0.10 \\
0.58\end{array}$ & & & & $\begin{array}{c}\frac{4}{0} \\
0 \\
0.03 \\
0\end{array}$ & $\begin{array}{l}\frac{5}{\underline{0}} \\
\underline{0} \\
\frac{0.41}{\underline{0}}\end{array}$ & \\
\hline $\begin{array}{l}\text { High } \\
\text { Velocity } \\
\text { Region }\end{array}$ & $\begin{array}{l}\frac{12}{0} \\
0 \\
0.83 \\
0.86\end{array}$ & $\frac{\frac{13}{1.50}}{\frac{0.67}{1.17}}$ & $\begin{array}{l}\frac{14}{\underline{0}} \\
\frac{0.13}{0.12} \\
\underline{0}\end{array}$ & $\begin{array}{l}\frac{15}{0} \\
0 \\
2.64 \\
0\end{array}$ & $\begin{array}{l}26 \\
0 \\
0 \\
0 \\
0 \\
-\end{array}$ & $\begin{array}{l}27 \\
0 \\
0 \\
0 \\
0.08 \\
0.21\end{array}$ & $\begin{array}{l}30 \\
0 \\
0 \\
0 \\
0.86 \\
0\end{array}$ & $\begin{array}{l}\frac{31}{0} \\
0 \\
0 \\
0.09 \\
0.14\end{array}$ & $\begin{array}{l}\frac{32}{\underline{0}} \\
\underline{0} \\
\underline{0} \\
\underline{0.65}\end{array}$ & $\begin{array}{l}\frac{41}{\underline{0}} \\
\underline{0} \\
\underline{0.64}\end{array}$ & $\frac{\frac{42}{0.15}}{\frac{0}{0}}$ & $\begin{array}{l}43 \\
0 \\
0 \\
0 \\
0 \\
0\end{array}$ & $\frac{44}{\frac{0}{0}} \frac{\underline{0}}{\underline{0}}$ & & $\frac{\frac{55}{0.45}}{\frac{0.82}{1.24}} \frac{0}{\underline{0}}$ & & & & & & & $\frac{\frac{2}{1.04}}{\frac{0}{0}}$ & $\begin{array}{l}\frac{3}{0} \\
0.03 \\
0.36 \\
0\end{array}$ & \\
\hline $\begin{array}{l}\text { Ring } \\
\text { Core }\end{array}$ & $\begin{array}{l}\frac{10}{\underline{0}} \\
\frac{0.65}{2.00} \\
\underline{0}\end{array}$ & $\begin{array}{l}\frac{11}{0} \\
0 \\
0 \\
6.43\end{array}$ & $\begin{array}{l}\frac{16}{0} \\
0 \\
4.00\end{array}$ & $\begin{array}{l}\frac{17}{\underline{0}} \\
\underline{2.11} \\
\underline{0.97}\end{array}$ & $\begin{array}{l}\frac{21}{\underline{0}} \\
\underline{0.80} \\
\frac{0.15}{1.25} \\
\underline{1.00}\end{array}$ & $\begin{array}{l}\frac{23}{\underline{0}} \\
\frac{1.73}{0.45} \\
\frac{0.77}{\underline{0}}\end{array}$ & $\begin{array}{l}\frac{24}{0} \\
0 \\
0 \\
1.56 \\
0\end{array}$ & $\begin{array}{l}28 \\
0 \\
0 \\
0.21 \\
1.65 \\
0\end{array}$ & & & & & & & $\begin{array}{l}\frac{56}{\underline{0}} \\
\underline{0} \\
\underline{0} \\
\underline{0} \\
\underline{0.66}\end{array}$ & $\begin{array}{l}\frac{57}{0} \\
\underline{0} \\
\underline{0} \\
\underline{0} \\
\underline{0.03}\end{array}$ & $\begin{array}{l}\frac{58}{0} \\
0 \\
0 \\
0 \\
1.32\end{array}$ & $\begin{array}{l}\frac{61}{\underline{0}} \\
\frac{0.61}{\underline{0}} \\
\underline{0.69} \\
\underline{3.68}\end{array}$ & $\begin{array}{l}\frac{62}{0} \\
0 \\
0 \\
0.24 \\
5.71\end{array}$ & $\begin{array}{l}\frac{63}{\frac{0.23}{0.43}} \\
\frac{0}{0} \\
\underline{1.54} \\
\underline{3.13}\end{array}$ & $\begin{array}{l}\frac{64}{0} \\
0 \\
0 \\
0.43 \\
2.31\end{array}$ & $\frac{\frac{1}{\frac{0.20}{0.60}}}{\frac{0.60}{0.69}}$ & & \\
\hline $\begin{array}{l}\text { Sargasso } \\
\text { Sea } \\
\& \\
\text { Gulf } \\
\text { Stream }\end{array}$ & & & & & & & & & & $\begin{array}{l}\frac{49}{0.16} \\
\frac{0.02}{\underline{0}} \\
\underline{0.36}\end{array}$ & $\begin{array}{l}\frac{50}{0} \\
0 \\
0 \\
0\end{array}$ & $\begin{array}{l}51 \\
0 \\
0 \\
0 \\
0\end{array}$ & $\frac{\frac{52}{0.43}}{\frac{0.39}{0.17}} \frac{0}{\underline{0}}$ & & & & & & & & & & & \\
\hline $\begin{array}{l}\text { Slope } \\
\text { Water }\end{array}$ & $\frac{8}{-}$ & $\frac{9}{0.17}$ & $\frac{18}{0}$ & & $\frac{19}{0}$ & $\frac{20}{0}$ & $\frac{33}{0.12}$ & $\frac{34}{0}$ & & $\frac{35}{0.33}$ & $\frac{47}{0}$ & $\frac{48}{0.02}$ & $\frac{53}{0.21}$ & $\frac{54}{0.10}$ & $\frac{59}{0}$ & $\frac{60}{0}$ & $\frac{65}{0.16}$ & $\frac{66}{0.18}$ & & & & $\frac{4}{0.01}$ & $\frac{5}{0.10}$ & $\frac{6}{0}$ \\
\hline $\begin{array}{l}\text { High } \\
\text { Velocity } \\
\text { Region }\end{array}$ & $\frac{12}{0.42}$ & $\frac{13}{1.13}$ & $\frac{14}{0.06}$ & $\frac{15}{0.66}$ & $\frac{27}{0.07}$ & $\frac{29}{0.11}$ & $\frac{30}{0.17}$ & $\frac{31}{0.05}$ & $\frac{32}{-}$ & $\frac{41}{0.16}$ & $\frac{42}{0.06}$ & $\frac{43}{0}$ & $\frac{44}{0}$ & & $\frac{55}{0.75}$ & & & & & & & $\frac{2}{1.54}$ & $\frac{3}{0.10}$ & \\
\hline $\begin{array}{l}\text { Ring } \\
\text { Core }\end{array}$ & $\frac{10}{0.66}$ & $\frac{11}{1.61}$ & $\frac{16}{-}$ & $\frac{17}{1.01}$ & $\frac{21}{0.76}$ & $\frac{23}{0.68}$ & $\frac{24}{0.31}$ & $\frac{28}{0.47}$ & & $\frac{37}{0.08}$ & $\frac{38}{0.14}$ & $\frac{39}{0.41}$ & & & $\frac{56}{0.13}$ & $\frac{57}{0.01}$ & $\frac{58}{0.26}$ & $\frac{61}{1.00}$ & $\frac{62}{1.19}$ & $\frac{63}{1.03}$ & $\frac{64}{0.55}$ & $\frac{1}{0.37}$ & & \\
\hline SS \& GS & & & & & & & & & & $\frac{49}{0.14}$ & $\frac{50}{0}$ & $\frac{51}{0}$ & $\frac{52}{0.50}$ & & & & & & & & & & & \\
\hline
\end{tabular}


Table 87. Volume of Lampanyctus macdonaldi $\left(\mathrm{ml} / 10,000 \mathrm{~m}^{3}\right)$.

\begin{tabular}{|c|c|c|c|c|c|c|c|c|c|c|c|c|c|c|c|c|c|c|c|c|c|c|c|c|}
\hline & \multicolumn{4}{|c|}{$\begin{array}{c}\text { Oceanus } 118 \\
\text { April }\end{array}$} & \multicolumn{5}{|c|}{$\begin{array}{c}\text { Oceanus } 121 \\
\text { June }\end{array}$} & \multicolumn{5}{|c|}{$\begin{array}{l}\text { Oceanus } 125 \\
\text { August }\end{array}$} & \multicolumn{7}{|c|}{$\begin{array}{l}\text { Knorr } 98 \\
\text { Sept-Oct }\end{array}$} & \multicolumn{3}{|c|}{$\begin{array}{c}\text { A2 } 110 \\
\text { Sept-0ct }\end{array}$} \\
\hline $\begin{array}{l}\text { Slope } \\
\text { Water }\end{array}$ & $\begin{array}{l}\frac{8}{\overline{0}} \\
\underline{0} \\
\underline{0} \\
\underline{0}\end{array}$ & $\begin{array}{l}9 \\
0 \\
0 \\
0 \\
0\end{array}$ & & & $\begin{array}{l}19 \\
\underline{0} \\
\underline{0} \\
\underline{0} \\
\underline{0} \\
\underline{0}\end{array}$ & $\begin{array}{l}20 \\
0 \\
0 \\
0 \\
0.36 \\
0\end{array}$ & $\begin{array}{l}\frac{33}{\underline{0}} \\
\underline{0} \\
\underline{0} \\
\underline{0} \\
\underline{0.68}\end{array}$ & $\begin{array}{l}\frac{34}{\underline{0}} \\
\underline{0} \\
\underline{0}\end{array}$ & & $\begin{array}{l}35 \\
0 \\
0 \\
0 \\
0 \\
0\end{array}$ & $\begin{array}{l}\frac{47}{0} \\
\underline{0} \\
\underline{0} \\
\underline{0}\end{array}$ & $\begin{array}{l}48 \\
0 \\
0 \\
0 \\
0\end{array}$ & $\begin{array}{l}53 \\
\underline{0} \\
\underline{0} \\
\underline{0} \\
\underline{0}\end{array}$ & $\begin{array}{c}54 \\
0 \\
0 \\
0.03 \\
0\end{array}$ & $\begin{array}{l}\frac{59}{0} \\
\underline{0} \\
\underline{0} \\
\underline{0} \\
\underline{0}\end{array}$ & $\begin{array}{l}\frac{60}{0} \\
0 \\
0 \\
0.09 \\
0\end{array}$ & $\begin{array}{l}\frac{65}{\underline{0}} \\
\underline{0} \\
\underline{0} \\
\underline{0} \\
\underline{0}\end{array}$ & $\begin{array}{l}66 \\
0 \\
0 \\
0 \\
0 \\
0\end{array}$ & & & & $\begin{array}{l}4 \\
0 \\
0 \\
0 \\
0\end{array}$ & $\begin{array}{l}\frac{5}{0} \\
\underline{0} \\
\underline{0} \\
\underline{0}\end{array}$ & \\
\hline $\begin{array}{l}\text { High } \\
\text { Velocity } \\
\text { Region }\end{array}$ & $\begin{array}{l}\frac{12}{0} \\
0 \\
0 \\
1.04\end{array}$ & $\begin{array}{l}\frac{13}{\underline{0}} \\
\frac{0}{0}\end{array}$ & $\begin{array}{l}14 \\
\underline{0} \\
\underline{0} \\
\underline{0} \\
\underline{0}\end{array}$ & $\begin{array}{c}\frac{15}{0} \\
0 \\
0 \\
13.33\end{array}$ & $\begin{array}{l}26 \\
0 \\
0 \\
0 \\
0 \\
-\end{array}$ & $\begin{array}{l}27 \\
0 \\
0 \\
0 \\
0 \\
0.61\end{array}$ & $\begin{array}{l}30 \\
0 \\
0 \\
0 \\
0 \\
0.14\end{array}$ & $\begin{array}{l}31 \\
0 \\
0 \\
0 \\
0 \\
0\end{array}$ & $\begin{array}{l}\frac{32}{\underline{0}} \\
\underline{0} \\
\underline{0} \\
\underline{0}\end{array}$ & $\begin{array}{l}\frac{41}{\underline{0}} \\
\frac{0.03}{\underline{0}}\end{array}$ & $\begin{array}{l}\frac{42}{\underline{0}} \\
\underline{0} \\
\underline{5.61} \\
\underline{0.33}\end{array}$ & $\begin{array}{l}\frac{43}{0} \\
0 \\
0 \\
0.04 \\
0\end{array}$ & $\begin{array}{l}\frac{44}{0} \\
\frac{0}{0} \\
\underline{0}\end{array}$ & & $\begin{array}{l}\frac{55}{0} \\
\underline{0} \\
\underline{0} \\
\underline{0}\end{array}$ & & & & & & & $\begin{array}{l}\frac{2}{0} \\
\underline{0} \\
\underline{0} \\
\underline{0}\end{array}$ & $\begin{array}{l}\frac{3}{0} \\
0 \\
0 \\
0.31\end{array}$ & \\
\hline $\begin{array}{l}\text { Ring } \\
\text { Core }\end{array}$ & $\begin{array}{l}10 \\
\underline{0} \\
\underline{0} \\
\underline{0} \\
\underline{0}\end{array}$ & $\begin{array}{l}11 \\
0 \\
0 \\
0 \\
0\end{array}$ & $\begin{array}{l}\frac{16}{0} \\
0 \\
0\end{array}$ & $\begin{array}{l}\frac{17}{\underline{0}} \\
\underline{0} \\
\underline{0}\end{array}$ & $\begin{array}{l}21 \\
\underline{0} \\
\underline{0} \\
\underline{0} \\
\underline{0} \\
\underline{0}\end{array}$ & $\begin{array}{l}\frac{23}{\underline{0}} \\
\underline{0} \\
\underline{0} \\
\underline{0} \\
\underline{0}\end{array}$ & $\begin{array}{l}24 \\
0 \\
0 \\
0 \\
0 \\
0\end{array}$ & $\begin{array}{l}28 \\
0 \\
0 \\
0 \\
0 \\
2.56\end{array}$ & & & & & & & $\begin{array}{l}56 \\
\underline{0} \\
\underline{0} \\
\underline{0} \\
\underline{0} \\
\underline{0}\end{array}$ & $\begin{array}{l}57 \\
\underline{0} \\
\underline{0} \\
\underline{0} \\
\underline{0} \\
\underline{0}\end{array}$ & $\begin{array}{l}58 \\
0 \\
0 \\
0 \\
0 \\
0\end{array}$ & $\begin{array}{l}61 \\
\underline{0} \\
0 \\
\underline{0} \\
\underline{0} \\
\underline{0}\end{array}$ & $\begin{array}{l}62 \\
0 \\
0 \\
0 \\
0 \\
0\end{array}$ & $\begin{array}{l}\frac{63}{0} \\
\underline{0} \\
\underline{0} \\
\underline{0} \\
\underline{0}\end{array}$ & $\begin{array}{l}64 \\
0 \\
0 \\
0 \\
0 \\
0\end{array}$ & $\begin{array}{l}\frac{1}{0} \\
\underline{0} \\
\underline{0} \\
\underline{0}\end{array}$ & & \\
\hline $\begin{array}{l}\text { Sargasso } \\
\text { Sea } \\
\& \\
\text { Gulf } \\
\text { Stream }\end{array}$ & & & & & & & & & & $\begin{array}{l}\frac{49}{\underline{0}} \\
\underline{0} \\
\underline{0} \\
0.03 \\
\end{array}$ & $\begin{array}{l}50 \\
0 \\
0 \\
0 \\
0\end{array}$ & $\begin{array}{l}\frac{51}{0} \\
0 \\
0 \\
0\end{array}$ & $\begin{array}{l}\frac{52}{\underline{0}} \\
\underline{0} \\
\underline{0} \\
\underline{0}\end{array}$ & & & & & & & & & & & \\
\hline $\begin{array}{l}\text { Slope } \\
\text { Water }\end{array}$ & $\frac{8}{-}$ & $\frac{9}{0}$ & $\frac{18}{0}$ & & $\frac{19}{0}$ & $\frac{20}{0.09}$ & $\frac{33}{0.34}$ & $\frac{34}{0}$ & & $\frac{35}{0}$ & $\frac{47}{0}$ & $\frac{48}{0}$ & $\frac{53}{0}$ & $\frac{54}{0.01}$ & $\frac{59}{0}$ & $\frac{60}{0.02}$ & $\frac{65}{0}$ & $\frac{66}{0}$ & & & & $\frac{4}{0}$ & $\frac{5}{0}$ & $\frac{6}{0.04}$ \\
\hline $\begin{array}{l}\text { High } \\
\text { Velocity } \\
\text { Region }\end{array}$ & $\frac{12}{0.26}$ & $\frac{13}{0}$ & $\frac{14}{0}$ & $\frac{15}{3.33}$ & $\frac{27}{0.15}$ & $\frac{29}{0}$ & $\frac{30}{0.03}$ & $\frac{31}{0}$ & $\frac{32}{-}$ & $\frac{41}{0.01}$ & $\frac{42}{1.19}$ & $\frac{43}{0.01}$ & $\frac{44}{0}$ & & $\frac{55}{0}$ & & & & & & & $\frac{2}{0}$ & $\frac{3}{0.08}$ & \\
\hline $\begin{array}{l}\text { Ring } \\
\text { Core }\end{array}$ & $\frac{10}{0}$ & $\frac{11}{0}$ & $\frac{16}{-}$ & $\frac{17}{0}$ & $\frac{21}{0}$ & $\frac{23}{0}$ & $\frac{24}{0}$ & $\frac{28}{0}$ & & $\frac{37}{0}$ & $\frac{38}{0}$ & $\frac{39}{0}$ & & & $\frac{56}{0}$ & $\frac{57}{0}$ & $\frac{58}{0}$ & $\frac{61}{0}$ & $\frac{62}{0}$ & $\frac{63}{0}$ & $\frac{64}{0}$ & $\frac{1}{0}$ & & \\
\hline SS \& GS & & & & & & & & & & $\frac{49}{0.01}$ & $\frac{50}{0}$ & $\frac{51}{0}$ & $\frac{52}{0}$ & & & & & & & & & & & \\
\hline
\end{tabular}


Table 88. Abundance of Lampanyctus photonotus (specimens $/ 10,000 \mathrm{~m}^{3}$ ).

\begin{tabular}{|c|c|c|c|c|c|c|c|c|c|c|c|c|c|c|c|c|c|c|c|c|c|c|c|c|}
\hline & \multicolumn{4}{|c|}{$\begin{array}{c}\text { Oceanus } 118 \\
\text { Aprif }\end{array}$} & \multicolumn{5}{|c|}{$\begin{array}{c}\text { Oceanus } 121 \\
\text { June }\end{array}$} & \multicolumn{5}{|c|}{$\begin{array}{c}\text { Oceanus } 125 \\
\text { August }\end{array}$} & \multicolumn{7}{|c|}{$\begin{array}{l}\text { Knorr } 98 \\
\text { Sept-0ct }\end{array}$} & \multicolumn{3}{|c|}{$\begin{array}{c}\text { A2 } 110 \\
\text { Sept-0ct }\end{array}$} \\
\hline $\begin{array}{l}\text { Slope } \\
\text { Water }\end{array}$ & $\begin{array}{l}\frac{8}{\bar{z}} \\
\underline{0} \\
\underline{0} \\
\underline{0}\end{array}$ & $\begin{array}{l}9 \\
0 \\
0 \\
0 \\
0\end{array}$ & & & $\begin{array}{l}\frac{19}{\underline{0}} \\
\underline{0} \\
\underline{0} \\
\underline{0} \\
\underline{0}\end{array}$ & $\begin{array}{c}20 \\
0 \\
0 \\
0 \\
0.5 \\
0\end{array}$ & $\begin{array}{r}33 \\
\underline{0} \\
\underline{0} \\
\underline{0} \\
\underline{0} \\
\underline{0}\end{array}$ & $\begin{array}{r}34 \\
\underline{0} \\
\underline{0} \\
\underline{0}\end{array}$ & & $\begin{array}{r}35 \\
0 \\
0 \\
0 \\
0 \\
0\end{array}$ & $\begin{array}{r}47 \\
\underline{0} \\
\underline{0} \\
\underline{0} \\
\underline{0}\end{array}$ & $\begin{array}{r}48 \\
0 \\
0 \\
0 \\
0\end{array}$ & $\begin{array}{r}\frac{53}{\underline{0}} \\
\underline{0} \\
\underline{0} \\
\underline{0}\end{array}$ & $\begin{array}{l}54 \\
0 \\
0 \\
0 \\
0\end{array}$ & $\begin{array}{r}59 \\
\underline{0} \\
\underline{0} \\
\underline{0} \\
\underline{0} \\
\underline{0}\end{array}$ & $\begin{array}{c}60 \\
0 \\
0 \\
0.3 \\
0 \\
0\end{array}$ & $\begin{array}{c}\frac{65}{1.7} \\
\underline{0} \\
\underline{0} \\
\frac{0.4}{\underline{0}}\end{array}$ & $\begin{array}{c}66 \\
0 \\
0 \\
0 \\
2.4 \\
0\end{array}$ & & & & $\begin{array}{c}\frac{4}{0} \\
0 \\
2.2 \\
0\end{array}$ & $\begin{array}{l}5 \\
\underline{0} \\
\underline{0} \\
\underline{0} \\
\underline{0}\end{array}$ & \\
\hline $\begin{array}{l}\text { High } \\
\text { Velocity } \\
\text { Region }\end{array}$ & $\begin{array}{r}12 \\
0 \\
0 \\
0 \\
0\end{array}$ & $\frac{13}{\underline{0}}$ & $\frac{14}{\frac{0.3}{\underline{0}}}$ & $\begin{array}{r}15 \\
0 \\
0 \\
0 \\
0\end{array}$ & $\begin{array}{r}26 \\
0 \\
0 \\
0 \\
0 \\
-\end{array}$ & $\begin{array}{r}27 \\
0 \\
0 \\
0 \\
0 \\
0\end{array}$ & $\begin{array}{c}30 \\
0 \\
0 \\
0 \\
0 \\
0.2\end{array}$ & $\begin{array}{c}31 \\
0 \\
0 \\
0 \\
0.3 \\
0\end{array}$ & $\begin{array}{l}32 \\
\underline{0} \\
\underline{0} \\
\underline{0} \\
\underline{0}\end{array}$ & $\begin{array}{r}41 \\
\underline{0} \\
\underline{0} \\
\underline{0}\end{array}$ & $\begin{array}{r}\frac{42}{\underline{0}} \\
\underline{0} \\
\underline{0} \\
\underline{0}\end{array}$ & $\begin{array}{r}43 \\
0 \\
0 \\
0 \\
0 \\
0\end{array}$ & $\begin{array}{l}\frac{44}{\underline{0}} \\
\frac{0.3}{\underline{0}}\end{array}$ & & $\begin{array}{c}\frac{55}{\underline{0.8}} \\
\underline{0.4} \\
\underline{0}\end{array}$ & & & & & & & $\frac{\frac{2}{1.0}}{\frac{0}{0}} \frac{0}{\underline{0}}$ & $\begin{array}{c}3 \\
0 \\
0 \\
0.4 \\
0\end{array}$ & \\
\hline $\begin{array}{l}\text { Ring } \\
\text { Core }\end{array}$ & $\begin{array}{l}10 \\
0.4 \\
\underline{0} \\
\underline{0} \\
\underline{0}\end{array}$ & $\begin{array}{r}11 \\
0 \\
0 \\
0 \\
0\end{array}$ & $\begin{array}{r}16 \\
0 \\
0 \\
0\end{array}$ & $\frac{\frac{17}{1.1}}{\frac{0}{0}}$ & $\begin{array}{c}21 \\
1.0 \\
\underline{0} \\
\underline{0} \\
\underline{0} \\
\underline{0}\end{array}$ & $\begin{array}{r}23 \\
\underline{0} \\
\underline{0} \\
\underline{0} \\
\underline{0} \\
\underline{0}\end{array}$ & $\begin{array}{c}24 \\
0.3 \\
0 \\
0 \\
0 \\
0\end{array}$ & $\begin{array}{c}28 \\
0 \\
0 \\
0 \\
0.8 \\
0\end{array}$ & & & & & & & $\begin{array}{l}\frac{56}{2.1} \\
\underline{0} \\
\underline{0} \\
\underline{0} \\
\underline{0}\end{array}$ & $\begin{array}{c}\frac{57}{1.6} \\
\frac{0.3}{\underline{0}} \\
\underline{0} \\
\underline{1.6}\end{array}$ & $\begin{array}{c}58 \\
0 \\
0 \\
0 \\
1.3 \\
1.6\end{array}$ & $\begin{array}{l}\frac{61}{0.5} \\
\frac{0.3}{0.3} \\
\underline{0.0} \\
\underline{1.3}\end{array}$ & $\begin{array}{c}62 \\
0 \\
0 \\
0.2 \\
1.4 \\
4.3\end{array}$ & $\begin{array}{l}\frac{63}{0.6} \\
\underline{0} \\
0.5 \\
\underline{0.4} \\
\underline{0.6}\end{array}$ & $\begin{array}{c}\frac{64}{0.2} \\
0 \\
0.7 \\
1.0 \\
0\end{array}$ & $\begin{array}{l}\frac{1}{0.6} \\
\underline{0} \\
\underline{0} \\
\underline{0.8}\end{array}$ & & \\
\hline $\begin{array}{l}\text { Sargasso } \\
\text { Sea } \\
\& \\
\text { Gulf } \\
\text { Stream }\end{array}$ & & & & & & & & & & $\begin{array}{l}49 \\
\underline{0} \\
\underline{0} \\
\underline{0} \\
\underline{0}\end{array}$ & $\begin{array}{c}50 \\
0 \\
0 \\
0 \\
0.6\end{array}$ & $\begin{array}{c}51 \\
0 \\
0 \\
0.2 \\
0\end{array}$ & $\begin{array}{l}52 \\
\underline{0} \\
\underline{0} \\
\underline{0}\end{array}$ & & & & & & & & & & & \\
\hline $\begin{array}{l}\text { Slope } \\
\text { Water }\end{array}$ & $\frac{8}{-}$ & $\frac{9}{0}$ & $\frac{18}{0}$ & & $\frac{19}{0}$ & $\frac{20}{0.1}$ & $\frac{33}{0}$ & $\frac{34}{0}$ & & $\frac{35}{0}$ & $\frac{47}{0}$ & $\frac{48}{0}$ & $\frac{53}{0}$ & $\frac{54}{0}$ & $\frac{59}{0}$ & $\frac{60}{0.1}$ & $\frac{65}{0.4}$ & $\frac{66}{0.5}$ & & & & $\frac{4}{0.6}$ & $\frac{5}{0}$ & $\frac{6}{0}$ \\
\hline $\begin{array}{l}\text { High } \\
\text { Ve1ocity } \\
\text { Region }\end{array}$ & $\frac{12}{0}$ & $\frac{13}{0}$ & $\frac{14}{0.1}$ & $\frac{15}{0}$ & $\frac{27}{0}$ & $\frac{29}{0.3}$ & $\frac{30}{0.1}$ & $\frac{31}{0.1}$ & $\frac{32}{-}$ & $\frac{41}{0}$ & $\frac{42}{0}$ & $\frac{43}{0}$ & $\frac{44}{0.1}$ & & $\frac{55}{0.3}$ & & & & & & & $\frac{2}{0.3}$ & $\frac{3}{0.1}$ & \\
\hline $\begin{array}{l}\text { Ring } \\
\text { Core }\end{array}$ & $\frac{10}{0.1}$ & $\frac{11}{0}$ & $\frac{16}{-}$ & $\frac{17}{0.4}$ & $\frac{21}{0.1}$ & $\frac{23}{0}$ & $\frac{24}{0.1}$ & $\frac{28}{0.2}$ & & $\frac{37}{0}$ & $\frac{38}{0}$ & $\frac{39}{0}$ & & & $\frac{56}{0.4}$ & $\frac{57}{0.7}$ & $\frac{58}{0.6}$ & $\frac{61}{1.4}$ & $\frac{62}{1.2}$ & $\frac{63}{0.7}$ & $\frac{64}{0.4}$ & $\frac{1}{0.4}$ & & \\
\hline SS \& GS & & & & & & & & & & $\frac{49}{0}$ & $\frac{50}{0.2}$ & $\frac{51}{0.1}$ & $\frac{52}{0}$ & & & & & & & & & & & \\
\hline
\end{tabular}


Table 89. Abundance of Lampanyctus pusillus (specimens $/ 10,000 \mathrm{~m}^{3}$ ).

\begin{tabular}{|c|c|c|c|c|c|c|c|c|c|c|c|c|c|c|c|c|c|c|c|c|c|c|c|c|}
\hline & & $\begin{array}{r}\text { Ocean } \\
\text { Apr }\end{array}$ & 118 & & & & $\begin{array}{l}\text { eanus } \\
\text { June }\end{array}$ & 121 & & & $\begin{array}{r}\text { Oce } \\
\mathrm{A}\end{array}$ & $\begin{array}{l}\text { anus } 1 \\
\text { ugust }\end{array}$ & & & & & $\begin{array}{l}\text { Knor } \\
\text { Sept }\end{array}$ & $\begin{array}{r}r 98 \\
-0 c t \\
\end{array}$ & & & & $\begin{array}{r}A \\
S e\end{array}$ & $\begin{array}{r}110 \\
t-0 c t\end{array}$ & \\
\hline $\begin{array}{l}\text { Slope } \\
\text { Water }\end{array}$ & $\begin{array}{l}\frac{8}{\overline{\overline{0}}} \\
\underline{0} \\
\underline{0}\end{array}$ & $\begin{array}{l}\frac{9}{0} \\
0.6 \\
0 \\
0\end{array}$ & & & $\frac{\frac{19}{11.2}}{\frac{0}{0}}$ & $\begin{array}{c}20 \\
0.5 \\
0 \\
0 \\
0 \\
0\end{array}$ & 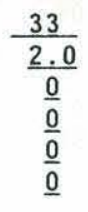 & $\frac{34}{\underline{0.5}} \frac{0.4}{\underline{0.5}}$ & & $\begin{array}{c}35 \\
0 \\
0 \\
2.3 \\
0 \\
0\end{array}$ & $\frac{47}{\frac{0.4}{\underline{0}}} \frac{\underline{0}}{\underline{0}}$ & $\begin{array}{c}48 \\
0 \\
0.3 \\
0 \\
0\end{array}$ & $\frac{53}{\frac{0.4}{\underline{0}}}$ & $\begin{array}{c}54 \\
0 \\
0 \\
3.0 \\
0\end{array}$ & 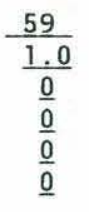 & $\begin{array}{c}60 \\
0 \\
0 \\
2.4 \\
0 \\
0\end{array}$ & $\frac{\frac{65}{2.2}}{\frac{\underline{0}}{\underline{0}}}$ & $\begin{array}{c}\frac{66}{0} \\
10.0 \\
1.5 \\
0 \\
0\end{array}$ & & & & $\begin{array}{c}4 \\
0 \\
0.4 \\
0.6 \\
0\end{array}$ & $\begin{array}{l}\frac{5}{0.3} \\
\frac{0}{0.3} \\
\underline{0}\end{array}$ & \\
\hline $\begin{array}{l}\text { High } \\
\text { Velocity } \\
\text { Region }\end{array}$ & $\begin{array}{c}12 \\
0 \\
0 \\
0.8 \\
0\end{array}$ & $\frac{13}{\underline{0}}$ & $\begin{array}{l}\frac{14}{\underline{0}} \\
\underline{0} \\
\underline{0} \\
\underline{0}\end{array}$ & $\begin{array}{r}15 \\
0 \\
0 \\
0 \\
0\end{array}$ & $\begin{array}{c}26 \\
0 \\
0 \\
0 \\
0.3 \\
-\end{array}$ & $\begin{array}{c}27 \\
0 \\
0 \\
0 \\
0.8 \\
0\end{array}$ & $\begin{array}{c}30 \\
0 \\
0 \\
3.1 \\
0 \\
0\end{array}$ & $\begin{array}{c}31 \\
0 \\
0 \\
1.4 \\
0 \\
0\end{array}$ & $\begin{array}{l}\frac{32}{\underline{0}} \\
\underline{0} \\
\underline{0.5} \\
\underline{0}\end{array}$ & $\frac{\frac{41}{1.3}}{\frac{0.6}{0}}$ & $\begin{array}{l}\frac{42}{1.1} \\
\underline{0} \underline{0.5} \\
\underline{0}\end{array}$ & $\begin{array}{c}43 \\
0 \\
0 \\
0 \\
0.9 \\
0\end{array}$ & $\frac{\frac{44}{0.4}}{\frac{0}{0}}$ & & $\begin{array}{l}\frac{55}{0.8} \\
\frac{0}{0.4} \\
\underline{0}\end{array}$ & & & & & & & $\frac{\frac{2}{1.5}}{\frac{0}{0}}$ & $\begin{array}{c}\frac{3}{0.7} \\
3.5 \\
0.4 \\
0\end{array}$ & \\
\hline $\begin{array}{l}\text { Ring } \\
\text { Core }\end{array}$ & $\frac{\frac{10}{\frac{1.6}{0.6}}}{\frac{0}{\underline{0}}}$ & $\begin{array}{c}11 \\
0 \\
0 \\
0 \\
0\end{array}$ & $\begin{array}{c}16 \\
0 \\
0 \\
4.0\end{array}$ & $\frac{17}{\frac{1.1}{\underline{0}}}$ & $\begin{array}{l}\frac{21}{\frac{4.0}{0.8}} \\
\frac{0.6}{2.1} \\
\frac{0}{\underline{0}}\end{array}$ & $\frac{\frac{23}{3.5}}{\frac{3.3}{1.3}} \frac{0.5}{\frac{4.1}{\underline{0}}}$ & $\begin{array}{c}\frac{24}{0.3} \\
0 \\
1.1 \\
0.6 \\
0.6\end{array}$ & $\begin{array}{c}28 \\
0.4 \\
0 \\
8.9 \\
0 \\
0\end{array}$ & & & & & & & $\begin{array}{l}\frac{56}{2.1} \\
\frac{0}{0} \\
\underline{0} \\
\frac{1.1}{0.3}\end{array}$ & $\begin{array}{l}\frac{57}{2.2} \\
\underline{0} \\
\underline{0.5} \\
\underline{4.7} \\
\underline{0.5}\end{array}$ & $\begin{array}{c}58 \\
0 \\
0 \\
0 \\
6.8 \\
3.2\end{array}$ & $\begin{array}{l}\frac{61}{\underline{0.2}} \\
\underline{0} \\
\underline{0} \\
\underline{6.5} \\
\underline{0.5}\end{array}$ & $\begin{array}{c}62 \\
0 \\
0 \\
0 \\
6.8 \\
1.8\end{array}$ & $\begin{array}{c}\frac{63}{1.1} \\
\underline{0} \\
\underline{0} \\
\underline{5.0} \\
\underline{0.3}\end{array}$ & $\begin{array}{c}64 \\
0 \\
0 \\
5.7 \\
2.9 \\
0\end{array}$ & $\begin{array}{l}\frac{1}{4.6} \\
\underline{0} \\
\underline{0.6} \\
\underline{0.8}\end{array}$ & & \\
\hline $\begin{array}{l}\text { Sargasso } \\
\text { Sea } \\
\& \\
\text { Gulf } \\
\text { Stream }\end{array}$ & & & & & & & & & & $\begin{array}{l}\frac{49}{\frac{4.7}{0}} \\
\frac{0.2}{\underline{0}}\end{array}$ & $\begin{array}{c}50 \\
0 \\
0 \\
3.1 \\
0\end{array}$ & $\begin{array}{c}51 \\
0 \\
0 \\
10.2 \\
0.3\end{array}$ & $\begin{array}{l}\frac{52}{\underline{0}} \\
\frac{0.6}{\underline{0}} \\
\underline{0}\end{array}$ & & & & & & & & & & & \\
\hline $\begin{array}{l}\text { Slope } \\
\text { Water }\end{array}$ & $\frac{8}{-}$ & $\frac{9}{0.2}$ & $\frac{18}{0}$ & & $\frac{19}{1.2}$ & $\frac{20}{0.1}$ & $\frac{33}{0.1}$ & $\frac{34}{0.5}$ & & $\frac{35}{0.5}$ & $\frac{47}{0.1}$ & $\frac{48}{0.1}$ & $\frac{53}{0.1}$ & $\frac{54}{0.8}$ & $\frac{59}{0.2}$ & $\frac{60}{0.5}$ & $\frac{65}{0.4}$ & $\frac{66}{1.3}$ & & & & $\frac{4}{0.3}$ & $\frac{5}{0.2}$ & $\frac{6}{0}$ \\
\hline $\begin{array}{l}\text { High } \\
\text { Velocity } \\
\text { Region }\end{array}$ & $\frac{12}{0.2}$ & $\frac{13}{0}$ & $\frac{14}{0}$ & $\frac{15}{0}$ & $\frac{27}{0.2}$ & $\frac{29}{1.2}$ & $\frac{30}{0.6}$ & $\frac{31}{0.3}$ & $\frac{32}{-}$ & $\frac{41}{0.8}$ & $\frac{42}{0.5}$ & $\frac{43}{0.2}$ & $\frac{44}{0.2}$ & & $\frac{55}{0.3}$ & & & & & & & $\frac{2}{0.4}$ & $\frac{3}{1.2}$ & \\
\hline $\begin{array}{l}\text { Ring } \\
\text { Core }\end{array}$ & $\frac{10}{0.6}$ & $\frac{11}{0}$ & $\frac{16}{-}$ & $\frac{17}{0.3}$ & $\frac{21}{1.0}$ & $\frac{23}{1.8}$ & $\frac{24}{0.6}$ & $\frac{28}{2.3}$ & & $\frac{37}{0.1}$ & $\frac{38}{0.3}$ & $\frac{39}{0.3}$ & & & $\frac{56}{0.7}$ & $\frac{57}{1.6}$ & $\frac{58}{2.0}$ & $\frac{61}{1.4}$ & $\frac{62}{1.7}$ & $\frac{63}{1.5}$ & $\frac{64}{1.2}$ & $\frac{1}{1.5}$ & & \\
\hline SS \& GS & & & & & & & & & & $\frac{49}{1.2}$ & $\frac{50}{0.8}$ & $\frac{51}{2.6}$ & $\frac{52}{0.2}$ & & & & & & & & & & & \\
\hline
\end{tabular}


Table 90. Volume of Lampanyctus pusillus (m1/10,000 $\left.\mathrm{m}^{3}\right)$.

\begin{tabular}{|c|c|c|c|c|c|c|c|c|c|c|c|c|c|c|c|c|c|c|c|c|c|c|c|c|}
\hline & \multicolumn{4}{|c|}{$\begin{array}{c}\text { Oceanus } 118 \\
\text { Apri1 }\end{array}$} & \multicolumn{5}{|c|}{$\begin{array}{l}\text { Oceanus } 121 \\
\text { June }\end{array}$} & \multicolumn{5}{|c|}{$\begin{array}{c}\text { Oceanus } 125 \\
\text { August }\end{array}$} & \multicolumn{7}{|c|}{$\begin{array}{l}\text { Knorr } 98 \\
\text { Sept-Oct }\end{array}$} & \multicolumn{3}{|c|}{$\begin{array}{c}\text { A2 } 110 \\
\text { Sept-0ct }\end{array}$} \\
\hline $\begin{array}{l}\text { Slope } \\
\text { Water }\end{array}$ & $\begin{array}{l}\frac{8}{\overline{0}} \\
\underline{0} \\
\underline{0}\end{array}$ & $\begin{array}{l}\frac{9}{0} \\
0.06 \\
0 \\
0\end{array}$ & & & $\frac{\frac{19}{1.10}}{\frac{0}{0}}$ & $\begin{array}{l}20 \\
0 \\
0 \\
0 \\
0 \\
0\end{array}$ & $\begin{array}{l}\frac{33}{0.17} \\
\frac{0}{0} \\
\underline{0} \\
\underline{0}\end{array}$ & $\frac{\frac{34}{0.05}}{\frac{0.04}{0.04}}$ & & $\begin{array}{l}\frac{35}{0} \\
0 \\
0.37 \\
0 \\
0\end{array}$ & $\frac{\frac{47}{0.02}}{\frac{0}{\underline{0}}}$ & $\begin{array}{l}\frac{48}{0} \\
0.03 \\
0 \\
0\end{array}$ & $\frac{\frac{53}{0.04}}{\frac{0.0}{\underline{0}}}$ & $\begin{array}{l}54 \\
0 \\
0 \\
0.06 \\
0\end{array}$ & $\begin{array}{l}\frac{59}{0.23} \\
\frac{0}{0} \\
\underline{0} \\
\underline{0}\end{array}$ & $\begin{array}{l}\frac{60}{0} \\
0 \\
0.45 \\
0 \\
0\end{array}$ & $\frac{\frac{65}{0.35}}{\frac{0}{0}}$ & $\begin{array}{l}\frac{66}{0} \\
0 \\
0.22 \\
0 \\
0\end{array}$ & & & & $\begin{array}{l}\frac{4}{0} \\
0.02 \\
0.03 \\
0\end{array}$ & $\begin{array}{l}\frac{5}{0.10} \\
\underline{0} \\
\frac{0.03}{\underline{0}}\end{array}$ & \\
\hline $\begin{array}{l}\text { High } \\
\text { Velocity } \\
\text { Region }\end{array}$ & $\begin{array}{l}\frac{12}{0} \\
0 \\
0.17 \\
0\end{array}$ & $\begin{array}{l}\frac{13}{\underline{0}} \\
\frac{0}{0} \\
\underline{0}\end{array}$ & $\begin{array}{l}14 \\
\underline{0} \\
\underline{0} \\
\underline{0} \\
\underline{0}\end{array}$ & $\begin{array}{l}\frac{15}{0} \\
0 \\
0 \\
0\end{array}$ & $\begin{array}{l}26 \\
0 \\
0 \\
0 \\
0.10 \\
-\end{array}$ & $\begin{array}{l}27 \\
0 \\
0 \\
0 \\
0.04 \\
0\end{array}$ & $\begin{array}{l}30 \\
0 \\
0 \\
0.31 \\
0 \\
0\end{array}$ & $\begin{array}{c}31 \\
0 \\
0 \\
0.04 \\
0 \\
0\end{array}$ & $\begin{array}{l}\frac{32}{0.03} \\
\underline{0} \\
\frac{0.05}{\underline{0}}\end{array}$ & $\frac{\frac{41}{0.14}}{\frac{0.03}{\underline{0}}}$ & $\begin{array}{l}\frac{42}{0.38} \\
\frac{0.02}{\underline{0}}\end{array}$ & $\begin{array}{l}\frac{43}{0} \\
0 \\
0 \\
0.04 \\
0\end{array}$ & $\frac{\frac{44}{0.08}}{\frac{0}{0}}$ & & $\begin{array}{l}\frac{55}{\underline{0.05}} \\
\underline{0} \\
\underline{0.08} \\
\underline{0}\end{array}$ & & & & & & & $\frac{\frac{2}{0.21}}{\underline{0}} \frac{0}{\underline{0}}$ & $\begin{array}{l}\frac{3}{0.04} \\
0.54 \\
0.04 \\
0\end{array}$ & \\
\hline $\begin{array}{l}\text { Ring } \\
\text { Core }\end{array}$ & $\frac{\frac{10}{0.48}}{\frac{0.12}{\underline{0}}}$ & $\begin{array}{l}11 \\
0 \\
0 \\
0 \\
0\end{array}$ & $\begin{array}{l}\frac{16}{0} \\
0 \\
0.93\end{array}$ & $\frac{\frac{17}{0.22}}{\frac{0}{0}}$ & $\frac{\frac{21}{0.50}}{\frac{0.36}{0.36}} \frac{0.03}{\frac{0.04}{0}}$ & $\frac{\frac{23}{0.47}}{\frac{0.07}{0.05}} \frac{0.05}{\underline{0.05}}$ & $\begin{array}{c}\frac{24}{0.03} \\
0 \\
0.14 \\
0.06 \\
0.25\end{array}$ & $\begin{array}{l}\frac{28}{0} \\
0 \\
0.31 \\
0 \\
0\end{array}$ & & & & & & & $\begin{array}{l}\frac{56}{0.06} \\
\frac{0}{0} \\
\underline{0.05} \\
\underline{0.03}\end{array}$ & $\begin{array}{l}\frac{57}{0.09} \\
\frac{0.03}{0.05} \\
\underline{0.38} \\
\underline{0.05}\end{array}$ & $\begin{array}{l}\frac{58}{0} \\
0 \\
0 \\
0.77 \\
0.26\end{array}$ & $\begin{array}{l}\frac{61}{0.05} \\
\frac{0.05}{0} \\
\underline{0.38} \\
\underline{0.05}\end{array}$ & $\begin{array}{l}\frac{62}{0} \\
0 \\
0 \\
0.54 \\
0.29\end{array}$ & $\begin{array}{l}\frac{63}{0.03} \\
\frac{0}{0} \\
\underline{0.54} \\
\underline{0.29}\end{array}$ & $\begin{array}{c}64 \\
0 \\
0 \\
0.21 \\
0.24 \\
0\end{array}$ & $\begin{array}{l}\frac{1}{0.68} \\
\underline{0} \\
\underline{0.03} \\
\underline{0.04}\end{array}$ & & \\
\hline $\begin{array}{l}\text { Sargasso } \\
\text { Sea } \\
\& \\
\text { Gulf } \\
\text { Stream }\end{array}$ & & & & & & & & & & $\begin{array}{l}\frac{49}{0.37} \\
\underline{0} \\
\underline{0.02} \\
\underline{0}\end{array}$ & $\begin{array}{l}\frac{50}{0} \\
0 \\
0.24 \\
0\end{array}$ & $\begin{array}{l}\frac{51}{0} \\
0 \\
1.15 \\
0.03\end{array}$ & $\frac{\frac{52}{0.14}}{\frac{0.03}{\underline{0}}}$ & & & & & & & & & & & \\
\hline $\begin{array}{l}\text { Slope } \\
\text { Water }\end{array}$ & $\frac{8}{-}$ & $\frac{9}{0.02}$ & $\frac{18}{0}$ & & $\frac{19}{0.11}$ & $\frac{20}{0}$ & $\frac{33}{0.01}$ & $\frac{34}{0.03}$ & & $\frac{35}{0.07}$ & $\frac{47}{0.01}$ & $\frac{48}{0.01}$ & $\frac{53}{0.01}$ & $\frac{54}{0.02}$ & $\frac{59}{0.05}$ & $\frac{60}{0.09}$ & $\frac{65}{0.07}$ & $\frac{66}{0.04}$ & & & & $\frac{4}{0.01}$ & $\frac{5}{0.03}$ & $\frac{6}{0}$ \\
\hline $\begin{array}{l}\text { High } \\
\text { Velocity } \\
\text { Region }\end{array}$ & $\frac{12}{0.04}$ & $\frac{13}{0}$ & $\frac{14}{0}$ & $\frac{15}{0}$ & $\frac{27}{0.01}$ & $\frac{29}{0.04}$ & $\frac{30}{0.06}$ & $\frac{31}{0.01}$ & $\frac{32}{-}$ & $\frac{41}{0.08}$ & $\frac{42}{0.15}$ & $\frac{43}{0.01}$ & $\frac{44}{0.05}$ & & $\frac{55}{0.04}$ & & & & & & & $\frac{2}{0.05}$ & $\frac{3}{0.16}$ & \\
\hline $\begin{array}{l}\text { Ring } \\
\text { Core }\end{array}$ & $\frac{10}{0.15}$ & $\frac{11}{0}$ & $\frac{16}{-}$ & $\frac{17}{0.06}$ & $\frac{21}{0.17}$ & $\frac{23}{0.11}$ & $\frac{24}{0.10}$ & $\frac{28}{0.18}$ & & $\frac{37}{0.01}$ & $\frac{38}{0.01}$ & $\frac{39}{0.06}$ & & & $\frac{56}{0.03}$ & $\frac{57}{0.12}$ & $\frac{58}{0.21}$ & $\frac{61}{0.10}$ & $\frac{62}{0.17}$ & $\frac{63}{0.13}$ & $\frac{64}{0.07}$ & $\frac{1}{0.19}$ & & \\
\hline SS \& GS & & & & & & & & & & $\frac{49}{0.10}$ & $\frac{50}{0.06}$ & $\frac{51}{0.30}$ & $\frac{52}{0.04}$ & & & & & & & & & & & \\
\hline
\end{tabular}


Table 91. Abundance of Lepidophanes gaussi (specimens $/ 10,000 \mathrm{~m}^{3}$ ).

\begin{tabular}{|c|c|c|c|c|c|c|c|c|c|c|c|c|c|c|c|c|c|c|c|c|c|c|c|c|}
\hline & \multicolumn{4}{|c|}{$\begin{array}{c}\text { Oceanus } \\
\text { April }\end{array}$} & \multicolumn{5}{|c|}{$\begin{array}{c}\text { Oceanus } 121 \\
\text { June }\end{array}$} & \multicolumn{5}{|c|}{$\begin{array}{c}\text { Oceanus } 125 \\
\text { August }\end{array}$} & \multicolumn{7}{|c|}{$\begin{array}{l}\text { Knorr } 98 \\
\text { Sept-0ct }\end{array}$} & \multicolumn{3}{|c|}{$\begin{array}{c}\text { A2 } 110 \\
\text { Sept-0ct }\end{array}$} \\
\hline $\begin{array}{l}\text { Slope } \\
\text { Water }\end{array}$ & $\begin{array}{l}\frac{8}{\overline{\overline{0}}} \\
\underline{0} \\
\underline{0}\end{array}$ & $\begin{array}{l}9 \\
0 \\
0 \\
0 \\
0\end{array}$ & & & 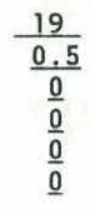 & $\begin{array}{r}20 \\
0 \\
0 \\
0 \\
0 \\
0\end{array}$ & $\begin{array}{l}\frac{33}{0.7} \\
\frac{0}{0} \\
\frac{0}{0} \\
\underline{0}\end{array}$ & $\frac{34}{\frac{0.5}{\underline{0}}}$ & & $\begin{array}{c}35 \\
0 \\
0 \\
0.3 \\
0 \\
0\end{array}$ & $\begin{array}{r}\frac{47}{\underline{0}} \\
\underline{0} \\
\underline{0} \\
\underline{0}\end{array}$ & $\begin{array}{c}48 \\
0.3 \\
0 \\
0 \\
0\end{array}$ & $\frac{53}{\frac{1.0}{\underline{0}}}$ & $\begin{array}{r}54 \\
0 \\
0 \\
0 \\
0\end{array}$ & $\frac{59}{\frac{5.3}{\underline{0}}}$ & $\begin{array}{r}60 \\
0 \\
0 \\
0 \\
0 \\
0\end{array}$ & $\begin{array}{r}65 \\
\underline{0} \\
\underline{0} \\
\underline{0} \\
\underline{0} \\
\underline{0}\end{array}$ & $\begin{array}{c}66 \\
0 \\
0 \\
0.4 \\
0 \\
0\end{array}$ & & & & $\begin{array}{l}4 \\
0 \\
0 \\
0 \\
0\end{array}$ & $\begin{array}{l}\frac{5}{0} \\
\underline{0} \\
\underline{0} \\
\underline{0}\end{array}$ & \\
\hline $\begin{array}{l}\text { High } \\
\text { Velocity } \\
\text { Region }\end{array}$ & $\begin{array}{r}12 \\
0 \\
0 \\
0 \\
0\end{array}$ & $\frac{13}{\frac{0}{0}}$ & $\frac{14}{\frac{0}{0}}$ & $\begin{array}{r}15 \\
0 \\
0 \\
0 \\
0\end{array}$ & $\begin{array}{c}26 \\
0 \\
0 \\
0 \\
0.3 \\
-\end{array}$ & $\begin{array}{r}27 \\
0 \\
0 \\
0 \\
0 \\
0\end{array}$ & $\begin{array}{c}30 \\
0 \\
0 \\
0.6 \\
0 \\
0\end{array}$ & $\begin{array}{r}31 \\
0 \\
0 \\
0 \\
0 \\
0\end{array}$ & $\begin{array}{r}32 \\
\underline{0} \\
\underline{0} \\
\underline{0} \\
\underline{0}\end{array}$ & $\frac{41}{\underline{0}}$ & $\begin{array}{r}\frac{42}{\underline{0}} \\
\underline{0} \\
\underline{0} \\
\underline{0}\end{array}$ & $\begin{array}{c}43 \\
0 \\
0 \\
0 \\
0 \\
0\end{array}$ & $\begin{array}{l}44 \\
\underline{0} \\
\underline{0} \\
\underline{0}\end{array}$ & & $\frac{55}{\frac{4.5}{\underline{0}}} \frac{\underline{0}}{\underline{0}}$ & & & & & & & $\begin{array}{l}\frac{2}{\underline{0}} \\
\underline{0} \\
\underline{0} \\
\underline{0}\end{array}$ & $\begin{array}{c}3 \\
0 \\
0.8 \\
0 \\
0\end{array}$ & \\
\hline $\begin{array}{l}\text { Ring } \\
\text { Core }\end{array}$ & $\frac{10}{\underline{0}}$ & $\begin{array}{r}11 \\
0 \\
0 \\
0 \\
0\end{array}$ & $\begin{array}{r}16 \\
0 \\
0 \\
0\end{array}$ & $\frac{17}{\frac{0}{0}}$ & $\begin{array}{r}21 \\
\underline{0} \\
\underline{0} \\
\underline{0} \\
\underline{0} \\
\underline{0}\end{array}$ & $\begin{array}{r}23 \\
\underline{0} \\
\underline{0} \\
\underline{0} \\
\underline{0} \\
\underline{0}\end{array}$ & $\begin{array}{r}24 \\
0 \\
0 \\
0 \\
0 \\
0\end{array}$ & $\begin{array}{c}28 \\
0 \\
0 \\
0.4 \\
0 \\
0\end{array}$ & & & & & & & $\begin{array}{l}\frac{56}{9.1} \\
\frac{0}{\underline{0}} \\
\underline{0} \\
\underline{0} \\
\underline{0}\end{array}$ & $\begin{array}{c}\frac{57}{\underline{0.3}} \\
\underline{0} \\
\underline{0} \\
\frac{0.3}{\underline{0}}\end{array}$ & $\begin{array}{c}58 \\
0 \\
0 \\
0 \\
4.5 \\
0\end{array}$ & $\begin{array}{c}\frac{61}{\frac{1.2}{\underline{0}}} \\
\underline{0} \\
\frac{1.5}{\underline{0}}\end{array}$ & $\begin{array}{c}62 \\
0 \\
0 \\
0 \\
1.4 \\
0\end{array}$ & 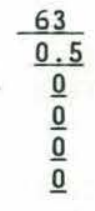 & $\begin{array}{c}64 \\
0 \\
0 \\
0 \\
2.9 \\
0\end{array}$ & $\begin{array}{l}\frac{1}{\underline{0}} \\
\underline{0} \\
\frac{0.6}{\underline{0}}\end{array}$ & & \\
\hline $\begin{array}{l}\text { Sargasso } \\
\text { Sea } \\
\& \\
\text { Gulf } \\
\text { Stream }\end{array}$ & & & & & & & & & & $\frac{49}{\frac{2.4}{\underline{0}}}$ & $\begin{array}{c}50 \\
0 \\
0 \\
4.5 \\
0.9\end{array}$ & $\begin{array}{c}51 \\
0 \\
0 \\
1.3 \\
0\end{array}$ & $\begin{array}{c}\frac{52}{0.2} \\
\underline{0} \\
\frac{0.9}{\underline{0}}\end{array}$ & & & & & & & & & & & \\
\hline $\begin{array}{l}\text { Slope } \\
\text { Water }\end{array}$ & $\frac{8}{-}$ & $\frac{9}{0}$ & $\frac{18}{0}$ & & $\frac{19}{0.1}$ & $\frac{20}{0}$ & $\frac{33}{0}$ & $\frac{34}{0}$ & & $\frac{35}{0.1}$ & $\frac{47}{0}$ & $\frac{48}{0.1}$ & $\frac{53}{0.3}$ & $\frac{54}{0}$ & $\frac{59}{0.1}$ & $\frac{60}{0}$ & $\frac{65}{0}$ & $\frac{66}{0.1}$ & & & & $\frac{4}{0}$ & $\frac{5}{0}$ & $\frac{6}{0}$ \\
\hline $\begin{array}{l}\text { High } \\
\text { Velocity } \\
\text { Region }\end{array}$ & $\frac{12}{0}$ & $\frac{13}{0}$ & $\frac{14}{0}$ & $\frac{15}{0}$ & $\frac{27}{0}$ & $\frac{29}{0}$ & $\frac{30}{0.1}$ & $\frac{31}{0}$ & $\frac{32}{-}$ & $\frac{41}{0}$ & $\frac{42}{0}$ & $\frac{43}{0}$ & $\frac{44}{0}$ & & $\frac{55}{0.9}$ & & & & & & & $\frac{2}{0}$ & $\frac{3}{0.2}$ & \\
\hline $\begin{array}{l}\text { Ring } \\
\text { Core }\end{array}$ & $\frac{10}{0}$ & $\frac{11}{0}$ & $\frac{16}{-}$ & $\frac{17}{0}$ & $\frac{21}{0}$ & $\frac{23}{0}$ & $\frac{24}{0}$ & $\frac{28}{0.1}$ & & $\frac{37}{0}$ & $\frac{38}{0}$ & $\frac{39}{0}$ & & & $\frac{56}{1.8}$ & $\frac{57}{0.1}$ & $\frac{58}{0.9}$ & $\frac{61}{0.5}$ & $\frac{62}{0.3}$ & $\frac{63}{0.2}$ & $\frac{64}{0.6}$ & $\frac{1}{0.2}$ & & \\
\hline SS \& GS & & & & & & & & & & $\frac{49}{0.6}$ & $\frac{50}{1.4}$ & $\frac{51}{0.3}$ & $\frac{52}{0.3}$ & & & & & & & & & & & \\
\hline
\end{tabular}


Table 92. Abundance of Lepidophanes quentheri (specimens $/ 10,000 \mathrm{~m}^{3}$ ).

\begin{tabular}{|c|c|c|c|c|c|c|c|c|c|c|c|c|c|c|c|c|c|c|c|c|c|c|c|c|}
\hline & \multicolumn{4}{|c|}{$\begin{array}{c}\text { Oceanus } 118 \\
\text { April }\end{array}$} & \multicolumn{5}{|c|}{$\begin{array}{c}\text { Oceanus } 121 \\
\text { June }\end{array}$} & \multicolumn{5}{|c|}{$\begin{array}{l}\text { Oceanus } 125 \\
\text { August }\end{array}$} & \multicolumn{7}{|c|}{$\begin{array}{l}\text { Knorr } 98 \\
\text { Sept-0ct }\end{array}$} & \multicolumn{3}{|c|}{$\begin{array}{c}\text { A2 } 110 \\
\text { Sept-0ct }\end{array}$} \\
\hline $\begin{array}{l}\text { Slope } \\
\text { Water }\end{array}$ & $\begin{array}{l}8 \\
\overline{\overline{0}} \\
\underline{0} \\
\underline{0}\end{array}$ & $\begin{array}{l}\frac{9}{0} \\
0.6 \\
0 \\
0\end{array}$ & & & 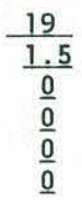 & $\begin{array}{c}20 \\
0 \\
0 \\
0.9 \\
0 \\
0\end{array}$ & $\begin{array}{l}\frac{33}{0.7} \\
\frac{0}{0} \\
\underline{0} \\
\underline{0} \\
\underline{0}\end{array}$ & $\frac{\frac{34}{1.4}}{\frac{0}{0}}$ & & $\begin{array}{r}35 \\
0 \\
0 \\
0 \\
0 \\
0\end{array}$ & $\frac{47}{\frac{0.6}{\underline{0}}} \frac{0}{\underline{0}}$ & $\begin{array}{c}48 \\
0 \\
0 \\
0 \\
0\end{array}$ & $\frac{53}{\frac{0.8}{\underline{0}}}$ & $\begin{array}{c}54 \\
0 \\
0 \\
0.3 \\
0\end{array}$ & $\frac{59}{\frac{1.7}{\underline{0}}}$ & $\begin{array}{c}60 \\
0 \\
0 \\
1.4 \\
0.4 \\
0 .\end{array}$ & $\begin{array}{c}\frac{65}{0.4} \\
\underline{0} \\
\underline{0} \\
\underline{0} \\
\underline{0}\end{array}$ & $\begin{array}{c}66 \\
0 \\
0 \\
2.6 \\
1.0 \\
0\end{array}$ & & & & $\begin{array}{l}\frac{4}{0} \\
1.5 \\
3.1 \\
0\end{array}$ & $\frac{\frac{5}{1.7}}{\frac{0}{0}} \frac{0}{0}$ & \\
\hline $\begin{array}{l}\text { High } \\
\text { Velocity } \\
\text { Region }\end{array}$ & $\begin{array}{c}12 \\
0 \\
0 \\
0.4 \\
0\end{array}$ & $\frac{\frac{13}{3.0}}{\frac{0}{0}}$ & $\frac{\frac{14}{0.3}}{\frac{0}{0}} \frac{0}{0}$ & $\begin{array}{c}15 \\
0 \\
0 \\
0 \\
0\end{array}$ & $\begin{array}{c}26 \\
0 \\
0 \\
0 \\
0.7 \\
-\end{array}$ & $\begin{array}{c}27 \\
0 \\
0 \\
0.5 \\
0 \\
0\end{array}$ & $\begin{array}{c}30 \\
0 \\
0 \\
0.3 \\
0 \\
0\end{array}$ & $\begin{array}{c}31 \\
0 \\
0 \\
1.1 \\
0.3 \\
0\end{array}$ & $\frac{\frac{32}{0.3}}{\frac{0}{0}}$ & $\frac{41}{\frac{0}{0}}$ & $\begin{array}{l}\frac{42}{\underline{0}} \\
\underline{0} \\
\underline{0} \\
\underline{0}\end{array}$ & $\begin{array}{r}43 \\
0 \\
0 \\
0 \\
0 \\
0\end{array}$ & $\frac{\frac{44}{0.4}}{\frac{0}{0}}$ & & $\frac{55}{\frac{4.5}{\underline{0}}}$ & & & & & & & $\frac{\frac{2}{0.4}}{\frac{0}{0}} \frac{\underline{0}}{\underline{0}}$ & $\begin{array}{l}\frac{3}{0} \\
3.0 \\
1.1 \\
0\end{array}$ & \\
\hline $\begin{array}{l}\text { Ring } \\
\text { Core }\end{array}$ & $\frac{\frac{10}{0.8}}{\frac{0}{0}} \frac{0}{\underline{0}}$ & $\begin{array}{r}11 \\
0 \\
0 \\
0 \\
0\end{array}$ & $\begin{array}{c}16 \\
0 \\
0 \\
1.3\end{array}$ & $\frac{\frac{17}{0.6}}{\frac{0}{0}}$ & $\frac{21}{\frac{1.0}{\underline{0}}}$ & 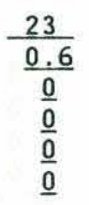 & $\begin{array}{r}24 \\
0 \\
0 \\
0 \\
0 \\
0\end{array}$ & $\begin{array}{r}28 \\
0 \\
0 \\
0 \\
0 \\
0\end{array}$ & & & & & & & $\begin{array}{l}\frac{56}{4.4} \\
\underline{0} \\
\underline{0} \\
\underline{0} \\
\underline{0}\end{array}$ & $\frac{57}{\frac{1.3}{\underline{0}}}$ & $\begin{array}{c}58 \\
0 \\
0 \\
0 \\
8.4 \\
0.3\end{array}$ & $\begin{array}{l}\frac{61}{1.2} \\
\underline{0} \\
\underline{0} \\
\underline{0.8}\end{array}$ & $\begin{array}{c}62 \\
0 \\
0 \\
0 \\
0 \\
0.4\end{array}$ & $\frac{\frac{63}{0.2}}{\underline{0}}$ & $\begin{array}{c}64 \\
0 \\
0 \\
0 \\
0.5 \\
0\end{array}$ & $\begin{array}{l}\frac{1}{\underline{0}} \\
\underline{0} \\
\underline{0} \\
\underline{0}\end{array}$ & & \\
\hline $\begin{array}{l}\text { Sargasso } \\
\text { Sea } \\
\& \\
\text { Gulf } \\
\text { Stream }\end{array}$ & & & & & & & & & & $\frac{49}{\frac{0.5}{\underline{0}}}$ & $\begin{array}{c}50 \\
0 \\
0 \\
0.7 \\
0\end{array}$ & $\begin{array}{c}51 \\
0 \\
0 \\
2.0 \\
0\end{array}$ & $\frac{52}{\frac{0.5}{\underline{0}}} \frac{0}{\underline{0}}$ & & & & & & & & & & & \\
\hline $\begin{array}{l}\text { Slope } \\
\text { Water }\end{array}$ & $\frac{8}{-}$ & $\frac{9}{0.2}$ & $\frac{18}{0}$ & & $\frac{19}{0.2}$ & $\frac{20}{0.2}$ & $\frac{33}{0.1}$ & $\frac{34}{0.1}$ & & $\frac{35}{0}$ & $\frac{47}{0.2}$ & $\frac{48}{0}$ & $\frac{53}{0.2}$ & $\frac{54}{0.1}$ & $\frac{59}{0.3}$ & $\frac{60}{0.4}$ & $\frac{65}{0.1}$ & $\frac{66}{0.7}$ & & & & $\frac{4}{1.2}$ & $\frac{5}{0.4}$ & $\frac{6}{0}$ \\
\hline $\begin{array}{l}\text { High } \\
\text { Velocity } \\
\text { Region }\end{array}$ & $\frac{12}{0.1}$ & $\frac{13}{0.8}$ & $\frac{14}{0.1}$ & $\frac{15}{0}$ & $\frac{27}{0.1}$ & $\frac{29}{0.1}$ & $\frac{30}{0.1}$ & $\frac{31}{0.3}$ & $\frac{32}{-}$ & $\frac{41}{0}$ & $\frac{42}{0}$ & $\frac{43}{0}$ & $\frac{44}{0.2}$ & & $\frac{55}{0.9}$ & & & & & & & $\frac{2}{0.1}$ & $\frac{3}{0.4}$ & \\
\hline $\begin{array}{l}\text { Ring } \\
\text { Core }\end{array}$ & $\frac{10}{0.2}$ & $\frac{11}{0}$ & $\frac{16}{-}$ & $\frac{17}{0.2}$ & $\frac{21}{0.1}$ & $\frac{23}{0.1}$ & $\frac{24}{0}$ & $\frac{28}{0}$ & & $\frac{37}{0.1}$ & $\frac{38}{0}$ & $\frac{39}{0}$ & & & $\frac{56}{0.9}$ & $\frac{57}{0.3}$ & $\frac{58}{1.7}$ & $\frac{61}{0.4}$ & $\frac{62}{0.1}$ & $\frac{63}{0.1}$ & $\frac{64}{0.1}$ & $\frac{1}{0}$ & & \\
\hline SS \& GS & & & & & & & & & & $\frac{49}{0.1}$ & $\frac{50}{0.2}$ & $\frac{51}{0}$ & $\frac{52}{0.1}$ & & & & & & & & & & & \\
\hline
\end{tabular}


Table 93. Volume of Lepidophanes quentheri $\left(\mathrm{ml} / 10,000 \mathrm{~m}^{3}\right)$.

\begin{tabular}{|c|c|c|c|c|c|c|c|c|c|c|c|c|c|c|c|c|c|c|c|c|c|c|c|c|}
\hline & \multicolumn{4}{|c|}{$\begin{array}{c}\text { Oceanus } 118 \\
\text { April }\end{array}$} & \multicolumn{5}{|c|}{$\begin{array}{l}\text { Oceanus } 121 \\
\text { June }\end{array}$} & \multicolumn{5}{|c|}{$\begin{array}{l}\text { Oceanus } 125 \\
\text { August }\end{array}$} & \multicolumn{7}{|c|}{$\begin{array}{l}\text { Knorr } 98 \\
\text { Sept-Oct }\end{array}$} & \multicolumn{3}{|c|}{$\begin{array}{c}\text { A2 } 110 \\
\text { Sept-0ct }\end{array}$} \\
\hline $\begin{array}{l}\text { Slope } \\
\text { Water }\end{array}$ & $\begin{array}{l}8 \\
\overline{0} \\
\underline{0} \\
\underline{0} \\
\underline{0}\end{array}$ & $\begin{array}{l}\frac{9}{0} \\
0.35 \\
0 \\
0\end{array}$ & & & $\frac{\frac{19}{0.10}}{\frac{0}{0}}$ & $\begin{array}{c}20 \\
0 \\
0 \\
0.09 \\
0 \\
0\end{array}$ & $\begin{array}{l}\frac{33}{0.07} \\
\frac{0}{0} \\
\frac{0}{0} \\
\underline{0}\end{array}$ & $\frac{\frac{34}{0.05}}{\frac{0}{0}}$ & . & $\begin{array}{l}35 \\
0 \\
0 \\
0 \\
0 \\
0\end{array}$ & $\frac{\frac{47}{0.04}}{\frac{0}{0}}$ & $\begin{array}{l}\frac{48}{0} \\
0 \\
0 \\
0\end{array}$ & $\frac{\frac{53}{0.17}}{\frac{0}{0}}$ & $\begin{array}{l}\frac{54}{0} \\
0 \\
0.06 \\
0\end{array}$ & $\begin{array}{l}\frac{59}{0.57} \\
\frac{0}{0} \\
\underline{0} \\
\underline{0}\end{array}$ & $\begin{array}{l}60 \\
0 \\
0 \\
0.28 \\
0.39 \\
0\end{array}$ & $\frac{\frac{65}{0.04}}{\frac{0}{0}}$ & $\begin{array}{l}\frac{66}{0} \\
0 \\
1.30 \\
0.19 \\
0\end{array}$ & & & & $\begin{array}{c}\frac{4}{0} \\
0.09 \\
1.03 \\
0\end{array}$ & $\frac{\frac{5}{1.07}}{\frac{0}{0}}$ & \\
\hline $\begin{array}{l}\text { High } \\
\text { Velocity } \\
\text { Region }\end{array}$ & $\begin{array}{l}\frac{12}{0} \\
0 \\
0.04 \\
0\end{array}$ & $\frac{\frac{13}{0.50}}{\frac{0}{0}}$ & $\frac{\frac{14}{2.43}}{\frac{0}{0}}$ & $\begin{array}{l}\frac{15}{0} \\
0 \\
0 \\
0\end{array}$ & $\begin{array}{l}26 \\
0 \\
0 \\
0 \\
0.07 \\
-\end{array}$ & $\begin{array}{l}\frac{27}{0} \\
0 \\
0.05 \\
0 \\
0\end{array}$ & $\begin{array}{c}\frac{30}{0} \\
0 \\
0.03 \\
0 \\
0.14\end{array}$ & $\begin{array}{c}31 \\
0 \\
0 \\
0.21 \\
0.57 \\
0\end{array}$ & $\frac{\frac{32}{0.50}}{\frac{0}{0}}$ & $\begin{array}{l}41 \\
\frac{0}{0} \\
\underline{0}\end{array}$ & $\begin{array}{l}\frac{42}{\underline{0}} \\
\underline{0} \\
\underline{0} \\
\underline{0}\end{array}$ & $\begin{array}{c}\frac{43}{0} \\
0 \\
0.04 \\
0 \\
0\end{array}$ & $\frac{\frac{44}{0.34}}{\frac{0}{0}}$ & & $\frac{\frac{55}{0.21}}{\frac{0}{0}}$ & & & & & & & $\frac{\frac{2}{0.77}}{\frac{0}{0}}$ & $\begin{array}{c}\frac{3}{0} \\
0.32 \\
0.89 \\
0\end{array}$ & \\
\hline $\begin{array}{l}\text { Ring } \\
\text { Core }\end{array}$ & $\frac{\frac{10}{0.12}}{\frac{0}{0}}$ & $\begin{array}{l}\frac{11}{0} \\
0 \\
0 \\
0\end{array}$ & $\begin{array}{l}\frac{16}{0} \\
0 \\
0.27\end{array}$ & $\frac{\frac{17}{0.78}}{\frac{0}{0}}$ & $\frac{\frac{21}{0.10}}{\frac{0}{0}}$ & $\begin{array}{l}\frac{23}{0.06} \\
\frac{0}{0} \\
\frac{0}{0} \\
\underline{0}\end{array}$ & $\begin{array}{l}24 \\
0 \\
0 \\
0 \\
0 \\
0\end{array}$ & $\begin{array}{l}\frac{28}{0} \\
0 \\
0 \\
0 \\
0\end{array}$ & & & & & & & $\begin{array}{l}\frac{56}{0.29} \\
\frac{0}{\underline{0}} \\
\underline{0} \\
\underline{0.03}\end{array}$ & $\frac{\frac{57}{0.50}}{\frac{0}{0}}$ & $\begin{array}{l}\frac{58}{0} \\
0 \\
0 \\
0.32 \\
0.11\end{array}$ & $\begin{array}{l}\frac{61}{0.07} \\
\frac{0}{0} \\
\underline{0.04} \\
\underline{0}\end{array}$ & $\begin{array}{l}\frac{62}{0} \\
0 \\
0 \\
0 \\
0.04\end{array}$ & $\begin{array}{l}\frac{63}{0.02} \\
\frac{0}{0} \\
\frac{0}{0} \\
\underline{0}\end{array}$ & $\begin{array}{l}\frac{64}{0} \\
0 \\
0 \\
0.05 \\
0\end{array}$ & $\begin{array}{l}\frac{1}{\underline{0}} \\
\underline{0} \\
\underline{0} \\
\underline{0}\end{array}$ & & \\
\hline $\begin{array}{l}\text { Sargasso } \\
\text { Sea } \\
\& \\
\text { Gulf } \\
\text { Stream }\end{array}$ & & & & & & & & & & $\frac{\frac{49}{0.02}}{\frac{0}{0}}$ & $\begin{array}{c}50 \\
0 \\
0 \\
0.03 \\
0\end{array}$ & $\begin{array}{l}\frac{51}{0} \\
0 \\
0.24 \\
0\end{array}$ & $\frac{\frac{52}{0.02}}{\frac{0}{0}}$ & & & & & & & & & & & \\
\hline $\begin{array}{l}\text { Slope } \\
\text { Water }\end{array}$ & $\frac{8}{-}$ & $\frac{9}{0.09}$ & $\frac{18}{0}$ & & $\frac{19}{0.01}$ & $\frac{20}{0.02}$ & $\frac{33}{0.01}$ & $\frac{34}{0.01}$ & & $\frac{35}{0}$ & $\frac{47}{0.01}$ & $\frac{48}{0}$ & $\frac{53}{0.04}$ & $\frac{54}{0.02}$ & $\frac{59}{0.11}$ & $\frac{60}{0.13}$ & $\frac{65}{0.01}$ & $\frac{66}{0.30}$ & & & & $\frac{4}{0.28}$ & $\frac{5}{0.27}$ & $\frac{6}{0}$ \\
\hline $\begin{array}{l}\text { High } \\
\text { Velocity } \\
\text { Region }\end{array}$ & $\frac{12}{0.01}$ & $\frac{13}{0.13}$ & $\frac{14}{0.61}$ & $\frac{15}{0}$ & $\frac{27}{0.01}$ & $\frac{29}{0.24}$ & $\frac{30}{0.03}$ & $\frac{31}{0.16}$ & $\frac{32}{-}$ & $\frac{41}{0}$ & $\frac{42}{0}$ & $\frac{43}{0.01}$ & $\frac{44}{0.20}$ & & $\frac{55}{0.04}$ & & & & & & & $\frac{2}{0.19}$ & $\frac{3}{0.30}$ & \\
\hline $\begin{array}{l}\text { Ring } \\
\text { Core }\end{array}$ & $\frac{10}{0.03}$ & $\frac{11}{0}$ & $\frac{16}{-}$ & $\frac{17}{0.20}$ & $\frac{21}{0.01}$ & $\frac{23}{0.01}$ & $\frac{24}{0}$ & $\frac{28}{0}$ & & $\frac{37}{0.08}$ & $\frac{38}{0}$ & $\frac{39}{0}$ & & & $\frac{56}{0.06}$ & $\frac{57}{0.10}$ & $\frac{58}{0.09}$ & $\frac{61}{0.02}$ & $\frac{62}{0.01}$ & $\frac{63}{0.01}$ & $\frac{64}{0.01}$ & $\frac{1}{0}$ & & \\
\hline SS \& GS & & & & & & & & & & $\frac{49}{0.01}$ & $\frac{50}{0.01}$ & $\frac{51}{0.06}$ & $\frac{52}{0.01}$ & & & & & & & & & & & \\
\hline
\end{tabular}


Table 94. Abundance of Lobianchia dofleini (specimens $/ 10,000 \mathrm{~m}^{3}$ ).

\begin{tabular}{|c|c|c|c|c|c|c|c|c|c|c|c|c|c|c|c|c|c|c|c|c|c|c|c|c|}
\hline & & $\begin{array}{r}\text { Oceanu } \\
\text { Apr }\end{array}$ & 118 & & & & $\begin{array}{l}\text { eanus } \\
\text { June }\end{array}$ & 121 & & & $\begin{array}{r}\text { Oce } \\
\mathrm{A}\end{array}$ & $\begin{array}{l}\text { anus } 1 \\
\text { ugust }\end{array}$ & & & & & $\begin{array}{l}\text { Knor } \\
\text { Sept }\end{array}$ & $\begin{array}{r}r 98 \\
-0 c t \\
\end{array}$ & & & & & $\begin{array}{c}110 \\
t-0 c t\end{array}$ & \\
\hline $\begin{array}{l}\text { Slope } \\
\text { Water }\end{array}$ & $\frac{8}{\overline{\overline{0}}}$ & $\begin{array}{l}9 \\
0 \\
0 \\
0 \\
0\end{array}$ & & & $\frac{\frac{19}{11.5}}{\underline{0}} \frac{0.2}{\frac{0}{0}}$ & $\begin{array}{c}20 \\
0 \\
1.6 \\
0 \\
0 \\
0\end{array}$ & $\frac{\frac{33}{13.0}}{\frac{1.7}{\frac{0}{0}}}$ & $\frac{\frac{34}{20.5}}{\frac{0.7}{\underline{0}}}$ & & $\begin{array}{c}35 \\
0 \\
8.7 \\
1.0 \\
0 \\
0\end{array}$ & $\begin{array}{l}\frac{47}{9.1} \\
\underline{0} \\
\frac{0.2}{\underline{0}}\end{array}$ & $\begin{array}{c}\frac{48}{0} \\
11.1 \\
0 \\
0.4\end{array}$ & $\frac{53}{\frac{2.1}{\underline{0}}}$ & $\begin{array}{c}54 \\
0 \\
9.1 \\
0 \\
0\end{array}$ & $\frac{59}{\frac{2.3}{\underline{0}}}$ & $\begin{array}{c}60 \\
0 \\
17.3 \\
0.7 \\
0 \\
0.3\end{array}$ & $\frac{\frac{65}{0.4}}{\frac{0.4}{\underline{0}}}$ & $\begin{array}{r}66 \\
0 \\
0 \\
0 \\
0 \\
0\end{array}$ & & & & $\begin{array}{c}\frac{4}{0} \\
6.7 \\
0 \\
0\end{array}$ & $\frac{\frac{5}{5.0}}{\frac{0}{0}}$ & \\
\hline $\begin{array}{l}\text { High } \\
\text { Velocity } \\
\text { Region }\end{array}$ & $\begin{array}{r}12 \\
0 \\
0 \\
0 \\
0\end{array}$ & $\frac{13}{\frac{0}{0}}$ & $\begin{array}{r}14 \\
\frac{0}{0} \\
\underline{0} \\
\underline{0}\end{array}$ & $\begin{array}{c}15 \\
0 \\
1.2 \\
0 \\
0\end{array}$ & $\begin{array}{c}\frac{26}{0} \\
3.2 \\
3.3 \\
0 \\
-\end{array}$ & $\begin{array}{c}27 \\
0 \\
0.6 \\
0 \\
0 \\
0\end{array}$ & $\begin{array}{c}\frac{30}{0} \\
30.8 \\
2.8 \\
0 \\
0\end{array}$ & $\begin{array}{c}31 \\
0 \\
0 \\
6.8 \\
0 \\
0\end{array}$ & $\frac{32}{\frac{8.7}{\underline{0}}}$ & $\frac{41}{\frac{1.4}{\frac{0}{0}}}$ & $\frac{42}{\frac{2.1}{\frac{0}{0}}}$ & $\begin{array}{c}43 \\
0 \\
0 \\
36.7 \\
0 \\
0\end{array}$ & $\frac{\frac{44}{2.0}}{\frac{0}{0}}$ & & $\frac{55}{\frac{1.1}{\underline{0}}} \frac{\underline{0}}{\underline{0}}$ & & & & & & & $\frac{\frac{2}{2.3}}{\frac{0}{0}}$ & $\begin{array}{c}\frac{3}{0.4} \\
0.8 \\
0 \\
0\end{array}$ & \\
\hline $\begin{array}{l}\text { Ring } \\
\text { Core }\end{array}$ & $\begin{array}{r}\frac{10}{\underline{0}} \\
\underline{0} \\
\underline{0} \\
\underline{0}\end{array}$ & $\begin{array}{c}11 \\
0 \\
0.6 \\
0 \\
0\end{array}$ & $\begin{array}{c}\frac{16}{0} \\
0.7 \\
0\end{array}$ & $\frac{17}{\frac{0.6}{\underline{0}}}$ & $\frac{\frac{21}{16.0}}{\frac{1.0}{\frac{0.3}{0}}}$ & $\frac{\frac{23}{20.6}}{\frac{0}{0}}$ & $\begin{array}{c}\frac{24}{2.6} \\
55.0 \\
3.5 \\
0 \\
0\end{array}$ & $\begin{array}{c}28 \\
0 \\
6.9 \\
0.4 \\
0 \\
0\end{array}$ & & & & & & & $\begin{array}{l}56 \\
\underline{0} \\
\underline{0} \\
\underline{0} \\
\underline{0} \\
\underline{0}\end{array}$ & $\begin{array}{r}57 \\
\underline{0} \\
\underline{0} \\
\underline{0} \\
\underline{0} \\
\underline{0}\end{array}$ & $\begin{array}{r}58 \\
0 \\
0 \\
0 \\
0 \\
0\end{array}$ & $\begin{array}{r}61 \\
\underline{0} \\
\underline{0} \\
\underline{0} \\
\underline{0} \\
\underline{0}\end{array}$ & $\begin{array}{r}62 \\
0 \\
0 \\
0 \\
0 \\
0\end{array}$ & $\begin{array}{r}6 \underline{63} \\
\underline{0} \\
\underline{0} \\
\underline{0} \\
\underline{0} \\
\underline{0}\end{array}$ & $\begin{array}{r}64 \\
0 \\
0 \\
0 \\
0 \\
0\end{array}$ & $\frac{\frac{1}{0.6}}{\underline{0}} \frac{\underline{0}}{\underline{0}}$ & & - \\
\hline $\begin{array}{l}\text { Sargasso } \\
\text { Sea } \\
\& \\
\text { Gulf } \\
\text { Stream }\end{array}$ & & & & & & & & & & $\frac{\frac{49}{0.3}}{\frac{0}{0}} \frac{0}{\underline{0}}$ & $\begin{array}{r}50 \\
0 \\
0 \\
0 \\
0\end{array}$ & $\begin{array}{c}51 \\
0 \\
0 \\
0.7 \\
0\end{array}$ & $\frac{52}{\frac{0.5}{\underline{0}}}$ & & & & & & & & & & & \\
\hline $\begin{array}{l}\text { Slope } \\
\text { Water }\end{array}$ & $\frac{8}{-}$ & $\frac{9}{0}$ & $\frac{18}{0}$ & & $\frac{19}{1.2}$ & $\frac{20}{0.4}$ & $\frac{33}{1.0}$ & $\frac{34}{1.9}$ & & $\frac{35}{2.0}$ & $\frac{47}{2.3}$ & $\frac{48}{2.9}$ & $\frac{53}{0.5}$ & $\frac{54}{2.3}$ & $\frac{59}{0.5}$ & $\frac{60}{1.9}$ & $\frac{65}{0.1}$ & $\frac{66}{0}$ & & & & $\frac{4}{1.7}$ & $\frac{5}{1.3}$ & $\frac{6}{5.8}$ \\
\hline $\begin{array}{l}\text { High } \\
\text { Velocity } \\
\text { Region }\end{array}$ & $\frac{12}{0}$ & $\frac{13}{0}$ & $\frac{14}{0}$ & $\frac{15}{0.3}$ & $\frac{27}{0.1}$ & $\frac{29}{2.3}$ & $\frac{30}{3.6}$ & $\frac{31}{1.4}$ & $\frac{32}{-}$ & $\frac{41}{0.4}$ & $\frac{42}{0.8}$ & $\frac{43}{7.3}$ & $\frac{44}{1.2}$ & & $\frac{55}{0.2}$ & & & & & & & $\frac{2}{0.6}$ & $\frac{3}{0.3}$ & \\
\hline $\begin{array}{l}\text { Ring } \\
\text { Core }\end{array}$ & $\frac{10}{0}$ & $\frac{11}{0.2}$ & $\frac{16}{-}$ & $\frac{17}{0.2}$ & $\frac{21}{1.3}$ & $\frac{23}{3.2}$ & $\frac{24}{2.7}$ & $\frac{28}{1.4}$ & & $\frac{37}{13.2}$ & $\frac{38}{0.4}$ & $\frac{39}{1.1}$ & & & $\frac{56}{0}$ & $\frac{57}{0}$ & $\frac{58}{0}$ & $\frac{61}{0}$ & $\frac{62}{0}$ & $\frac{63}{0}$ & $\frac{64}{0}$ & $\frac{1}{0.2}$ & & \\
\hline SS \& GS & & & & & & & & & & $\frac{49}{0.1}$ & $\frac{50}{0}$ & $\frac{51}{0.2}$ & $\frac{52}{0.1}$ & & & & & & & & & & & \\
\hline
\end{tabular}


Table 95. Volume of Lobianchia dofleini $\left(\mathrm{ml} / 10,000 \mathrm{~m}^{3}\right)$.

\begin{tabular}{|c|c|c|c|c|c|c|c|c|c|c|c|c|c|c|c|c|c|c|c|c|c|c|c|c|}
\hline & \multicolumn{4}{|c|}{$\begin{array}{c}\text { Oceanus } \\
\text { April }\end{array}$} & \multicolumn{5}{|c|}{$\begin{array}{c}\text { Oceanus } 121 \\
\text { June }\end{array}$} & \multicolumn{5}{|c|}{$\begin{array}{c}\text { Oceanus } 125 \\
\text { August }\end{array}$} & \multicolumn{7}{|c|}{$\begin{array}{l}\text { Knorr } 98 \\
\text { Sept-Oct }\end{array}$} & \multicolumn{3}{|c|}{$\begin{array}{c}\text { A2 } 110 \\
\text { Sept- }-0 c t\end{array}$} \\
\hline $\begin{array}{l}\text { Slope } \\
\text { Water }\end{array}$ & $\begin{array}{l}\frac{8}{\overline{\overline{0}}} \\
\underline{0} \\
\underline{0}\end{array}$ & $\begin{array}{l}9 \\
0 \\
0 \\
0 \\
0\end{array}$ & & & $\begin{array}{l}\frac{19}{2.44} \\
\frac{0.21}{\underline{0}} \\
\underline{0} \\
\underline{0}\end{array}$ & $\begin{array}{l}\frac{20}{0} \\
0.37 \\
0 \\
0 \\
0\end{array}$ & $\frac{\frac{33}{4.00}}{\frac{0.63}{\frac{0}{0}}}$ & $\frac{\frac{34}{5.00}}{\frac{0.37}{\underline{0}}}$ & & $\begin{array}{l}\frac{35}{0} \\
1.91 \\
0.10 \\
0 \\
0\end{array}$ & $\begin{array}{l}\frac{47}{4.26} \\
\underline{0} \\
\frac{0.04}{\underline{0}}\end{array}$ & $\begin{array}{c}\frac{48}{0} \\
4.00 \\
0 \\
0.10\end{array}$ & $\frac{\frac{53}{0.58}}{\underline{0}}$ & $\begin{array}{c}\frac{54}{0} \\
2.65 \\
0 \\
0\end{array}$ & $\begin{array}{l}\frac{59}{0.83} \\
\underline{0} \\
\underline{0} \\
\underline{0} \\
\underline{0}\end{array}$ & $\begin{array}{c}\frac{60}{0} \\
6.36 \\
0.34 \\
0 \\
0.09\end{array}$ & $\frac{\frac{65}{0.13}}{\frac{0}{\underline{0}}}$ & $\begin{array}{c}\frac{66}{0} \\
2.71 \\
0 \\
0 \\
0\end{array}$ & & & & $\begin{array}{c}\frac{4}{0} \\
2.39 \\
0 \\
0\end{array}$ & $\frac{\frac{5}{2.00}}{\frac{0}{0}}$ & \\
\hline $\begin{array}{l}\text { High } \\
\text { Velocity } \\
\text { Region }\end{array}$ & $\begin{array}{l}12 \\
0 \\
0 \\
0 \\
0\end{array}$ & $\frac{13}{\frac{0}{0}}$ & $\begin{array}{l}\frac{14}{\underline{0}} \\
\frac{0}{0} \\
\underline{0}\end{array}$ & $\begin{array}{c}\frac{15}{0} \\
0.06 \\
0 \\
0\end{array}$ & $\begin{array}{c}\frac{26}{0} \\
0.79 \\
0.95 \\
0 \\
-\end{array}$ & $\begin{array}{l}\frac{27}{0} \\
0.15 \\
0 \\
0 \\
0\end{array}$ & $\begin{array}{c}\frac{30}{0} \\
6.00 \\
0.38 \\
0 \\
0\end{array}$ & $\begin{array}{l}31 \\
0 \\
0 \\
1.36 \\
0 \\
0\end{array}$ & $\frac{\frac{32}{1.84}}{\frac{0}{0}}$ & $\frac{\frac{41}{0.52}}{\frac{0}{0}}$ & $\frac{\frac{42}{1.03}}{\frac{0}{0}}$ & $\begin{array}{c}43 \\
0 \\
0 \\
13.33 \\
0 \\
0\end{array}$ & $\frac{\frac{44}{0.65}}{\frac{0}{0}}$ & & $\frac{\frac{55}{0.21}}{\underline{0}}$ & & & & & & & $\frac{\frac{2}{0.83}}{\frac{0}{0}}$ & $\begin{array}{l}\frac{3}{0.11} \\
0.49 \\
0 \\
0\end{array}$ & \\
\hline $\begin{array}{l}\text { Ring } \\
\text { Core }\end{array}$ & $\begin{array}{l}10 \\
\underline{0} \\
\underline{0} \\
\underline{0} \\
\underline{0}\end{array}$ & $\begin{array}{c}\frac{11}{0} \\
0.06 \\
0 \\
0\end{array}$ & $\begin{array}{c}\frac{16}{0} \\
0.07 \\
0\end{array}$ & $\frac{\frac{17}{0.28}}{\frac{0}{0}}$ & $\frac{\frac{21}{0.60}}{\frac{0.20}{0.03}} \frac{\underline{0}}{\underline{0}}$ & $\begin{array}{l}\frac{23}{2.35} \\
\frac{0}{0} \\
\underline{0} \\
\underline{0.06}\end{array}$ & $\begin{array}{l}\frac{24}{0.21} \\
3.00 \\
0.35 \\
0 \\
0\end{array}$ & $\begin{array}{l}\frac{28}{0} \\
1.15 \\
0.04 \\
0 \\
0\end{array}$ & & & & & & & $\begin{array}{l}\frac{56}{0} \\
\underline{0} \\
\underline{0} \\
\underline{0} \\
\underline{0}\end{array}$ & $\begin{array}{l}\frac{57}{\underline{0}} \\
\underline{0} \\
\underline{0} \\
\underline{0} \\
\underline{0}\end{array}$ & $\begin{array}{l}58 \\
0 \\
0 \\
0 \\
0 \\
0\end{array}$ & $\begin{array}{l}61 \\
\underline{0} \\
\underline{0} \\
\underline{0} \\
\underline{0} \\
\underline{0}\end{array}$ & $\begin{array}{l}\frac{62}{0} \\
0 \\
0 \\
0 \\
0\end{array}$ & $\begin{array}{l}\frac{6}{\underline{0}}- \\
\underline{0} \\
\underline{0} \\
\underline{0} \\
\underline{0}\end{array}$ & $\begin{array}{l}64 \\
0 \\
0 \\
0 \\
0 \\
0\end{array}$ & $\frac{\frac{1}{0.24}}{\frac{0}{0}}$ & & \\
\hline $\begin{array}{l}\text { Sargasso } \\
\text { Sea } \\
\& \\
\text { Gulf } \\
\text { Stream }\end{array}$ & & & & & & & & & & $\frac{\frac{49}{0.03}}{\frac{0}{0}}$ & $\begin{array}{l}50 \\
0 \\
0 \\
0 \\
0\end{array}$ & $\begin{array}{l}51 \\
0 \\
0 \\
0.11 \\
0\end{array}$ & $\frac{\frac{52}{0.16}}{\frac{0}{0}}$ & & & & & & & & & & & \\
\hline $\begin{array}{l}\text { Slope } \\
\text { Water }\end{array}$ & $\frac{8}{-}$ & $\frac{9}{0}$ & $\frac{18}{0}$ & & $\frac{19}{0.28}$ & $\frac{20}{0.09}$ & $\frac{33}{0.33}$ & $\frac{34}{0.52}$ & & $\frac{35}{0.40}$ & $\frac{47}{1.08}$ & $\frac{48}{1.03}$ & $\frac{53}{0.15}$ & $\frac{54}{0.66}$ & $\frac{59}{0.17}$ & $\frac{60}{0.72}$ & $\frac{65}{0.03}$ & $\frac{66}{0.27}$ & & & & $\frac{4}{0.60}$ & $\frac{5}{0.50}$ & $\frac{6}{2.62}$ \\
\hline $\begin{array}{l}\text { High } \\
\text { Velocity } \\
\text { Region }\end{array}$ & $\frac{12}{0}$ & $\frac{13}{0}$ & $\frac{14}{0}$ & $\frac{15}{0.02}$ & $\frac{27}{0.02}$ & $\frac{29}{0.34}$ & $\frac{30}{0.68}$ & $\frac{31}{0.27}$ & $\frac{32}{-}$ & $\frac{41}{0.26}$ & $\frac{42}{0.39}$ & $\frac{43}{2.67}$ & $\frac{44}{0.39}$ & & $\frac{55}{0.04}$ & & & & & & & $\frac{2}{0.21}$ & $\frac{3}{0.15}$ & \\
\hline $\begin{array}{l}\text { Ring } \\
\text { Core }\end{array}$ & $\frac{10}{0}$ & $\frac{11}{0.02}$ & $\frac{16}{-}$ & $\frac{17}{0.07}$ & $\frac{21}{0.11}$ & $\frac{23}{0.36}$ & $\frac{24}{0.21}$ & $\frac{28}{0.22}$ & & $\frac{37}{4.54}$ & $\frac{38}{0.08}$ & $\frac{39}{0.41}$ & & & $\frac{56}{0}$ & $\frac{57}{0}$ & $\frac{58}{0}$ & $\frac{61}{0}$ & $\frac{62}{0}$ & $\frac{63}{0}$ & $\frac{64}{0}$ & $\frac{1}{0.06}$ & & \\
\hline SS \& GS & & & & & & & & & & $\frac{49}{0.01}$ & $\frac{50}{0}$ & $\frac{51}{0.03}$ & $\frac{52}{0.04}$ & & & & & & & & & & & \\
\hline
\end{tabular}


Table 96. Abundance of Lobianchia gemellarii (specimens/10,000 $\mathrm{m}^{3}$ ).

\begin{tabular}{|c|c|c|c|c|c|c|c|c|c|c|c|c|c|c|c|c|c|c|c|c|c|c|c|c|}
\hline & \multicolumn{4}{|c|}{$\begin{array}{c}\text { Oceanus } 118 \\
\text { April }\end{array}$} & \multicolumn{5}{|c|}{$\begin{array}{l}\text { Oceanus } 121 \\
\text { June }\end{array}$} & \multicolumn{5}{|c|}{$\begin{array}{c}\text { Oceanus } 125 \\
\text { August }\end{array}$} & \multicolumn{7}{|c|}{$\begin{array}{l}\text { Knorr } 98 \\
\text { Sept-oct }\end{array}$} & \multicolumn{3}{|c|}{$\begin{array}{c}\text { A2 } 110 \\
\text { Sept-0ct }\end{array}$} \\
\hline $\begin{array}{l}\text { Slope } \\
\text { Water }\end{array}$ & $\begin{array}{l}8 \underline{\bar{z}} \\
\underline{0} \\
\underline{0} \\
\underline{0}\end{array}$ & $\begin{array}{l}\frac{9}{0} \\
0 \\
0 \\
0\end{array}$ & & & $\frac{\frac{19}{0.2}}{\frac{0.3}{0.0}} \frac{\underline{0}}{\underline{0}}$ & $\begin{array}{c}20 \\
0 \\
0 \\
0 \\
0 \\
0\end{array}$ & $\begin{array}{l}\frac{33}{\underline{0}} \\
\underline{0} \\
\underline{0} \\
\underline{0} \\
\underline{0}\end{array}$ & $\begin{array}{r}34 \\
\underline{0} \\
\underline{0} \\
\underline{0}\end{array}$ & & $\begin{array}{r}35 \\
0 \\
0 \\
0 \\
0 \\
0\end{array}$ & $\begin{array}{l}\frac{47}{\underline{0}} \\
\underline{0} \\
\underline{0} \\
\underline{0}\end{array}$ & $\begin{array}{r}48 \\
0 \\
0 \\
0 \\
0\end{array}$ & $\frac{53}{\frac{0}{0}}$ & $\begin{array}{r}54 \\
0 \\
0 \\
0 \\
0\end{array}$ & $\begin{array}{l}\frac{59}{0.3} \\
\frac{0}{0} \\
\frac{0}{0} \\
\underline{0}\end{array}$ & $\begin{array}{c}60 \\
0 \\
0.9 \\
0.3 \\
0 \\
0\end{array}$ & $\begin{array}{r}65 \\
\underline{0} \\
\underline{0} \\
\underline{0} \\
\underline{0} \\
\underline{0}\end{array}$ & $\begin{array}{r}66 \\
0 \\
0 \\
0 \\
0 \\
0\end{array}$ & & & & $\begin{array}{c}\frac{4}{0} \\
0.4 \\
0 \\
0\end{array}$ & $\begin{array}{l}\frac{5}{0} \\
\underline{0} \\
\underline{0} \\
\underline{0}\end{array}$ & \\
\hline $\begin{array}{l}\text { High } \\
\text { Velocity } \\
\text { Region }\end{array}$ & $\begin{array}{r}12 \\
0 \\
0 \\
0 \\
0\end{array}$ & $\frac{\frac{13}{4.0}}{\frac{0}{0}}$ & $\frac{\frac{14}{\frac{1.1}{0.3}}}{\frac{0}{0}}$ & $\begin{array}{c}\frac{15}{1.7} \\
0 \\
0 \\
0\end{array}$ & $\begin{array}{c}26 \\
0 \\
0 \\
0 \\
0 \\
-\end{array}$ & $\begin{array}{r}27 \\
0 \\
0 \\
0 \\
0 \\
0\end{array}$ & $\begin{array}{c}30 \\
0 \\
0.4 \\
0.6 \\
0 \\
0\end{array}$ & $\begin{array}{c}31 \\
0 \\
0 \\
0 \\
0 \\
0\end{array}$ & $\frac{32}{\frac{0.3}{\underline{0}}} \frac{\underline{0}}{\underline{0}}$ & $\begin{array}{r}41 \\
\underline{0} \\
\underline{0} \\
\underline{0}\end{array}$ & $\begin{array}{l}\frac{42}{\underline{0}} \\
\underline{0} \\
\underline{0} \\
\underline{0}\end{array}$ & $\begin{array}{r}43 \\
0 \\
0 \\
0 \\
0 \\
0\end{array}$ & $\frac{\frac{44}{0.4}}{\frac{0}{0}}$ & & $\frac{55}{\frac{0.3}{\underline{0}}} \frac{\underline{0}}{\underline{0}}$ & & & & & & & $\frac{\frac{2}{0.4}}{\frac{0}{0}} \frac{0}{0}$ & $\begin{array}{c}3 \\
0 \\
0.3 \\
0 \\
0\end{array}$ & \\
\hline $\begin{array}{l}\text { Ring } \\
\text { Core }\end{array}$ & $\begin{array}{r}10 \\
\underline{0} \\
\underline{0} \\
\underline{0} \\
\underline{0}\end{array}$ & $\begin{array}{r}11 \\
0 \\
0 \\
0 \\
0\end{array}$ & $\begin{array}{c}\frac{16}{0} \\
0.7 \\
0\end{array}$ & $\frac{\frac{17}{0.6}}{\frac{0}{0}}$ & $\begin{array}{c}\frac{21}{\underline{0}} \\
\frac{0.2}{0.3} \\
\frac{0}{\underline{0}}\end{array}$ & $\begin{array}{l}\frac{23}{0.6} \\
\frac{0}{0} \\
\underline{0} \\
\underline{0}\end{array}$ & $\begin{array}{c}24 \\
0 \\
5.0 \\
0.5 \\
0 \\
0\end{array}$ & $\begin{array}{c}28 \\
0 \\
0.4 \\
0.4 \\
0 \\
0\end{array}$ & & & & & & & $\begin{array}{l}\frac{56}{\underline{0}} \\
\underline{0} \\
\underline{0} \\
\underline{0} \\
\underline{0}\end{array}$ & $\begin{array}{l}57 \\
\underline{0} \\
\underline{0} \\
\underline{0} \\
\underline{0} \\
\underline{0}\end{array}$ & $\begin{array}{r}58 \\
0 \\
0 \\
0 \\
0 \\
0\end{array}$ & $\begin{array}{l}61 \\
\underline{0} \\
\underline{0} \\
\underline{0} \\
\underline{0} \\
\underline{0}\end{array}$ & $\begin{array}{r}62 \\
0 \\
0 \\
0 \\
0 \\
0\end{array}$ & $\begin{array}{r}63 \\
\underline{0} \\
\underline{0} \\
\underline{0} \\
\underline{0} \\
\underline{0}\end{array}$ & $\begin{array}{r}64 \\
0 \\
0 \\
0 \\
0 \\
0\end{array}$ & $\begin{array}{l}\frac{1}{0} \\
\underline{0} \\
\underline{0} \\
\underline{0} \\
\underline{0}\end{array}$ & & \\
\hline $\begin{array}{l}\text { Sargasso } \\
\text { Sea } \\
\& \\
\text { Gulf } \\
\text { Stream }\end{array}$ & & & & & & & & & & $\begin{array}{l}\frac{49}{\underline{0}} \\
\underline{0} \\
\underline{0} \\
\underline{0}\end{array}$ & $\begin{array}{r}50 \\
0 \\
0 \\
0 \\
0\end{array}$ & $\begin{array}{r}51 \\
0 \\
0 \\
0 \\
0\end{array}$ & $\frac{52}{\frac{0.5}{\underline{0}}}$ & & & & & & & & & & & \\
\hline $\begin{array}{l}\text { Slope } \\
\text { Water }\end{array}$ & $\frac{8}{-}$ & $\frac{9}{0}$ & $\frac{18}{0.1}$ & & $\frac{19}{0.1}$ & $\frac{20}{0}$ & $\frac{33}{0}$ & $\frac{34}{0}$ & & $\frac{35}{0}$ & $\frac{47}{0}$ & $\frac{48}{0}$ & $\frac{53}{0}$ & $\frac{54}{0}$ & $\frac{59}{0.1}$ & $\frac{60}{0.2}$ & $\frac{65}{0}$ & $\frac{66}{0}$ & & & & $\frac{4}{0.1}$ & $\frac{5}{0}$ & $\frac{6}{0.1}$ \\
\hline $\begin{array}{l}\text { High } \\
\text { Velocity } \\
\text { Region }\end{array}$ & $\frac{12}{0}$ & $\frac{13}{1.0}$ & $\frac{14}{0.4}$ & $\frac{15}{0.4}$ & $\frac{27}{0}$ & $\frac{29}{0}$ & $\frac{30}{0.2}$ & $\frac{31}{0}$ & $\frac{32}{-}$ & $\frac{41}{0}$ & $\frac{42}{0}$ & $\frac{43}{0}$ & $\frac{44}{0.2}$ & & $\frac{55}{0.1}$ & & & & & & & $\frac{2}{0.1}$ & $\frac{3}{0.1}$ & \\
\hline $\begin{array}{l}\text { Ring } \\
\text { Core }\end{array}$ & $\frac{10}{0}$ & $\frac{11}{0}$ & $\frac{16}{-}$ & $\frac{17}{0.1}$ & $\frac{21}{0.1}$ & $\frac{23}{0.1}$ & $\frac{24}{0.2}$ & $\frac{28}{0.2}$ & & $\frac{37}{0.2}$ & $\frac{38}{0.1}$ & $\frac{39}{0}$ & & & $\frac{56}{0}$ & $\begin{array}{r}57 \\
0\end{array}$ & $\frac{58}{0}$ & $\frac{61}{0}$ & $\frac{62}{0}$ & $\frac{63}{0}$ & $\frac{64}{0}$ & $\frac{1}{0}$ & & \\
\hline SS \& GS & & & & & & & & & & $\frac{49}{0}$ & $\frac{50}{0}$ & $\frac{51}{0}$ & $\frac{52}{0.1}$ & & & & & & & & & & & \\
\hline
\end{tabular}


Table 97. Volume of Malacosteus niger $\left(\mathrm{ml} / 10,000 \mathrm{~m}^{3}\right)$.

\begin{tabular}{|c|c|c|c|c|c|c|c|c|c|c|c|c|c|c|c|c|c|c|c|c|c|c|c|c|}
\hline & \multicolumn{4}{|c|}{$\begin{array}{c}\text { Oceanus } \\
\text { April }\end{array}$} & \multicolumn{5}{|c|}{$\begin{array}{c}\text { Oceanus } 121 \\
\text { June }\end{array}$} & \multicolumn{5}{|c|}{$\begin{array}{c}\text { Oceanus } 125 \\
\text { August }\end{array}$} & \multicolumn{7}{|c|}{$\begin{array}{l}\text { Knorr } 98 \\
\text { Sept-0ct }\end{array}$} & \multicolumn{3}{|c|}{$\begin{array}{c}\text { A2 } 110 \\
\text { Sept-0ct }\end{array}$} \\
\hline $\begin{array}{l}\text { Slope } \\
\text { Water }\end{array}$ & $\begin{array}{c}\frac{8}{\overline{\overline{0}}} \\
\frac{40.00}{\underline{0}}\end{array}$ & $\begin{array}{l}9 \\
0 \\
0 \\
0 \\
0\end{array}$ & & & $\begin{array}{l}19 \\
\underline{0} \\
\underline{0} \\
\underline{0} \\
\underline{0} \\
\underline{0}\end{array}$ & $\begin{array}{l}20 \\
0 \\
0 \\
0 \\
0 \\
0\end{array}$ & $\begin{array}{l}33 \\
\underline{0} \\
\underline{0} \\
\underline{0} \\
\underline{0} \\
\underline{0}\end{array}$ & $\begin{array}{l}\frac{34}{\underline{0}} \\
\underline{0} \\
\underline{0}\end{array}$ & & $\begin{array}{l}35 \\
0 \\
0 \\
0 \\
0 \\
0\end{array}$ & $\begin{array}{l}47 \\
\frac{0}{0} \\
\frac{0}{0} \\
\underline{0}\end{array}$ & $\begin{array}{l}48 \\
0 \\
0 \\
0 \\
0\end{array}$ & $\frac{\frac{53}{2.88}}{\underline{0}}$ & $\begin{array}{c}54 \\
0 \\
0 \\
4.85 \\
0\end{array}$ & $\begin{array}{l}\frac{59}{\underline{0}} \\
\underline{0} \\
\underline{0} \\
\frac{4.14}{\underline{0}}\end{array}$ & $\begin{array}{l}\frac{60}{0} \\
0 \\
0 \\
7.39 \\
0\end{array}$ & $\begin{array}{l}\frac{65}{0} \\
\underline{0} \\
\underline{0} \\
\underline{0} \\
\underline{0}\end{array}$ & $\begin{array}{l}\frac{66}{0} \\
0 \\
0 \\
0 \\
3.85\end{array}$ & & & & $\begin{array}{c}\frac{4}{0} \\
0 \\
8.44 \\
10.00\end{array}$ & $\begin{array}{l}\frac{5}{\underline{0}} \\
\underline{0} \\
\frac{5.94}{\underline{0}}\end{array}$ & \\
\hline $\begin{array}{l}\text { High } \\
\text { Velocity } \\
\text { Region }\end{array}$ & $\begin{array}{c}12 \\
0 \\
0 \\
0 \\
31.72 \\
\end{array}$ & $\begin{array}{l}\frac{13}{0} \\
\frac{0}{0} \\
\underline{0}\end{array}$ & $\begin{array}{l}\frac{14}{\underline{0}} \\
\underline{0} \\
\frac{5.88}{0}\end{array}$ & $\begin{array}{l}15 \\
0 \\
0 \\
0 \\
0\end{array}$ & $\begin{array}{l}\frac{26}{0} \\
0 \\
0 \\
0 \\
-\end{array}$ & $\begin{array}{l}27 \\
0 \\
0 \\
0 \\
0 \\
3.68\end{array}$ & $\begin{array}{l}30 \\
0 \\
0 \\
0 \\
0 \\
0\end{array}$ & $\begin{array}{l}31 \\
0 \\
0 \\
0 \\
0 \\
0\end{array}$ & $\begin{array}{l}\frac{32}{0} \\
\underline{0} \\
\underline{0} \\
\underline{0}\end{array}$ & $\begin{array}{l}\frac{41}{\underline{0}} \\
\underline{0} \\
\underline{0}\end{array}$ & $\begin{array}{l}\frac{42}{\underline{0}} \\
\frac{0}{0} \\
\underline{0} \\
\underline{0}\end{array}$ & $\begin{array}{l}43 \\
0 \\
0 \\
0 \\
0 \\
0\end{array}$ & $\begin{array}{l}\frac{44}{0} \\
\underline{0} \\
\underline{0}\end{array}$ & & $\begin{array}{l}\frac{55}{\underline{0}} \\
\underline{0} \\
\underline{0} \\
\underline{0}\end{array}$ & & & & & & & $\begin{array}{l}\frac{2}{0} \\
\underline{0} \\
\underline{0} \\
\underline{0}\end{array}$ & $\begin{array}{c}\frac{3}{0} \\
0 \\
5.43 \\
0\end{array}$ & \\
\hline $\begin{array}{l}\text { Ring } \\
\text { Core }\end{array}$ & $\begin{array}{l}\frac{10}{\underline{0}} \\
\underline{0} \\
\underline{\underline{0}} .06 \\
\end{array}$ & $\begin{array}{l}11 \\
0 \\
0 \\
0 \\
0\end{array}$ & $\begin{array}{l}\frac{16}{0} \\
0 \\
1.47\end{array}$ & $\begin{array}{l}\frac{17}{\underline{0}} \\
\underline{0} \\
\underline{3.73}\end{array}$ & $\begin{array}{l}21 \\
\underline{0} \\
\underline{0} \\
\underline{0} \\
\underline{0} \\
\underline{0}\end{array}$ & $\begin{array}{l}23 \\
\underline{0} \\
\underline{0} \\
\underline{0} \\
\underline{0} \\
\underline{0}\end{array}$ & $\begin{array}{l}24 \\
0 \\
0 \\
0 \\
0 \\
0\end{array}$ & $\begin{array}{c}\frac{28}{0} \\
0 \\
3.57 \\
0 \\
6.67\end{array}$ & & & & & & & $\begin{array}{l}\frac{56}{\underline{0}} \\
\underline{0} \\
\underline{0} \\
\underline{0} \\
\underline{2.50} \\
\end{array}$ & $\begin{array}{l}\frac{57}{0} \\
\underline{0} \\
\underline{0} \\
\underline{0} \\
\underline{1.71}\end{array}$ & $\begin{array}{l}58 \\
0 \\
0 \\
0 \\
0 \\
0\end{array}$ & $\begin{array}{l}\frac{61}{\underline{0}} \\
\underline{0} \\
\underline{0} \\
\underline{0.31} \\
\underline{0.03}\end{array}$ & $\begin{array}{l}\frac{62}{0} \\
0 \\
0 \\
0 \\
0.04\end{array}$ & $\begin{array}{l}\frac{63}{\underline{0}} \\
\underline{0} \\
\underline{0} \\
\underline{0} \\
\underline{2.34}\end{array}$ & $\begin{array}{l}64 \\
0 \\
0 \\
0 \\
0 \\
0\end{array}$ & $\begin{array}{l}\frac{1}{0} \\
\underline{0} \\
\underline{0} \\
\underline{0}\end{array}$ & & \\
\hline $\begin{array}{l}\text { Sargasso } \\
\text { Sea } \\
\& \\
\text { Gulf } \\
\text { Stream }\end{array}$ & & & & & & & & & & $\begin{array}{l}\frac{49}{\underline{0}} \\
\underline{0} \\
\underline{0} \\
\underline{2.82}\end{array}$ & $\begin{array}{l}50 \\
0 \\
0 \\
0 \\
0\end{array}$ & $\begin{array}{l}51 \\
0 \\
0 \\
0 \\
0\end{array}$ & $\begin{array}{l}\frac{52}{\underline{0}} \\
\underline{0} \\
\underline{0} \\
\underline{0}\end{array}$ & & & & & & & & & & & \\
\hline $\begin{array}{l}\text { Slope } \\
\text { Water }\end{array}$ & $\frac{8}{-}$ & $\frac{9}{0}$ & $\frac{18}{0}$ & & $\frac{19}{0}$ & $\frac{20}{0}$ & $\frac{33}{0}$ & $\frac{34}{0}$ & & $\frac{35}{0}$ & $\frac{47}{0}$ & $\frac{48}{0}$ & $\frac{53}{0.72}$ & $\frac{54}{1.21}$ & $\frac{59}{0.83}$ & $\frac{60}{1.48}$ & $\frac{65}{0}$ & $\frac{66}{0.77}$ & & & & $\frac{4}{4.61}$ & $\frac{5}{1.49}$ & $\frac{6}{0.52}$ \\
\hline $\begin{array}{l}\text { High } \\
\text { Velocity } \\
\text { Region }\end{array}$ & $\frac{12}{7.93}$ & $\frac{13}{0}$ & $\frac{14}{1.47}$ & $\frac{15}{0}$ & $\frac{27}{0.92}$ & $\frac{29}{0}$ & $\frac{30}{0}$ & $\frac{31}{0}$ & $\frac{32}{-}$ & $\frac{41}{0}$ & $\frac{42}{0}$ & $\frac{43}{0}$ & $\frac{44}{0}$ & & $\frac{55}{0}$ & & & & & & & $\frac{2}{0}$ & $\frac{3}{1.36}$ & \\
\hline $\begin{array}{l}\text { Ring } \\
\text { Core }\end{array}$ & $\frac{10}{0.52}$ & $\frac{11}{0}$ & $\frac{16}{-}$ & $\frac{17}{1.87}$ & $\frac{21}{0}$ & $\frac{23}{0}$ & $\frac{24}{0}$ & $\frac{28}{0.89}$ & & $\frac{37}{0}$ & $\frac{38}{0}$ & $\frac{39}{0}$ & & & $\frac{56}{0.50}$ & $\frac{57}{0.34}$ & $\frac{58}{0}$ & $\frac{61}{0.07}$ & $\frac{62}{0.01}$ & $\frac{63}{0.47}$ & $\frac{64}{0}$ & $\frac{1}{0}$ & & \\
\hline SS \& GS & & & & & & & & & & $\frac{49}{0.71}$ & $\frac{50}{0}$ & $\frac{51}{0}$ & $\frac{52}{0}$ & & & & & & & & & & & \\
\hline
\end{tabular}


Table 98. Abundance of Maurolicus muelleri (specimens $/ 10,000 \mathrm{~m}^{3}$ ).

\begin{tabular}{|c|c|c|c|c|c|c|c|c|c|c|c|c|c|c|c|c|c|c|c|c|c|c|c|c|}
\hline & & $\begin{array}{r}\text { Ocean } \\
A D\end{array}$ & $\begin{array}{l}5118 \\
\text { i1 }\end{array}$ & & & & $\begin{array}{l}\text { eanus } \\
\text { June }\end{array}$ & 121 & & & $\begin{array}{r}\text { Oce } \\
\mathrm{A}\end{array}$ & $\begin{array}{l}\text { anus } 1 \\
\text { ugust }\end{array}$ & & & & & $\begin{array}{l}\text { Knor } \\
\text { Sept }\end{array}$ & $\begin{array}{r}r 98 \\
-0 c t \\
\end{array}$ & & & & st & $\begin{array}{c}110 \\
t-0 c t\end{array}$ & \\
\hline $\begin{array}{l}\text { Slope } \\
\text { Water }\end{array}$ & $\begin{array}{l}\frac{8}{\overline{0}} \\
\underline{0} \\
\underline{0} \\
\underline{0}\end{array}$ & $\begin{array}{l}9 \\
0 \\
0 \\
0 \\
0\end{array}$ & & & $\frac{\frac{19}{18.8}}{\frac{0}{0}}$ & $\begin{array}{c}20 \\
1.1 \\
0 \\
0 \\
0 \\
0\end{array}$ & $\frac{\frac{33}{12.3}}{\frac{0}{0}}$ & $\frac{34}{\underline{0}}$ & & $\begin{array}{r}35 \\
0 \\
0 \\
0 \\
0 \\
0\end{array}$ & $\frac{47}{\frac{1.7}{\frac{0}{0}}}$ & $\begin{array}{c}\frac{48}{1.5} \\
0 \\
0 \\
0\end{array}$ & $\frac{53}{\frac{1.5}{\underline{0}}}$ & $\begin{array}{c}54 \\
0.6 \\
0 \\
0 \\
0\end{array}$ & $\frac{59}{\frac{1.0}{\underline{0}}}$ & $\begin{array}{r}60 \\
0 \\
0 \\
0 \\
0 \\
0\end{array}$ & $\begin{array}{c}\frac{65}{\underline{0.4}} \\
\underline{0} \\
\underline{0} \\
\underline{0} \\
\underline{0}\end{array}$ & $\begin{array}{c}\frac{66}{0.4} \\
0 \\
0 \\
0 \\
0\end{array}$ & & & & $\begin{array}{l}4 \\
0 \\
0 \\
0 \\
0\end{array}$ & $\frac{\frac{5}{0.3}}{\frac{0}{0}}$ & \\
\hline $\begin{array}{l}\text { High } \\
\text { Velocity } \\
\text { Region }\end{array}$ & $\begin{array}{r}12 \\
0 \\
0 \\
0 \\
0\end{array}$ & $\frac{13}{\underline{0}}$ & $\frac{\frac{14}{0.9}}{\frac{0.9}{0}}$ & $\begin{array}{r}15 \\
0 \\
0 \\
0 \\
0\end{array}$ & $\begin{array}{c}\frac{26}{0.3} \\
0 \\
0 \\
0 \\
-\end{array}$ & $\begin{array}{c}27 \\
0.3 \\
0 \\
0 \\
0 \\
0\end{array}$ & $\begin{array}{r}30 \\
0 \\
0 \\
0 \\
0 \\
0\end{array}$ & $\begin{array}{r}31 \\
0 \\
0 \\
0 \\
0 \\
0\end{array}$ & $\begin{array}{l}32 \\
\underline{0} \\
\underline{0} \\
\underline{0} \\
\underline{0}\end{array}$ & $\frac{41}{\underline{0}}$ & $\begin{array}{r}\frac{42}{\underline{0}} \\
\underline{0} \\
\underline{0} \\
\underline{0}\end{array}$ & $\begin{array}{r}43 \\
0 \\
0 \\
0 \\
0 \\
0\end{array}$ & $\begin{array}{r}\frac{44}{0} \\
\underline{0} \\
\underline{0}\end{array}$ & & $\begin{array}{l}55 \\
\underline{0} \\
\underline{0} \\
\underline{0} \\
\underline{0}\end{array}$ & & & & . & & & $\begin{array}{l}\frac{2}{0} \\
\underline{0} \\
\underline{0} \\
\underline{0}\end{array}$ & $\begin{array}{l}3 \\
0 \\
0 \\
0 \\
0\end{array}$ & \\
\hline $\begin{array}{l}\text { Ring } \\
\text { Core }\end{array}$ & $\frac{10}{\frac{0.4}{\underline{0}}} \frac{0}{\underline{0}}$ & $\begin{array}{r}11 \\
0 \\
0 \\
0 \\
0\end{array}$ & $\begin{array}{r}16 \\
0 \\
0 \\
0\end{array}$ & $\frac{17}{\underline{0}}$ & $\begin{array}{r}21 \\
\underline{0} \\
\underline{0} \\
\underline{0} \\
\underline{0} \\
\underline{0}\end{array}$ & $\begin{array}{l}23 \\
\underline{0} \\
\underline{0} \\
\underline{0} \\
\underline{0} \\
\underline{0}\end{array}$ & $\begin{array}{r}24 \\
0 \\
0 \\
0 \\
0 \\
0\end{array}$ & $\begin{array}{r}28 \\
0 \\
0 \\
0 \\
0 \\
0\end{array}$ & & & & & & & $\begin{array}{l}\frac{56}{\underline{0}} \\
\underline{0} \\
\underline{0} \\
\underline{0} \\
\underline{0}\end{array}$ & $\begin{array}{l}57 \\
\underline{0} \\
\underline{0} \\
\underline{0} \\
\underline{0} \\
\underline{0}\end{array}$ & $\begin{array}{r}58 \\
0 \\
0 \\
0 \\
0 \\
0\end{array}$ & $\begin{array}{l}61 \\
\underline{0} \\
\underline{0} \\
\underline{0} \\
\underline{0} \\
\underline{0}\end{array}$ & $\begin{array}{r}62 \\
0 \\
0 \\
0 \\
0 \\
0\end{array}$ & $\begin{array}{l}63 \\
\underline{0} \\
\underline{0} \\
\underline{0} \\
\underline{0} \\
\underline{0}\end{array}$ & $\begin{array}{r}64 \\
0 \\
0 \\
0 \\
0 \\
0\end{array}$ & $\begin{array}{l}1 \\
\underline{0} \\
\underline{0} \\
\underline{0} \\
\underline{0}\end{array}$ & & \\
\hline $\begin{array}{l}\text { Sargasso } \\
\text { Sea } \\
\& \\
\text { Gulf } \\
\text { Stream }\end{array}$ & & & & & & & & & & $\begin{array}{r}\frac{49}{\underline{0}} \\
\underline{0} \\
\underline{0} \\
\underline{0}\end{array}$ & $\begin{array}{r}50 \\
0 \\
0 \\
0 \\
0\end{array}$ & $\begin{array}{l}51 \\
0 \\
0 \\
0 \\
0\end{array}$ & $\begin{array}{r}52 \\
\underline{0} \\
\underline{0} \\
\underline{0} \\
\underline{0}\end{array}$ & & & & & & & & & & & \\
\hline $\begin{array}{l}\text { Slope } \\
\text { Water }\end{array}$ & $\frac{8}{-}$ & $\frac{9}{0}$ & $\frac{18}{0.1}$ & & $\frac{19}{1.9}$ & $\frac{20}{0.3}$ & $\frac{33}{0.7}$ & $\frac{34}{0}$ & & $\frac{35}{0}$ & $\frac{47}{0.4}$ & $\frac{48}{0.4}$ & $\frac{53}{0.4}$ & $\frac{54}{0.2}$ & $\frac{59}{0.2}$ & $\frac{60}{0}$ & $\frac{65}{0.1}$ & $\frac{66}{0.1}$ & & & & $\frac{4}{0}$ & $\frac{5}{0.1}$ & $\frac{6}{0.1}$ \\
\hline $\begin{array}{l}\text { High } \\
\text { Velocity } \\
\text { Region }\end{array}$ & $\frac{12}{0}$ & $\frac{13}{0}$ & $\frac{14}{0.2}$ & $\frac{15}{0}$ & $\frac{27}{0.1}$ & $\frac{29}{0}$ & $\frac{30}{0}$ & $\frac{31}{0}$ & $\frac{32}{-}$ & $\frac{41}{0}$ & $\frac{42}{0}$ & $\frac{43}{0}$ & $\frac{44}{0}$ & & $\frac{55}{0}$ & & & & & & & $\frac{2}{0}$ & $\frac{3}{0}$ & \\
\hline $\begin{array}{l}\text { Ring } \\
\text { Core }\end{array}$ & $\frac{10}{0.1}$ & $\frac{11}{0}$ & $\frac{16}{-}$ & $\frac{17}{0}$ & $\frac{21}{0}$ & $\frac{23}{0}$ & $\frac{24}{0}$ & $\frac{28}{0}$ & & $\frac{37}{0.3}$ & $\frac{38}{0}$ & $\frac{39}{0.1}$ & & & $\frac{56}{0}$ & $\frac{57}{0}$ & $\frac{58}{0}$ & $\frac{61}{0}$ & $\frac{62}{0}$ & $\frac{63}{0}$ & $\frac{64}{0}$ & $\frac{1}{0}$ & & \\
\hline SS \& GS & & & & & & & & & & $\frac{49}{0}$ & $\frac{50}{0}$ & $\frac{51}{0}$ & $\frac{52}{0}$ & & & & & & & & & & & \\
\hline
\end{tabular}


Table 99. Abundance of Melamphaes pumilus (specimens $/ 10,000 \mathrm{~m}^{3}$ ).

\begin{tabular}{|c|c|c|c|c|c|c|c|c|c|c|c|c|c|c|c|c|c|c|c|c|c|c|c|c|}
\hline & \multicolumn{4}{|c|}{$\begin{array}{c}\text { Oceanus } 118 \\
\text { April }\end{array}$} & \multicolumn{5}{|c|}{$\begin{array}{c}\text { Oceanus } 121 \\
\text { June }\end{array}$} & \multicolumn{5}{|c|}{$\begin{array}{l}\text { Oceanus } 125 \\
\text { Auqust }\end{array}$} & \multicolumn{7}{|c|}{$\begin{array}{l}\text { Knorr } 98 \\
\text { Sept-0ct }\end{array}$} & \multicolumn{3}{|c|}{$\begin{array}{c}\text { A2 } 110 \\
\text { Sept-0ct }\end{array}$} \\
\hline $\begin{array}{l}\text { Slope } \\
\text { Water }\end{array}$ & $\begin{array}{l}\frac{8}{\overline{0}} \\
\underline{0} \\
\underline{0} \\
\underline{0}\end{array}$ & $\begin{array}{l}9 \\
0 \\
0 \\
0 \\
0\end{array}$ & & & $\frac{\frac{19}{0.2}}{\frac{0.2}{\underline{0}}} \frac{\underline{0}}{\underline{0}}$ & $\begin{array}{c}20 \\
0 \\
0 \\
0 \\
0.5 \\
0\end{array}$ & $\begin{array}{c}\frac{33}{\underline{0}} \\
\frac{0.4}{\underline{0}} \\
\frac{0}{0}\end{array}$ & $\begin{array}{l}\frac{34}{\underline{0}} \\
\underline{0} \\
\underline{0}\end{array}$ & & $\begin{array}{c}35 \\
0 \\
0 \\
0 \\
0 \\
0\end{array}$ & $\begin{array}{r}47 \\
\underline{0} \\
\underline{0} \\
\underline{0} \\
\underline{0}\end{array}$ & $\begin{array}{r}48 \\
0 \\
0 \\
0 \\
0\end{array}$ & $\begin{array}{l}5 \underline{53} \\
\underline{0} \\
\underline{0} \\
\underline{0} \\
\underline{0}\end{array}$ & $\begin{array}{c}54 \\
0 \\
0 \\
0 \\
0.3\end{array}$ & 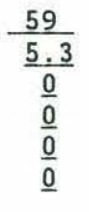 & $\begin{array}{c}60 \\
0.3 \\
0 \\
1.0 \\
0 \\
0\end{array}$ & $\frac{\frac{65}{1.3}}{\frac{0}{\underline{0}}}$ & $\begin{array}{c}66 \\
0 \\
0 \\
0 \\
0.5 \\
0\end{array}$ & & & & $\begin{array}{l}4 \\
0 \\
0 \\
0 \\
0\end{array}$ & $\frac{\frac{5}{0.3}}{\frac{0}{0}}$ & \\
\hline $\begin{array}{l}\text { High } \\
\text { Velocity } \\
\text { Region }\end{array}$ & $\begin{array}{c}12 \\
0 \\
0.4 \\
0.4 \\
0\end{array}$ & $\frac{\frac{13}{1.0}}{\frac{1.1}{\underline{0}}}$ & $\frac{\frac{14}{0.3}}{\frac{0.0}{\underline{0}}}$ & $\begin{array}{r}15 \\
0 \\
0 \\
0 \\
0\end{array}$ & $\begin{array}{c}26 \\
0 \\
0 \\
0 \\
0 \\
-\end{array}$ & $\begin{array}{r}27 \\
0 \\
0 \\
0 \\
0 \\
0\end{array}$ & $\begin{array}{r}30 \\
0 \\
0 \\
0 \\
0 \\
0\end{array}$ & $\begin{array}{r}31 \\
0 \\
0 \\
0 \\
0 \\
0\end{array}$ & $\frac{32}{\frac{0.8}{\underline{0}}}$ & $\frac{\frac{41}{0.4}}{\frac{0}{0}}$ & $\frac{\frac{42}{0.6}}{\frac{0}{0}} \frac{\underline{0}}{\underline{0}}$ & $\begin{array}{r}43 \\
0 \\
0 \\
0 \\
0 \\
0\end{array}$ & $\frac{\frac{44}{0.6}}{\frac{0}{\underline{0}}}$ & & $\frac{\frac{55}{4.0}}{\frac{2.7}{\underline{0}}}$ & & & & & & & $\begin{array}{l}2 \\
\underline{0} \\
\underline{0} \\
\underline{0} \\
\underline{0}\end{array}$ & $\begin{array}{c}3 \\
0 \\
1.1 \\
0 \\
0\end{array}$ & \\
\hline $\begin{array}{l}\text { Ring } \\
\text { Core }\end{array}$ & $\frac{\frac{10}{4.0}}{\frac{0.6}{\frac{0}{0}}}$ & $\begin{array}{c}11 \\
0 \\
0 \\
0 \\
0.4\end{array}$ & $\begin{array}{c}16 \\
0 \\
0 \\
0\end{array}$ & $\frac{\frac{17}{0.6}}{\frac{0.1}{\underline{0}}}$ & $\begin{array}{c}\frac{21}{\underline{0}} \\
\frac{0.2}{\underline{0}} \\
\underline{0} \\
\underline{0}\end{array}$ & $\begin{array}{c}\frac{23}{\underline{0}} \\
\frac{1.3}{\underline{0}} \\
\frac{0}{0}\end{array}$ & $\begin{array}{c}24 \\
0 \\
0 \\
0 \\
0 \\
0\end{array}$ & $\begin{array}{c}28 \\
0 \\
0 \\
0 \\
0 \\
0.5\end{array}$ & & & & & & & $\frac{\frac{56}{8.8}}{\frac{8.3}{\underline{0}}}$ & $\frac{\frac{57}{17.2}}{\frac{10.3}{\frac{0.8}{0}}}$ & $\begin{array}{c}58 \\
0.3 \\
0 \\
1.0 \\
10.7 \\
8.7\end{array}$ & $\frac{\frac{61}{2.7}}{\frac{\frac{20.9}{0.1}}{\frac{0}{0}}}$ & $\begin{array}{c}62 \\
0 \\
0 \\
0.2 \\
9.7 \\
8.2\end{array}$ & $\frac{\frac{63}{14.4}}{\frac{2.2}{1.4}} \frac{\underline{0}}{\underline{0}}$ & $\begin{array}{r}64 \\
0 \\
0 \\
1.4 \\
12.9 \\
3.1\end{array}$ & $\frac{\frac{1}{13.2}}{\frac{0}{0}}$ & & \\
\hline $\begin{array}{l}\text { Sargasso } \\
\text { Sea } \\
\& \\
\text { Gu1f } \\
\text { Stream }\end{array}$ & & & & & & & & & & $\frac{\frac{49}{16.1}}{\frac{0.7}{\underline{0}}}$ & $\begin{array}{c}50 \\
0 \\
0 \\
3.1 \\
6.9\end{array}$ & $\begin{array}{r}51 \\
0 \\
0 \\
21.1 \\
4.8\end{array}$ & $\frac{\frac{52}{17.7}}{\frac{1.1}{0.4}}$ & & & & & & & & & & & \\
\hline $\begin{array}{l}\text { Slope } \\
\text { Water }\end{array}$ & $\frac{8}{-}$ & $\frac{9}{0}$ & $\frac{18}{0}$ & & $\frac{19}{0}$ & $\frac{20}{0.1}$ & $\frac{33}{0.1}$ & $\frac{34}{0}$ & & $\frac{35}{0}$ & $\frac{47}{0}$ & $\frac{48}{0}$ & $\frac{53}{0}$ & $\frac{54}{0.1}$ & $\frac{59}{1.1}$ & $\frac{60}{0.3}$ & $\frac{65}{0.3}$ & $\frac{66}{0.1}$ & & & & $\frac{4}{0}$ & $\frac{5}{0.1}$ & $\frac{6}{0}$ \\
\hline $\begin{array}{l}\text { High } \\
\text { Velocity } \\
\text { Region }\end{array}$ & $\frac{12}{0.2}$ & $\frac{13}{0.5}$ & $\frac{14}{0.3}$ & $\frac{15}{0}$ & $\frac{27}{0}$ & $\frac{29}{0.6}$ & $\frac{30}{0}$ & $\frac{31}{0}$ & $\frac{32}{-}$ & $\frac{41}{0.2}$ & $\frac{42}{0.2}$ & $\frac{43}{0}$ & $\frac{44}{0.4}$ & & $\frac{55}{1.3}$ & & & & & & & $\frac{2}{0}$ & $\frac{3}{0.3}$ & \\
\hline $\begin{array}{l}\text { Ring } \\
\text { Core }\end{array}$ & $\frac{10}{1.2}$ & $\frac{11}{0.1}$ & $\frac{16}{-}$ & $\frac{17}{0.7}$ & $\frac{21}{0.1}$ & $\frac{23}{0.3}$ & $\frac{24}{0}$ & $\frac{28}{0}$ & & $\frac{37}{0}$ & $\frac{38}{0}$ & $\frac{39}{0}$ & & & $\frac{56}{2.4}$ & $\frac{57}{5.7}$ & $\frac{58}{4.2}$ & $\frac{61}{2.9}$ & $\frac{62}{3.6}$ & $\frac{63}{5.7}$ & $\frac{64}{3.3}$ & $\frac{1}{3.3}$ & & \\
\hline SS \& GS & & & & & & & & & & $\frac{49}{4.2}$ & $\frac{50}{2.5}$ & $\frac{51}{6.5}$ & $\frac{52}{4.8}$ & & & & & & & & & & & \\
\hline
\end{tabular}


Table 100. Volume of Melamphaes pumilus $\left(\mathrm{ml} / 10,000 \mathrm{~m}^{3}\right)$.

\begin{tabular}{|c|c|c|c|c|c|c|c|c|c|c|c|c|c|c|c|c|c|c|c|c|c|c|c|c|}
\hline \multirow[b]{2}{*}{$\begin{array}{l}\text { Slope } \\
\text { Water }\end{array}$} & \multicolumn{4}{|c|}{$\begin{array}{c}\text { Oceanus } 118 \\
\text { Apri1 }\end{array}$} & \multicolumn{5}{|c|}{$\begin{array}{c}\text { Oceanus } 121 \\
\text { June }\end{array}$} & \multicolumn{5}{|c|}{$\begin{array}{l}\text { Oceanus } 125 \\
\text { August }\end{array}$} & \multicolumn{7}{|c|}{$\begin{array}{l}\text { Knorr } 98 \\
\text { Sept-0ct }\end{array}$} & \multicolumn{3}{|c|}{$\begin{array}{c}\text { A2 } 110 \\
\text { Sept-0ct }\end{array}$} \\
\hline & $\begin{array}{l}\frac{8}{\bar{a}} \\
\underline{0} \\
\underline{0} \\
\underline{0}\end{array}$ & $\begin{array}{l}\frac{9}{0} \\
0 \\
0 \\
0\end{array}$ & & & $\begin{array}{l}\frac{19}{0.02} \\
\frac{0}{0} \\
\frac{0}{0} \\
\underline{0}\end{array}$ & $\begin{array}{l}20 \\
0 \\
0 \\
0 \\
0.07 \\
0\end{array}$ & $\begin{array}{l}\frac{33}{\underline{0}} \\
\underline{0.08} \\
\underline{0} \\
\underline{0} \\
\underline{0}\end{array}$ & $\begin{array}{l}\frac{34}{\underline{0}} \\
\underline{0} \\
\underline{0}\end{array}$ & & $\begin{array}{l}35 \\
0 \\
0 \\
0 \\
0 \\
0\end{array}$ & $\begin{array}{l}\frac{47}{0} \\
\underline{0} \\
\underline{0} \\
\underline{0}\end{array}$ & $\begin{array}{l}48 \\
0 \\
0 \\
0 \\
0\end{array}$ & $\begin{array}{l}\frac{53}{0} \\
\underline{0} \\
\underline{0} \\
\underline{0} \\
\underline{0}\end{array}$ & $\begin{array}{c}\frac{54}{0} \\
0 \\
0 \\
0.03\end{array}$ & $\begin{array}{l}\frac{59}{0.27} \\
\frac{0}{0} \\
\underline{0} \\
\underline{0}\end{array}$ & $\begin{array}{c}\frac{60}{0.03} \\
0 \\
0.03 \\
0 \\
0\end{array}$ & $\begin{array}{l}\frac{65}{0.22} \\
\frac{0}{0} \\
\frac{0}{0} \\
\underline{0}\end{array}$ & $\begin{array}{l}\frac{66}{0} \\
0 \\
0 \\
0.05 \\
0\end{array}$ & & & & $\begin{array}{l}\frac{4}{0} \\
0 \\
0 \\
0\end{array}$ & $\frac{\frac{5}{0.03}}{\frac{0}{0}}$ & \\
\hline $\begin{array}{l}\text { High } \\
\text { Velocity } \\
\text { Region }\end{array}$ & $\begin{array}{l}\frac{12}{0} \\
0.08 \\
0.04 \\
0\end{array}$ & $\frac{\frac{13}{0.20}}{\frac{0.22}{\underline{0}}}$ & $\begin{array}{l}\frac{14}{\underline{0}} \\
\underline{0.23} \\
\underline{0} \\
\underline{0}\end{array}$ & $\begin{array}{l}\frac{15}{0} \\
0 \\
0 \\
0\end{array}$ & $\begin{array}{l}26 \\
0 \\
0 \\
0 \\
0 \\
-\end{array}$ & $\begin{array}{l}27 \\
0 \\
0 \\
0 \\
0 \\
0\end{array}$ & $\begin{array}{l}30 \\
0 \\
0 \\
0 \\
0 \\
0\end{array}$ & $\begin{array}{l}31 \\
0 \\
0 \\
0 \\
0 \\
0\end{array}$ & $\frac{\frac{32}{0.11}}{\frac{0}{0}}$ & $\begin{array}{l}\frac{41}{\underline{0}} \\
\underline{0} \\
\underline{0}\end{array}$ & $\frac{\frac{42}{0.13}}{\frac{0}{\underline{0}}}$ & $\begin{array}{l}43 \\
0 \\
0 \\
0 \\
0 \\
0\end{array}$ & $\frac{\frac{44}{0.12}}{\frac{0}{0}}$ & & $\frac{\frac{55}{0.26}}{\frac{0.45}{\underline{0}}}$ & & & & & & & $\begin{array}{l}\frac{2}{0} \\
\underline{0} \\
\underline{0} \\
\underline{0}\end{array}$ & $\begin{array}{l}\frac{3}{0} \\
0.11 \\
0 \\
0\end{array}$ & \\
\hline $\begin{array}{l}\text { Ring } \\
\text { Core }\end{array}$ & $\frac{\frac{10}{0.80}}{\frac{0.06}{\underline{0}}}$ & $\begin{array}{l}\frac{11}{0} \\
0 \\
0.13 \\
0.07\end{array}$ & $\begin{array}{l}\frac{16}{0} \\
0 \\
0\end{array}$ & $\frac{\frac{17}{0.06}}{\frac{0}{0}}$ & $\begin{array}{l}\frac{21}{\underline{0}} \\
\frac{0.04}{\underline{0}} \\
\underline{0} \\
\underline{0}\end{array}$ & $\begin{array}{l}\frac{23}{\underline{0}} \\
\frac{0.27}{\underline{0}} \\
\underline{0} \\
\underline{0}\end{array}$ & $\begin{array}{c}\frac{24}{0.07} \\
0 \\
0 \\
0 \\
0\end{array}$ & $\begin{array}{l}28 \\
0 \\
0 \\
0 \\
0 \\
0.10\end{array}$ & & & & & & 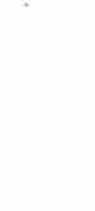 & $\begin{array}{l}\frac{\frac{56}{0.44}}{0.44} \\
\frac{0.0}{\underline{0}} \\
\underline{0}\end{array}$ & $\begin{array}{l}\frac{57}{0.94} \\
\frac{0.13}{1.13} \\
\frac{0.05}{\underline{0}} \\
\underline{0}\end{array}$ & $\begin{array}{c}\frac{58}{0.03} \\
0 \\
0.05 \\
0.32 \\
0.66\end{array}$ & $\begin{array}{l}\frac{61}{0.24} \\
\frac{0.79}{0.04} \\
\frac{0}{\underline{0}}\end{array}$ & $\begin{array}{l}\frac{62}{0} \\
0 \\
0.02 \\
0.38 \\
0.54\end{array}$ & $\frac{\frac{63}{1.06}}{\frac{0.35}{0.10}} \frac{0.0}{\underline{0}}$ & $\begin{array}{l}\frac{64}{0} \\
0 \\
0.29 \\
0.57 \\
0.46\end{array}$ & $\frac{\frac{1}{1.40}}{\frac{0}{0}}$ & & \\
\hline $\begin{array}{l}\text { Sargasso } \\
\text { Sea } \\
\& \\
\text { Gulf } \\
\text { Stream }\end{array}$ & & & & & & & & & & $\frac{\frac{49}{0.81}}{\frac{0.09}{\underline{0}}}$ & $\begin{array}{l}\frac{50}{0} \\
0 \\
0.07 \\
0.63\end{array}$ & $\begin{array}{l}51 \\
0 \\
0 \\
1.09 \\
0.34\end{array}$ & $\frac{\frac{52}{1.02}}{\frac{0.19}{0.04}} \frac{\underline{0}}{\underline{0}}$ & & & & & & & & & & & \\
\hline $\begin{array}{l}\text { Slope } \\
\text { Water }\end{array}$ & $\frac{8}{-}$ & $\frac{9}{0}$ & $\frac{18}{0}$ & & $\frac{19}{0.01}$ & $\frac{20}{0.02}$ & $\frac{33}{0.01}$ & $\frac{34}{0}$ & & $\frac{35}{0}$ & $\frac{47}{0}$ & $\frac{48}{0}$ & $\frac{53}{0}$ & $\frac{54}{0.01}$ & $\frac{59}{0.05}$ & $\frac{60}{0.02}$ & $\frac{65}{0.04}$ & $\frac{66}{0.01}$ & & & & $\frac{4}{0}$ & $\frac{5}{0.01}$ & $\frac{6}{0}$ \\
\hline $\begin{array}{l}\text { High } \\
\text { Velocity } \\
\text { Region }\end{array}$ & $\frac{12}{0.03}$ & $\frac{13}{0.11}$ & $\frac{14}{0.06}$ & $\frac{15}{0}$ & $\frac{27}{0}$ & $\frac{29}{0.12}$ & $\frac{30}{0}$ & $\frac{31}{0}$ & $\frac{32}{-}$ & $\frac{41}{0}$ & $\frac{42}{0.05}$ & $\frac{43}{0}$ & $\frac{44}{0.07}$ & & $\frac{55}{0.14}$ & & & & & & & $\frac{2}{0}$ & $\frac{3}{0.03}$ & \\
\hline $\begin{array}{l}\text { Ring } \\
\text { Core }\end{array}$ & $\frac{10}{0.22}$ & $\frac{11}{0.05}$ & $\frac{16}{-}$ & $\frac{17}{0.01}$ & $\frac{21}{0.01}$ & $\frac{23}{0.07}$ & $\frac{24}{0}$ & $\frac{28}{0}$ & & $\frac{37}{0.01}$ & $\frac{38}{0.09}$ & $\frac{39}{0.13}$ & & & $\frac{56}{0.18}$ & $\frac{57}{0.42}$ & $\frac{58}{0.22}$ & $\frac{61}{0.21}$ & $\frac{62}{0.19}$ & $\frac{63}{0.42}$ & $\frac{64}{0.26}$ & $\frac{1}{0.35}$ & & \\
\hline SS \& GS & & & & & & & & & & $\frac{49}{0.23}$ & $\frac{50}{0.18}$ & $\frac{51}{0.36}$ & $\frac{52}{0.31}$ & & & & & & & & & & & \\
\hline
\end{tabular}


Table 101. Volume of Melamphaes suborbitalis $\left(\mathrm{ml} / 10,000 \mathrm{~m}^{3}\right)$.

\begin{tabular}{|c|c|c|c|c|c|c|c|c|c|c|c|c|c|c|c|c|c|c|c|c|c|c|c|c|}
\hline & \multicolumn{4}{|c|}{$\begin{array}{c}\text { Oceanus } \\
\text { April }\end{array}$} & \multicolumn{5}{|c|}{$\begin{array}{c}\text { Oceanus } 121 \\
\text { June }\end{array}$} & \multicolumn{5}{|c|}{$\begin{array}{l}\text { Oceanus } 125 \\
\text { August }\end{array}$} & \multicolumn{7}{|c|}{$\begin{array}{l}\text { Knorr } 98 \\
\text { Sept-0ct }\end{array}$} & \multicolumn{3}{|c|}{$\begin{array}{c}\text { A2 } 110 \\
\text { Sept-0ct }\end{array}$} \\
\hline $\begin{array}{l}\text { Slope } \\
\text { Water }\end{array}$ & $\begin{array}{l}\frac{8}{\overline{\overline{0}}} \\
\underline{0} \\
\underline{0}\end{array}$ & $\begin{array}{l}9 \\
0 \\
0 \\
0 \\
0\end{array}$ & & & $\begin{array}{l}19 \\
\frac{0}{0} \\
\underline{0} \\
\underline{0} \\
\underline{0} \\
\underline{0}\end{array}$ & $\begin{array}{l}20 \\
0 \\
0 \\
0 \\
0 \\
0\end{array}$ & $\begin{array}{l}33 \\
\underline{0} \\
\underline{0} \\
\underline{0} \\
\underline{0} \\
\underline{0}\end{array}$ & $\begin{array}{l}\frac{34}{\underline{0}} \\
\underline{0} \\
\underline{0}\end{array}$ & & $\begin{array}{l}35 \\
0 \\
0 \\
0 \\
0 \\
0\end{array}$ & $\begin{array}{l}47 \\
\underline{0} \\
\underline{0} \\
\underline{0} \\
\underline{0}\end{array}$ & $\begin{array}{l}48 \\
0 \\
0 \\
0 \\
0\end{array}$ & $\begin{array}{l}\frac{53}{0} \\
\frac{0}{0} \\
\frac{0}{0}\end{array}$ & $\begin{array}{l}54 \\
0 \\
0 \\
0 \\
0\end{array}$ & $\begin{array}{l}59 \\
\underline{0} \\
\underline{0} \\
\underline{0} \\
\underline{0} \\
\underline{0}\end{array}$ & $\begin{array}{l}60 \\
0 \\
0 \\
0 \\
0 \\
0\end{array}$ & $\begin{array}{l}\frac{65}{\underline{0}} \\
\underline{0} \\
\underline{0} \\
\underline{0} \\
\underline{0}\end{array}$ & $\begin{array}{l}\frac{66}{0} \\
0 \\
0 \\
0 \\
0.58\end{array}$ & & & & $\begin{array}{l}4 \\
0 \\
0 \\
0 \\
0\end{array}$ & $\begin{array}{c}\frac{5}{\underline{0}} \\
\frac{14.69}{\underline{0}}\end{array}$ & \\
\hline $\begin{array}{l}\text { High } \\
\text { Velocity } \\
\text { Region }\end{array}$ & $\begin{array}{l}12 \\
0 \\
0 \\
0 \\
0\end{array}$ & $\frac{13}{\frac{0}{0}}$ & $\begin{array}{l}\frac{14}{0} \\
\frac{0}{0} \\
\frac{0}{0} \\
\underline{0}\end{array}$ & $\begin{array}{l}15 \\
0 \\
0 \\
0 \\
0\end{array}$ & $\begin{array}{l}26 \\
0 \\
0 \\
0 \\
0 \\
-\end{array}$ & $\begin{array}{l}27 \\
0 \\
0 \\
0 \\
0 \\
0\end{array}$ & $\begin{array}{l}30 \\
0 \\
0 \\
0 \\
0.05 \\
0.05\end{array}$ & $\begin{array}{l}31 \\
0 \\
0 \\
0 \\
0 \\
0\end{array}$ & $\begin{array}{l}32 \\
\frac{0}{0} \\
\frac{0}{0} \\
\underline{0}\end{array}$ & $\begin{array}{l}41 \\
\underline{0} \\
\underline{0} \\
\underline{0}\end{array}$ & $\begin{array}{l}42 \\
\underline{0} \\
\frac{0}{0} \\
\underline{0}\end{array}$ & $\begin{array}{l}43 \\
0 \\
0 \\
0 \\
0 \\
0\end{array}$ & $\begin{array}{l}\frac{44}{0} \\
\frac{0}{0} \\
\underline{0}\end{array}$ & & $\begin{array}{l}55 \\
\underline{0} \\
\underline{0} \\
\underline{0} \\
\underline{0}\end{array}$ & & & & & & & $\begin{array}{c}\frac{2}{\frac{0}{0}} \\
\frac{15.33}{\underline{0}}\end{array}$ & $\begin{array}{l}3 \\
0 \\
0 \\
0 \\
0\end{array}$ & \\
\hline $\begin{array}{l}\text { Ring } \\
\text { Core }\end{array}$ & $\begin{array}{l}10 \\
\underline{0} \\
\underline{0} \\
\underline{0} \\
\underline{0}\end{array}$ & $\begin{array}{l}11 \\
0 \\
0 \\
0 \\
0\end{array}$ & $\begin{array}{l}16 \\
0 \\
0 \\
0\end{array}$ & $\begin{array}{l}17 \\
\underline{0} \\
\underline{0} \\
\underline{0}\end{array}$ & $\begin{array}{l}21 \\
\frac{0}{0} \\
\underline{0} \\
\frac{0}{0} \\
\underline{0}\end{array}$ & $\begin{array}{l}\frac{23}{\underline{0}} \\
\underline{0} \\
\underline{0} \\
\frac{9.55}{\underline{0}}\end{array}$ & $\begin{array}{l}24 \\
0 \\
0 \\
0 \\
0 \\
0\end{array}$ & $\begin{array}{c}28 \\
0 \\
0 \\
0 \\
0 \\
10.00\end{array}$ & & & & & & & $\begin{array}{l}56 \\
\underline{0} \\
\underline{0} \\
\underline{0} \\
\underline{0} \\
\underline{0}\end{array}$ & $\begin{array}{l}57 \\
\frac{0}{0} \\
\underline{0} \\
\underline{0} \\
\underline{0} \\
\underline{0}\end{array}$ & $\begin{array}{l}58 \\
0 \\
0 \\
0 \\
0 \\
0\end{array}$ & $\begin{array}{l}\frac{61}{0} \\
\underline{0} \\
\underline{0} \\
\underline{0} \\
\underline{0}\end{array}$ & $\begin{array}{l}\frac{62}{0} \\
0 \\
0 \\
0 \\
0\end{array}$ & $\begin{array}{l}\frac{63}{0} \\
\underline{0} \\
\underline{0} \\
\underline{0} \\
\underline{0}\end{array}$ & $\begin{array}{l}64 \\
0 \\
0 \\
0 \\
0 \\
0\end{array}$ & $\begin{array}{l}\frac{1}{0} \\
\underline{0} \\
\underline{0} \\
\underline{0}\end{array}$ & & \\
\hline $\begin{array}{l}\text { Sargasso } \\
\text { Sea } \\
\& \\
\text { Gulf } \\
\text { Stream }\end{array}$ & & & & & & & & & & $\begin{array}{l}49 \\
\underline{0} \\
\underline{0} \\
\underline{0} \\
\underline{0}\end{array}$ & $\begin{array}{l}50 \\
0 \\
0 \\
0 \\
0\end{array}$ & $\begin{array}{l}51 \\
0 \\
0 \\
0 \\
0\end{array}$ & $\begin{array}{l}52 \\
\frac{0}{0} \\
\underline{0} \\
\underline{0} \\
\underline{0}\end{array}$ & & & & & & & & & & & \\
\hline $\begin{array}{l}\text { Slope } \\
\text { Water }\end{array}$ & $\frac{8}{-}$ & $\frac{9}{0}$ & $\frac{18}{0}$ & & $\frac{19}{0}$ & $\frac{20}{0}$ & $\frac{33}{0}$ & $\frac{34}{0}$ & & $\frac{35}{0}$ & $\frac{47}{0}$ & $\frac{48}{0}$ & $\frac{53}{0}$ & $\frac{54}{0}$ & $\frac{59}{0}$ & $\frac{60}{0}$ & $\frac{65}{0}$ & $\frac{66}{0.12}$ & & & & $\frac{4}{0}$ & $\frac{5}{3.67}$ & $\frac{6}{0}$ \\
\hline $\begin{array}{l}\text { High } \\
\text { Velocity } \\
\text { Region }\end{array}$ & $\frac{12}{0}$ & $\frac{13}{0}$ & $\frac{14}{0}$ & $\frac{15}{0}$ & $\frac{27}{0}$ & $\frac{29}{0.93}$ & $\frac{30}{0.02}$ & $\frac{31}{0}$ & $\frac{32}{-}$ & $\frac{41}{0}$ & $\frac{42}{0}$ & $\frac{43}{0}$ & $\frac{44}{0}$ & & $\frac{55}{0}$ & & & & & & & $\frac{2}{3.83}$ & $\frac{3}{0}$ & \\
\hline $\begin{array}{l}\text { Ring } \\
\text { Core }\end{array}$ & $\frac{10}{0}$ & $\frac{11}{0}$ & $\frac{16}{-}$ & $\frac{17}{0}$ & $\frac{21}{0}$ & $\frac{23}{1.91}$ & $\frac{24}{0}$ & $\frac{28}{0}$ & & $\frac{37}{0}$ & $\frac{38}{0}$ & $\frac{39}{0}$ & & & $\frac{56}{0}$ & $\frac{57}{0}$ & $\frac{58}{0}$ & $\frac{61}{0}$ & $\frac{62}{0}$ & $\frac{63}{0}$ & $\frac{64}{0}$ & $\frac{1}{0}$ & & \\
\hline SS \& GS & & & & & & & & & & $\frac{49}{0}$ & $\frac{50}{0}$ & $\frac{51}{0}$ & $\frac{52}{0}$ & & & & & & & & & & & \\
\hline
\end{tabular}


Table 102. Volume of Melanostomias bartonbeani $\left(\mathrm{ml} / 10,000 \mathrm{~m}^{3}\right)$.

\begin{tabular}{|c|c|c|c|c|c|c|c|c|c|c|c|c|c|c|c|c|c|c|c|c|c|c|c|c|}
\hline \multirow[b]{2}{*}{$\begin{array}{l}\text { Slope } \\
\text { Water }\end{array}$} & \multicolumn{4}{|c|}{$\begin{array}{c}\text { Oceanus } \\
\text { April }\end{array}$} & \multicolumn{5}{|c|}{$\begin{array}{c}\text { Oceanus } 121 \\
\text { June }\end{array}$} & \multicolumn{5}{|c|}{$\begin{array}{l}\text { Oceanus } 125 \\
\text { Auqust }\end{array}$} & \multicolumn{7}{|c|}{$\begin{array}{l}\text { Knorr } 98 \\
\text { Sept-0ct }\end{array}$} & \multicolumn{3}{|c|}{$\begin{array}{c}\text { A2 } 110 \\
\text { Sept-0ct }\end{array}$} \\
\hline & $\begin{array}{l}\frac{8}{\overline{\overline{0}}} \\
\underline{0} \\
\underline{0}\end{array}$ & $\begin{array}{l}9 \\
0 \\
0 \\
0 \\
0\end{array}$ & & & $\frac{\frac{19}{0.10}}{\frac{0}{0}}$ & $\begin{array}{l}20 \\
0 \\
0 \\
0 \\
0 \\
0\end{array}$ & $\begin{array}{c}\frac{33}{0} \\
\frac{25.00}{\underline{0}} \\
\underline{0}\end{array}$ & $\begin{array}{l}\frac{34}{0} \\
\frac{0}{0} \\
\underline{0}\end{array}$ & & $\begin{array}{l}35 \\
0 \\
0 \\
0 \\
0 \\
0\end{array}$ & $\frac{\frac{47}{0.39}}{\frac{0}{\underline{0}}}$ & $\begin{array}{l}\frac{48}{0} \\
0 \\
0 \\
0\end{array}$ & $\begin{array}{l}\frac{53}{\underline{0}} \\
\underline{0} \\
\underline{0} \\
\underline{0}\end{array}$ & $\begin{array}{l}\frac{54}{0} \\
0 \\
0.03 \\
0\end{array}$ & $\begin{array}{l}\frac{59}{\underline{0}} \\
\underline{0} \\
\underline{0} \\
\underline{0} \\
\underline{0}\end{array}$ & $\begin{array}{c}\frac{60}{0.05} \\
0 \\
0.41 \\
0 \\
0\end{array}$ & $\begin{array}{l}\frac{65}{\underline{0}} \\
\underline{0} \\
\underline{0} \\
\underline{0} \\
\underline{0}\end{array}$ & $\begin{array}{c}\frac{66}{0} \\
0 \\
14.07 \\
0 \\
0\end{array}$ & & & & $\begin{array}{l}4 \\
0 \\
0 \\
0 \\
0\end{array}$ & $\begin{array}{l}\frac{5}{0} \\
\frac{0}{0} \\
\underline{0} \\
\underline{0}\end{array}$ & \\
\hline $\begin{array}{l}\text { High } \\
\text { Velocity } \\
\text { Region }\end{array}$ & $\begin{array}{l}12 \\
0 \\
0 \\
0 \\
0\end{array}$ & $\begin{array}{l}\frac{13}{\underline{0}} \\
\frac{0}{0}\end{array}$ & $\frac{\frac{14}{0.26}}{\frac{0}{0}}$ & $\begin{array}{l}\frac{15}{0} \\
0 \\
0 \\
0\end{array}$ & $\begin{array}{l}26 \\
0 \\
0 \\
0 \\
0 \\
-\end{array}$ & $\begin{array}{l}27 \\
0 \\
0 \\
0 \\
0 \\
0\end{array}$ & $\begin{array}{l}30 \\
0 \\
0 \\
0 \\
0 \\
0\end{array}$ & $\begin{array}{l}31 \\
0 \\
0 \\
0 \\
0 \\
0\end{array}$ & $\begin{array}{l}32 \\
\underline{0} \\
\underline{0} \\
\underline{0} \\
\underline{0}\end{array}$ & $\begin{array}{l}41 \\
\frac{0}{0} \\
\underline{0}\end{array}$ & $\begin{array}{l}\frac{42}{0} \\
\underline{0} \\
\underline{0} \\
\underline{0}\end{array}$ & $\begin{array}{l}43 \\
0 \\
0 \\
0 \\
0 \\
0\end{array}$ & $\begin{array}{l}\frac{44}{\underline{0}} \\
\frac{0}{0} \\
\underline{0}\end{array}$ & & $\begin{array}{l}55 \\
\underline{0} \\
\underline{0} \\
\underline{0} \\
\underline{0}\end{array}$ & & & & & & & $\begin{array}{l}\frac{2}{0} \\
\frac{0}{0} \\
\underline{0} \\
\underline{0}\end{array}$ & $\begin{array}{l}3 \\
0 \\
0 \\
0 \\
0\end{array}$ & \\
\hline $\begin{array}{l}\text { Ring } \\
\text { Core }\end{array}$ & $\begin{array}{l}10 \\
\underline{0} \\
\underline{0} \\
\underline{0} \\
\underline{0}\end{array}$ & $\begin{array}{l}11 \\
0 \\
0 \\
0 \\
0\end{array}$ & $\begin{array}{l}16 \\
0 \\
0 \\
0\end{array}$ & $\begin{array}{l}17 \\
\underline{0} \\
\underline{0} \\
\underline{0}\end{array}$ & $\begin{array}{l}21 \\
\underline{0} \\
\underline{0} \\
\underline{0} \\
\underline{0} \\
\underline{0}\end{array}$ & $\begin{array}{l}23 \\
\underline{0} \\
\underline{0} \\
\underline{0} \\
\underline{0} \\
\underline{0}\end{array}$ & $\begin{array}{l}24 \\
0 \\
0 \\
0 \\
0 \\
0\end{array}$ & $\begin{array}{l}28 \\
0 \\
0 \\
0 \\
0 \\
0\end{array}$ & & & & & & & $\begin{array}{l}56 \\
\frac{0}{0} \\
\underline{0} \\
\underline{0} \\
\underline{0} \\
\underline{0}\end{array}$ & $\begin{array}{l}\frac{57}{0} \\
\underline{0} \\
\underline{0} \\
\underline{0} \\
\underline{0} \\
\underline{0}\end{array}$ & $\begin{array}{c}58 \\
0 \\
0 \\
0 \\
0.13 \\
13.16\end{array}$ & $\begin{array}{l}61 \\
\underline{0} \\
\underline{0} \\
\underline{0} \\
\underline{0} \\
\underline{0}\end{array}$ & $\begin{array}{l}62 \\
0 \\
0 \\
0 \\
0 \\
0\end{array}$ & $\begin{array}{l}\frac{63}{\underline{0}} \\
\underline{0} \\
\underline{0} \\
\underline{0} \\
\underline{0}\end{array}$ & $\begin{array}{l}64 \\
0 \\
0 \\
0 \\
0 \\
0\end{array}$ & $\begin{array}{l}\frac{1}{0} \\
\underline{0} \\
\underline{0} \\
\underline{0}\end{array}$ & & \\
\hline $\begin{array}{l}\text { Sargasso } \\
\text { Sea } \\
\& \\
\text { Gulf } \\
\text { Stream }\end{array}$ & & & & & & & & & & $\frac{\frac{49}{0.08}}{\frac{0}{0}}$ & $\begin{array}{c}50 \\
0 \\
0 \\
0.03 \\
0\end{array}$ & $\begin{array}{l}\frac{51}{0} \\
0 \\
0.02 \\
0\end{array}$ & $\begin{array}{l}\frac{52}{\underline{0}} \\
\underline{0} \\
\underline{0} \\
\underline{0}\end{array}$ & & & & & & & & & & & \\
\hline $\begin{array}{l}\text { Slope } \\
\text { Water }\end{array}$ & $\frac{8}{-}$ & $\frac{9}{0}$ & $\frac{18}{0}$ & & $\frac{19}{0.01}$ & $\frac{20}{0}$ & $\frac{33}{3.50}$ & $\frac{34}{0}$ & & $\frac{35}{0}$ & $\frac{47}{0.10}$ & $\frac{48}{0}$ & $\frac{53}{0}$ & $\frac{54}{0.01}$ & $\frac{59}{0}$ & $\frac{60}{0.11}$ & $\frac{65}{0}$ & $\frac{66}{2.81}$ & & & & $\frac{4}{0}$ & $\frac{5}{0}$ & $\frac{6}{0}$ \\
\hline $\begin{array}{l}\text { High } \\
\text { Velocity } \\
\text { Region }\end{array}$ & $\frac{12}{0}$ & $\frac{13}{0}$ & $\frac{14}{0.07}$ & $\frac{15}{0}$ & $\frac{27}{0}$ & $\frac{29}{0}$ & $\frac{30}{0}$ & $\frac{31}{0}$ & $\frac{32}{-}$ & $\frac{41}{0}$ & $\frac{42}{0}$ & $\frac{43}{0}$ & $\frac{44}{0}$ & & $\frac{55}{0}$ & & & & & & & $\frac{2}{0}$ & $\frac{3}{0}$ & \\
\hline $\begin{array}{l}\text { Ring } \\
\text { Core }\end{array}$ & $\frac{10}{0}$ & $\frac{11}{0}$ & $\frac{16}{-}$ & $\frac{17}{0}$ & $\frac{21}{0}$ & $\frac{23}{0}$ & $\frac{24}{0}$ & $\frac{28}{0}$ & & $\frac{37}{0}$ & $\frac{38}{0}$ & $\frac{39}{0}$ & & & $\frac{56}{0}$ & $\frac{57}{0}$ & $\frac{58}{2.66}$ & $\frac{61}{0}$ & $\frac{62}{0}$ & $\frac{63}{0}$ & $\frac{64}{0}$ & $\frac{1}{0}$ & & \\
\hline SS \& GS & & & & & & & & & & $\frac{49}{0.02}$ & $\frac{50}{0.01}$ & $\frac{51}{0.01}$ & $\frac{52}{0}$ & & & & & & & & & & & \\
\hline
\end{tabular}


Table 103. Abundance of Myctophum affine (specimens $/ 10,000 \mathrm{~m}^{3}$ ).

\begin{tabular}{|c|c|c|c|c|c|c|c|c|c|c|c|c|c|c|c|c|c|c|c|c|c|c|c|c|}
\hline & & $\begin{array}{r}\text { Oceanu } \\
\text { Apr }\end{array}$ & $i^{118}$ & & & & $\begin{array}{l}\text { eanus } \\
\text { June }\end{array}$ & 121 & & & $\begin{array}{r}\text { Oce } \\
A\end{array}$ & $\begin{array}{l}\text { anus } 1 \\
\text { ugust }\end{array}$ & & & & & $\begin{array}{l}\text { Knor } \\
\text { Sept }\end{array}$ & $\begin{array}{r}98 \\
-0 c t \\
\end{array}$ & & & & & $\begin{array}{l}110 \\
t-0 c t\end{array}$ & \\
\hline $\begin{array}{l}\text { Slope } \\
\text { Water }\end{array}$ & $\begin{array}{l}\frac{8}{\overline{\overline{0}}} \\
\underline{0} \\
\underline{0}\end{array}$ & $\begin{array}{c}\frac{9}{0} \\
0.6 \\
0 \\
0\end{array}$ & & & $\begin{array}{r}\frac{19}{\underline{0}} \\
\underline{0} \\
\underline{0} \\
\underline{0} \\
\underline{0}\end{array}$ & $\begin{array}{r}20 \\
0 \\
0 \\
0 \\
0 \\
0\end{array}$ & $\begin{array}{c}\frac{33}{\underline{0}} \\
\underline{0} \\
\underline{0} \\
\underline{0} \\
\underline{0.3}\end{array}$ & $\frac{34}{\frac{1.8}{\frac{0}{0}}}$ & & $\begin{array}{c}35 \\
0 \\
0 \\
1.7 \\
1.7 \\
0\end{array}$ & $\frac{\frac{47}{0.9}}{\frac{0}{0}}$ & $\begin{array}{c}48 \\
0 \\
0.3 \\
0.3 \\
0\end{array}$ & $\frac{53}{\frac{1.4}{\underline{0}}}$ & $\begin{array}{c}54 \\
0 \\
0.6 \\
0 \\
0\end{array}$ & $\begin{array}{r}59 \\
\underline{0} \\
\underline{0} \\
\underline{0} \\
\underline{0} \\
\underline{0}\end{array}$ & $\begin{array}{c}60 \\
0 \\
0 \\
1.4 \\
0 \\
0\end{array}$ & $\begin{array}{r}\frac{65}{0} \\
\frac{0}{0} \\
\underline{0} \\
\underline{0} \\
\underline{0}\end{array}$ & $\begin{array}{c}66 \\
0 \\
0 \\
1.1 \\
0 \\
0\end{array}$ & & & & $\begin{array}{c}\frac{4}{0} \\
7.0 \\
3.8 \\
0\end{array}$ & $\frac{\frac{5}{0.7}}{\frac{0}{0}}$ & \\
\hline $\begin{array}{l}\text { High } \\
\text { Velocity } \\
\text { Region }\end{array}$ & $\begin{array}{r}12 \\
0 \\
0 \\
0 \\
0\end{array}$ & $\frac{\frac{13}{0.5}}{\frac{0}{0}}$ & $\begin{array}{c}\frac{14}{0.6} \\
\frac{0}{0} \\
1.4\end{array}$ & $\begin{array}{c}15 \\
0 \\
1.2 \\
0 \\
0\end{array}$ & $\begin{array}{c}26 \\
0 \\
0 \\
0 \\
0 \\
-\end{array}$ & $\begin{array}{c}27 \\
0 \\
0 \\
0.5 \\
0 \\
0\end{array}$ & $\begin{array}{c}30 \\
0 \\
0 \\
0.3 \\
0 \\
0\end{array}$ & $\begin{array}{c}31 \\
0 \\
0 \\
0.6 \\
0 \\
0\end{array}$ & $\begin{array}{c}32 \\
\underline{0} \\
\underline{0} \\
\underline{0} \\
\underline{0}\end{array}$ & $\frac{41}{\frac{0.1}{\underline{0}}}$ & $\begin{array}{l}\frac{42}{0.3} \\
\frac{0.9}{0.7} \\
\underline{0.5}\end{array}$ & $\begin{array}{c}43 \\
0 \\
0 \\
0.4 \\
0 \\
0\end{array}$ & $\frac{\frac{44}{0.3}}{\frac{0}{0}}$ & & $\begin{array}{l}55 \\
\underline{0} \\
\underline{0} \\
\underline{0} \\
\underline{0}\end{array}$ & & & & & & & $\begin{array}{c}\frac{2}{0.2} \\
\underline{0} \\
\underline{0} \\
\underline{1.3}\end{array}$ & $\begin{array}{l}\frac{3}{0} \\
0.3 \\
0.7 \\
1.4\end{array}$ & \\
\hline $\begin{array}{l}\text { Ring } \\
\text { Core }\end{array}$ & $\begin{array}{r}10 \\
\frac{0}{0} \\
\underline{0} \\
\underline{0}\end{array}$ & $\begin{array}{r}11 \\
0 \\
0 \\
0 \\
0\end{array}$ & $\begin{array}{r}16 \\
0 \\
0 \\
0\end{array}$ & $\begin{array}{l}17 \\
\underline{0} \\
\underline{0} \\
0.2\end{array}$ & $\frac{21}{\frac{1.0}{\underline{0}}} \frac{\underline{0}}{\underline{0}}$ & $\begin{array}{l}\frac{23}{\underline{0}} \\
\underline{0} \\
\underline{0} \\
\underline{0} \\
\underline{0}\end{array}$ & $\begin{array}{r}24 \\
0 \\
0 \\
0 \\
0 \\
0\end{array}$ & $\begin{array}{r}28 \\
0 \\
0 \\
0 \\
0 \\
0\end{array}$ & & & & & & & $\begin{array}{l}\frac{56}{0} \\
\frac{0}{0} \\
\underline{0} \\
\underline{0} \\
\underline{0}\end{array}$ & $\begin{array}{l}\frac{57}{0.3} \\
\frac{0}{0} \\
\underline{0} \\
\underline{0} \\
\underline{0.8}\end{array}$ & $\begin{array}{c}58 \\
0 \\
0 \\
0 \\
0.3 \\
0.3\end{array}$ & $\begin{array}{r}61 \\
\underline{0} \\
\underline{0} \\
\underline{0} \\
\underline{0} \\
\underline{0}\end{array}$ & $\begin{array}{c}62 \\
0 \\
0 \\
0 \\
0.5 \\
0\end{array}$ & $\begin{array}{c}\frac{63}{\underline{0}} \\
\underline{0} \\
\underline{0} \\
\underline{0.4} \\
\underline{0}\end{array}$ & $\begin{array}{r}64 \\
0 \\
0 \\
0 \\
0 \\
0\end{array}$ & $\begin{array}{c}\frac{1}{0.6} \\
\underline{0} \\
\underline{0.8}\end{array}$ & & \\
\hline $\begin{array}{l}\text { Sargasso } \\
\text { Sea } \\
\& \\
\text { Gulf } \\
\text { Stream }\end{array}$ & & & & & & & & & & $\frac{49}{\frac{0.7}{\underline{0}}}$ & $\begin{array}{r}50 \\
0 \\
0 \\
0 \\
0\end{array}$ & $\begin{array}{r}51 \\
0 \\
0 \\
0 \\
0\end{array}$ & $\begin{array}{c}\frac{52}{\underline{0}} \\
\underline{0} \\
\underline{0.4} \\
\underline{0.8}\end{array}$ & & & & & & & & & & & \\
\hline $\begin{array}{l}\text { Slope } \\
\text { Water }\end{array}$ & $\frac{8}{-}$ & $\frac{9}{0.2}$ & $\frac{18}{0}$ & & $\frac{19}{0}$ & $\frac{20}{0}$ & $\frac{33}{0.1}$ & $\frac{34}{1.1}$ & & $\frac{35}{0.7}$ & $\frac{47}{0.2}$ & $\frac{48}{0.2}$ & $\frac{53}{0.3}$ & $\frac{54}{0.2}$ & $\frac{59}{0}$ & $\frac{60}{0.3}$ & $\frac{65}{0}$ & $\frac{66}{0.2}$ & & & & $\frac{4}{2.7}$ & $\frac{5}{0.2}$ & $\frac{6}{0.9}$ \\
\hline $\begin{array}{l}\text { High } \\
\text { Velocity } \\
\text { Region }\end{array}$ & $\frac{12}{0}$ & $\frac{13}{0.1}$ & $\frac{14}{0.5}$ & $\frac{15}{0.3}$ & $\frac{27}{0.1}$ & $\frac{29}{0}$ & $\frac{30}{0.1}$ & $\frac{31}{0.1}$ & $\frac{32}{-}$ & $\frac{41}{0.1}$ & $\frac{42}{0.5}$ & $\frac{43}{0.1}$ & $\frac{44}{0.2}$ & & $\frac{55}{0}$ & & & & & & & $\frac{2}{0.4}$ & $\frac{3}{0.6}$ & \\
\hline $\begin{array}{l}\text { Ring } \\
\text { Core }\end{array}$ & $\frac{10}{0}$ & $\frac{11}{0}$ & $\frac{16}{-}$ & $\frac{17}{0.1}$ & $\frac{21}{0.1}$ & $\frac{23}{0}$ & $\frac{24}{0}$ & $\frac{28}{0}$ & & $\frac{37}{0}$ & $\frac{38}{0}$ & $\frac{39}{0.1}$ & & & $\frac{56}{0}$ & $\frac{57}{0.2}$ & $\frac{58}{0.1}$ & $\frac{61}{0}$ & $\frac{62}{0.1}$ & $\frac{63}{0.1}$ & $\frac{64}{0}$ & $\frac{1}{0.4}$ & & \\
\hline SS \& GS & & & & & & & & & & $\frac{49}{0.2}$ & $\frac{50}{0}$ & $\frac{51}{0}$ & $\frac{52}{0.3}$ & & & & & & & & & & & \\
\hline
\end{tabular}


Table 104. Abundance of Myctophum punctatum (specimens $/ 10,000 \mathrm{~m}^{3}$ ).

\begin{tabular}{|c|c|c|c|c|c|c|c|c|c|c|c|c|c|c|c|c|c|c|c|c|c|c|c|c|}
\hline & \multicolumn{4}{|c|}{$\begin{array}{c}\text { Oceanus } \\
\text { April }\end{array}$} & \multicolumn{5}{|c|}{$\begin{array}{c}\text { Oceanus } 121 \\
\text { June }\end{array}$} & \multicolumn{5}{|c|}{$\begin{array}{l}\text { Oceanus } 125 \\
\text { August }\end{array}$} & \multicolumn{7}{|c|}{$\begin{array}{l}\text { Knorr } 98 \\
\text { Sept-0ct }\end{array}$} & \multicolumn{3}{|c|}{$\begin{array}{c}\text { A2 } 110 \\
\text { Sept-0ct }\end{array}$} \\
\hline $\begin{array}{l}\text { Slope } \\
\text { Water }\end{array}$ & $\begin{array}{l}\frac{8}{\bar{\alpha}} \\
\underline{0} \\
\underline{0} \\
\underline{0}\end{array}$ & $\begin{array}{l}9 \\
0 \\
0 \\
0 \\
0\end{array}$ & & & $\frac{19}{\frac{0.5}{0}}$ & $\begin{array}{r}20 \\
0 \\
0 \\
0 \\
0 \\
0\end{array}$ & $\begin{array}{l}\frac{33}{\underline{0}} \\
\underline{0} \\
\underline{0} \\
\underline{0} \\
\underline{0}\end{array}$ & $\begin{array}{c}\frac{34}{\underline{0}} \\
\underline{0} \\
0.2\end{array}$ & & $\begin{array}{c}35 \\
0 \\
0 \\
0.3 \\
0.6 \\
0\end{array}$ & $\begin{array}{r}47 \\
\underline{0} \\
\underline{0} \\
\underline{0} \\
\underline{0}\end{array}$ & $\begin{array}{r}48 \\
0 \\
0 \\
0 \\
0\end{array}$ & $\begin{array}{r}53 \\
\underline{0} \\
\underline{0} \\
\underline{0} \\
\underline{0}\end{array}$ & $\begin{array}{r}54 \\
0 \\
0 \\
0 \\
0\end{array}$ & $\begin{array}{l}59 \\
\underline{0} \\
\underline{0} \\
\underline{0} \\
\underline{0} \\
\underline{0}\end{array}$ & $\begin{array}{r}60 \\
0 \\
0 \\
0 \\
0 \\
0\end{array}$ & $\begin{array}{r}\frac{65}{\underline{0}} \\
\underline{0} \\
\underline{0} \\
\underline{0} \\
\underline{0}\end{array}$ & $\begin{array}{r}66 \\
0 \\
0 \\
0 \\
0 \\
0\end{array}$ & & & & $\begin{array}{l}4 \\
0 \\
0 \\
0 \\
0\end{array}$ & $\begin{array}{l}\frac{5}{0} \\
\underline{0} \\
\underline{0} \\
\underline{0}\end{array}$ & \\
\hline $\begin{array}{l}\text { High } \\
\text { Velocity } \\
\text { Region }\end{array}$ & $\begin{array}{r}12 \\
0 \\
0 \\
0 \\
0\end{array}$ & $\frac{13}{\frac{0}{0}}$ & $\frac{14}{\underline{0}}$ & $\begin{array}{r}15 \\
0 \\
0 \\
0 \\
0\end{array}$ & $\begin{array}{c}26 \\
0 \\
0 \\
0.5 \\
13.4 \\
-\end{array}$ & $\begin{array}{c}27 \\
0 \\
0.6 \\
2.7 \\
0.8 \\
0\end{array}$ & $\begin{array}{c}\frac{30}{0.3} \\
0 \\
2.2 \\
1.9 \\
0\end{array}$ & $\begin{array}{c}31 \\
0 \\
0 \\
0.4 \\
2.0 \\
0\end{array}$ & $\begin{array}{l}\frac{32}{\frac{0.5}{0}} \\
\frac{0.5}{\underline{0}}\end{array}$ & $\frac{41}{\frac{0}{0}}$ & $\begin{array}{r}\frac{42}{\underline{0}} \\
\underline{0} \\
\underline{0} \\
\underline{0}\end{array}$ & $\begin{array}{r}43 \\
0 \\
0 \\
0 \\
0 \\
0\end{array}$ & $\begin{array}{r}\frac{44}{\underline{0}} \\
\underline{0} \\
\underline{0}\end{array}$ & & $\begin{array}{r}55 \\
\underline{0} \\
\underline{0} \\
\underline{0} \\
\underline{0}\end{array}$ & & & & & & & $\begin{array}{l}\frac{2}{0} \\
\underline{0} \\
\underline{0} \\
\underline{0}\end{array}$ & $\begin{array}{l}3 \\
0 \\
0 \\
0 \\
0\end{array}$ & \\
\hline $\begin{array}{l}\text { Ring } \\
\text { Core }\end{array}$ & $\begin{array}{r}10 \\
\frac{0}{0} \\
\underline{0} \\
\underline{0}\end{array}$ & $\begin{array}{l}11 \\
0 \\
0 \\
0 \\
0\end{array}$ & $\begin{array}{r}16 \\
0 \\
0 \\
0\end{array}$ & $\frac{17}{\underline{0}}$ & $\frac{\frac{21}{33.0}}{\frac{2.6}{7.6}} \frac{\frac{6.7}{0}}{\frac{0}{2.0}}$ & 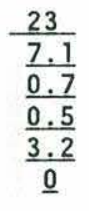 & $\begin{array}{c}24 \\
0 \\
0 \\
0.3 \\
8.8 \\
0\end{array}$ & $\begin{array}{c}\frac{28}{0.2} \\
\frac{0.9}{0} \\
\frac{8}{0}\end{array}$ & & & & & & & $\begin{array}{r}\frac{56}{\underline{0}} \\
\underline{0} \\
\underline{0} \\
\underline{0} \\
\underline{0}\end{array}$ & $\begin{array}{r}57 \\
\underline{0} \\
\underline{0} \\
\underline{0} \\
\underline{0} \\
\underline{0}\end{array}$ & $\begin{array}{r}58 \\
0 \\
0 \\
0 \\
0 \\
0\end{array}$ & $\begin{array}{r}61 \\
\underline{0} \\
\underline{0} \\
\underline{0} \\
\underline{0} \\
\underline{0}\end{array}$ & $\begin{array}{r}62 \\
0 \\
0 \\
0 \\
0 \\
0\end{array}$ & $\begin{array}{r}63 \\
\underline{0} \\
\underline{0} \\
\underline{0} \\
\underline{0} \\
\underline{0}\end{array}$ & $\begin{array}{r}64 \\
0 \\
0 \\
0 \\
0 \\
0\end{array}$ & $\begin{array}{l}\frac{1}{\underline{0}} \\
\underline{0} \\
\underline{0} \\
\underline{0}\end{array}$ & & \\
\hline $\begin{array}{l}\text { Sargasso } \\
\text { Sea } \\
\& \\
\text { Gulf } \\
\text { Stream }\end{array}$ & & & & & & & & & & $\frac{49}{\underline{0}}$ & $\begin{array}{r}50 \\
0 \\
0 \\
0 \\
0\end{array}$ & $\begin{array}{r}51 \\
0 \\
0 \\
0 \\
0\end{array}$ & $\begin{array}{r}52 \\
\underline{0} \\
\underline{0} \\
\underline{0} \\
\underline{0}\end{array}$ & & & & & & & & & & & \\
\hline $\begin{array}{l}\text { Slope } \\
\text { Water }\end{array}$ & $\frac{8}{-}$ & $\frac{9}{0}$ & $\frac{18}{0}$ & & $\frac{19}{0.1}$ & $\frac{20}{0}$ & $\frac{33}{0}$ & $\frac{34}{0.1}$ & & $\frac{35}{0.2}$ & $\frac{47}{0}$ & $\frac{48}{0}$ & $\frac{53}{0}$ & $\frac{54}{0}$ & $\frac{59}{0}$ & $\frac{60}{0}$ & $\frac{65}{0}$ & $\frac{66}{0}$ & & & & $\frac{4}{0}$ & $\frac{5}{0}$ & $\frac{6}{0}$ \\
\hline $\begin{array}{l}\text { High } \\
\text { Velocity } \\
\text { Region }\end{array}$ & $\frac{12}{0}$ & $\frac{13}{0}$ & $\frac{14}{0}$ & $\frac{15}{0}$ & $\frac{27}{0.8}$ & $\frac{29}{0.8}$ & $\frac{30}{0.9}$ & $\frac{31}{0.5}$ & $\frac{32}{-}$ & $\frac{41}{0}$ & $\frac{42}{0}$ & $\frac{43}{0}$ & $\frac{44}{0}$ & & $\frac{55}{0}$ & & & & & & & $\frac{2}{0}$ & $\frac{3}{0}$ & \\
\hline $\begin{array}{l}\text { Ring } \\
\text { Core }\end{array}$ & $\frac{10}{0}$ & $\frac{11}{0}$ & $\frac{16}{-}$ & $\frac{17}{0}$ & $\frac{21}{5.5}$ & $\frac{23}{2.0}$ & $\frac{24}{1.8}$ & $\frac{28}{2.9}$ & & $\frac{37}{0}$ & $\frac{38}{0}$ & $\frac{39}{0}$ & & & $\frac{56}{0}$ & $\frac{57}{0}$ & $\frac{58}{0}$ & $\frac{61}{0}$ & $\frac{62}{0}$ & $\frac{63}{0}$ & $\frac{64}{0}$ & $\frac{1}{0}$ & & \\
\hline SS \& GS & & & & & & & & & & $\frac{49}{0}$ & $\frac{50}{0}$ & $\frac{51}{0}$ & $\frac{52}{0}$ & & & & & & & & & & & \\
\hline
\end{tabular}


Table 105. Abundance of Myctophum selenops (specimens $/ 10,000 \mathrm{~m}^{3}$ ).

\begin{tabular}{|c|c|c|c|c|c|c|c|c|c|c|c|c|c|c|c|c|c|c|c|c|c|c|c|c|}
\hline & \multicolumn{4}{|c|}{$\begin{array}{c}\text { Oceanus } 118 \\
\text { April }\end{array}$} & \multicolumn{5}{|c|}{$\begin{array}{c}\text { Oceanus } 121 \\
\text { June }\end{array}$} & \multicolumn{5}{|c|}{$\begin{array}{l}\text { Oceanus } 125 \\
\text { August }\end{array}$} & \multicolumn{7}{|c|}{$\begin{array}{l}\text { Knorr } 98 \\
\text { Sept-0ct }\end{array}$} & \multicolumn{3}{|c|}{$\begin{array}{c}\text { A2 } 110 \\
\text { Sept-Oct }\end{array}$} \\
\hline $\begin{array}{l}\text { Slope } \\
\text { Water }\end{array}$ & $\begin{array}{l}\frac{8}{\bar{\alpha}} \\
\underline{0} \\
\underline{0} \\
\underline{0}\end{array}$ & $\begin{array}{l}9 \\
0 \\
0 \\
0 \\
0\end{array}$ & & & $\frac{\frac{19}{0.5}}{\frac{0}{0}}$ & $\begin{array}{r}20 \\
0 \\
0 \\
0 \\
0 \\
0\end{array}$ & $\begin{array}{l}3 \underline{3} \\
\underline{0} \\
\underline{0} \\
\underline{0} \\
\underline{0} \\
\underline{0}\end{array}$ & $\begin{array}{r}\frac{34}{\underline{0}} \\
\underline{0} \\
\underline{0}\end{array}$ & & $\begin{array}{c}35 \\
0 \\
0 \\
0 \\
0 \\
0\end{array}$ & $\begin{array}{r}47 \\
\underline{0} \\
\underline{0} \\
\underline{0} \\
\underline{0}\end{array}$ & $\begin{array}{c}48 \\
0.3 \\
0 \\
0 \\
0\end{array}$ & $\begin{array}{r}53 \\
\underline{0} \\
\underline{0} \\
\underline{0} \\
\underline{0}\end{array}$ & $\begin{array}{r}54 \\
0 \\
0 \\
0 \\
0\end{array}$ & $\begin{array}{l}59 \\
\underline{0} \\
\underline{0} \\
\underline{0} \\
\underline{0} \\
\underline{0}\end{array}$ & $\begin{array}{r}60 \\
0 \\
0 \\
0 \\
0 \\
0\end{array}$ & $\begin{array}{c}\frac{65}{0.4} \\
\frac{0}{\underline{0}} \\
\underline{0} \\
\underline{0} \\
\underline{0}\end{array}$ & $\begin{array}{r}66 \\
0 \\
0 \\
0 \\
0 \\
0\end{array}$ & & & & $\begin{array}{l}\frac{4}{0} \\
0 \\
0 \\
0 \\
0\end{array}$ & $\begin{array}{l}\frac{5}{0} \\
\underline{0} \\
\underline{0} \\
\underline{0}\end{array}$ & \\
\hline $\begin{array}{l}\text { High } \\
\text { Velocity } \\
\text { Region }\end{array}$ & $\begin{array}{r}12 \\
0 \\
0 \\
0 \\
0\end{array}$ & $\frac{13}{\underline{0}}$ & $\frac{14}{\frac{0}{0}}$ & $\begin{array}{r}15 \\
0 \\
0 \\
0 \\
0\end{array}$ & $\begin{array}{r}26 \\
0 \\
0 \\
0 \\
0 \\
-\end{array}$ & $\begin{array}{l}27 \\
0 \\
0 \\
0 \\
0 \\
0\end{array}$ & $\begin{array}{r}30 \\
0 \\
0 \\
0 \\
0 \\
0\end{array}$ & $\begin{array}{r}31 \\
0 \\
0 \\
0 \\
0 \\
0\end{array}$ & $\begin{array}{r}\frac{32}{\underline{0}} \\
\underline{0} \\
\underline{0} \\
\underline{0}\end{array}$ & $\frac{\frac{41}{1.4}}{\frac{0}{0}}$ & $\begin{array}{r}42 \\
\underline{0} \\
\underline{0} \\
\underline{0} \\
\underline{0}\end{array}$ & $\begin{array}{l}\frac{43}{0.6} \\
1.0 \\
0 \\
0 \\
0\end{array}$ & $\frac{\frac{44}{0.5}}{\frac{0}{0}}$ & & $\frac{55}{\frac{0.5}{\underline{0}}}$ & & & & & & & $\frac{\frac{2}{0.6}}{\frac{0}{0}} \frac{0}{\underline{0}}$ & $\begin{array}{l}3 \\
0 \\
0 \\
0 \\
0\end{array}$ & \\
\hline $\begin{array}{l}\text { Ring } \\
\text { Core }\end{array}$ & $\begin{array}{l}\frac{10}{\underline{0}} \\
\underline{0} \\
\underline{0} \\
\underline{0}\end{array}$ & $\begin{array}{r}11 \\
0 \\
0 \\
0 \\
0\end{array}$ & $\begin{array}{r}16 \\
0 \\
0 \\
0\end{array}$ & $\frac{17}{\underline{0}}$ & $\begin{array}{r}21 \\
\underline{0} \\
\underline{0} \\
\underline{0} \\
\underline{0} \\
\underline{0}\end{array}$ & $\begin{array}{r}23 \\
\underline{0} \\
\underline{0} \\
\underline{0} \\
\underline{0} \\
\underline{0}\end{array}$ & $\begin{array}{r}24 \\
0 \\
0 \\
0 \\
0 \\
0\end{array}$ & $\begin{array}{r}28 \\
0 \\
0 \\
0 \\
0 \\
0\end{array}$ & & & & & & & $\begin{array}{l}56 \\
\underline{0} \\
\underline{0} \\
\underline{0} \\
\underline{0} \\
\underline{0}\end{array}$ & $\begin{array}{l}\frac{57}{0.3} \\
\frac{0}{\underline{0}} \\
\underline{0} \\
\underline{0} \\
\underline{0}\end{array}$ & $\begin{array}{r}58 \\
0 \\
0 \\
0 \\
0 \\
0\end{array}$ & $\frac{61}{\frac{0.2}{\underline{0}}}$ & $\begin{array}{r}62 \\
0 \\
0 \\
0 \\
0 \\
0\end{array}$ & $\begin{array}{l}\frac{63}{\underline{0.2}} \\
\underline{0} \\
\underline{0} \\
\underline{0} \\
\underline{0}\end{array}$ & $\begin{array}{r}64 \\
0 \\
0 \\
0 \\
0 \\
0\end{array}$ & $\begin{array}{l}\frac{1}{0} \\
\underline{0} \\
\underline{0} \\
\underline{0}\end{array}$ & & \\
\hline $\begin{array}{l}\text { Sargasso } \\
\text { Sea } \\
\& \\
\text { Gu1f } \\
\text { Stream }\end{array}$ & & & & & & & & & & $\begin{array}{l}49 \\
\underline{0} \\
\underline{0} \\
\underline{0} \\
\underline{0}\end{array}$ & $\begin{array}{r}50 \\
0 \\
0 \\
0 \\
0\end{array}$ & $\begin{array}{r}51 \\
0 \\
0 \\
0 \\
0\end{array}$ & $\frac{52}{\frac{0.2}{\underline{0}}}$ & & & & & & & & & & & \\
\hline $\begin{array}{l}\text { Slope } \\
\text { Water }\end{array}$ & $\frac{8}{-}$ & $\frac{9}{0}$ & $\frac{18}{0}$ & & $\frac{19}{0.1}$ & $\frac{20}{0}$ & $\frac{33}{0}$ & $\frac{34}{0}$ & & $\frac{35}{0}$ & $\frac{47}{0}$ & $\frac{48}{0.1}$ & $\frac{53}{0}$ & $\frac{54}{0}$ & $\frac{59}{0}$ & $\frac{60}{0}$ & $\frac{65}{0.1}$ & $\frac{66}{0}$ & & & & $\frac{4}{0}$ & $\frac{5}{0}$ & $\frac{6}{0}$ \\
\hline $\begin{array}{l}\text { High } \\
\text { Velocity } \\
\text { Region }\end{array}$ & $\frac{12}{0}$ & $\frac{13}{0}$ & $\frac{14}{0}$ & $\frac{15}{0}$ & $\frac{27}{0}$ & $\frac{29}{0}$ & $\frac{30}{0}$ & $\frac{31}{0}$ & $\frac{32}{-}$ & $\frac{41}{0.7}$ & $\frac{42}{0}$ & $\frac{43}{0.3}$ & $\frac{44}{0.3}$ & & $\frac{55}{0.1}$ & & & & & & & $\frac{2}{0.2}$ & $\frac{3}{0}$ & \\
\hline $\begin{array}{l}\text { Ring } \\
\text { Core }\end{array}$ & $\frac{10}{0}$ & $\frac{11}{0}$ & $\frac{16}{-}$ & $\frac{17}{0}$ & $\frac{21}{0}$ & $\frac{23}{0}$ & $\frac{24}{0}$ & $\frac{28}{0}$ & & $\frac{37}{0.1}$ & $\frac{38}{0.7}$ & $\frac{39}{0.2}$ & & & $\frac{56}{0}$ & $\frac{57}{0.1}$ & $\frac{58}{0}$ & $\frac{61}{0.1}$ & $\frac{62}{0}$ & $\frac{63}{0.1}$ & $\frac{64}{0}$ & $\frac{1}{0}$ & & \\
\hline SS \& GS & & & & & & & & & & $\frac{49}{0}$ & $\frac{50}{0}$ & $\frac{51}{0}$ & $\frac{52}{0.1}$ & & & & & & & & & & & \\
\hline
\end{tabular}


Table 106. Abundance of Nemichthys scolopaceus (specimens $/ 10,000 \mathrm{~m}^{3}$ ).

\begin{tabular}{|c|c|c|c|c|c|c|c|c|c|c|c|c|c|c|c|c|c|c|c|c|c|c|c|c|}
\hline & \multicolumn{4}{|c|}{$\begin{array}{c}\text { Oceanus } 118 \\
\text { Apri1 }\end{array}$} & \multicolumn{5}{|c|}{$\begin{array}{c}\text { Oceanus } 121 \\
\text { June }\end{array}$} & \multicolumn{5}{|c|}{$\begin{array}{l}\text { Oceanus } 125 \\
\text { Auqust }\end{array}$} & \multicolumn{7}{|c|}{$\begin{array}{l}\text { Knorr } 98 \\
\text { Sept-0ct }\end{array}$} & \multicolumn{3}{|c|}{$\begin{array}{c}\text { A2 } 110 \\
\text { Sept-0ct }\end{array}$} \\
\hline $\begin{array}{l}\text { Slope } \\
\text { Water }\end{array}$ & $\begin{array}{l}8 \\
\overline{\overline{0}} \\
\underline{0} \\
\underline{0} \\
\underline{0}\end{array}$ & $\begin{array}{c}\frac{9}{0} \\
1.8 \\
0 \\
0\end{array}$ & & & 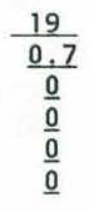 & $\begin{array}{c}20 \\
0 \\
1.1 \\
0 \\
0 \\
0\end{array}$ & $\begin{array}{l}\frac{33}{1.0} \\
\frac{0.4}{\underline{0}} \\
\frac{0}{0} \\
\underline{0}\end{array}$ & $\frac{\frac{34}{0.9}}{\frac{0.7}{0}}$ & & $\begin{array}{c}35 \\
0 \\
0 \\
0 \\
0 \\
0\end{array}$ & $\begin{array}{l}\frac{47}{0.4} \\
\underline{0} \\
\frac{0.4}{\underline{0}}\end{array}$ & $\begin{array}{c}\frac{48}{0.5} \\
0 \\
0.6 \\
0\end{array}$ & $\begin{array}{r}53 \\
\underline{0} \\
\underline{0} \\
\underline{0} \\
\underline{0}\end{array}$ & $\begin{array}{c}\frac{54}{0.3} \\
0 \\
0.3 \\
0\end{array}$ & $\begin{array}{r}\frac{59}{\underline{0}} \\
\underline{0} \\
\underline{0} \\
\underline{0} \\
\underline{0}\end{array}$ & $\begin{array}{c}60 \\
0 \\
0 \\
0.3 \\
0 \\
0\end{array}$ & $\begin{array}{r}65 \\
\underline{0} \\
\underline{0} \\
\underline{0} \\
\underline{0} \\
\underline{0}\end{array}$ & $\begin{array}{c}66 \\
0 \\
0 \\
0 \\
0.5 \\
0\end{array}$ & & & & $\begin{array}{l}4 \\
0 \\
0 \\
0 \\
0\end{array}$ & $\begin{array}{l}\frac{5}{0} \\
\underline{0} \\
\underline{0} \\
\underline{0}\end{array}$ & \\
\hline $\begin{array}{l}\text { High } \\
\text { Velocity } \\
\text { Region }\end{array}$ & $\begin{array}{r}12 \\
0 \\
0 \\
0 \\
0\end{array}$ & $\frac{13}{\frac{0}{0}}$ & $\begin{array}{l}\frac{14}{\underline{0}} \\
\underline{0} \\
\underline{0}\end{array}$ & $\begin{array}{r}15 \\
0 \\
0 \\
0 \\
0\end{array}$ & $\begin{array}{c}26 \\
0 \\
0 \\
0 \\
0 \\
-\end{array}$ & $\begin{array}{r}27 \\
0 \\
0 \\
0 \\
0 \\
0\end{array}$ & $\begin{array}{r}30 \\
0 \\
0 \\
0 \\
0 \\
0\end{array}$ & $\begin{array}{c}31 \\
0 \\
0 \\
0 \\
0.3 \\
0\end{array}$ & $\begin{array}{l}\frac{32}{\underline{0}} \\
\underline{0} \\
\underline{0}\end{array}$ & $\frac{41}{\frac{0}{0}}$ & $\begin{array}{l}\frac{42}{\underline{0}} \\
\underline{0} \\
\underline{0} \\
\underline{0.5}\end{array}$ & $\begin{array}{c}43 \\
0 \\
0 \\
0 \\
0 \\
0\end{array}$ & $\frac{44}{\underline{0}} \frac{0.3}{\underline{0}}$ & & $\begin{array}{r}5 \underline{5} \\
\underline{0} \\
\underline{0} \\
\underline{0} \\
\underline{0}\end{array}$ & & & & & & & $\begin{array}{l}\frac{2}{0} \\
\underline{0} \\
\underline{0} \\
\underline{0}\end{array}$ & $\begin{array}{c}3 \\
0 \\
0 \\
0.4 \\
0\end{array}$ & \\
\hline $\begin{array}{l}\text { Ring } \\
\text { Core }\end{array}$ & $\begin{array}{l}\frac{10}{\underline{0}} \\
\frac{0}{0.9} \\
\underline{0}\end{array}$ & $\begin{array}{c}11 \\
0 \\
0 \\
0 \\
0\end{array}$ & $\begin{array}{r}16 \\
0 \\
0 \\
0\end{array}$ & $\frac{17}{\underline{0}}$ & $\begin{array}{c}\frac{21}{\underline{0}} \\
\frac{0.2}{\underline{0}} \\
\underline{0} \\
\underline{0}\end{array}$ & $\begin{array}{c}23 \\
\underline{0} \\
\underline{0} \\
\underline{0} \\
\underline{0} \\
\underline{0}\end{array}$ & $\begin{array}{r}24 \\
0 \\
0 \\
0 \\
0 \\
0\end{array}$ & $\begin{array}{c}28 \\
0 \\
0 \\
0 \\
0.4 \\
0\end{array}$ & & & & & & & $\begin{array}{c}\frac{56}{0.3} \\
\frac{0}{\underline{0}} \\
\underline{0} \underline{0} \\
\underline{0.3}\end{array}$ & $\begin{array}{c}57 \\
\underline{0} \\
\underline{0} \\
\underline{0} \\
\underline{0} \\
\underline{0.3}\end{array}$ & $\begin{array}{c}58 \\
0 \\
0 \\
0 \\
0 \\
0.3\end{array}$ & $\begin{array}{c}\frac{61}{\underline{0}} \\
\underline{0} \\
\underline{0} \\
\underline{0.4} \\
\underline{0}\end{array}$ & $\begin{array}{c}62 \\
0 \\
0 \\
0 \\
0 \\
0.4\end{array}$ & $\begin{array}{c}\frac{63}{\underline{0}} \\
\underline{0} \\
\underline{0} \\
\underline{0} \\
\underline{0.9}\end{array}$ & $\begin{array}{c}64 \\
0 \\
0 \\
0 \\
0 \\
1.2\end{array}$ & $\begin{array}{l}\frac{1}{0} \\
\frac{0}{0} \\
\frac{0}{0}\end{array}$ & & \\
\hline $\begin{array}{l}\text { Sargasso } \\
\text { Sea } \\
\& \\
\text { Guif } \\
\text { Stream }\end{array}$ & & & & & & & & & & $\begin{array}{c}49 \\
\underline{0} \\
\underline{0} \\
\underline{0} \\
\underline{0.3}\end{array}$ & $\begin{array}{c}50 \\
0 \\
0 \\
0.3 \\
0\end{array}$ & $\begin{array}{c}51 \\
0 \\
0 \\
0 \\
0.3\end{array}$ & $\begin{array}{l}\frac{52}{\underline{0}} \\
\frac{0}{0} \\
\underline{0}\end{array}$ & & & & & & & & & & & \\
\hline $\begin{array}{l}\text { Slope } \\
\text { Water }\end{array}$ & $\frac{8}{-}$ & $\frac{9}{0.4}$ & $\frac{18}{0}$ & & $\frac{19}{0.1}$ & $\frac{20}{0.3}$ & $\frac{33}{0.1}$ & $\frac{34}{0.3}$ & & $\frac{35}{0}$ & $\frac{47}{0.2}$ & $\frac{48}{0.3}$ & $\frac{53}{0}$ & $\frac{54}{0.2}$ & $\frac{59}{0}$ & $\frac{60}{0.1}$ & $\frac{65}{0}$ & $\frac{66}{0.1}$ & & & & $\frac{4}{0}$ & $\frac{5}{0}$ & $\frac{6}{0}$ \\
\hline $\begin{array}{l}\text { High } \\
\text { Velocity } \\
\text { Region }\end{array}$ & $\frac{12}{0}$ & $\frac{13}{0}$ & $\frac{14}{0}$ & $\frac{15}{0}$ & $\frac{27}{0}$ & $\frac{29}{0}$ & $\frac{30}{0}$ & $\frac{31}{0.1}$ & $\frac{32}{-}$ & $\frac{41}{0}$ & $\frac{42}{0.1}$ & $\frac{43}{0}$ & $\frac{44}{0.1}$ & & $\frac{55}{0}$ & & & & & & & $\frac{2}{0}$ & $\frac{3}{0.1}$ & \\
\hline $\begin{array}{l}\text { Ring } \\
\text { Core }\end{array}$ & $\frac{10}{0.2}$ & $\frac{11}{0}$ & $\frac{16}{-}$ & $\frac{17}{0}$ & $\frac{21}{0.1}$ & $\frac{23}{0}$ & $\frac{24}{0}$ & $\frac{28}{0.1}$ & & $\frac{37}{0}$ & $\frac{38}{0}$ & $\frac{39}{0.1}$ & & & $\frac{56}{0.1}$ & $\frac{57}{0.1}$ & $\frac{58}{0.1}$ & $\frac{61}{0.1}$ & $\frac{62}{0.1}$ & $\frac{63}{0.2}$ & $\frac{64}{0.2}$ & $\frac{1}{0}$ & & \\
\hline SS \& GS & & & & & & & & & & $\frac{49}{0.1}$ & $\frac{50}{0.1}$ & $\frac{51}{0.1}$ & $\frac{52}{0}$ & & & & & & & & & & & \\
\hline
\end{tabular}


Table 107. Volume of Nemichthys scolopaceus $\left(\mathrm{m} 1 / 10,000 \mathrm{~m}^{3}\right)$.

\begin{tabular}{|c|c|c|c|c|c|c|c|c|c|c|c|c|c|c|c|c|c|c|c|c|c|c|c|c|}
\hline & \multicolumn{4}{|c|}{$\begin{array}{c}\text { Oceanus } \\
\text { April }\end{array}$} & \multicolumn{5}{|c|}{$\begin{array}{c}\text { Oceanus } 121 \\
\text { June }\end{array}$} & \multicolumn{5}{|c|}{$\begin{array}{c}\text { Oceanus } 125 \\
\text { Auqust }\end{array}$} & \multicolumn{7}{|c|}{$\begin{array}{l}\text { Knorr } 98 \\
\text { Sept-0ct }\end{array}$} & \multicolumn{3}{|c|}{$\begin{array}{c}\text { A2 } 110 \\
\text { Sept-0ct }\end{array}$} \\
\hline $\begin{array}{l}\text { Slope } \\
\text { Water }\end{array}$ & $\begin{array}{l}\frac{8}{\overline{0}} \\
\underline{0} \\
\underline{0} \\
\underline{0}\end{array}$ & $\begin{array}{c}\frac{9}{0} \\
16.47 \\
0 \\
0\end{array}$ & & & $\frac{\frac{19}{9,02}}{\frac{0}{0}}$ & $\begin{array}{c}\frac{20}{0} \\
4.21 \\
0 \\
0 \\
0\end{array}$ & $\frac{\frac{33}{31.33}}{\frac{1.67}{\frac{0}{0}}}$ & $\frac{\frac{34}{3.18}}{\frac{3.33}{0}}$ & & $\begin{array}{l}35 \\
0 \\
0 \\
0 \\
0 \\
0\end{array}$ & $\begin{array}{l}\frac{47}{3.15} \\
\frac{0}{6.08} \\
\underline{0}\end{array}$ & $\begin{array}{c}\frac{48}{0.75} \\
0 \\
7.94 \\
0\end{array}$ & $\begin{array}{l}\frac{53}{0} \\
\underline{0} \\
\underline{0} \\
\underline{0}\end{array}$ & $\begin{array}{c}\frac{54}{0.59} \\
0 \\
3.64 \\
0\end{array}$ & $\begin{array}{l}\frac{59}{0} \\
\underline{0} \\
\underline{0} \\
\underline{0} \\
\underline{0}\end{array}$ & $\begin{array}{l}\frac{60}{0} \\
0 \\
0.14 \\
0 \\
0\end{array}$ & $\begin{array}{l}\frac{65}{0} \\
\underline{0} \\
\underline{0} \\
\underline{0} \\
\underline{0}\end{array}$ & $\begin{array}{l}\frac{66}{0} \\
0 \\
0 \\
0.07 \\
0\end{array}$ & & & & $\begin{array}{l}4 \\
0 \\
0 \\
0 \\
0\end{array}$ & $\begin{array}{l}\frac{5}{\underline{0}} \\
\underline{0} \\
\underline{0} \\
\underline{0}\end{array}$ & \\
\hline $\begin{array}{l}\text { High } \\
\text { Velocity } \\
\text { Region }\end{array}$ & $\begin{array}{l}\frac{12}{0} \\
0 \\
0 \\
0\end{array}$ & $\begin{array}{l}\frac{13}{0} \\
\underline{0} \\
\underline{0}\end{array}$ & $\begin{array}{l}14 \\
\frac{0}{0} \\
\underline{0} \\
\underline{0}\end{array}$ & $\begin{array}{l}15 \\
0 \\
0 \\
0 \\
0\end{array}$ & $\begin{array}{l}26 \\
0 \\
0 \\
0 \\
0 \\
-\end{array}$ & $\begin{array}{l}27 \\
0 \\
0 \\
0 \\
0 \\
0\end{array}$ & $\begin{array}{l}30 \\
0 \\
0 \\
0 \\
0 \\
0\end{array}$ & $\begin{array}{l}31 \\
0 \\
0 \\
0 \\
3.71 \\
0\end{array}$ & $\begin{array}{l}\frac{32}{0} \\
\frac{0}{0} \\
\underline{0} \\
\underline{0}\end{array}$ & $\begin{array}{l}\frac{41}{\underline{0}} \\
\underline{0} \\
\underline{0}\end{array}$ & $\begin{array}{l}\frac{42}{\underline{0}} \\
\underline{0} \\
\underline{0} \\
\underline{3.81}\end{array}$ & $\begin{array}{l}43 \\
0 \\
0 \\
0 \\
0 \\
0\end{array}$ & $\begin{array}{l}\frac{44}{\underline{0}} \\
\frac{1.33}{\underline{0}}\end{array}$ & & $\begin{array}{l}\frac{55}{\underline{0}} \\
\underline{0} \\
\underline{0} \\
\underline{0}\end{array}$ & & & & & & & $\begin{array}{l}\frac{2}{0} \\
\frac{0}{0} \\
\underline{0} \\
\underline{0}\end{array}$ & $\begin{array}{c}\frac{3}{0} \\
0 \\
2.86 \\
0\end{array}$ & \\
\hline $\begin{array}{l}\text { Ring } \\
\text { Core }\end{array}$ & $\begin{array}{c}\frac{10}{\frac{0}{0}} \\
\frac{13.64}{\underline{0}}\end{array}$ & $\begin{array}{l}11 \\
0 \\
0 \\
0 \\
0\end{array}$ & $\begin{array}{l}16 \\
0 \\
0 \\
0\end{array}$ & $\begin{array}{l}17 \\
\underline{0} \\
\underline{0} \\
\underline{0}\end{array}$ & $\begin{array}{l}\frac{21}{\underline{0}} \\
\frac{3.00}{\underline{0}} \\
\underline{0} \\
\underline{0}\end{array}$ & $\begin{array}{l}\frac{23}{\underline{0}} \\
\underline{0} \\
\underline{0} \\
\underline{0} \\
\underline{0}\end{array}$ & $\begin{array}{l}24 \\
0 \\
0 \\
0 \\
0 \\
0\end{array}$ & $\begin{array}{c}\frac{28}{0} \\
0 \\
0 \\
5.77 \\
0\end{array}$ & & & & & & & $\begin{array}{l}\frac{56}{0.35} \\
\frac{0}{0} \\
\frac{0}{0} \\
\underline{0.13}\end{array}$ & $\begin{array}{l}\frac{57}{0} \\
\frac{0}{0} \\
\underline{0} \\
\underline{0} \\
0.24\end{array}$ & $\begin{array}{l}\frac{58}{0} \\
0 \\
0 \\
0 \\
0.24\end{array}$ & $\begin{array}{l}\frac{61}{\underline{0}} \\
\underline{0} \\
\underline{0} \\
\frac{0.19}{\underline{0}}\end{array}$ & $\begin{array}{l}\frac{62}{0} \\
0 \\
0 \\
0 \\
0.05\end{array}$ & $\begin{array}{l}\frac{63}{\underline{0}} \\
\underline{0} \\
\underline{0} \\
\underline{0} \\
\underline{0.63}\end{array}$ & $\begin{array}{l}\frac{64}{0} \\
0 \\
0 \\
0 \\
0.54\end{array}$ & $\begin{array}{l}\frac{1}{0} \\
\underline{0} \\
\underline{0} \\
\underline{0}\end{array}$ & & \\
\hline $\begin{array}{l}\text { Sargasso } \\
\text { Sea } \\
\& \\
\text { Gulf } \\
\text { Stream }\end{array}$ & & & & & & & & & & $\begin{array}{l}\frac{49}{\underline{0}} \\
\underline{0} \\
\underline{0} \\
\underline{0.03}\end{array}$ & $\begin{array}{c}50 \\
0 \\
0 \\
5.86 \\
0\end{array}$ & $\begin{array}{l}\frac{51}{0} \\
0 \\
0 \\
0.17\end{array}$ & $\begin{array}{l}\frac{52}{\underline{0}} \\
\underline{0} \\
\underline{0} \\
\underline{0}\end{array}$ & & & & & & & & & & & \\
\hline $\begin{array}{l}\text { Slope } \\
\text { Water }\end{array}$ & $\frac{8}{-}$ & $\frac{9}{4.12}$ & $\frac{18}{0}$ & & $\frac{19}{0.90}$ & $\frac{20}{1.05}$ & $\frac{33}{2.11}$ & $\frac{34}{1.32}$ & & $\frac{35}{0}$ & $\frac{47}{2.31}$ & $\frac{48}{2.17}$ & $\frac{53}{0}$ & $\frac{54}{1.06}$ & $\frac{59}{0}$ & $\frac{60}{0.03}$ & $\frac{65}{0}$ & $\frac{66}{0.01}$ & & & & $\frac{4}{0}$ & $\frac{5}{0}$ & $\frac{6}{0}$ \\
\hline $\begin{array}{l}\text { High } \\
\text { Velocity } \\
\text { Region }\end{array}$ & $\frac{12}{0}$ & $\frac{13}{0}$ & $\frac{14}{0}$ & $\frac{15}{0}$ & $\frac{27}{0}$ & $\frac{29}{0}$ & $\frac{30}{0}$ & $\frac{31}{0.74}$ & $\frac{32}{-}$ & $\frac{41}{0}$ & $\frac{42}{0.76}$ & $\frac{43}{0}$ & $\frac{44}{0.27}$ & & $\frac{55}{0}$ & & & & & & & $\frac{2}{0}$ & $\frac{3}{0.72}$ & \\
\hline $\begin{array}{l}\text { Ring } \\
\text { Core }\end{array}$ & $\frac{10}{3.41}$ & $\frac{11}{0}$ & $\frac{16}{-}$ & $\frac{17}{0}$ & $\frac{21}{1.05}$ & $\frac{23}{0}$ & $\frac{24}{0}$ & $\frac{28}{1.44}$ & & $\frac{37}{0}$ & $\frac{38}{0}$ & $\frac{39}{0.78}$ & & & $\frac{56}{0.10}$ & $\frac{57}{0.05}$ & $\frac{58}{0.05}$ & $\frac{61}{0.04}$ & $\frac{62}{0.01}$ & $\frac{63}{0.13}$ & $\frac{64}{0.11}$ & $\frac{1}{0}$ & & \\
\hline SS \& GS & & & & & & & & & & $\frac{49}{0.01}$ & $\frac{50}{1.47}$ & $\frac{51}{0.04}$ & $\frac{52}{0}$ & & & & & & & & & & & \\
\hline
\end{tabular}


Table 108. Abundance of Notolychnus valdiviae (specimens $/ 10,000 \mathrm{~m}^{3}$ ).

\begin{tabular}{|c|c|c|c|c|c|c|c|c|c|c|c|c|c|c|c|c|c|c|c|c|c|c|c|c|}
\hline & \multicolumn{4}{|c|}{$\begin{array}{c}\text { Oceanus } 118 \\
\text { April }\end{array}$} & \multicolumn{5}{|c|}{$\begin{array}{c}\text { Oceanus } 121 \\
\text { June }\end{array}$} & \multicolumn{5}{|c|}{$\begin{array}{c}\text { Oceanus } 125 \\
\text { August }\end{array}$} & \multicolumn{7}{|c|}{$\begin{array}{l}\text { Knorr } 98 \\
\text { Sept-0ct }\end{array}$} & \multicolumn{3}{|c|}{$\begin{array}{c}\text { A2 } 110 \\
\text { Sept-0ct }\end{array}$} \\
\hline $\begin{array}{l}\text { Slope } \\
\text { Water }\end{array}$ & $\begin{array}{l}\frac{8}{\overline{0}} \\
\underline{0} \\
\underline{0} \\
\underline{0}\end{array}$ & $\begin{array}{l}\frac{9}{0} \\
1.8 \\
0 \\
0\end{array}$ & & & $\begin{array}{l}\frac{19}{4.1} \\
\frac{0.2}{0.2} \\
\frac{0}{0}\end{array}$ & $\begin{array}{c}20 \\
0 \\
1.1 \\
0 \\
0 \\
0\end{array}$ & $\begin{array}{l}\frac{33}{2.3} \\
\frac{2.4}{0 .} \\
\underline{0.6} \\
\underline{0}\end{array}$ & $\frac{\frac{34}{1.4}}{\frac{0}{0}}$ & & $\begin{array}{c}35 \\
0 \\
0.4 \\
3.0 \\
0 \\
0\end{array}$ & $\frac{\frac{47}{0.6}}{\frac{0.3}{0}}$ & $\begin{array}{c}\frac{48}{0} \\
2.3 \\
0 \\
0\end{array}$ & $\begin{array}{r}53 \\
\underline{0} \\
\underline{0} \\
\underline{0} \\
\underline{0}\end{array}$ & $\begin{array}{c}54 \\
0 \\
0.6 \\
0 \\
0\end{array}$ & $\frac{\frac{59}{1.0}}{\frac{1.4}{1.8}} \frac{\underline{0}}{\underline{0}}$ & $\begin{array}{c}60 \\
0 \\
0 \\
7.6 \\
0 \\
0\end{array}$ & $\begin{array}{r}65 \\
\underline{0} \\
\underline{0} \\
\underline{0} \\
\underline{0} \\
\underline{0}\end{array}$ & $\begin{array}{c}\frac{66}{0} \\
6.4 \\
3.0 \\
0 \\
0\end{array}$ & & & & $\begin{array}{c}\frac{4}{0} \\
1.1 \\
0 \\
0\end{array}$ & $\begin{array}{l}\frac{5}{0} \\
\underline{0} \\
\underline{0} \\
\underline{0} \\
\underline{0}\end{array}$ & \\
\hline $\begin{array}{l}\text { High } \\
\text { Velocity } \\
\text { Region }\end{array}$ & $\begin{array}{c}12 \\
0 \\
0.4 \\
0.8 \\
0\end{array}$ & $\frac{\frac{13}{3.0}}{\frac{0}{0}}$ & $\frac{\frac{14}{\frac{2.3}{1.7}}}{\frac{0}{0}}$ & $\begin{array}{c}15 \\
0 \\
4.7 \\
0 \\
0\end{array}$ & $\begin{array}{c}26 \\
0 \\
0 \\
2.4 \\
0.3 \\
-\end{array}$ & $\begin{array}{r}27 \\
0 \\
0 \\
0 \\
0 \\
0\end{array}$ & $\begin{array}{c}30 \\
0 \\
0 \\
38.4 \\
0 \\
0\end{array}$ & $\begin{array}{c}31 \\
0 \\
0 \\
2.9 \\
0 \\
0\end{array}$ & $\frac{\frac{32}{0.5}}{\frac{0.4}{\underline{0}}}$ & $\begin{array}{r}41 \\
\underline{0} \\
\underline{0} \\
\underline{0}\end{array}$ & $\frac{\frac{42}{0.3}}{\frac{0.6}{\underline{0}}}$ & $\begin{array}{c}43 \\
0 \\
0 \\
3.3 \\
0 \\
0\end{array}$ & $\frac{44}{\frac{0.6}{\underline{0}}}$ & & $\frac{\frac{55}{4.7}}{\frac{1.4}{2.0}} \frac{0}{\frac{0}{2}}$ & & & & & & & $\frac{\frac{2}{0.8}}{\frac{0}{0}} \frac{\underline{0}}{\underline{0}}$ & $\begin{array}{c}\frac{3}{0} \\
1.4 \\
0 \\
0\end{array}$ & \\
\hline $\begin{array}{l}\text { Ring } \\
\text { Core }\end{array}$ & $\frac{\frac{10}{\frac{1.2}{2.9}}}{\frac{0}{0}}$ & $\begin{array}{c}11 \\
0 \\
3.9 \\
0.6 \\
0\end{array}$ & $\begin{array}{c}16 \\
0 \\
4.3 \\
0\end{array}$ & $\frac{\frac{17}{\frac{1.1}{0.5}}}{\underline{0}}$ & $\frac{21}{\underline{0}}$ & $\begin{array}{c}\frac{23}{\underline{0}} \\
\underline{2.7} \\
\underline{0} \\
\underline{0} \\
\underline{0}\end{array}$ & $\begin{array}{c}24 \\
0 \\
2.7 \\
0 \\
0 \\
0\end{array}$ & $\begin{array}{c}28 \\
0 \\
4.6 \\
2.9 \\
0 \\
0\end{array}$ & & & & & & & $\frac{\frac{56}{14.1}}{\frac{0}{\underline{0}}}$ & $\frac{\frac{57}{25.0}}{\frac{0.6}{0.3}} \frac{\frac{0.3}{2.2}}{\underline{0}}$ & $\begin{array}{c}58 \\
0 \\
0 \\
5.2 \\
2.3 \\
0\end{array}$ & $\begin{array}{c}\frac{61}{17.6} \\
\frac{1.2}{\underline{0}} \\
\frac{7.7}{\underline{0}}\end{array}$ & $\begin{array}{c}\frac{62}{0.2} \\
0 \\
18.6 \\
3.2 \\
0\end{array}$ & $\begin{array}{c}\frac{63}{8.0} \\
\underline{0} \\
\frac{1.0}{\underline{0}} \\
\underline{0}\end{array}$ & $\begin{array}{c}64 \\
0 \\
8.8 \\
2.9 \\
1.4 \\
0\end{array}$ & $\frac{\frac{1}{1.8}}{\frac{0.2}{\frac{0}{0}}}$ & & \\
\hline $\begin{array}{l}\text { Sargasso } \\
\text { Sea } \\
\& \\
\text { Guif } \\
\text { Stream }\end{array}$ & & & & & & & & & & $\frac{\frac{49}{\frac{0.5}{2.2}}}{\frac{0}{0}}$ & $\begin{array}{c}50 \\
0 \\
2.4 \\
8.6 \\
0\end{array}$ & $\begin{array}{c}51 \\
0 \\
0 \\
5.0 \\
0.3\end{array}$ & $\frac{\frac{52}{1.6}}{\frac{1.9}{\frac{0}{0}}}$ & & & & & & & & & & & \\
\hline $\begin{array}{l}\text { Slope } \\
\text { Water }\end{array}$ & $\frac{8}{-}$ & $\frac{9}{0.5}$ & $\frac{18}{0}$ & & $\frac{19}{0.5}$ & $\frac{20}{0.3}$ & $\frac{33}{0.3}$ & $\frac{34}{0.1}$ & & $\frac{35}{0.7}$ & $\frac{47}{0.2}$ & $\frac{48}{0.6}$ & $\frac{53}{0}$ & $\frac{54}{0.2}$ & $\frac{59}{1.0}$ & $\frac{60}{1.5}$ & $\frac{65}{0}$ & $\frac{66}{1.2}$ & & & & $\frac{4}{0.3}$ & $\frac{5}{0}$ & $\frac{6}{0.1}$ \\
\hline $\begin{array}{l}\text { High } \\
\text { Velocity } \\
\text { Region }\end{array}$ & $\frac{12}{0.3}$ & $\frac{13}{0.8}$ & $\frac{14}{1.0}$ & $\frac{15}{1.2}$ & $\frac{27}{0}$ & $\frac{29}{2.8}$ & $\frac{30}{7.6}$ & $\frac{31}{0.6}$ & $\frac{32}{-}$ & $\frac{41}{0}$ & $\frac{42}{0.3}$ & $\frac{43}{0.7}$ & $\frac{44}{0.4}$ & & $\frac{55}{2.0}$ & & & & & & & $\frac{2}{0.2}$ & $\frac{3}{0.4}$ & \\
\hline $\begin{array}{l}\text { Ring } \\
\text { Core }\end{array}$ & $\frac{10}{1.0}$ & $\frac{11}{1.1}$ & $\frac{16}{-}$ & $\frac{17}{0.4}$ & $\frac{21}{0.4}$ & $\frac{23}{0.7}$ & $\frac{24}{0.5}$ & $\frac{28}{1.6}$ & & $\frac{37}{0.5}$ & $\frac{38}{0.4}$ & $\frac{39}{0.1}$ & & & $\frac{56}{2.8}$ & $\frac{57}{5.6}$ & $\frac{58}{1.5}$ & $\frac{61}{5.3}$ & $\frac{62}{4.4}$ & $\frac{63}{3.1}$ & $\frac{64}{1.5}$ & $\frac{1}{0.5}$ & & \\
\hline SS \& GS & & & & & & & & & & $\frac{49}{1.8}$ & $\frac{50}{2.8}$ & $\frac{51}{1.3}$ & $\frac{52}{0.9}$ & & & & & & & & & & & \\
\hline
\end{tabular}


Table 109. Abundance of Notescopelus resplendens (specimens $/ 10,000 \mathrm{~m}^{3}$ ).

\begin{tabular}{|c|c|c|c|c|c|c|c|c|c|c|c|c|c|c|c|c|c|c|c|c|c|c|c|c|}
\hline & & $\begin{array}{r}\text { Oceanu } \\
\text { Apr }\end{array}$ & 118 & & & & $\begin{array}{l}\text { anus } \\
\text { June }\end{array}$ & 121 & & & $\begin{array}{r}\text { Oce } \\
\mathrm{A}\end{array}$ & $\begin{array}{l}\text { inus } 1 \\
\text { Igust }\end{array}$ & & & & & $\begin{array}{l}\text { Knor } \\
\text { Sept }\end{array}$ & $\begin{array}{r}98 \\
0 c t \\
\end{array}$ & & & & & $\begin{array}{l}110 \\
t-0 c t\end{array}$ & \\
\hline $\begin{array}{l}\text { Slope } \\
\text { Water }\end{array}$ & $\begin{array}{l}\frac{8}{\overline{\overline{0}}} \\
\underline{\underline{0}} \\
\underline{0} \\
\underline{0}\end{array}$ & $\begin{array}{l}\frac{9}{0} \\
0 \\
0 \\
0\end{array}$ & & & 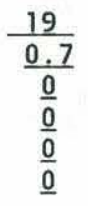 & $\begin{array}{c}20 \\
0 \\
0 \\
0 \\
0 \\
0.4\end{array}$ & $\frac{\frac{33}{2.0}}{\frac{2.4}{0.4}} \frac{\underline{0}}{\underline{0}}$ & $\frac{34}{\underline{0}} \frac{0}{\underline{0}}$ & & $\begin{array}{r}35 \\
0 \\
0 \\
0 \\
0 \\
0\end{array}$ & $\begin{array}{r}47 \\
\underline{0} \\
\underline{0} \\
\underline{0}\end{array}$ & $\begin{array}{c}48 \\
0 \\
0 \\
0.3 \\
0\end{array}$ & $\begin{array}{l}53 \\
\underline{0} \\
\underline{0} \\
\underline{0}\end{array}$ & $\begin{array}{r}54 \\
0 \\
0 \\
0 \\
0\end{array}$ & $\begin{array}{l}59 \\
\underline{0} \\
\underline{0} \\
\underline{0} \\
\underline{0} \\
\underline{0}\end{array}$ & $\begin{array}{c}60 \\
0 \\
0 \\
0 \\
0 \\
0.9\end{array}$ & $\begin{array}{r}65 \\
\underline{0} \\
\underline{0} \\
\underline{0} \\
\underline{0} \\
\underline{0}\end{array}$ & $\begin{array}{r}66 \\
0 \\
0 \\
0 \\
0 \\
0\end{array}$ & & & & $\begin{array}{l}4 \\
0 \\
0 \\
0 \\
0\end{array}$ & $\frac{\frac{5}{0.7}}{\frac{0}{0}} \frac{0}{\underline{0}}$ & \\
\hline $\begin{array}{l}\text { High } \\
\text { Velocity } \\
\text { Region }\end{array}$ & $\begin{array}{r}12 \\
0 \\
0 \\
0 \\
0\end{array}$ & $\frac{\frac{13}{0.5}}{\frac{0.6}{\underline{0}}}$ & $\frac{\frac{14}{0.3}}{\frac{0}{0}} \frac{\underline{0}}{\underline{0}}$ & $\begin{array}{r}15 \\
0 \\
0 \\
0 \\
0\end{array}$ & $\begin{array}{c}26 \\
0 \\
0 \\
0 \\
0 \\
-\end{array}$ & $\begin{array}{c}27 \\
0 \\
0 \\
0 \\
0 \\
0.7\end{array}$ & $\begin{array}{c}30 \\
0 \\
0 \\
0 \\
0 \\
0.7\end{array}$ & $\begin{array}{r}31 \\
0 \\
0 \\
0 \\
0 \\
0\end{array}$ & $\frac{\frac{32}{1.1}}{\frac{0}{0}}$ & $\frac{41}{\frac{0.1}{\underline{0}}}$ & $\frac{\frac{42}{0.1}}{\frac{0}{\underline{0}}}$ & $\begin{array}{c}43 \\
0 \\
0 \\
0 \\
0 \\
1.0\end{array}$ & $\frac{\frac{44}{0.5}}{\frac{0}{0}}$ & & $\begin{array}{l}\frac{55}{\underline{0}} \\
\underline{0} \\
\underline{0} \\
\underline{0}\end{array}$ & & & & & & & $\frac{\frac{2}{1.0}}{\frac{0}{0}} \frac{\underline{0}}{\underline{1}}$ & $\begin{array}{l}3 \\
0 \\
0 \\
0 \\
0\end{array}$ & \\
\hline $\begin{array}{l}\text { Ring } \\
\text { Core }\end{array}$ & $\begin{array}{l}\frac{10}{0} \\
\underline{0} \\
\underline{0} \\
\underline{0}\end{array}$ & $\begin{array}{l}11 \\
0 \\
0 \\
0 \\
0\end{array}$ & $\begin{array}{r}16 \\
0 \\
0 \\
0\end{array}$ & $\frac{17}{\underline{0}}$ & $\begin{array}{l}\frac{21}{1.0} \\
\frac{0}{0.3} \\
\frac{0}{0}\end{array}$ & $\frac{23}{\frac{1.8}{\underline{0}}}$ & $\begin{array}{c}24 \\
0.9 \\
0 \\
0 \\
0 \\
0\end{array}$ & $\begin{array}{c}28 \\
0 \\
0 \\
0.4 \\
0.4 \\
0\end{array}$ & & & & & & & $\begin{array}{r}56 \\
\underline{0} \\
\underline{0} \\
\underline{0} \\
\underline{0} \\
\underline{0}\end{array}$ & $\begin{array}{l}57 \\
\underline{0} \\
\underline{0} \\
\underline{0} \\
\underline{0} \\
\underline{0}\end{array}$ & $\begin{array}{c}58 \\
0 \\
0 \\
0 \\
0 \\
0\end{array}$ & $\begin{array}{r}61 \\
\underline{0} \\
\underline{0} \\
\underline{0} \\
\underline{0} \\
\underline{0}\end{array}$ & $\begin{array}{r}62 \\
0 \\
0 \\
0 \\
0 \\
0\end{array}$ & $\begin{array}{r}63 \\
\underline{0} \\
\underline{0} \\
\underline{0} \\
\underline{0} \\
\underline{0}\end{array}$ & $\begin{array}{r}64 \\
0 \\
0 \\
0 \\
0 \\
0\end{array}$ & $\frac{\frac{1}{0.4}}{\frac{0}{0}} \frac{0}{\underline{0}}$ & & \\
\hline $\begin{array}{l}\text { Sargasso } \\
\text { Sea } \\
\& \\
\text { Gulf } \\
\text { Stream }\end{array}$ & & & & & & & & & & $\frac{\frac{49}{0.3}}{\frac{0}{0}}$ & $\begin{array}{r}50 \\
0 \\
0 \\
0 \\
0\end{array}$ & $\begin{array}{l}51 \\
0 \\
0 \\
0 \\
0\end{array}$ & $\begin{array}{l}\frac{52}{\underline{0}} \\
\underline{0} \\
\underline{0} \\
\underline{0}\end{array}$ & & & & & & & & & & & \\
\hline $\begin{array}{l}\text { Slope } \\
\text { Water }\end{array}$ & $\frac{8}{-}$ & $\frac{9}{0}$ & $\frac{18}{0}$ & & $\frac{19}{0.1}$ & $\frac{20}{0}$ & $\frac{33}{0.2}$ & $\frac{34}{0}$ & & $\frac{35}{0}$ & $\frac{47}{0}$ & $\frac{48}{0.1}$ & $\frac{53}{0}$ & $\frac{54}{0}$ & $\frac{59}{0}$ & $\frac{60}{0.2}$ & $\frac{65}{0}$ & $\frac{66}{0}$ & & & & $\frac{4}{0}$ & $\frac{5}{0.2}$ & $\frac{6}{0.1}$ \\
\hline $\begin{array}{l}\text { High } \\
\text { Velocity } \\
\text { Region }\end{array}$ & $\frac{12}{0}$ & $\frac{13}{0.4}$ & $\frac{14}{0.1}$ & $\frac{15}{0}$ & $\frac{27}{0.2}$ & $\frac{29}{0.1}$ & $\frac{30}{0.2}$ & $\frac{31}{0}$ & $\frac{32}{-}$ & $\frac{41}{0.1}$ & $\frac{42}{0}$ & $\frac{43}{0.2}$ & $\frac{44}{0.3}$ & & $\frac{55}{0}$ & & & & & & & $\frac{2}{0.3}$ & $\frac{3}{0}$ & \\
\hline $\begin{array}{l}\text { Ring } \\
\text { Core }\end{array}$ & $\frac{10}{0}$ & $\frac{11}{0}$ & $\frac{16}{-}$ & $\frac{17}{0}$ & $\frac{21}{0.1}$ & $\frac{23}{0.3}$ & $\frac{24}{0.3}$ & $\frac{28}{0.2}$ & & $\frac{37}{0.1}$ & $\frac{38}{0.5}$ & $\frac{39}{0.3}$ & & & $\frac{56}{0}$ & $\frac{57}{0}$ & $\frac{58}{0}$ & $\frac{61}{0}$ & $\frac{62}{0}$ & $\frac{63}{0}$ & $\frac{64}{0}$ & $\frac{1}{0.1}$ & & \\
\hline SS \& GS & & & & & & & & & & $\frac{49}{0.1}$ & $\frac{50}{0}$ & $\frac{51}{0}$ & $\frac{52}{0}$ & & & . & & & & & & & & \\
\hline
\end{tabular}


Table 110. Volume of Notoscopelus resplendens $\left(\mathrm{ml} / 10,000 \mathrm{~m}^{3}\right)$.

\begin{tabular}{|c|c|c|c|c|c|c|c|c|c|c|c|c|c|c|c|c|c|c|c|c|c|c|c|c|}
\hline & \multicolumn{4}{|c|}{$\underset{\substack{\text { Oceanus } \\
\text { Apri1 }}}{118}$} & \multicolumn{5}{|c|}{$\begin{array}{c}\text { Oceanus } \\
\text { June }\end{array}$} & \multicolumn{5}{|c|}{$\begin{array}{c}\text { Oceanus } 125 \\
\text { August }\end{array}$} & \multicolumn{7}{|c|}{$\begin{array}{l}\text { Knorr } 98 \\
\text { Sept-0ct }\end{array}$} & \multicolumn{3}{|c|}{$\begin{array}{c}\text { A2 } 110 \\
\text { Sept-0ct }\end{array}$} \\
\hline $\begin{array}{l}\text { Slope } \\
\text { Water }\end{array}$ & $\begin{array}{l}\frac{8}{\overline{\overline{0}}} \\
\underline{0} \\
\underline{0} \\
\underline{0}\end{array}$ & $\begin{array}{l}\frac{9}{0} \\
0 \\
0 \\
0\end{array}$ & & & $\frac{\frac{19}{0.98}}{\frac{0}{0}}$ & $\begin{array}{c}20 \\
0 \\
0 \\
0 \\
0 \\
0.24\end{array}$ & $\frac{\frac{33}{5.00}}{\frac{1.25}{\frac{0}{0}}}$ & $\begin{array}{l}\frac{34}{0} \\
\frac{0}{0} \\
\underline{0}\end{array}$ & & $\begin{array}{l}35 \\
0 \\
0 \\
0 \\
0 \\
0\end{array}$ & $\begin{array}{l}\frac{47}{0} \\
\frac{0}{0} \\
\frac{0}{0} \\
\underline{0}\end{array}$ & $\begin{array}{l}48 \\
0 \\
0 \\
0 \\
0\end{array}$ & $\begin{array}{l}\frac{53}{0} \\
\underline{0} \\
\underline{0} \\
\underline{0}\end{array}$ & $\begin{array}{l}54 \\
0 \\
0 \\
0 \\
0\end{array}$ & $\begin{array}{l}\frac{59}{0} \\
\underline{0} \\
\underline{0} \\
\underline{0} \\
\underline{0}\end{array}$ & $\begin{array}{c}\frac{60}{0} \\
0 \\
0 \\
0 \\
0.91\end{array}$ & $\begin{array}{l}\frac{65}{\underline{0}} \\
\underline{0} \\
\underline{0} \\
\underline{0} \\
\underline{0}\end{array}$ & $\begin{array}{l}\frac{66}{0} \\
0 \\
0 \\
0 \\
0\end{array}$ & & & & $\begin{array}{l}4 \\
0 \\
0 \\
0 \\
0\end{array}$ & $\frac{\frac{5}{0.90}}{\frac{0}{0}} \frac{0}{0}$ & \\
\hline $\begin{array}{l}\text { High } \\
\text { Velocity } \\
\text { Region }\end{array}$ & $\begin{array}{l}\frac{12}{0} \\
0 \\
0 \\
0\end{array}$ & $\frac{\frac{13}{0.05}}{\frac{0.06}{0}}$ & $\frac{\frac{14}{0.03}}{\frac{0}{0}}$ & $\begin{array}{l}\frac{15}{15} \\
0 \\
0 \\
0\end{array}$ & $\begin{array}{l}26 \\
0 \\
0 \\
0 \\
0 \\
-\end{array}$ & $\begin{array}{c}27 \\
0 \\
0 \\
0 \\
0 \\
0.35\end{array}$ & $\begin{array}{c}30 \\
0 \\
0 \\
0 \\
0 \\
0.19\end{array}$ & $\begin{array}{l}\frac{31}{0} \\
0 \\
0 \\
0 \\
0\end{array}$ & $\frac{\frac{32}{0.68}}{\frac{0}{0}}$ & $\frac{\frac{41}{0.01}}{\frac{0}{0}}$ & $\frac{\frac{42}{0.08}}{\frac{0}{0}}$ & $\begin{array}{l}43 \\
0 \\
0 \\
0 \\
0 \\
0.95\end{array}$ & $\frac{\frac{44}{0.79}}{\frac{0}{0}}$ & & $\begin{array}{l}55 \\
\underline{0} \\
\underline{0} \\
\underline{0} \\
\underline{0}\end{array}$ & & & & & & & $\frac{\frac{2}{1.67}}{\frac{0}{0}}$ & $\begin{array}{l}3 \\
0 \\
0 \\
0 \\
0\end{array}$ & \\
\hline $\begin{array}{l}\text { Ring } \\
\text { Core }\end{array}$ & $\begin{array}{l}\frac{10}{0} \\
\frac{0}{0} \\
\frac{0}{0} \\
\underline{0}\end{array}$ & $\begin{array}{l}11 \\
0 \\
0 \\
0 \\
0\end{array}$ & $\begin{array}{l}\frac{16}{16} \\
0 \\
0\end{array}$ & $\begin{array}{l}\frac{17}{0} \\
\frac{0}{0}\end{array}$ & $\begin{array}{l}\frac{21}{0.10} \\
\underline{0} \\
\frac{0.03}{\underline{0}} \\
\underline{0}\end{array}$ & 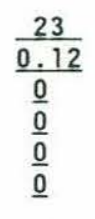 & $\begin{array}{l}\frac{24}{0.03} \\
0 \\
0 \\
0 \\
0\end{array}$ & $\begin{array}{l}28 \\
0 \\
0 \\
0 \\
0.04 \\
0.03\end{array}$ & & & & & & & $\begin{array}{l}\frac{56}{\underline{0}} \\
\underline{0} \\
\underline{0} \\
\underline{0} \\
\underline{0}\end{array}$ & $\begin{array}{l}\frac{57}{\underline{0}} \\
\underline{0} \\
\underline{0} \\
\underline{0} \\
\underline{0}\end{array}$ & $\begin{array}{l}58 \\
0 \\
0 \\
0 \\
0 \\
0\end{array}$ & $\begin{array}{l}\frac{61}{\underline{0}} \\
0 \\
\underline{0} \\
\underline{0} \\
\underline{0}\end{array}$ & $\begin{array}{l}62 \\
0 \\
0 \\
0 \\
0 \\
0\end{array}$ & $\begin{array}{l}\frac{63}{0} \\
\frac{0}{0} \\
\underline{0} \\
\underline{0} \\
\underline{0}\end{array}$ & $\begin{array}{l}\frac{64}{0} \\
0 \\
0 \\
0 \\
0\end{array}$ & $\frac{\frac{1}{0.50}}{\frac{0}{0}} \frac{0}{\underline{0}}$ & & \\
\hline $\begin{array}{l}\text { Sargasso } \\
\text { Sea } \\
\& \\
\text { Gulf } \\
\text { Stream }\end{array}$ & & & & & & & & & & $\frac{\frac{49}{0.18}}{\frac{0}{0}}$ & $\begin{array}{l}50 \\
0 \\
0 \\
0 \\
0\end{array}$ & $\begin{array}{l}51 \\
0 \\
0 \\
0 \\
0\end{array}$ & $\begin{array}{l}\frac{52}{0} \\
\underline{0} \\
\underline{0} \\
\underline{0}\end{array}$ & & & & & & & & & & & \\
\hline $\begin{array}{l}\text { Slope } \\
\text { Water }\end{array}$ & $\frac{8}{-}$ & $\frac{9}{0}$ & $\frac{18}{0}$ & & $\frac{19}{0.10}$ & $\frac{20}{0}$ & $\frac{33}{0.48}$ & $\frac{34}{0}$ & & $\frac{35}{0}$ & $\frac{47}{0}$ & $\frac{48}{0}$ & $\frac{53}{0}$ & $\frac{54}{0}$ & $\frac{59}{0}$ & $\frac{60}{0.18}$ & $\frac{65}{0}$ & $\frac{66}{0}$ & & & & $\frac{4}{0}$ & $\frac{5}{0.23}$ & $\frac{6}{0.16}$ \\
\hline $\begin{array}{l}\text { High } \\
\text { Velocity } \\
\text { Region }\end{array}$ & $\frac{12}{0}$ & $\frac{13}{0.03}$ & $\frac{14}{0.01}$ & $\frac{15}{0}$ & $\frac{27}{0.09}$ & $\frac{29}{0.01}$ & $\frac{30}{0.04}$ & $\frac{31}{0}$ & $\frac{32}{-}$ & $\frac{41}{0.01}$ & $\frac{42}{0.03}$ & $\frac{43}{0.19}$ & $\frac{44}{0.47}$ & & $\frac{55}{0}$ & & & & & & & $\frac{2}{0.42}$ & $\frac{3}{0}$ & \\
\hline $\begin{array}{l}\text { Ring } \\
\text { Core }\end{array}$ & $\frac{10}{0}$ & $\frac{11}{0}$ & $\frac{16}{-}$ & $\frac{17}{0}$ & $\frac{21}{0.01}$ & $\frac{23}{0.02}$ & $\frac{24}{0.01}$ & $\frac{28}{0.01}$ & & $\frac{37}{0.02}$ & $\frac{38}{0.52}$ & $\frac{39}{0.71}$ & & & $\frac{56}{0}$ & $\frac{57}{0}$ & $\frac{58}{0}$ & $\frac{61}{0}$ & $\frac{62}{0}$ & $\frac{63}{0}$ & $\frac{64}{0}$ & $\frac{1}{0.13}$ & & \\
\hline SS \& GS & & & & & & & & & & $\frac{49}{0.05}$ & $\frac{50}{0}$ & $\frac{51}{0}$ & $\frac{52}{0}$ & & & & & & & & & & & \\
\hline
\end{tabular}


Table 111. Volume of Photonectes margarita $\left(\mathrm{ml} / 10,000 \mathrm{~m}^{3}\right)$.

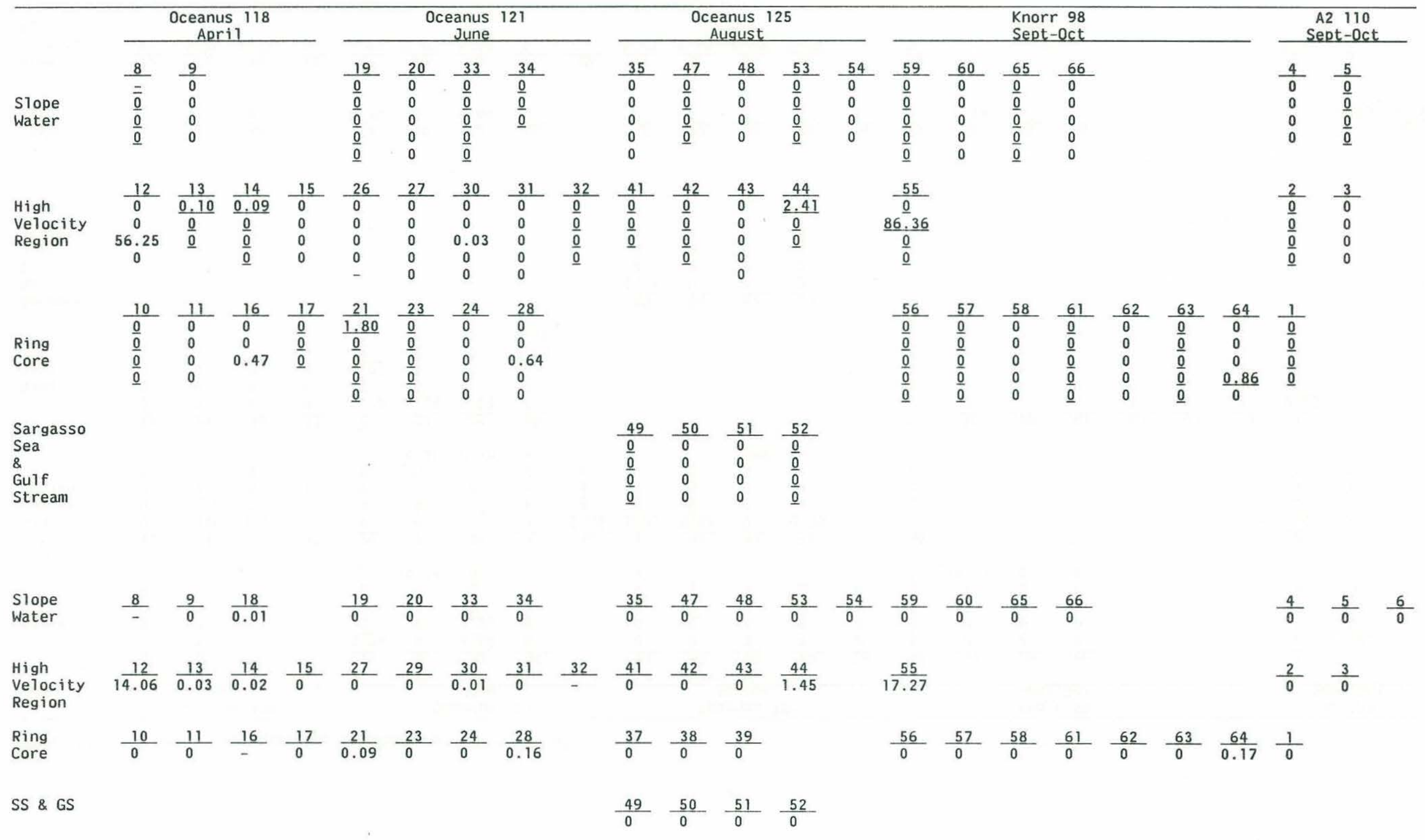


Table 112. Volume of Photostomias guernei $\left(\mathrm{m} 1 / 10,000 \mathrm{~m}^{3}\right)$.

\begin{tabular}{|c|c|c|c|c|c|c|c|c|c|c|c|c|c|c|c|c|c|c|c|c|c|c|c|c|}
\hline & \multicolumn{4}{|c|}{$\begin{array}{c}\text { Oceanus } 118 \\
\text { Aprif }\end{array}$} & \multicolumn{5}{|c|}{$\begin{array}{c}\text { Oceanus } \\
\text { June }\end{array}$} & \multicolumn{5}{|c|}{$\begin{array}{c}\text { Oceanus } 125 \\
\text { Auqust }\end{array}$} & \multicolumn{7}{|c|}{$\begin{array}{l}\text { Knorr } 98 \\
\text { Sept-0ct }\end{array}$} & \multicolumn{3}{|c|}{$\begin{array}{c}\text { A2 } 110 \\
\text { Sept-0ct }\end{array}$} \\
\hline $\begin{array}{l}\text { Slope } \\
\text { Water }\end{array}$ & $\begin{array}{l}\frac{8}{\overline{\overline{0}}} \\
\underline{\underline{0} .27} \\
\underline{0}\end{array}$ & $\begin{array}{l}\frac{9}{0} \\
0 \\
0 \\
0\end{array}$ & & & $\begin{array}{l}\frac{19}{\underline{0}} \\
\frac{5.86}{\underline{0}} \\
\underline{0} \underline{0}\end{array}$ & $\begin{array}{l}20 \\
0 \\
0 \\
0 \\
0 \\
0\end{array}$ & $\begin{array}{l}\frac{33}{0} \\
\frac{0}{0} \\
\frac{0}{0} \\
\frac{0}{0}\end{array}$ & $\begin{array}{l}\frac{34}{\underline{0}} \\
\underline{0} \\
\underline{0}\end{array}$ & & $\begin{array}{l}35 \\
0 \\
0 \\
0 \\
0 \\
0\end{array}$ & $\begin{array}{l}47 \\
\frac{0}{0} \\
\frac{0}{0} \\
\underline{0}\end{array}$ & $\begin{array}{l}48 \\
0 \\
0 \\
0 \\
0\end{array}$ & $\frac{\frac{53}{1.54}}{\frac{0}{0}}$ & $\begin{array}{l}\frac{54}{0} \\
0 \\
0 \\
0\end{array}$ & $\begin{array}{l}-59 \\
\underline{0} \\
\underline{0} \\
\underline{0} \\
\underline{0} \\
\underline{0}\end{array}$ & $\begin{array}{l}60 \\
0 \\
0 \\
0 \\
0 \\
0\end{array}$ & $\begin{array}{l}\frac{65}{0} \\
\frac{0}{0} \\
\underline{0} \\
\frac{0}{0}\end{array}$ & $\begin{array}{l}\frac{66}{0} \\
0 \\
0.15 \\
1.90 \\
1.54\end{array}$ & & & & $\begin{array}{l}\frac{4}{0} \\
0 \\
0 \\
0\end{array}$ & $\frac{\frac{5}{1.03}}{\frac{0}{0}}$ & \\
\hline $\begin{array}{l}\text { High } \\
\text { Velocity } \\
\text { Region }\end{array}$ & $\begin{array}{l}\frac{12}{0} \\
0 \\
0 \\
0\end{array}$ & $\begin{array}{l}\frac{13}{0} \\
\frac{0}{0} \\
\underline{0}\end{array}$ & $\frac{\frac{14}{0.71}}{\frac{0}{0}}$ & $\begin{array}{l}\frac{15}{0} \\
0 \\
0 \\
0\end{array}$ & $\begin{array}{l}26 \\
0 \\
0 \\
0 \\
0 \\
-\end{array}$ & $\begin{array}{l}\frac{27}{0} \\
0 \\
0 \\
0 \\
0\end{array}$ & $\begin{array}{l}30 \\
0 \\
0 \\
0 \\
1.76 \\
0\end{array}$ & $\begin{array}{l}31 \\
0 \\
0 \\
0 \\
0 \\
0\end{array}$ & $\begin{array}{l}\frac{32}{0} \\
\underline{0} \\
\underline{0} \\
\underline{0}\end{array}$ & $\begin{array}{l}\frac{41}{0} \\
\underline{0} \\
\underline{0}\end{array}$ & $\begin{array}{l}\frac{42}{0} \\
\frac{0}{0} \\
\underline{0} \\
\underline{0}\end{array}$ & $\begin{array}{l}\frac{43}{0} \\
0 \\
0 \\
0 \\
0\end{array}$ & $\frac{\frac{44}{0.32}}{\frac{0}{0}}$ & & $\begin{array}{l}\frac{55}{0} \\
\underline{0} \\
\underline{0} \\
\underline{0}\end{array}$ & & & & & & & $\begin{array}{l}\frac{2}{0} \\
\underline{0} \\
\underline{0} \\
\underline{0}\end{array}$ & $\begin{array}{c}3 \\
0 \\
0 \\
0.43 \\
0\end{array}$ & \\
\hline $\begin{array}{l}\text { Ring } \\
\text { Core }\end{array}$ & $\begin{array}{l}\frac{10}{\underline{0}} \\
\underline{0} \\
\underline{0} \\
\underline{0}\end{array}$ & $\begin{array}{l}\frac{11}{0} \\
0 \\
0 \\
0\end{array}$ & $\begin{array}{l}\frac{16}{0} \\
0 \\
0.67\end{array}$ & $\begin{array}{l}\frac{17}{0} \\
\underline{0} \\
1.08 \\
\end{array}$ & $\begin{array}{l}\frac{21}{\underline{0}} \\
\underline{0} \\
\underline{0} \\
\underline{4.17} \\
\underline{0}\end{array}$ & $\begin{array}{l}\frac{23}{\underline{0}} \\
\underline{0} \\
\underline{0} \\
\underline{0} \\
\underline{0}\end{array}$ & $\begin{array}{l}24 \\
0 \\
0 \\
0 \\
0 \\
0\end{array}$ & $\begin{array}{l}\frac{28}{0} \\
0 \\
0 \\
1.54 \\
0\end{array}$ & & & & & & & $\begin{array}{l}\frac{56}{0.15} \\
\frac{0}{0} \\
\frac{0}{0} \\
\underline{0}\end{array}$ & $\begin{array}{l}\frac{57}{0} \\
\underline{0} \\
\underline{0} \\
\underline{0} \\
\underline{0.13} \\
\underline{0}\end{array}$ & $\begin{array}{l}58 \\
0 \\
0 \\
0 \\
0 \\
1.18\end{array}$ & $\begin{array}{l}\frac{61}{\underline{0}} \\
\frac{0.15}{\underline{0}} \\
\underline{0} \\
\underline{0}\end{array}$ & $\begin{array}{c}\frac{62}{0} \\
0 \\
0 \\
0.05 \\
0.18\end{array}$ & $\begin{array}{l}\frac{63}{0} \\
\underline{0} \\
\underline{0} \\
\underline{0} \\
\underline{0}\end{array}$ & $\begin{array}{l}64 \\
0 \\
0 \\
0 \\
0 \\
0\end{array}$ & $\frac{\frac{1}{0.28}}{\frac{0}{0}}$ & & \\
\hline $\begin{array}{l}\text { Sargasso } \\
\text { Sea } \\
\& \\
\text { Gulf } \\
\text { Stream }\end{array}$ & & & & & & & & & & $\begin{array}{l}\frac{49}{0} \\
\underline{0} \\
\underline{0} \\
\underline{0}\end{array}$ & $\begin{array}{l}50 \\
0 \\
0 \\
0 \\
0\end{array}$ & $\begin{array}{l}\frac{51}{0} \\
0 \\
0 \\
0\end{array}$ & $\begin{array}{l}\frac{52}{0} \\
\frac{0}{1.11} \\
\underline{0} \\
\underline{1.79}\end{array}$ & & & & & & & & & & & \\
\hline $\begin{array}{l}\text { Slope } \\
\text { Water }\end{array}$ & $\frac{8}{-}$ & $\frac{9}{0}$ & $\frac{18}{0}$ & & $\frac{19}{0.88}$ & $\frac{20}{0}$ & $\frac{33}{0}$ & $\frac{34}{0}$ & & $\frac{35}{0}$ & $\frac{47}{0}$ & $\frac{48}{0}$ & $\frac{53}{0.39}$ & $\frac{54}{0}$ & $\frac{59}{0}$ & $\frac{60}{0}$ & $\frac{65}{0}$ & $\frac{66}{0.72}$ & & & & $\frac{4}{0}$ & $\frac{5}{0.26}$ & $\frac{6}{0}$ \\
\hline $\begin{array}{l}\text { High } \\
\text { Velocity } \\
\text { Region }\end{array}$ & $\frac{12}{0}$ & $\frac{13}{0}$ & $\frac{14}{0.18}$ & $\frac{15}{0}$ & $\frac{27}{0}$ & $\frac{29}{0.31}$ & $\frac{30}{0.35}$ & $\frac{31}{0}$ & $\frac{32}{-}$ & $\frac{41}{0}$ & $\frac{42}{0}$ & $\frac{43}{0}$ & $\frac{44}{0.19}$ & & $\frac{55}{0}$ & & & & & & & $\frac{2}{0}$ & $\frac{3}{0.11}$ & \\
\hline $\begin{array}{l}\text { Ring } \\
\text { Core }\end{array}$ & $\frac{10}{0}$ & $\frac{11}{0}$ & $\frac{16}{-}$ & $\frac{17}{0.80}$ & $\frac{21}{0.83}$ & $\frac{23}{0}$ & $\frac{24}{0}$ & $\frac{28}{0.39}$ & & $\frac{37}{0}$ & $\frac{38}{0.23}$ & $\frac{39}{0}$ & & & $\frac{56}{0.03}$ & $\frac{57}{0.03}$ & $\frac{58}{0.24}$ & $\frac{61}{0.03}$ & $\frac{62}{0.05}$ & $\frac{63}{0}$ & $\frac{64}{0}$ & $\frac{1}{0.07}$ & & \\
\hline SS \& GS & & & & & & & & & & $\frac{49}{0}$ & $\frac{50}{0}$ & $\frac{51}{0}$ & $\frac{52}{0.73}$ & & & & & & & & & & & \\
\hline
\end{tabular}


Table 113. Abundance of Pollichthys mauli (specimens $/ 10,000 \mathrm{~m}^{3}$ ).

\begin{tabular}{|c|c|c|c|c|c|c|c|c|c|c|c|c|c|c|c|c|c|c|c|c|c|c|c|c|}
\hline & & $\begin{array}{r}\text { ceant } \\
\text { Apr }\end{array}$ & $1^{118}$ & & & & $\begin{array}{l}\text { eanus } \\
\text { June }\end{array}$ & 121 & & & & $\begin{array}{l}\text { nus } 1 \\
\text { Iqust }\end{array}$ & & & & & $\begin{array}{l}\text { Knor } \\
\text { Sept }\end{array}$ & $\begin{array}{r}98 \\
-0 c t \\
\end{array}$ & & & & & $\begin{array}{l}110 \\
-0 c t\end{array}$ & \\
\hline $\begin{array}{l}\text { Slope } \\
\text { Water }\end{array}$ & $\begin{array}{l}8 \underline{\overline{0}} \\
\underline{\overline{0}} \\
\underline{0} \\
\underline{0}\end{array}$ & $\begin{array}{l}9 \\
0 \\
0 \\
0 \\
0\end{array}$ & & & 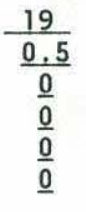 & $\begin{array}{c}20 \\
0 \\
0 \\
0 \\
0 \\
0\end{array}$ & $\frac{\frac{33}{0.3}}{\frac{0.3}{\underline{0}}}$ & $\begin{array}{r}34 \\
\underline{0} \\
\underline{0} \\
\underline{0}\end{array}$ & & $\begin{array}{c}35 \\
0 \\
0.2 \\
0 \\
0 \\
0\end{array}$ & $\frac{47}{\frac{0.4}{\underline{0}}}$ & $\begin{array}{r}48 \\
0 \\
0 \\
0 \\
0\end{array}$ & $\begin{array}{r}53 \\
\underline{0} \\
\underline{0} \\
\underline{0} \\
\underline{0}\end{array}$ & $\begin{array}{r}54 \\
0 \\
0 \\
0 \\
0\end{array}$ & $\begin{array}{r}59 \\
\underline{0} \\
\underline{0} \\
\underline{0} \\
\underline{0} \\
\underline{0}\end{array}$ & $\begin{array}{c}60 \\
0.3 \\
0 \\
0 \\
0 \\
0\end{array}$ & $\begin{array}{l}\frac{65}{\underline{0}} \\
\underline{0} \\
\underline{0} \\
\underline{0} \\
\underline{0}\end{array}$ & $\begin{array}{c}66 \\
0 \\
0 \\
0 \\
0 \\
0\end{array}$ & & & & $\begin{array}{l}4 \\
0 \\
0 \\
0 \\
0\end{array}$ & $\begin{array}{l}\frac{5}{0} \\
\underline{0} \\
\underline{0} \\
\underline{0} \\
\underline{0}\end{array}$ & \\
\hline $\begin{array}{l}\text { High } \\
\text { Velocity } \\
\text { Region }\end{array}$ & $\begin{array}{r}12 \\
0 \\
0 \\
0 \\
0\end{array}$ & $\frac{13}{\frac{0}{0}}$ & $\begin{array}{l}14 \\
\frac{0}{0} \\
\underline{0} \\
\underline{0}\end{array}$ & $\begin{array}{r}15 \\
0 \\
0 \\
0 \\
0\end{array}$ & $\begin{array}{c}26 \\
0 \\
0 \\
0 \\
0 \\
-\end{array}$ & $\begin{array}{c}27 \\
0 \\
0 \\
0 \\
0 \\
0\end{array}$ & $\begin{array}{c}30 \\
0 \\
0 \\
0 \\
0 \\
0.2\end{array}$ & $\begin{array}{c}31 \\
0 \\
0 \\
0 \\
0 \\
0\end{array}$ & $\frac{\frac{32}{0.8}}{\frac{0}{0}}$ & $\frac{\frac{41}{0.4}}{\frac{0}{0}}$ & $\begin{array}{l}\frac{42}{\underline{0}} \\
\underline{0} \\
\underline{0} \\
\underline{0}\end{array}$ & $\begin{array}{r}43 \\
0 \\
0 \\
0 \\
0 \\
0\end{array}$ & $\frac{44}{\frac{0.1}{\underline{0}}}$ & & $\frac{\frac{55}{0.8}}{\frac{0.8}{\underline{0}}}$ & & & & & & & $\begin{array}{l}\frac{2}{0} \\
\underline{0} \\
\underline{0} \\
\underline{0}\end{array}$ & $\begin{array}{l}3 \\
0 \\
0 \\
0 \\
0\end{array}$ & \\
\hline $\begin{array}{l}\text { Ring } \\
\text { Core }\end{array}$ & $\frac{\frac{10}{0.4}}{\frac{0}{\underline{0}}} \frac{\underline{0}}{\underline{0}}$ & $\begin{array}{c}11 \\
0 \\
0.6 \\
0 \\
0\end{array}$ & $\begin{array}{r}16 \\
0 \\
0 \\
0\end{array}$ & $\frac{17}{\underline{0}} \frac{0.5}{\underline{0}}$ & $\begin{array}{l}\frac{21}{\underline{0}} \\
\frac{0.4}{\underline{0}} \\
\underline{0} \\
\underline{0}\end{array}$ & $\begin{array}{l}\frac{23}{2.9} \\
\frac{0}{0} \\
\underline{0} \\
\underline{0}\end{array}$ & $\begin{array}{c}24 \\
0.3 \\
0 \\
0 \\
0 \\
0\end{array}$ & $\begin{array}{r}28 \\
0 \\
0 \\
0 \\
0 \\
0\end{array}$ & & & & & & & $\frac{56}{\frac{5.2}{\underline{0}}}$ & $\frac{57}{\frac{2.8}{\underline{0}}}$ & $\begin{array}{c}58 \\
0 \\
0 \\
9.5 \\
0 \\
0\end{array}$ & $\frac{\frac{61}{2.7}}{\frac{\underline{0}}{\underline{0}}}$ & $\begin{array}{c}62 \\
0 \\
0 \\
1.2 \\
0 \\
0\end{array}$ & $\frac{\frac{63}{1.1}}{\frac{0.2}{\underline{0}}}$ & $\begin{array}{c}64 \\
2.5 \\
0 \\
0 \\
0 \\
0\end{array}$ & $\begin{array}{l}\frac{1}{\underline{0}} \\
\underline{0} \\
\underline{0} \\
\underline{0}\end{array}$ & & \\
\hline $\begin{array}{l}\text { Sargasso } \\
\text { Sea } \\
\& \\
\text { Gulf } \\
\text { Stream }\end{array}$ & & & & & & & & & & $\frac{\frac{49}{3.2}}{\frac{0}{0}} \frac{\underline{0}}{\underline{0}}$ & $\begin{array}{c}50 \\
0 \\
3.9 \\
0 \\
0\end{array}$ & $\begin{array}{c}51 \\
0 \\
0.7 \\
0 \\
0\end{array}$ & $\frac{52}{\frac{3.4}{\frac{0}{0}}}$ & & & & & & & & & & & \\
\hline $\begin{array}{l}\text { Slope } \\
\text { Water }\end{array}$ & $\frac{8}{-}$ & $\frac{9}{0}$ & $\frac{18}{0}$ & & $\frac{19}{0.1}$ & $\frac{20}{0}$ & $\frac{33}{0}$ & $\frac{34}{0}$ & & $\frac{35}{0}$ & $\frac{47}{0.1}$ & $\frac{48}{0}$ & $\frac{53}{0}$ & $\frac{54}{0}$ & $\frac{59}{0}$ & $\frac{60}{0.1}$ & $\frac{65}{0}$ & $\frac{66}{0}$ & & & & $\frac{4}{0}$ & $\frac{5}{0}$ & $\frac{6}{0}$ \\
\hline $\begin{array}{l}\text { High } \\
\text { Velocity } \\
\text { Region }\end{array}$ & $\frac{12}{0}$ & $\frac{13}{0}$ & $\frac{14}{0}$ & $\frac{15}{0}$ & $\frac{27}{0}$ & $\frac{29}{0.2}$ & $\frac{30}{0.1}$ & $\frac{31}{0}$ & $\frac{32}{-}$ & $\frac{41}{0.2}$ & $\frac{42}{0}$ & $\frac{43}{0}$ & $\frac{44}{0.1}$ & & $\frac{55}{0.2}$ & & & & & & & $\frac{2}{0}$ & $\frac{3}{0}$ & \\
\hline $\begin{array}{l}\text { Ring } \\
\text { Core }\end{array}$ & $\frac{10}{0.1}$ & $\frac{11}{0.2}$ & $\frac{16}{-}$ & $\frac{17}{0.1}$ & $\frac{21}{0.1}$ & $\frac{23}{0.4}$ & $\frac{24}{0.1}$ & $\frac{28}{0}$ & & $\frac{37}{0.1}$ & $\frac{38}{0.2}$ & $\frac{39}{0}$ & & & $\frac{56}{1.2}$ & $\frac{57}{0.6}$ & $\frac{58}{1.9}$ & $\frac{61}{0.4}$ & $\frac{62}{0.2}$ & $\frac{63}{0.4}$ & $\frac{64}{1.0}$ & $\frac{1}{0}$ & & \\
\hline SS \& GS & & & & & & & & & & $\frac{49}{0.8}$ & $\frac{50}{1.0}$ & $\frac{51}{0.2}$ & $\frac{52}{0.9}$ & & & & & & & & & & & \\
\hline
\end{tabular}


Tab1e 114. Volume of Poromitra capito $\left(\mathrm{ml} / 10,000 \mathrm{~m}^{3}\right)$.

\begin{tabular}{|c|c|c|c|c|c|c|c|c|c|c|c|c|c|c|c|c|c|c|c|c|c|c|c|c|}
\hline & \multicolumn{4}{|c|}{$\begin{array}{c}\text { Oceanus } 118 \\
\text { April }\end{array}$} & \multicolumn{5}{|c|}{$\begin{array}{c}\text { Oceanus } 121 \\
\text { June }\end{array}$} & \multicolumn{5}{|c|}{$\begin{array}{c}\text { Oceanus } 125 \\
\text { August }\end{array}$} & \multicolumn{7}{|c|}{$\begin{array}{l}\text { Knorr } 98 \\
\text { Sept-Oct }\end{array}$} & \multicolumn{3}{|c|}{$\begin{array}{c}\text { A2 } 110 \\
\text { Sept-0ct }\end{array}$} \\
\hline $\begin{array}{l}\text { Slope } \\
\text { Water }\end{array}$ & $\begin{array}{l}\frac{8}{\overline{0}} \\
\underline{0} \\
\underline{0} \\
\underline{0}\end{array}$ & $\begin{array}{l}9 \\
0 \\
0 \\
0 \\
0\end{array}$ & & & $\begin{array}{l}\frac{19}{\underline{0}} \\
\frac{0.31}{\underline{0}} \\
\underline{0} \\
\underline{0}\end{array}$ & $\begin{array}{l}20 \\
0 \\
0 \\
0 \\
0 \\
0\end{array}$ & $\begin{array}{l}\frac{33}{\underline{0}} \\
\underline{0} \\
\underline{0} \\
\underline{0} \\
\underline{0}\end{array}$ & $\begin{array}{l}\frac{34}{\underline{0}} \\
\underline{0} \\
\underline{0}\end{array}$ & & $\begin{array}{l}35 \\
0 \\
0 \\
0 \\
0 \\
0\end{array}$ & $\begin{array}{l}47 \\
\underline{0} \\
\underline{0} \\
\underline{0} \\
\underline{0}\end{array}$ & $\begin{array}{l}48 \\
0 \\
0 \\
0 \\
0\end{array}$ & $\begin{array}{l}\frac{53}{0} \\
\underline{0} \\
\underline{0} \\
\underline{0}\end{array}$ & $\begin{array}{l}54 \\
0 \\
0 \\
0 \\
0\end{array}$ & $\begin{array}{l}\frac{59}{\underline{0}} \\
\frac{1.20}{\underline{0}} \\
\underline{0} \\
\underline{0}\end{array}$ & $\begin{array}{l}60 \\
0 \\
0 \\
0 \\
0 \\
0\end{array}$ & $\begin{array}{l}\frac{65}{\underline{0}} \\
\underline{0} \\
\underline{0} \\
\underline{0} \\
\underline{0}\end{array}$ & $\begin{array}{l}\frac{66}{0} \\
0 \\
0 \\
0 \\
1.92\end{array}$ & & & & $\begin{array}{l}4 \\
0 \\
0 \\
0 \\
0\end{array}$ & $\begin{array}{l}\frac{5}{0} \\
\underline{0} \\
\underline{0} \\
\underline{0}\end{array}$ & \\
\hline $\begin{array}{l}\text { High } \\
\text { Velocity } \\
\text { Region }\end{array}$ & $\begin{array}{l}\frac{12}{0} \\
0 \\
0 \\
0.34\end{array}$ & $\frac{13}{\frac{0}{0}}$ & $\begin{array}{l}\frac{14}{0} \\
\frac{0}{0} \\
\frac{0}{0}\end{array}$ & $\begin{array}{l}\frac{15}{0} \\
0 \\
0 \\
0\end{array}$ & $\begin{array}{l}26 \\
0 \\
0 \\
0 \\
0 \\
-\end{array}$ & $\begin{array}{l}27 \\
0 \\
0 \\
0 \\
0 \\
0\end{array}$ & $\begin{array}{l}30 \\
0 \\
0 \\
0 \\
0.49 \\
0.14\end{array}$ & $\begin{array}{l}31 \\
0 \\
0 \\
0 \\
0 \\
0.41\end{array}$ & $\begin{array}{l}\frac{32}{\underline{0}} \\
\underline{0} \\
\underline{0} \\
\underline{0}\end{array}$ & $\frac{\frac{41}{0.21}}{\frac{0}{0}}$ & $\frac{\frac{42}{0.25}}{\frac{0}{0}}$ & $\begin{array}{l}43 \\
0 \\
0 \\
0 \\
0 \\
0\end{array}$ & $\begin{array}{l}\frac{44}{\underline{0}} \\
\frac{0}{0} \\
\underline{0}\end{array}$ & & $\begin{array}{l}\frac{55}{0} \\
\underline{0} \\
\underline{0} \\
\underline{0}\end{array}$ & & & & & & & $\frac{\frac{2}{0.73}}{\frac{1.22}{\underline{0}}}$ & $\begin{array}{c}\frac{3}{0} \\
0 \\
0 \\
3.85\end{array}$ & \\
\hline $\begin{array}{l}\text { Ring } \\
\text { Core }\end{array}$ & $\frac{\frac{10}{0.36}}{\frac{0}{0}}$ & $\begin{array}{c}\frac{11}{0} \\
0 \\
0 \\
0.18\end{array}$ & $\begin{array}{l}16 \\
0 \\
0 \\
0\end{array}$ & $\begin{array}{l}17 \\
\frac{0}{0} \\
0 \\
0\end{array}$ & $\begin{array}{l}21 \\
\frac{0}{0} \\
\underline{0} \\
\frac{0}{0} \\
\underline{0}\end{array}$ & $\begin{array}{l}\frac{23}{0} \\
\frac{0}{0} \\
\frac{0}{0} \\
\underline{0} \\
\underline{0}\end{array}$ & $\begin{array}{l}24 \\
0 \\
0 \\
0 \\
0 \\
0.89\end{array}$ & $\begin{array}{l}\frac{28}{0} \\
0 \\
0 \\
0 \\
0\end{array}$ & & & & & & & $\begin{array}{l}\frac{56}{\underline{0}} \\
\underline{0} \\
\underline{0} \\
\underline{0} \\
\underline{0}\end{array}$ & $\begin{array}{l}57 \\
\underline{0} \\
\underline{0} \\
\underline{0} \\
\underline{0} \\
\underline{0}\end{array}$ & $\begin{array}{l}\frac{58}{0} \\
0 \\
0 \\
0 \\
8.16\end{array}$ & $\begin{array}{l}\frac{61}{0} \\
\underline{0} \\
\underline{0} \\
\underline{0} \\
\underline{0}\end{array}$ & $\begin{array}{l}62 \\
0 \\
0 \\
0 \\
0 \\
0\end{array}$ & $\begin{array}{l}\frac{63}{0} \\
\underline{0} \\
\underline{0} \\
\underline{0} \\
\underline{0}\end{array}$ & $\begin{array}{l}64 \\
0 \\
0 \\
0 \\
0 \\
1.15\end{array}$ & $\begin{array}{l}\frac{1}{0} \\
\underline{0} \\
\underline{0} \\
\underline{0}\end{array}$ & & \\
\hline $\begin{array}{l}\text { Sargasso } \\
\text { Sea } \\
\& \\
\text { Gulf } \\
\text { Stream }\end{array}$ & & & & & & & & & & $\begin{array}{l}\frac{49}{0} \\
\frac{0}{0} \\
\underline{0} \\
\underline{0}\end{array}$ & $\begin{array}{l}50 \\
0 \\
0 \\
0 \\
0\end{array}$ & $\begin{array}{l}51 \\
0 \\
0 \\
0 \\
0\end{array}$ & $\begin{array}{l}\frac{52}{\underline{0}} \\
\underline{0} \\
\underline{0} \\
\underline{0}\end{array}$ & & & & & & & & & & & \\
\hline $\begin{array}{l}\text { Slope } \\
\text { Water }\end{array}$ & $\frac{8}{-}$ & $\frac{9}{0}$ & $\frac{18}{0}$ & & $\frac{19}{0.05}$ & $\frac{20}{0}$ & $\frac{33}{0}$ & $\frac{34}{0}$ & & $\frac{35}{0}$ & $\frac{47}{0}$ & $\frac{48}{0}$ & $\frac{53}{0}$ & $\frac{54}{0}$ & $\frac{59}{0.24}$ & $\frac{60}{0}$ & $\frac{65}{0}$ & $\frac{66}{0.38}$ & & & & $\frac{4}{0}$ & $\frac{5}{0}$ & $\frac{6}{0}$ \\
\hline $\begin{array}{l}\text { High } \\
\text { Velocity } \\
\text { Region }\end{array}$ & $\frac{12}{0.09}$ & $\frac{13}{0}$ & $\frac{14}{0}$ & $\frac{15}{0}$ & $\frac{27}{0}$ & $\frac{29}{0}$ & $\frac{30}{0.13}$ & $\frac{31}{0.08}$ & $\frac{32}{-}$ & $\frac{41}{0.11}$ & $\frac{42}{0.10}$ & $\frac{43}{0}$ & $\frac{44}{0}$ & & $\frac{55}{0}$ & & & & & & & $\frac{2}{0.49}$ & $\frac{3}{0.96}$ & \\
\hline $\begin{array}{l}\text { Ring } \\
\text { Core }\end{array}$ & $\frac{10}{0.09}$ & $\frac{11}{0.05}$ & $\frac{16}{-}$ & $\frac{17}{0}$ & $\frac{21}{0}$ & $\frac{23}{0}$ & $\frac{24}{0.18}$ & $\frac{28}{0}$ & & $\frac{37}{0}$ & $\frac{38}{0}$ & $\frac{39}{0.06}$ & & & $\frac{56}{0}$ & $\frac{57}{0}$ & $\frac{58}{1.63}$ & $\frac{61}{0}$ & $\frac{62}{0}$ & $\frac{63}{0}$ & $\frac{64}{0.23}$ & $\frac{1}{0}$ & & \\
\hline SS \& GS & & & & & & & & & & $\frac{49}{0}$ & $\frac{50}{0}$ & $\frac{51}{0}$ & $\frac{52}{0}$ & & & & & & & & & & & \\
\hline
\end{tabular}


Table 115. Abundance of Scopeloberyx opisthopterus (specimens $/ 10,000 \mathrm{~m}^{3}$ ).

\begin{tabular}{|c|c|c|c|c|c|c|c|c|c|c|c|c|c|c|c|c|c|c|c|c|c|c|c|c|}
\hline & & $\begin{array}{r}\text { ceanu } \\
\text { Apr }\end{array}$ & $\begin{array}{l}118 \\
i 1\end{array}$ & & & & $\begin{array}{l}\text { anus } \\
\text { June }\end{array}$ & 121 & & & $\begin{array}{r}\text { Oce } \\
A\end{array}$ & $\begin{array}{l}\text { anus } \\
\text { dqust }\end{array}$ & & & & & $\begin{array}{l}\text { Knor } \\
\text { Sept }\end{array}$ & $\begin{array}{r}98 \\
-0 c t \\
\end{array}$ & & & & Se & $\begin{array}{l}110 \\
t-0 c t\end{array}$ & \\
\hline $\begin{array}{l}\text { Slope } \\
\text { Water }\end{array}$ & $\begin{array}{l}\frac{8}{\overline{\overline{0}}} \\
\underline{0} \\
\underline{\underline{0}} \\
\underline{\underline{2} .3}\end{array}$ & $\begin{array}{c}\frac{9}{0} \\
0 \\
0 \\
2.7\end{array}$ & & & $\begin{array}{c}\frac{19}{\underline{0}} \\
\underline{0} \\
\underline{0} \\
\underline{0} \\
\underline{0.5}\end{array}$ & $\begin{array}{c}20 \\
0 \\
0 \\
0.4 \\
0.9 \\
0\end{array}$ & $\begin{array}{c}\frac{33}{0} \\
\underline{0} 0 \\
\underline{0} \\
\underline{0} \\
\underline{0.9}\end{array}$ & $\begin{array}{c}\frac{34}{\underline{0}} \\
\underline{0.2} \\
\underline{0.2}\end{array}$ & & $\begin{array}{c}35 \\
0 \\
0 \\
0 \\
0.6 \\
0\end{array}$ & $\begin{array}{l}\frac{47}{\underline{0}} \\
\underline{0} \\
\underline{0.2} \\
\underline{0.2}\end{array}$ & $\begin{array}{r}48 \\
0 \\
0 \\
0 \\
0\end{array}$ & $\begin{array}{l}\frac{53}{\underline{0}} \\
\underline{0} \\
0.5 \\
\underline{0.2}\end{array}$ & $\begin{array}{c}54 \\
0 \\
0 \\
0 \\
1.3\end{array}$ & $\begin{array}{c}\frac{59}{0} \\
\underline{0} \\
\underline{0} \\
\underline{0} \\
\underline{0.5}\end{array}$ & $\begin{array}{c}60 \\
0 \\
0 \\
0 \\
0 \\
0.3\end{array}$ & $\begin{array}{c}65 \\
\underline{0} \\
\underline{0} \\
\underline{0} \\
\underline{0} \\
\underline{0.6}\end{array}$ & $\begin{array}{c}66 \\
0 \\
0 \\
0 \\
1.0 \\
0.4\end{array}$ & & & & $\begin{array}{l}4 \\
0 \\
0 \\
0 \\
1.2\end{array}$ & $\begin{array}{l}\frac{5}{\underline{0}} \\
\underline{0} \\
\frac{0.3}{\underline{0}}\end{array}$ & \\
\hline $\begin{array}{l}\text { High } \\
\text { Velocity } \\
\text { Region }\end{array}$ & $\begin{array}{c}12 \\
0 \\
0 \\
0.4 \\
0.7\end{array}$ & $\begin{array}{c}\frac{13}{\underline{0}} \\
\underline{0} \\
1.0 \\
\end{array}$ & $\begin{array}{l}\frac{14}{0} \\
\frac{0}{0} \\
0.7\end{array}$ & $\begin{array}{c}15 \\
0 \\
0 \\
0.6 \\
3.8\end{array}$ & $\begin{array}{c}26 \\
0 \\
0 \\
0 \\
0 \\
-\end{array}$ & $\begin{array}{c}27 \\
0 \\
0 \\
0 \\
0 \\
0.5\end{array}$ & $\begin{array}{c}30 \\
0 \\
0 \\
0 \\
0 \\
0.5\end{array}$ & $\begin{array}{c}31 \\
0 \\
0 \\
0 \\
0.3 \\
1.7\end{array}$ & $\begin{array}{c}32 \\
\underline{0} \\
\underline{0} \\
\underline{0} \\
\underline{0}\end{array}$ & $\begin{array}{c}\frac{41}{\underline{0}} \\
\underline{0} \\
1.7\end{array}$ & $\begin{array}{c}\frac{42}{\underline{0}} \\
\underline{0} \\
\underline{0.2} \\
\underline{0.5}\end{array}$ & $\begin{array}{c}43 \\
0 \\
0 \\
0 \\
0 \\
1.4\end{array}$ & $\begin{array}{l}\frac{44}{0} \\
\underline{0.3} \\
\underline{0.8}\end{array}$ & & $\begin{array}{c}\frac{55}{\underline{0}} \\
\underline{0} \\
\underline{0} \\
\underline{3} .3 \\
\end{array}$ & & & & & & & $\begin{array}{l}\frac{2}{\underline{0}} \\
\underline{0} \\
\underline{0} \\
\underline{0.6}\end{array}$ & $\begin{array}{l}3 \\
0 \\
0 \\
0 \\
0\end{array}$ & \\
\hline $\begin{array}{l}\text { Ring } \\
\text { Core }\end{array}$ & $\begin{array}{r}10 \\
\underline{0} \\
\underline{0} \\
\underline{0} \\
\underline{0}\end{array}$ & $\begin{array}{c}11 \\
0 \\
0 \\
0 \\
0.7\end{array}$ & $\begin{array}{l}\frac{16}{0} \\
0.7 \\
1.3\end{array}$ & $\begin{array}{r}17 \\
\underline{0} \\
\underline{0} \\
\underline{2.1}\end{array}$ & $\begin{array}{c}\frac{21}{\underline{0}} \\
\underline{0} \\
\underline{0} \\
\underline{0.8} \\
\underline{0.6}\end{array}$ & $\begin{array}{c}\frac{23}{\underline{0}} \\
\underline{0} \\
\underline{0} \\
\underline{0.5} \\
\underline{2.9}\end{array}$ & $\begin{array}{c}24 \\
0 \\
0 \\
0 \\
0 \\
1.1\end{array}$ & $\begin{array}{c}28 \\
0 \\
0 \\
0 \\
0.8 \\
0\end{array}$ & & & & & & & $\begin{array}{c}56 \\
\underline{0} \\
\underline{0} \\
\underline{0} \\
\underline{0} \\
\underline{1.9} \\
\end{array}$ & $\begin{array}{c}57 \\
\underline{0} \\
\underline{0} \\
\underline{0} \\
\underline{0} \\
\underline{1.3}\end{array}$ & $\begin{array}{c}58 \\
0 \\
0 \\
0 \\
0 \\
0.5\end{array}$ & $\begin{array}{c}61 \\
\underline{0} \\
\underline{0} \\
\underline{0} \\
\underline{0} \\
\underline{2.1}\end{array}$ & $\begin{array}{c}62 \\
0 \\
0 \\
0 \\
0 \\
0\end{array}$ & $\begin{array}{c}\frac{63}{\underline{0}} \\
\underline{0} \\
\underline{0.5} \\
\underline{0} \\
\underline{0.6}\end{array}$ & $\begin{array}{c}64 \\
0 \\
0 \\
0 \\
0 \\
1.5\end{array}$ & $\begin{array}{c}\frac{1}{0} \\
\underline{0} \\
\underline{0} \\
\underline{0.8}\end{array}$ & & \\
\hline $\begin{array}{l}\text { Sargasso } \\
\text { Sea } \\
\& \\
\text { Gu1f } \\
\text { Stream }\end{array}$ & & & & & & & & & & $\begin{array}{l}\frac{49}{\underline{0}} \\
\underline{0} \\
\underline{0} \\
\underline{0}\end{array}$ & $\begin{array}{r}50 \\
0 \\
0 \\
0 \\
0\end{array}$ & $\begin{array}{c}51 \\
0 \\
0 \\
0 \\
1.4\end{array}$ & $\begin{array}{l}\frac{52}{\underline{0}} \\
\underline{0} \\
\underline{0} \\
\underline{2.1}\end{array}$ & & & & & & & & & & & \\
\hline $\begin{array}{l}\text { Slope } \\
\text { Water }\end{array}$ & $\frac{8}{-}$ & $\frac{9}{0.7}$ & $\frac{18}{0.2}$ & & $\frac{19}{0.1}$ & $\frac{20}{0.4}$ & $\frac{33}{0.4}$ & $\frac{34}{0.1}$ & & $\frac{35}{0.1}$ & $\frac{47}{0.1}$ & $\frac{48}{0}$ & $\frac{53}{0.2}$ & $\frac{54}{0.3}$ & $\frac{59}{0.1}$ & $\frac{60}{0.1}$ & $\frac{65}{0.1}$ & $\frac{66}{0.3}$ & & & & $\frac{4}{0.3}$ & $\frac{5}{0.1}$ & $\frac{6}{0.1}$ \\
\hline $\begin{array}{l}\text { High } \\
\text { Velocity } \\
\text { Region }\end{array}$ & $\frac{12}{0.3}$ & $\frac{13}{0.3}$ & $\frac{14}{0.2}$ & $\frac{15}{1.1}$ & $\frac{27}{0.1}$ & $\frac{29}{0.2}$ & $\frac{30}{0.1}$ & $\frac{31}{0.4}$ & $\frac{32}{-}$ & $\frac{41}{0.4}$ & $\frac{42}{0.1}$ & $\frac{43}{0.3}$ & $\frac{44}{0.2}$ & & $\frac{55}{0.7}$ & & & & & & & $\frac{2}{0.2}$ & $\frac{3}{0}$ & \\
\hline $\begin{array}{l}\text { Ring } \\
\text { Core }\end{array}$ & $\frac{10}{0}$ & $\frac{11}{0.2}$ & $\frac{16}{-}$ & $\frac{17}{1.1}$ & $\frac{21}{0.3}$ & $\frac{23}{0.7}$ & $\frac{24}{0.2}$ & $\frac{28}{0.2}$ & & $\frac{37}{0.1}$ & $\frac{38}{0.1}$ & $\frac{39}{0}$ & & & $\frac{56}{0.4}$ & $\frac{57}{0.3}$ & $\frac{58}{0.1}$ & $\frac{61}{0.4}$ & $\frac{62}{0}$ & $\frac{63}{0.2}$ & $\frac{64}{0.3}$ & $\frac{1}{0.2}$ & & \\
\hline SS \& GS & & & & & & & & & & $\frac{49}{0}$ & $\frac{50}{0}$ & $\frac{51}{0.4}$ & $\frac{52}{0.5}$ & & & & & & & & & & & \\
\hline
\end{tabular}


Table 116. Volume of Scopeloberyx opisthopterus $\left(\mathrm{ml} / 10,000 \mathrm{~m}^{3}\right)$.

\begin{tabular}{|c|c|c|c|c|c|c|c|c|c|c|c|c|c|c|c|c|c|c|c|c|c|c|c|c|}
\hline & \multicolumn{4}{|c|}{$\begin{array}{c}\text { Oceanus } 118 \\
\text { April }\end{array}$} & \multicolumn{5}{|c|}{$\begin{array}{c}\text { Oceanus } 121 \\
\text { June }\end{array}$} & \multicolumn{5}{|c|}{$\begin{array}{c}\text { Oceanus } 125 \\
\text { August }\end{array}$} & \multicolumn{7}{|c|}{$\begin{array}{l}\text { Knorr } 98 \\
\text { Sept-0ct }\end{array}$} & \multicolumn{3}{|c|}{$\begin{array}{c}\text { A2 } 110 \\
\text { Sept-0ct }\end{array}$} \\
\hline $\begin{array}{l}\text { Slope } \\
\text { Water }\end{array}$ & $\begin{array}{l}\frac{8}{\overline{0}} \\
\underline{0} \\
\underline{0} \\
\underline{0.64} \\
\end{array}$ & $\begin{array}{l}\frac{9}{0} \\
0 \\
0 \\
1.33\end{array}$ & & & $\begin{array}{l}\frac{19}{\underline{0}} \\
\frac{0}{0} \\
\underline{0} \\
\underline{0.11}\end{array}$ & $\begin{array}{l}\frac{20}{0} \\
0 \\
0 \\
0.09 \\
0.45\end{array}$ & $\begin{array}{l}\frac{33}{0} \\
\frac{0}{0} \\
\frac{0}{0} \\
\underline{0.49}\end{array}$ & $\begin{array}{l}\frac{34}{\underline{0}} \\
\underline{0} \\
\underline{0.11}\end{array}$ & & $\begin{array}{l}\frac{35}{0} \\
0 \\
0 \\
0.11 \\
0\end{array}$ & $\begin{array}{l}\frac{47}{\underline{0}} \\
\underline{0} \\
0.02 \\
0.16 \\
0.03\end{array}$ & $\begin{array}{l}48 \\
0 \\
0 \\
0 \\
0\end{array}$ & $\begin{array}{l}\frac{53}{\frac{0}{0}} \\
\underline{0.10} \\
\underline{0.04}\end{array}$ & $\begin{array}{l}\frac{54}{0} \\
0 \\
0 \\
0.60\end{array}$ & $\begin{array}{l}\frac{59}{\underline{0}} \\
\underline{0} \\
\underline{0} \\
\underline{0.07} \\
\underline{0.14}\end{array}$ & $\begin{array}{l}\frac{60}{0} \\
0 \\
0 \\
0 \\
0.12\end{array}$ & $\begin{array}{l}\frac{65}{\underline{0}} \\
\underline{0} \\
\underline{0} \\
\underline{0} \\
\underline{0.13}\end{array}$ & $\begin{array}{l}\frac{66}{0} \\
0 \\
0 \\
0.19 \\
0.08\end{array}$ & & & & $\begin{array}{c}\frac{4}{0} \\
0 \\
0 \\
0.26\end{array}$ & $\begin{array}{l}\frac{5}{\underline{0}} \\
\underline{0} \\
\frac{0.03}{\underline{0}}\end{array}$ & \\
\hline $\begin{array}{l}\text { High } \\
\text { Velocity } \\
\text { Region }\end{array}$ & $\begin{array}{l}\frac{12}{0} \\
0 \\
0.04 \\
0.34\end{array}$ & $\begin{array}{l}\frac{13}{\underline{0}} \\
\underline{0} \\
\underline{0.29}\end{array}$ & $\begin{array}{l}\frac{14}{\underline{0}} \\
\underline{0} \\
\underline{0} \\
\underline{0.07}\end{array}$ & $\begin{array}{l}\frac{15}{0} \\
0 \\
0.12 \\
2.14\end{array}$ & $\begin{array}{l}26 \\
0 \\
0 \\
0 \\
0 \\
-\end{array}$ & $\begin{array}{l}\frac{27}{0} \\
0 \\
0 \\
0 \\
0.26\end{array}$ & $\begin{array}{l}30 \\
0 \\
0 \\
0 \\
0 \\
0.02\end{array}$ & $\begin{array}{l}31 \\
0 \\
0 \\
0 \\
0.06 \\
0.48\end{array}$ & $\begin{array}{l}\frac{32}{\underline{0}} \\
\underline{0} \\
\underline{0} \\
\underline{0}\end{array}$ & $\begin{array}{l}\frac{41}{\underline{0}} \\
\underline{0} \\
0.53 \\
\end{array}$ & $\begin{array}{l}\frac{42}{\underline{0}} \\
\underline{0} \\
0.12 \\
0.29 \\
\end{array}$ & $\begin{array}{l}\frac{43}{0} \\
0 \\
0 \\
0 \\
0.57\end{array}$ & $\begin{array}{l}\frac{44}{0} \\
\underline{0.03} \\
0.46 \\
\end{array}$ & & $\begin{array}{l}\frac{55}{\underline{0}} \\
\underline{0} \\
\underline{0} \\
\underline{0.33}\end{array}$ & & & & & & & $\begin{array}{l}\frac{2}{0} \\
\frac{0}{0} \\
\underline{0.31}\end{array}$ & $\begin{array}{l}3 \\
0 \\
0 \\
0 \\
0\end{array}$ & \\
\hline $\begin{array}{l}\text { Ring } \\
\text { Core }\end{array}$ & $\begin{array}{l}\frac{10}{\underline{0}} \\
\underline{0} \\
\underline{0} \\
\underline{0}\end{array}$ & $\begin{array}{c}\frac{11}{0} \\
0 \\
0 \\
0.25\end{array}$ & $\begin{array}{l}\frac{16}{0} \\
0.57 \\
0.13\end{array}$ & $\begin{array}{l}\frac{17}{\underline{0}} \\
\underline{0} \\
1.00 \\
\end{array}$ & $\begin{array}{l}\frac{21}{\underline{0}} \\
\underline{0} \\
\underline{0} \\
\underline{0.08} \\
\underline{0.17}\end{array}$ & $\begin{array}{l}\frac{23}{\underline{0}} \\
\underline{0} \\
\underline{0} \\
\underline{0.09} \\
\underline{1.47}\end{array}$ & $\begin{array}{l}24 \\
0 \\
0 \\
0 \\
0 \\
0.83\end{array}$ & $\begin{array}{l}\frac{28}{0} \\
0 \\
0 \\
0.27 \\
0.38\end{array}$ & & & & & & & $\begin{array}{l}\frac{56}{\underline{0}} \\
\underline{0} \\
\underline{0} \\
\underline{0} \\
\underline{0.19}\end{array}$ & $\begin{array}{l}\frac{57}{0} \\
\frac{0}{0} \\
\underline{0} \\
\underline{0} \\
0.16\end{array}$ & $\begin{array}{l}\frac{58}{0} \\
0 \\
0 \\
0 \\
0.08\end{array}$ & $\begin{array}{l}\frac{61}{0} \\
\underline{0} \\
\underline{0} \\
\underline{0} \\
\underline{0.26}\end{array}$ & $\begin{array}{l}\frac{62}{0} \\
0 \\
0 \\
0 \\
0.21\end{array}$ & $\begin{array}{l}\frac{63}{\underline{0}} \\
\underline{0} \\
\underline{0.14} \\
\underline{0} \\
\underline{0.03}\end{array}$ & $\begin{array}{l}\frac{64}{0} \\
0 \\
0 \\
0 \\
0.19\end{array}$ & $\begin{array}{l}\frac{1}{\underline{0}} \\
\underline{0} \\
\underline{0} \\
\underline{0.08}\end{array}$ & & \\
\hline $\begin{array}{l}\text { Sargasso } \\
\text { Sea } \\
\& \\
\text { Gulf } \\
\text { Stream }\end{array}$ & & & & & & & & & & $\begin{array}{l}\frac{49}{0} \\
\frac{0}{0} \\
\frac{0}{0}\end{array}$ & $\begin{array}{l}50 \\
0 \\
0 \\
0 \\
0\end{array}$ & $\begin{array}{l}51 \\
0 \\
0 \\
0 \\
0.17\end{array}$ & $\begin{array}{l}\frac{52}{\underline{0}} \\
\underline{0} \\
\underline{0} \\
\underline{0.64}\end{array}$ & & & & & & & & & & & \\
\hline $\begin{array}{l}\text { Slope } \\
\text { Water }\end{array}$ & $\frac{8}{-}$ & $\frac{9}{0.33}$ & $\frac{18}{0.09}$ & & $\frac{19}{0.03}$ & $\frac{20}{0.14}$ & $\frac{33}{0.25}$ & $\frac{34}{0.07}$ & & $\frac{35}{0.02}$ & $\frac{47}{0.05}$ & $\frac{48}{0}$ & $\frac{53}{0.04}$ & $\frac{54}{0.15}$ & $\frac{59}{0.04}$ & $\frac{60}{0.02}$ & $\frac{65}{0.03}$ & $\frac{66}{0.05}$ & & & & $\frac{4}{0.07}$ & $\frac{5}{0.01}$ & $\frac{6}{0.02}$ \\
\hline $\begin{array}{l}\text { High } \\
\text { Velocity } \\
\text { Region }\end{array}$ & $\frac{12}{0.10}$ & $\frac{13}{0.07}$ & $\frac{14}{0.02}$ & $\frac{15}{0.57}$ & $\frac{27}{0.07}$ & $\frac{29}{0.16}$ & $\frac{30}{0.01}$ & $\frac{31}{0.11}$ & $\frac{32}{-}$ & $\frac{41}{0.13}$ & $\frac{42}{0.08}$ & $\frac{43}{0.11}$ & $\frac{44}{0.10}$ & & $\frac{55}{0.07}$ & & & & & & & $\frac{2}{0.08}$ & $\frac{3}{0}$ & \\
\hline $\begin{array}{l}\text { Ring } \\
\text { Core }\end{array}$ & $\frac{10}{0}$ & $\frac{11}{0.06}$ & $\frac{16}{-}$ & $\frac{17}{0.25}$ & $\frac{21}{0.05}$ & $\frac{23}{0.31}$ & $\frac{24}{0.17}$ & $\frac{28}{0.07}$ & & $\frac{37}{0.02}$ & $\frac{38}{0.01}$ & $\frac{39}{0}$ & & & $\frac{56}{0.04}$ & $\frac{57}{0.03}$ & $\frac{58}{0.02}$ & $\frac{61}{0.05}$ & $\frac{62}{0.04}$ & $\frac{63}{0.03}$ & $\frac{64}{0.04}$ & $\frac{1}{0.02}$ & & \\
\hline SS \& GS & & & & & & & & & & $\frac{49}{0}$ & $\frac{50}{0}$ & $\frac{51}{0.04}$ & $\frac{52}{0.16}$ & & & & & & & & & & & \\
\hline
\end{tabular}


Table 117. Abundance of Scopelogadus beanii (specimens $/ 10,000 \mathrm{~m}^{3}$ ).

\begin{tabular}{|c|c|c|c|c|c|c|c|c|c|c|c|c|c|c|c|c|c|c|c|c|c|c|c|c|}
\hline & & $\begin{array}{r}\text { Ocean } \\
\mathrm{Ap}\end{array}$ & $\begin{array}{l}\text { S } 118 \\
\text { if }\end{array}$ & & & & $\begin{array}{l}\text { anus } \\
\text { June }\end{array}$ & 121 & & & $\begin{array}{r}\text { Oce } \\
\text { A }\end{array}$ & $\begin{array}{l}\text { nus } 1 \\
\text { Igust }\end{array}$ & & & & & $\begin{array}{l}\text { Knor } \\
\text { Sept }\end{array}$ & $\begin{array}{r}98 \\
-0 c t \\
\end{array}$ & & & & Se & $\begin{array}{c}110 \\
t-0 c t\end{array}$ & \\
\hline $\begin{array}{l}\text { Slope } \\
\text { Water }\end{array}$ & $\begin{array}{c}\frac{8}{\overline{\overline{0}}} \\
\underline{0.9} \\
\underline{0.5}\end{array}$ & $\begin{array}{l}9 \\
0 \\
0 \\
0 \\
0\end{array}$ & & & $\begin{array}{r}\frac{19}{\underline{0}} \\
\underline{0} \\
\underline{0} \\
\underline{0} \\
\underline{0}\end{array}$ & $\begin{array}{r}20 \\
0 \\
0 \\
0 \\
0 \\
0\end{array}$ & $\begin{array}{r}33 \\
\underline{0} \\
\underline{0} \\
\underline{0} \\
\underline{0} \\
\underline{0}\end{array}$ & $\begin{array}{l}\frac{34}{\underline{0}} \\
\underline{0} \\
\underline{0.2}\end{array}$ & & $\begin{array}{c}35 \\
0 \\
0 \\
0 \\
0 \\
0.7\end{array}$ & $\frac{47}{\frac{0.2}{\underline{0}}} \frac{\underline{0}}{\underline{0}}$ & $\begin{array}{c}48 \\
0 \\
0 \\
0.6 \\
0\end{array}$ & $\frac{53}{\underline{0}}$ & $\begin{array}{l}54 \\
0 \\
0 \\
0 \\
0\end{array}$ & $\begin{array}{l}\frac{59}{\underline{0}} \\
\underline{0} \\
\underline{0.6} \\
\underline{1.0} \\
\underline{0.2}\end{array}$ & $\begin{array}{r}60 \\
0 \\
0 \\
0 \\
0 \\
0\end{array}$ & $\begin{array}{c}\frac{65}{\underline{0}} \\
\frac{1.0}{0.9} \\
\frac{0.8}{\underline{0.8}}\end{array}$ & $\begin{array}{c}66 \\
0 \\
0 \\
0.4 \\
0 \\
0.4\end{array}$ & & & & $\begin{array}{l}4 \\
0 \\
0 \\
0 \\
0\end{array}$ & $\frac{\frac{5}{0}}{\frac{0.4}{0.3}}$ & \\
\hline $\begin{array}{l}\text { High } \\
\text { Velocity } \\
\text { Region }\end{array}$ & $\begin{array}{c}12 \\
0 \\
0 \\
0 \\
0\end{array}$ & $\frac{13}{\underline{0}}$ & $\frac{14}{\underline{0}} \frac{0.7}{\frac{0}{0}}$ & $\begin{array}{c}15 \\
0 \\
0 \\
0.6 \\
0.5\end{array}$ & $\begin{array}{c}26 \\
0 \\
0 \\
0 \\
0.7 \\
-\end{array}$ & $\begin{array}{c}27 \\
0 \\
0 \\
0 \\
0.8 \\
0.7\end{array}$ & $\begin{array}{c}30 \\
0 \\
0 \\
0 \\
1.9 \\
0\end{array}$ & $\begin{array}{c}31 \\
0 \\
0 \\
0 \\
0.3 \\
0\end{array}$ & $\frac{\frac{32}{2.1}}{\frac{3.2}{1.0}}$ & $\frac{\frac{41}{0.1}}{\frac{0.9}{\underline{0}}}$ & $\frac{42}{\underline{0}}$ & $\begin{array}{r}43 \\
0 \\
0 \\
0 \\
0 \\
0\end{array}$ & $\frac{44}{\frac{0.1}{\underline{0}}}$ & & $\begin{array}{l}55 \\
\underline{0} \\
\underline{0} \\
\underline{0} \\
\underline{0}\end{array}$ & & & & & & & $\begin{array}{l}\frac{2}{\underline{0}} \\
\underline{0} \\
\frac{0.7}{\underline{0}}\end{array}$ & $\begin{array}{l}3 \\
0 \\
0 \\
0 \\
0\end{array}$ & \\
\hline $\begin{array}{l}\text { Ring } \\
\text { Core }\end{array}$ & $\begin{array}{r}10 \\
\frac{0}{0} \\
\underline{0} \\
\underline{0}\end{array}$ & $\begin{array}{l}11 \\
0 \\
0 \\
0 \\
0\end{array}$ & $\begin{array}{c}16 \\
0 \\
0 \\
0\end{array}$ & $\frac{17}{\underline{0}}$ & $\begin{array}{l}\frac{21}{\underline{0}} \\
\underline{0} \\
\frac{4.6}{2.5} \\
\underline{0}\end{array}$ & $\begin{array}{l}\frac{23}{\underline{0}} \\
\underline{0} \\
\frac{2.5}{1.8} \\
\underline{0}\end{array}$ & $\begin{array}{c}24 \\
0 \\
0 \\
0 \\
3.8 \\
0\end{array}$ & $\begin{array}{c}28 \\
0 \\
0 \\
1.4 \\
0 \\
0\end{array}$ & & & & & & & $\begin{array}{c}\frac{56}{\underline{0}} \\
\underline{0} \\
\underline{0} \\
\underline{0} \\
\underline{0.6}\end{array}$ & $\begin{array}{c}\frac{57}{0} \\
\underline{0} \\
\underline{0} \\
\underline{0} \\
\underline{0} \\
\underline{0.3}\end{array}$ & $\begin{array}{r}58 \\
0 \\
0 \\
0 \\
0 \\
0\end{array}$ & $\begin{array}{c}61 \\
\underline{0} \\
\underline{0} \\
\underline{0} \\
\underline{0} \\
\underline{0.3}\end{array}$ & $\begin{array}{c}62 \\
0 \\
0 \\
0 \\
0 \\
0.4\end{array}$ & $\begin{array}{c}\frac{63}{\underline{0}} \\
\underline{0} \\
\underline{0} \\
\underline{0.4} \\
\underline{0}\end{array}$ & $\begin{array}{c}64 \\
0 \\
0 \\
0 \\
0.5 \\
0.8\end{array}$ & $\begin{array}{c}\frac{1}{\underline{0}} \\
\underline{0} \\
\underline{0} \\
\underline{0.8}\end{array}$ & & \\
\hline $\begin{array}{l}\text { Sargasso } \\
\text { Sea } \\
\& \\
\text { Gulf } \\
\text { Stream }\end{array}$ & & & & & & & & & & $\frac{\frac{49}{0.2}}{\frac{0}{0}} \frac{\underline{0}}{\underline{0}}$ & $\begin{array}{c}50 \\
0 \\
0 \\
0.3 \\
0\end{array}$ & $\begin{array}{c}51 \\
0 \\
0 \\
0.2 \\
0.3\end{array}$ & $\begin{array}{r}52 \\
\underline{0} \\
\underline{0} \\
\underline{0} \\
\underline{0}\end{array}$ & & & & & & & & & & & \\
\hline $\begin{array}{l}\text { Slope } \\
\text { Water }\end{array}$ & $\frac{8}{-}$ & $\frac{9}{0}$ & $\frac{18}{0.2}$ & & $\frac{19}{0}$ & $\frac{20}{0}$ & $\frac{33}{0}$ & $\frac{34}{0.1}$ & & $\frac{35}{0.1}$ & $\frac{47}{0.1}$ & $\frac{48}{0.2}$ & $\frac{53}{0.1}$ & $\frac{54}{0}$ & $\frac{59}{0.4}$ & $\frac{60}{0}$ & $\frac{65}{0.6}$ & $\frac{66}{0.2}$ & & & & $\frac{4}{0}$ & $\frac{5}{0.2}$ & $\frac{6}{0}$ \\
\hline $\begin{array}{l}\text { High } \\
\text { Velocity } \\
\text { Region }\end{array}$ & $\frac{12}{0}$ & $\frac{13}{0}$ & $\frac{14}{0.2}$ & $\frac{15}{0.3}$ & $\frac{27}{0.4}$ & $\frac{29}{1.2}$ & $\frac{30}{0.4}$ & $\frac{31}{0.1}$ & $\frac{32}{-}$ & $\frac{41}{0.3}$ & $\frac{42}{0}$ & $\frac{43}{0}$ & $\frac{44}{0.1}$ & & $\frac{55}{0}$ & & & & & & & $\frac{2}{0.2}$ & $\frac{3}{0}$ & \\
\hline $\begin{array}{l}\text { Ring } \\
\text { Core }\end{array}$ & $\frac{10}{0}$ & $\frac{11}{0}$ & $\frac{16}{-}$ & $\frac{17}{0.1}$ & $\frac{21}{1.4}$ & $\frac{23}{0.9}$ & $\frac{24}{0.8}$ & $\frac{28}{0.4}$ & & $\frac{37}{0}$ & $\frac{38}{0}$ & $\frac{39}{0}$ & & & $\frac{56}{0.1}$ & $\frac{57}{0.1}$ & $\frac{58}{0}$ & $\frac{61}{0.1}$ & $\frac{62}{0.1}$ & $\frac{63}{0.1}$ & $\frac{64}{0.3}$ & $\frac{1}{0.2}$ & & \\
\hline SS \& GS & & & & & & & & & & $\frac{49}{0.1}$ & $\frac{50}{0.1}$ & $\frac{51}{0.1}$ & $\frac{52}{0}$ & & & & & & & & & & & \\
\hline
\end{tabular}


Table 118. Volume of Scopelogadus beanii $\left(\mathrm{ml} / 10,000 \mathrm{~m}^{3}\right)$.

\begin{tabular}{|c|c|c|c|c|c|c|c|c|c|c|c|c|c|c|c|c|c|c|c|c|c|c|c|c|}
\hline & \multicolumn{4}{|c|}{$\begin{array}{c}\text { Oceanus } \\
\text { Apri1 }\end{array}$} & \multicolumn{5}{|c|}{$\begin{array}{c}\text { Oceanus } 121 \\
\text { June }\end{array}$} & \multicolumn{5}{|c|}{$\begin{array}{l}\text { Oceanus } 125 \\
\text { August }\end{array}$} & \multicolumn{7}{|c|}{$\begin{array}{l}\text { Knorr } 98 \\
\text { Sept-0ct }\end{array}$} & \multicolumn{3}{|c|}{$\begin{array}{c}\text { A2 } 110 \\
\text { Sept-0ct }\end{array}$} \\
\hline $\begin{array}{l}\text { Slope } \\
\text { Water }\end{array}$ & $\begin{array}{c}\frac{8}{\overline{\overline{0}}} \\
\frac{11.82}{20.00} \\
\end{array}$ & $\begin{array}{l}9 \\
0 \\
0 \\
0 \\
0\end{array}$ & & & $\begin{array}{l}\frac{19}{0} \\
\frac{0}{0} \\
\underline{0} \\
\underline{0} \\
\underline{0}\end{array}$ & $\begin{array}{l}20 \\
0 \\
0 \\
0 \\
0 \\
0\end{array}$ & $\begin{array}{l}\frac{33}{0} \\
\underline{0} \\
\underline{0} \\
\underline{0} \\
\underline{0}\end{array}$ & $\begin{array}{l}\frac{34}{\underline{0}} \\
\underline{0} \\
7.27\end{array}$ & & $\begin{array}{l}\frac{35}{0} \\
0 \\
0 \\
0 \\
0.21\end{array}$ & $\begin{array}{l}\frac{47}{\underline{0}} \\
\underline{0} \\
\underline{0} \\
\underline{0}\end{array}$ & $\begin{array}{c}\frac{48}{0} \\
0 \\
0.09 \\
0\end{array}$ & $\begin{array}{l}\frac{53}{\underline{0}} \\
\frac{0.02}{\underline{0}} \\
\underline{0}\end{array}$ & $\begin{array}{l}54 \\
0 \\
0 \\
0 \\
0\end{array}$ & $\begin{array}{r}\frac{59}{0} \\
\underline{0} \\
\frac{0.44}{16.55} \\
10.91 \\
\end{array}$ & $\begin{array}{l}60 \\
0 \\
0 \\
0 \\
0 \\
0\end{array}$ & $\begin{array}{l}\frac{65}{\underline{0}} \\
\frac{0.10}{9.09} \\
\frac{16.00}{\underline{0}}\end{array}$ & $\begin{array}{l}\frac{66}{0} \\
0 \\
0.04 \\
0 \\
1.54\end{array}$ & & & & $\begin{array}{l}4 \\
0 \\
0 \\
0 \\
0\end{array}$ & $\frac{\frac{5}{0}}{\frac{\underline{\underline{2} .41}}{5.00}}$ & \\
\hline $\begin{array}{l}\text { High } \\
\text { Velocity } \\
\text { Region }\end{array}$ & $\begin{array}{l}\frac{12}{0} \\
0 \\
0 \\
0\end{array}$ & $\frac{13}{\frac{0}{0}}$ & $\frac{\frac{14}{\underline{0}}}{\frac{0.67}{\underline{0}}}$ & $\begin{array}{l}\frac{15}{0} \\
0 \\
0.18 \\
5.24\end{array}$ & $\begin{array}{l}26 \\
0 \\
0 \\
0 \\
0.03 \\
-\end{array}$ & $\begin{array}{l}\frac{27}{0} \\
0 \\
0 \\
0.04 \\
2.81\end{array}$ & $\begin{array}{l}\frac{30}{0} \\
0 \\
0 \\
0.05 \\
0\end{array}$ & $\begin{array}{l}31 \\
0 \\
0 \\
0 \\
0.03 \\
0\end{array}$ & $\frac{\frac{32}{0.08}}{\frac{0.12}{\frac{0}{0}}}$ & $\frac{\frac{41}{0.01}}{\frac{0}{0}}$ & $\begin{array}{l}\frac{42}{\underline{0}} \\
\frac{0}{0} \\
\underline{0}\end{array}$ & $\begin{array}{l}43 \\
0 \\
0 \\
0 \\
0 \\
0\end{array}$ & $\frac{\frac{44}{0.01}}{\frac{0}{0}}$ & & $\begin{array}{l}\frac{55}{\underline{0}} \\
\frac{0}{0} \\
\underline{0}\end{array}$ & & & & & & & $\begin{array}{c}\frac{2}{\underline{0}} \\
\frac{22.00}{\underline{0}}\end{array}$ & $\begin{array}{l}3 \\
0 \\
0 \\
0 \\
0\end{array}$ & \\
\hline $\begin{array}{l}\text { Ring } \\
\text { Core }\end{array}$ & $\begin{array}{l}10 \\
\frac{0}{0} \\
\underline{0} \\
\underline{0} \\
\underline{0}\end{array}$ & $\begin{array}{l}11 \\
0 \\
0 \\
0 \\
0\end{array}$ & $\begin{array}{l}\frac{16}{0} \\
0 \\
0\end{array}$ & $\begin{array}{l}\frac{17}{\underline{0}} \\
\underline{0} \\
0.16\end{array}$ & $\begin{array}{l}\frac{21}{\underline{0}} \\
\frac{0}{0.18} \\
\frac{0.17}{\underline{0}}\end{array}$ & $\begin{array}{l}\frac{23}{\underline{0}} \\
\underline{\underline{0}} \\
\frac{0.10}{0.05} \\
\underline{0}\end{array}$ & $\begin{array}{l}24 \\
0 \\
0 \\
0 \\
0.13 \\
0\end{array}$ & $\begin{array}{l}28 \\
0 \\
0 \\
0.04 \\
0 \\
0\end{array}$ & & & & & & & $\begin{array}{l}\frac{56}{\underline{0}} \\
\underline{0} \\
\underline{0} \\
\underline{0} \\
\underline{0.06}\end{array}$ & $\begin{array}{l}\frac{57}{\underline{0}} \\
\underline{0} \\
\underline{0} \\
\underline{0} \\
\underline{0.03} \\
\end{array}$ & $\begin{array}{l}58 \\
0 \\
0 \\
0 \\
0 \\
0\end{array}$ & $\begin{array}{l}\frac{61}{\underline{0}} \\
\frac{0}{0} \\
\underline{0} \\
\underline{0.03} \\
\end{array}$ & $\begin{array}{l}\frac{62}{0} \\
0 \\
0 \\
0 \\
0.04\end{array}$ & $\begin{array}{l}\frac{63}{\underline{0}} \\
\underline{0} \\
\underline{0} \\
\frac{0.04}{\underline{0}}\end{array}$ & $\begin{array}{l}\frac{64}{0} \\
0 \\
0 \\
0.05 \\
0.04\end{array}$ & $\begin{array}{l}\frac{1}{\frac{0}{0}} \\
\frac{0}{0} \\
\underline{0.17} \\
\end{array}$ & & \\
\hline $\begin{array}{l}\text { Sargasso } \\
\text { Sea } \\
\& \\
\text { Gulf } \\
\text { Stream }\end{array}$ & & & & & & & & & & $\frac{\frac{49}{0.02}}{\frac{0}{0}}$ & $\begin{array}{c}50 \\
0 \\
0 \\
0.03 \\
7.19\end{array}$ & $\begin{array}{l}\frac{51}{0} \\
0 \\
0.02 \\
0.03\end{array}$ & $\begin{array}{l}\frac{52}{\underline{0}} \\
\underline{0} \\
\underline{0} \\
\underline{0}\end{array}$ & & & & & & & & & & & \\
\hline $\begin{array}{l}\text { Slope } \\
\text { Water }\end{array}$ & $\frac{8}{-}$ & $\frac{9}{0}$ & $\frac{18}{0.16}$ & & $\frac{19}{0}$ & $\frac{20}{0}$ & $\frac{33}{0}$ & $\frac{34}{4.36}$ & & $\frac{35}{0.04}$ & $\frac{47}{0}$ & $\frac{48}{0.02}$ & $\frac{53}{0.01}$ & $\frac{54}{0}$ & $\frac{59}{5.58}$ & $\frac{60}{0}$ & $\frac{65}{5.04}$ & $\frac{66}{0.32}$ & & & & $\frac{4}{0}$ & $\frac{5}{1.85}$ & $\frac{6}{0}$ \\
\hline $\begin{array}{l}\text { High } \\
\text { Velocity } \\
\text { Region }\end{array}$ & $\frac{12}{0}$ & $\frac{13}{0}$ & $\frac{14}{0.17}$ & $\frac{15}{1.36}$ & $\frac{27}{0.71}$ & $\frac{29}{0.05}$ & $\frac{30}{0.01}$ & $\frac{31}{0.01}$ & $\frac{32}{-}$ & $\frac{41}{0.01}$ & $\frac{42}{0}$ & $\frac{43}{0}$ & $\frac{44}{0.01}$ & & $\frac{55}{0}$ & & & & & & & $\frac{2}{5.50}$ & $\frac{3}{0}$ & \\
\hline $\begin{array}{l}\text { Ring } \\
\text { Core }\end{array}$ & $\frac{10}{0}$ & $\frac{11}{0}$ & $\frac{16}{-}$ & $\frac{17}{0.08}$ & $\frac{21}{0.07}$ & $\frac{23}{0.03}$ & $\frac{24}{0.03}$ & $\frac{28}{0.01}$ & & $\frac{37}{0}$ & $\frac{38}{0}$ & $\frac{39}{0}$ & & & $\frac{56}{0.01}$ & $\frac{57}{0.01}$ & $\frac{58}{0}$ & $\frac{61}{0.01}$ & $\frac{62}{0.01}$ & $\frac{63}{0.01}$ & $\frac{64}{0.02}$ & $\frac{1}{0.04}$ & & \\
\hline SS \& GS & & & & & & & & & & $\frac{49}{0.01}$ & $\frac{50}{1.81}$ & $\frac{51}{0.01}$ & $\frac{52}{0}$ & & & & & & & & & & & \\
\hline
\end{tabular}


Table 119. Volume of Scopelogadus mizolepis $\left(\mathrm{ml} / 10,000 \mathrm{~m}^{3}\right)$.

\begin{tabular}{|c|c|c|c|c|c|c|c|c|c|c|c|c|c|c|c|c|c|c|c|c|c|c|c|c|}
\hline & \multicolumn{4}{|c|}{$\begin{array}{c}\text { Oceanus } \\
\text { April }\end{array}$} & \multicolumn{5}{|c|}{$\begin{array}{c}\text { Oceanus } 121 \\
\text { June }\end{array}$} & \multicolumn{5}{|c|}{$\begin{array}{l}\text { Oceanus } 125 \\
\text { August }\end{array}$} & \multicolumn{7}{|c|}{$\begin{array}{l}\text { Knorr } 98 \\
\text { Sept-Oct }\end{array}$} & \multicolumn{3}{|c|}{$\begin{array}{c}\text { A2 } 110 \\
\text { Sept-0ct }\end{array}$} \\
\hline $\begin{array}{l}\text { Slope } \\
\text { Water }\end{array}$ & $\begin{array}{l}\frac{8}{\overline{0}} \\
\underline{0} \\
\underline{0} \\
\underline{0}\end{array}$ & $\begin{array}{l}\frac{9}{0} \\
0 \\
0 \\
0\end{array}$ & & & $\begin{array}{l}\frac{19}{\underline{0}} \\
\underline{0} \\
\underline{0} \\
\frac{1.05}{\underline{0}}\end{array}$ & $\begin{array}{l}20 \\
0 \\
0 \\
0 \\
0 \\
0\end{array}$ & $\begin{array}{l}33 \\
\underline{0} \\
\underline{0} \\
\underline{0} \\
\underline{0} \\
\underline{0}\end{array}$ & $\begin{array}{l}\frac{34}{\underline{0}} \\
\underline{0} \\
\underline{4.09} \\
\end{array}$ & & $\begin{array}{l}35 \\
0 \\
0 \\
0 \\
0 \\
0\end{array}$ & $\begin{array}{l}\frac{47}{0.02} \\
\underline{0.06} \\
\underline{0.067} \\
\underline{0.57}\end{array}$ & $\begin{array}{c}\frac{48}{0} \\
0 \\
0.79 \\
10.82\end{array}$ & $\begin{array}{l}\frac{53}{\underline{0}} \\
\underline{0} \\
\underline{0} \\
\underline{0}\end{array}$ & $\begin{array}{l}54 \\
0 \\
0 \\
0 \\
0.83\end{array}$ & $\begin{array}{l}\frac{59}{\frac{0}{0}} \\
\frac{8.24}{\underline{0}} \\
11.36 \\
\end{array}$ & $\begin{array}{l}60 \\
0 \\
0 \\
0 \\
0 \\
0\end{array}$ & $\begin{array}{l}\frac{65}{0} \\
\underline{0} \\
\underline{0} \\
\underline{0} \\
\underline{0} \\
\underline{0}\end{array}$ & $\begin{array}{l}\frac{66}{0} \\
0 \\
0 \\
0 \\
0\end{array}$ & & & & $\begin{array}{l}\frac{4}{0} \\
0 \\
0.03 \\
0.09\end{array}$ & $\begin{array}{l}\frac{5}{\frac{0}{0}} \\
\frac{0}{0} \\
\underline{0.38}\end{array}$ & \\
\hline $\begin{array}{l}\text { High } \\
\text { Velocity } \\
\text { Region }\end{array}$ & $\begin{array}{c}\frac{12}{0} \\
0 \\
0 \\
13.10\end{array}$ & $\begin{array}{l}\frac{13}{0} \\
\frac{0}{0} \\
\underline{0}\end{array}$ & $\begin{array}{l}\frac{14}{0} \\
\frac{0}{0} \\
\frac{0}{0} \\
\underline{0}\end{array}$ & $\begin{array}{l}15 \\
0 \\
0 \\
0 \\
0\end{array}$ & $\begin{array}{l}26 \\
0 \\
0 \\
0 \\
0 \\
-\end{array}$ & $\begin{array}{l}\frac{27}{0} \\
0 \\
0 \\
0 \\
1.14\end{array}$ & $\begin{array}{l}30 \\
0 \\
0 \\
0 \\
0 \\
0\end{array}$ & $\begin{array}{l}31 \\
0 \\
0 \\
0 \\
0 \\
0\end{array}$ & $\frac{32}{\frac{0}{0}}$ & $\begin{array}{r}\frac{41}{0} \\
\frac{28.57}{0.11} \\
\end{array}$ & $\frac{\frac{42}{0}}{\frac{0}{1.18}}$ & $\begin{array}{l}43 \\
0 \\
0 \\
0 \\
0 \\
0\end{array}$ & $\begin{array}{l}\frac{44}{5.74} \\
\underline{0} \\
\underline{0.38}\end{array}$ & & $\begin{array}{l}\frac{55}{\underline{0}} \\
\underline{0} \\
\underline{0} \\
\underline{0}\end{array}$ & & & & & & & $\frac{\frac{2}{\underline{0}}}{\frac{0.03}{\underline{0}}}$ & $\begin{array}{l}\frac{3}{0} \\
0 \\
0.32 \\
0\end{array}$ & \\
\hline $\begin{array}{l}\text { Ring } \\
\text { Core }\end{array}$ & $\begin{array}{l}\frac{10}{0} \\
\underline{0} \\
\underline{0} \\
\underline{0}\end{array}$ & $\begin{array}{l}11 \\
0 \\
0 \\
0 \\
0\end{array}$ & $\begin{array}{l}\frac{16}{0} \\
0 \\
0\end{array}$ & $\begin{array}{l}\frac{17}{\underline{0}} \\
\underline{0} \\
\underline{0}\end{array}$ & $\begin{array}{l}21 \\
\underline{0} \\
\underline{0} \\
\underline{0} \\
\underline{0} \\
\underline{0}\end{array}$ & $\begin{array}{l}23 \\
\underline{0} \\
\underline{0} \\
\underline{0} \\
\underline{0} \\
\underline{0}\end{array}$ & $\begin{array}{l}24 \\
0 \\
0 \\
0 \\
0 \\
0\end{array}$ & $\begin{array}{l}\frac{28}{0} \\
0 \\
0 \\
2.31 \\
0\end{array}$ & & & & & & & $\begin{array}{l}56 \\
\underline{0} \\
\underline{0} \\
\underline{0} \\
\underline{0} \\
\underline{0}\end{array}$ & $\begin{array}{l}\frac{57}{0} \\
\underline{0} \\
\underline{0} \\
\underline{0} \\
\underline{0} \\
\underline{0}\end{array}$ & $\begin{array}{l}\frac{58}{0} \\
0 \\
0 \\
0 \\
0.18\end{array}$ & $\begin{array}{l}61 \\
\underline{0} \\
\underline{0} \\
\underline{0} \\
\underline{0} \\
\underline{0}\end{array}$ & $\begin{array}{l}62 \\
0 \\
0 \\
0 \\
0 \\
0\end{array}$ & $\begin{array}{l}\frac{63}{0} \\
\underline{0} \\
\underline{0} \\
\underline{0} \\
\underline{0}\end{array}$ & $\begin{array}{l}64 \\
0 \\
0 \\
0 \\
0 \\
0\end{array}$ & $\begin{array}{l}\frac{1}{0} \\
\underline{0} \\
\underline{0} \\
\underline{0}\end{array}$ & & \\
\hline $\begin{array}{l}\text { Sargasso } \\
\text { Sea } \\
\& \\
\text { Gulf } \\
\text { Stream }\end{array}$ & & & & & & & & & & $\begin{array}{l}\frac{49}{\underline{0}} \\
\underline{0} \\
\underline{0} \\
\underline{0}\end{array}$ & $\begin{array}{l}\frac{50}{0} \\
0 \\
0 \\
0.09\end{array}$ & $\begin{array}{l}\frac{51}{0} \\
0 \\
0 \\
0\end{array}$ & $\begin{array}{l}\frac{52}{0} \\
\underline{0} \\
\underline{0} \\
\underline{0}\end{array}$ & & & & & & & & & & & \\
\hline $\begin{array}{l}\text { Slope } \\
\text { Water }\end{array}$ & $\frac{8}{-}$ & $\frac{9}{0}$ & $\frac{18}{0}$ & & $\frac{19}{0.26}$ & $\frac{20}{0}$ & $\frac{33}{0}$ & $\frac{34}{2.45}$ & & $\frac{35}{0}$ & $\frac{47}{0.13}$ & $\frac{48}{2.90}$ & $\frac{53}{0}$ & $\frac{54}{0.21}$ & $\frac{59}{3.92}$ & $\frac{60}{0}$ & $\frac{65}{0}$ & $\frac{66}{0}$ & & & & $\frac{4}{0.03}$ & $\frac{5}{0.10}$ & $\frac{6}{3.81}$ \\
\hline $\begin{array}{l}\text { High } \\
\text { Velocity } \\
\text { Region }\end{array}$ & $\frac{12}{3.28}$ & $\frac{13}{0}$ & $\frac{14}{0}$ & $\frac{15}{0}$ & $\frac{27}{0.29}$ & $\frac{29}{0.10}$ & $\frac{30}{0}$ & $\frac{31}{0}$ & $\frac{32}{-}$ & $\frac{41}{7.17}$ & $\frac{42}{0.26}$ & $\frac{43}{0}$ & $\frac{44}{3.52}$ & & $\frac{55}{0}$ & & & & & & & $\frac{2}{0.01}$ & $\frac{3}{0.08}$ & \\
\hline $\begin{array}{l}\text { Ring } \\
\text { Core }\end{array}$ & $\frac{10}{0}$ & $\frac{11}{0}$ & $\frac{16}{-}$ & $\frac{17}{0}$ & $\frac{21}{0}$ & $\frac{23}{0}$ & $\frac{24}{0}$ & $\frac{28}{0.58}$ & & $\frac{37}{2.31}$ & $\frac{38}{0}$ & $\frac{39}{0}$ & & & $\frac{56}{0}$ & $\frac{57}{0}$ & $\frac{58}{0.04}$ & $\frac{61}{0}$ & $\frac{62}{0}$ & $\frac{63}{0}$ & $\frac{64}{0}$ & $\frac{1}{0}$ & & \\
\hline SS \& GS & & & & & & & & & & $\frac{49}{0}$ & $\frac{50}{0.02}$ & $\frac{51}{0}$ & $\frac{52}{0}$ & & & & & & & & & & & \\
\hline
\end{tabular}


Table 120. Abundance of Serrivomer beanii (specimens $/ 10,000 \mathrm{~m}^{3}$ ).

\begin{tabular}{|c|c|c|c|c|c|c|c|c|c|c|c|c|c|c|c|c|c|c|c|c|c|c|c|c|}
\hline & \multicolumn{4}{|c|}{$\begin{array}{c}\text { Oceanus } \\
\text { Aprif }\end{array}$} & \multicolumn{5}{|c|}{$\begin{array}{c}\text { Oceanus } 121 \\
\text { June }\end{array}$} & \multicolumn{5}{|c|}{$\begin{array}{c}\text { Oceanus } 125 \\
\text { August }\end{array}$} & \multicolumn{7}{|c|}{$\begin{array}{l}\text { Knorr } 98 \\
\text { Sept-Oct }\end{array}$} & \multicolumn{3}{|c|}{$\begin{array}{c}\text { A2 } 110 \\
\text { Sept-Oct }\end{array}$} \\
\hline $\begin{array}{l}\text { Slope } \\
\text { Water }\end{array}$ & $\begin{array}{l}8 \\
\overline{\overline{0}} \\
\underline{0} \\
\underline{0.5}\end{array}$ & $\begin{array}{l}9 \\
0 \\
0 \\
0 \\
0\end{array}$ & & & $\begin{array}{l}\frac{19}{0} \\
\underline{0} \\
\underline{0} \\
\underline{1.1} \\
\underline{0.5}\end{array}$ & $\begin{array}{c}20 \\
0 \\
0 \\
0.4 \\
0 \\
0\end{array}$ & $\begin{array}{c}33 \\
\underline{0} \\
\underline{0} \\
\underline{0} \\
\underline{0.1} \\
\underline{0}\end{array}$ & $\begin{array}{l}\frac{34}{\underline{0}} \\
\underline{0} \\
\underline{0}\end{array}$ & & $\begin{array}{c}35 \\
0 \\
0 \\
1.0 \\
0 \\
0\end{array}$ & $\begin{array}{l}\frac{47}{\underline{0}} \\
\underline{0} \\
\underline{0.2} \\
\underline{0.2}\end{array}$ & $\begin{array}{c}48 \\
0.3 \\
0 \\
0 \\
0.2\end{array}$ & $\frac{\frac{53}{0.2}}{\frac{0.4}{0.3}} \frac{0}{\underline{0}}$ & $\begin{array}{l}54 \\
0 \\
0 \\
0 \\
0\end{array}$ & $\begin{array}{c}59 \\
\underline{0} \\
\underline{0} \\
\underline{0} \\
\underline{0.3} \\
\underline{0.2} \\
\end{array}$ & $\begin{array}{l}60 \\
0 \\
0 \\
0 \\
0 \\
0\end{array}$ & 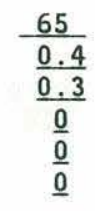 & $\begin{array}{l}66 \\
0 \\
0 \\
0 \\
0 \\
0\end{array}$ & & & & $\begin{array}{c}4 \\
0 \\
0 \\
0 \\
0.3\end{array}$ & $\begin{array}{l}\frac{5}{\underline{0}} \\
\underline{0.4} \\
\underline{0.6} \\
\underline{0.3}\end{array}$ & \\
\hline $\begin{array}{l}\text { High } \\
\text { Velocity } \\
\text { Region }\end{array}$ & $\begin{array}{c}12 \\
0 \\
0 \\
0 \\
0.3\end{array}$ & $\begin{array}{c}\frac{13}{0} \\
\underline{0} \\
\underline{0.2}\end{array}$ & $\frac{14}{\underline{0}} \frac{0.3}{\underline{0}}$ & $\begin{array}{r}15 \\
0 \\
0 \\
0 \\
0\end{array}$ & $\begin{array}{c}26 \\
0 \\
0 \\
0 \\
1.4 \\
-\end{array}$ & $\begin{array}{c}27 \\
0 \\
0 \\
0.5 \\
0 \\
0\end{array}$ & $\begin{array}{c}30 \\
0 \\
0 \\
0 \\
0.5 \\
0.2\end{array}$ & $\begin{array}{l}31 \\
0 \\
0 \\
0 \\
0 \\
0\end{array}$ & $\begin{array}{l}\frac{32}{\underline{0}} \\
\frac{0}{1.0} \\
\underline{0}\end{array}$ & $\frac{\frac{41}{0.1}}{\frac{0}{0}}$ & $\begin{array}{l}\frac{42}{0} \\
\frac{0.9}{0.5} \\
\underline{0}\end{array}$ & $\begin{array}{c}43 \\
0 \\
0 \\
0 \\
0.4 \\
0.5\end{array}$ & $\frac{\frac{44}{0.1}}{\frac{0.3}{\underline{0}}}$ & & $\begin{array}{l}\frac{55}{\underline{0}} \\
\underline{0} \\
\underline{0} \\
\underline{0}\end{array}$ & & & & & & & $\frac{\frac{2}{0.2}}{\frac{0.3}{0.7}} \frac{0}{\underline{0}}$ & $\begin{array}{l}3 \\
0 \\
0.3 \\
0.4 \\
0.8\end{array}$ & \\
\hline $\begin{array}{l}\text { Ring } \\
\text { Core }\end{array}$ & $\begin{array}{l}\frac{10}{\underline{0}} \\
\underline{0} \\
\underline{0} \\
\underline{0}\end{array}$ & $\begin{array}{r}11 \\
0 \\
0 \\
0 \\
0\end{array}$ & $\begin{array}{r}16 \\
0 \\
0 \\
0\end{array}$ & $\begin{array}{c}\frac{17}{\underline{0}} \\
\underline{0} \\
\underline{0.3}\end{array}$ & $\begin{array}{l}\frac{21}{\underline{0}} \\
\underline{0} \\
\underline{0} \\
\frac{0.4}{\underline{0}}\end{array}$ & $\begin{array}{l}\frac{23}{\underline{0}} \\
\underline{0} \\
\frac{1.5}{\underline{0}} \\
\underline{0}\end{array}$ & $\begin{array}{c}24 \\
0 \\
0 \\
0.3 \\
0.6 \\
0\end{array}$ & $\begin{array}{c}28 \\
0 \\
0 \\
0 \\
0 \\
0.3\end{array}$ & & & & & & & $\begin{array}{l}\frac{56}{\underline{0}} \\
\underline{0} \\
\frac{0}{0.5} \\
\underline{0}\end{array}$ & $\begin{array}{l}\frac{57}{\underline{0}} \\
\underline{0} \\
\underline{0} \\
\underline{0.9} \\
\underline{0.8}\end{array}$ & $\begin{array}{c}58 \\
0 \\
0 \\
0 \\
0.7 \\
0\end{array}$ & $\begin{array}{l}\frac{61}{\underline{0}} \\
\underline{0} \\
\underline{0} \\
\underline{0.4} \\
\underline{0.3} \\
\end{array}$ & $\begin{array}{c}62 \\
0 \\
0 \\
0 \\
1.6 \\
1.1\end{array}$ & $\begin{array}{l}\frac{63}{\underline{0}} \\
\underline{0} \\
\underline{0} \\
\frac{0.8}{\underline{0}}\end{array}$ & $\begin{array}{c}64 \\
0 \\
0 \\
0 \\
0 \\
0.4\end{array}$ & $\begin{array}{l}\frac{1}{\underline{0}} \\
\frac{0.2}{0.3} \\
\underline{0}\end{array}$ & & \\
\hline $\begin{array}{l}\text { Sargasso } \\
\text { Sea } \\
\& \\
\text { Gulf } \\
\text { Stream }\end{array}$ & & & & & & & & & & $\begin{array}{l}\frac{49}{\underline{0}} \\
\underline{0} \\
\underline{0.7} \\
\underline{0.3} \\
\end{array}$ & $\begin{array}{c}50 \\
0 \\
0 \\
0.7 \\
0.3\end{array}$ & $\begin{array}{c}51 \\
0 \\
0 \\
0.9 \\
0\end{array}$ & $\begin{array}{l}\frac{52}{0} \\
\underline{0} \\
\underline{0} \\
\underline{0}\end{array}$ & & & & & & & & & & & \\
\hline $\begin{array}{l}\text { Slope } \\
\text { Water }\end{array}$ & $\frac{8}{-}$ & $\frac{9}{0}$ & $\frac{18}{0}$ & & $\frac{19}{0.4}$ & $\frac{20}{0.1}$ & $\frac{33}{0.1}$ & $\frac{34}{0}$ & & $\frac{35}{0.2}$ & $\frac{47}{0.1}$ & $\frac{48}{0.1}$ & $\frac{53}{0.2}$ & $\frac{54}{0}$ & $\frac{59}{0.1}$ & $\frac{60}{0}$ & $\frac{65}{0.2}$ & $\frac{66}{0}$ & & & & $\frac{4}{0.1}$ & $\frac{5}{0.3}$ & $\frac{6}{0.2}$ \\
\hline $\begin{array}{l}\text { High } \\
\text { Velocity } \\
\text { Region }\end{array}$ & $\frac{12}{0.1}$ & $\frac{13}{0.1}$ & $\frac{14}{0.1}$ & $\frac{15}{0}$ & $\frac{27}{0.1}$ & $\frac{29}{0.1}$ & $\frac{30}{0.2}$ & $\frac{31}{0}$ & $\frac{32}{-}$ & $\frac{41}{0.1}$ & $\frac{42}{0.1}$ & $\frac{43}{0.2}$ & $\frac{44}{0.3}$ & & $\frac{55}{0}$ & & & & & & & $\frac{2}{0.3}$ & $\frac{3}{0.4}$ & \\
\hline $\begin{array}{l}\text { Ring } \\
\text { Core }\end{array}$ & $\frac{10}{0}$ & $\frac{11}{0}$ & $\frac{16}{-}$ & $\frac{17}{0.2}$ & $\frac{21}{0.1}$ & $\frac{23}{0.3}$ & $\frac{24}{0.2}$ & $\frac{28}{0}$ & & $\frac{37}{0}$ & $\frac{38}{0}$ & $\frac{39}{0.1}$ & & & $\frac{56}{0.1}$ & $\frac{57}{0.4}$ & $\frac{58}{0.1}$ & $\frac{61}{0.1}$ & $\frac{62}{0.5}$ & $\frac{63}{0.2}$ & $\frac{64}{0.1}$ & $\frac{1}{0.1}$ & & \\
\hline SS \& GS & & & & & & & & & & $\frac{49}{0.2}$ & $\frac{50}{0.3}$ & $\frac{51}{0.2}$ & $\frac{52}{0}$ & & & & & & & & & & & \\
\hline
\end{tabular}


Table 121. Volume of Serrivomer beanii $\left(\mathrm{ml} / 10,000 \mathrm{~m}^{3}\right)$.

\begin{tabular}{|c|c|c|c|c|c|c|c|c|c|c|c|c|c|c|c|c|c|c|c|c|c|c|c|c|}
\hline & \multicolumn{4}{|c|}{$\begin{array}{c}\text { Oceanus } 118 \\
\text { April }\end{array}$} & \multicolumn{5}{|c|}{$\begin{array}{c}\text { Oceanus } 121 \\
\text { June }\end{array}$} & \multicolumn{5}{|c|}{$\begin{array}{l}\text { Oceanus } 125 \\
\text { Auqust }\end{array}$} & \multicolumn{7}{|c|}{$\begin{array}{l}\text { Knorr } 98 \\
\text { Sept-0ct }\end{array}$} & \multicolumn{3}{|c|}{$\begin{array}{c}\text { A2 } 110 \\
\text { Sept-0ct }\end{array}$} \\
\hline $\begin{array}{l}\text { Slope } \\
\text { Water }\end{array}$ & $\begin{array}{l}\frac{8}{\overline{\overline{0}}} \\
\underline{0} \\
\underline{0} .68\end{array}$ & $\begin{array}{l}\frac{9}{0} \\
0 \\
0 \\
0\end{array}$ & & & $\begin{array}{c}\frac{19}{\frac{0}{0}} \\
\frac{0}{0} \\
24.74 \\
7.63 \\
\end{array}$ & $\begin{array}{l}20 \\
0 \\
0 \\
0.52 \\
0 \\
0\end{array}$ & $\begin{array}{l}\frac{33}{0} \\
\underline{0} \\
\underline{0} \\
\underline{0} \\
\underline{0}\end{array}$ & $\begin{array}{l}\frac{34}{0} \\
\underline{0} \\
\underline{0}\end{array}$ & & $\begin{array}{c}\frac{35}{0} \\
0 \\
0.33 \\
0 \\
0\end{array}$ & $\begin{array}{l}\frac{47}{\underline{0}} \\
\underline{0} \\
\underline{0.88} \\
\underline{0.89}\end{array}$ & $\begin{array}{c}\frac{48}{0.25} \\
0 \\
0 \\
2.04\end{array}$ & $\frac{\frac{53}{0.04}}{\frac{0.24}{0.03}} \frac{\underline{0}}{\frac{0.03}{4}}$ & $\begin{array}{l}54 \\
0 \\
0 \\
0 \\
0\end{array}$ & $\begin{array}{c}\frac{59}{\underline{0}} \\
\underline{0} \\
\underline{0} \\
17.24 \\
\underline{0.80}\end{array}$ & $\begin{array}{l}60 \\
0 \\
0 \\
0 \\
0 \\
0\end{array}$ & $\frac{\frac{65}{0.04}}{\frac{0.09}{0.09}}$ & $\begin{array}{l}66 \\
0 \\
0 \\
0 \\
0 \\
0\end{array}$ & & & & $\begin{array}{l}\frac{4}{0} \\
0 \\
0 \\
0.35\end{array}$ & $\begin{array}{r}\frac{5}{\underline{0}} \\
\frac{12.96}{0.63} \\
16.67 \\
\end{array}$ & \\
\hline $\begin{array}{l}\text { High } \\
\text { Velocity } \\
\text { Region }\end{array}$ & $\begin{array}{l}\frac{12}{0} \\
0 \\
0 \\
1.03\end{array}$ & $\begin{array}{l}\frac{13}{\underline{0}} \\
\underline{0} \\
0.20 \\
\end{array}$ & $\begin{array}{l}\frac{14}{\underline{0}} \\
\frac{0.03}{\underline{0}} \\
\underline{0}\end{array}$ & $\begin{array}{l}15 \\
0 \\
0 \\
0 \\
0\end{array}$ & $\begin{array}{l}26 \\
0 \\
0 \\
0 \\
7.24 \\
-\end{array}$ & $\begin{array}{l}27 \\
0 \\
0 \\
0.05 \\
0 \\
0\end{array}$ & $\begin{array}{l}30 \\
0 \\
0 \\
0 \\
0.59 \\
2.56\end{array}$ & $\begin{array}{l}31 \\
0 \\
0 \\
0.14 \\
0 \\
0\end{array}$ & $\begin{array}{l}\frac{32}{\underline{0}} \\
\underline{0} \\
\frac{3.50}{\underline{0}}\end{array}$ & $\frac{\frac{41}{1.56}}{\frac{0}{0}}$ & $\begin{array}{l}\frac{42}{\underline{0}} \\
\frac{0.44}{7.80} \\
\underline{0}\end{array}$ & $\begin{array}{l}\frac{43}{0} \\
0 \\
0 \\
4.35 \\
0.10\end{array}$ & $\frac{\frac{44}{1.48}}{\frac{19.00}{0}}$ & & $\begin{array}{l}\frac{55}{0} \\
\underline{0} \\
\underline{0} \\
\underline{0}\end{array}$ & & & & & & & $\frac{\frac{2}{0.02}}{\frac{0.05}{0.07}} \frac{\underline{0}}{\underline{0}}$ & $\begin{array}{c}\frac{3}{0} \\
0.03 \\
0.18 \\
12.31\end{array}$ & \\
\hline $\begin{array}{l}\text { Ring } \\
\text { Core }\end{array}$ & $\begin{array}{l}10 \\
\frac{0}{0} \\
\underline{0} \\
\underline{0} \\
\underline{0}\end{array}$ & $\begin{array}{l}11 \\
0 \\
0 \\
0 \\
0\end{array}$ & $\begin{array}{l}\frac{16}{16} \\
0 \\
0\end{array}$ & $\begin{array}{l}\frac{17}{\underline{0}} \\
\underline{0} \\
0.27\end{array}$ & $\begin{array}{c}\frac{21}{\underline{0}} \\
\underline{0} \\
\underline{0} \\
\frac{0.21}{\underline{0}}\end{array}$ & $\begin{array}{l}\frac{23}{\underline{0}} \\
\underline{0} \\
\frac{0.25}{\underline{0}} \\
\underline{0}\end{array}$ & $\begin{array}{c}24 \\
0 \\
0 \\
0.03 \\
0.19 \\
0\end{array}$ & $\begin{array}{l}28 \\
0 \\
0 \\
0 \\
0 \\
0.26\end{array}$ & & & & & & & $\begin{array}{l}\frac{56}{\underline{0}} \\
\underline{0} \\
\underline{0} \\
\frac{0.37}{\underline{0}}\end{array}$ & $\begin{array}{l}\frac{57}{\underline{0}} \\
\underline{0} \\
\underline{0} \\
\underline{0.19} \\
\underline{0.32}\end{array}$ & $\begin{array}{l}58 \\
0 \\
0 \\
0 \\
0.08 \\
0\end{array}$ & $\begin{array}{l}\frac{61}{\underline{0}} \\
\underline{0} \\
\underline{0} \\
\underline{0.38} \\
\underline{0.05}\end{array}$ & $\begin{array}{l}\frac{62}{0} \\
0 \\
0 \\
0.32 \\
0.21\end{array}$ & $\begin{array}{l}\frac{63}{\underline{0}} \\
\underline{0} \\
\underline{0} \\
\underline{0.19} \\
\underline{0}\end{array}$ & $\begin{array}{l}\frac{64}{0} \\
0 \\
0 \\
0 \\
0.27\end{array}$ & $\begin{array}{l}\frac{1}{\underline{0}} \\
\frac{0.04}{0.06} \\
\underline{0}\end{array}$ & & \\
\hline $\begin{array}{l}\text { Sargasso } \\
\text { Sea } \\
\& \\
\text { Gulf } \\
\text { Stream }\end{array}$ & & & & & & & & & & $\begin{array}{l}\frac{49}{\underline{0}} \\
\underline{0} \\
0.07 \\
\underline{0.05}\end{array}$ & $\begin{array}{l}\frac{50}{0} \\
0 \\
0.03 \\
0.03\end{array}$ & $\begin{array}{c}\frac{51}{0} \\
0 \\
0.20 \\
0\end{array}$ & $\begin{array}{l}\frac{52}{\underline{0}} \\
\underline{0} \\
\underline{0} \\
\underline{0}\end{array}$ & & & & & & & & & & & \\
\hline $\begin{array}{l}\text { Slope } \\
\text { Water }\end{array}$ & $\frac{8}{-}$ & $\frac{9}{0}$ & $\frac{18}{0}$ & & $\frac{19}{8.09}$ & $\frac{20}{0.13}$ & $\frac{33}{0}$ & $\frac{34}{0}$ & & $\frac{35}{0.07}$ & $\frac{47}{0.44}$ & $\frac{48}{0.57}$ & $\frac{53}{0.08}$ & $\frac{54}{0}$ & $\frac{59}{3.61}$ & $\frac{60}{0}$ & $\frac{65}{0.03}$ & $\frac{66}{0}$ & & & & $\frac{4}{0.09}$ & $\frac{5}{7.57}$ & $\frac{6}{0.40}$ \\
\hline $\begin{array}{l}\text { High } \\
\text { Velocity } \\
\text { Region }\end{array}$ & $\frac{12}{0.26}$ & $\frac{13}{0.10}$ & $\frac{14}{0.01}$ & $\frac{15}{0}$ & $\frac{27}{0.01}$ & $\frac{29}{0.55}$ & $\frac{30}{0.63}$ & $\frac{31}{0.03}$ & $\frac{32}{-}$ & $\frac{41}{0.78}$ & $\frac{42}{1.66}$ & $\frac{43}{0.89}$ & $\frac{44}{4.69}$ & & $\frac{55}{0}$ & & & & & & & $\frac{2}{0.04}$ & $\frac{3}{3.18}$ & \\
\hline $\begin{array}{l}\text { Ring } \\
\text { Core }\end{array}$ & $\frac{10}{0}$ & $\frac{11}{0}$ & $\frac{16}{-}$ & $\frac{17}{0.14}$ & $\frac{21}{0.04}$ & $\frac{23}{0.05}$ & $\frac{24}{0.04}$ & $\frac{28}{0}$ & & $\frac{37}{0}$ & $\frac{38}{0}$ & $\frac{39}{1.41}$ & & & $\frac{56}{0.07}$ & $\frac{57}{0.10}$ & $\frac{58}{0.02}$ & $\frac{61}{0.09}$ & $\frac{62}{0.11}$ & $\frac{63}{0.04}$ & $\frac{64}{0.05}$ & $\frac{1}{0.03}$ & & \\
\hline SS \& GS & & & & & & & & & & $\frac{49}{0.03}$ & $\frac{50}{0.02}$ & $\frac{51}{0.05}$ & $\frac{52}{0}$ & & & & & & & & & & & \\
\hline
\end{tabular}


Table 122. Abundance of Sternoptyx diaphana (specimens $/ 10,000 \mathrm{~m}^{3}$ ).

\begin{tabular}{|c|c|c|c|c|c|c|c|c|c|c|c|c|c|c|c|c|c|c|c|c|c|c|c|c|}
\hline & \multicolumn{4}{|c|}{$\begin{array}{c}\text { Oceanus } \\
\text { April }\end{array}$} & \multicolumn{5}{|c|}{$\begin{array}{c}\text { Oceanus } 121 \\
\text { June }\end{array}$} & \multicolumn{5}{|c|}{$\begin{array}{c}\text { Oceanus } 125 \\
\text { August }\end{array}$} & \multicolumn{7}{|c|}{$\begin{array}{l}\text { Knorr } 98 \\
\text { Sept-0ct }\end{array}$} & \multicolumn{3}{|c|}{$\begin{array}{c}\text { A2 } 110 \\
\text { Sept-Oct }\end{array}$} \\
\hline $\begin{array}{l}\text { Slope } \\
\text { Water }\end{array}$ & $\frac{8}{\overline{\overline{0}}}$ & $\begin{array}{c}9 \\
0 \\
0 \\
2.1 \\
0\end{array}$ & & & $\begin{array}{l}\frac{19}{\underline{0}} \\
\underline{0} \\
\underline{0} \\
\underline{\underline{0} .1} \\
\underline{0.5}\end{array}$ & $\begin{array}{c}20 \\
0 \\
0 \\
0.9 \\
0.5 \\
0\end{array}$ & $\begin{array}{c}\frac{33}{0} \\
\frac{0}{0} \\
\underline{0} \\
\underline{0} \\
\underline{0,6}\end{array}$ & $\begin{array}{l}\frac{34}{\underline{0}} \\
\underline{0} \\
\underline{0.9}\end{array}$ & & $\begin{array}{c}\frac{35}{1.0} \\
0 \\
2.3 \\
5.6 \\
0.7\end{array}$ & $\begin{array}{c}\frac{47}{\underline{0}} \\
\underline{0} \\
0.6 \\
0.4\end{array}$ & $\begin{array}{c}\frac{48}{0} \\
0.3 \\
1.5 \\
0\end{array}$ & $\begin{array}{l}\frac{53}{\underline{0}} \\
\underline{0} \\
\underline{2.8} \\
\underline{0.2}\end{array}$ & $\begin{array}{l}54 \\
0 \\
0.3 \\
2.4 \\
0.3\end{array}$ & $\begin{array}{l}\frac{59}{\underline{0}} \\
\underline{0} \\
\frac{0.3}{\underline{0}} \\
\underline{0}\end{array}$ & $\begin{array}{r}60 \\
0 \\
0 \\
0 \\
0 \\
0\end{array}$ & $\frac{\frac{65}{0}}{\frac{0.3}{0.0}} \frac{10}{\underline{3.2}}$ & $\begin{array}{c}66 \\
0 \\
0 \\
5.2 \\
1.9 \\
0\end{array}$ & & & & $\begin{array}{l}\frac{4}{0} \\
0 \\
4.1 \\
0.3\end{array}$ & $\begin{array}{l}\frac{5}{\underline{0}} \\
\frac{1.1}{0.6} \\
\underline{0}\end{array}$ & \\
\hline $\begin{array}{l}\text { High } \\
\text { Velocity } \\
\text { Region }\end{array}$ & $\begin{array}{l}\frac{12}{0} \\
0 \\
0.8 \\
0.3\end{array}$ & 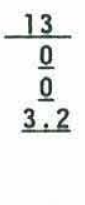 & $\begin{array}{l}\frac{14}{\underline{0}} \\
\underline{0} \\
\frac{0.6}{\underline{0}}\end{array}$ & $\begin{array}{c}15 \\
0 \\
0 \\
1.8 \\
0\end{array}$ & $\begin{array}{c}26 \\
0 \\
0 \\
0 \\
1.4 \\
-\end{array}$ & $\begin{array}{c}27 \\
0 \\
0 \\
0 \\
2.4 \\
1.9\end{array}$ & $\begin{array}{c}30 \\
0 \\
0 \\
0 \\
1.6 \\
0.5\end{array}$ & $\begin{array}{c}31 \\
0 \\
0 \\
0 \\
0.6 \\
0\end{array}$ & $\begin{array}{c}\frac{32}{\underline{0}} \\
\underline{0} \\
1.0 \\
\underline{0.4}\end{array}$ & $\begin{array}{l}\frac{41}{\underline{0}} \\
\underline{2.6} \\
\underline{0.2}\end{array}$ & $\frac{\frac{42}{\underline{0}}}{\frac{1.8}{2.7}} \frac{\underline{0}}{\underline{1.8}}$ & $\begin{array}{c}\frac{43}{0} \\
\frac{0.2}{0} \\
\frac{1.3}{0}\end{array}$ & $\begin{array}{c}\frac{44}{0.2} \\
1.7 \\
\underline{0}\end{array}$ & & $\begin{array}{l}\frac{55}{\underline{0}} \\
\underline{0} \\
\underline{6.4}\end{array}$ & & & & & & & $\begin{array}{l}\frac{2}{\underline{0}} \\
\frac{1.1}{6.7} \\
\underline{0}\end{array}$ & $\begin{array}{c}\frac{3}{0.4} \\
2.4 \\
6.8 \\
0\end{array}$ & \\
\hline $\begin{array}{l}\text { Ring } \\
\text { Core }\end{array}$ & $\begin{array}{l}\frac{10}{\underline{0}} \\
\underline{\underline{0}} \\
\frac{\underline{0} .6}{\underline{0}}\end{array}$ & $\begin{array}{l}11 \\
0 \\
0 \\
3.1 \\
1.4\end{array}$ & $\begin{array}{c}\frac{16}{0} \\
0 \\
10.7\end{array}$ & $\begin{array}{r}17 \\
\underline{0} \\
\underline{0} \\
\underline{4.8}\end{array}$ & $\begin{array}{l}\frac{21}{\underline{0}} \\
\underline{0} \\
\underline{0.3} \\
\underline{1.7} \\
\underline{0.6}\end{array}$ & $\begin{array}{c}\frac{23}{\underline{0}} \\
\underline{0} \\
\underline{0} \\
\underline{0.9} \\
\underline{0}\end{array}$ & $\begin{array}{c}24 \\
0 \\
0 \\
0 \\
2.5 \\
0\end{array}$ & $\begin{array}{c}28 \\
0 \\
0 \\
1.8 \\
0.4 \\
0\end{array}$ & & & & & & & $\begin{array}{c}\frac{56}{\underline{0}} \\
\frac{0.6}{\underline{0}} \\
11.6 \\
\underline{4.7}\end{array}$ & $\begin{array}{c}\frac{57}{\underline{0}} \\
\underline{0} \underline{0} \\
10.9 \\
\underline{7.4}\end{array}$ & $\begin{array}{r}\frac{58}{0.5} \\
0.4 \\
1.4 \\
13.5 \\
6.8\end{array}$ & $\begin{array}{r}\frac{61}{\underline{0}} \\
\underline{0} \\
\frac{0.7}{2.7} \\
1.3 \\
\end{array}$ & $\begin{array}{c}\frac{62}{0.4} \\
0 \\
0.8 \\
9.5 \\
16.1\end{array}$ & $\begin{array}{l}\frac{63}{\underline{0}} \\
\frac{0.2}{1.0} \\
\frac{9.2}{4.7} \\
\end{array}$ & $\begin{array}{c}64 \\
0 \\
0 \\
0 \\
10.5 \\
0.8\end{array}$ & $\begin{array}{r}\frac{1}{\underline{0}} \\
\frac{0.2}{18.4} \\
\underline{3.3}\end{array}$ & & \\
\hline $\begin{array}{l}\text { Sargasso } \\
\text { Sea } \\
\& \\
\text { Gulf } \\
\text { Stream }\end{array}$ & & & & & & & & & & $\begin{array}{r}\frac{49}{0} \\
\underline{0} \\
\underline{2.2} \\
\underline{23.1} \\
\end{array}$ & $\begin{array}{c}50 \\
0 \\
0 \\
3.4 \\
15.9\end{array}$ & $\begin{array}{c}51 \\
0 \\
0 \\
6.1 \\
0\end{array}$ & $\begin{array}{c}\frac{52}{\underline{0}} \\
\underline{0} \\
16.1 \\
\underline{0.5}\end{array}$ & & & & & & & & & & & \\
\hline $\begin{array}{l}\text { Slope } \\
\text { Water }\end{array}$ & $\frac{8}{0.6}$ & $\frac{9}{0.5}$ & $\frac{18}{1.0}$ & & $\frac{19}{0.7}$ & $\frac{20}{0.4}$ & $\frac{33}{0.3}$ & $\frac{34}{0.5}$ & & $\frac{35}{1.9}$ & $\frac{47}{0.3}$ & $\frac{48}{0.5}$ & $\frac{53}{0.8}$ & $\frac{54}{0.8}$ & $\frac{59}{0.1}$ & $\frac{60}{0}$ & $\frac{65}{2.7}$ & $\frac{66}{1.4}$ & & & & $\frac{4}{1.1}$ & $\frac{5}{0.4}$ & $\frac{6}{0.1}$ \\
\hline $\begin{array}{l}\text { High } \\
\text { Velocity } \\
\text { Region }\end{array}$ & $\frac{12}{0.3}$ & $\frac{13}{1.6}$ & $\frac{14}{0.2}$ & $\frac{15}{0.5}$ & $\frac{27}{1.1}$ & $\frac{29}{0.3}$ & $\frac{30}{0.4}$ & $\frac{31}{0.1}$ & $\frac{32}{0.3}$ & $\frac{41}{0.7}$ & $\frac{42}{1.6}$ & $\frac{43}{0.3}$ & $\frac{44}{0.5}$ & & $\frac{55}{2.6}$ & & & & & & & $\frac{2}{2.0}$ & $\frac{3}{2.4}$ & \\
\hline $\begin{array}{l}\text { Ring } \\
\text { Core }\end{array}$ & $\frac{10}{0.9}$ & $\frac{11}{1.1}$ & $\frac{16}{-}$ & $\frac{17}{2.4}$ & $\frac{21}{0.5}$ & $\frac{23}{0.2}$ & $\frac{24}{0.5}$ & $\frac{28}{0.6}$ & & $\frac{37}{0.2}$ & $\frac{38}{0.4}$ & $\frac{39}{0.4}$ & & & $\frac{56}{3.4}$ & $\frac{57}{3.7}$ & $\frac{58}{4.5}$ & $\frac{61}{1.1}$ & $\frac{62}{5.4}$ & $\frac{63}{3.0}$ & $\frac{64}{2.3}$ & $\frac{1}{5.5}$ & & \\
\hline SS \& GS & & & & & & & & & & $\frac{49}{6.3}$ & $\frac{50}{4.8}$ & $\frac{51}{1.5}$ & $\frac{52}{4.2}$ & & & & & & & & & & & \\
\hline
\end{tabular}


Table 123. Volume of Sternoptyx diaphana $\left(\mathrm{ml} / 10,000 \mathrm{~m}^{3}\right)$.

\begin{tabular}{|c|c|c|c|c|c|c|c|c|c|c|c|c|c|c|c|c|c|c|c|c|c|c|c|c|}
\hline & \multicolumn{4}{|c|}{$\begin{array}{c}\text { Oceanus } \\
\text { April }\end{array}$} & \multicolumn{5}{|c|}{$\begin{array}{c}\text { Oceanus } 121 \\
\text { June }\end{array}$} & \multicolumn{5}{|c|}{$\begin{array}{c}\text { Oceanus } 125 \\
\text { August }\end{array}$} & \multicolumn{7}{|c|}{$\begin{array}{l}\text { Knorr } 98 \\
\text { Sept-0ct }\end{array}$} & \multicolumn{3}{|c|}{$\begin{array}{c}\text { A2 } 110 \\
\text { Sept-0ct }\end{array}$} \\
\hline $\begin{array}{l}\text { Slope } \\
\text { Water }\end{array}$ & $\begin{array}{l}\frac{8}{\overline{\overline{0}}} \\
\underline{\underline{0}} \\
\underline{0.23}\end{array}$ & $\begin{array}{l}\frac{9}{0} \\
0 \\
1.14 \\
0\end{array}$ & & & $\begin{array}{l}\frac{19}{\underline{0}} \\
\underline{0} \\
\underline{0} \\
\underline{0.21} \\
\underline{0.05}\end{array}$ & $\begin{array}{l}20 \\
0 \\
0 \\
0.48 \\
0.05 \\
0\end{array}$ & $\begin{array}{l}\frac{33}{\underline{0}} \\
\frac{0}{0} \\
\underline{0} \\
\underline{0.70}\end{array}$ & $\begin{array}{l}\frac{34}{\underline{0}} \\
\underline{0} \\
\underline{0.20} \\
\end{array}$ & & $\begin{array}{c}\frac{35}{0.10} \\
0 \\
0.20 \\
1.86 \\
0.57\end{array}$ & $\begin{array}{l}\frac{47}{\underline{0}} \\
\underline{0} \\
\underline{0.41} \\
\underline{0.23}\end{array}$ & $\begin{array}{c}\frac{48}{0} \\
0.14 \\
0.25 \\
0\end{array}$ & $\begin{array}{l}\frac{53}{\underline{0}} \\
\underline{0} \\
\underline{0.26} \\
0.22 \\
\end{array}$ & $\begin{array}{l}\frac{54}{0} \\
0.03 \\
0.64 \\
0.30\end{array}$ & $\begin{array}{l}\frac{59}{\underline{0}} \\
\underline{0} \\
\frac{0.03}{\underline{0}} \\
\underline{0}\end{array}$ & $\begin{array}{l}60 \\
0 \\
0 \\
0 \\
0 \\
0\end{array}$ & $\begin{array}{l}\frac{65}{0} \\
\underline{0.03} \\
\frac{1.14}{0.17} \\
\underline{0.02}\end{array}$ & $\begin{array}{c}\frac{66}{0} \\
0.96 \\
0 \\
0.24 \\
0\end{array}$ & & & & $\begin{array}{c}\frac{4}{0} \\
0 \\
1.19 \\
0.09\end{array}$ & $\frac{\frac{5}{\underline{0}}}{\frac{0.44}{1.00}}$ & \\
\hline $\begin{array}{l}\text { High } \\
\text { Velocity } \\
\text { Region }\end{array}$ & $\begin{array}{l}\frac{12}{0} \\
0 \\
0.42 \\
0.03\end{array}$ & $\begin{array}{l}\frac{13}{\underline{0}} \\
\underline{0} \\
1.02\end{array}$ & $\begin{array}{l}\frac{14}{\underline{0}} \\
\underline{0} \\
\frac{0.59}{\underline{0}}\end{array}$ & $\begin{array}{c}\frac{15}{0} \\
0 \\
0.29 \\
0\end{array}$ & $\begin{array}{l}26 \\
0 \\
0 \\
0 \\
0.55 \\
-\end{array}$ & $\begin{array}{l}\frac{27}{0} \\
0 \\
0 \\
2.54 \\
0.70\end{array}$ & $\begin{array}{l}30 \\
0 \\
0 \\
0 \\
0.84 \\
0.58\end{array}$ & $\begin{array}{l}\frac{31}{0} \\
0 \\
0 \\
0.19 \\
0\end{array}$ & $\begin{array}{l}\frac{32}{\underline{0}} \\
\underline{0} \\
0.55 \\
0.09 \\
\end{array}$ & $\begin{array}{l}\frac{41}{\underline{0}} \\
\frac{1.49}{0.11}\end{array}$ & $\frac{\frac{42}{\underline{0}}}{\frac{0.21}{1.49}} \frac{\underline{0}}{}$ & $\begin{array}{c}\frac{43}{0} \\
0.02 \\
0 \\
0.11 \\
0\end{array}$ & $\frac{\frac{44}{0.02}}{\frac{0.22}{\underline{0}}}$ & & $\begin{array}{l}\frac{55}{\underline{0}} \\
\frac{0.16}{\underline{0}}\end{array}$ & & & & & & & $\begin{array}{l}\frac{2}{\underline{0}} \\
\frac{0.05}{1.80} \\
\underline{0}\end{array}$ & $\begin{array}{c}\frac{3}{0.04} \\
0.05 \\
1.25 \\
0\end{array}$ & \\
\hline $\begin{array}{l}\text { Ring } \\
\text { Core }\end{array}$ & $\begin{array}{l}\frac{10}{\underline{0}} \\
\underline{0} \\
\frac{0.45}{\underline{0}}\end{array}$ & $\begin{array}{l}\frac{11}{0} \\
0 \\
0.50 \\
0.75\end{array}$ & $\begin{array}{l}\frac{16}{0} \\
0 \\
4.73\end{array}$ & $\begin{array}{l}\frac{17}{\underline{0}} \\
\underline{\underline{0}} \\
\underline{2.58}\end{array}$ & $\begin{array}{l}\frac{21}{\underline{0}} \\
\underline{0.06} \\
\underline{0.30} \\
\underline{0.38} \\
\underline{0.06}\end{array}$ & $\begin{array}{l}\frac{23}{\underline{0}} \\
\underline{0} \\
\frac{0.65}{0.13} \\
\frac{0}{\underline{0}}\end{array}$ & $\begin{array}{l}\frac{24}{0} \\
0 \\
0 \\
0.63 \\
0\end{array}$ & $\begin{array}{l}28 \\
0 \\
0 \\
0.50 \\
0.31 \\
0\end{array}$ & & . & & & & & $\begin{array}{l}\frac{56}{\underline{0}} \\
\underline{0.06} \\
\underline{0} \\
\underline{0.11} \\
\underline{0.88}\end{array}$ & $\begin{array}{l}\frac{57}{\underline{0}} \\
\underline{0} \\
\underline{0} \\
\underline{0.16} \\
\underline{2.53}\end{array}$ & $\begin{array}{l}\frac{58}{0.05} \\
0.04 \\
0.05 \\
0.19 \\
1.74\end{array}$ & $\begin{array}{l}\frac{61}{\underline{0}} \\
\underline{0} \\
\underline{0.19} \\
\underline{0.85} \\
\underline{0.53}\end{array}$ & $\begin{array}{c}\frac{62}{0.02} \\
0 \\
0.02 \\
0.30 \\
2.93\end{array}$ & $\begin{array}{l}\frac{63}{\underline{0}} \\
\underline{0.02} \\
\underline{0.05} \\
\underline{0.20} \\
\underline{0.69}\end{array}$ & $\begin{array}{c}\frac{64}{0.02} \\
0 \\
0 \\
0.20 \\
0.12\end{array}$ & $\begin{array}{l}\frac{1}{\underline{0}} \\
\underline{0.02} \\
\underline{0.66} \\
\underline{2.25}\end{array}$ & & \\
\hline $\begin{array}{l}\text { Sargasso } \\
\text { Sea } \\
\& \\
\text { Gulf } \\
\text { Stream }\end{array}$ & & & & & & & & & & $\begin{array}{l}\frac{49}{\underline{0}} \\
\underline{0} \\
0.02 \\
1.23 \\
\end{array}$ & $\begin{array}{l}\frac{50}{0} \\
0 \\
0.06 \\
2.00\end{array}$ & $\begin{array}{l}51 \\
0 \\
0 \\
0.85 \\
0\end{array}$ & $\begin{array}{l}\frac{52}{\underline{0}} \\
\underline{0} \\
\underline{1.00} \\
\underline{0.36}\end{array}$ & & & & & & & & & & & \\
\hline $\begin{array}{l}\text { Slope } \\
\text { Water }\end{array}$ & $\frac{8}{-}$ & $\frac{9}{0.29}$ & $\frac{18}{0.07}$ & & $\frac{19}{0.07}$ & $\frac{20}{0.13}$ & $\frac{33}{0.35}$ & $\frac{34}{0.12}$ & & $\frac{35}{0.55}$ & $\frac{47}{0.16}$ & $\frac{48}{0.10}$ & $\frac{53}{0.12}$ & $\frac{54}{0.24}$ & $\frac{59}{0.01}$ & $\frac{60}{0}$ & $\frac{65}{0.27}$ & $\frac{66}{0.14}$ & 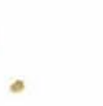 & & & $\frac{4}{0.32}$ & $\frac{5}{0.36}$ & $\frac{6}{0.32}$ \\
\hline $\begin{array}{l}\text { High } \\
\text { Velocity } \\
\text { Region }\end{array}$ & $\frac{12}{0.11}$ & $\frac{13}{0.26}$ & $\frac{14}{0.15}$ & $\frac{15}{0.07}$ & $\frac{27}{0.81}$ & $\frac{29}{0.01}$ & $\frac{30}{0.28}$ & $\frac{31}{0.04}$ & $\frac{32}{-}$ & $\frac{41}{0.40}$ & $\frac{42}{0.34}$ & $\frac{43}{0.02}$ & $\frac{44}{0.06}$ & & $\frac{55}{0.06}$ & & & & & & & $\frac{2}{0.46}$ & $\frac{3}{0.34}$ & \\
\hline $\begin{array}{l}\text { Ring } \\
\text { Core }\end{array}$ & $\frac{10}{0.11}$ & $\frac{11}{0.31}$ & $\frac{16}{-}$ & $\frac{17}{1.29}$ & $\frac{21}{0.17}$ & $\frac{23}{0.16}$ & $\frac{24}{0.13}$ & $\frac{28}{0.20}$ & & $\frac{37}{0.24}$ & $\frac{38}{0.05}$ & $\frac{39}{0.04}$ & & & $\frac{56}{0.21}$ & $\frac{57}{0.54}$ & $\frac{58}{0.42}$ & $\frac{61}{0.31}$ & $\frac{62}{0.66}$ & $\frac{63}{0.19}$ & $\frac{64}{0.07}$ & $\frac{1}{0.73}$ & & \\
\hline SS \& GS & & & & & & & & & & $\frac{49}{0.31}$ & $\frac{50}{0.52}$ & $\frac{51}{0.21}$ & $\frac{52}{0.34}$ & & & & & & & & & & & \\
\hline
\end{tabular}


Table 124. Abundance of Stomias boa ferox (specimens $/ 10,000 \mathrm{~m}^{3}$ ).

\begin{tabular}{|c|c|c|c|c|c|c|c|c|c|c|c|c|c|c|c|c|c|c|c|c|c|c|c|c|}
\hline & \multicolumn{4}{|c|}{ 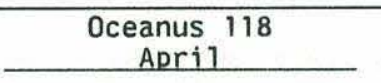 } & \multicolumn{5}{|c|}{$\begin{array}{c}\text { Oceanus } 121 \\
\text { June }\end{array}$} & \multicolumn{5}{|c|}{$\begin{array}{l}\text { Oceanus } 125 \\
\text { August }\end{array}$} & \multicolumn{7}{|c|}{$\begin{array}{l}\text { Knorr } 98 \\
\text { Sept-0ct }\end{array}$} & \multicolumn{3}{|c|}{$\begin{array}{c}\text { A2 } 110 \\
\text { Sept-Oct }\end{array}$} \\
\hline $\begin{array}{l}\text { Slope } \\
\text { Water }\end{array}$ & $\begin{array}{l}\frac{8}{\bar{z}} \\
\underline{0} \\
\underline{0} \\
\underline{0}\end{array}$ & $\begin{array}{l}\frac{9}{0} \\
0 \\
1.4 \\
0\end{array}$ & & & $\frac{\frac{19}{0.5}}{\frac{0.3}{0.0}}$ & $\begin{array}{r}20 \\
0 \\
0 \\
0 \\
0 \\
0\end{array}$ & $\begin{array}{c}\frac{33}{0.3} \\
\frac{0.8}{\underline{0}} \\
\underline{0} \\
0.1\end{array}$ & $\begin{array}{l}\frac{34}{\underline{0}} \\
\underline{0.4} \\
\underline{2.7}\end{array}$ & & $\begin{array}{c}35 \\
0 \\
0 \\
3.7 \\
1.1 \\
0\end{array}$ & $\begin{array}{l}\frac{47}{\underline{0.7}} \\
\frac{0.2}{\underline{0}}\end{array}$ & $\begin{array}{c}48 \\
0 \\
0.9 \\
1.2 \\
0.2\end{array}$ & $\frac{\frac{53}{0.4}}{\frac{0}{0}}$ & $\begin{array}{c}\frac{54}{0} \\
0 \\
2.1 \\
0.7\end{array}$ & $\begin{array}{c}\frac{59}{\underline{0}} \\
\underline{0} \\
\underline{0} \\
\underline{0.3} \\
\underline{0}\end{array}$ & $\begin{array}{c}60 \\
0 \\
0 \\
0 \\
1.3 \\
0\end{array}$ & 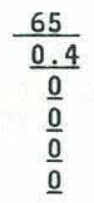 & $\begin{array}{c}66 \\
0 \\
0 \\
2.2 \\
0.5 \\
0\end{array}$ & & & & $\begin{array}{c}\frac{4}{0} \\
1.5 \\
1.3 \\
0\end{array}$ & $\begin{array}{l}\frac{5}{1.7} \\
\underline{0} \\
\frac{0.3}{\underline{0}}\end{array}$ & \\
\hline $\begin{array}{l}\text { High } \\
\text { Velocity } \\
\text { Region }\end{array}$ & $\begin{array}{c}12 \\
0 \\
0 \\
0.4 \\
0\end{array}$ & $\begin{array}{l}\frac{13}{\underline{0}} \\
\underline{0.5}\end{array}$ & $\frac{\frac{14}{0.3}}{\frac{0.7}{\underline{0}}}$ & $\begin{array}{c}15 \\
0 \\
1.2 \\
0 \\
0\end{array}$ & $\begin{array}{c}26 \\
0.3 \\
0 \\
0 \\
0 \\
-\end{array}$ & $\begin{array}{c}27 \\
0 \\
0 \\
2.7 \\
0 \\
0\end{array}$ & $\begin{array}{c}30 \\
0 \\
0 \\
11.6 \\
0.3 \\
0\end{array}$ & $\begin{array}{c}31 \\
0 \\
0 \\
3.6 \\
0.3 \\
0\end{array}$ & $\begin{array}{c}\frac{32}{\underline{0}} \\
\frac{0.8}{1.0} \\
\underline{0}\end{array}$ & $\frac{\frac{41}{0.1}}{\frac{0.9}{\underline{0}}}$ & $\frac{42}{\frac{42}{\underline{0}}} \frac{\underline{2.1}}{\underline{0}}$ & $\begin{array}{c}43 \\
0 \\
0 \\
2.9 \\
0 \\
0\end{array}$ & $\frac{\frac{44}{0.3}}{\underline{0}}$ & & $\begin{array}{l}55 \\
\underline{0} \\
\underline{0} \\
\underline{0}\end{array}$ & & & & & & & $\begin{array}{l}\frac{2}{\underline{0}} \\
\underline{0} \\
\frac{1,3}{\underline{0}}\end{array}$ & $\begin{array}{c}\frac{3}{0} \\
0.3 \\
0 \\
0\end{array}$ & \\
\hline $\begin{array}{l}\text { Ring } \\
\text { Core }\end{array}$ & $\begin{array}{l}\frac{10}{\underline{0}} \\
\underline{0} \\
\frac{0.9}{\underline{0}}\end{array}$ & $\begin{array}{r}11 \\
0 \\
0 \\
0 \\
0\end{array}$ & $\begin{array}{c}16 \\
0 \\
0.7 \\
0\end{array}$ & $\frac{17}{\underline{0}}$ & $\frac{\frac{21}{8.0}}{\frac{0.6}{17.3}} \frac{1.7}{\underline{0}}$ & $\frac{\frac{23}{1.2}}{\frac{1.3}{1.5}} \frac{\frac{9.5}{0.5}}{\underline{0}}$ & $\begin{array}{c}\frac{24}{0.3} \\
5.0 \\
33.8 \\
0.6 \\
0\end{array}$ & $\begin{array}{c}28 \\
0 \\
0 \\
16.8 \\
0 \\
0\end{array}$ & & & & & & & $\begin{array}{l}56 \\
\underline{0} \\
\underline{0} \\
\underline{0} \\
\underline{0} \\
\underline{0}\end{array}$ & $\begin{array}{r}57 \\
\underline{0} \\
\underline{0} \\
\underline{0} \\
\underline{0} \\
\underline{0}\end{array}$ & $\begin{array}{r}58 \\
0 \\
0 \\
0 \\
0 \\
0\end{array}$ & $\begin{array}{l}61 \\
\underline{0} \\
\underline{0} \\
\underline{0} \\
\underline{0} \\
\underline{0}\end{array}$ & $\begin{array}{r}62 \\
0 \\
0 \\
0 \\
0 \\
0\end{array}$ & $\begin{array}{r}63 \\
\underline{0} \\
\underline{0} \\
\underline{0} \\
\underline{0} \\
\underline{0}\end{array}$ & $\begin{array}{r}64 \\
0 \\
0 \\
0 \\
0 \\
0\end{array}$ & $\begin{array}{l}\frac{1}{\underline{0}} \\
\underline{0} \\
\underline{0} \\
\underline{0}\end{array}$ & & \\
\hline $\begin{array}{l}\text { Sargasso } \\
\text { Sea } \\
\& \\
\text { Gu1f } \\
\text { Stream }\end{array}$ & & & & & & & & & & $\begin{array}{l}\frac{49}{0} \\
\underline{0} \\
\underline{0} \\
\underline{0}\end{array}$ & $\begin{array}{r}50 \\
0 \\
0 \\
0 \\
0\end{array}$ & $\begin{array}{r}51 \\
0 \\
0 \\
0 \\
0\end{array}$ & $\begin{array}{l}\frac{52}{\underline{0}} \\
\underline{0} \\
\underline{0} \\
\underline{0.3}\end{array}$ & & & & & & & & & & & \\
\hline $\begin{array}{l}\text { Slope } \\
\text { Water }\end{array}$ & $\frac{8}{-}$ & $\frac{9}{0.4}$ & $\frac{18}{0.4}$ & & $\frac{19}{0.1}$ & $\frac{20}{0}$ & $\frac{33}{0.2}$ & $\frac{34}{1.8}$ & & $\frac{35}{1.0}$ & $\frac{47}{0.2}$ & $\frac{48}{0.6}$ & $\frac{53}{0.1}$ & $\frac{54}{0.7}$ & $\frac{59}{0.1}$ & $\frac{60}{0.3}$ & $\frac{65}{0.1}$ & $\frac{66}{0.5}$ & & & & $\frac{4}{0.7}$ & $\frac{5}{0.5}$ & $\frac{6}{0.5}$ \\
\hline $\begin{array}{l}\text { High } \\
\text { Velocity } \\
\text { Region }\end{array}$ & $\frac{12}{0.1}$ & $\frac{13}{0.3}$ & $\frac{14}{0.2}$ & $\frac{15}{0.3}$ & $\frac{27}{0.6}$ & $\frac{29}{1.0}$ & $\frac{30}{2.4}$ & $\frac{31}{0.8}$ & $\frac{32}{-}$ & $\frac{41}{0.3}$ & $\frac{42}{0.5}$ & $\frac{43}{0.6}$ & $\frac{44}{0.2}$ & & $\frac{55}{0}$ & & & & & & & $\frac{2}{0.3}$ & $\frac{3}{0.1}$ & \\
\hline $\begin{array}{l}\text { Ring } \\
\text { Core }\end{array}$ & $\frac{10}{0.2}$ & $\frac{11}{0}$ & $\frac{16}{-}$ & $\frac{17}{0}$ & $\frac{21}{4.4}$ & $\frac{23}{2.5}$ & $\frac{24}{7.1}$ & $\frac{28}{4.2}$ & & $\frac{37}{0}$ & $\frac{38}{0.2}$ & $\frac{39}{0.2}$ & & & $\frac{56}{0}$ & $\frac{57}{0}$ & $\frac{58}{0}$ & $\frac{61}{0}$ & $\frac{62}{0}$ & $\frac{63}{0}$ & $\frac{64}{0}$ & $\frac{1}{0}$ & & \\
\hline SS \& GS & & & & & & & & & & $\frac{49}{0}$ & $\frac{50}{0}$ & $\frac{51}{0}$ & $\frac{52}{0.1}$ & & & & & & & & & & & \\
\hline
\end{tabular}


Table 125. Volume of Stomias boa ferox $\left(\mathrm{ml} / 10,000 \mathrm{~m}^{3}\right)$.

\begin{tabular}{|c|c|c|c|c|c|c|c|c|c|c|c|c|c|c|c|c|c|c|c|c|c|c|c|c|}
\hline & \multicolumn{4}{|c|}{$\begin{array}{c}\text { Oceanus } 118 \\
\text { Aprif }\end{array}$} & \multicolumn{5}{|c|}{$\begin{array}{c}\text { Oceanus } 121 \\
\text { June }\end{array}$} & \multicolumn{5}{|c|}{$\begin{array}{l}\text { Oceanus } 125 \\
\text { August }\end{array}$} & \multicolumn{7}{|c|}{$\begin{array}{l}\text { Knorr } 98 \\
\text { Sept-0ct }\end{array}$} & \multicolumn{3}{|c|}{$\begin{array}{c}\text { A2 } 110 \\
\text { Sept-0ct }\end{array}$} \\
\hline $\begin{array}{l}\text { Slope } \\
\text { Water }\end{array}$ & $\begin{array}{l}\frac{8}{\overline{0}} \\
\underline{0} \\
\underline{0} \\
\underline{0}\end{array}$ & $\begin{array}{l}\frac{9}{0} \\
0 \\
5.71 \\
0\end{array}$ & & & $\frac{\frac{19}{0.73}}{\frac{0.41}{\underline{0}}}$ & $\begin{array}{l}20 \\
0 \\
0 \\
0 \\
0 \\
0\end{array}$ & $\begin{array}{l}\frac{33}{\frac{0.03}{5.42}} \\
\frac{0}{\underline{0}} \\
\underline{0.01}\end{array}$ & $\begin{array}{l}\frac{34}{\underline{0}} \\
\underline{1.85} \\
\underline{3.86}\end{array}$ & & $\begin{array}{c}\frac{35}{0} \\
0 \\
0.27 \\
2.22 \\
0\end{array}$ & $\begin{array}{l}\frac{47}{0.83} \\
\frac{0}{0.02} \\
\underline{0}\end{array}$ & $\begin{array}{r}\frac{48}{0} \\
0.03 \\
14.12 \\
0.51\end{array}$ & $\frac{\frac{53}{0.77}}{\frac{0}{0}}$ & $\begin{array}{c}\frac{54}{0} \\
0 \\
6.67 \\
19.33\end{array}$ & $\begin{array}{l}\frac{59}{\underline{0}} \\
\underline{0} \\
\underline{0} \\
\underline{1.03} \\
\underline{0}\end{array}$ & $\begin{array}{l}\frac{60}{0} \\
0 \\
0 \\
8.70 \\
0\end{array}$ & $\frac{\frac{65}{0.09}}{\frac{0}{0}}$ & $\begin{array}{c}\frac{66}{0} \\
0 \\
0.30 \\
0 \\
3.85\end{array}$ & & & & $\begin{array}{c}\frac{4}{0} \\
0.22 \\
2.50 \\
0\end{array}$ & $\frac{\frac{5}{11.00}}{\underline{0}} \frac{0.53}{\underline{0}}$ & \\
\hline $\begin{array}{l}\text { High } \\
\text { Velocity } \\
\text { Region }\end{array}$ & $\begin{array}{l}\frac{12}{0} \\
0 \\
4.16 \\
0\end{array}$ & $\begin{array}{l}\frac{13}{\underline{0}} \\
\underline{\underline{0}} \\
\underline{2.68}\end{array}$ & $\frac{\frac{14}{\frac{0.03}{12.67}}}{\frac{0}{0}}$ & $\begin{array}{l}\frac{15}{0} \\
0.06 \\
0 \\
0\end{array}$ & $\begin{array}{c}\frac{26}{0.04} \\
0 \\
0 \\
0 \\
-\end{array}$ & $\begin{array}{c}27 \\
0 \\
0 \\
0.77 \\
0 \\
0\end{array}$ & $\begin{array}{c}30 \\
0 \\
0 \\
0.84 \\
0.68 \\
0\end{array}$ & $\begin{array}{c}31 \\
0 \\
0 \\
0.18 \\
10.86 \\
0\end{array}$ & $\begin{array}{l}\frac{32}{\underline{0}} \\
\frac{0.05}{0.05} \\
\underline{0}\end{array}$ & $\frac{\frac{41}{0.01}}{\frac{0.06}{\underline{0}}}$ & $\begin{array}{l}\frac{42}{\underline{0}} \\
\frac{0.04}{\underline{0}} \\
\underline{0}\end{array}$ & $\begin{array}{l}\frac{43}{0} \\
0 \\
0.25 \\
0 \\
0\end{array}$ & $\frac{\frac{44}{0.56}}{\frac{0}{0}}$ & & $\begin{array}{l}\frac{55}{0} \\
\underline{0} \\
\underline{0} \\
\underline{0}\end{array}$ & & & & & & & $\begin{array}{l}\frac{2}{\frac{0}{0}} \\
\frac{21.33}{\underline{0}}\end{array}$ & $\begin{array}{c}\frac{3}{0} \\
0.03 \\
0 \\
0\end{array}$ & \\
\hline $\begin{array}{l}\text { Ring } \\
\text { Core }\end{array}$ & $\begin{array}{l}\frac{10}{\underline{0}} \\
\underline{0} \\
\frac{0.09}{\underline{0}}\end{array}$ & $\begin{array}{l}\frac{11}{0} \\
0 \\
0 \\
0\end{array}$ & $\begin{array}{c}\frac{16}{0} \\
0.07 \\
0\end{array}$ & $\begin{array}{l}\frac{17}{0} \\
\frac{0}{0} \\
\underline{0}\end{array}$ & $\begin{array}{l}\frac{21}{0.50} \\
\underline{0} \\
\frac{0.91}{0.08} \\
\frac{0}{\underline{0}}\end{array}$ & $\begin{array}{l}\frac{23}{0.12} \\
\frac{0.07}{\underline{0}} \\
\frac{0.09}{\underline{0}}\end{array}$ & $\begin{array}{c}\frac{24}{0.04} \\
0.50 \\
2.30 \\
0.06 \\
0\end{array}$ & $\begin{array}{l}28 \\
0 \\
0 \\
1.61 \\
0 \\
0\end{array}$ & & & & & & & $\begin{array}{l}\frac{56}{0} \\
\underline{0} \\
\underline{0} \\
\underline{0} \\
\underline{0}\end{array}$ & $\begin{array}{l}57 \\
\underline{0} \\
\underline{0} \\
\underline{0} \\
\underline{0} \\
\underline{0}\end{array}$ & $\begin{array}{l}\frac{58}{0} \\
0 \\
0 \\
0 \\
0\end{array}$ & $\begin{array}{l}\frac{61}{0} \\
\underline{0} \\
\underline{0} \\
\underline{0} \\
\underline{0}\end{array}$ & $\begin{array}{l}62 \\
0 \\
0 \\
0 \\
0 \\
0\end{array}$ & $\begin{array}{l}\frac{63}{0} \\
\underline{0} \\
\underline{0} \\
\underline{0} \\
\underline{0}\end{array}$ & $\begin{array}{l}64 \\
0 \\
0 \\
0 \\
0 \\
0\end{array}$ & $\begin{array}{l}\frac{1}{\underline{0}} \\
\underline{0} \\
\underline{0.03} \\
\underline{0}\end{array}$ & & \\
\hline $\begin{array}{l}\text { Sargasso } \\
\text { Sea } \\
\& \\
\text { Gulf } \\
\text { Stream }\end{array}$ & & & & & & & & & & $\begin{array}{l}\frac{49}{\underline{0}} \\
\underline{0} \\
\underline{0} \\
\underline{0}\end{array}$ & $\begin{array}{l}50 \\
0 \\
0 \\
0 \\
0\end{array}$ & $\begin{array}{l}\frac{51}{0} \\
0 \\
0 \\
0\end{array}$ & $\begin{array}{l}\frac{52}{\underline{0}} \\
\underline{0} \\
\underline{0} \\
\underline{0.05}\end{array}$ & & & & & & & & & & & \\
\hline $\begin{array}{l}\text { Slope } \\
\text { Water }\end{array}$ & $\frac{8}{-}$ & $\frac{9}{1.43}$ & $\frac{18}{3.11}$ & & $\frac{19}{0.13}$. & $\frac{20}{0}$ & $\frac{33}{0.77}$ & $\frac{34}{2.91}$ & & $\frac{35}{0.50}$ & $\frac{47}{0.21}$ & $\frac{48}{3.67}$ & $\frac{53}{0.19}$ & $\frac{54}{6.50}$ & $\frac{59}{0.21}$ & $\frac{60}{1.74}$ & $\frac{65}{0.02}$ & $\frac{66}{0.83}$ & & & & $\frac{4}{0.68}$ & $\frac{5}{2.88}$ & $\frac{6}{5.00}$ \\
\hline $\begin{array}{l}\text { High } \\
\text { Velocity } \\
\text { Region }\end{array}$ & $\frac{12}{1.04}$ & $\frac{13}{1.34}$ & $\frac{14}{3.18}$ & $\frac{15}{0.02}$ & $\frac{27}{0.15}$ & $\frac{29}{0.06}$ & $\frac{30}{0.30}$ & $\frac{31}{2.19}$ & $\frac{32}{-}$ & $\frac{41}{0.02}$ & $\frac{42}{0.01}$ & $\frac{43}{0.05}$ & $\frac{44}{0.34}$ & & $\frac{55}{0}$ & & & & & & & $\frac{2}{5.33}$ & $\frac{3}{0.01}$ & \\
\hline $\begin{array}{l}\text { Ring } \\
\text { Core }\end{array}$ & $\frac{10}{0.02}$ & $\frac{11}{0}$ & $\frac{16}{-}$ & $\frac{17}{0}$ & $\frac{21}{0.22}$ & $\frac{23}{0.05}$ & $\frac{24}{0.50}$ & $\frac{28}{0.40}$ & & $\frac{37}{0}$ & $\frac{38}{5.15}$ & $\frac{39}{0.26}$ & & & $\frac{56}{0}$ & $\frac{57}{0}$ & $\frac{58}{0}$ & $\frac{61}{0}$ & $\frac{62}{0}$ & $\frac{63}{0}$ & $\frac{64}{0}$ & $\frac{1}{0.01}$ & & \\
\hline SS \& GS & & & & & & & & & & $\frac{49}{0}$ & $\frac{50}{0}$ & $\frac{51}{0}$ & $\frac{52}{0.01}$ & & & & & & & & & & & \\
\hline
\end{tabular}


Table 126. Abundance of Symbolophorus veranyi (specimens $/ 10,000 \mathrm{~m}^{3}$ ).

\begin{tabular}{|c|c|c|c|c|c|c|c|c|c|c|c|c|c|c|c|c|c|c|c|c|c|c|c|c|}
\hline & \multicolumn{4}{|c|}{$\begin{array}{c}\text { Oceanus } 118 \\
\text { April }\end{array}$} & \multicolumn{5}{|c|}{$\begin{array}{c}\text { Oceanus } 121 \\
\text { June }\end{array}$} & \multicolumn{5}{|c|}{$\begin{array}{l}\text { Oceanus } 125 \\
\text { Auqust }\end{array}$} & \multicolumn{7}{|c|}{$\begin{array}{l}\text { Knorr } 98 \\
\text { Sept-0ct }\end{array}$} & \multicolumn{3}{|c|}{$\begin{array}{c}\text { A2 } 110 \\
\text { Sept-0ct }\end{array}$} \\
\hline $\begin{array}{l}\text { Slope } \\
\text { Water }\end{array}$ & $\begin{array}{l}8 \\
\overline{\overline{0}} \\
\underline{0} \\
\underline{0}\end{array}$ & $\begin{array}{l}9 \\
0 \\
0 \\
0 \\
0\end{array}$ & & & $\begin{array}{l}\frac{19}{\underline{0}} \\
\underline{0} \\
\underline{0} \\
\underline{0} \\
\underline{0}\end{array}$ & $\begin{array}{r}20 \\
0 \\
0 \\
0 \\
0 \\
0\end{array}$ & $\begin{array}{c}33 \\
\underline{0} \\
\underline{0} \\
\underline{0} \\
\underline{0} \\
0.1\end{array}$ & $\begin{array}{l}\frac{34}{\underline{0}} \\
\underline{0} \\
\underline{0}\end{array}$ & & $\begin{array}{c}35 \\
0 \\
0 \\
0 \\
0 \\
0\end{array}$ & $\begin{array}{c}\frac{47}{\underline{0}} \\
\underline{0} \\
0.2 \\
0.4\end{array}$ & $\begin{array}{c}48 \\
0 \\
0 \\
0.3 \\
0\end{array}$ & $\begin{array}{l}\frac{53}{0} \\
\frac{0}{0} \\
\underline{0} \\
\underline{0}\end{array}$ & $\begin{array}{c}54 \\
0 \\
0 \\
0 \\
0.3\end{array}$ & $\begin{array}{l}\frac{59}{\underline{0}} \\
\frac{0}{0} \\
\underline{0} \\
\underline{0}\end{array}$ & $\begin{array}{c}60 \\
0 \\
0 \\
0 \\
0 \\
0.3\end{array}$ & $\begin{array}{r}\frac{65}{\underline{0}} \\
\underline{0} \\
\underline{0} \\
\underline{0} \\
\underline{0}\end{array}$ & $\begin{array}{c}66 \\
0 \\
0 \\
0 \\
0.5 \\
0\end{array}$ & & & & $\begin{array}{l}4 \\
0 \\
0 \\
0 \\
0\end{array}$ & $\begin{array}{l}\frac{5}{0} \\
\underline{0} \\
\underline{0} \\
\underline{0}\end{array}$ & \\
\hline $\begin{array}{l}\text { High } \\
\text { Velocity } \\
\text { Region }\end{array}$ & $\begin{array}{r}12 \\
0 \\
0 \\
0 \\
0\end{array}$ & $\frac{13}{\frac{0}{0}}$ & $\frac{14}{\frac{0}{0}}$ & $\begin{array}{r}15 \\
0 \\
0 \\
0 \\
0\end{array}$ & $\begin{array}{c}\frac{26}{0.3} \\
0 \\
0 \\
0 \\
-\end{array}$ & $\begin{array}{c}27 \\
0 \\
0 \\
0 \\
0 \\
0.2\end{array}$ & $\begin{array}{c}30 \\
0.8 \\
0 \\
0 \\
0 \\
0.9\end{array}$ & $\begin{array}{c}31 \\
0.9 \\
0 \\
0 \\
0 \\
0\end{array}$ & $\frac{\frac{32}{0.3}}{\frac{0}{0}} \frac{\underline{0}}{\underline{0}}$ & $\begin{array}{l}41 \\
\underline{0} \\
\underline{0} \\
\underline{0.4}\end{array}$ & $\begin{array}{l}\frac{42}{\underline{0}} \\
\underline{\underline{0}} \\
\underline{0.5}\end{array}$ & $\begin{array}{c}43 \\
0 \\
0 \\
0 \\
0.9 \\
0\end{array}$ & $\begin{array}{l}\frac{44}{\underline{0}} \\
\underline{0} \\
0.4\end{array}$ & & $\begin{array}{l}\frac{55}{0} \\
\underline{0} \\
\underline{0} \\
\underline{0}\end{array}$ & & & & & & & $\begin{array}{l}\frac{2}{0} \\
\underline{0} \\
\underline{0} \\
\underline{0}\end{array}$ & $\begin{array}{l}3 \\
0 \\
0 \\
0 \\
0\end{array}$ & \\
\hline $\begin{array}{l}\text { Ring } \\
\text { Core }\end{array}$ & $\begin{array}{r}10 \\
\underline{0} \\
\underline{0} \\
\underline{0} \\
\underline{0}\end{array}$ & $\begin{array}{r}11 \\
0 \\
0 \\
0 \\
0\end{array}$ & $\begin{array}{r}16 \\
0 \\
0 \\
0\end{array}$ & $\begin{array}{l}\frac{17}{\underline{0}} \\
\underline{0} \\
\underline{0}\end{array}$ & $\frac{\frac{21}{2.0}}{\frac{0.2}{0.0}}$ & $\begin{array}{l}\frac{23}{1.8} \\
\frac{0}{0} \\
\underline{0} \\
\underline{0} .1\end{array}$ & $\begin{array}{c}24 \\
0.3 \\
0 \\
0 \\
0.6 \\
1.1\end{array}$ & $\begin{array}{c}28 \\
0 \\
0 \\
0 \\
1.2 \\
0\end{array}$ & & & & & & & $\begin{array}{c}56 \\
\underline{0} \\
\underline{0} \\
\underline{0} \\
\underline{0} \\
\underline{0.3}\end{array}$ & $\begin{array}{l}\frac{57}{\underline{0}} \\
\underline{0} \\
\underline{0} \\
\underline{0} \\
\underline{0}\end{array}$ & $\begin{array}{r}58 \\
0 \\
0 \\
0 \\
0 \\
0\end{array}$ & $\begin{array}{l}61 \\
\underline{0} \\
\underline{0} \\
\underline{0} \\
\underline{0} \\
\underline{0}\end{array}$ & $\begin{array}{r}62 \\
0 \\
0 \\
0 \\
0 \\
0\end{array}$ & 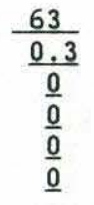 & $\begin{array}{r}64 \\
0 \\
0 \\
0 \\
0 \\
0\end{array}$ & $\begin{array}{l}\frac{1}{0} \\
\underline{0} \\
\underline{0} \\
\underline{0}\end{array}$ & & \\
\hline $\begin{array}{l}\text { Sargasso } \\
\text { Sea } \\
\& \\
\text { Gulf } \\
\text { Stream }\end{array}$ & & & & & & & & & & $\begin{array}{r}\frac{49}{\underline{0}} \\
\underline{0} \\
\underline{0} \\
\underline{0}\end{array}$ & $\begin{array}{r}50 \\
0 \\
0 \\
0 \\
0\end{array}$ & $\begin{array}{r}51 \\
0 \\
0 \\
0 \\
0\end{array}$ & $\begin{array}{l}\frac{52}{\underline{0}} \\
\underline{0} \\
\underline{0} \\
\underline{0}\end{array}$ & & & & & & & & & & & \\
\hline $\begin{array}{l}\text { Slope } \\
\text { Water }\end{array}$ & $\frac{8}{-}$ & $\frac{9}{0}$ & $\frac{18}{0}$ & & $\frac{19}{0}$ & $\frac{20}{0}$ & $\frac{33}{0.1}$ & $\frac{34}{0}$ & & $\frac{35}{0}$ & $\frac{47}{0.2}$ & $\frac{48}{0.1}$ & $\frac{53}{0}$ & $\frac{54}{0.1}$ & $\frac{59}{0}$ & $\frac{60}{0.1}$ & $\frac{65}{0}$ & $\frac{66}{0}$ & & & & $\frac{4}{0}$ & $\frac{5}{0}$ & $\frac{6}{0}$ \\
\hline $\begin{array}{l}\text { High } \\
\text { Velocity } \\
\text { Region }\end{array}$ & $\frac{12}{0}$ & $\frac{13}{0}$ & $\frac{14}{0}$ & $\frac{15}{0}$ & $\frac{27}{0.1}$ & $\frac{29}{0.2}$ & $\frac{30}{0.5}$ & $\frac{31}{0.3}$ & $\frac{32}{-}$ & $\frac{41}{0.1}$ & $\frac{42}{0.1}$ & $\frac{43}{0.2}$ & $\frac{44}{0.1}$ & & $\frac{55}{0}$ & & & & & & & $\frac{2}{0}$ & $\frac{3}{0}$ & \\
\hline $\begin{array}{l}\text { Ring } \\
\text { Core }\end{array}$ & $\frac{10}{0}$ & $\frac{11}{0}$ & $\frac{16}{-}$ & $\frac{17}{0}$ & $\frac{21}{0.2}$ & $\frac{23}{1.1}$ & $\frac{24}{0.5}$ & $\frac{28}{0.3}$ & & $\frac{37}{0}$ & $\frac{38}{0.2}$ & $\frac{39}{0}$ & & & $\frac{56}{0.1}$ & $\frac{57}{0}$ & $\frac{58}{0}$ & $\frac{61}{0}$ & $\frac{62}{0}$ & $\frac{63}{0.1}$ & $\frac{64}{0}$ & $\frac{1}{0}$ & & \\
\hline SS \& GS & & & & & & & & & & $\frac{49}{0}$ & $\frac{50}{0}$ & $\frac{51}{0}$ & $\frac{52}{0}$ & & & & & & & & & & & \\
\hline
\end{tabular}


Table 127. Abundance of Valenciennellus tripunctulatus (specimens $/ 10,000 \mathrm{~m}^{3}$ ).

\begin{tabular}{|c|c|c|c|c|c|c|c|c|c|c|c|c|c|c|c|c|c|c|c|c|c|c|c|c|}
\hline & & $\begin{array}{l}\text { ceanu } \\
\text { Apr }\end{array}$ & $1^{118}$ & & & & $\begin{array}{l}\text { eanus } \\
\text { June }\end{array}$ & 121 & & & $\begin{array}{r}\text { Oce } \\
\mathrm{A}\end{array}$ & $\begin{array}{l}\text { inus } 1 \\
\text { Igust }\end{array}$ & & & & & $\begin{array}{l}\text { Knor } \\
\text { Sept }\end{array}$ & $\begin{array}{r}98 \\
-0 c t \\
\end{array}$ & & & & $\begin{array}{r}\mathrm{A} \\
\mathrm{Se}\end{array}$ & $\begin{array}{c}110 \\
t-0 c t\end{array}$ & \\
\hline $\begin{array}{l}\text { Slope } \\
\text { Water }\end{array}$ & $\begin{array}{l}\frac{8}{\overline{0}} \\
\underline{0} \\
\underline{0} \\
\underline{0}\end{array}$ & $\begin{array}{c}\frac{9}{1.3} \\
0 \\
0 \\
0\end{array}$ & & & $\frac{\frac{19}{\underline{0}}}{\frac{15.9}{0.5}} \frac{0.5}{\underline{0}}$ & $\begin{array}{l}20 \\
0.5 \\
2.6 \\
0 \\
0 \\
0\end{array}$ & $\frac{\frac{33}{\underline{0}}}{\frac{2.1}{2.2}} \frac{\underline{0}}{\underline{0}}$ & $\frac{\frac{34}{0.5}}{\frac{0.1}{\underline{0}}}$ & & $\begin{array}{c}35 \\
0 \\
1.9 \\
0 \\
0 \\
0\end{array}$ & $\frac{\frac{47}{2.8}}{\frac{0.3}{\underline{0}}}$ & $\begin{array}{c}\frac{48}{0} \\
1.4 \\
0.3 \\
0\end{array}$ & $\frac{\frac{53}{1.5}}{\frac{0.2}{\underline{0}}}$ & $\begin{array}{r}54 \\
0 \\
0 \\
0 \\
0\end{array}$ & $\frac{\frac{59}{1.3}}{\frac{\frac{5.2}{0}}{\frac{0}{0}}}$ & $\begin{array}{c}60 \\
1.1 \\
10.5 \\
0 \\
0 \\
0\end{array}$ & $\frac{\frac{65}{1.3}}{\frac{1.1}{2.1}} \frac{0}{\frac{0}{0}}$ & $\begin{array}{c}\frac{66}{0.8} \\
2.9 \\
0 \\
0 \\
0\end{array}$ & & & & $\begin{array}{l}\frac{4}{0} \\
0.9 \\
0 \\
0\end{array}$ & $\frac{\frac{5}{1.0}}{\frac{0}{0}}$ & \\
\hline $\begin{array}{l}\text { High } \\
\text { Velocity } \\
\text { Region }\end{array}$ & $\begin{array}{c}\frac{12}{0.3} \\
1.5 \\
0 \\
0\end{array}$ & $\frac{\frac{13}{2.0}}{\frac{1.7}{\underline{0}}}$ & $\frac{\frac{14}{1.7}}{\frac{1.7}{\frac{0}{0}}}$ & $\begin{array}{c}15 \\
0 \\
3.5 \\
0 \\
0\end{array}$ & $\begin{array}{c}26 \\
0 \\
1.1 \\
0.5 \\
0 \\
-\end{array}$ & $\begin{array}{c}27 \\
0 \\
0.5 \\
0 \\
0 \\
0\end{array}$ & $\begin{array}{c}30 \\
0 \\
2.8 \\
0 \\
0 \\
0\end{array}$ & $\begin{array}{c}31 \\
0 \\
0.5 \\
2.1 \\
0 \\
0\end{array}$ & $\frac{\frac{32}{1.8}}{\frac{0}{0}}$ & $\frac{\frac{41}{0.1}}{\frac{0}{0}}$ & $\frac{\frac{42}{0.5}}{\frac{0.3}{\frac{0}{0}}}$ & $\begin{array}{c}\frac{43}{0} \\
0.2 \\
0 \\
0 \\
0\end{array}$ & $\frac{\frac{44}{0.5}}{\frac{0}{0}}$ & & $\begin{array}{c}\frac{55}{\underline{0}} \\
\underline{0} \\
\underline{0.8} \\
\underline{0}\end{array}$ & & & & & & & $\frac{\frac{2}{2.1}}{\frac{0}{0}}$ & $\begin{array}{c}\frac{3}{0.7} \\
0 \\
0 \\
0\end{array}$ & \\
\hline $\begin{array}{l}\text { Ring } \\
\text { Core }\end{array}$ & $\frac{10}{\frac{0}{1.8}} \frac{0}{\underline{0}}$ & $\begin{array}{c}11 \\
0 \\
1.7 \\
0 \\
0\end{array}$ & $\begin{array}{c}16 \\
0 \\
0 \\
0\end{array}$ & $\frac{17}{\underline{0}} \frac{2.1}{\underline{0}}$ & $\frac{21}{\frac{1.0}{\underline{0}}}$ & $\frac{23}{\underline{0}} \frac{\underline{\underline{0}} .0}{\underline{0}}$ & $\begin{array}{c}24 \\
1.2 \\
5.0 \\
0 \\
0 \\
0\end{array}$ & $\begin{array}{c}28 \\
0 \\
0 \\
0 \\
0 \\
0\end{array}$ & & & & & & & $\begin{array}{c}\frac{56}{\underline{0}} \\
\frac{0.6}{3.2} \\
\frac{0}{0} \\
\underline{0}\end{array}$ & $\begin{array}{c}\frac{57}{\underline{0}} \\
\frac{0.3}{0.3} \\
\underline{0} \\
\underline{0}\end{array}$ & $\begin{array}{c}\frac{58}{0.3} \\
0.4 \\
1.0 \\
0 \\
0\end{array}$ & 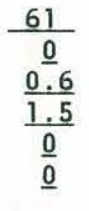 & $\begin{array}{c}62 \\
0 \\
0 \\
0.4 \\
0 \\
0\end{array}$ & $\begin{array}{c}\frac{63}{\underline{0}} \\
\underline{0} \\
\frac{1.4}{\underline{0}} \\
\underline{0}\end{array}$ & $\begin{array}{c}\frac{64}{0.4} \\
1.3 \\
0 \\
0 \\
0\end{array}$ & $\frac{\frac{1}{0}}{\frac{\underline{0}}{1.2}} \frac{\underline{0}}{\underline{0}}$ & & \\
\hline $\begin{array}{l}\text { Sargasso } \\
\text { Sea } \\
\& \\
\text { Gulf } \\
\text { Stream }\end{array}$ & & & & & & & & & & $\frac{\frac{49}{0.3}}{\frac{0.9}{0.4}} \frac{0}{\underline{0}}$ & $\begin{array}{c}\frac{50}{0.3} \\
1.2 \\
0 \\
0\end{array}$ & $\begin{array}{c}51 \\
0 \\
0.4 \\
0 \\
0\end{array}$ & $\begin{array}{c}\frac{52}{\underline{0}} \\
\frac{0.3}{\underline{0}} \\
\underline{0}\end{array}$ & & & & & & & & & & & \\
\hline $\begin{array}{l}\text { Slope } \\
\text { Water }\end{array}$ & $\frac{8}{-}$ & $\frac{9}{0.3}$ & $\frac{18}{0.3}$ & & $\frac{19}{2.6}$ & $\frac{20}{0.8}$ & $\frac{33}{0.7}$ & $\frac{34}{0.4}$ & & $\frac{35}{0.4}$ & $\frac{47}{0.8}$ & $\frac{48}{0.4}$ & $\frac{53}{0.4}$ & $\frac{54}{0}$ & $\frac{59}{1.3}$ & $\frac{60}{1.4}$ & $\frac{65}{0.7}$ & $\frac{66}{0.5}$ & & & & $\frac{4}{0.2}$ & $\frac{5}{0.3}$ & $\frac{6}{0.5}$ \\
\hline $\begin{array}{l}\text { High } \\
\text { Velocity } \\
\text { Region }\end{array}$ & $\frac{12}{0.5}$ & $\frac{13}{0.9}$ & $\frac{14}{0.9}$ & $\frac{15}{0.9}$ & $\frac{27}{0.1}$ & $\frac{29}{0.8}$ & $\frac{30}{0.3}$ & $\frac{31}{0.5}$ & $\frac{32}{-}$ & $\frac{41}{0.1}$ & $\frac{42}{0.3}$ & $\frac{43}{0.1}$ & $\frac{44}{0.3}$ & & $\frac{55}{0.3}$ & & & & & & & $\frac{2}{0.5}$ & $\frac{3}{0.2}$ & \\
\hline $\begin{array}{l}\text { Ring } \\
\text { Core }\end{array}$ & $\frac{10}{0.5}$ & $\frac{11}{0.4}$ & $\frac{16}{-}$ & $\frac{17}{0.5}$ & $\frac{21}{0.1}$ & $\frac{23}{0.5}$ & $\frac{24}{0.6}$ & $\frac{28}{0}$ & & $\frac{37}{0.5}$ & $\frac{38}{0.9}$ & $\frac{39}{0.4}$ & & & $\frac{56}{0.8}$ & $\frac{57}{0.1}$ & $\frac{58}{0.3}$ & $\frac{61}{0.2}$ & $\frac{62}{0.1}$ & $\frac{63}{0.3}$ & $\frac{64}{0.3}$ & $\frac{1}{0.3}$ & & \\
\hline SS \& GS & & & & & & & & & & $\frac{49}{0.4}$ & $\frac{50}{0.4}$ & $\frac{51}{0.1}$ & $\frac{52}{0.1}$ & & & & & & & & & & & \\
\hline
\end{tabular}


Table 128. Abundance of Vinciguerria attenuata (specimens $/ 10,000 \mathrm{~m}^{3}$ ).

\begin{tabular}{|c|c|c|c|c|c|c|c|c|c|c|c|c|c|c|c|c|c|c|c|c|c|c|c|c|}
\hline & \multicolumn{4}{|c|}{$\begin{array}{c}\text { Oceanus } 118 \\
\text { April }\end{array}$} & \multicolumn{5}{|c|}{$\begin{array}{c}\text { Oceanus } 121 \\
\text { June }\end{array}$} & \multicolumn{5}{|c|}{$\begin{array}{c}\text { Oceanus } 125 \\
\text { Auqust }\end{array}$} & \multicolumn{7}{|c|}{$\begin{array}{l}\text { Knorr } 98 \\
\text { Sept-0ct }\end{array}$} & \multicolumn{3}{|c|}{$\begin{array}{c}\text { A2 } 110 \\
\text { Sept-0ct }\end{array}$} \\
\hline $\begin{array}{l}\text { Slope } \\
\text { Water }\end{array}$ & $\begin{array}{l}\frac{8}{\overline{0}} \\
\underline{0} \\
\underline{0} \\
\underline{0}\end{array}$ & $\begin{array}{l}9 \\
0 \\
0 \\
0 \\
0\end{array}$ & & & $\begin{array}{l}\frac{19}{\underline{0}} \\
\underline{0} \\
\underline{0} \\
\underline{0}\end{array}$ & $\begin{array}{c}20 \\
0 \\
0.5 \\
0 \\
0 \\
0\end{array}$ & $\begin{array}{l}33 \\
\underline{0} \\
\underline{0} \\
\underline{0} \\
\underline{0} \\
\underline{0}\end{array}$ & $\begin{array}{l}\frac{34}{\underline{0}} \\
\underline{0} \\
\underline{0}\end{array}$ & & $\begin{array}{c}35 \\
0 \\
0.4 \\
0 \\
0 \\
0\end{array}$ & $\begin{array}{r}47 \\
\underline{0} \\
\underline{0} \\
\underline{0} \\
\underline{0}\end{array}$ & $\begin{array}{r}48 \\
0 \\
0 \\
0 \\
0\end{array}$ & $\begin{array}{c}\frac{53}{\underline{0}} \\
\frac{0.4}{\underline{0}} \\
\underline{0}\end{array}$ & $\begin{array}{r}54 \\
0 \\
0 \\
0 \\
0\end{array}$ & 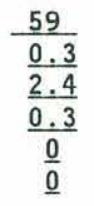 & $\begin{array}{c}60 \\
0.5 \\
8.2 \\
0 \\
0 \\
0\end{array}$ & $\frac{\frac{65}{1.3}}{\frac{0}{0}} \frac{\underline{0}}{\underline{0}}$ & $\begin{array}{c}66 \\
1.5 \\
3.6 \\
0 \\
0 \\
0\end{array}$ & & & & $\begin{array}{c}\frac{4}{0} \\
0.9 \\
0 \\
0\end{array}$ & $\begin{array}{l}\frac{5}{0} \\
\underline{0} \\
\underline{0} \\
\underline{0}\end{array}$ & \\
\hline $\begin{array}{l}\text { High } \\
\text { Velocity } \\
\text { Region }\end{array}$ & $\begin{array}{r}12 \\
0 \\
0 \\
0 \\
0\end{array}$ & $\begin{array}{r}13 \\
\underline{0} \\
\underline{0} \\
\underline{0}\end{array}$ & $\frac{14}{\frac{0}{0}}$ & $\begin{array}{r}15 \\
0 \\
0 \\
0 \\
0\end{array}$ & $\begin{array}{c}\frac{26}{0} \\
0.5 \\
1.4 \\
0 \\
-\end{array}$ & $\begin{array}{c}27 \\
0 \\
0.5 \\
0 \\
0 \\
0\end{array}$ & $\begin{array}{c}30 \\
0 \\
3.6 \\
12.2 \\
0 \\
0\end{array}$ & $\begin{array}{c}31 \\
0 \\
0.5 \\
2.1 \\
0.3 \\
0\end{array}$ & $\frac{\frac{32}{3.4}}{\frac{\frac{3.2}{0}}{\underline{0}}}$ & $\frac{\frac{41}{0.6}}{\frac{0}{0}}$ & $\frac{\frac{42}{1.0}}{\frac{0.9}{\underline{0}}}$ & $\begin{array}{c}43 \\
0 \\
0.2 \\
0 \\
0 \\
0\end{array}$ & $\frac{44}{\frac{0.7}{\underline{0}}}$ & & $\begin{array}{c}55 \\
\underline{0} \\
\underline{0} \\
\underline{0} \\
\underline{0}\end{array}$ & & & & & & & $\frac{\frac{2}{1.3}}{\frac{0}{0}}$ & $\begin{array}{c}\frac{3}{1.4} \\
0.3 \\
0 \\
0\end{array}$ & \\
\hline $\begin{array}{l}\text { Ring } \\
\text { Core }\end{array}$ & $\frac{10}{\frac{0}{0}}$ & $\begin{array}{r}11 \\
0 \\
0 \\
0 \\
0\end{array}$ & $\begin{array}{r}16 \\
0 \\
0 \\
0\end{array}$ & $\frac{\frac{17}{1.7}}{\frac{0}{0}}$ & $\begin{array}{c}\frac{21}{23.0} \\
\frac{1.2}{8.8} \\
\underline{0} \\
\underline{0.6}\end{array}$ & $\begin{array}{l}\frac{23}{1.8} \\
\frac{0}{0.5} \\
\frac{0}{0}\end{array}$ & $\begin{array}{c}\frac{24}{1.5} \\
0 \\
3.2 \\
0 \\
0\end{array}$ & $\begin{array}{c}28 \\
0.6 \\
3.8 \\
2.1 \\
0 \\
0\end{array}$ & & & & & & & $\frac{56}{\underline{0}}$ & $\begin{array}{c}\frac{57}{\underline{0}} \\
\frac{2.8}{1.0} \\
\underline{0} \\
\underline{0}\end{array}$ & $\begin{array}{c}\frac{58}{0.3} \\
2.9 \\
6.7 \\
0 \\
0\end{array}$ & $\begin{array}{c}\frac{61}{\underline{0}} \\
\frac{1.8}{5.6} \\
\frac{0}{0}\end{array}$ & $\begin{array}{c}\frac{62}{0} \\
0.3 \\
1.0 \\
0 \\
0\end{array}$ & $\frac{\frac{63}{2.0}}{\frac{4.1}{1.9}} \frac{\underline{0}}{\underline{0}}$ & $\begin{array}{c}\frac{64}{0.8} \\
0.6 \\
0.7 \\
0 \\
0\end{array}$ & $\frac{\frac{1}{\underline{0}}}{\frac{1.0}{\underline{0}}}$ & & \\
\hline $\begin{array}{l}\text { Sargasso } \\
\text { Sea } \\
\& \\
\text { Gulf } \\
\text { Stream }\end{array}$ & & & & & & & . & & & $\begin{array}{l}\frac{49}{\underline{0}} \\
\frac{5.3}{\underline{0}} \\
\underline{0}\end{array}$ & $\begin{array}{r}50 \\
0 \\
0 \\
0 \\
0\end{array}$ & $\begin{array}{c}51 \\
0 \\
0.7 \\
0 \\
0\end{array}$ & $\begin{array}{l}\frac{52}{\underline{0}} \\
\frac{3.9}{\underline{0}} \\
\underline{0}\end{array}$ & & & & & & & & & & & \\
\hline $\begin{array}{l}\text { Slope } \\
\text { Water }\end{array}$ & $\frac{8}{-}$ & $\frac{9}{0}$ & $\frac{18}{0}$ & & $\frac{19}{0}$ & $\frac{20}{0.1}$ & $\frac{33}{0}$ & $\frac{34}{0}$ & & $\frac{35}{0.1}$ & $\frac{47}{0}$ & $\frac{48}{0}$ & $\frac{53}{0.1}$ & $\frac{54}{0}$ & $\frac{59}{0.6}$ & $\frac{60}{1.0}$ & $\frac{65}{0.3}$ & $\frac{66}{0.8}$ & & & & $\frac{4}{0.2}$ & $\frac{5}{0}$ & $\frac{6}{0.2}$ \\
\hline $\begin{array}{l}\text { High } \\
\text { Velocity } \\
\text { Region }\end{array}$ & $\frac{12}{0}$ & $\frac{13}{0}$ & $\frac{14}{0}$ & $\frac{15}{0}$ & $\frac{27}{0.1}$ & $\frac{29}{0.8}$ & $\frac{30}{2.8}$ & $\frac{31}{0.5}$ & $\frac{32}{-}$ & $\frac{41}{0.3}$ & $\frac{42}{0.6}$ & $\frac{43}{0.1}$ & $\frac{44}{0.4}$ & & $\frac{55}{0}$ & & & & & & & $\frac{2}{0.3}$ & $\frac{3}{0.4}$ & \\
\hline $\begin{array}{l}\text { Ring } \\
\text { Core }\end{array}$ & $\frac{10}{0}$ & $\frac{11}{0}$ & $\frac{16}{-}$ & $\frac{17}{0.4}$ & $\frac{21}{3.5}$ & $\frac{23}{0.4}$ & $\frac{24}{1.2}$ & $\frac{28}{1.4}$ & & $\frac{37}{4.4}$ & $\frac{38}{0.9}$ & $\frac{39}{0.7}$ & & & $\frac{56}{0.3}$ & $\frac{57}{0.8}$ & $\frac{58}{1.7}$ & $\frac{61}{0.6}$ & $\frac{62}{0.2}$ & $\frac{63}{1.2}$ & $\frac{64}{0.5}$ & $\frac{1}{0.3}$ & & \\
\hline SS \& GS & & & & & & & & & & $\frac{49}{1.3}$ & $\frac{50}{0}$ & $\frac{51}{0.2}$ & $\frac{52}{1.0}$ & & & & & & & & & & & \\
\hline
\end{tabular}


Table 129. Abundance of Vinciguerria poweriae (specimens $/ 10,000 \mathrm{~m}^{3}$ ).

\begin{tabular}{|c|c|c|c|c|c|c|c|c|c|c|c|c|c|c|c|c|c|c|c|c|c|c|c|c|}
\hline & & $\begin{array}{r}\text { ceanu } \\
\text { Apr }\end{array}$ & $1^{118}$ & & & & $\begin{array}{l}\text { eanus } \\
\text { June }\end{array}$ & 121 & & & $\begin{array}{r}0 c e \\
A\end{array}$ & $\begin{array}{l}\text { anus } 1 \\
\text { ugust }\end{array}$ & & & & & $\begin{array}{l}\text { Knor } \\
\text { Sep }\end{array}$ & $\begin{array}{r}98 \\
-0 c t \\
\end{array}$ & & & & S & $\begin{array}{c}110 \\
t-0 c t\end{array}$ & \\
\hline $\begin{array}{l}\text { Slope } \\
\text { Water }\end{array}$ & $\begin{array}{l}8 \\
\overline{\overline{0}} \\
\frac{0}{0} \\
\underline{0}\end{array}$ & $\begin{array}{l}9 \\
0 \\
0 \\
0 \\
0\end{array}$ & & & $\frac{\frac{19}{21,7}}{\frac{0}{0}}$ & $\begin{array}{r}20 \\
0 \\
0 \\
0 \\
0 \\
0\end{array}$ & $\frac{\frac{33}{\frac{1.3}{0.4}}}{\frac{0.4}{\underline{0}}}$ & $\frac{\frac{34}{13.2}}{\frac{0}{0}}$ & & $\begin{array}{c}35 \\
0 \\
0.6 \\
0.3 \\
0 \\
0\end{array}$ & $\frac{47}{\frac{1.5}{\underline{0}}}$ & $\begin{array}{c}48 \\
0 \\
0.3 \\
0 \\
0\end{array}$ & $\frac{\frac{53}{\frac{1.3}{0.4}}}{\frac{0}{\underline{0}}}$ & $\begin{array}{r}54 \\
0 \\
0 \\
0 \\
0\end{array}$ & $\begin{array}{l}\frac{59}{4.3} \\
\frac{0}{0} \\
\underline{0} \\
\underline{0}\end{array}$ & $\begin{array}{c}60 \\
0 \\
0.5 \\
0 \\
0 \\
0\end{array}$ & $\frac{\frac{65}{1.3}}{\frac{0}{\underline{0}}} \frac{\underline{0}}{\underline{0}}$ & $\begin{array}{r}66 \\
0 \\
0 \\
0 \\
0 \\
0\end{array}$ & & & & $\begin{array}{l}4 \\
0 \\
0 \\
0 \\
0\end{array}$ & $\frac{\frac{5}{1.3}}{\frac{0}{0}} \frac{0}{\underline{0}}$ & \\
\hline $\begin{array}{l}\text { High } \\
\text { Velocity } \\
\text { Region }\end{array}$ & $\begin{array}{r}12 \\
0 \\
0 \\
0 \\
0\end{array}$ & $\frac{13}{\frac{0}{0}}$ & $\frac{\frac{14}{0.3}}{\frac{0.3}{\underline{0}}}$ & $\begin{array}{c}15 \\
0 \\
0 \\
0 \\
0\end{array}$ & $\begin{array}{c}26 \\
0 \\
0 \\
0 \\
0 \\
-\end{array}$ & $\begin{array}{r}27 \\
0 \\
0 \\
0 \\
0 \\
0\end{array}$ & $\begin{array}{c}30 \\
0 \\
0 \\
0 \\
0 \\
0\end{array}$ & $\begin{array}{c}31 \\
0 \\
0 \\
0.4 \\
0 \\
0\end{array}$ & $\frac{\frac{32}{0.3}}{\frac{0}{0}}$ & $\frac{\frac{41}{1.7}}{\frac{0}{0}}$ & $\frac{\frac{42}{2.6}}{\frac{0}{0}}$ & $\begin{array}{c}43 \\
0 \\
0 \\
0.4 \\
0 \\
0\end{array}$ & $\frac{44}{\frac{0.7}{\underline{0}}}$ & & $\begin{array}{c}\frac{55}{\frac{0.8}{0}} \\
\frac{0.4}{\underline{0}}\end{array}$ & & & & & & & $\frac{\frac{2}{0.8}}{\frac{0}{0}}$ & $\begin{array}{c}\frac{3}{0.4} \\
0.3 \\
0 \\
0\end{array}$ & \\
\hline $\begin{array}{l}\text { Ring } \\
\text { Core }\end{array}$ & $\frac{10}{\frac{0}{0}}$ & $\begin{array}{r}11 \\
0 \\
0 \\
0 \\
0\end{array}$ & $\begin{array}{r}16 \\
0 \\
0 \\
0\end{array}$ & $\frac{17}{\frac{0}{0.5}}$ & $\frac{\frac{21}{8.0}}{\frac{8.6}{\underline{0}}}$ & $\begin{array}{l}\frac{23}{2.4} \\
\frac{\underline{0}}{\underline{0}} \\
\underline{0} \\
\underline{0.6}\end{array}$ & $\begin{array}{c}\frac{24}{0.3} \\
0 \\
0 \\
0 \\
0\end{array}$ & $\begin{array}{c}28 \\
0 \\
0.8 \\
0 \\
0 \\
0\end{array}$ & & & & & & & $\frac{\frac{56}{0.9}}{\frac{0.6}{1.1}} \frac{\underline{0}}{\underline{0}}$ & $\frac{\frac{57}{0.6}}{\underline{0}}$ & $\begin{array}{r}58 \\
0 \\
0 \\
0 \\
0 \\
0\end{array}$ & $\frac{61}{\frac{0.5}{\underline{0}}}$ & $\begin{array}{c}\frac{62}{0.2} \\
0 \\
0 \\
0 \\
0\end{array}$ & $\frac{\frac{63}{0.3}}{\frac{0}{\underline{0}}}$ & $\begin{array}{c}64 \\
0.2 \\
0 \\
0.7 \\
0 \\
0\end{array}$ & $\frac{\frac{1}{0.6}}{\frac{0}{0}}$ & & \\
\hline $\begin{array}{l}\text { Sargasso } \\
\text { Sea } \\
\& \\
\text { Gulf } \\
\text { Stream }\end{array}$ & & & & & & & & & & $\frac{\frac{49}{1.0}}{\frac{0}{0}} \frac{0}{\underline{0}}$ & $\begin{array}{c}50 \\
0 \\
0.7 \\
0 \\
0\end{array}$ & $\begin{array}{c}51 \\
0 \\
4.8 \\
0 \\
0\end{array}$ & $\frac{\frac{52}{1.6}}{\frac{\frac{1.8}{2.8}}{\frac{0}{0}}}$ & & & & & & & & & & & \\
\hline $\begin{array}{l}\text { Slope } \\
\text { Water }\end{array}$ & $\frac{8}{-}$ & $\frac{9}{0}$ & $\frac{18}{0}$ & & $\frac{19}{2.2}$ & $\frac{20}{0}$ & $\frac{33}{0.1}$ & $\frac{34}{1.1}$ & & $\frac{35}{0.2}$ & $\frac{47}{0.4}$ & $\frac{48}{0.1}$ & $\frac{53}{0.4}$ & $\frac{54}{0}$ & $\frac{59}{0.9}$ & $\frac{60}{0.1}$ & $\frac{65}{0.3}$ & $\frac{66}{0}$ & & & & $\frac{4}{0}$ & $\frac{5}{0.3}$ & $\frac{6}{0}$ \\
\hline $\begin{array}{l}\text { High } \\
\text { Velocity } \\
\text { Region }\end{array}$ & $\frac{12}{0}$ & $\frac{13}{0}$ & $\frac{14}{0.2}$ & $\frac{15}{0}$ & $\frac{27}{0}$ & $\frac{29}{0.5}$ & $\frac{30}{0}$ & $\frac{31}{0.1}$ & $\frac{32}{-}$ & $\frac{41}{0.9}$ & $\frac{42}{1.0}$ & $\frac{43}{0.1}$ & $\frac{44}{0.4}$ & & $\frac{55}{0.3}$ & & & & & & & $\frac{2}{0.2}$ & $\frac{3}{0.2}$ & \\
\hline $\begin{array}{l}\text { Ring } \\
\text { Core }\end{array}$ & $\frac{10}{0}$ & $\frac{11}{0}$ & $\frac{16}{-}$ & $\frac{17}{0.1}$ & $\frac{21}{1.0}$ & $\frac{23}{0.5}$ & $\frac{24}{0.1}$ & $\frac{28}{0.2}$ & & $\frac{37}{0.8}$ & $\frac{38}{1.3}$ & $\frac{39}{0.9}$ & & & $\frac{56}{0.5}$ & $\frac{57}{0.1}$ & $\frac{58}{0}$ & $\frac{61}{0.1}$ & $\frac{62}{0.1}$ & $\frac{63}{0.1}$ & $\frac{64}{0.3}$ & $\frac{1}{0.2}$ & & \\
\hline SS \& GS & & & & & & & & & & $\frac{49}{0.3}$ & $\frac{50}{0.2}$ & $\frac{51}{1.2}$ & $\frac{52}{1.1}$ & & & & & & & & & & & \\
\hline
\end{tabular}




\section{DOCUMENT LIBRARY}

August 21, 1987

Distribution List for Technical Report Exchange

Attn: Stella Sanchez-Wade

Documents Section

Scripps Institution of Oceanography

Library, Mail Code C-075C

La Jolla, CA 92093

Hancock Library of Biology \& Oceanography

Alan Hancock Laboratory

University of Southern California

University Park

Los Angeles, CA 90089-0371

Gifts \& Exchanges

Library

Bedford Institute of Oceanography

P.O. Box 1006

Dartmouth, NS, B2Y 4A2, CANADA

Office of the International Ice Patrol

c/o Coast Guard R \& D Center

Avery Point

Groton, CT 06340

Library

Physical Oceanographic Laboratory

Nova University

8000 N. Ocean Drive

Dania, FL 33304

NOAA/EDIS Miami Library Center 4301 Rickenbacker Causeway

Miami, FL 33149

Library

Skidaway Institute of Oceanography

P.O. Box 13687

Savannah, GA 31416

Institute of Geophysics

University of Hawaii

Library Room 252

2525 Correa Road

Honolulu, HI 96822

Library

Chesapeake Bay Institute

4800 Atwell Road

Shady Side, MD 20876

MIT Libraries

Serial Journal Room 14E-210

Cambridge, MA 02139
Director, Ralph M. Parsons Laboratory

Room 48-311

MIT

Cambridge, MA 02139

Marine Resources Information Center

Building E38-320

MIT

Cambridge, MA 02139

Library

Lamont-Doherty Geological Observatory

Colombia University

Palisades, NY 10964

Library

Serials Department

Oregon State University

Corvallis, OR 97331

Pell Marine Science Library

University of Rhode Island

Narragansett Bay Campus

Narragansett, RI 02882

Working Collection

Texas A\&M University

Dept. of Oceanography

College Station, TX 77843

Library

Virginia Institute of Marine Science

Gloucester Point, VA 23062

Fisheries-Oceanography Library

151 Oceanography Teaching Bldg.

University of Washington

Seattle, WA 98195

Library

R.S.M.A.S.

University of Miami

4600 Rickenbacker Causeway

Miami, FL 33149

Maury Oceanographic Library

Naval Oceanographic Office

Bay St. Louis

NSTL, MS 39522-5001 

REPORT DOCUMENTATION

PAGE

4. Title and Subtitle

Midwater Fish Data Report for Warm-Core Gulf Stream Rings Cruises 1981-1982

7. Author(s)

James E. Craddock, Richard H. Backus and Mary Ann Daher

9. Performing Organization Name and Address

Woods Hole Oceanographic Institution

Woods Hole, Massachusetts 02543

National Science Foundation

15. Supplementary Notes

This report should be cited as: Woods Hole Oceanog. Inst. Tech. Rept., WHOI-87-42.

16. Abstract (Limit: 200 words)

During the multidisciplinary Warm-Core Rings Program in 1981 and 1982, 312 collections were made at 64 stations with a $20 \mathrm{~m}^{2}$ MOCNESS midwater trawl. This report presents collection and station data, a list of species collected, and catch data (specimens and volume per $10^{4} \mathrm{~m}^{3}$, respectively) for the important families and species of midwater fishes.

17. Document Analysis a. Descriptors

1. MOCNESS-20

2. Midwater Fishes

3. Warm-Core Gulf Stream Rings

b. Identifiers/Open-Ended Terms

c. COSATI FIeld/Group

18. Availability Statemen:

Approved for publication; distribution unlimited.

19. Security Class (This Report) UNCLASSIFIED

20. Security Class (This Page)
21. No. of Pages 140 
